\title{
Teoria de Nielsen de raízes e teoria do grau de Hopf
}

\author{
Paulo Takashi Taneda
}

DISSERTAÇÃO APRESENTADA

$\mathrm{AO}$

INSTITUTO DE MATEMÁTICA E ESTATÍSTICA

DA

UNIVERSIDADE DE SÃO PAULO

PARA

OBTENÇÃO DO TÍTULO DE MESTRE

EM

CIÊNCIAS

Área de Concentração: Matemática

Orientadora: Prof. ${ }^{.}$Dr. ${ }^{\underline{a}}$ Lucília Daruiz Borsari

Durante a elaboração deste trabalho o autor recebeu o auxílio financeiro da CAPES

São Paulo, fevereiro de 2007. 



\section{Teoria de Nielsen de raízes e teoria do grau de Hopf}

Este exemplar corresponde à redação final da dissertação devidamente corrigida e defendida por Paulo Takashi Taneda, e aprovada pela comissão julgadora.

São Paulo, 21 de março de 2007.

Banca Examinadora:

Prof. ${ }^{\underline{a}}$ Dr. ${ }^{-}$Lucília Daruiz Borsari (orientadora) - IME/USP

Prof. ${ }^{-}$Dr. ${ }^{-}$Fernanda Soares Pinto Cardona - IME/USP

Prof. Dr. Daniel Vendrúsculo - DM/UFSCar 



\section{Dedicatória}

Dedico este trabalho a meus queridos pais, Shunji Taneda e Heiko Uemura Taneda, por todo o apoio durante esta empreitada, e à Tatyana Maya Okano, cujo carinho me foi de grande inspiração. 



\section{Agradecimentos}

Quero registrar aqui meus sinceros agradecimentos a todos os que contribuíram, direta ou indiretamente, para a realização deste trabalho: à minha orientadora Lucília Daruiz Borsari, pela paciência e compreensão com que sempre está disposta a auxiliar seus alunos; à Tatyana Maya Okano, pelo companheirismo e encorajamento na horas mais difíceis; a meus colegas e professores da área de Topologia Algébrica (em particular, às alunas de doutorado Hildebrane, Fernanda e Natália, e aos professores Fernanda S. P. Cardona, Daciberg L. Gonçalves e Daniel Vendrúsculo), cuja convivência me foi muito enriquecedora; e a meus caros amigos do IME, Márcio M. Onodera, Rodnei R. da Silva, Carlos H. Griese, Débora C. Brandt, Ednei F. Reis, Maria Cristina D. N. do Amaral, João R. Sato, Paula M. Yamakawa e Marcelo H. Ogava, por todos os momentos compartilhados. 



\section{Resumo}

Este trabalho é baseado num artigo de Robert F. Brown e Helga Schirmer, intitulado Nielsen Root Theory and Hopf Degree Theory, publicado em 2001 no Pacific Journal of Mathematics (vide [BS1]). Para aplicações $f: M \longrightarrow N$, entre variedades topológicas compactas, orientáveis e de mesma dimensão, existe um número, conhecido como número de Nielsen de raízes (de $f$ num ponto $c \in N$ ), que constitui um invariante homotópico e é um limitante inferior para o número de classes de raízes de $f$ em $c$. Neste trabalho, veremos que a noção de número de Nielsen pode ser estendida para aplicações entre variedades não necessariamente orientáveis ou compactas, com ou sem fronteira. Este novo número, denominado número de Nielsen transverso de raízes, também é um invariante homotópico e constitui um limitante inferior para o número de classes de raízes na categoria em que se define. Esta extensão da teoria de Nielsen é então aplicada à teoria do grau, obtendo-se a relação entre o grau absoluto de Hopf e o grau geométrico.

\section{Abstract}

This work is based on Robert F. Brown and Helga Schirmer's article, named Nielsen Root Theory and Hopf Degree Theory, published in 2001, in the Pacific Journal of Mathematics (see [BS1]). For maps $f: M \longrightarrow N$, between orientable and compact topological manifolds of the same dimension, there exists a number, called the Nielsen root number (of $f$ at $c \in N$ ), that is a homotopy invariant and a lower bound for the number of root classes of $f$ at $c$. In this work, we are going to see that the concept of Nielsen root number can be extended to maps between not necessarily orientable nor compact manifolds, with or without boundary. This new number, the transverse Nielsen root number, is a homotopy invariant and a lower bound for the number of root classes os maps which are transverse to $c$. This extension of the Nielsen theory is applied to degree theory, to obtain the relationship between the Hopf's absolute degree and the geometric degree. 



\section{Sumário}

Dedicatória

Agradecimentos

$\mathbf{v}$

Resumo/Abstract vii

$\begin{array}{ll}\text { Introdução } & 1\end{array}$

1 Preliminares 5

1.1 Orientabilidade de Variedades Topológicas . . . . . . . . . . . . . . 5

1.2 O Recobrimento Orientado de uma Variedade Topológica . . . . . . . . . 28

1.3 A Multiplicidade de uma Classe de Raízes . . . . . . . . . . . . . . . . . 41

1.4 O Grau Cohomológico . . . . . . . . . . . . . . . . . 57

2 Teoria de Nielsen de Raízes $\quad 76$

2.1 A Propriedade Fundamental da Teoria de Raízes . . . . . . . . . . . . . . . 76

2.2 O Cálculo da Multiplicidade de uma Classe de Raízes . . . . . . . . . . . 84

2.3 Os Números de Nielsen de Raízes . . . . . . . . . . . . . . . . . . . . . . 99

2.4 A Realização dos Números de Nielsen de Raízes . . . . . . . . . . . . . . 115

3 Aplicações à Teoria do Grau de Hopf 153

3.1 Os Graus Absoluto e Geométrico . . . . . . . . . . . . . . . . . . . . . . 153

3.2 Os Números de Nielsen de Raízes para Aplicações entre Superfícies . . . 156

$\begin{array}{ll}\text { A Limites Diretos } & 159\end{array}$

B Um Homeomorfismo para Variedades Topológicas $\quad 171$

C Deformações Propriamente Homotópicas que Preservam Raízes 176

$\begin{array}{ll}\text { Referências Bibliográficas } & 186\end{array}$ 



\section{Introdução}

O trabalho a seguir baseia-se no artigo intitulado Nielsen Root Theory and Hopf Degree Theory, de Robert F. Brown e Helga Schirmer, publicado em 2001 (vide [BS1]). Nele, vamos estender alguns resultados da teoria de Nielsen de raízes, que originalmente tratam de aplicações entre variedades topológicas orientáveis, de modo a remover a hipótese de orientabilidade. A teoria obtida será, então, utilizada para restabelecer a conexão entre a teoria de Nielsen de raízes e duas diferentes versões do grau de uma aplicação, a saber, os graus absoluto e geométrico. Assim, os métodos da teoria de Nielsen nos proporcionarão uma nova maneira de compreender alguns conceitos básicos da teoria de Hopf, bem como fornecerão demonstrações mais diretas para alguns de seus resultados. No que segue, vamos descrever os objetivos acima mais detalhadamente.

Se $f: M \longrightarrow N$ é uma aplicação contínua entre duas variedades topológicas (de mesma dimensão), e $c \in N$, então uma raiz de $f$ em $c$ é um ponto da imagem inversa $f^{-1}(c)$. Por outro lado, o número de Nielsen de raízes (de $f$ em $c$ ), denotado por $\mathrm{N}(f, c)$, é um limitante inferior para a cardinalidade de $f^{-1}(c)$, e constitui um invariante homotópico. Embora seja possível definir $\mathrm{N}(f, c)$, mesmo $M$ e $N$ não sendo variedades topológicas, é usualmente muito difícil (quando possível) calcular este número com tal generalidade. Entretanto, se $M$ e $N$ são variedades orientáveis, existe uma fórmula para se determinar $\mathrm{N}(f, c)$. Denotando por $\mathrm{j}$ a cardinalidade do grupo quociente $\pi_{1}(N) / f_{\#}\left(\pi_{1}(M)\right)$, onde $f_{\#}: \pi_{1}(M) \longrightarrow \pi_{1}(N)$ é o homomorfismo induzido por $f$, H. Hopf ([H2]) e X. Lin ([L]) obtiveram o seguinte resultado (que constitui uma versão parcial do corolário 2.3.1, a ser demonstrado mais adiante):

Teorema. Se $f: M \longrightarrow N$ é uma aplicação contínua entre duas variedades topológicas de mesma dimensão $n$, fechadas, conexas e orientadas, então $\mathrm{N}(f, c)=0$ se o grau de $f$ é zero, ou $\mathrm{N}(f, c)=\mathrm{j}$ caso contrário. 
Na seção 2.3 (teorema 2.3.1), veremos que este teorema pode ser estendido para aplicações entre variedades não-necessariamente orientáveis, não-compactas e com fronteira (desde que $f$ seja uma aplicação própria, que leve a fronteira de $M$ na fronteira de $N)$.

Em 1930, Hopf utilizou a teoria de Nielsen de raízes como base para a sua teoria do grau (vide [H2]), a qual, por este motivo, distinguia-se das demais existentes em sua época. O grau mencionado no teorema acima, refere-se ao conceito clássico introduzido por Brouwer ([Bw], página 105), que é definido em função do homomorfismo em homologia (com coeficientes em $\mathbb{Z}$ ) induzido por $f$. Esta definição pode ainda ser estendida para aplicações próprias que preservam fronteira, entre variedades topológicas de mesma dimensão, orientáveis, com fronteira e não-compactas. No entanto, se ao menos uma das variedades $M$ ou $N$ for não-orientável, o grau homológico só poderá ser definido utilizando-se homologia com coeficientes em $\mathbb{Z}_{2}$, e, neste caso, o grau resultante não reflete satisfatoriamente as propriedades geométricas da aplicação $f$. Deste modo, a fim de obter as informações geométricas desejadas, e, em particular, procurando uma interpretação algébrica que tivesse em vista as imagens inversas pontuais de $f$, Hopf introduziu um conceito de grau denominado Absolutgrad (ou grau absoluto); este, por não depender da orientabilidade das variedades topológicas envolvidas, mostrou-se muito mais fiel à geometria da aplicação $f$ que o grau homológico a coeficientes em $\mathbb{Z}_{2}$. O grau absoluto de Hopf pode ser considerado uma variante do número de Nielsen de raízes: com efeito, ele é exatamente o que chamamos de "número de Nielsen transverso de raízes", denotado por $\mathrm{N}_{\phi}(f, c)$, e constitui um limitante inferior para a cardinalidade do conjunto de raízes das aplicações que são "tranversas" ao ponto $c$, num sentido que tornaremos mais preciso na definição 2.3.2 (vide teorema 3.1.1). Em geral, tem-se $\mathrm{N}_{\phi}(f, c) \geqslant \mathrm{N}(f, c)$, sendo que esta igualdade não é necessariamente válida; na seção 2.3, veremos como calcular estes números de acordo com as propriedades geométricas de $f$.

Uma importante motivação para o cálculo do número de Nielsen de raízes $\mathrm{N}(f, c)$, é que ele contém informações relacionadas à geometria da aplicação $f$ : se a dimensão $n$ das variedades $M$ e $N$ for diferente de 2, este número constitui um limitante inferior preciso, ou seja, existe uma aplicação $g: M \longrightarrow N$, homotópica à $f$, tal que a imagem inversa $g^{-1}(c)$ contém exatamente $\mathrm{N}(f, c)$ pontos (por "precisão", estamos nos referindo à propriedade do limitante ser efetivamente realizado). Por outro lado, o número de Nielsen transverso $\mathrm{N}_{\pitchfork}(f, c)$ também é um limitante inferior preciso: em outras palavras, existe uma aplicação $g: M \longrightarrow N$, homotópica à $f$ e "transversa" à $c$, tal que $g^{-1}(c)$ possui $\mathrm{N}_{\pitchfork}(f, c)$ pontos. Na seção 3.1, veremos que isto equivale a dizer que o limite inferior $\mathrm{N}_{\phi}(f, c)$ é realizado por uma aplicação $g: M \longrightarrow N$ que possui $\mathrm{N}_{\phi}(f, c)$ como seu "grau geométrico" (vide definição 3.1.2); logo, a realização do número de Nielsen 
transverso $\mathrm{N}_{\uparrow}(f, c)$ implica na igualdade entre os graus absoluto e geométrico de $f$.

Assim sendo, o trabalho que será apresentado a seguir pode ser visto, em parte, como um re-exame da teoria do grau de Hopf segundo uma perspectiva matemática contemporânea. Muitos dos resultados contidos em [BS1] (que, conforme mencionamos de início, é o artigo no qual se baseia esta dissertação) não eram absolutamente inéditos à época de sua publicação: os primeiros estudos neste sentido podem ser encontrados em dois importantes artigos, escritos respectivamente por P. Olum ([O]) e D. B. A. Epstein ([E]). Estes autores classificaram as aplicações entre variedades topológicas de mesma dimensão em três tipos distintos, e então definiram o grau absoluto separadamente para cada caso. Entretanto, embora facilite o cálculo do grau absoluto, esta definição obscurece seu significado geométrico. Mais recentemente, foram publicadas outras extensões da teoria de Hopf: em 1986, X. Lin ([L]) introduziu uma definição moderna para a multiplicidade de uma classe de raízes, e forneceu uma nova demonstração para a realização do número de Nielsen de raízes, no caso específico em que $f$ é uma aplicação entre variedades topológicas fechadas de dimensão maior ou igual a 3. No entanto, Lin não considerou a não-orientabilidade das variedades, nem restabeleceu a conexão entre a teoria de Nielsen de raízes e a teoria do grau de Hopf. Por outro lado, em 1987, R. Skora ([Sk]) apresentou uma versão geométrica para a relação entre o grau absoluto de Hopf e o grau geométrico, mas também não conectou seus resultados à teoria de Nielsen de raízes. Já em 1992, Y. Rong e S. Wang ([RW]) desenvolveram uma extensão da teoria de Nielsen de raízes para aplicações próprias $f: M \longrightarrow N$, que pode ser utilizada para demonstrar a realização do número de Nielsen transverso de raízes, no caso particular em que as variedades $M$ e $N$ são fechadas e orientáveis; contudo, o artigo que publicaram não faz referências ao grau absoluto de Hopf. Isto posto, a diferença crucial entre estas abordagens e à de [BS1], é que, além de restabelecer a ligação entre as teorias de Hopf e de Nielsen, Brown e Schirmer apresentam demonstrações (bem como definições) muito diferentes daquelas que se encontram nos artigos acima mencionados: para definir a multiplicidade de uma classe de raízes (de uma aplicação $f: M \longrightarrow N$, entre variedades de mesma dimensão), eles utilizam as propriedades do "grau local" de $f$, e, então, definem o número de Nielsen de raízes, $\mathrm{N}(f, c)$, como sendo o número de classes de raízes (de $f$ em $c \in \operatorname{Int} N$ ) que têm multiplicidades não-nulas; ademais, eles também introduzem o conceito de número de Nielsen transverso de raízes, $\mathrm{N}_{\uparrow}(f, c)$, definido como a soma das multiplicidades de todas as classes de raízes de $f$ em $c$, e mostram que $\mathrm{N}(f, c)$ e $\mathrm{N}_{\phi}(f, c)$ podem ser calculados a partir dos graus locais de determinados levantamentos de $f$, provando ainda que estes números são efetivamente realizáveis quando a dimensão das variedades $M$ e $N$ é diferente de 2. Em particular, a demonstração de que $\mathrm{N}_{\phi}(f, c)$ é realizável requer o uso de uma certa versão do "lema de Whitney", adaptado ao conceito de "transversalidade 
de microfibrados", assunto cujo desenvolvimento é relativamente recente.

Com estes objetivos em mente, nosso trabalho será organizado da seguinte forma: no capítulo 1, iremos introduzir os conceitos e resultados que servirão de suporte para os capítulos seguintes (mais especificamente, as definições de orientabilidade de variedades, o grau local, a multiplicidade de uma classe de raízes, e o grau cohomológico); já no capítulo 2, procedemos ao cálculo da multiplicidade de uma classe de raízes, através do qual poderemos determinar os números de Nielsen de raízes e demonstrar suas respectivas realizações, e finalmente, no capítulo 3, mostraremos a relação da teoria do grau de Hopf com a teoria de Nielsen desenvolvida. 


\section{Preliminares}

O objetivo deste capítulo é introduzir os conceitos básicos que serão utilizados nas definições dos números de Nielsen de raízes. No que segue, consideraremos que um espaço topológico de Hausdorff $M$ é uma variedade topológica de dimensão $\boldsymbol{n}$ sem fronteira, se $M$ possui uma base enumerável de abertos e todo ponto de $M$ admite uma vizinhança homeomorfa a um subconjunto aberto de $\mathbb{R}^{n}$; analogamente, dizemos que um espaço de Hausdorff $M$ é uma variedade topológica de dimensão $n$ com fronteira, se $M$ possui uma base enumerável de abertos e todo ponto de $M$ admite uma vizinhança homeomorfa, ou a subconjunto aberto de $\mathbb{R}^{n}$, ou a um subconjunto aberto de $\mathbb{R}_{+}^{n}=\left\{\left(x_{1}, \ldots, x_{n}\right) \in \mathbb{R}^{n}: x_{n} \geqslant 0\right\}$.

\subsection{Orientabillidadle de Variedades Topológicas}

Nesta seção, vamos estabelecer um critério que nos permitirá subdividir as aplicações contínuas entre variedades topológicas de mesma dimensão em três tipos distintos. Primeiramente, no entanto, convém apresentarmos alguns resultados preliminares.

Definição 1.1.1. Seja M uma variedade topológica de dimensão n, com ou sem fronteira. Dado um ponto $x \in \operatorname{Int} M$, dizemos que um elemento $\mu_{x} \in \mathrm{H}_{n}(M, M \backslash\{x\})$ é uma orientação local de $M$ em $x$ se $\mu_{x}$ é um gerador de $\mathrm{H}_{n}(M, M \backslash\{x\})$; evidentemente, como $\mathrm{H}_{n}(M, M \backslash\{x\}) \simeq \mathbb{Z}$ para todo $x \in \operatorname{Int} M$, segue que cada ponto de Int $M$ admite exatamente duas orientações locais.

Agora, tendo em vista a definição acima, veremos como definir uma "orientação global" numa variedade topológica conexa $M$, sem fronteira. Intuitivamente, tal 
orientação (quando existir) será uma "escolha contínua" de orientações locais para cada ponto de $M$, sendo que, por "continuidade", devemos subentender que, escolhida uma orientação local num ponto $x \in M$, esta será, de algum modo, coerente com a orientação local num outro ponto $y \in M$ (de maneira que, globalmente, possamos definir uma única "função" para escolher orientações locais em cada ponto de $M$ ).

Seja, pois, $M$ uma variedade topológica conexa de dimensão $n$, sem fronteira; considerando o conjunto

$$
\Theta(M)=\bigcup_{x \in M} \mathrm{H}_{n}(M, M \backslash\{x\})
$$

vamos introduzir uma estrutura de espaço topológico em $\Theta(M)$. Para tanto, definimos uma n-bola própria em $M$ como sendo um aberto $V \subseteq M$, tal que existe um homeomorfismo do disco unitário fechado $\mathbb{D}^{n}$ em $\bar{V}$, que leva $\mathbb{S}^{n-1}=\partial\left(\mathbb{D}^{n}\right)$ sobre $\bar{V} \backslash V=\partial V$ (note que toda n-bola própria em $M$ é, portanto, um subconjunto aberto e conexo de $M$ ). Assim, a coleção de todas as n-bolas próprias em $M$ constitui uma base para a topologia de $M$. Por outro lado, se $V$ é um aberto conexo em $M$, e $x \in V$, sabemos que $M \backslash V$ é um retrato por deformação de $M \backslash\{x\}$, o que implica que a inclusão $j_{x}:(M, M \backslash V) \longrightarrow(M, M \backslash\{x\})$ induz um isomorfismo em homologia singular $\left(j_{x}\right)_{*}: \mathrm{H}_{n}(M, M \backslash V) \longrightarrow \mathrm{H}_{n}(M, M \backslash\{x\})$. Deste modo, denotando

$$
U(z, V)=\left\{\left(j_{x}\right)_{*}(z): x \in V\right\},
$$

onde $V \subseteq M$ é uma n-bola própria e $z \in \mathrm{H}_{n}(M, M \backslash V)$, temos que a coleção

$$
\mathcal{C}=\left\{U(z, V): V \subseteq M \text { é uma n-bola própria e } z \in \mathrm{H}_{n}(M, M \backslash V)\right\}
$$

constitui uma base para a topologia de $\Theta(M)$.

Isto posto, para cada $x \in M$, seja $\phi_{x}: \mathrm{H}_{n}(M, M \backslash\{x\}) \longrightarrow \mathbb{Z}$ um isomorfismo (lembrese que $\left.\mathrm{H}_{n}(M, M \backslash\{x\}) \simeq \mathbb{Z}\right)$, e considere a aplicação $\gamma: \Theta(M) \longrightarrow \mathbb{Z}$ definida por $\gamma(w)=\left|\phi_{x}(w)\right|$, para cada $w \in \mathrm{H}_{n}(M, M \backslash\{x\}) \subseteq \Theta(M)$. Note que, como $\gamma(w)$ é o valor absoluto de $\phi_{x}(w)$, segue que $\gamma$ não depende de uma particular escolha do isomorfismo $\phi_{x}: \mathrm{H}_{n}(M, M \backslash\{x\}) \longrightarrow \mathbb{Z}$, de modo que a aplicação $\gamma$ está, portanto, bem definida. Vamos mostrar então que $\gamma$ é constante em cada aberto $U(z, V)$ da coleção $\mathcal{C}$ que constitui a base para a topologia de $\Theta(M)$. Com efeito, dada uma n-bola própria $V \subseteq M$ e um elemento $z \in \mathrm{H}_{n}(M, M \backslash V)$, sejam $x, y \in V$ e considere os isomorfismos $\left(j_{x}\right)_{*}: \mathrm{H}_{n}(M, M \backslash V) \longrightarrow \mathrm{H}_{n}(M, M \backslash\{x\})$ e $\left(j_{y}\right)_{*}: \mathrm{H}_{n}(M, M \backslash V) \longrightarrow \mathrm{H}_{n}(M, M \backslash\{y\})$ induzidos pelas respectivas inclusões; temos, pois, que $\left(j_{x}\right)_{*}(z) \in \mathrm{H}_{n}(M, M \backslash\{x\})$ e $\left(j_{y}\right)_{*}(z) \in \mathrm{H}_{n}(M, M \backslash\{y\})$ são tais que $\left(j_{x}\right)_{*}(z),\left(j_{y}\right)_{*}(z) \in U(z, V)$. Assim, tendo em vista que $\mathrm{H}_{n}(M, M \backslash V) \simeq \mathbb{Z}$, seja $\mu_{V}$ um dos dois geradores de $\mathrm{H}_{n}(M, M \backslash V)$, e denote por $\mu_{x}$ e $\mu_{y}$ os geradores de $\mathrm{H}_{n}(M, M \backslash\{x\})$ e $\mathrm{H}_{n}(M, M \backslash\{y\})$, respectivamente, tais que $\left(j_{x}\right)_{*}\left(\mu_{V}\right)=\mu_{x}$ e $\left(j_{y}\right)_{*}\left(\mu_{V}\right)=\mu_{y}$. Deste modo, representando $z \in \mathrm{H}_{n}(M, M \backslash V)$ como 
sendo $z=k \cdot \mu_{V}$, onde $k \in \mathbb{Z}$, e observando que $\phi_{x}\left(\mu_{x}\right)= \pm 1$ e $\phi_{y}\left(\mu_{y}\right)= \pm 1$ (uma vez que $\phi_{x}$ e $\phi_{y}$ são isomorfismos), obtemos que:

$$
\begin{aligned}
\gamma\left(\left(j_{x}\right)_{*}(z)\right) & =\gamma\left(\left(j_{x}\right)_{*}\left(k \cdot \mu_{V}\right)\right)=\gamma\left(k \cdot\left(j_{x}\right)_{*}\left(\mu_{V}\right)\right) \\
& =\gamma\left(k \cdot \mu_{x}\right)=\left|\phi_{x}\left(k \cdot \mu_{x}\right)\right| \\
& =\left|k \cdot \phi_{x}\left(\mu_{x}\right)\right| \\
& =|k|
\end{aligned}
$$

e (analogamente) $\gamma\left(\left(j_{y}\right)_{*}(z)\right)=|k|$. Logo, temos que $\gamma\left(w_{1}\right)=\gamma\left(w_{2}\right)$ para quaisquer $w_{1}, w_{2} \in U(z, V)$, o que prova que $\gamma$ é constante em cada $U(z, V) \in \mathcal{C}$ (e, portanto, $\gamma: \Theta(M) \longrightarrow \mathbb{Z}$ é contínua, supondo que $\mathbb{Z}$ está munido da topologia discreta). Note ainda que, dado $w \in \mathrm{H}_{n}(M, M \backslash\{x\})$ para algum $x \in M$, temos que $\gamma(w)=\left|\phi_{x}(w)\right|=1$ se, e somente se, $w$ é um gerador de $\mathrm{H}_{n}(M, M \backslash\{x\})$, pois $\phi_{x}: \mathrm{H}_{n}(M, M \backslash\{x\}) \longrightarrow \mathbb{Z}$ é um isomorfismo; por conseguinte, o subconjunto $\gamma^{-1}(1) \subseteq \Theta(M)$ é constituído exatamente das orientações locais nos pontos $x \in M$. Além disso, o fato de $\gamma$ ser constante em cada conjunto de $\mathcal{C}$, implica, em particular, que o espaço topológico $\Theta(M)$ se decompõe numa união disjunta,

$$
\Theta(M)=\tilde{M}(0) \cup \tilde{M}(1) \cup \tilde{M}(2) \cup \ldots,
$$

onde, para cada $k \in\{0,1,2, \ldots\}$, denotamos $\tilde{M}(k)=\gamma^{-1}(k)$.

Agora, dada uma n-bola própria $V \subseteq M$, seja $\mu_{V}$ um gerador de $\mathrm{H}_{n}(M, M \backslash V) \simeq \mathbb{Z}$, e, para cada $x \in V$, considere o gerador $\mu_{x}$ de $\mathrm{H}_{n}(M, M \backslash\{x\})$ tal que $\left(j_{x}\right)_{*}\left(\mu_{V}\right)=\mu_{x}$, onde $\left(j_{x}\right)_{*}: \mathrm{H}_{n}(M, M \backslash V) \longrightarrow \mathrm{H}_{n}(M, M \backslash\{x\})$ é o isomorfismo induzido pela inclusão. Como todo elemento de $\mathrm{H}_{n}(M, M \backslash\{x\})$ pode ser representado de maneira única por $k \cdot \mu_{x}$, onde $k \in \mathbb{Z}$, segue que a projeção $p_{\Theta(M)}: \Theta(M) \longrightarrow M$, definida por $p_{\Theta(M)}(z)=x$ para cada $z \in \mathrm{H}_{n}(M, M \backslash\{x\})$, $\operatorname{com} x \in M$, é tal que:

$$
\begin{aligned}
p_{\Theta(M)}^{-1}(V) & =\left\{z \in \Theta(M)=\bigcup_{x \in M} \mathrm{H}_{n}(M, M \backslash\{x\}): p_{\Theta(M)}(z) \in V\right\} \\
& =\left\{z \in \mathrm{H}_{n}(M, M \backslash\{x\}): x \in V\right\} \\
& =\left\{k \cdot \mu_{x}: k \in \mathbb{Z} \text { e } x \in V\right\} \\
& =\left\{k \cdot\left(j_{x}\right)_{*}\left(\mu_{V}\right): k \in \mathbb{Z} \text { e } x \in V\right\} \\
& =\left\{\left(j_{x}\right)_{*}\left(k \cdot \mu_{V}\right): k \in \mathbb{Z} \text { e } x \in V\right\} \\
& =\bigcup_{k \in \mathbb{Z}} U\left(k \cdot \mu_{V}, V\right) .
\end{aligned}
$$

Entretanto, se $k_{1}, k_{2} \in \mathbb{Z}$ são tais que $k_{1} \neq k_{2}$, segue que $U\left(k_{1} \cdot \mu_{V}, V\right) \cap U\left(k_{2} \cdot \mu_{V}, V\right)=\varnothing$, uma vez que:

$$
U\left(k \cdot \mu_{V}, V\right)=\left\{\left(j_{x}\right)_{*}\left(k \cdot \mu_{V}\right): x \in V\right\}=\left\{k \cdot \mu_{x}: x \in V\right\},
$$


para todo $k \in \mathbb{Z}$; assim, $p_{\Theta(M)}^{-1}(V)$ é a união disjunta dos abertos $U\left(k \cdot \mu_{V}, V\right) \in \mathcal{C}$ de $\Theta(M)$, onde $k \in \mathbb{Z}$. Isto posto, observe que cada $U\left(k \cdot \mu_{V}, V\right), k \in \mathbb{Z}$, é levado homeomorficamente sobre $V$ pela projeção $p_{\Theta(M)}: \Theta(M) \longrightarrow M$, pois $p_{\Theta(M)}\left(k \cdot \mu_{x}\right)=x$ para todo $x \in V$. Conseqüentemente, como a coleção de todas as n-bolas próprias em $M$ constitui uma base para a topologia de $M$, segue que todo ponto $x \in M$ admite uma vizinhança aberta $V \subseteq M$ (a saber, uma n-bola própria contendo $x$ ), tal que $p_{\Theta(M)}^{-1}(V)$ é uma união disjunta $\bigcup_{k \in \mathbb{Z}} U_{k}$ de abertos em $\Theta(M)$ (a saber, $U_{k}=U\left(k \cdot \mu_{V}, V\right)$ ), cada um dos quais se aplica por $p_{\Theta(M)}$ homeomorficamente sobre $V$; em outras palavras, a restrição de $p_{\Theta(M)}: \Theta(M) \longrightarrow M$ à cada componente conexa de $\Theta(M)$ é um recobrimento de $M, \mathrm{e}$, portanto, denotando por $\tilde{p}: \tilde{M} \longrightarrow M$ a restrição de $p_{\Theta(M)}$ ao conjunto

$$
\tilde{M}=\tilde{M}(1) \subseteq \Theta(M)=\bigcup_{k \in \mathbb{Z}} \tilde{M}(k),
$$

temos que a restrição de $\tilde{p}$ a cada componente conexa de $\tilde{M}=\tilde{M}(1)$ é um recobrimento de $M$ (note que, embora os conjuntos $\tilde{M}(k)=\gamma^{-1}(k), k \in \mathbb{Z}$, sejam dois a dois disjuntos, cada um deles pode não ser necessariamente uma componente conexa de $\Theta(M)$ ). Convém mencionarmos ainda que, pelo fato de $\tilde{p}: \tilde{M} \longrightarrow M$ ser um homeomorfismo local, segue que o espaço $\tilde{M}=\tilde{M}(1)$ possui as mesmas propriedades topológicas locais de $M$; em particular, temos que $\tilde{M}$ é uma variedade topológica de dimensão $n$, sem fronteira.

Finalmente, lembrando que $\tilde{M}=\tilde{M}(1)$ é constituído exclusivamente dos geradores $\mu_{x},-\mu_{x} \in \mathrm{H}_{n}(M, M \backslash\{x\}) \simeq \mathbb{Z}$, onde $x \in M$, vamos mostrar que, dado $x \in M$, se $\mu_{x} \mathrm{e}$ $-\mu_{x}$ pertencem à uma mesma componente conexa $\tilde{C}$ de $\tilde{M}$, então, para todo $y \in M$, os geradores $\mu_{y}$ e $-\mu_{y}$ de $\mathrm{H}_{n}(M, M \backslash\{y\})$ também pertencem a $\tilde{C}$. De fato, como a variedade $M$ é conexa (e, portanto, conexa por caminhos), sabemos que existe um caminho $\omega: I \longrightarrow M$ tal que $\omega(0)=y$ e $\omega(1)=x$; logo, como $\tilde{p}\left(\mu_{y}\right)=\tilde{p}\left(-\mu_{y}\right)=y$, segue que existem caminhos $\tilde{\omega}_{1}: I \longrightarrow \tilde{C}$ e $\tilde{\omega}_{2}: I \longrightarrow \tilde{C}$ tais que $\tilde{\omega}_{1}(0)=\mu_{y}, \tilde{\omega}_{2}(0)=-\mu_{y}$ e $\tilde{p} \circ \tilde{\omega}_{1}=\tilde{p} \circ \tilde{\omega}_{2}=\omega$, isto é, $\omega$ admite levantamentos $\tilde{\omega}_{1}$ e $\tilde{\omega}_{2}$, relativamente ao recobrimento $\left.\tilde{p}\right|_{\tilde{C}}: \tilde{C} \longrightarrow M$, com início nos pontos $\mu_{y} \mathrm{e}-\mu_{y}$, respectivamente (note que $\tilde{C}$ é conexo por caminhos, pois é uma componente conexa da variedade topológica $\tilde{M}$ ). Assim, como $\tilde{\omega}_{1}(1), \tilde{\omega}_{2}(1) \in \tilde{p}^{-1}(x)=\left\{\mu_{x},-\mu_{x}\right\}$, temos que, ou $\tilde{\omega}_{1}(1)=\tilde{\omega}_{2}(1)= \pm \mu_{x}$, ou $\tilde{\omega}_{1}(1)=\mu_{x}$ e $\tilde{\omega}_{2}(1)=-\mu_{x} ;$ no primeiro caso, segue que o caminho justaposto $\tilde{\omega}_{1} \vee \tilde{\omega}_{2}^{-1}: I \longrightarrow \tilde{C}$ liga os pontos $\mu_{y},-\mu_{y} \mathrm{e} \pm \mu_{x}$, enquanto que, no segundo caso, os caminhos $\tilde{\omega}_{1} \mathrm{e} \tilde{\omega}_{2}$ ligam $\mu_{y} \mathrm{e}$ $-\mu_{y}$ aos pontos $\mu_{x} \mathrm{e}-\mu_{x}$, respectivamente. Deste modo, concluímos que, para qualquer que seja $y \in M$, os geradores $\mu_{y}$ e $-\mu_{y}$ de $\mathrm{H}_{n}(M, M \backslash\{y\}) \simeq \mathbb{Z}$ também pertencem à mesma componente conexa $\tilde{C} \subseteq \tilde{M}$ que contém $\mu_{x} \mathrm{e}-\mu_{x}$; isto, por sua vez, implica que a variedade $\tilde{M}$ (que contém somente os geradores dos grupos $\mathrm{H}_{n}(M, M \backslash\{x\}$ ), onde $x \in M$ ) é conexa, e, conseqüentemente, a aplicação $\tilde{p}: \tilde{M} \longrightarrow M$ é um recobrimento duplo de $M$ 
(ou seja, um recobrimento de duas folhas). Em contrapartida, observe agora que, se os geradores $\mu_{x} \mathrm{e}-\mu_{x}$ de $\mathrm{H}_{n}(M, M \backslash\{x\}) \simeq \mathbb{Z}$ pertencem a componentes conexas distintas de $\tilde{M}$, digamos $\mu_{x} \in \tilde{C}_{1}$ e $-\mu_{x} \in \tilde{C}_{2}$, então, para todo $y \in M$, os geradores $\mu_{y}$ e $-\mu_{y}$ de $\mathrm{H}_{n}(M, M \backslash\{y\})$ também são tais que $\mu_{y} \in \tilde{C}_{1} \mathrm{e}-\mu_{y} \in \tilde{C}_{2}$ : com efeito, sendo $\lambda: I \longrightarrow M$ um caminho tal que $\lambda(0)=x$ e $\lambda(1)=y$, sabemos que $\lambda$ admite levantamentos $\tilde{\lambda}_{1}: I \longrightarrow \tilde{C}_{1} \mathrm{e}$ $\tilde{\lambda}_{2}: I \longrightarrow \tilde{C}_{2}$, relativos aos recobrimentos $\left.\tilde{p}\right|_{\tilde{C}_{1}}$ e $\left.\tilde{p}\right|_{\tilde{C}_{2}}$, respectivamente, tais que $\tilde{\lambda}_{1}(0)=\mu_{x}$ e $\tilde{\lambda}_{2}(0)=-\mu_{x}$; logo, como $\tilde{\lambda}_{1}(1), \tilde{\lambda}_{2}(1) \in \tilde{p}^{-1}(y)=\left\{\mu_{y},-\mu_{y}\right\}$, segue que $\tilde{\lambda}_{1}(1)=\mu_{y}$ e $\tilde{\lambda}_{2}(1)=-\mu_{y}$ (uma vez que não podemos ter $\tilde{\lambda}_{1}(1)=\tilde{\lambda}_{2}(1)$, pois, do contrário, os pontos $\mu_{x}=\tilde{\lambda}_{1}(0)$ e $-\mu_{x}=\tilde{\lambda}_{2}(0)$ pertenceriam à mesma componente conexa de $\left.\tilde{M}\right)$, o que mostra que $\mu_{y} \in \tilde{C}_{1} \mathrm{e}-\mu_{y} \in \tilde{C}_{2}$. Neste caso, temos que a variedade $\tilde{M}$ é a união de duas componentes conexas distintas $\tilde{C}_{1}$ e $\tilde{C}_{2}$, cada qual contendo exatamente um dos dois geradores de $\mathrm{H}_{n}(M, M \backslash\{x\})$ para cada $x \in M$, sendo que as restrições $\left.\tilde{p}\right|_{\tilde{C}_{1}}: \tilde{C}_{1} \longrightarrow M \mathrm{e}$ $\left.\tilde{p}\right|_{\tilde{C}_{2}}: \tilde{C}_{2} \longrightarrow M$ são recobrimentos simples de $M$ (isto é, com uma única folha).

Deste modo, podemos introduzir a seguinte

Definição 1.1.2 . Seja M uma variedade topológica conexa, de dimensão n e sem fronteira. $\grave{A}$ variedade $\tilde{M}$ obtida acima, constituída das orientações locais nos pontos $x \in M$, denominamos fibrado orientado de $M$, ao passo que a aplicação $\tilde{p}: \tilde{M} \longrightarrow M$, definida por $\tilde{p}\left(\mu_{x}\right)=x$ para cada gerador $\mu_{x} \in \mathrm{H}_{n}(M, M \backslash\{x\})$, com $x \in M$, é chamada projeção orientada de $M$. Além disso, se $\tilde{M}$ for conexa (e, conseqüentemente, $\tilde{p}$ for um recobrimento duplo de $M$ ), dizemos que $M$ é uma variedade não-orientável; por outro lado, se $\tilde{M}$ for a união disjunta de duas componentes conexas $\tilde{C}_{1}$ e $\tilde{C}_{2}$ (e, portanto, cada restrição $\left.\tilde{p}\right|_{\tilde{C}_{1}}$ e $\left.\tilde{p}\right|_{\tilde{C}_{2}}$ for um recobrimento simples de $M$ ), a variedade $M$ é dita orientável, sendo que uma orientação em $M$ é uma aplicação contínua $s: M \longrightarrow \tilde{M}$ tal que $\tilde{p} \circ \mathrm{s}=\operatorname{Id}_{M}$, onde $\operatorname{Id}_{M}: M \longrightarrow M$ é a identidade.

A respeito da definição acima, temos o seguinte

Lema 1.1.1. Se Mé uma variedade topológica conexa, de dimensão ne sem fronteira, então: (i) se s : $M \longrightarrow \tilde{M}$ é uma orientação em $M$, então, para cada $x \in M$, s(x) é um gerador de $\mathrm{H}_{n}(M, M \backslash\{x\}) \simeq \mathbb{Z}$ (ou seja, para cada $x \in M, s(x)$ é uma orientação local de $M$ em $x$ );

(ii) Mé orientável se, e somente se, ela admite uma orientação s : $\longrightarrow \tilde{M}$;

(iii) denotando por $\tilde{p}: \tilde{M} \longrightarrow M$ a projeção orientada de $M$, e sendo s $: M \longrightarrow \tilde{M}$ uma aplicação tal que $\tilde{p} \circ \mathrm{s}=\operatorname{Id}_{M}$, onde $\operatorname{Id}_{M}: M \longrightarrow M$ é a identidade, segue que s é contínua (e, portanto, define uma orientação em $M$ ) se, e somente se, para todo $x \in M$, existem uma n-bola própria $V \subseteq M$, contendo $x$, e um gerador $\mu_{V}$ de $\mathrm{H}_{n}(M, M \backslash V) \simeq \mathbb{Z}$, tais que $\left(j_{y}\right)_{*}\left(\mu_{V}\right)=s(y)$ para cada $y \in V$, onde $\left(j_{y}\right)_{*}: \mathrm{H}_{n}(M, M \backslash V) \longrightarrow \mathrm{H}_{n}(M, M \backslash\{y\})$ é o isomorfismo induzido pela inclusão. 
DemonstraçÃo. (i) Com efeito, sabemos que o fibrado orientado $\tilde{M}$ é constituído somente dos geradores dos grupos $\mathrm{H}_{n}(M, M \backslash\{x\}) \simeq \mathbb{Z}$, onde $x \in M$, enquanto que a projeção orientada $\tilde{p}: \tilde{M} \longrightarrow M$ é definida como sendo a aplicação que leva cada gerador de $\mathrm{H}_{n}(M, M \backslash\{x\})$ no ponto $x \in M$; conseqüentemente, dado $x \in M$, temos que a imagem inversa $\tilde{p}^{-1}(x)$ é o conjunto constituído unicamente dos dois geradores de $\mathrm{H}_{n}(M, M \backslash\{x\}) \simeq \mathbb{Z}$. Por outro lado, se $s: M \longrightarrow \tilde{M}$ é uma orientação em $M$, segue (por definição) que s é contínua, e $\tilde{p} \circ s=\operatorname{Id}_{M}$, onde $\operatorname{Id}_{M}: M \longrightarrow M$ é a identidade. Logo, dado $x \in M$, temos que $\tilde{p}(s(x))=x$, isto é, $s(x) \in \tilde{p}^{-1}(x)$, o que implica que $s(x)$ é um gerador de $\mathrm{H}_{n}(M, M \backslash\{x\}) \simeq \mathbb{Z}$.

(ii) De fato, se $M$ é orientável, temos que o fibrado orientado $\tilde{M}$ é a união de duas componentes conexas distintas $\tilde{C}_{1}$ e $\tilde{C}_{2}$, e as restrições $\left.\tilde{p}\right|_{\tilde{C}_{1}}: \tilde{C}_{1} \longrightarrow M$ e $\left.\tilde{p}\right|_{\tilde{C}_{2}}$ : $\tilde{C}_{2} \longrightarrow M\left(\right.$ da projeção orientada $\tilde{p}: \tilde{M} \longrightarrow M$ às componentes $\tilde{C}_{1}$ e $\tilde{C}_{2}$ ) constituem recobrimentos simples de $M$. Logo, temos que $\left.\tilde{p}\right|_{\tilde{C}_{1}}$ e $\left.\tilde{p}\right|_{\tilde{C}_{2}}$ são homeomorfismos sobre $M$, e, portanto, os homeomorfismos inversos $s_{1}=\left(\left.\tilde{p}\right|_{\tilde{C}_{1}}\right)^{-1}: M \longrightarrow \tilde{C}_{1} \subseteq \tilde{M}$ e $s_{2}=\left(\left.\tilde{p}\right|_{\tilde{C}_{2}}\right)^{-1}: M \longrightarrow \tilde{C}_{2} \subseteq \tilde{M}$ definem orientações distintas em $M$ (note que $\tilde{p} \circ s_{1}=\tilde{p} \circ s_{2}=\operatorname{Id}_{M}$, onde $\operatorname{Id}_{M}: M \longrightarrow M$ é a identidade). Reciprocamente, seja $s: M \longrightarrow \tilde{M}$ uma orientação em $M$, e suponhamos (por absurdo) que $M$ seja nãoorientável; neste caso, temos que o fibrado orientado $\tilde{M}$ é uma variedade topológica conexa (e, portanto, conexa por caminhos), e a projeção orientada $\tilde{p}: \tilde{M} \longrightarrow M$ é um recobrimento duplo de $M$. Por outro lado, dado $x \in M$, sabemos que $\mu_{x}=s(x)$ é um gerador de $\mathrm{H}_{n}(M, M \backslash\{x\}) \simeq \mathbb{Z}$ (conforme vimos no item anterior); assim, denotando por $-\mu_{x}$ o outro gerador de $\mathrm{H}_{n}(M, M \backslash\{x\}) \simeq \mathbb{Z}$, distinto de $\mu_{x}=s(x)$, seja $\tilde{\omega}: I \longrightarrow \tilde{M}$ um caminho tal que $\tilde{\omega}(0)=\mu_{x}$ e $\tilde{\omega}(1)=-\mu_{x}$. Temos então que a imagem $\omega=\tilde{p} \circ \tilde{\omega}: I \longrightarrow M$ é um laço com base em $x=\tilde{p}\left(\mu_{x}\right)=\tilde{p}\left(-\mu_{x}\right)$, e $\tilde{\omega}$ é o único levantamento de $\omega$ (relativamente ao recobrimento $\tilde{p}: \tilde{M} \longrightarrow M$ ), com início em $\mu_{x}$. Além disso, como a composição $\tilde{p} \circ s: M \longrightarrow M$ é a identidade em $M$, segue que o caminho $s \circ \omega: I \longrightarrow \tilde{M}$ coincide com $\tilde{\omega}$ (pois, como $\tilde{p} \circ(s \circ \omega)=\omega$, segue que so $\omega: I \longrightarrow \tilde{M}$ também é um levantamento de $\omega$ com início em $(s \circ \omega)(0)=s(x)=\mu_{x}$, o que, pela unicidade do levantamento $\tilde{\omega}$, implica que $s \circ \omega=\tilde{\omega})$. Logo, a aplicação $s: M \longrightarrow \tilde{M}$ leva o laço $\omega: I \longrightarrow M$ no caminho (não-fechado) $\tilde{\omega}: I \longrightarrow \tilde{M}, \mathrm{o}$ que contradiz o fato de $s$ ser contínua. Portanto, se $M$ admite uma orientação $s: M \longrightarrow \tilde{M}$, segue que o fibrado orientado $\tilde{M}$ não pode ser conexo, isto é, $M$ deve ser orientável.

(iii) Com efeito, dado $x_{0} \in M$ arbitrário, sabemos que $s: M \longrightarrow \tilde{M}$ é contínua em $x_{0}$ se, e somente se, para toda vizinhança aberta $\tilde{W} \subseteq \tilde{M}$ de $s\left(x_{0}\right)$, existe uma vizinhança $W \subseteq M$ de $x_{0}$, tal que $s(W) \subseteq \tilde{W}$. Por outro lado, sabemos também que a coleção $\mathcal{C}=\left\{U\left(\mu_{V}, V\right): V \subseteq M\right.$ é n-bola própria e $\mu_{V}$ é gerador de $\left.\mathrm{H}_{n}(M, M \backslash V) \simeq \mathbb{Z}\right\}$ 
constitui uma base para a topologia de $\tilde{M}$ (lembre-se que, para cada n-bola própria $V \subseteq M$ e cada gerador $\mu_{V} \in \mathrm{H}_{n}(M, M \backslash V) \simeq \mathbb{Z}$, denotamos $U\left(\mu_{V}, V\right)$ como sendo o conjunto $\left\{\left(j_{y}\right)_{*}\left(\mu_{V}\right): y \in V\right\}$, onde $\left(j_{y}\right)_{*}: \mathrm{H}_{n}(M, M \backslash V) \longrightarrow \mathrm{H}_{n}(M, M \backslash\{y\})$ é o isomorfismo induzido pela inclusão). Logo, dada uma vizinhança aberta qualquer $\tilde{W} \subseteq \tilde{M}$ de $s\left(x_{0}\right)$, segue que existem uma n-bola própria $V \subseteq M$ e um gerador $\mu_{V} \in \mathrm{H}_{n}(M, M \backslash V)$, tais que $s\left(x_{0}\right) \in U\left(\mu_{V}, V\right) \subseteq \tilde{W}$. Deste modo, como a coleção de todas as n-bolas próprias em $M$ constitui uma base para a topologia de $M$, temos que a aplicação $s: M \longrightarrow \tilde{M}$ é contínua em $x_{0}$ se, e somente se, para toda n-bola própria $V \subseteq M$, e todo gerador $\mu_{V} \in \mathrm{H}_{n}(M, M \backslash V)$ satisfazendo $s\left(x_{0}\right) \in U\left(\mu_{V}, V\right)$ [isto é, para todo aberto básico $U\left(\mu_{V}, V\right) \in \mathcal{C}$, contendo $s\left(x_{0}\right)$ ], existe uma n-bola própria $W \subseteq M$, contendo $x_{0}$, tal que $s(W) \subseteq U\left(\mu_{V}, V\right)$. Isto posto, utilizando a continuidade de $s: M \longrightarrow \tilde{M}$ em $x_{0}$, vamos mostrar que, a fim de que $s$ seja contínua em $x_{0}$, é necessário e suficiente que existam uma n-bola própria $V_{0} \subseteq M$, contendo $x_{0}$, e um gerador $\mu_{V_{0}}$ de $H_{n}\left(M, M \backslash V_{0}\right) \simeq \mathbb{Z}$, tais que $\left(j_{y_{0}}^{V_{0}}\right)_{*}\left(\mu_{V_{0}}\right)=s\left(y_{0}\right)$ para todo $y_{0} \in V_{0}$, onde $\left(j_{y_{0}}^{V_{0}}\right)_{*}: \mathrm{H}_{n}\left(M, M \backslash V_{0}\right) \longrightarrow \mathrm{H}_{n}\left(M, M \backslash\left\{y_{0}\right\}\right)$ é o isomorfismo induzido pela inclusão.

De fato, sejam $V \subseteq M$ uma n-bola própria e $\mu_{V}$ um gerador de $\mathrm{H}_{n}(M, M \backslash V)$, com $s\left(x_{0}\right) \in U\left(\mu_{V}, V\right)$; supondo $s: M \longrightarrow \tilde{M}$ contínua em $x_{0}$, temos então que existe uma n-bola própria $W \subseteq M$, contendo $x_{0}$, tal que $s(W) \subseteq U\left(\mu_{V}, V\right)$. No entanto, se $s\left(x_{0}\right) \in U\left(\mu_{V}, V\right)$, segue que existe um $y \in V$ tal que $s\left(x_{0}\right)=\left(j_{y}^{V}\right)_{*}\left(\mu_{V}\right)$; logo, como a composição $\tilde{p} \circ s: M \longrightarrow M$ é a identidade em $M$ (onde $\tilde{p}: \tilde{M} \longrightarrow M$ é a projeção orientada de $M$ ), segue que $x_{0}=\tilde{p}\left(s\left(x_{0}\right)\right)=\tilde{p}\left(\left(j_{y}^{V}\right)_{*}\left(\mu_{V}\right)\right)=y$ (uma vez que $\left(j_{y}^{V}\right)_{*}\left(\mu_{V}\right)$ é um gerador de $\left.H_{n}(M, M \backslash\{y\}) \simeq \mathbb{Z}\right)$, ou seja, $x_{0} \in V$. Agora, considere uma n-bola própria $V_{0} \subseteq M$ tal que $x_{0} \in V_{0} \subseteq V \cap W$ (a qual existe, pois a coleção de todas as n-bolas próprias em $M$ é uma base para a topologia de $M$ ); como $M \backslash V$ é um retrato por deformação de $M \backslash V_{0}$, temos que o homomorfismo $i_{*}: \mathrm{H}_{n}(M, M \backslash V) \longrightarrow \mathrm{H}_{n}\left(M, M \backslash V_{0}\right)$, induzido pela inclusão $i:(M, M \backslash V) \longrightarrow$ $\left(M, M \backslash V_{0}\right)$, constitui um isomorfismo. Seja, pois, $\mu_{V_{0}}$ o gerador $\operatorname{de~}_{n}\left(M, M \backslash V_{0}\right) \simeq$ $\mathbb{Z}$ tal que $i_{*}\left(\mu_{V}\right)=\mu_{V_{0}}$, e, para cada $y \in V$, continuemos a denotar por $\left(j_{y}^{V}\right)_{*}$ : $\mathrm{H}_{n}(M, M \backslash V) \longrightarrow \mathrm{H}_{n}(M, M \backslash\{y\})$ o isomorfismo induzido pela inclusão. Como $s\left(V_{0}\right) \subseteq s(W) \subseteq U\left(\mu_{V}, V\right)=\left\{\left(j_{y}^{V}\right)_{*}\left(\mu_{V}\right): y \in V\right\}$, segue que, para cada $y_{0} \in V_{0}$, existe um $y \in V$ tal que $s\left(y_{0}\right)=\left(j_{y}^{V}\right)_{*}\left(\mu_{V}\right)$; logo, como $\tilde{p} \circ s: M \longrightarrow M$ é a identidade em $M$, obtemos que $y_{0}=\tilde{p}\left(s\left(y_{0}\right)\right)=\tilde{p}\left(\left(j_{y}^{V}\right)_{*}\left(\mu_{V}\right)\right)=y$, isto é, $s\left(y_{0}\right)=\left(j_{y_{0}}^{V}\right)_{*}\left(\mu_{V}\right)$ para cada $y_{0} \in V_{0} \subseteq V$. Portanto, denotando por $\left(j_{y_{0}}^{V_{0}}\right)_{*}: \mathrm{H}_{n}\left(M, M \backslash V_{0}\right) \longrightarrow \mathrm{H}_{n}\left(M, M \backslash\left\{y_{0}\right\}\right)$ o isomorfismo induzido pela inclusão, onde $y_{0} \in V_{0}$, segue, da comutatividade do diagrama abaixo, que $\left(j_{y_{0}}^{V_{0}}\right)_{*}\left(\mu_{V_{0}}\right)=\left[\left(j_{y_{0}}^{V}\right)_{*} \circ i_{*}^{-1}\right]\left(\mu_{V_{0}}\right)=\left(j_{y_{0}}^{V}\right)_{*}\left(\mu_{V}\right)=s\left(y_{0}\right)$ para todo $y_{0} \in V_{0}$. Em outras palavras, a continuidade de $s: M \longrightarrow \tilde{M}$ no ponto $x_{0}$ 
implica que existem uma n-bola própria $V_{0} \subseteq M$, contendo $x_{0}$, e um gerador $\mu_{V_{0}}$ de $\mathrm{H}_{n}\left(M, M \backslash V_{0}\right)$, tais que $\left(j_{y_{0}}^{V_{0}}\right)_{*}\left(\mu_{V_{0}}\right)=s\left(y_{0}\right)$ para cada $y_{0} \in V_{0}$.

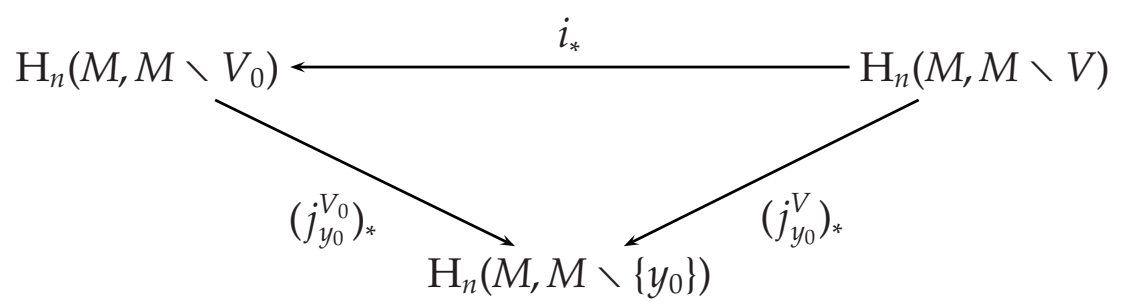

Reciprocamente, suponhamos então que existam uma n-bola própria $V_{0} \subseteq M$, contendo $x_{0}$, e um gerador $\mu_{V_{0}} \in \mathrm{H}_{n}\left(M, M \backslash V_{0}\right)$, tais que $\left(j_{y_{0}}\right)_{*}\left(\mu_{V_{0}}\right)=s\left(y_{0}\right)$ para todo $y_{0} \in V_{0}$ (onde $\left(j_{y_{0}}^{V_{0}}\right)_{*}: \mathrm{H}_{n}\left(M, M \backslash V_{0}\right) \longrightarrow \mathrm{H}_{n}\left(M, M \backslash\left\{y_{0}\right\}\right)$ é o isomorfismo induzido pela inclusão), e seja $V \subseteq M$ uma n-bola própria satisfazendo $s\left(x_{0}\right) \in U\left(\mu_{V}, V\right)$, para algum gerador $\mu_{V} \in \mathrm{H}_{n}(M, M \backslash V) \simeq \mathbb{Z}$. Conforme vimos há pouco, o fato de termos $s\left(x_{0}\right) \in U\left(\mu_{V}, V\right)$ nos garante que $x_{0} \in V$. Considere, pois, uma n-bola própria $W \subseteq M$ tal que $x_{0} \in W \subseteq V \cap V_{0}$ (a qual existe, uma vez que a coleção de todas as n-bolas próprias em $M$ é uma base para a topologia de $M$ ); como $\left(j_{y_{0}}^{V_{0}}\right)_{*}\left(\mu_{V_{0}}\right)=s\left(y_{0}\right)$ para todo $y_{0} \in V_{0}$, segue, em particular, que $\left(j_{x_{0}}^{V_{0}}\right)_{*}\left(\mu_{V_{0}}\right)=s\left(x_{0}\right)$ e $\left(j_{w}^{V_{0}}\right)_{*}\left(\mu_{V_{0}}\right)=s(w)$ para cada $w \in W \subseteq V_{0}$. Agora, observando que $M \backslash V$ e $M \backslash V_{0}$ são retratos por deformação de $M \backslash W$, segue que os homomorfismos $\left(i_{V}\right)_{*}: \mathrm{H}_{n}(M, M \backslash$ $V) \longrightarrow \mathrm{H}_{n}(M, M \backslash W)$ e $\left(i_{V_{0}}\right)_{*}: \mathrm{H}_{n}\left(M, M \backslash V_{0}\right) \longrightarrow \mathrm{H}_{n}(M, M \backslash W)$, induzidos pelas inclusões $i_{V}:(M, M \backslash V) \longrightarrow(M, M \backslash W)$ e $i_{V_{0}}:\left(M, M \backslash V_{0}\right) \longrightarrow(M, M \backslash W)$, constituem isomorfismos. Isto posto, para cada $y \in V$ e cada $w \in W$, denote por $\left(j_{y}^{V}\right)_{*}: \mathrm{H}_{n}(M, M \backslash V) \longrightarrow \mathrm{H}_{n}(M, M \backslash\{y\})$ e $\left(j_{w}^{W}\right)_{*}: \mathrm{H}_{n}(M, M \backslash W) \longrightarrow \mathrm{H}_{n}(M, M \backslash\{w\})$ os isomorfismos induzidos pelas respectivas inclusões, e, dado $w \in W \subseteq V \cap V_{0}$, considere o seguinte diagrama comutativo:

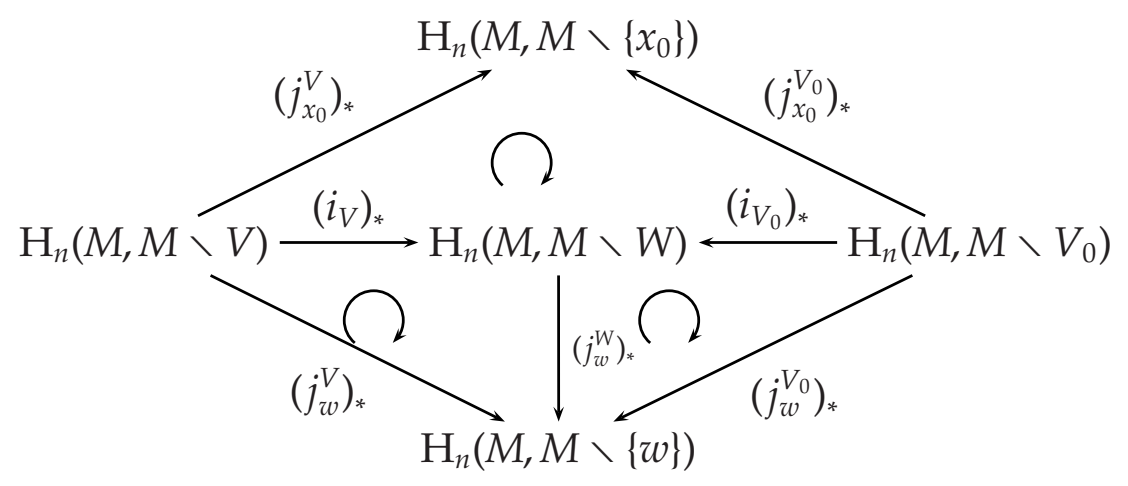

(note que a comutatividade do diagrama segue do fato de que os isomorfismos representados são todos induzidos por inclusões). Assim, como $\left(j_{x_{0}}^{V}\right)_{*}\left(\mu_{V}\right)=s\left(x_{0}\right)$ (pois $s\left(x_{0}\right) \in U\left(\mu_{V}, V\right)$ e $\tilde{p} \circ s: M \longrightarrow M$ é a identidade em $M$ ), podemos concluir 
que:

$$
\begin{aligned}
\left(j_{w}^{V}\right)_{*}\left(\mu_{V}\right) & =\left[\left(j_{w}^{W}\right)_{*} \circ\left(i_{V}\right)_{*}\right]\left(\mu_{V}\right)=\left\{\left(j_{w}^{W}\right)_{*} \circ\left[\left(i_{V_{0}}\right)_{*} \circ\left(j_{x_{0}}^{V_{0}}\right)_{*}^{-1} \circ\left(j_{x_{0}}^{V}\right)_{*}\right]\right\}\left(\mu_{V}\right) \\
& =\left[\left(j_{w}^{W}\right)_{*} \circ\left(i_{V_{0}}\right)_{*} \circ\left(j_{x_{0}}^{V_{0}}\right)_{*}^{-1}\right]\left(s\left(x_{0}\right)\right)=\left[\left(j_{w}^{W}\right)_{*} \circ\left(i_{V_{0}}\right)_{*}\right]\left(\mu_{V_{0}}\right) \\
& =\left(j_{w}^{V_{0}}\right)_{*}\left(\mu_{V_{0}}\right)=s(w)
\end{aligned}
$$

isto é, $\left(j_{w}^{V}\right)_{*}\left(\mu_{V}\right)=s(w)$ para todo $w \in W$. Portanto:

$$
s(W) \subseteq\left\{\left(j_{w}^{V}\right)_{*}\left(\mu_{V}\right): w \in W \subseteq V\right\} \subseteq\left\{\left(j_{y}^{V}\right)_{*}\left(\mu_{V}\right): y \in V\right\}=U\left(\mu_{V}, V\right),
$$

o que mostra que, para toda n-bola própria $V \subseteq M$, e todo gerador $\mu_{V} \in \mathrm{H}_{n}(M, M \backslash$ $V$ ) satisfazendo $s\left(x_{0}\right) \in U\left(\mu_{V}, V\right)$, existe uma n-bola própria $W \subseteq M$, contendo $x_{0}$, tal que $s(W) \subseteq U\left(\mu_{V}, V\right)$, o que significa que $s: M \longrightarrow \tilde{M}$ é contínua em $x_{0}$. Em resumo, temos então que $s$ é contínua em $x_{0}$ se, e somente se, existem uma n-bola própria $V_{0} \subseteq M$, contendo $x_{0}$, e um gerador $\mu_{V_{0}}$ de $\mathrm{H}_{n}\left(M, M \backslash V_{0}\right)$, tais que $\left(j_{y_{0}}^{V_{0}}\right)_{*}\left(\mu_{V_{0}}\right)=s\left(y_{0}\right)$ para cada $y_{0} \in V_{0}$, conforme queríamos provar.

Finalmente, como $s: M \longrightarrow \tilde{M}$ é contínua se, e só se, ela for contínua em cada ponto de $M$, segue que $s$ será contínua se, e somente se, para todo ponto $x \in M$, existirem uma n-bola própria $V \subseteq M$, contendo $x$, e um gerador $\mu_{V} \in \mathrm{H}_{n}(M, M \backslash V) \simeq \mathbb{Z}$, tais que $\left(j_{y}\right)_{*}\left(\mu_{V}\right)=s(y)$ para cada $y \in V$ (onde $\left(j_{y}\right)_{*}: \mathrm{H}_{n}(M, M \backslash V) \longrightarrow \mathrm{H}_{n}(M, M \backslash\{y\})$ é o isomorfismo induzido pela inclusão), o que encerra a demonstração.

Intuitivamente, uma orientação $s: M \longrightarrow \tilde{M}$ pode ser vista como uma "escolha contínua" de orientações locais para os pontos de $M$, de tal forma que o diagrama abaixo seja comutativo (note que $s$ é um levantamento da aplicação identidade $\operatorname{Id}_{M}: M \longrightarrow M$, relativamente à projeção orientada $\tilde{p}: \tilde{M} \longrightarrow M$ ).

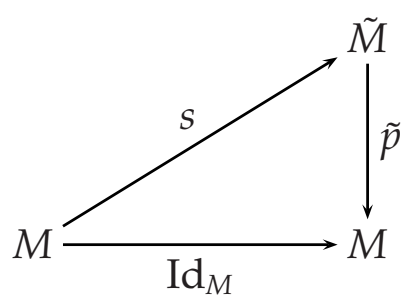

Convém observarmos ainda que, se $M$ for uma variedade topológica, de dimensão $n$ e sem fronteira, mas não-conexa, a orientabilidade de $M$ fica condicionada à orientabilidade de cada uma de suas componentes conexas, isto é, $M$ será considerada orientável se, e somente se, todas as suas componentes conexas assim o forem; neste caso, denotando por $m \geqslant 1$ o número de componentes de $M$, podemos ver que há $2^{m}$ maneiras distintas de orientar $M$, posto que cada componente conexa $C$ de $M$ admitirá 
duas orientações distintas (cada uma assumindo valores numa das duas componentes do fibrado orientado $\tilde{C}$ de $C$ ).

Exemplo 1.1.1. Pode-se verificar (embora não o faremos aqui) que o espaço euclidiano $\mathbb{R}^{n}$, a esfera unitária n-dimensional e o toro $\mathbb{T}^{2}$ são exemplos de variedades topológicas orientáveis, enquanto que o plano projetivo real $\mathbb{R P}^{2}$ e a garrafa de Klein $\mathbb{K}$ são variedades não-orientáveis.

Exemplo 1.1.2. Se Mé uma variedade topológica conexa, contrátil e sem fronteira, então $M$ é orientável: com efeito, se $M$ é contrátil, segue que $M$ é simplesmente conexa, e, portanto, todo recobrimento de $M$ constitui um homeomorfismo (vide [Li], proposição 11, página 136); isto, por sua vez, implica que o fibrado orientado de $M$ não pode ser conexo, pois, neste caso, a projeção orientada de $M$ seria um recobrimento de duas folhas (e não um homeomorfismo).

Proposição 1.1.1. Sejam $M$ e N variedades topológicas de dimensão n, conexas e sem fronteira. Se $f: M \longrightarrow N$ é um homeomorfismo local, e N é orientável, então M também é orientável.

Demonstração. Sendo $N$ orientável, considere uma orientação $s_{N}: N \longrightarrow \tilde{C}_{N}$, onde $\tilde{C}_{N}$ é uma das duas componentes conexas do fibrado orientado $\tilde{N}$. Dado um ponto $x \in M$, sabemos (pela continuidade de $s_{N}$ ) que existem uma n-bola própria $V \subseteq N$, com $f(x) \in V$, e um gerador $\mu_{V} \in \mathrm{H}_{n}(N, N \backslash V)$, tais que $\left(i_{y}^{V}\right)_{*}\left(\mu_{V}\right)=s_{N}(y)$ para todo $y \in V$, onde $\left(i_{y}^{V}\right)_{*}: \mathrm{H}_{n}(N, N \backslash V) \longrightarrow \mathrm{H}_{n}(N, N \backslash\{y\})$ é o isomorfismo induzido pela inclusão (vide item (iii) do lema 1.1.1). Seja, pois, $U \subseteq f^{-1}(V)$ uma n-bola própria contendo $x$, tal que $W=f(U) \subseteq V$ é aberto (e conexo) em $M$, contendo $f(x)$, e $\left.f\right|_{U}$ é um homeomorfismo sobre $W$. Denotando por $i_{*}: \mathrm{H}_{n}(N, N \backslash V) \longrightarrow \mathrm{H}_{n}(N, N \backslash W)$ o homomorfismo induzido pela inclusão, temos que $\mu_{W}=i_{*}\left(\mu_{V}\right) \in \mathrm{H}_{n}(N, N \backslash W)$ é tal que $\left(i_{y}\right)_{*}\left(\mu_{W}\right)=s_{N}(y)$ para todo $y \in V \cap W$, onde $\left(i_{y}\right)_{*}: \mathrm{H}_{n}(N, N \backslash W) \longrightarrow \mathrm{H}_{n}(N, N \backslash\{y\})$ é o isomorfismo induzido pela inclusão: com efeito, da comutatividade do diagrama abaixo, segue que $\left(i_{y}\right)_{*}\left(\mu_{W}\right)=\left(i_{y}\right)_{*}\left(i_{*}\left(\mu_{V}\right)\right)=\left(i_{y}^{V}\right)_{*}\left(\mu_{V}\right)=s_{N}(y)$, para cada $y \in V \cap W$.

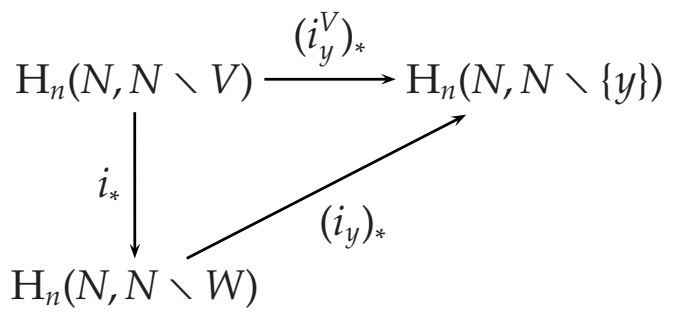

Além disso, do teorema da excisão em homologia singular, sabemos que os homomorfismos $\left(j_{x}^{U}\right)_{*}: \mathrm{H}_{n}(U, U \backslash\{x\}) \longrightarrow \mathrm{H}_{n}(M, M \backslash\{x\})$ e $\left(i_{f(x)}^{W}\right)_{*}: \mathrm{H}_{n}(W, W \backslash\{f(x)\}) \longrightarrow$ 
$\mathrm{H}_{n}(N, N \backslash\{f(x)\})$, induzidos pelas respectivas inclusões, são isomorfismos, assim como o homomorfismo $\left(\left.f\right|_{U}\right)_{*}^{x}: \mathrm{H}_{n}(U, U \backslash\{x\}) \longrightarrow \mathrm{H}_{n}(W, W \backslash\{f(x)\})$ induzido por $\left.f\right|_{U}$. Logo, vamos definir a orientação de $M$ no ponto $x$ como sendo o único gerador $\mu_{x}$ de $\mathrm{H}_{n}(M, M \backslash\{x\})$ que é levado no gerador $s_{N}(f(x))$ de $\mathrm{H}_{n}(N, N \backslash\{f(x)\})$ pela seguinte cadeia de isomorfismos:

$$
\begin{aligned}
\mathrm{H}_{n}(M, M \backslash\{x\}) & \\
\left.\left(j_{x}^{U}\right)_{*}^{-1}\right|_{n}(U, U \backslash\{x\}) \stackrel{\left(\left.f\right|_{U}\right)_{*}^{x}}{\longrightarrow} & \mathrm{H}_{n}(W, W \backslash\{f(x)\}) \\
& \left.\downarrow i_{f(x)}^{W}\right)_{*} \\
& \mathrm{H}_{n}(N, N \backslash\{f(x)\})
\end{aligned}
$$

(note que $s_{N}(f(x))$ é, de fato, um gerador de $\mathrm{H}_{n}(N, N \backslash\{f(x)\})$, conforme nos garante o item (i) do lema 1.1.1). Considere ainda o gerador $\mu_{U} \in \mathrm{H}_{n}(M, M \backslash U)$ que é levado em $\mu_{W} \in \mathrm{H}_{n}(N, N \backslash W)$ pelos isomorfismos dados no diagrama abaixo:

$$
\begin{aligned}
& \mathrm{H}_{n}(M, M \backslash U) \\
& \mathrm{H}_{n}(M, M \backslash\{x\}) \stackrel{\left.\left.\left(j_{x}\right)_{*}\right|_{*} ^{U}\right)_{*}^{-1}}{\longrightarrow} \mathrm{H}_{n}(U, U \backslash\{x\}) \\
& \left(\left.f\right|_{U}\right)_{*}^{x} \mathrm{H}_{n}(W, W \backslash\{f(x)\}) \stackrel{\left(i_{f(x)}^{W}\right)_{*}}{\longrightarrow} \mathrm{H}_{n}(N, N \backslash\{f(x)\}) \\
& \downarrow\left(i_{f(x)}\right)_{*}^{-1} \\
& \mathrm{H}_{n}(N, N \backslash W)
\end{aligned}
$$

Temos então que $\left(j_{z}\right)_{*}\left(\mu_{U}\right)=\mu_{z}$ para cada $z \in U$, onde $\left(j_{z}\right)_{*}: \mathrm{H}_{n}(M, M \backslash U) \longrightarrow \mathrm{H}_{n}(M, M \backslash$ $\{z\})$ é o isomorfismo induzido pela inclusão (obviamente, estamos denotando por $\mu_{z}$ a orientação de $M$ no ponto $z$, obtida de maneira análoga à $\mu_{x}$ ). De fato, dado $z \in U$ arbitrário (e observando que $f(z) \in W$, pois $\left.f\right|_{u}$ é um homeomorfismo sobre $W$ ), temos 
o seguinte diagrama comutativo:

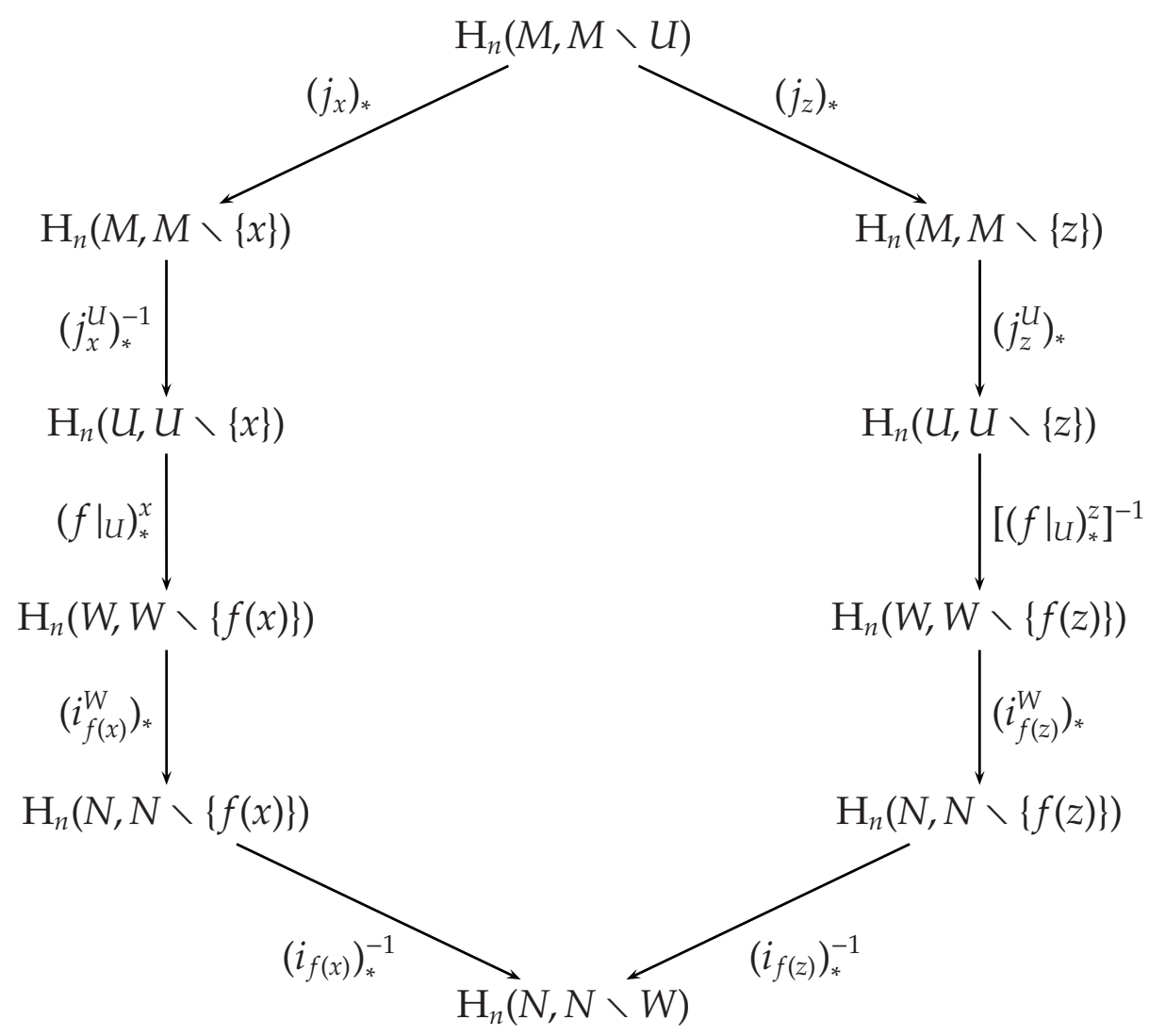

Utilizando este diagrama, podemos ver que os isomorfismos do ramo esquerdo levam $\mu_{U} \in \mathrm{H}_{n}(M, M \backslash U)$ em $\mu_{W} \in \mathrm{H}_{n}(N, N \backslash W)$, o qual, por sua vez, é levado por $\left(i_{f(z)}\right)_{*}$ em $s_{N}(f(z)) \in \mathrm{H}_{n}(N, N \backslash\{f(z)\})$; entretanto, sabemos (por definição) que a composição $\left(j_{z}^{U}\right)_{*}^{-1} \circ\left[\left(\left.f\right|_{U}\right)_{*}^{z}\right] \circ\left(i_{f(z)}^{W}\right)_{*}$ leva $s_{N}(f(z))$ no gerador $\mu_{z} \in \mathrm{H}_{n}(M, M \backslash\{z\})$. Portanto, como o resultado deste procedimento coincide com a imagem de $\mu_{U}$ por $\left(j_{z}\right)_{*}$, concluímos que $\left(j_{z}\right)_{*}\left(\mu_{U}\right)=\mu_{z}$.

Agora, suponha que $U^{\prime} \subseteq f^{-1}(V)$ seja outra n-bola própria contendo $x$, tal que $W^{\prime}=f\left(U^{\prime}\right) \subseteq V$ é aberto (e conexo) em $M$, com $f(x) \in W^{\prime}$, e $f l_{U^{\prime}}$ é um homeomorfismo sobre $W^{\prime}$. Neste caso, a orientação de $M$ no ponto $x$ poderia ser o gerador $\mu_{x}^{\prime}$ de $\mathrm{H}_{n}(M, M \backslash\{x\})$, que é levado em $s_{N}(f(x)) \in \mathrm{H}_{n}(N, N \backslash\{f(x)\})$ pela seguinte cadeia de isomorfismos:

$$
\begin{aligned}
& \mathrm{H}_{n}(M, M \backslash\{x\}) \stackrel{\left(j_{x}^{U^{\prime}}\right)_{*}^{-1}}{\longrightarrow} \mathrm{H}_{n}\left(U^{\prime}, U^{\prime} \backslash\{x\}\right) \\
& \downarrow\left(\left.f\right|_{U^{\prime}}\right)_{*}^{x} \\
& \mathrm{H}_{n}\left(W^{\prime}, W^{\prime} \backslash\{f(x)\}\right) \underset{\left(i_{f(x)}^{W^{\prime}}\right)_{*}}{\longrightarrow} \mathrm{H}_{n}(N, N \backslash\{f(x)\})
\end{aligned}
$$


Entretanto, a comutatividade do diagrama abaixo nos garante que $\mu_{x}^{\prime}=\mu_{x}$.

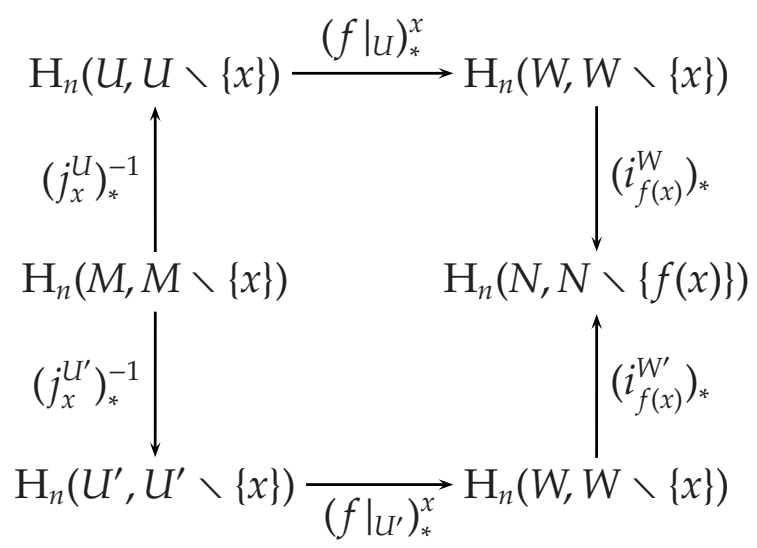

Finalmente, sendo $\tilde{M}$ o fibrado orientado de $M$, considere a aplicação $s_{M}: M \longrightarrow \tilde{M}$, que, a cada $x \in M$, associa o gerador $\mu_{x} \in \mathrm{H}_{n}(M, M \backslash\{x\})$ obtido conforme descrito acima. Denotando por $\tilde{p}_{M}: \tilde{M} \longrightarrow M$ a projeção orientada de $M$, segue que a composição $\tilde{p}_{M} \circ s_{M}: M \longrightarrow M$ é a identidade em $M$; além disso, pelos fatos que acabamos de demonstrar, temos que $s_{M}$ está bem definida e é contínua em $M$ (vide lema 1.1.1). Logo, $s_{M}$ define uma orientação em $M$, o que significa que $M$ é uma variedade orientável, como queríamos provar.

Proposição 1.1.2 . Sendo M uma variedade topológica de dimensão n, conexa e sem fronteira, temos que o fibrado orientado $\tilde{M}$ é uma variedade orientável, independentemente da orientabilidade de $M$.

DemonstraçÃo. Primeiramente, suponhamos que $M$ seja orientável, e, denotando por $\tilde{C}_{1}$ e $\tilde{C}_{2}$ as componentes conexas do fibrado orientado $\tilde{M}$, considere os recobrimentos $\tilde{p}_{1}=\left.\tilde{p}\right|_{\tilde{C}_{1}}: \tilde{C}_{1} \longrightarrow M$ e $\tilde{p}_{2}=\left.\tilde{p}\right|_{\tilde{C}_{2}}: \tilde{C}_{2} \longrightarrow M$, obtidos pela restrição da projeção orientada $\tilde{p}: \tilde{M} \longrightarrow M$ a $\tilde{C}_{1}$ e $\tilde{C}_{2}$, respectivamente. Como $\tilde{p}_{1}$ e $\tilde{p}_{2}$ são homeomorfismos locais, segue (da proposição 1.1.1) que $\tilde{C}_{1}$ e $\tilde{C}_{2}$ são orientáveis, e, portanto, o fibrado orientado $\tilde{M}=\tilde{C}_{1} \cup \tilde{C}_{2}$ é orientável. Suponhamos então que $M$ seja não-orientável; neste caso, temos que $\tilde{M}$ é conexo, e a projeção orientada $\tilde{p}: \tilde{M} \longrightarrow M$ é um recobrimento de duas folhas. Isto posto, dado $\tilde{x} \in \tilde{M}$, seja $x=\tilde{p}(\tilde{x}) \in M$ (de modo, que $\tilde{x}$ é, portanto, um gerador de $\left.\mathrm{H}_{n}(M, M \backslash\{x\})\right)$, e considere uma n-bola própria $V \subseteq M$, contendo $x$, que constitui uma vizinhança elementar em relação ao espaço de recobrimento $\tilde{M}$ (isto é, tal que $\tilde{p}^{-1}(V)$ é uma união disjunta de abertos em $\tilde{M}$, cada um dos quais se aplica por $\tilde{p}$ homeomorficamente sobre $V$ ). Sendo $\tilde{V} \subseteq \tilde{M}$ a componente conexa de $\tilde{p}^{-1}(V)$ contendo $\tilde{x}$, temos que $\left.\tilde{p}\right|_{\tilde{V}}: \tilde{V} \longrightarrow V$ é um homeomorfismo sobre $V$, que leva $\tilde{x}$ em $x$; logo, o homomorfismo $\left(\left.\tilde{p}\right|_{\tilde{V}}\right)_{*}: \mathrm{H}_{n}(\tilde{V}, \tilde{V} \backslash\{\tilde{x}\}) \longrightarrow \mathrm{H}_{n}(V, V \backslash\{x\})$, induzido 
por $\left.\tilde{p}\right|_{\tilde{V}}$, é um isomorfismo. Por outro lado, do teorema da excisão em homologia

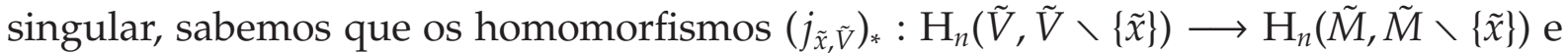
$\left(j_{x, V}\right)_{*}: \mathrm{H}_{n}(V, V \backslash\{x\}) \longrightarrow \mathrm{H}_{n}(M, M \backslash\{x\})$, induzidos pelas inclusões $j_{\tilde{x}, \tilde{V}}:(\tilde{V}, \tilde{V} \backslash\{\tilde{x}\}) \longrightarrow$ $(\tilde{M}, \tilde{M} \backslash\{\tilde{x}\})$ e $j_{x, V}:(V, V \backslash\{x\}) \longrightarrow(M, M \backslash\{x\})$, também são isomorfismos. Assim, definimos a orientação de $\tilde{M}$ em $\tilde{x}$ como sendo o gerador $\tilde{s}(\tilde{x})$ de $\mathrm{H}_{n}(\tilde{M}, \tilde{M} \backslash\{\tilde{x}\})$ que é levado no gerador $\tilde{x} \in \mathrm{H}_{n}(M, M \backslash\{x\})$ pela seguinte cadeia de isomorfismos:

$$
\begin{aligned}
\mathrm{H}_{n}(\tilde{M}, \tilde{M} \backslash\{\tilde{x}\}) \stackrel{\left(j_{\tilde{x}, \tilde{V})_{*}^{-1}}^{\longrightarrow}\right.}{ } & \mathrm{H}_{n}(\tilde{V}, \tilde{V} \backslash\{\tilde{x}\}) \\
& \stackrel{\downarrow}{\left(\left.\tilde{p}\right|_{\tilde{V}}\right)_{*}} \\
& \mathrm{H}_{n}(V, V \backslash\{x\}) \underset{\left(j_{x, V}\right)_{*}}{\longrightarrow} \mathrm{H}_{n}(M, M \backslash\{x\})
\end{aligned}
$$

Note que $\tilde{s}(\tilde{x})$ não depende da n-bola própria $V \subseteq M$ que tomamos inicialmente: de fato, dada outra n-bola própria $V^{\prime} \subseteq M$, com $x \in V^{\prime}$, que constitui uma vizinhança elementar relativamente ao espaço de recobrimento $\tilde{M}$, e denotando por $\tilde{V}^{\prime} \subseteq \tilde{M}$ a componente conexa de $\tilde{p}^{-1}\left(V^{\prime}\right)$ contendo $\tilde{x}$, temos que a restrição $\left.\tilde{p}\right|_{\tilde{V}^{\prime}}: \tilde{V}^{\prime} \longrightarrow V^{\prime}$ é um homeomorfismo sobre $V^{\prime}$ (que leva $\tilde{x}$ em $x$ ), e, conseqüentemente, o homomorfismo induzido $\left(\left.\tilde{p}\right|_{\tilde{V}^{\prime}}\right)_{*}: \mathrm{H}_{n}\left(\tilde{V}^{\prime}, \tilde{V}^{\prime} \backslash\{\tilde{x}\}\right) \longrightarrow \mathrm{H}_{n}\left(V^{\prime}, V^{\prime} \backslash\{x\}\right)$ é um isomorfismo; logo, sendo $\left(j_{\tilde{x}, \tilde{V}^{\prime}}\right)_{*}: \mathrm{H}_{n}\left(\tilde{V}^{\prime}, \tilde{V}^{\prime} \backslash\{\tilde{x}\}\right) \longrightarrow \mathrm{H}_{n}(\tilde{M}, \tilde{M} \backslash\{\tilde{x}\})$ e $\left(j_{x, V^{\prime}}\right)_{*}: \mathrm{H}_{n}\left(V^{\prime}, V^{\prime} \backslash\{x\}\right) \longrightarrow \mathrm{H}_{n}(M, M \backslash\{x\})$ os isomorfismos induzidos pelas respectivas inclusões, segue da comutatividade do diagrama abaixo que o gerador $\tilde{s}(\tilde{x}) \in \mathrm{H}_{n}(\tilde{M}, \tilde{M} \backslash\{\tilde{x}\})$, correspondente a $\tilde{x} \in \mathrm{H}_{n}(M, M \backslash$ $\{x\})$ pelo isomorfismo $\left(j_{x, V}\right)_{*} \circ\left(\left.\tilde{p}\right|_{\tilde{V}}\right)_{*} \circ\left(j_{\tilde{x}, \tilde{V}}\right)_{*}^{-1}$, coincide com o gerador de $\mathrm{H}_{n}(\tilde{M}, \tilde{M} \backslash\{\tilde{x}\})$ que é levado em $\tilde{x}$ pelo isomorfismo $\left(j_{x, V^{\prime}}\right)_{*} \circ\left(\left.\tilde{p}\right|_{\tilde{V}^{\prime}}\right)_{*} \circ\left(j_{\tilde{x}, \tilde{V}^{\prime}}\right)_{*}^{-1}$.

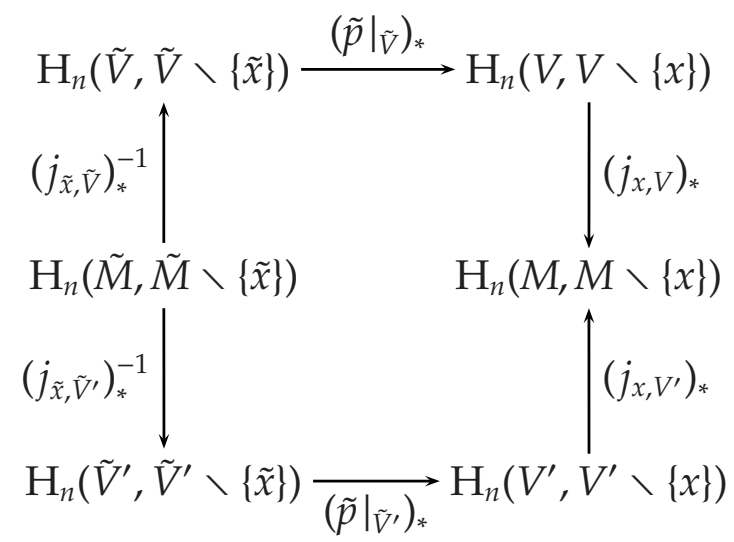

Deste modo, denotando por $\widetilde{\widetilde{M}}$ o fibrado orientado de $\tilde{M}$ (que é a variedade topológica constituída exclusivamente dos geradores dos grupos $\mathrm{H}_{n}(\tilde{M}, \tilde{M} \backslash\{\tilde{x}\})$, onde $\tilde{x} \in \tilde{M})$, seja $\tilde{s}: \tilde{M} \longrightarrow \widetilde{\widetilde{M}}$ a aplicação que, a cada $\tilde{x} \in \tilde{M}$, associa o gerador $\tilde{s}(\tilde{x}) \in$ 
$\mathrm{H}_{n}(\tilde{M}, \tilde{M} \backslash\{\tilde{x}\})$ segundo o procedimento acima; vamos mostrar então que $\tilde{s}$ define uma orientação em $\tilde{M}$. Com efeito, sendo $\tilde{\tilde{p}}: \widetilde{\widetilde{M}} \longrightarrow \tilde{M}$ a fibração orientada de $\tilde{M}$ (dada por $\tilde{\tilde{p}}(\tilde{\tilde{x}})=\tilde{x}$ para cada gerador $\tilde{\tilde{x}} \in \mathrm{H}_{n}(\tilde{M}, \tilde{M} \backslash\{\tilde{x}\})$, onde $\left.\tilde{x} \in \tilde{M}\right)$, temos de imediato que $\tilde{\tilde{p}} \circ \tilde{s}=\operatorname{Id}_{\tilde{M}}$, onde $\operatorname{Id}_{\tilde{M}}: \tilde{M} \longrightarrow \tilde{M}$ é a identidade. Agora, a fim de mostrarmos que $\tilde{s}$ é contínua em $\tilde{M}$, tomemos um ponto $\tilde{x} \in \tilde{M}$ arbitrário, e consideremos uma n-bola própria $V \subseteq M$, contendo o ponto $x=\tilde{p}(\tilde{x})$, que constitui uma vizinhança elementar em relação ao espaço de recobrimento $\tilde{M}$. Sendo $\tilde{V} \subseteq \tilde{M}$ a componente conexa de $\tilde{p}^{-1}(V)$ que contém $\tilde{x}$, temos, pois, que a restrição $\left.\tilde{p}\right|_{\tilde{V}}: \tilde{V} \longrightarrow V$ é um homeomorfismo sobre $V ; \operatorname{logo}$, dado outro ponto $\tilde{y} \in \tilde{V}$, segue que $\tilde{y}$ é o único gerador de $\mathrm{H}_{n}(M, M \backslash\{y\})$ tal que $\tilde{y} \in \tilde{V}$, onde $y=\tilde{p}(\tilde{y}) \in V$. Por outro lado, denotando por $\left(j_{x}\right)_{*}: \mathrm{H}_{n}(M, M \backslash V) \longrightarrow \mathrm{H}_{n}(M, M \backslash\{x\})$ e $\left(j_{\tilde{x}}\right)_{*}: \mathrm{H}_{n}(\tilde{M}, \tilde{M} \backslash \tilde{V}) \longrightarrow \mathrm{H}_{n}(\tilde{M}, \tilde{M} \backslash\{\tilde{x}\})$ os isomorfismos induzidos pelas inclusões $j_{x}:(M, M \backslash V) \longrightarrow(M, M \backslash\{x\})$ e $j_{\tilde{x}}:(\tilde{M}, \tilde{M} \backslash \tilde{V}) \longrightarrow(\tilde{M}, \tilde{M} \backslash\{\tilde{x}\})$, respectivamente, sejam $\mu_{V} \in \mathrm{H}_{n}(M, M \backslash V)$ e $\mu_{\tilde{V}} \in \mathrm{H}_{n}(\tilde{M}, \tilde{M} \backslash \tilde{V})$ os geradores tais que $\left(j_{x}\right)_{*}\left(\mu_{V}\right)=\tilde{x}$ e $\left(j_{\tilde{x}}\right)_{*}\left(\mu_{\tilde{V}}\right)=\tilde{s}(\tilde{x})$, onde $\tilde{s}(\tilde{x})$ é o gerador de $\mathrm{H}_{n}(\tilde{M}, \tilde{M} \backslash\{\tilde{x}\})$ definido há pouco (note que $\tilde{x}$ é um gerador de $\mathrm{H}_{n}(M, M \backslash\{x\})$ ). Considere ainda o diagrama comutativo abaixo, onde $\left(j_{x, V}\right)_{*}\left(j_{\tilde{x}, \tilde{V}}\right)_{*},\left(j_{y, V}\right)_{*},\left(j_{\tilde{y}, \tilde{V}}\right)_{*},\left(j_{y}\right)_{*}$ e $\left(j_{\tilde{y}}\right)_{*}$ são os isomorfismos induzidos pelas respectivas inclusões:

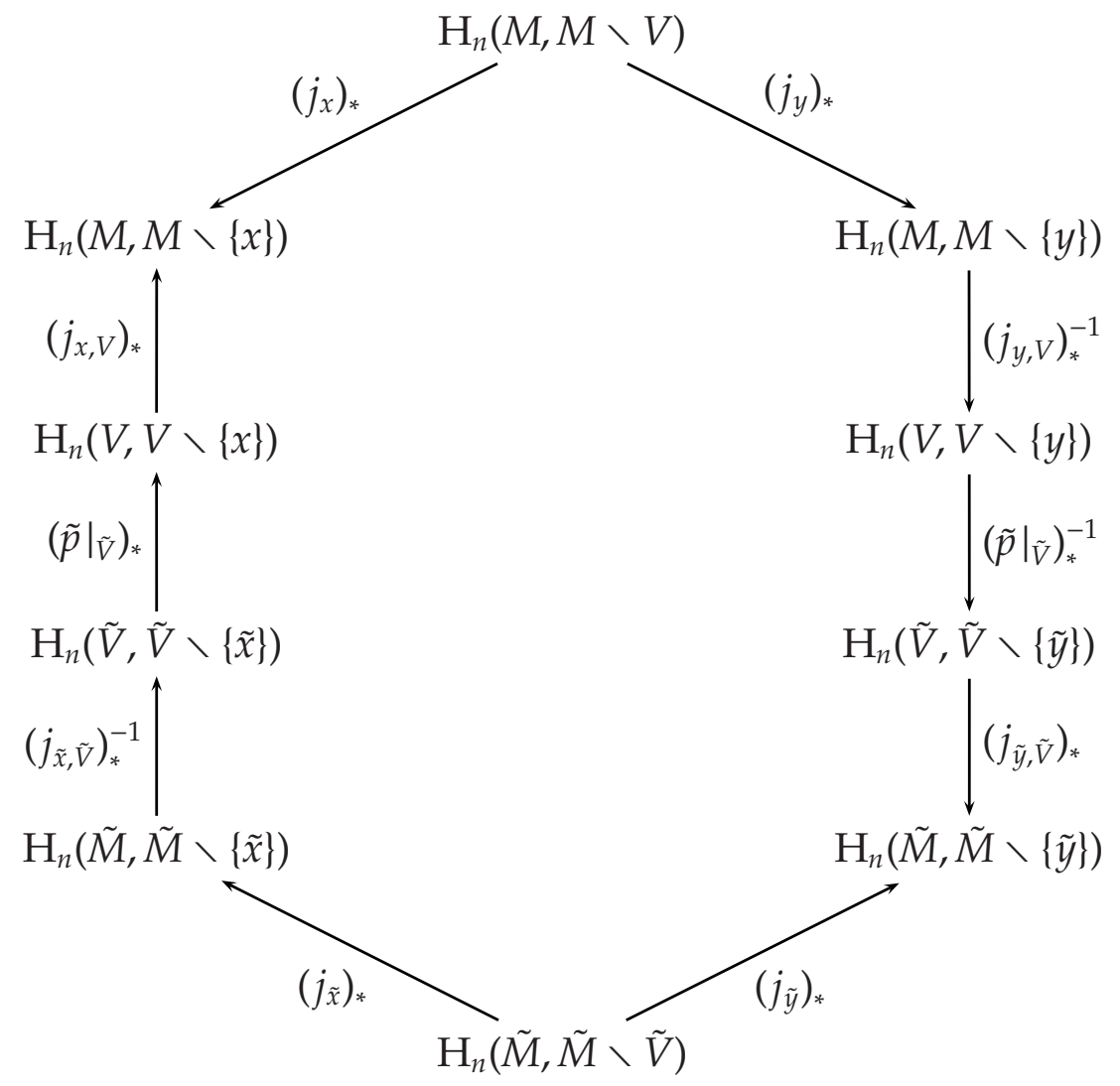

Por definição, sabemos que $\tilde{x}=\left[\left(j_{x, V}\right)_{*} \circ\left(\left.\tilde{p}\right|_{\tilde{V}}\right)_{*} \circ\left(j_{\tilde{x}, \tilde{V}}\right)_{*}^{-1}\right](\tilde{s}(\tilde{x}))$ e $\tilde{s}\left(\left(j_{y}\right)_{*}\left(\mu_{V}\right)\right) \tilde{x}=\left[\left(j_{\tilde{y}, \tilde{V}}\right)_{*} \circ\right.$ $\left.\left(\left.\tilde{p}\right|_{\tilde{V}}\right)_{*}^{-1} \circ\left(j_{y, V}\right)_{*}^{-1}\right]\left(\left(j_{y}\right)_{*}\left(\mu_{V}\right)\right)$; conseqüentemente, pela comutatividade do diagrama acima, 
obtemos que:

$$
\begin{aligned}
& \left(j_{\tilde{y}}\right)_{*}\left(\mu_{\tilde{V}}\right)=\left[\left(j_{\tilde{y}, \tilde{V}}\right)_{*} \circ\left(\left.\tilde{p}\right|_{\tilde{V}}\right)_{*}^{-1} \circ\left(j_{y, V}\right)_{*}^{-1} \circ\left(j_{y}\right)_{*} \circ\left(j_{x}\right)_{*}^{-1} \circ\right. \\
& \left.\left(j_{x, V}\right)_{*} \circ\left(\left.\tilde{p}\right|_{\tilde{V}}\right)_{*} \circ\left(j_{\tilde{x}, \tilde{V}}\right)_{*}^{-1} \circ\left(j_{\tilde{x}}\right)_{*}\right]\left(\mu_{\tilde{V}}\right) \\
& =\left[\left(j_{\tilde{y}, \tilde{V}}\right)_{*} \circ\left(\left.\tilde{p}\right|_{\tilde{V}}\right)_{*}^{-1} \circ\left(j_{y, V}\right)_{*}^{-1} \circ\left(j_{y}\right)_{*} \circ\left(j_{x}\right)_{*}^{-1} \circ\right. \\
& \left.\left(j_{x, V}\right)_{*} \circ\left(\left.\tilde{p}\right|_{\tilde{V}}\right)_{*} \circ\left(j_{\tilde{x}, \tilde{V}}\right)_{*}^{-1}\right](\tilde{s}(\tilde{x})) \\
& =\left[\left(j_{\tilde{y}, \tilde{V}}\right)_{*} \circ\left(\left.\tilde{p}\right|_{\tilde{V}}\right)_{*}^{-1} \circ\left(j_{y, V}\right)_{*}^{-1} \circ\left(j_{y}\right)_{*} \circ\left(j_{x}\right)_{*}^{-1}\right](\tilde{x}) \\
& =\left[\left(j_{\tilde{y}, \tilde{V}}\right)_{*} \circ\left(\left.\tilde{p}\right|_{\tilde{V}}\right)_{*}^{-1} \circ\left(j_{y, V}\right)_{*}^{-1} \circ\left(j_{y}\right)_{*}\right]\left(\mu_{V}\right) \\
& =\tilde{s}\left(\left(j_{y}\right)_{*}\left(\mu_{V}\right)\right) \text {. }
\end{aligned}
$$

Assim, como o ponto $\tilde{y} \in \tilde{V}$ é arbitrário, podemos concluir que $\tilde{s}\left(\left(j_{y}\right)_{*}\left(\mu_{V}\right)\right)=\left(j_{\tilde{y}}\right)_{*}\left(\mu_{\tilde{V}}\right)$ para todo $\tilde{y} \in \tilde{V}$, onde $y=\tilde{p}(\tilde{y})$; em outras palavras, lembrando que $U\left(\mu_{V}, V\right)=$ $\left\{\left(j_{y}\right)_{*}\left(\mu_{V}\right): y \in V\right\}$ e $U\left(\mu_{\tilde{V}}, \tilde{V}\right)=\left\{\left(j_{\tilde{y}}\right)_{*}\left(\mu_{\tilde{V}}\right): \tilde{y} \in \tilde{V}\right\}$, temos $\tilde{s}\left[U\left(\mu_{V}, V\right)\right] \subseteq U\left(\mu_{\tilde{V}}, \tilde{V}\right)$.

Finalmente, observe então que $\tilde{x} \in U\left(\mu_{V}, V\right)$ e $\tilde{s}(\tilde{x}) \in U\left(\mu_{\tilde{V}}, \tilde{V}\right)$, pois os pontos $x \in V$ e $\tilde{x} \in \tilde{V}$ são tais que $\tilde{x}=\left(j_{x}\right)_{*}\left(\mu_{V}\right)$ e $\tilde{s}(\tilde{x})=\left(j_{\tilde{x}}\right)_{*}\left(\mu_{\tilde{V}}\right)$. Logo, $U\left(\mu_{V}, V\right) \subseteq \tilde{M}$ e $U\left(\mu_{\tilde{V}}, \tilde{V}\right) \subseteq \widetilde{\widetilde{M}}$ são vizinhanças abertas de $\tilde{x}$ e $\tilde{s}(\tilde{x})$, respectivamente, tais que $\tilde{s}\left[U\left(\mu_{V}, V\right)\right] \subseteq U\left(\mu_{\tilde{V}}, \tilde{V}\right)$. Portanto, como a coleção

$\mathcal{C}=\left\{U\left(\mu_{\tilde{B}}, \tilde{B}\right): \tilde{B} \subseteq \tilde{M}\right.$ é uma n-bola própria e $\mu_{\tilde{B}}$ é um gerador de $\left.\mathrm{H}_{n}(\tilde{M}, \tilde{M} \backslash \tilde{B}) \simeq \mathbb{Z}\right\}$

constitui uma base para a topologia de $\widetilde{\widetilde{M}}$, e como $\tilde{V} \subseteq \tilde{M}$ é uma n-bola própria contendo $\tilde{x}$ (pois $\tilde{V}$ é homeomorfo à n-bola própria $V \subseteq M$ pela restrição $\left.\tilde{p}\right|_{\tilde{V}}: \tilde{V} \longrightarrow V$ ), segue que, para toda vizinhança aberta $\widetilde{\widetilde{W}}$ de $\tilde{s}(\tilde{x})$ em $\widetilde{\widetilde{M}}$, existem n-bolas próprias $V \subseteq M$ e $\tilde{V} \subseteq \tilde{M}$, contendo $x=\tilde{p}(\tilde{x})$ e $\tilde{x}$, respectivamente, e existem geradores $\mu_{V} \in \mathrm{H}_{n}(M, M \backslash V)$ e $\mu_{\tilde{V}} \in \mathrm{H}_{n}(\tilde{M}, \tilde{M} \backslash \tilde{V})$, tais que $\tilde{x} \in U\left(\mu_{V}, V\right), \tilde{s}(\tilde{x}) \in U\left(\mu_{\tilde{V}}, \tilde{V}\right)$ e $\tilde{s}\left[U\left(\mu_{V}, V\right)\right] \subseteq U\left(\mu_{\tilde{V}}, \tilde{V}\right) \subseteq \widetilde{\widetilde{W}}$ (lembre-se que $\tilde{s}(\tilde{x})$ não depende da n-bola própria $V \subseteq M$ utilizada para defini-lo, desde que ela contenha $x=\tilde{p}(\tilde{x})$ e seja uma vizinhança elementar em relação ao recobrimento $\tilde{p}: \tilde{M} \longrightarrow M)$. Deste modo, mostramos que, para toda vizinhança aberta $\widetilde{\widetilde{W}} \subseteq \widetilde{\widetilde{M}}$ de $\tilde{s}(\tilde{x})$, existe uma vizinhança aberta $\tilde{W} \subseteq \tilde{M}$ de $\tilde{x}$ tal que $\tilde{s}(\tilde{W}) \subseteq \widetilde{\widetilde{W}}$, o que implica que $\tilde{s}: \tilde{M} \longrightarrow \widetilde{\widetilde{M}}$ é contínua em $\tilde{x}$. Logo, como $\tilde{x} \in \tilde{M}$ é arbitrário, segue que a aplicação $\tilde{s}: \tilde{M} \longrightarrow \widetilde{\widetilde{M}}$ é contínua, e, por conseguinte, define uma orientação em $\tilde{M}$ (vide lema 1.1.1).

Isto posto, dada uma variedade topológica conexa $M$, de dimensão $n$ e sem fronteira, considere agora um subconjunto aberto e conexo $U \subseteq M$. Temos então que $U$ também é uma variedade topológica de dimensão $n$ (conexa e sem fronteira), e, para cada $x \in U$, o homomorfismo $\left(i_{x}\right)_{*}: \mathrm{H}_{n}(U, U \backslash\{x\}) \longrightarrow \mathrm{H}_{n}(M, M \backslash\{x\})$, induzido pela inclusão $i_{x}:(U, U \backslash\{x\}) \longrightarrow(M, M \backslash\{x\})$, constitui um isomorfismo (conforme nos garante o 
teorema da excisão em homologia singular). Deste modo, supondo $M$ orientável, e sendo $s: M \longrightarrow \tilde{M}$ uma orientação em $M$, vamos mostrar que a aplicação $s_{U}: U \longrightarrow \tilde{U}$, dada por $s_{U}(x)=\left[\left(i_{x}\right)_{*}^{-1} \circ s\right](x)$ para cada $x \in U$, define uma orientação em $U$. Com efeito, dado $x_{0} \in U$, temos que $s_{U}\left(x_{0}\right)$ é o único gerador de $H_{n}\left(U, U \backslash\left\{x_{0}\right\}\right)$ que é levado no gerador $s\left(x_{0}\right)$ de $\mathrm{H}_{n}\left(M, M \backslash\left\{x_{0}\right\}\right)$ pelo isomorfismo $\left(i_{x_{0}}\right)_{*}$ [note que, pelo item (i) do lema 1.1.1, temos que $s\left(x_{0}\right)$ é de fato um gerador de $\left.\mathrm{H}_{n}\left(M, M \backslash\left\{x_{0}\right\}\right)\right]$; além disso, a projeção orientada $\tilde{p}_{U}: \tilde{U} \longrightarrow U$, que leva cada gerador $\mu_{x} \in \mathrm{H}_{n}(U, U \backslash\{x\})$ no ponto $x \in U$, é tal que $\tilde{p}_{U} \circ s_{U}=\operatorname{Id}_{U}$, onde $\operatorname{Id}_{U}: U \longrightarrow U$ é a identidade (lembre-se que $\tilde{U}$ é o fibrado orientado de $U$, que contém somente as orientações locais dos pontos de $U$ ). Por outro lado, a continuidade de $s: M \longrightarrow \tilde{M}$ no ponto $x_{0} \in U$, implica que existem uma n-bola própria $V \subseteq M$, contendo $x_{0}$, e um gerador $\mu_{V} \in \mathrm{H}_{n}(M, M \backslash V)$, tais que $\left(j_{x}\right)_{*}\left(\mu_{V}\right)=s(x)$ para todo $x \in V$, onde $\left(j_{x}\right)_{*}: \mathrm{H}_{n}(M, M \backslash V) \longrightarrow \mathrm{H}_{n}(M, M \backslash\{x\})$ é o isomorfismo induzido pela inclusão (vide item (iii) do lema 1.1.1). Seja, pois, $W \subseteq M$ uma n-bola própria contendo $x_{0}$, tal que $W \subseteq U \cap V$ (a qual existe, pois a coleção de todas as n-bolas próprias em $M$ é uma base para a topologia de $M$ ), e, para cada $x \in W \subseteq V$, considere os isomorfismos ilustrados no diagrama comutativo abaixo, induzidos pelas respectivas inclusões:

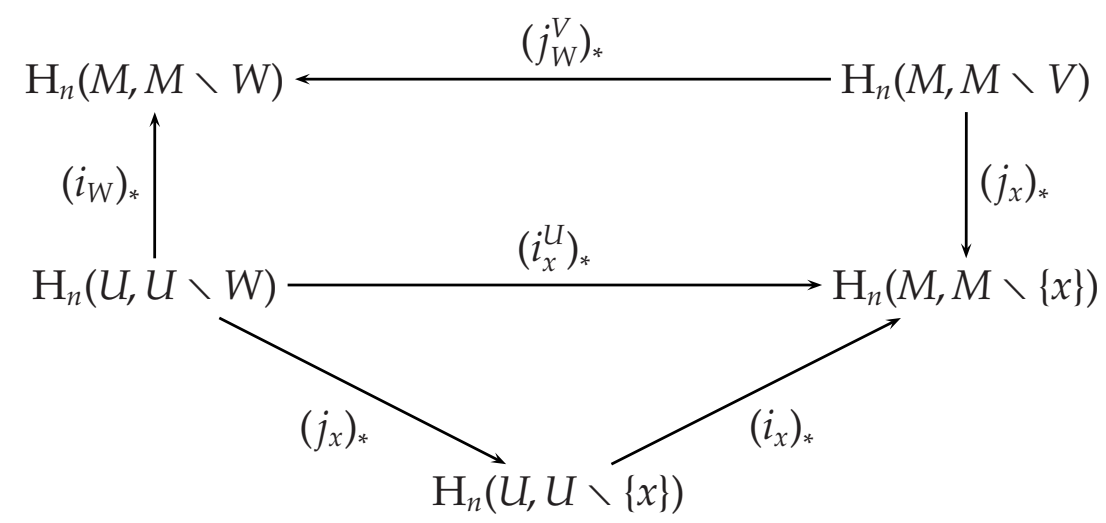

(note que $M \backslash V$ e $U \backslash W$ são retratos por deformação de $M \backslash W$ e $U \backslash\{x\}$, respectivamente). Deste modo, sendo $\mu_{W}$ o gerador de $\mathrm{H}_{n}(U, U \backslash W)$ tal que $\left[\left(j_{W}^{V}\right)_{*}^{-1} \circ\left(i_{W}\right)_{*}\right]\left(\mu_{W}\right)=\mu_{V} \in$ $\mathrm{H}_{n}(M, M \backslash V)$, segue (da comutatividade do diagrama acima) que:

$$
\begin{aligned}
\left(j_{x}^{U}\right)_{*}\left(\mu_{W}\right) & =\left[\left(i_{x}\right)_{*}^{-1} \circ\left(i_{x}^{U}\right)_{*}\right]\left(\mu_{W}\right)=\left\{\left(i_{x}\right)_{*}^{-1} \circ\left[\left(j_{x}\right)_{*} \circ\left(j_{W}^{V}\right)_{*}^{-1} \circ\left(i_{W}\right)_{*}\right]\right\}\left(\mu_{W}\right) \\
& =\left[\left(i_{x}\right)_{*}^{-1} \circ\left(j_{x}\right)_{*}\right]\left(\mu_{V}\right)=\left(i_{x}\right)_{*}^{-1}(s(x))=s_{U}(x)
\end{aligned}
$$

para todo $x \in W \subseteq U \cap V$. Assim, podemos concluir que existem uma n-bola própria $W \subseteq U$, contendo $x_{0}$, e um gerador $\mu_{W} \in \mathrm{H}_{n}(U, U \backslash W)$, tais que $\left(j_{x}^{U}\right)_{*}\left(\mu_{W}\right)=s_{U}(x)$ para todo $x \in W$, o que, por sua vez, significa que a aplicação $s_{U}: U \longrightarrow \tilde{U}$ é contínua no ponto $x_{0} \in U$ (vide item (iii) do lema 1.1.1). Portanto, temos que $s_{U}$ é, de fato, uma orientação em $U$, de modo que podemos introduzir a seguinte 
Definição 1.1.3 . Sendo M uma variedade topológica de dimensão n, conexa, orientável e sem fronteira, denotemos por $s: M \longrightarrow \tilde{M}$ uma orientação em $M, e$, dado um subconjunto aberto e conexo $U \subseteq M$, consideremos a orientação $s_{U}: U \longrightarrow \tilde{U}$ definida por $s_{U}(x)=\left[\left(i_{x}\right)_{*}^{-1} \circ s\right](x)$ para cada $x \in U$, onde $\left(i_{x}\right)_{*}: \mathrm{H}_{n}(U, U \backslash\{x\}) \longrightarrow \mathrm{H}_{n}(M, M \backslash\{x\})$ é o isomorfismo induzido pela inclusão. Utilizando um certo abuso de notação, dizemos então que $s_{U}$ é a restrição da orientação $s: M \longrightarrow \tilde{M}$ ao conjunto $U \subseteq M$, ou ainda, que $s_{U}$ é a orientação induzida em $U$ pela orientação s $: M \longrightarrow \tilde{M}$.

Na definição acima, note que, mesmo que o aberto $U \subseteq M$ não seja conexo, a orientação $s: M \longrightarrow \tilde{M}$ induz uma orientação $s_{U^{\prime}}: U^{\prime} \longrightarrow \tilde{U}^{\prime}$ em cada componente conexa $U^{\prime}$ de $U$, o que torna $U$ uma variedade orientada.

Definição 1.1.4 . Sendo M uma variedade topológica de dimensão n, conexa e com fronteira $\partial M$, dizemos que $M$ é orientável se Int $M=M \backslash \partial M$ for uma variedade orientável, isto é, se o fibrado orientado de Int $M$ for a união de duas componentes conexas distintas. Neste caso, sendo $\tilde{p}: \overparen{\operatorname{Int} M} \longrightarrow \operatorname{Int} M$ a projeção orientada de Int $M$, definimos uma orientação em $M$ como sendo uma aplicação contínua $s: \operatorname{Int} M \longrightarrow \overparen{\operatorname{Int} M}$, tal que $\tilde{p} \circ s=\operatorname{Id}_{\operatorname{Int} M}$, onde $\operatorname{Id}_{\text {Int } M}: \operatorname{Int} M \longrightarrow \operatorname{Int} M$ é a identidade.

Note que a definição acima não leva em consideração os pontos da fronteira de $M$; entretanto, como $\partial M$ constitui uma variedade topológica de dimensão $n-1$ (sem fronteira), segue que o conceito de orientabilidade se aplica a cada componente conexa de $\partial M$. Além disso, pode-se provar (vide [D], página 257) que, se $M$ é orientável, então qualquer orientação em Int $M$ induz uma orientação em $\partial M$; convém lembrarmos apenas que o fato de Int $M$ ser não-orientável não significa que $\partial M$ também o seja (a faixa de Möbius é um exemplo de variedade conexa não-orientável, cuja fronteira é orientável).

Definição 1.1.5 . Seja M uma variedade topológica de dimensão $n$, conexa e com fronteira, $e$ seja $\tilde{p}: \tilde{N} \longrightarrow N$ a projeção orientada de $N=\operatorname{Int} M$. Sendo $\tilde{C}$ uma componente conexa de $\tilde{N}$, sabemos que a restrição $\left.\tilde{p}\right|_{\tilde{C}}: \tilde{C} \longrightarrow N$ é um recobrimento de $N$ (eventualmente, $\tilde{C}=\tilde{N}$ se $\tilde{N}$ é conexo, isto é, se $N=\operatorname{Int} M$ é não-orientável). Logo, dado um laço $\omega: I \longrightarrow N$, $\operatorname{com} \omega(0)=\omega(1)=x_{0} \in N$, e denotando por $\mu_{x_{0}}$ o gerador de $\mathrm{H}_{n}\left(N, N \backslash\left\{x_{0}\right\}\right) \simeq \mathbb{Z}$ tal que $\mu_{x_{0}} \in \tilde{C}$, segue que existe um único levantamento $\tilde{\omega}: I \longrightarrow \tilde{C}$ (isto é, $\left.\tilde{p}\right|_{\tilde{C}} \circ \tilde{\omega}=\omega$ ) satisfazendo $\tilde{\omega}(0)=\mu_{x_{0}}$. Assim, temos que $\tilde{\omega}(1)= \pm \mu_{x_{0}}$; dizemos, pois, que $\omega$ preserva orientação (respectivamente, inverte orientação) se $\tilde{\omega}(1)=\mu_{x_{0}}$ (respectivamente, $\left.\tilde{\omega}(1)=-\mu_{x_{0}}\right)$. 
Exemplo 1.1.3 . Intuitivamente, podemos ver que o círculo central de uma faixa de Möbius constitui um laço que inverte orientação, pois, dada uma orientação $s\left(x_{0}\right)$ num ponto $x_{0}$ deste círculo, e estendendo-se $s\left(x_{0}\right)$ continuamente ao longo do círculo, obtemos, após uma volta completa, uma orientação $s^{\prime}\left(x_{0}\right)$ que é oposta à $s\left(x_{0}\right)$.

Proposição 1.1.3 . Seja $M$ uma variedade topológica de dimensão n, conexa e com fronteira não necessariamente vazia. Então, $M$ é orientável se, e somente se, todo laço $\omega: I \longrightarrow \operatorname{Int} M$ preserva orientação (ou, equivalentemente, $M$ é não-orientável se, e somente se, existe pelo menos um laço $\omega: I \longrightarrow$ Int $M$ que inverte orientação).

DemonstraÇÃo. Primeiramente, suponhamos que $M$ seja orientável, e consideremos o recobrimento $\left.\tilde{p}\right|_{\tilde{C}}: \tilde{C} \longrightarrow N$ de $N=\operatorname{Int} M$, relativo a uma componente conexa $\tilde{C}$ de $\tilde{N}$ (lembre-se que a orientabilidade de $M$ significa que $\tilde{N}$ é constituído de duas componentes conexas distintas). Sendo $s: N \longrightarrow \tilde{C}$ uma orientação em $N$, temos que $\tilde{p} \circ s=\mathrm{Id}$ (onde Id $: N \longrightarrow N$ é a identidade), e $s(x)$ é um gerador de $\mathrm{H}_{n}(N, N \backslash\{x\})$ para cada $x \in N$. Agora, dado um laço $\omega: I \longrightarrow N$, com $\omega(0)=\omega(1)=x_{0} \in N$, seja $\mu_{x_{0}} \in \tilde{C}$ o gerador de $\mathrm{H}_{n}\left(N, N \backslash\left\{x_{0}\right\}\right) \simeq \mathbb{Z}$ tal que $s\left(x_{0}\right)=\mu_{x_{0}}$, e considere o (único) levantamento $\tilde{\omega}: I \longrightarrow \tilde{C}$ de $\omega$ (isto é, $\left.\tilde{p}\right|_{\tilde{C}} \circ \tilde{\omega}=\omega$ ) tal que $\tilde{\omega}(0)=\mu_{x_{0}}$. Temos então que $\tilde{\omega}(1)=\mu_{x_{0}}$ (pois $\mu_{x_{0}}$ é o único gerador de $\mathrm{H}_{n}\left(N, N \backslash\left\{x_{0}\right\}\right)$ pertencente à componente conexa $\tilde{C}$ de $N)$, o que implica que $\omega$ é um laço que preserva orientação.

Reciprocamente, suponhamos que todo laço em $N=\operatorname{Int} M$ preserve orientação, e seja $\left.\tilde{p}\right|_{\tilde{C}}: \tilde{C} \longrightarrow N$ o recobrimento de $N$, relativo a uma componente conexa $\tilde{C}$ de $\tilde{N}$. Dado um laço $\omega: I \longrightarrow N$, com $\omega(0)=\omega(1)=x_{0} \in N$, e sendo $\tilde{\omega}: I \longrightarrow \tilde{C}$ o único levantamento de $\omega$ tal que $\tilde{\omega}(0)=\mu_{x_{0}}$, onde $\mu_{x_{0}} \in \tilde{C}$ é um dos dois geradores de $\mathrm{H}_{n}\left(N, N \backslash\left\{x_{0}\right\}\right) \simeq \mathbb{Z}$, segue então que $\tilde{\omega}(1)=\mu_{x_{0}}$. Ora, se $\tilde{\omega}(0)=\tilde{\omega}(1)=\mu_{x_{0}}$, temos que $\tilde{\omega}$ é um laço em $\tilde{C}$; deste modo, concluímos que todo laço $\omega: I \longrightarrow N$ é levantado para um laço em $\tilde{C}$ (pelo recobrimento $\left.\tilde{p}\right|_{\tilde{C}}$ ). Isto posto, vamos mostrar que $\tilde{C}$ não é a única componente conexa de $\tilde{N}$. Com efeito, se assim fosse, ambos os geradores $\mu_{x_{0}} \mathrm{e}-\mu_{x_{0}}$ de $\mathrm{H}_{n}\left(N, N \backslash\left\{x_{0}\right\}\right) \simeq \mathbb{Z}$ pertenceriam a $\tilde{C}$, qualquer que fosse $x_{0} \in N$; neste caso, sendo $\tilde{\alpha}: I \longrightarrow \tilde{C}$ um caminho tal que $\tilde{\alpha}(0)=\mu_{x_{0}}$ e $\tilde{\alpha}(1)=-\mu_{x_{0}}$ (o qual existe, pois o espaço de recobrimento $\tilde{C}$ é conexo por caminhos), segue que $\alpha=\tilde{p} \circ \tilde{\alpha}: I \longrightarrow N$ seria um laço com base no ponto $x_{0}$, cujo levantamento $\tilde{\alpha}$ não é um laço em $\tilde{C}$, contradizendo a conclusão acima. Portanto, se todo laço em $N$ preserva orientação, temos que $\tilde{N}$ é constituído de duas componentes conexas distintas, o que, por definição, significa que $N=\operatorname{Int} M$ é orientável, conforme queríamos provar.

Definição 1.1.6 . Sejam $M$ e $N$ variedades topológicas de dimensão n, não necessariamente orientáveis, e com fronteira (eventualmente vazias). Dizemos que uma aplicação contínua 
$f: M \longrightarrow N$ é fiel à orientação se ela leva laços que preservam (respectivamente, invertem) orientação em laços que preservam (respectivamente, invertem) orientação.

A proposição a seguir constitui uma conseqüência direta da proposição 1.1.3.

Proposição 1.1.4 . Seja $f: M \longrightarrow N$ uma aplicação contínua entre variedades topológicas conexas, ambas de dimensão n (não necessariamente orientáveis e com fronteiras possivelmente não-vazias). Então:

(i) se Me N são orientáveis, $f$ é fiel à orientação;

(ii) se Mé não-orientável e $N$ é orientável, $f$ não é fiel à orientação.

Exemplo 1.1.4 . Considere a aplicação quociente $\pi: \mathbb{S}^{2} \longrightarrow \mathbb{R P}^{2}$. Como todo laço $\omega: I \longrightarrow \mathbb{S}^{2}$ preserva orientação (pois $\mathbb{S}^{2}$ é orientável), e a imagem $\pi \circ \omega: I \longrightarrow \mathbb{R P}^{2}$ é um laço contrátil (e, portanto, preserva orientação), segue que $\pi$ é um exemplo de aplicação fiel à orientação entre uma variedade orientável $\left(\mathbb{S}^{2}\right)$ e uma variedade nãoorientável $\left(\mathbb{R P}^{2}\right)$. Observe que os laços que invertem orientação em $\mathbb{R P}^{2}$ são imagens (pela aplicação quociente $\pi$ ) de caminhos homotópicos a semi-circulos máximos em $\mathbb{S}^{2}$; logo, tais laços em $\mathbb{R} P^{2}$ não constituem imagens de laços em $\mathbb{S}^{2}$.

Proposição 1.1.5 . Se M é uma variedade topológica de dimensão n, conexa e com fronteira (eventualmente vazia), então todo recobrimento $p: M^{\prime} \longrightarrow M$ é uma aplicação fiel à orientação.

Demonstração. Primeiramente, observe que, como $p: M^{\prime} \longrightarrow M$ é um homeomorfismo local, segue (do teorema da invariância do domínio) que $p\left(\partial M^{\prime}\right) \subseteq \partial M$ e $p\left(\operatorname{Int} M^{\prime}\right) \subseteq \operatorname{Int} M$; conseqüentemente, temos que a restrição de $p$ a Int $M^{\prime}$ é um recobrimento de Int $M$. Seja, pois, $\bar{p}: N^{\prime} \longrightarrow N$ a restrição de $p$ a $N^{\prime}=\operatorname{Int} M^{\prime}$ sobre $N=\operatorname{Int} M$; para provarmos que $p: M^{\prime} \longrightarrow M$ é uma aplicação fiel à orientação, basta mostrarmos que $\bar{p}$ é fiel à orientação (uma vez que os conceitos de laços que preservam ou invertem orientação estão definidos apenas para laços no interior de uma variedade), sendo que, para tanto, basta verificarmos que um laço $\alpha: I \longrightarrow N^{\prime}$ preserva orientação se, e somente se, sua imagem $\bar{p} \circ \alpha: I \longrightarrow N$ é um laço que preserva orientação. Entretanto, se $M$ é orientável (isto é, se $N=\operatorname{Int} M$ é orientável), este fato torna-se evidente, pois, pela proposição 1.1.1, a orientabilidade de $N$ implica na orientabilidade de $N^{\prime}$, e, conseqüentemente, todos os laços em $N^{\prime}$ e $N$ preservam orientação (conforme nos garante a proposição 1.1.3). 


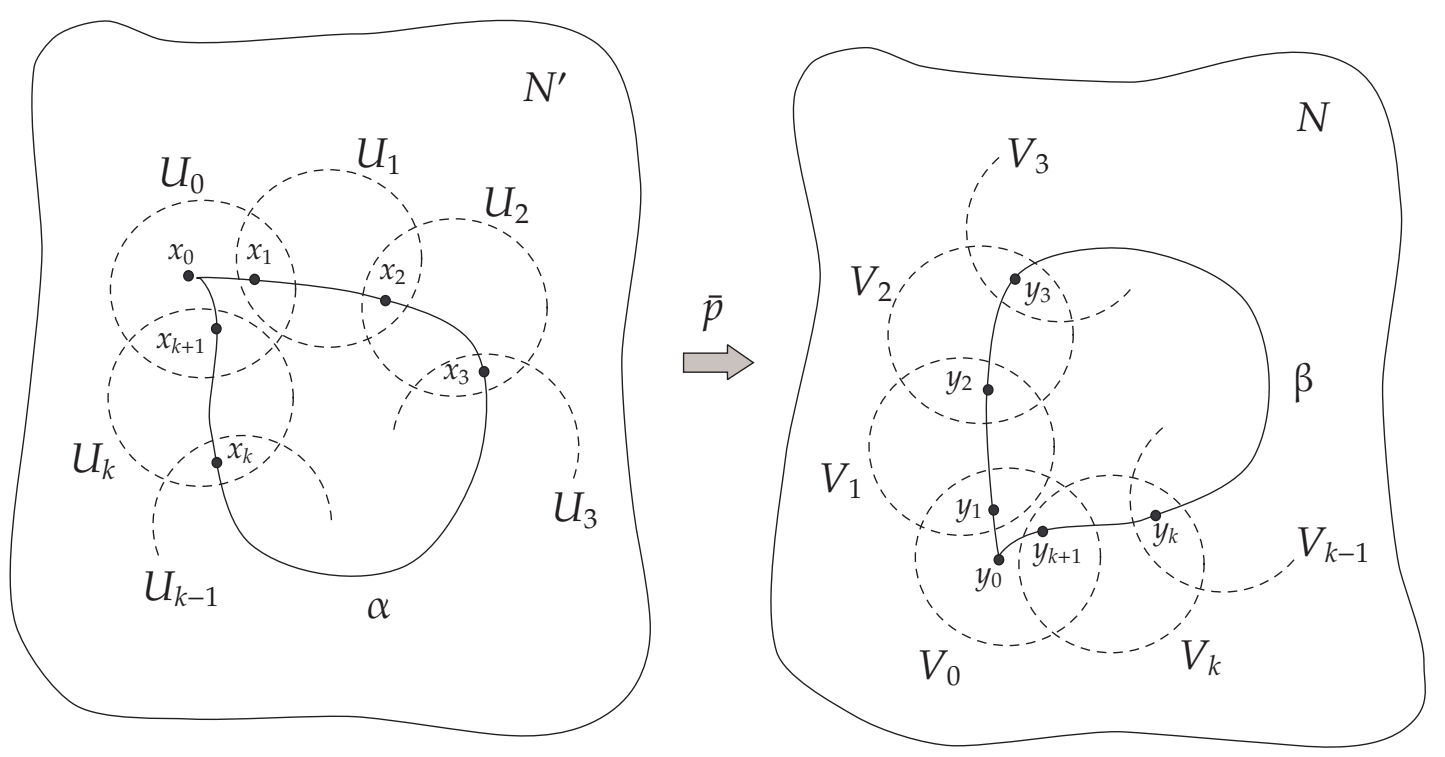

Figura 1.1

Assim sendo, suponhamos que $N=$ Int $M$ seja não-orientável, e considere um laço $\alpha: I \longrightarrow N^{\prime}$, com base num ponto $x_{0} \in N^{\prime}$, que preserva orientação. Denotando por $\beta: I \longrightarrow N$ o laço, com base em $y_{0} \in \bar{p}\left(x_{0}\right) \in N$, dado por $\beta=\bar{p} \circ \alpha$, seja $\mathcal{C}(\beta)=\left\{V_{0}, V_{1}, \ldots, V_{k}\right\}$ uma cobertura finita de $\beta(I) \subseteq N$, onde $V_{0}, V_{1}, \ldots, V_{k}$ são subconjuntos abertos contráteis (e, portanto, orientáveis) de $N$, que constituem vizinhanças elementares em relação ao espaço de recobrimento $N^{\prime}$. Se, para cada $\lambda \in\{0,1, \ldots, k\}$, denotarmos por $U_{\lambda}$ a componente conexa de $\bar{p}^{-1}\left(V_{\lambda}\right)$ tal que $U_{\lambda} \cap \alpha(I) \neq \varnothing$, temos que $\mathcal{C}(\alpha)=\left\{U_{0}, U_{1}, \ldots, U_{k}\right\}$ é uma cobertura de $\alpha(I)$, constituída de subconjuntos abertos contráteis de $N^{\prime}$, e cada restrição $\left.\bar{p}\right|_{U_{\lambda}}: U_{\lambda} \longrightarrow V_{\lambda}$ é um homeomorfismo sobre $V_{\lambda}$. Sejam ainda $x_{1}, x_{2}, \ldots, x_{k+1} \in \alpha(I)$ e $y_{1}, y_{2}, \ldots, y_{k+1} \in \beta(I)$ tais que:

- $\bar{p}\left(x_{\lambda}\right)=y_{\lambda}$ para cada $\lambda \in\{1,2, \ldots, k+1\}$;

- $x_{\lambda} \in U_{\lambda-1} \cap U_{\lambda}$ e $y_{\lambda} \in V_{\lambda-1} \cap V_{\lambda}$ para cada $\lambda \in\{1,2, \ldots, k\}$;

- $x_{k+1} \in U_{k} \cap U_{0}$ e $y_{k+1} \in V_{k} \cap V_{0}$

(vide figura 1.1 acima), e considere os caminhos $\alpha_{0}, \alpha_{1}, \ldots, \alpha_{k+1}: I \longrightarrow \alpha(I) \subseteq N^{\prime} \mathrm{e}$ $\beta_{0}, \beta_{1}, \ldots, \beta_{k+1}: I \longrightarrow \beta(I) \subseteq N$, cujas imagens estão contidas nas imagens dos laços $\alpha \mathrm{e}$ $\beta$, satifazendo:

- $\beta_{\lambda}=\bar{p} \circ \alpha_{\lambda}$ para cada $\lambda \in\{0,1, \ldots, k+1\}$;

- $\alpha_{\lambda}(0)=x_{\lambda}, \alpha_{\lambda}(1)=x_{\lambda+1}$ e $\alpha_{\lambda}(I) \subseteq U_{\lambda}$ para cada $\lambda \in\{0,1, \ldots, k\}$;

- $\alpha_{k+1}(0)=x_{k+1}, \alpha_{k+1}(1)=x_{0}$ e $\alpha_{k+1}(I) \subseteq U_{0}$;

- $\beta_{\lambda}(0)=y_{\lambda}, \beta_{\lambda}(1)=y_{\lambda+1}$ e $\beta_{\lambda}(I) \subseteq V_{\lambda}$ para cada $\lambda \in\{0,1, \ldots, k\}$; 
- $\beta_{k+1}(0)=y_{k+1}, \beta_{k+1}(1)=y_{0}$ e $\beta_{k+1}(I) \subseteq V_{0}$.

Isto posto, sendo $\tilde{p}^{\prime}: \tilde{N}^{\prime} \longrightarrow N^{\prime}$ o recobrimento obtido de uma componente conexa do fibrado orientado de $N^{\prime}$, vamos levantar os caminhos $\alpha_{\lambda}, \lambda \in\{0,1, \ldots, k+1\}$, relativamente a $\tilde{p}^{\prime}$, da seguinte maneira: dado um gerador $\tilde{x}_{0} \in \mathrm{H}_{n}\left(N^{\prime}, N^{\prime} \backslash\left\{x_{0}\right\}\right) \subseteq \tilde{N}^{\prime}$, e sendo $\tilde{\alpha}_{0}: I \longrightarrow \tilde{N}^{\prime}$ o único levantamento de $\alpha_{0}$ tal que $\tilde{\alpha}_{0}(0)=\tilde{x}_{0}$, considere o único levantamento $\tilde{\alpha}_{1}: I \longrightarrow \tilde{N}^{\prime}$ de $\alpha_{1}$, com $\tilde{\alpha}_{1}(0)=\tilde{x}_{1}$, onde $\tilde{x}_{1}=\tilde{\alpha}_{0}(1)$; então, tomando $\tilde{x}_{2}=\tilde{\alpha}_{1}(1)$, definimos $\tilde{\alpha}_{2}: I \longrightarrow \tilde{N}^{\prime}$ como o único levantamento de $\alpha_{2}$ tal que $\tilde{\alpha}_{2}(0)=\tilde{x}_{2}, \mathrm{e}$ assim sucessivamente (em outras palavras, escolhida uma orientação $\tilde{x}_{0}$ para $\alpha(0)=x_{0}$, estendêmo-la ao longo do laço $\alpha$, até o ponto $\alpha(1))$. Deste modo, observando que $\alpha=\alpha_{0} \vee \alpha_{1} \vee \ldots \vee \alpha_{k+1}$, obtemos:

$$
\begin{aligned}
\tilde{p}^{\prime} \circ\left(\tilde{\alpha}_{0} \vee \tilde{\alpha}_{1} \vee \ldots \vee \tilde{\alpha}_{k+1}\right) & =\left(\tilde{p}^{\prime} \circ \tilde{\alpha}_{0}\right) \vee\left(\tilde{p}^{\prime} \circ \tilde{\alpha}_{1}\right) \vee \ldots \vee\left(\tilde{p}^{\prime} \circ \tilde{\alpha}_{k+1}\right) \\
& =\alpha_{0} \vee \alpha_{1} \vee \ldots \vee \alpha_{k+1}=\alpha,
\end{aligned}
$$

isto é, o caminho $\tilde{\alpha}=\tilde{\alpha}_{0} \vee \tilde{\alpha}_{1} \vee \ldots \vee \tilde{\alpha}_{k+1}: I \longrightarrow \tilde{N}^{\prime}$, constituído da justaposição de $\tilde{\alpha}_{0}$, $\tilde{\alpha}_{1}, \ldots, \tilde{\alpha}_{k+1}$, é um levantamento de $\alpha$ (relativamente a $\left.\tilde{p}^{\prime}\right)$, com início em $\tilde{\alpha}_{0}(0)=\tilde{x}_{0}$. Entretanto, como o laço $\alpha: I \longrightarrow N^{\prime}$ preserva orientação (conforme nossa suposição inicial), temos que $\alpha$ pode ser levantado para um único laço em $\tilde{N}^{\prime}$ com início em $\tilde{x}_{0}$; logo, o levantamento $\tilde{\alpha}=\tilde{\alpha}_{0} \vee \tilde{\alpha}_{1} \vee \ldots \vee \tilde{\alpha}_{k+1}$ é um laço em $\tilde{N}^{\prime}$, ou seja, $\tilde{x}_{0}=\tilde{\alpha}(1)=\tilde{\alpha}_{k+1}(1)$.

Agora, utilizando o teorema da excisão em homologia singular, considere os isomorfismos $\left(i_{0}\right)_{*}: \mathrm{H}_{n}\left(U_{0}, U_{0} \backslash\left\{x_{0}\right\}\right) \longrightarrow \mathrm{H}_{n}\left(N^{\prime}, N^{\prime} \backslash\left\{x_{0}\right\}\right)$ e $\left(j_{0}\right)_{*}: \mathrm{H}_{n}\left(V_{0}, V_{0} \backslash\left\{y_{0}\right\}\right) \longrightarrow$ $\mathrm{H}_{n}\left(N, N \backslash\left\{y_{0}\right\}\right)$, induzidos pelas respectivas inclusões $i_{0}: U_{0} \longrightarrow N^{\prime}$ e $j_{0}: V_{0} \longrightarrow N$. Lembrando que a restrição $\left.\bar{p}\right|_{U_{0}}: U_{0} \longrightarrow V_{0}$ é um homeomorfismo sobre $V_{0}$, considere também o isomorfismo $\left(\bar{p}_{0}\right)_{*}: \mathrm{H}_{n}\left(U_{0}, U_{0} \backslash\left\{x_{0}\right\}\right) \longrightarrow \mathrm{H}_{n}\left(V_{0}, V_{0} \backslash\left\{y_{0}\right\}\right)$ induzido por $\left.\bar{p}\right|_{u_{0}}, \mathrm{e}$ seja $\tilde{y}_{0}$ o gerador de $\mathrm{H}_{n}\left(N, N \backslash\left\{y_{0}\right\}\right)$ que é levado em $\tilde{x}_{0} \in \mathrm{H}_{n}\left(N^{\prime}, N^{\prime} \backslash\left\{x_{0}\right\}\right)$ pela seguinte cadeia de isomorfismos:

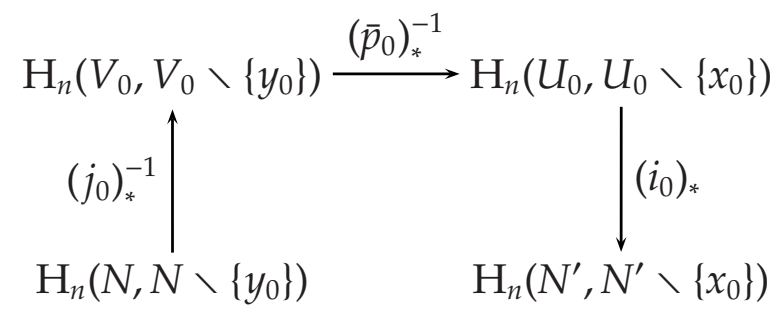

Por outro lado, denotemos por $\tilde{p}: \tilde{N} \longrightarrow N$ a projeção orientada de $N$ (que é um recobrimento de $N$, pois estamos supondo $N$ não-orientável), e, para cada $\lambda \in\{0,1,2, \ldots, k\}$ e cada $t \in I$, seja $\left(\bar{p}_{\lambda}^{t}\right)_{*}: \mathrm{H}_{n}\left(U_{\lambda}, U_{\lambda} \backslash\left\{\alpha_{\lambda}(t)\right\}\right) \longrightarrow \mathrm{H}_{n}\left(V_{\lambda}, V_{\lambda} \backslash\left\{\beta_{\lambda}(t)\right\}\right)$ o isomorfismo induzido pela restrição $\left.\bar{p}\right|_{u_{\lambda}}: U_{\lambda} \longrightarrow V_{\lambda}$ (lembre-se que $\left.\bar{p}\right|_{U_{\lambda}}$ é um homeomorfismo sobre $V_{\lambda}$, e $\bar{p} \circ \alpha_{\lambda}=\beta_{\lambda}$, com $\alpha_{\lambda}(I) \subseteq U_{\lambda}$ e $\beta_{\lambda}(I) \subseteq V_{\lambda}$, para cada $\left.\lambda \in\{0,1, \ldots, k\}\right)$. Além disso, para cada $t \in I$, considere ainda o isomorfismo $\left(\bar{p}_{k+1}^{t}\right)_{*}: \mathrm{H}_{n}\left(U_{0}, U_{0} \backslash\left\{\alpha_{k+1}(t)\right\}\right) \longrightarrow$ 
$\mathrm{H}_{n}\left(V_{0}, V_{0} \backslash\left\{\beta_{k+1}(t)\right\}\right)$, induzido pela restrição $\left.\bar{p}\right|_{U_{0}}: U_{0} \longrightarrow V_{0}$ (o qual está bem definido, pois $\alpha_{k+1}(I) \subseteq U_{0}, \beta_{k+1}(I) \subseteq V_{0}$ e $\left.\bar{p} \circ \alpha_{k+1}=\beta_{k+1}\right)$, e, utilizando o teorema da excisão em homologia singular, sejam $\left(i_{\lambda}^{t}\right)_{*}: \mathrm{H}_{n}\left(U_{\lambda}, U_{\lambda} \backslash\left\{\alpha_{\lambda}(t)\right\}\right) \longrightarrow \mathrm{H}_{n}\left(N^{\prime}, N^{\prime} \backslash\left\{\alpha_{\lambda}(t)\right\}\right)$ e $\left(j_{\lambda}^{t}\right)_{*}: \mathrm{H}_{n}\left(V_{\lambda}, V_{\lambda} \backslash\left\{\beta_{\lambda}(t)\right\}\right) \longrightarrow \mathrm{H}_{n}\left(N, N \backslash\left\{\beta_{\lambda}(t)\right\}\right)$ os isomorfismos induzidos pelas inclusões $i_{\lambda}: U_{\lambda} \longrightarrow N^{\prime}$ e $j_{\lambda}: V_{\lambda} \longrightarrow N$, onde $\lambda \in\{0,1, \ldots, k\}$. Então, sendo $\left(i_{k+1}^{t}\right)_{*}: \mathrm{H}_{n}\left(U_{0}, U_{0} \backslash\left\{\alpha_{k+1}(t)\right\}\right) \longrightarrow \mathrm{H}_{n}\left(N^{\prime}, N^{\prime} \backslash\left\{\alpha_{k+1}(t)\right\}\right)$ e $\left(j_{k+1}^{t}\right)_{*}: \mathrm{H}_{n}\left(V_{0}, V_{0} \backslash\left\{\beta_{k+1}(t)\right\}\right) \longrightarrow$ $\mathrm{H}_{n}\left(N, N \backslash\left\{\beta_{k+1}(t)\right\}\right)$ os isomorfismos induzidos pelas inclusões $i_{k+1}=i_{0}: U_{0} \longrightarrow N^{\prime} \mathrm{e}$ $j_{k+1}=j_{0}: V_{0} \longrightarrow N$, vamos definir os caminhos $\tilde{\beta}_{0}, \tilde{\beta}_{1}, \ldots, \tilde{\beta}_{k+1}: I \longrightarrow \tilde{N}$ da seguinte maneira: se $\lambda \in\{0,1, \ldots, k\}, \tilde{\beta}_{\lambda}$ é dado por $\tilde{\beta}_{\lambda}(t)=\left[\left(j_{\lambda}^{t}\right)_{*} \circ\left(\bar{p}_{\lambda}^{t}\right)_{*} \circ\left(i_{\lambda}^{t}\right)_{*}^{-1}\right]\left(\tilde{\alpha}_{\lambda}(t)\right)$, para cada $t \in I$, conforme ilustra o diagrama abaixo:

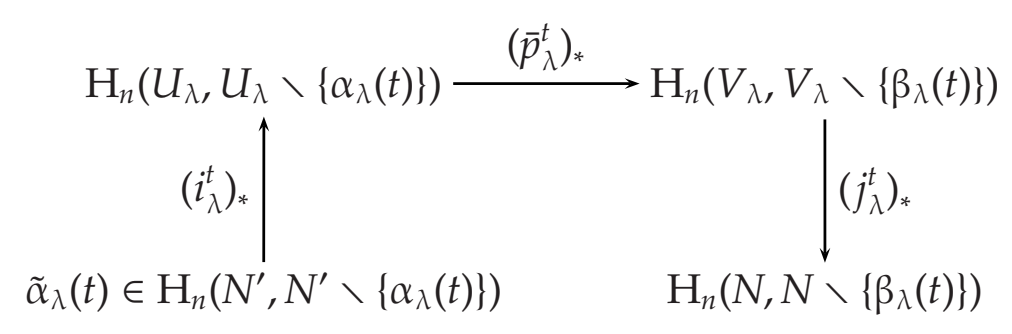

Por outro lado, se $\lambda=k+1$, defina $\tilde{\beta}_{k+1}$ como sendo $\tilde{\beta}_{k+1}(t)=\left[\left(j_{k+1}^{t}\right)_{*} \circ\left(\bar{p}_{k+1}^{t}\right)_{*} \circ\right.$ $\left.\left(i_{k+1}^{t}\right)_{*}^{-1}\right]\left(\tilde{\alpha}_{k+1}(t)\right)$, de acordo com o diagrama a seguir:

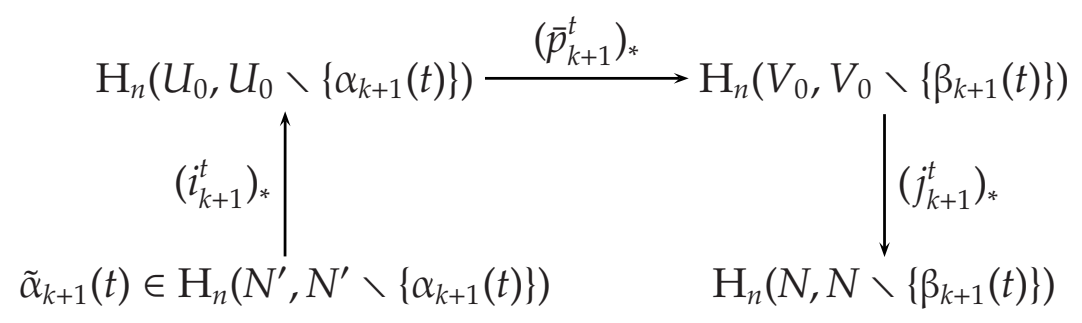

Note que, como $\tilde{\alpha}_{\eta}: I \longrightarrow \tilde{N}^{\prime}$ é um levantamento de $\alpha_{\eta}: I \longrightarrow N^{\prime}$, relativamente ao recobrimento $\tilde{p}^{\prime}: \tilde{N}^{\prime} \longrightarrow N^{\prime}$, para cada $\eta \in\{0,1, \ldots, k+1\}$, temos de fato que $\tilde{\alpha}_{\eta}(t) \in \mathrm{H}_{n}\left(N^{\prime}, N^{\prime} \backslash\left\{\alpha_{\eta}(t)\right\}\right) \subseteq \tilde{N}^{\prime}$ para cada $t \in I$ (pois $\tilde{p}^{\prime}$ é a projeção que leva cada ponto $\tilde{z} \in \mathrm{H}_{n}\left(N^{\prime}, N^{\prime} \backslash\{z\}\right)$ em $\left.z \in N^{\prime}\right)$.

Isto posto, observe que, como $\tilde{\beta}_{\eta}(t) \in \mathrm{H}_{n}\left(N, N \backslash\left\{\beta_{\eta}(t)\right\}\right)$ para cada $\eta \in\{0,1, \ldots, k+1\}$ e cada $t \in I$, e como $\tilde{p}: \tilde{N} \longrightarrow N$ é a projeção orientada de $N$, temos $\left(\tilde{p} \circ \tilde{\beta}_{\eta}\right)(t)=\beta_{\eta}(t)$, ou seja, $\tilde{p} \circ \tilde{\beta}_{\eta}=\beta_{\eta}$; isto, por sua vez, significa que $\tilde{\beta}_{\eta}: I \longrightarrow \tilde{N}$ é um levantamento de $\beta_{\eta}$ relativamente a $\tilde{p}$, para cada $\eta \in\{0,1, \ldots, k+1\}$. Logo, como $\beta=\beta_{0} \vee \beta_{1} \vee \ldots \vee \beta_{k+1}$, segue que:

$$
\begin{aligned}
\tilde{p} \circ\left(\tilde{\beta}_{0} \vee \tilde{\beta}_{1} \vee \ldots \vee \tilde{\beta}_{k+1}\right) & =\left(\tilde{p} \circ \tilde{\beta}_{0}\right) \vee\left(\tilde{p} \circ \tilde{\beta}_{1}\right) \vee \ldots \vee\left(\tilde{p} \circ \tilde{\beta}_{k+1}\right) \\
& =\beta_{0} \vee \beta_{1} \vee \ldots \vee \beta_{k+1}=\beta,
\end{aligned}
$$


e, conseqüentemente, o caminho justaposto $\tilde{\beta}=\tilde{\beta}_{0} \vee \tilde{\beta}_{1} \vee \ldots \vee \tilde{\beta}_{k+1}: I \longrightarrow \tilde{N}$ é um levantamento de $\beta$ relativamente à projeção orientada $\tilde{p}: \tilde{N} \longrightarrow N$. Além disso, como $\alpha_{0}(0)=x_{0}$ e $\beta_{0}(0)=y_{0}$, segue que, para $\lambda=0$ e $t=0$, temos $\left(i_{0}^{0}\right)_{*}=\left(i_{0}\right)_{*}$ : $\mathrm{H}_{n}\left(U_{0}, U_{0} \backslash\left\{x_{0}\right\}\right) \longrightarrow \mathrm{H}_{n}\left(N^{\prime}, N^{\prime} \backslash\left\{x_{0}\right\}\right),\left(j_{0}^{0}\right)_{*}=\left(j_{0}\right)_{*}: \mathrm{H}_{n}\left(V_{0}, V_{0} \backslash\left\{y_{0}\right\}\right) \longrightarrow \mathrm{H}_{n}\left(N, N \backslash\left\{y_{0}\right\}\right)$ e $\left(\bar{p}_{0}^{0}\right)_{*}=\left(\bar{p}_{0}\right)_{*}: \mathrm{H}_{n}\left(U_{0}, U_{0} \backslash\left\{x_{0}\right\}\right) \longrightarrow \mathrm{H}_{n}\left(V_{0}, V_{0} \backslash\left\{y_{0}\right\}\right)$, e, portanto:

$$
\tilde{\beta}(0)=\tilde{\beta}_{0}(0)=\left[\left(j_{0}\right)_{*} \circ\left(\bar{p}_{0}\right)_{*} \circ\left(i_{0}\right)_{*}^{-1}\right]\left(\tilde{\alpha}_{0}(0)\right)=\left[\left(j_{0}\right)_{*} \circ\left(\bar{p}_{0}\right)_{*} \circ\left(i_{0}\right)_{*}^{-1}\right]\left(\tilde{x}_{0}\right)=\tilde{y}_{0} .
$$

Agora, como $\alpha_{k+1}(1)=x_{0}$ e $\beta_{k+1}(1)=y_{0}$, segue que, para $\lambda=k+1$ e $t=1$, também temos $\left(i_{k+1}^{1}\right)_{*}=\left(i_{0}\right)_{*}: \mathrm{H}_{n}\left(U_{0}, U_{0} \backslash\left\{x_{0}\right\}\right) \longrightarrow \mathrm{H}_{n}\left(N^{\prime}, N^{\prime} \backslash\left\{x_{0}\right\}\right),\left(j_{k+1}^{1}\right)_{*}=\left(j_{0}\right)_{*}: \mathrm{H}_{n}\left(V_{0}, V_{0} \backslash\left\{y_{0}\right\}\right) \longrightarrow$ $\mathrm{H}_{n}\left(N, N \backslash\left\{y_{0}\right\}\right)$ e $\left(\bar{p}_{k+1}^{1}\right)_{*}=\left(\bar{p}_{0}\right)_{*}: \mathrm{H}_{n}\left(U_{0}, U_{0} \backslash\left\{x_{0}\right\}\right) \longrightarrow \mathrm{H}_{n}\left(V_{0}, V_{0} \backslash\left\{y_{0}\right\}\right) ;$ logo, como $\tilde{\alpha}=\tilde{\alpha}_{0} \vee \tilde{\alpha}_{1} \vee \ldots \vee \tilde{\alpha}_{k+1}: I \longrightarrow \tilde{N}^{\prime}$ é um laço com base em $\tilde{x}_{0}$, segue que:

$$
\tilde{\beta}(1)=\tilde{\beta}_{k+1}(1)=\left[\left(j_{0}\right)_{*} \circ\left(\bar{p}_{0}\right)_{*} \circ\left(i_{0}\right)_{*}^{-1}\right]\left(\tilde{\alpha}_{k+1}(1)\right)=\left[\left(j_{0}\right)_{*} \circ\left(\bar{p}_{0}\right)_{*} \circ\left(i_{0}\right)_{*}^{-1}\right]\left(\tilde{x}_{0}\right)=\tilde{y}_{0} .
$$

Deste modo, concluímos que $\tilde{\beta}: I \longrightarrow \tilde{N}$ é um laço com base em $\tilde{y}_{0}$, que constitui o único levantamento de $\beta$, relativamente ao recobrimento $\tilde{p}: \tilde{N} \longrightarrow N$, com início em $\tilde{y}_{0}$, o que, por definição, significa que $\beta: I \longrightarrow N$ é um laço que preserva orientação.

Em resumo, supondo $N$ não-orientável, mostramos que, se um laço $\alpha: I \longrightarrow N^{\prime}$ preserva orientação, então o laço $\beta=\bar{p} \circ \alpha: I \longrightarrow N$ também o faz. Assim, demonstrando-se a recíproca de maneira análoga, obtemos que o recobrimento $\bar{p}: N^{\prime}=\operatorname{Int} M^{\prime} \longrightarrow N=$ Int $M$ é uma aplicação fiel à orientação, conforme queríamos provar.

\section{2 (1) Recobrimento Orientado dle urma Variedadle Topolo̊gica}

Seja $M$ uma variedade topológica conexa e sem fronteira, e denotemos por $\tilde{p}: \tilde{M} \longrightarrow$ $M$ a projeção orientada de $M$. Na seção anterior, vimos que, dada uma componente conexa $\tilde{C}$ de $\tilde{M}$, a restrição $\left.\tilde{p}\right|_{\tilde{C}}: \tilde{C} \longrightarrow M$ constitui um recobrimento de uma ou duas folhas, conforme $M$ seja respectivamente orientável ou não. De maneira análoga, veremos a seguir que, ainda que $M$ seja uma variedade topológica com fronteira, existe uma aplicação $\widehat{p}: \widehat{M} \longrightarrow M$ tal que $\hat{p}$ é um recobrimento simples se $M$ é orientável, ou um recobrimento duplo se $M$ é não-orientável, o que mostrará que o recobrimento $\left.\tilde{p}\right|_{\tilde{c}}$ pode (de um certo modo) ser "generalizado" para variedades com fronteira. Entretanto, a fim de definirmos $\hat{p}$, precisaremos recordar alguns conceitos da teoria de recobrimentos.

Seja $M$ uma variedade topológica conexa (e, portanto, conexa por caminhos), e considere um subgrupo $H \subseteq \pi_{1}\left(M, x_{0}\right)$, onde $x_{0} \in M$ (como usualmente, denotaremos por 
$\pi_{1}\left(M, x_{0}\right)$ o grupo fundamental de $M$ com base no ponto $\left.x_{0}\right)$; vamos mostrar então (resumidamente) que existem um recobrimento $\hat{p}: \hat{M} \longrightarrow M$, com $\hat{M}$ conexo, e um ponto $\hat{x_{0}} \in \hat{M}$, tais que $\hat{p}_{\#}\left(\pi_{1}\left(\hat{M}, \hat{x_{0}}\right)\right)=H$, onde $\hat{p}_{\#}: \pi_{1}\left(\hat{M}, \hat{x_{0}}\right) \longrightarrow \pi_{1}\left(M, x_{0}\right)$ é o homomorfismo induzido por $\hat{p}$.

Com efeito, dados dois caminhos $\alpha, \beta: I \longrightarrow M$, com $\alpha(0)=\beta(0)=x_{0}$, diremos que $\alpha$ e $\beta$ são equivalentes, e escreveremos $\alpha \equiv \beta$, quando $\alpha(1)=\beta(1)$ e $\left[\alpha \vee \beta^{-1}\right] \in H$ (onde o símbolo $\vee$ denota a justaposição de caminhos). Como $H$ é um subgrupo de $\pi_{1}\left(M, x_{0}\right)$, temos que $\equiv$ constitui uma relação de equivalência; assim, dado um caminho $\alpha: I \longrightarrow M, \operatorname{com} \alpha(0)=x_{0}$, utilizaremos a notação $\langle\alpha\rangle$ para nos referirmos à classe de equivalência de $\alpha$, segundo a relação $\equiv$. Seja, pois, $\hat{M}$ o conjunto de todas as classes de equivalência $\langle\alpha\rangle$ dos caminhos $\alpha: I \longrightarrow M$ que começam no ponto $x_{0}$, e definamos $\hat{p}: \hat{M} \longrightarrow M$ como sendo $\hat{p}(\langle\alpha\rangle)=\alpha(1)$. A fim de introduzir uma topologia em $\hat{M}$, consideremos a base $\mathcal{B}$ de $M$, constituída dos abertos $U \subseteq M$, conexos por caminhos, e tais que todo laço em $U$ é homotópico a uma constante em $M$ (lembre-se que, por ser uma variedade topológica, $M$ é localmente conexa por caminhos e localmente simplesmente conexa), e, para cada $\langle\alpha\rangle \in \hat{M}$ e cada aberto $U \in \mathcal{B}$, com $\alpha(1) \in U$, tomemos:

$$
\hat{U}\langle\alpha\rangle=\{\langle\alpha \vee \beta\rangle: \beta: I \longrightarrow U \text { é um caminho tal que } \beta(0)=\alpha(1)\} \text {. }
$$

Pode-se provar então que os conjuntos $\hat{U}\langle\alpha\rangle$ constituem a base de uma topologia em $\hat{M}$, segundo a qual $\hat{p}: \hat{M} \longrightarrow M$ é contínua e aberta (para mais detalhes, vide [R], paginas 295 a 299). Além disso, para cada $U \in \mathcal{B}$ e cada $\langle\alpha\rangle \in \hat{M}$, $\operatorname{com} \alpha(1) \in U$, a restrição $\left.\hat{p}\right|_{\hat{U}\langle\alpha\rangle}: \hat{U}\langle\alpha\rangle \longrightarrow U$ é uma bijeção (e, portanto, um homeomorfismo) sobre $U$. Observe ainda que, para cada $U \in \mathcal{B}$, a imagem inversa $\hat{p}^{-1}(U)$ é a reunião dos conjuntos $\hat{U}\langle\alpha\rangle$, onde $\alpha$ varia entre os caminhos em $M$ com origem $x_{0}$ e extremidade em $U$; logo, como dois desses conjuntos $\hat{U}\langle\alpha\rangle$, ou coincidem ou são disjuntos, segue que $\hat{p}: \hat{M} \longrightarrow M$ é uma aplicação de recobrimento. Agora, dado um caminho $\alpha: I \longrightarrow M$ com origem $\alpha(0)=x_{0}$, consideremos, para cada $t \in I$, o caminho $\alpha_{t}: I \longrightarrow M$ definido por $\alpha_{t}(s)=\alpha(s t)$; como $\alpha_{0}(s)=\alpha(0)=x_{0}$ e $\alpha_{1}(s)=\alpha(s)$ para todo $s \in I$, segue que $\alpha_{0}$ é o caminho constante igual a $x_{0}$, denotado por $\varepsilon_{x_{0}}: I \longrightarrow M$, enquanto que $\alpha_{1}=\alpha$. Assim, o caminho $\gamma_{\alpha}: I \longrightarrow \hat{M}$, dado por $\gamma_{\alpha}(t)=\left\langle\alpha_{t}\right\rangle$, é tal que $\gamma_{\alpha}(0)=\left\langle\alpha_{0}\right\rangle=\left\langle\varepsilon_{x_{0}}\right\rangle$ e $\gamma_{\alpha}(1)=\left\langle\alpha_{1}\right\rangle=\langle\alpha\rangle$; em outras palavras, todo ponto $\langle\alpha\rangle \in \hat{M}$ pode ser ligado ao ponto $\hat{x_{0}}=\left\langle\varepsilon_{x_{0}}\right\rangle \in \hat{M}$ por um caminho $\gamma_{\alpha}: I \longrightarrow \hat{M}$, o que prova que $\hat{M}$ é conexo por caminhos (e, portanto, conexo). Finalmente, sendo $\hat{\alpha}: I \longrightarrow \hat{M}$ o caminho definido por $\hat{\alpha}(t)=\left\langle\alpha_{t}\right\rangle$ (onde $\langle\alpha\rangle \in \hat{M})$, temos que $\hat{\alpha}(0)=\left\langle\alpha_{0}\right\rangle=\left\langle\varepsilon_{x_{0}}\right\rangle=\hat{x_{0}}$ e $(\hat{p} \circ \hat{\alpha})(t)=\hat{p}\left(\left\langle\alpha_{t}\right\rangle\right)=\alpha_{t}(1)=\alpha(t)$; logo, pela propriedade do levantamento único de caminhos, segue que $\hat{\alpha}$ é o único levantamento do caminho $\alpha: I \longrightarrow M$ (relativamente a $\hat{p}$ ), com início no ponto $\hat{x_{0}}=$ $\left\langle\varepsilon_{x_{0}}\right\rangle \in \hat{M}$. Por outro lado, dado um caminho $\alpha: I \longrightarrow M \operatorname{com} \alpha(0)=x_{0}$, e sendo $\hat{p}_{\#}: \pi_{1}\left(\hat{M}, \hat{x}_{0}\right) \longrightarrow \pi_{1}\left(M, x_{0}\right)$ o homomorfismo induzido pela aplicação de recobrimento $\hat{p}: \hat{M} \longrightarrow M$, temos que $[\alpha] \in \hat{p}_{\#}\left(\pi_{1}\left(\hat{M}, \hat{x_{0}}\right)\right)$ se, e somente se, o levantamento $\hat{\alpha}: I \longrightarrow \hat{M}$ 
for um laço com base em $\hat{x}_{0}$ (uma vez que $[\alpha]=[\hat{p} \circ \hat{\alpha}]=\hat{p}_{\#}([\alpha])$ ); isto, por sua vez, ocorre se, e somente se, $\hat{x_{0}}=\hat{\alpha}(1)=\left\langle\alpha_{1}\right\rangle=\langle\alpha\rangle$. Ora, por definição, sabemos que as classes de equivalência $\hat{x}_{0}=\left\langle\varepsilon_{x_{0}}\right\rangle$ e $\langle\alpha\rangle$ coincidem se, e só se, $[\alpha]=\left[\alpha \vee \varepsilon_{x_{0}}^{-1}\right] \in H$. Deste modo, concluímos que $[\alpha] \in \hat{p}_{\#}\left(\pi_{1}\left(\hat{M}, \hat{x}_{0}\right)\right)$ se, e somente se, $[\alpha] \in H$, o que prova que $H=\hat{p}_{\#}\left(\pi_{1}\left(\hat{M}, \hat{x}_{0}\right)\right)$, completando a demonstração.

Definição 1.2.1. Seja M uma variedade topológica conexa. Dado um subgrupo $H \subseteq \pi_{1}\left(M, x_{0}\right)$, onde $x_{0} \in M$, o recobrimento $\hat{p}: \hat{M} \longrightarrow M$ obtido acima é denominado recobrimento de $\mathbf{M}$ associado $a \mathrm{H}$.

Observe que, como $\hat{p}: \hat{M} \longrightarrow M$ é um homeomorfismo local sobrejetivo, o espaço de recobrimento $\hat{M}$ herda as propriedades topológicas locais de $M$, e vice-versa; em particular, temos que $\hat{M}$ é uma variedade topológica, de dimensão igual à de $M$, e (pelo teorema da invariância do domínio) $\hat{p}(\partial \hat{M})=\partial M$; além disso, da proposição 1.1.1, segue que, se $M$ for uma variedade orientável, então $\hat{M}$ também o será.

Lema 1.2.1. Dadas variedades topológicas conexas $M e N$, de mesma dimensão, considere uma aplicação contínua $f: M \longrightarrow N$, e sejam $x_{0} \in M$ e $c \in N$ tais que $f\left(x_{0}\right)=c$. Denotando por $\hat{q}: \hat{N} \longrightarrow N$ o recobrimento de $N$ associado ao subgrupo $H=f_{\#}\left(\pi_{1}\left(M, x_{0}\right)\right) \subseteq \pi_{1}(N, c)$, onde $f_{\#}: \pi_{1}\left(M, x_{0}\right) \longrightarrow \pi_{1}(N, c)$ é o homomorfismo induzido por $f$, segue que existe uma aplicação continua $\hat{f}: M \longrightarrow \hat{N}$ tal que $f=\hat{q} \circ \hat{f}$ (isto é, $f$ admite um levantamento $\hat{f}: M \longrightarrow \hat{N}$ relativamente a $\hat{q})$.

Demonstração. Com efeito, se $\hat{q}: \hat{N} \longrightarrow N$ é o recobrimento de $N$ associado à $f_{\#}\left(\pi_{1}\left(M, x_{0}\right)\right)$, temos que $\hat{q}_{\#}\left(\pi_{1}(\hat{N}, \hat{C})\right)=f_{\#}\left(\pi_{1}\left(M, x_{0}\right)\right)$, onde $\hat{c} \in \hat{q}^{-1}(c) ; \log \mathrm{o}$, da proposição 8 , página 162 , de [Li], segue que existe uma aplicação contínua $\hat{f}: M \longrightarrow \hat{N}$, com $\hat{f}\left(x_{0}\right)=\hat{c}$, tal que $f=\hat{q} \circ \hat{f}$.

Proposição 1.2.1 . Seja $M$ uma variedade topológica de dimensão n, conexa e sem fronteira, e denote por $\tilde{p}: \tilde{C} \longrightarrow M$ o recobrimento de $M$ obtido de uma componente conexa $\tilde{C}$ do fibrado orientado $\tilde{M}$ (em outras palavras, $\tilde{p}$ é a restrição da projeção orientada $\pi: \tilde{M} \longrightarrow M$ à componente $\tilde{C} \subseteq \tilde{M})$. Dado um ponto $x_{0} \in M$, considere ainda o conjunto $H \subseteq \pi_{1}\left(M, x_{0}\right)$ constituído das classes de homotopia dos laços $\alpha: I \longrightarrow M$, com base em $x_{0}$, que preservam orientação. Temos então que Hé um subgrupo de $\pi_{1}\left(M, x_{0}\right)$, e $\tilde{p}$ é o recobrimento de $M$ associado a $H$ (isto é, $\tilde{p}_{\#}\left(\pi_{1}\left(\tilde{C}, \tilde{x_{0}}\right)\right)=H$, onde $\left.\tilde{x_{0}} \in \tilde{p}^{-1}\left(x_{0}\right)\right)$.

DemonstraçÃo. De fato, considere dois laços $\alpha, \beta: I \longrightarrow M$, com base no ponto $x_{0}$; vamos mostrar primeiramente que, se $\alpha$ e $\beta$ são homotópicos, por uma homotopia que 
fixa o ponto base $x_{0}$, então $\alpha$ preserva orientação se, e somente se, $\beta$ também o faz. Com efeito, denotando por $\mu_{x_{0}}$ um dos dois geradores de $H_{n}\left(M, M \backslash\left\{x_{0}\right\}\right) \simeq \mathbb{Z}$, com $\mu_{x_{0}} \in \tilde{C}$, sabemos que $\alpha$ admite um único levantamento $\tilde{\alpha}: I \longrightarrow \tilde{C}$, relativamente ao recobrimento $\tilde{p}$, tal que $\tilde{\alpha}(0)=\mu_{x_{0}}$. Logo, supondo que $\alpha$ preserve orientação, temos que $\tilde{\alpha}(1)=\mu_{x_{0}}$, ou seja, $\tilde{\alpha}$ é um laço em $\tilde{C}$ com base em $\mu_{x_{0}}$. Isto posto, seja $\tilde{\beta}: I \longrightarrow \tilde{C}$ o único levantamento de $\beta$ (relativamente a $\tilde{p}$ ), satisfazendo $\tilde{\beta}(0)=\mu_{x_{0}}$, e denotemos por $H: I \times I \longrightarrow M$ a homotopia entre $\alpha$ e $\beta$ que fixa o ponto base $x_{0}$. Temos, pois, que $H$ admite um único levantamento $\tilde{H}: I \times I \longrightarrow \tilde{C}$, que constitui uma homotopia entre $\tilde{\alpha}$ e $\tilde{\beta}$ e mantém fixo o ponto $\mu_{x_{0}} \in \tilde{C}$ (isto é, $\tilde{H}(s, 0)=\tilde{\alpha}(s), \tilde{H}(s, 1)=\tilde{\beta}(1)$ e $\tilde{H}(0, t)=\tilde{H}(1, t)=\mu_{x_{0}}$ para quaisquer $s, t \in I)$. Em particular, segue que $\tilde{\beta}(1)=\tilde{H}(1,1)=\tilde{H}(0,1)=\tilde{\beta}(0)=\mu_{x_{0}}, \mathrm{o}$ que significa que o laço $\beta: I \longrightarrow M$ também preserva orientação.

Agora, observe que, se dois laços $\alpha, \beta: I \longrightarrow M$, com base no ponto $x_{0}$, preservam orientação, o mesmo se dá com o laço justaposto $\alpha \vee \beta: I \longrightarrow M$ e com os inversos $\alpha^{-1}$ e $\beta^{-1}$. Destas propriedades, podemos concluir então que o conjunto $H$ (das classes de homotopia dos caminhos que preservam orientação e têm base no ponto $x_{0}$ ) constitui um subgrupo de $\pi_{1}\left(M, x_{0}\right)$. Finalmente, para verificar que $\tilde{p}$ é o recobrimento de $M$ associado a $H$, considere um laço $\alpha: I \longrightarrow M$ com base no ponto $x_{0}$, e seja $\tilde{\alpha}: I \longrightarrow \tilde{C}$ o único levantamento de $\alpha$ (relativamente a $\tilde{p}$ ) tal que $\tilde{\alpha}(0)=\mu_{x_{0}}$. Suponto $[\alpha] \in H$, temos que $\alpha$ preserva orientação, isto é, $\tilde{\alpha}$ é um laço com base em $\mu_{x_{0}}$ (pois $\left.\tilde{\alpha}(0)=\tilde{\alpha}(1)=\mu_{x_{0}}\right)$; $\operatorname{logo}, \operatorname{como} \alpha=\tilde{p} \circ \tilde{\alpha}$, segue que $[\alpha]=[\tilde{p} \circ \tilde{\alpha}]=\tilde{p}_{\#}([\tilde{\alpha}]) \in \tilde{p}_{\#}\left(\pi_{1}\left(\tilde{C}, \mu_{x_{0}}\right)\right)$. Reciprocamente, se $[\alpha] \in \tilde{p}_{\#}\left(\pi_{1}\left(\tilde{C}, \mu_{x_{0}}\right)\right)$, segue que existe um laço $\tilde{\beta}: I \longrightarrow \tilde{C}$, com base em $\mu_{x_{0}}$, tal que $[\alpha]=\tilde{p}_{\#}([\tilde{\beta}])=[\tilde{p} \circ \tilde{\beta}]$; assim, denotando por $\beta: I \longrightarrow M$ o laço (com base em $\left.x_{0}\right)$ dado por $\beta=\tilde{p} \circ \tilde{\beta}$, temos que $[\alpha]=[\beta]$, sendo que $\tilde{\beta}$ é o único levantamento de $\beta$ (relativamente a $\tilde{p}$ ) tal que $\tilde{\beta}(0)=\mu_{x_{0}}$. Além disso, como $\tilde{\beta}(0)=\tilde{\beta}(1)=\mu_{x_{0}}$, segue que $\beta$ preserva orientação, e, portanto, $[\alpha]=[\beta] \in H$. Deste modo, concluímos que $\tilde{p}_{\#}\left(\pi_{1}\left(\tilde{C}, \mu_{x_{0}}\right)\right)=H$, conforme queríamos provar.

Definição 1.2.2 . Sendo M uma variedade topológica de dimensão n, conexa e com fronteira, sabemos que existem uma vizinhança aberta $U$ de $\partial M$ em $M$, e um homeomorfismo $h: U \longrightarrow$ $\partial M \times(0,1]$, tais que $h(x)=(x, 0)$ para todo $x \in \partial M$ (vide [Bwn1], teorema 2, página 339). Isto, por sua vez, significa que a inclusão $i:$ Int $M \longrightarrow M$ é um equivalência homotópica: com efeito, sendo $V \subseteq U$ tal que $V=h^{-1}\left(\partial M \times\left[0, \frac{1}{2}\right]\right)$, e denotando por $\rho: \partial M \times\left[0, \frac{1}{2}\right] \longrightarrow \partial M \times\left[\frac{1}{4}, \frac{1}{2}\right]$ o homeomorfismo dado por $\rho\left(y_{0}, t_{0}\right)=\left(y_{0}, \frac{t_{0}}{2}+\frac{1}{4}\right)$, temos que a aplicação $r: M \longrightarrow \operatorname{Int} M$, definida por

$$
r(x)=\left\{\begin{array}{ll}
x & \text { se } x \in M \backslash V \\
h^{-1}\left(x, \frac{1}{4}\right) & \text { se } x \in \partial M \\
h^{-1}(\rho(h(x))) & \text { se } x \in V \backslash \partial M
\end{array},\right.
$$


é uma inversa homotópica de $i$. Logo, o homomorfismo induzido $i_{\#}: \pi_{1}\left(\operatorname{Int} M, x_{0}\right) \longrightarrow \pi_{1}\left(M, x_{0}\right)$ constitui um isomorfismo, qualquer que seja $x_{0} \in \operatorname{Int} M$. Por outro lado, se Mé não-orientável, segue que o fibrado orientado $\overparen{\operatorname{Int} M}$ de Int $M$ é conexo, e a projeção orientada $\tilde{p}: \overparen{\operatorname{Int} M} \longrightarrow \operatorname{Int} M$ é um recobrimento de duas folhas. Assim, definimos o recobrimento orientado de $M$ como sendo a aplicação $\hat{p}: \widehat{M} \longrightarrow M$ tal que:

- se Mé orientável, $\widehat{p}: \widehat{M} \longrightarrow$ Mé a identidade;

- se $M$ é não-orientável, $\widehat{p}: \widehat{M} \longrightarrow M$ é o recobrimento de $M$ associado ao subgrupo ( $i_{\#} \circ$ $\left.\tilde{p}_{\#}\right)\left(\pi_{1}\left(\widetilde{\operatorname{Int} M}, \tilde{x_{0}}\right)\right) \subseteq \pi_{1}\left(M, x_{0}\right)$, isto é, $\widehat{p}_{\#}\left(\pi_{1}\left(\widehat{M}, \widehat{x_{0}}\right)\right)=\left(i_{\#} \circ \tilde{p}_{\#}\right)\left(\pi_{1}\left(\widetilde{\operatorname{Int} M}, \tilde{x_{0}}\right)\right)$, onde $x_{0} \in \operatorname{Int} M$, $\tilde{x_{0}} \in \tilde{p}^{-1}\left(x_{0}\right)$ e $\widehat{x_{0}} \in \widehat{p}^{-1}\left(x_{0}\right)$.

Note que, como $\hat{p}: \widehat{M} \longrightarrow M$ é um homeomorfismo local, segue que o espaço $\widehat{M}$ é uma variedade topológica de dimensão $n$, com fronteira, tal que $\widehat{p}(\partial \widehat{M})=\partial M$.

O lema a seguir nos mostra que, para variedades topológicas sem fronteira, o espaço de recobrimento orientado coincide com o fibrado orientado (a menos de homeomorfismos).

Lema 1.2.2. Seja M uma variedade topológica de dimensão n, conexa e com fronteira (eventualmente vazia), e denote por $\hat{p}: \widehat{M} \longrightarrow$ Mo recobrimento orientado de $M$. Sendo $\tilde{p}: \tilde{C} \longrightarrow \operatorname{Int} M$ o recobrimento obtido de uma componente conexa $\tilde{C}$ do fibrado orientado de Int $M$, segue que existe um homeomorfismo $\varphi: \tilde{C} \longrightarrow$ Int $\widehat{M}$ que comuta o seguinte diagrama:

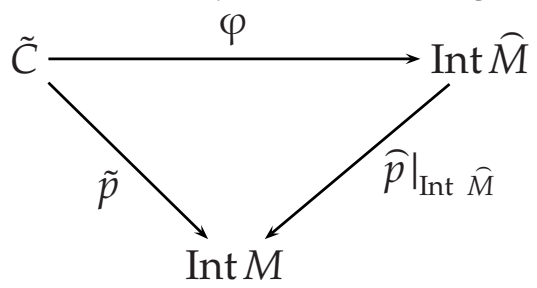

(note que a restrição de $\widehat{p}$ a Int $\widehat{M}$ é um recobrimento de $\operatorname{Int} M$ ).

DemonstraçÃo. Com efeito, se $M$ é orientável, temos que $\widehat{p}: \widehat{M} \longrightarrow M$ é a identidade, isto é, $\widehat{M}=M$, e, portanto, o homeomorfismo $\varphi$ é dado pelo recobrimento $\tilde{p}: \tilde{C} \longrightarrow$ Int $M=\operatorname{Int} \widehat{M}$ (o qual, pelo fato de $M$ ser orientável, constitui um homeomorfismo, cuja inversa é uma das duas possíveis orientações em Int $M$ ). Por outro lado, se $M$ é não-orientável, temos que $\tilde{p}: \tilde{C}=\overparen{\operatorname{Int} M} \longrightarrow \operatorname{Int} M$ é a projeção orientada de $\operatorname{Int} M$, e $\widehat{p}: \widehat{M} \longrightarrow M$ é o recobrimento de $M$ associado ao subgrupo $\left(i_{\#} \circ \tilde{p}_{\#}\right)\left(\pi_{1}\left(\widetilde{\operatorname{Int} M}, \tilde{x}_{0}\right)\right) \subseteq$ $\pi_{1}\left(M, x_{0}\right)$, onde $i_{\#}: \pi_{1}\left(\operatorname{Int} M, x_{0}\right) \longrightarrow \pi_{1}\left(M, x_{0}\right)$ é o isomorfismo induzido pela inclusão, com $x_{0} \in \operatorname{Int} M$ e $\tilde{x}_{0} \in \tilde{p}^{-1}\left(x_{0}\right)$ (vide definição 1.2.2); logo, dado $\widehat{x}_{0} \in \widehat{p}^{-1}\left(x_{0}\right) \subseteq \operatorname{Int} \widehat{M}$, segue que $\widehat{p}_{\#}\left(\pi_{1}\left(\operatorname{Int} \widehat{M} \widehat{x}_{0}\right)\right)=\tilde{p}_{\#}\left(\pi_{1}\left(\overparen{\operatorname{Int} M}, \tilde{x}_{0}\right)\right)$, o que, por sua vez, implica que existe um homeomorfismo $\varphi: \overparen{\operatorname{Int} M} \longrightarrow \operatorname{Int} \widehat{M}$ satisfazendo $\left(\left.\hat{p}\right|_{\text {Int } \widehat{M}}\right) \circ \varphi=\tilde{p}$ e $\varphi\left(\tilde{x}_{0}\right)=\widehat{x}_{0}$ (vide [Vi], proposições 4.18 e 4.19 , página 104), conforme queríamos demosntrar. 
Observe ainda que a existência do homeomorfismo $\varphi: \tilde{C} \longrightarrow \operatorname{Int} \widehat{M}$, mencionado no lema anterior, significa que, se $U \subseteq \operatorname{Int} M$ é um aberto orientável, então uma orientação $s: U \longrightarrow \tilde{C}$ induz uma aplicação contínua $\widehat{s}: U \longrightarrow \operatorname{Int} \widehat{M}$, dada por $\widehat{s}(x)=(\varphi \circ s)(x)$ para cada $x \in U$; assim, como $\hat{p} \circ \varphi=\tilde{p}$, temos que $\hat{p} \circ \widehat{s}=\hat{p} \circ(\varphi \circ s)=\tilde{p} \circ s=\operatorname{Id}_{U}$, onde $\operatorname{Id}_{U}: U \longrightarrow U$ é a identidade. Por este motivo, à aplicação $\widehat{s}: U \longrightarrow \operatorname{Int} \widehat{M}$, denominamos orientação induzida por $s$ no espaço de recobrimento orientado de $M$ (ou, simplesmente, orientação induzida por s em $\widehat{M}$ ).

Proposição 1.2.2. Sendo $M$ uma variedade topológica de dimensão $n$, conexa e com fronteira (eventualmente vazia), e denotando por $\hat{p}: \widehat{M} \longrightarrow M$ o recobrimento orientado de $M$, temos que:

(i) $\bar{p}$ possui uma ou duas folhas, conforme $M$ seja respectivamente orientável ou não;

(ii) a variedade $\widehat{M}$ é sempre orientável (independentemente da orientabilidade de $M$ ).

DemonstraÇão. (i) De fato, sendo $\tilde{p}: \tilde{C} \longrightarrow$ Int $M$ o recobrimento obtido de uma componente conexa do fibrado orientado de $\operatorname{Int} M$, sabemos que $\tilde{p}$ possui uma ou duas folhas conforme Int $M$ (e, conseqüentemente, $M$ ) é orientável ou não, respectivamente. Logo, como $\tilde{C}$ é homeomorfo a Int $\widehat{M}$ (pelo lema anterior), segue que a restrição $\left(\left.\widehat{p}\right|_{\text {Int } \widehat{M}}\right): \operatorname{Int} \widehat{M} \longrightarrow$ Int $M$ é um recobrimento de uma folha se $M$ é orientável, ou de duas folhas caso $M$ seja não-orientável. Portanto, como todas as fibras $\hat{p}^{-1}(x)$, com $x \in M$, possuem o mesmo número cardinal (uma vez que $M$ é uma variedade topológica conexa), concluímos que $\widehat{p}: \widehat{M} \longrightarrow M$ é um recobrimento de uma ou duas folhas, caso $M$ seja respectivamente orientável ou não.

(ii) Com efeito, da proposição 1.1.2, sabemos que o fibrado orientado de Int $M$ é sempre orientável, independentemente da orientabilidade de $M$. Logo, sendo $\tilde{p}: \tilde{C} \longrightarrow$ Int $M$ o recobrimento obtido de uma componente conexa do fibrado orientado de Int $M$, temos que $\tilde{C}$ é orientável, e, portanto, a existência do homeomorfismo $\varphi: \tilde{C} \longrightarrow \operatorname{Int} \widehat{M}$ (dado pelo lema 1.2.2) nos garante que Int $\widehat{M}$ é orientável (vide proposição 1.1.1, substituindo $f$ por $\varphi^{-1}$ ). Deste modo, concluímos que $\operatorname{Int} \widehat{M}$, e, conseqüentemente, $\widehat{M}$, é sempre orientável, quer $M$ seja orientável ou não.

A seguir, veremos que as noções de laços que preservam ou invertem orientação (dadas na definição 1.1.4) podem ser estendidas para laços cujas imagens não se encontram necessariamente no interior de uma variedade. 
Definição 1.2.3 . Seja $M$ uma variedade topológica de dimensão $n$, conexa e com fronteira, $e$ denote por $\hat{p}: \widehat{M} \longrightarrow$ Mo recobrimento orientado de $M$. Dado um laço $\omega: I \longrightarrow M$, com base num ponto $x_{0} \in M$, dizemos que $\omega$ preserva orientação relativamente ao recobrimento orientado de $\boldsymbol{M}$ (ou, simplesmente, preserva orientação em $\widehat{M})$ se existe um laço $\widehat{\omega}: I \longrightarrow \widehat{M}$ tal que $\hat{p} \circ \widehat{\omega}=\omega$, isto é, se $\omega$ pode ser levantado para um laço em $\widehat{M}$ relativamente ao recobrimento orientado $\hat{p}$; caso contrário, diremos que $\omega$ inverte orientação relativamente ao recobrimento orientado de $M$ (ou, simplesmente, inverte orientação em $\widehat{M}$ ).

Proposição 1.2.3 . Seja $M$ uma variedade topológica de dimensão n, conexa e com fronteira (eventualmente vazia), e denote por $\hat{p}: \widehat{M} \longrightarrow M$ o recobrimento orientado de $M$. Dado um laço $\omega: I \longrightarrow$ Int $M$, temos que $\omega$ preserva (respectivamente, inverte) orientação em $\widehat{M}$ se, e somente se, ele preserva (respectivamente, inverte) orientação.

Demonstração. Com efeito, seja $\tilde{p}: \tilde{C} \longrightarrow$ Int $M$ o recobrimento obtido de uma componente conexa do fibrado orientado de Int $M$, e considere o homeomorfismo $\varphi: \tilde{C} \longrightarrow$ Int $\widehat{M}$ tal que $\tilde{p}=\left.\hat{p}\right|_{\text {Int } \widehat{M}} \circ \varphi$ (cuja existência é garantida pelo lema 1.2.2). Por definição, sabemos que $\omega: I \longrightarrow$ Int $M$ é um laço que preserva orientação se, e somente se, ele pode ser levantado para um laço $\tilde{\omega}: I \longrightarrow \tilde{C}$ relativamente a $\tilde{p}$; entretanto, como $\tilde{p}=\left.\hat{p}\right|_{\text {Int } \hat{M}} \circ \varphi$, segue que isto ocorre se, e somente se, $\omega$ admite um levantamento $\widehat{\omega}: I \longrightarrow \operatorname{Int} \widehat{M}$ relativamente ao recobrimento $\left.\hat{p}\right|_{\text {Int } \widehat{M}}: \operatorname{Int} \widehat{M} \longrightarrow \operatorname{Int} M$. Portanto, $\omega$ preserva ou inverte orientação se, e só se, ele respectivamente preserva ou inverte orientação em $\widehat{M}$, conforme queríamos provar.

Na proposição 1.2.1, vimos que, se $M$ é uma variedade sem fronteira, o recobrimento $\tilde{p}: \tilde{C} \longrightarrow M$, obtido de uma componente conexa $\tilde{C}$ do fibrado orientado $\tilde{M}$, constitui o recobrimento de $M$ associado ao subgrupo $H \subseteq \pi_{1}\left(M, x_{0}\right)$, gerado pelas classes de homotopia dos laços que preservam orientação. Por outro lado, se $M$ for uma variedade com fronteira, a proposição a seguir (cuja demonstração é análoga à da proposição 1.2.1, e, portanto, será omitida) nos mostra que o recobrimento orientado $\widehat{p}: \widehat{M} \longrightarrow M$ constitui o recobrimento de $M$ associado ao subgrupo $\widehat{H} \subseteq \pi_{1}\left(M, x_{0}\right)$, gerado pelas classs de homotopia dos laços que preservam orientação em $\widehat{M}$. Assim, $\hat{p}$ é, de certo modo, uma generalização do recobrimento $\tilde{p}$ para variedades com fronteira.

Proposição 1.2.4. Sendo M uma variedade topológica de dimensão n, conexa e com fronteira (eventualmente vazia), denote por $\widehat{p}: \widehat{M} \longrightarrow M$ o recobrimento orientado de $M$, e considere o subconjunto $\hat{H} \subseteq \pi_{1}\left(M, x_{0}\right)$, constituído das classes de homotopia dos laços $\alpha: I \longrightarrow M$, com base em $x_{0} \in M$, que preservam orientação em $\widehat{M}$. Temos então que $\widehat{H}$ é um subgrupo de $\pi_{1}\left(M, x_{0}\right)$, e $\widehat{p}$ é o recobrimento de Massociado a $\widehat{H}\left(\right.$ isto é, $\widehat{p}_{\#}\left(\pi_{1}\left(\widehat{M}, \widehat{x}_{0}\right)\right)=\widehat{H}$, onde $\widehat{x}_{0} \in \widehat{p}^{-1}\left(x_{0}\right)$ ). 
Proposição 1.2.5 . Dada uma variedade topológica $M$, conexa e com fronteira (eventualmente vazia), temos que $M$ é orientável se, e somente se, todo laço em M preserva orientação relativamente ao recobrimento orientado de $M$.

DemonstraçÃo. Com efeito, se $M$ é orientável, segue que o recobrimento orientado $\widehat{p}: \widehat{M} \longrightarrow M$ é a identidade, e, portanto, todo laço em $M$ pode ser levantado para um laço em $\widehat{M}$ (ou seja, todo laço em $M$ preserva orientação em $\widehat{M}$ ). Reciprocamente, suponhamos que $M$ seja não-orientável; neste caso, o recobrimento orientado $\widehat{p}: \widehat{M} \longrightarrow$ $M$ é um recobrimento de duas folhas, o que, pelo fato de $\widehat{M}$ ser uma variedade conexa por caminhos, implica que $\hat{p}: \widehat{M} \longrightarrow M$ é um recobrimento regular (vide [Li], corolário 2 , página 152). Isto, por sua vez, significa que, dado um laço qualquer $\omega: I \longrightarrow M$, com base num ponto $x \in M$, ou todos os levantamentos de $\omega$ a partir dos pontos $\widehat{x} \in \hat{p}^{-1}(x)$ são laços, ou nenhum é. Agora, observe que, como $\widehat{p}: \widehat{M} \longrightarrow M$ é um recobrimento de duas folhas, segue que existem $\widehat{x}_{0}, \widehat{x}_{1} \in \widehat{p}^{-1}(x)$ tais que $\widehat{x}_{0} \neq \widehat{x}_{1} ; \operatorname{logo}$, sendo $\widehat{\omega}: I \longrightarrow \widehat{M}$ um caminho tal que $\widehat{\omega}(0)=\widehat{x}_{0}$ e $\widehat{\omega}(1)=\widehat{x}_{1}$, e denotando por $\omega: I \longrightarrow M$ o laço com base em $x$ dado por $\omega=\widehat{p} \circ \widehat{\omega}$, temos que $\widehat{\omega}$ é um levantamento de $\omega$ que não constitui um laço em $\widehat{M}$. Portanto, do que vimos acima, concluímos que $\omega$ é um laço em $M$ que não pode ser levantado para um laço em $\widehat{M}$ (isto é, $\omega$ inverte orientação em $\widehat{M}$ ), o que mostra que, se $M$ é não-orientável, nem todo laço em $M$ preserva orientação em $\widehat{M}$. Daí, segue então que, se todo laço em $M$ preserva orientação em $\widehat{M}$, a variedade $M$ deve ser orientável.

Agora, observe que, uma vez definidos os conceitos de laços que preservam ou invertem orientação relativamente ao recobrimento orientado, podemos utilizá-los para introduzir a seguinte

Definição 1.2.4 . Sejam $M$ e $N$ variedades topológicas de dimensão n, com fronteira, e denote por $\widehat{p}_{M}: \widehat{M} \longrightarrow M e \hat{p}_{N}: \widehat{N} \longrightarrow N$ os recobrimentos orientados de $M$ e $N$, respectivamente. Dada uma aplicação contínua $f: M \longrightarrow N$, dizemos que $f$ é fiel à orientação relativamente aos recobrimentos orientados de $M$ e $N$ (ou, simplesmente, fiel à orientação em $\widehat{M} e \hat{N}$ ) se a imagem $f \circ \alpha: I \longrightarrow N$, de todo laço $\alpha: I \longrightarrow M$ que preserva (respectivamente, inverte) orientação em $\widehat{M}$, for um laço que preserva (respectivamente, inverte) orientação em $\widehat{N}$.

A proposição a seguir mostra que o conceito de aplicação fiel à orientação relativamente a recobrimentos orientados é, de fato, uma generalização da definição 1.1.5.

Proposição 1.2.6. Se Me N são variedades topológicas de dimensão n, conexas e com fronteira (eventualmente vazias), e $f: M \longrightarrow N$ é uma aplicação fiel à orientação relativamente aos recobrimentos orientados de $M$ e $N$, tal que $f(\operatorname{Int} M) \subseteq \operatorname{Int} N$, então f é fiel à orientação. 
Demonstração. De fato, se $f: M \longrightarrow N$ é fiel à orientação relativamente aos recobrimentos orientados de $M$ e $N$, com $f(\operatorname{Int} M) \subseteq \operatorname{Int} N$, segue (da proposição 1.2.3) que um laço $\omega: I \longrightarrow$ Int $M$ preserva ou inverte orientação se, e somente se, o laço $f \circ \omega: I \longrightarrow$ Int $N$ respectivamente preserva ou inverte orientação, o que (pela definição 1.1.5) significa que $f$ e fiel à orientação.

Proposição 1.2.7. Se M é uma variedade topológica de dimensão $n$, conexa e com fronteira (eventualmente vazia), então todo recobrimento $p: N \longrightarrow M$ é uma aplicação fiel à orientação relativamente aos recobrimentos orientados de $N$ e $M$.

DemonstraÇÃo. Denotemos por $\widehat{p}_{N}: \widehat{N} \longrightarrow N$ e $\widehat{p}_{M}: \widehat{M} \longrightarrow M$ os recobrimentos orientados de $N$ e $M$. Por definição, sabemos que $M$ é orientável se, e somente se, Int $M$ é orientável; $\operatorname{logo}$, como a restrição $\left.p\right|_{\operatorname{Int} N}: \operatorname{Int} N \longrightarrow \operatorname{Int} M$ é um homeomorfismo local sobre Int $M$, segue (da proposição 1.1.1) que a orientabilidade de $M$ implica na orientabilidade de Int $N$, o que mostra que, se $M$ é orientável, $N$ também o é. Entretanto, pela proposição 1.2.5, sabemos que, se as variedades $M$ e $N$ são orientáveis, então ambas possuem apenas laços que preservam orientação relativamente a seus recobrimentos orientados. Assim, concluímos que, caso $M$ seja orientável, o recobrimento $p: N \longrightarrow M$ é fiel à orientação relativamente aos recobrimentos orientados de $N$ e $M$.

Suponhamos agora que $M$ seja não-orientável. Para provarmos que o recobrimento $p: N \longrightarrow M$ é uma aplicação fiel à orientação relativamente aos recobrimentos orientados de $N$ e $M$, basta mostrarmos que um laço $\alpha: I \longrightarrow N$ preserva orientação em $\widehat{N}$ se, e somente se, o laço $\beta=p \circ \alpha: I \longrightarrow M$ preserva orientação em $\widehat{M}$.

Isto posto, considere um laço $\alpha: I \longrightarrow N$, com base num ponto $y \in N$, e suponhamos que $\alpha$ preserve orientação em $\widehat{N}$. Como $N$ e $\widehat{N}$ são variedades topológicas conexas (e, portanto, conexas por caminhos), sabemos que, dados $y_{0} \in \operatorname{Int} N, \widehat{y}_{0} \in \widehat{p}_{N}^{-1}\left(y_{0}\right) \subseteq \operatorname{Int} \widehat{N}$ e $\widehat{y} \in \widehat{p}_{N}^{-1}(y)$, os homomorfismos $\phi_{N}: \pi_{1}\left(N, y_{0}\right) \longrightarrow \pi_{1}(N, y)$ e $\widehat{\phi}_{N}: \pi_{1}\left(\widehat{N}, \widehat{y}_{0}\right) \longrightarrow \pi_{1}(\widehat{N}, \widehat{y})$ definidos por $\phi_{N}([\gamma])=\left[\omega \vee \gamma \vee \omega^{-1}\right]$ e $\widehat{\phi}_{N}([\hat{\gamma}])=\left[\widehat{\omega} \vee \hat{\gamma} \vee \widehat{\omega}^{-1}\right]$, para cada $[\gamma] \in \pi_{1}\left(N, y_{0}\right)$ e cada $[\widehat{\gamma}] \in \pi_{1}\left(\widehat{N}, \widehat{y}_{0}\right)$, constituem isomorfismos, onde $\omega: I \longrightarrow N$ e $\widehat{\omega}: I \longrightarrow \widehat{N}$ são caminhos ligando $y$ a $y_{0}$, e $\hat{y}$ a $\hat{y}_{0}$, respectivamente. Sejam, pois, $i: \operatorname{Int} N \longrightarrow N$ e $\widehat{i}:$ Int $\widehat{N} \longrightarrow \widehat{N}$ as inclusões, e denote por $\tilde{p}_{N}: \tilde{C}_{N} \longrightarrow$ Int $N$ o recobrimento obtido de uma componente conexa $\tilde{C}_{N}$ do fibrado orientado de $\operatorname{Int} N$. Dado $\tilde{y}_{0} \in \tilde{p}_{N}^{-1}\left(y_{0}\right)$, tal que $\tilde{y}_{0}$ é um gerador de $\mathrm{H}_{n}\left(\operatorname{Int} N\right.$, Int $\left.N \backslash\left\{y_{0}\right\}\right)$ contido em $\tilde{C}_{N}$, considere ainda a aplicação $\varphi_{N}: \tilde{C}_{N} \longrightarrow \operatorname{Int} \widehat{N}$ dada por:

- se $N$ é orientável (e, conseqüentemente, o recobrimento orientado $\widehat{p}_{N}: \widehat{N} \longrightarrow N$ é a identidade), $\varphi_{N}=\tilde{p}_{N}$;

- se $N$ é não-orientável, $\varphi_{N}$ é o homeomorfismo dado pelo lema 1.2.2.

Conforme vimos na definição 1.2.2, temos que as inclusões $i$ e $\hat{i}$ são equivalências 
homotópicas, e, portanto, os homomorfismos induzidos $i_{\#}: \pi_{1}\left(\operatorname{Int} N, y_{0}\right) \longrightarrow \pi_{1}\left(N, y_{0}\right)$ e $\widehat{i}_{\#}: \pi_{1}\left(\operatorname{Int} \widehat{N}, \widehat{y}_{0}\right) \longrightarrow \pi_{1}\left(\widehat{N}, \widehat{y}_{0}\right)$ são isomorfismos que comutam o seguinte diagrama:

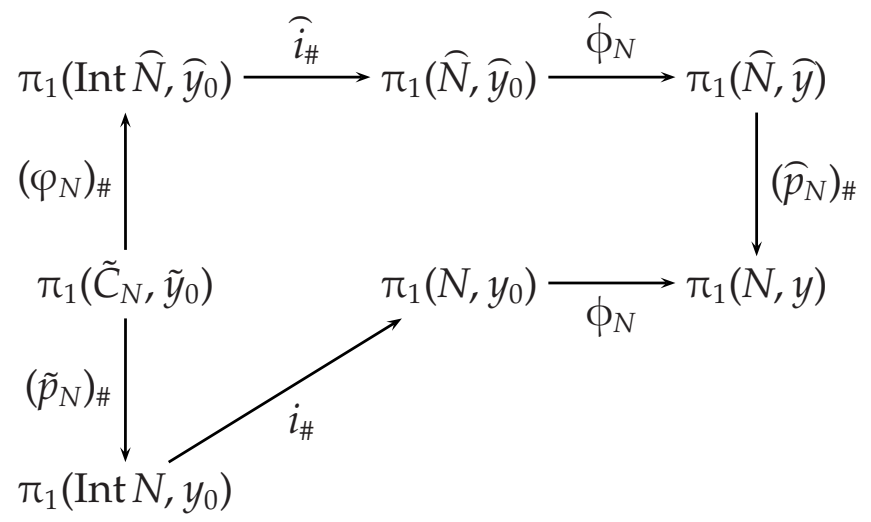

Agora, lembrando que $\alpha: I \longrightarrow N$ é um laço que preserva orientação em $\widehat{N}$, segue que existe um laço $\widehat{\alpha}: I \longrightarrow \widehat{N}$, com base em $\widehat{y}$, tal que $\widehat{p}_{N} \circ \widehat{\alpha}=\alpha$. Por outro lado, como $i_{\#}$ e $\phi_{N}$ são isomorfismos, segue que existe um laço $\alpha^{\prime}: I \longrightarrow \operatorname{Int} N$, com base em $y_{0}$, que satisfaz $\left(\phi_{N} \circ i_{\#}\right)\left(\left[\alpha^{\prime}\right]\right)=[\alpha]$. Vamos mostrar então que $\alpha^{\prime}$ preserva orientação (ou seja, $\alpha^{\prime}$ pode ser levantado para um laço em $\tilde{C}_{N}$, relativamente ao recobrimento $\tilde{p}_{N}$ ).

Com efeito, se $N$ é orientável, a conclusão é imediata (pois, pela proposição 1.1.2, teremos que $\alpha^{\prime}$ preserva orientação). Caso contrário, isto é, se $N$ é não-orientável, temos que $\varphi_{N}: \tilde{C}_{N}=\overparen{\operatorname{Int} N} \longrightarrow \operatorname{Int} \widehat{N}$ é um homeomorfismo que leva $\tilde{y}_{0}$ em $\widehat{y}_{0}$, e, conseqüentemente, o homomorfismo induzido $\left(\varphi_{N}\right)_{\#}: \pi_{1}\left(\widetilde{\operatorname{Int} N}, \tilde{y}_{0}\right) \longrightarrow \pi_{1}\left(\operatorname{Int} \widehat{N}, \widehat{y}_{0}\right)$ é um isomorfismo; isto posto, seja $\tilde{\xi}: I \longrightarrow \overparen{\operatorname{Int} N}$ um laço, com base em $\tilde{y}_{0}$, tal que $\left[\widehat{\phi}_{N} \circ \widehat{i}_{\#} \circ\left(\varphi_{N}\right)_{\#}\right]([\tilde{\xi}])=[\widehat{\alpha}] \in \pi_{1}(\widehat{N}, \widehat{y})$. Da comutatividade do diagrama acima, segue que:

$$
\begin{aligned}
{\left[\tilde{p}_{N} \circ \tilde{\xi}\right] } & =\left(\tilde{p}_{N}\right)_{\#}([\tilde{\xi}])=\left[\left(\phi_{N} \circ i_{\#}\right)^{-1} \circ\left(\widehat{p}_{N}\right)_{\#} \circ\left(\widehat{\phi}_{N} \circ \widehat{i}_{\#} \circ\left(\varphi_{N}\right)_{\#}\right)\right]([\tilde{\xi}]) \\
& =\left[\left(\phi_{N} \circ i_{\#}\right)^{-1} \circ\left(\hat{p}_{N}\right)_{\#}\right]([\alpha])=\left(\phi_{N} \circ i_{\#}\right)^{-1}([\alpha]) \\
& =\left(\phi_{N} \circ i_{\#}\right)^{-1}\left[\left(\phi_{N} \circ i_{\#}\right)\left(\left[\alpha^{\prime}\right]\right)\right]=\left[\alpha^{\prime}\right] .
\end{aligned}
$$

Logo, denotando por $\xi: I \longrightarrow$ Int $N$ o laço (com base em $y_{0}$ ) dado por $\xi=\tilde{p}_{N} \circ \tilde{\xi}$, temos que $[\xi]=\left[\alpha^{\prime}\right]$, sendo que $\tilde{\xi}$ é o único levantamento de $\xi$ (relativamente a $\tilde{p}_{N}$ ) tal que $\tilde{\xi}(0)=\tilde{\xi}(1)=\tilde{y}_{0}$. Isto, por sua vez, significa que $\xi$ é um laço que preserva orientação, e, portanto (como $[\xi]=\left[\alpha^{\prime}\right]$ ), segue que $\alpha^{\prime}$ também o é (pois qualquer homotopia entre $\xi$ e $\alpha^{\prime}$ pode ser levantada para uma homotopia entre $\tilde{\xi}$ e um levantamento de $\alpha^{\prime}$ ).

Agora, considerando $x_{0}=p\left(y_{0}\right) \in \operatorname{Int} M$, seja $\left.p\right|_{\operatorname{Int} N}: \operatorname{Int} N \longrightarrow \operatorname{Int} M$ o recobrimento de Int $M$ obtido pela restrição de $p$ a Int $M$, e denote por $\tilde{p}_{M}: \overparen{\operatorname{Int} M} \longrightarrow \operatorname{Int} M$ a projeção 
orientada de Int $M$ (que é um recobrimento de duas folhas, pois estamos supondo $M$ não-orientável). Pela proposição 1.1.5, temos que $\left.p\right|_{\operatorname{Int} N}$ é uma aplicação fiel à orientação; logo, como $\alpha^{\prime}: I \longrightarrow \operatorname{Int} N$ é um laço que preserva orientação, segue que o laço $\beta^{\prime}=\left(\left.p\right|_{\text {Int } N}\right) \circ \alpha^{\prime}=p \circ \alpha^{\prime}: I \longrightarrow \operatorname{Int} M\left(\right.$ com base em $\left.p\left(y_{0}\right)=x_{0}\right)$ também o é, ou seja, $\beta^{\prime}$ pode ser levantado para um laço $\tilde{\beta}^{\prime}: I \longrightarrow \widetilde{\operatorname{Int} M}$, relativamente ao recobrimento $\tilde{p}_{M}$, com base num ponto $\tilde{x}_{0} \in \tilde{p}_{M}^{-1}\left(x_{0}\right) \subseteq \widetilde{\operatorname{Int} M}$. Assim sendo, vamos mostrar então que o laço $\beta=p \circ \alpha: I \longrightarrow M$ pode ser levantado para um laço em $\widehat{M}$, relativamente ao recobrimento orientado $\widehat{p}: \widehat{M} \longrightarrow M$.

De fato, dados $\widehat{x}_{0} \in \widehat{p}_{M}^{-1}\left(x_{0}\right) \subseteq \operatorname{Int} \widehat{M}$ e $\widehat{x} \in \widehat{p}_{M}^{-1}(x)$, onde $x=p(y)$, considere as seguintes aplicações:

- $j_{\#}: \pi_{1}\left(\operatorname{Int} M, x_{0}\right) \longrightarrow \pi_{1}\left(M, x_{0}\right)$ e $\widehat{j_{\#}}: \pi_{1}\left(\operatorname{Int} \widehat{M}, \widehat{x}_{0}\right) \longrightarrow \pi_{1}\left(\widehat{M}, \widehat{x}_{0}\right)$ são os homomorfismos induzidos pelas inclusões $j:$ Int $M \longrightarrow M$ e $\hat{j}: \operatorname{Int} \widehat{M} \longrightarrow \widehat{M}$;

- $\left(\varphi_{M}\right)_{\#}: \pi_{1}\left(\widetilde{\operatorname{Int} M}, \tilde{x}_{0}\right) \longrightarrow \pi_{1}\left(\operatorname{Int} \widehat{M}, \widehat{x}_{0}\right)$ é o homomorfismo induzido pelo homeomorfismo $\varphi_{M}: \overparen{\operatorname{Int} M} \longrightarrow \operatorname{Int} \widehat{M}$, dado pelo lema 1.2.2;

- $\phi_{M}: \pi_{1}\left(M, x_{0}\right) \longrightarrow \pi_{1}(M, x)$ e $\widehat{\phi}_{M}: \pi_{1}\left(\widehat{M}, \widehat{x}_{0}\right) \longrightarrow \pi_{1}(\widehat{M}, \widehat{x})$ são os isomorfismos definidos por $\phi_{M}([\delta])=\left[\eta \vee \delta \vee \eta^{-1}\right]$ e $\widehat{\phi}_{M}([\widehat{\delta}])=\left[\hat{\eta} \vee \widehat{\delta} \vee \widehat{\eta}^{-1}\right]$, para cada $[\delta] \in \pi_{1}\left(M, x_{0}\right)$ e cada $[\widehat{\delta}] \in \pi_{1}\left(\widehat{M}, \widehat{x}_{0}\right)$, onde $\delta: I \longrightarrow M$ e $\widehat{\delta}: I \longrightarrow \widehat{M}$ são caminhos ligando $x$ a $x_{0}$, e $\widehat{x}$ a $\widehat{x}_{0}$, respectivamente.

Pelo que vimos na definição 1.2.2, temos que as inclusões $j$ e $\widehat{j}$ são equivalências homotópicas, e, portanto, $j_{\#}$ e $\hat{j}_{\#}$ são isomorfismos; assim, denotando por $p_{\#}: \pi_{1}(N, y) \longrightarrow$ $\pi_{1}(M, x)$ e $\left(\left.p\right|_{\operatorname{Int} N}\right)_{\#}: \pi_{1}\left(\operatorname{Int} N, y_{0}\right) \longrightarrow \pi_{1}\left(\operatorname{Int} M, x_{0}\right)$ os homomorfismos induzidos pelos recobrimentos $p: N \longrightarrow M$ e $\left.p\right|_{\operatorname{Int} N}: \operatorname{Int} N \longrightarrow \operatorname{Int} M$, obtemos o diagrama comutativo ilustrado na figura 1.3 .

Como $\left(\phi_{N} \circ i_{\#}\right)\left(\left[\alpha^{\prime}\right]\right)=[\alpha], p \circ \alpha=\beta$ e $\left(\left.p\right|_{\operatorname{IntN}}\right) \circ \alpha^{\prime}=\beta^{\prime}$, a comutatividade deste diagrama nos mostra que:

$$
\left(\phi_{M} \circ j_{\#}\right)\left(\left[\beta^{\prime}\right]\right)=\left(\phi_{M} \circ j_{\#}\right)\left(\left(\left.p\right|_{\operatorname{Int} N}\right)_{\#}\left(\left[\alpha^{\prime}\right]\right)\right)=\left(p_{\#} \circ \phi_{N} \circ i_{\#}\right)\left(\left[\alpha^{\prime}\right]\right)=p_{\#}([\alpha])=[\beta] .
$$

Isto posto, lembrando que o laço $\tilde{\beta}^{\prime}: I \longrightarrow \widetilde{\operatorname{Int} M}$, com base em $\tilde{x}_{0} \in \widetilde{\operatorname{Int} M}$, é um levantamento de $\beta^{\prime}$ relativamente ao recobrimento $\tilde{p}_{M}$, seja $\widehat{\mu}: I \longrightarrow \widehat{M}$ um laço com base em $\widehat{x}$ tal que $[\widehat{\mu}]=\left(\widehat{\phi}_{M} \circ{\widehat{j_{\#}}}_{0}\left(\varphi_{M}\right)_{\#}\right)\left(\left[\tilde{\beta}^{\prime}\right]\right)$. Do diagrama comutativo da figura 1.2, segue então que:

$$
\begin{aligned}
\left(\widehat{p}_{M}\right)_{\#}([\hat{\mu}]) & =\left[\left(\phi_{M} \circ j_{\#}\right) \circ\left(\tilde{p}_{M}\right)_{\#} \circ\left(\left(\varphi_{M}\right)_{\#}^{-1} \circ j_{\#}^{-1} \circ \widehat{\phi}_{M}^{-1}\right)\right]([\hat{\mu}]) \\
& =\left[\left(\phi_{M} \circ j_{\#}\right) \circ\left(\tilde{p}_{M}\right)_{\#}\right]\left(\left[\tilde{\beta}^{\prime}\right]\right)=\left(\phi_{M} \circ j_{\#}\right)\left(\left[\beta^{\prime}\right]\right)=[\beta] .
\end{aligned}
$$

Logo, denotando por $\mu: I \longrightarrow M$ o laço (com base em $x=p(y)$ ) dado por $\mu=\widehat{p}_{M} \circ \widehat{\mu}$, temos que $[\mu]=[\beta]$, sendo que $\widehat{\mu}$ é o único levantamento de $\mu$, relativamente ao recobrimento orientado $\widehat{p}_{M}$, tal que $\widehat{\mu}(0)=\widehat{\mu}(1)=\widehat{x}$. Isto, por sua vez, significa que 


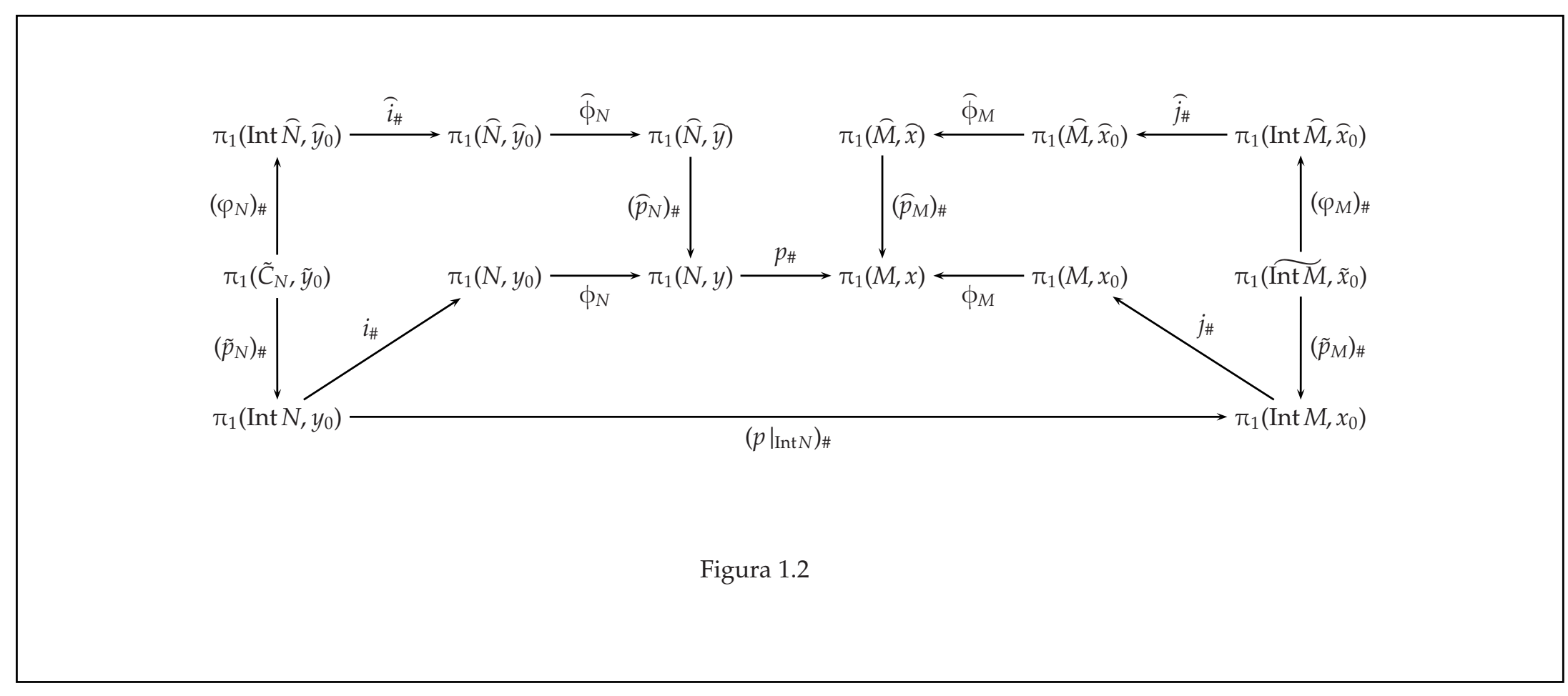


$\mu$ é um laço que preserva orientação em $\widehat{M}$, e, portanto, como $[\mu]=[\beta]$, segue que $\beta$ também o é (pois toda homotopia entre $\mu$ e $\beta$ pode ser levantada, relativamente ao recobrimento $\hat{p}_{M}$, para uma homotopia entre $\hat{\mu}$ e um levantamento de $\beta$ ). Deste modo, podemos concluir que existe um laço $\widehat{\beta}: I \longrightarrow \widehat{M}$, com base em $\widehat{x}$, tal que $\widehat{p}_{M} \circ \widehat{\beta}=\beta$.

Resumindo, supondo $M$ não-orientável, mostramos que, se $\alpha: I \longrightarrow N$ é um laço que preserva orientação em $\widehat{N}$, então o laço $\beta=p \circ \alpha: I \longrightarrow M$ preserva orientação em $\hat{M}$. Provando-se a recíproca de maneira análoga, concluímos então que o laço $\alpha$ preserva orientação em $\widehat{N}$ se, e somente se, o laço $\beta=p \circ \alpha$ preserva orientação em $\widehat{M}$, o que implica que o recobrimento $p: N \longrightarrow M$ é fiel à orientação relativamente aos recobrimentos orientados de $N$ e $M$.

Definição 1.2.5. Dada uma aplicação contínua $f: M \longrightarrow N$, entre variedades topológicas de mesma dimensão n, conexas e com fronteira (eventualmente vazias), dizemos que $f$ :

(1) é do tipo I, se ela é fiel à orientação relativamente aos recobrimentos orientados de $\mathrm{M}$ e $\mathrm{N}$;

(2) é do tipo II, se ela não é fiel à orientação relativamente aos recobrimentos orientados de $\mathrm{Me}$ $N$, e não leva laços que invertem orientação relativamente ao recobrimento orientado de $M$ em laços contráteis em $N$;

(3) é do tipo III, se ela leva um laço que inverte orientação relativamente ao recobrimento orientado de $M$ num laço contrátil em $N$.

Além disso, se f for dos tipos I ou II, dizemos que ela é orientável; caso contrário, é dita não-orientável.

Convém observarmos que alguns autores utilizam o termo orientável apenas para aplicações do tipo I (vide [D], exercício 6, página 271). Mais adiante, veremos que as aplicações dos dois primeiros tipos acima possuem muitas propriedades relacionadas à teoria de Nielsen de raízes.

Para encerrar esta seção, apresentaremos alguns exemplos destes três tipos de aplicações.

Exemplo 1.2.1 (Tipo I). (a) Se $M$ e $N$ são variedades conexas orientáveis (de mesma dimensão), então toda aplicação contínua $f: M \longrightarrow N$ é fiel à orientação (vide item (i) da proposição 1.1.4).

(b) A aplicação identidade numa variedade não-orientável é um exemplo de aplicação do tipo I entre variedades não-orientáveis.

(c) A aplicação quociente $\pi: \mathbb{S}^{2} \longrightarrow \mathbb{R} P^{2}$ é do tipo I (vide exemplo 1.1.4). 
Exemplo 1.2.2 (Tipo II). Seja $M_{+}$a faixa de Möbius, e considere a aplicação $p: M_{+} \longrightarrow$ $\mathbb{S}^{1}$, obtida retraindo-se $M_{+}$ao seu círculo central. Seja ainda $i: \mathbb{S}^{1} \longrightarrow \mathbb{S}^{1} \times I=N_{+}$definida por $i(x)=(x, 0)$ para cada $x \in \mathbb{S}^{1}$. Temos, pois, que a aplicação $f_{+}=i \circ p: M_{+} \longrightarrow N_{+}$ leva a fronteira de $M_{+}$na fronteira de $N_{+}$. Logo, sendo $f=2 f_{+}: M=2 M_{+} \longrightarrow 2 N_{+}=N$ o dobro da aplicação $f_{+}$, segue que $M$ é a garrafa de Klein e $N$ é o toro bidimensional. Agora, dado $x_{0} \in M$, e sendo $c=f\left(x_{0}\right) \in N$, observe que os laços que representam os elementos do núcleo do homomorfismo induzido $f_{\#}: \pi_{1}\left(M, x_{0}\right) \longrightarrow \pi_{1}(N, c)$ preservam orientação, o que implica que $f$ não é do tipo III. Como uma aplicação, de uma variedade não-orientável $(M)$ numa variedade orientável $(N)$, não pode ser fiel à orientação (vide proposição 1.1.4), concluímos então que $f$ é do tipo II.

Exemplo 1.2.3 (Tipo III). (a) Se $M$ é uma variedade não-orientável, então uma aplicação constante $f: M \longrightarrow N$ é obviamente do tipo III (independentemente da variedade $N$ ser ou não orientável).

(b) Sejam $D_{1} \subseteq \mathbb{T}^{2}$ e $D_{2} \subseteq \mathbb{R P}^{2}$ discos fechados, e considere a soma conexa $\mathbb{T}^{2} \# \mathbb{R} P^{2}$, obtida identificando-se os pontos de $\partial D_{1} \subseteq \mathbb{T}^{2}$ com os pontos de $\partial D_{2} \subseteq \mathbb{R P}^{2}$, por meio de um homeomorfismo $h: \partial D_{1} \longrightarrow \partial D_{2}$. Isto posto, sendo Id : $\mathbb{T}^{2} \backslash$ Int $D_{1} \longrightarrow \mathbb{T}^{2} \backslash$ Int $D_{1}$ a aplicação identidade, vamos estender (continuamente) Id à uma aplicação $f: \mathbb{T}^{2} \# \mathbb{R} P^{2} \longrightarrow \mathbb{T}^{2}$. Para tanto, observe que, como $\partial D_{2}$ é fechado em $\mathbb{R P}^{2} \backslash$ Int $D_{2}$, e como $D_{1} \subseteq \mathbb{T}^{2}$ é um subconjunto contrátil do tipo ENR, segue que a aplicação Id $\circ h^{-1}: \partial D_{2} \longrightarrow D_{1} \subseteq \mathbb{T}^{2}$ estende-se continuamente a uma aplicação $g: \mathbb{R P}^{2} \backslash \operatorname{Int} D_{2} \longrightarrow D_{1}$ (vide [Li], corolário 1, página 18); logo, como $\partial D_{1}=\operatorname{Id}\left(\partial D_{1}\right)=\operatorname{Id}\left(h^{-1}\left(\partial D_{2}\right)\right)=g\left(\partial D_{2}\right)$, temos que a aplicação $f: \mathbb{T}^{2} \# \mathbb{R} P^{2} \longrightarrow \mathbb{T}^{2}$, dada por:

$$
f(x)=\left\{\begin{array}{lll}
\operatorname{Id}(x) & \text { se } & x \in \mathbb{T}^{2} \backslash \operatorname{Int} D_{1} \\
g(x) & \text { se } & x \in \mathbb{R P}^{2} \backslash \operatorname{Int} D_{2}
\end{array},\right.
$$

encontra-se bem definida e constitui uma extensão contínua de Id à soma conexa $\mathbb{T}^{2} \# \mathbb{R} P^{2}$. Agora, note que $\mathbb{T}^{2} \# \mathbb{R} P^{2}$ possui um laço que inverte orientação (pois $\mathbb{R P}^{2}$ é não-orientável), o qual é levado por $f$ no subconjunto contrátil $D_{1} \subseteq \mathbb{T}^{2}$. Deste modo, temos que $f$ é um exemplo de aplicação do tipo III.

\section{3 $\mathbb{A}$ Mullipiplicidadle de unma Classe de $\mathbb{R a i} z e s$}

Nesta seção, vamos introduzir o conceito de multiplicidade de uma classe de raízes, para aplicações próprias que preservam fronteira. Para tanto, precisaremos de algumas 
definições preliminares.

Definição 1.3.1. Sejam X e Y espaços topológicos. Uma aplicação $f: X \longrightarrow Y$ é dita própria se, para todo subconjunto compacto $K \subseteq Y$, a imagem inversa $f^{-1}(K)$ é um subconjunto compacto de $X$. Além disso, dizemos que duas aplicações contínuas $f, g: X \longrightarrow Y$ são propriamente homotópicas se existe uma homotopia própria $h: X \times I \longrightarrow Y$ entre $f$ e g, isto é, uma homotopia que constitui uma aplicação própria. Por outro lado, se $M$ e $N$ são variedades topológicas de dimensão $n$, dizemos que uma aplicação $f: M \longrightarrow N$ preserva fronteira se $f(\partial M) \subseteq \partial N$ (supondo, obviamente, que $M$ e $N$ sejam variedades com fronteira); neste caso, denotamos $f$ como uma aplicação entre pares, ou seja, $f:(M, \partial M) \longrightarrow(N, \partial N)$. Assim, se $h: M \times I \longrightarrow N$ for uma homotopia entre duas aplicações $f, g:(M, \partial M) \longrightarrow(N, \partial N)$, tal que $h(\partial M \times I) \subseteq \partial N$, escrevemos $h:(M \times I, \partial M \times I) \longrightarrow(N, \partial N)$.

Exemplo 1.3.1 . Todo homeomorfismo é evidentemente uma aplicação própria. Outro exemplo: dado um subconjunto fechado $A$ de um espaço topológico $X$, a inclusão $i: A \longrightarrow X$ é própria. Com efeito, se $A$ é um subconjunto fechado de $X$, então, para todo compacto $K \subseteq X$, a imagem inversa $i^{-1}(K)=A \cap K$ é um subconjunto fechado de $K$, e, portanto, é compacto. Mais geralmente, a restrição de uma aplicação própria $f: X \longrightarrow Y$, a qualquer subconjunto fechado de $X$, constitui uma aplicação própria.

Exemplo 1.3.2 . Se $X$ é um espaço topológico compacto, então toda aplicação contínua $f: X \longrightarrow Y$, num espaço de Hausdorff $Y$, é própria: com efeito, se $K \subseteq Y$ é compacto, segue (do fato de $Y$ ser um espaço de Hausdorff) que $K$ é fechado em $Y$, e, portanto, $f^{-1}(K)$ é um subconjunto fechado de $X$; logo, sendo $X$ um espaço compacto, temos que $f^{-1}(K)$ é compacto (pois constitui um subconjunto fechado de um espaço compacto).

Exemplo 1.3.3 . Se $M$ é uma variedade topológica conexa, então todo recobrimento $p: M^{\prime} \longrightarrow M$, que possui um número finito de folhas, constitui uma aplicação própria (vide [Li], proposição 4, página 121).

Definição 1.3.2 . Dada uma aplicação contínua $f: X \longrightarrow Y$, entre dois espaços topológicos $X$ e $Y$, dizemos que um ponto $x \in X$ é uma raiz de $f$ em $y \in Y$, se $f(x)=y$; conseqüentemente, as raízes de $f$ num ponto $y \in Y$ são os pontos da imagem inversa $f^{-1}(y)$. Por outro lado, sendo $f: M \longrightarrow N$ uma aplicação contínua entre variedades topológicas conexas (e, portanto, conexas por caminhos), e considerando $c \in N$ tal que $f^{-1}(c) \neq \varnothing$, dizemos que dois pontos $x_{1}, x_{2} \in f^{-1}(c)$ pertencem à mesma classe de raízes de $\mathbf{f}$ em $\mathrm{c}$ se existe um caminho $\omega: I \longrightarrow M$, com 
$\omega(0)=x_{1}$ e $\omega(1)=x_{2}$, tal que $f \circ \omega: I \longrightarrow N$ é um laço contrátil em c; em outras palavras, uma classe de raízes de $f$ em c é um subconjunto não-vazio de $f^{-1}(c)$, tal que quaisquer dois de seus pontos podem ser ligados por um caminho (em $M$ ) que é levado por $f$ num laço contrátil em $N$.

Da definição anterior, podemos ver que a relação de pertencer à mesma classe de raízes constitui uma relação de equivalência, e, portanto, o conjunto $f^{-1}(c)$ é a união disjunta das classes de raízes de $f$ em $c$. Note que, como estamos supondo (por definição) que uma classe de raízes de $f$ em $c$ é um conjunto não-vazio, segue que, se $f^{-1}(c)=\varnothing$, então não existem classes de raízes de $f$ em $c$ (e, reciprocamente, se existir uma classe de raízes de $f$ em $c$, segue que $f^{-1}(c) \neq \varnothing$ ). Convém observarmos, no entanto, que nem toda classe de raízes (de $f$ em $c$ ) é um subconjunto conexo de $M$, conforme ilustra o seguinte

Exemplo 1.3.4 . Considere a aplicação $f: \mathbb{R} \longrightarrow \mathbb{R}_{+}$dada por $f(x)=x^{2}$, e seja $c=1$. Como $f^{-1}(c)=\{1,-1\}$, e $\mathbb{R}_{+}$é simplesmente conexo, segue que a única classe de raízes de $f$ em $c$ é o conjunto $R=\{-1,1\}$.

Exemplo 1.3.5. Dado $n \neq 0$, seja $f: \mathbb{S}^{1} \longrightarrow \mathbb{S}^{1}$ a aplicação dada por $f(z)=z^{n}$, onde $\mathbb{S}^{1}$ é a circunferência unitária no plano complexo. Então, sendo $c=1 \in \mathbb{S}^{1}$, temos que:

$$
f^{-1}(c)=\left\{\exp \left(\frac{2 \pi i r}{|n|}\right): r=1,2, \ldots,|n|\right\} .
$$

Agora, dados $z_{1}, z_{2} \in f^{-1}(c)$ distintos, note que qualquer caminho ligando $z_{1}$ a $z_{2}$ em $\mathbb{S}^{1}$ é levado por $f$ num laço que completa uma ou mais voltas ao redor de $\mathbb{S}^{1}$; logo, como tais laços em $\mathbb{S}^{1}$ não são contráteis, concluímos que as classes de raízes de $f$ em $c$ são da forma $R=\{z\}$, onde $z \in f^{-1}(c)$.

Proposição 1.3.1. Seja $f: M \longrightarrow N$ uma aplicação contínua entre variedades topológicas conexas. Se $c \in \operatorname{Int} N$, então toda classe de raízes de $f$ em $c$ constitui um subconjunto aberto do subespaço $f^{-1}(c) \subseteq M$ (isto é, toda classe de raízes de $f$ em c é a intersecção de um aberto $\left.U \subseteq M \operatorname{com} f^{-1}(c)\right)$.

DemonstraçÃo. Com efeito, sendo $R$ uma classe de raízes de $f$ em $c$, e dado $y \in R$ qualquer, considere uma vizinhança contrátil $V$ de $c$ em $N$ (tal vizinhança de fato existe, pois toda variedade topológica é localmente simplesmente conexa). Temos então que $f^{-1}(V)$ é uma vizinhança aberta de $y$ em $M$. Logo, como toda variedade topológica é localmente conexa por caminhos, segue que y admite uma vizinhança 
aberta $U_{y} \subseteq f^{-1}(V)$, conexa por caminhos. Assim, observe que, se $y^{\prime} \in U_{y} \cap f^{-1}(c)$, temos $y^{\prime} \in R$ : de fato, como $U_{y}$ é conexo por caminhos, existe um caminho $\omega: I \longrightarrow U_{y}$ tal que $\omega(0)=y$ e $\omega(1)=y^{\prime} ; \log$, como $V$ é contrátil, segue que $f \circ \omega: I \longrightarrow V$ é um laço contrátil com base no ponto $c$, o que significa que $y^{\prime} \in R$. Deste modo, concluímos que $U_{y} \cap f^{-1}(c) \subseteq R$. Isto posto, seja $U=\bigcup\left\{U_{y}: y \in R\right\}$, onde, para cada $y \in R$, $U_{y} \subseteq f^{-1}(V)$ é uma vizinhança aberta de $y$, conexa por caminhos. Temos, pois, que $U$ é um subconjunto aberto de $M$, tal que $U \cap f^{-1}(c)=R$ (uma vez que $U_{y} \cap f^{-1}(c)=R$ para cada $y \in R$, e $R \subseteq U \cap f^{-1}(c)$ ); em outras palavras, $R$ é um subconjunto aberto do subespaço $f^{-1}(c) \subseteq M$, como queríamos mostrar.

Proposição 1.3.2. Se $f: M \longrightarrow N$ é uma aplicação contínua própria entre variedades topológicas conexas, e $c \in \operatorname{Int} N$, então toda classe de raízes de $f$ em $c$ é um subconjunto compacto de $M$. Além disso, $f^{-1}(c)$ contém apenas uma quantidade finita de classes de raizes.

Demonstração. Primeiramente, observe que $f^{-1}(c)$ é um subconjunto compacto de $M$, pois $f: M \longrightarrow N$ é uma aplicação própria (e o conjunto unitário $\{c\} \subseteq \operatorname{Int} N$ é compacto). Logo, como toda classe de raízes de $f$ em $c$ é um subconjunto aberto de $f^{-1}(c)$ (conforme vimos na proposição anterior), e como $f^{-1}(c)$ é a união disjunta das classes de raízes de $f$ em $c$, segue que $f^{-1}(c)$ deve conter apenas uma quantidade finita de classes de raízes (uma vez que estas constituem uma cobertura aberta de $f^{-1}(c)$ ). Por outro lado, seja $R$ uma classe de raízes de $f$ em $c$, arbitrária; da compacidade de $f^{-1}(c)$, segue que, para provarmos que $R$ é um subconjunto compacto de $M$, basta mostrarmos que $M \backslash R$ é aberto em $M$ (pois isto implicará que $R$ é fechado em $M$, e, portanto, compacto, uma vez que $\left.R \subseteq f^{-1}(c)\right)$.

Assim, considere $y \in M \backslash R$; se $y \notin f^{-1}(c)$, segue que existe um aberto $U \subseteq M$ tal que $y \in U$ e $U \cap f^{-1}(c)=\varnothing$ (posto que $f^{-1}(c)$ é fechado em $M$ ). Como $R \subseteq f^{-1}(c)$, segue então que $U$ é uma vizinhança aberta de $y \operatorname{em~} M$, tal que $U \subseteq M \backslash R$. Agora, suponhamos que $y \in f^{-1}(c)$; ora, como $y \notin R$, segue que, para todo $x \in R$ e todo caminho $\omega: I \longrightarrow M, \operatorname{com} \omega(0)=x$ e $\omega(1)=y$, o laço $f \circ \omega: I \longrightarrow N$ (com base no ponto $c$ ) não é contrátil. Entretanto, como toda variedade topológica é localmente simplesmente conexa, sabemos que existe uma vizinhança aberta $V$ de $c \in \operatorname{Int} N$ em $N$, tal que todo laço em $V$ é contrátil. Além disso, como toda variedade topológica é localmente conexa por caminhos (e observando que $f^{-1}(V)$ é uma vizinhança aberta de $y$ em $M$ ), segue que $y$ admite uma vizinhança aberta $U \subseteq f^{-1}(V)$ em $M$, conexa por caminhos. Isto posto, note que $U \cap R=\varnothing$ : de fato, se existisse $x \in R$ tal que $x \in U$, também existiria um caminho $\omega: I \longrightarrow U$, com $\omega(0)=x$ e $\omega(1)=y$ (pois $U$ é conexo por caminhos), tal que $f \circ \omega: I \longrightarrow f(U) \subseteq V$ é um laço contrátil em $N$ (uma vez que $V$ é uma vizinhança contrátil de $c$ em $N$ ), contradizendo a hipótese de que $y \notin R$. Logo, $U$ é uma vizinhança 
aberta de $y$ em $M$, tal que $U \cap R=\varnothing$. Deste modo, concluímos que, para todo $y \in M \backslash R$, existe um aberto $U \subseteq M$ tal que $y \in U$ e $U \subseteq M \backslash R$, o que significa que $M \backslash R$ é aberto em $M$, conforme queríamos mostrar.

Agora, para o que faremos em seguida, precisaremos recordar as definições de classe fundamental e grau local.

Definição (e Proposição) 1.3.3 . Seja M uma variedade topológica orientável de dimensão $n$ (sem fronteira), com orientação $s: M \longrightarrow \tilde{M}$, e considere um conjunto compacto $K \subseteq M$. Então, existe uma única classe $\theta_{K} \in \mathrm{H}_{n}(M, M \backslash K)$ tal que $\left(j_{p}\right)_{*}\left(\theta_{K}\right)=s(p)$ para cada $p \in K$, onde $\left(j_{p}\right)_{*}: \mathrm{H}_{n}(M, M \backslash K) \longrightarrow \mathrm{H}_{n}(M, M \backslash\{p\})$ é o homomorfismo induzido pela inclusão (para maiores detalhes, vide [D], definição 4.1, página 267). À esta classe $\theta_{K}$, denominamos classe fundamental em $\mathrm{K}$. Em particular, se a variedade $M$ é compacta, existe uma classe fundamental $\theta_{M} \in \mathrm{H}_{n}(M, M \backslash M) \simeq \mathrm{H}_{n}(M)$, a qual é denominada classe fundamental de $\mathbf{M}$.

Na definição acima, convém observarmos que, se o conjunto compacto $K \subseteq M$ é conexo (e não-vazio), temos $\mathrm{H}_{n}(M, M \backslash K) \simeq \mathbb{Z}$ (vide [D], corolário 3.4, página 260), e, neste caso, a classe fundamental $\theta_{K}$ é um gerador de $\mathrm{H}_{n}(M, M \backslash K)$.

Definição 1.3.4 . Seja $f: M \longrightarrow N$ uma aplicação contínua entre variedades topológicas orientáveis (sem fronteira), ambas de dimensão $n$, e seja $K \subseteq N$ um subconjunto compacto, conexo e não-vazio, tal que $f^{-1}(K) \subseteq M$ é compacto. Como a classe fundamental $\theta_{K}$ é um gerador de $\mathrm{H}_{n}(M, M \backslash K) \simeq \mathbb{Z}$, segue que o homomorfismo $f_{*}: \mathrm{H}_{n}\left(M, M \backslash f^{-1}(K)\right) \longrightarrow$ $\mathrm{H}_{n}(N, N \backslash K) \simeq \mathbb{Z}$, induzido por $f$, leva a classe fundamental $\theta_{f^{-1}(K)}$ num múltiplo inteiro de $\theta_{K}$, o qual denominamos grau local de $\mathbf{f}$ em $\mathbf{K}$, e denotamos por $\operatorname{deg}_{K} f$; em outras palavras, o número $\operatorname{deg}_{K} f \in \mathbb{Z}$ é tal que $f_{*}\left(\theta_{f^{-1}(K)}\right)=\operatorname{deg}_{K} f \cdot \theta_{K}$.

Na definição acima, note que, se $f^{-1}(K)=\varnothing$ (por exemplo, se $K=\{c\} \subseteq N$ e $c \notin \operatorname{Im}(f)$ ), segue que $f_{*}: \mathrm{H}_{n}\left(M, M \backslash f^{-1}(K)\right) \longrightarrow \mathrm{H}_{n}(M, M \backslash K) \simeq \mathbb{Z}$ é o homomorfismo trivial, e, portanto, $\operatorname{deg}_{K}(f)=0$.

Exemplo 1.3.6 . Se $M$ é uma variedade topológica orientável de dimensão $n$ (sem fronteira), e Id $: M \longrightarrow M$ é a aplicação identidade, então, para todo subconjunto compacto, conexo e não-vazio $K \subseteq M$, temos $\operatorname{deg}_{K}(\mathrm{Id})=1$. De fato, como as classes fundamentais $\theta_{K} \in \mathrm{H}_{n}(M, M \backslash K)$ e $\theta_{\mathrm{Id}^{-1}(K)} \in \mathrm{H}_{n}\left(M, M \backslash \operatorname{Id}^{-1}(K)\right)=\mathrm{H}_{n}(M, M \backslash K)$ coincidem, e o homomorfismo $\mathrm{Id}_{*}: \mathrm{H}_{n}\left(M, M \backslash \mathrm{Id}^{-1}(K)\right)=\mathrm{H}_{n}(M, M \backslash K) \longrightarrow \mathrm{H}_{n}(M, M \backslash K)$ é a identidade, segue que $\operatorname{Id}_{*}\left(\theta_{\operatorname{Id}^{-1}(K)}\right)=\operatorname{Id}_{*}\left(\theta_{K}\right)=\theta_{K}$, e, portanto, $\operatorname{deg}_{K}(\mathrm{Id})=1$. 
Exemplo 1.3.7 . Se $M$ e $N$ são variedades topológicas conexas de mesma dimensão $n$, fechadas (isto é, compactas e sem fronteira) e orientáveis, definimos o grau de uma aplicação contínua $f: M \longrightarrow N$ como sendo o número inteiro $\mathrm{d}(f)$ tal que $f_{*}\left(\theta_{M}\right)=$ $\mathrm{d}(f) \cdot \theta_{N}$, onde $\theta_{M}$ e $\theta_{N}$ são as classes fundamentais de $M$ e $N$, respectivamente (e $f_{*}: \mathrm{H}_{n}(M) \longrightarrow \mathrm{H}_{n}(N)$ é o homomorfismo induzido por $f$ ). Da definição 1.3.4, temos então que $\mathrm{d}(f)=\operatorname{deg}_{N}(f)$, o que mostra que o conceito de grau local é uma generalização para o grau de uma aplicação contínua entre variedades fechadas de mesma dimensão. Em particular, se $f: \mathbb{S}^{n} \longrightarrow \mathbb{S}^{n}$ é uma aplicação contínua entre esferas de dimensão $n$ (com $n \geqslant 1$ ), segue que $\mathrm{d}(f)=\operatorname{deg}_{\mathbb{S}^{n}}(f)$ (para a definição de grau de uma aplicação entre esferas de mesma dimensão, vide também [Vi], página 25).

O conceito de grau local possui muitas propriedades importantes, dentre as quais destacaremos algumas que nos serão particularmente úteis.

Lema 1.3.1. Sejam M uma variedade topológica orientável de dimensão n (sem fronteira), $U \subseteq$ $M$ um aberto, e $K \subseteq U$ um subconjunto compacto não-vazio. Então, denotando por $i: U \longrightarrow M$ a inclusão, temos que o homomorfismo $i_{*}: \mathrm{H}_{n}(U, U \backslash K) \rightarrow \mathrm{H}_{n}(M, M \backslash K)$, induzido por $i$, leva a classe fundamental $\theta_{K}^{U} \in \mathrm{H}_{n}(U, U \backslash K)$ na classe fundamental $\theta_{K}^{M} \in \mathrm{H}_{n}(M, M \backslash K)$, isto é, $i_{*}\left(\theta_{K}^{U}\right)=\theta_{K}^{M}$. Conseqüentemente, se o compacto $K \subseteq U$ for conexo (e, portanto, a classe fundamental $\theta_{K}^{M}$ for um gerador de $\mathrm{H}_{n}(M, M \backslash K)$ ), teremos que $\operatorname{deg}_{K} i=1$.

DemonstraÇÃo. Com efeito, dado $x \in K$, considere os homomorfismos

$$
\begin{gathered}
\left(j_{M}\right)_{*}: \mathrm{H}_{n}(M, M \backslash K) \longrightarrow \mathrm{H}_{n}(M, M \backslash\{x\}), \\
\left(j_{U}\right)_{*}: \mathrm{H}_{n}(U, U \backslash K) \longrightarrow \mathrm{H}_{n}(U, U \backslash\{x\}) \mathrm{e} \\
\left(j_{U}^{M}\right)_{*}: \mathrm{H}_{n}(U, U \backslash\{x\}) \longrightarrow \mathrm{H}_{n}(M, M \backslash\{x\}),
\end{gathered}
$$

induzidos pelas respectivas inclusões $j_{M}:(M, M \backslash K) \rightarrow(M, M \backslash\{x\}), j_{U}:(U, U \backslash$ $K) \longrightarrow(U, U \backslash\{x\})$ e $j_{U}^{M}:(U, U \backslash\{x\}) \longrightarrow(M, M \backslash\{x\})$. Como $\overline{M \backslash U}=M \backslash U \subseteq$ $\operatorname{Int}(M \backslash\{x\})=M \backslash\{x\}$, segue (do teorema da excisão em homologia singular) que $\left(j_{U}^{M}\right)_{*}$ é um isomorfismo. Por outro lado, como $\overline{M \backslash U}=M \backslash U \subseteq \operatorname{Int}(M \backslash K)=M \backslash K$, temos que o homomorfismo $i_{*}: \mathrm{H}_{n}(U, U \backslash K)=\mathrm{H}_{n}\left(U, U \backslash i^{-1}(K)\right) \longrightarrow \mathrm{H}_{n}(M, M \backslash K)$, induzido pela inclusão $i: U \longrightarrow M$, também constitui um isomorfismo, o qual comuta o seguinte diagrama:

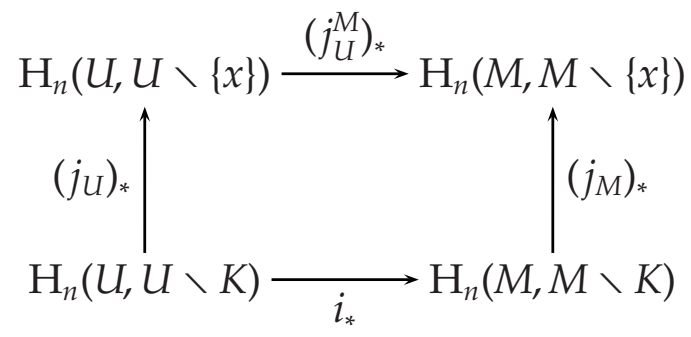


Isto posto, sejam $\theta_{K}^{U} \in \mathrm{H}_{n}(U, U \backslash K)$ e $\theta_{K}^{M} \in \mathrm{H}_{n}(M, M \backslash K)$ as classes fundamentais, e, adotando-se uma orientação $s_{M}: M \longrightarrow \tilde{M}$ em $M$, considere $s_{U}: U \longrightarrow \tilde{U}$ a orientação de $U$ obtida pela restrição da orientação de $M$ (lembre-se que, por hipótese, $M$ é orientável). Pela definição de classe fundamental, temos então que $\left(j_{U}\right)_{*}\left(\theta_{K}^{U}\right)=s_{U}(x)$ e $\left(j_{M}\right)_{*}\left(\theta_{K}^{M}\right)=s_{M}(x)$. Logo, como $\left(j_{U}^{M}\right)_{*}\left(s_{U}(x)\right)=s_{M}(x)$ (pois $U$ é orientado pela restrição da orientação de $M)$, a comutatividade do diagrama acima nos garante que:

$$
\left(j_{M}\right)_{*}\left(i_{*}\left(\theta_{K}^{U}\right)\right)=\left(j_{U}^{M}\right)_{*}\left[\left(j_{U}\right)_{*}\left(\theta_{K}^{U}\right)\right]=\left(j_{U}^{M}\right)_{*}\left[s_{U}(x)\right]=s_{M}(x)
$$

Deste modo, podemos concluir que, para todo $x \in K$, o homomorfismo $\left(j_{M}\right)_{*}: \mathrm{H}_{n}(M, M \backslash$ $K) \longrightarrow \mathrm{H}_{n}(M, M \backslash\{x\})$ é tal que $\left(j_{M}\right)_{*}\left(i_{*}\left(\theta_{K}^{U}\right)\right)=s_{M}(x)$. Assim, como a classe fundamental $\theta_{K}^{M}$ é o único elemento de $\mathrm{H}_{n}(M, M \backslash K)$ tal que $\left(j_{M}\right)_{*}\left(\theta_{K}^{M}\right)=s_{M}(x)$, para cada $x \in K$, segue que $i_{*}\left(\theta_{K}^{U}\right)=\theta_{K}^{M}$. Conseqüentemente, se o compacto $K \subseteq U$ for conexo, temos que $\theta_{K}^{M}$ é um gerador de $\mathrm{H}_{n}(M, M \backslash K)$, o que (pela definição de grau local) implica que $\operatorname{deg}_{K} i=1$, conforme queríamos demonstrar.

Proposição 1.3.3. Sejam $M, M^{\prime}$ e $M^{\prime \prime}$ variedades topológicas orientáveis de dimensão n (sem fronteira), e considere duas aplicações contínuas $f: M^{\prime \prime} \longrightarrow M^{\prime}$ e $g: M^{\prime} \longrightarrow M$. Dado um subconjunto compacto, conexo e não-vazio $K \subseteq M$, tal que $g^{-1}(K) \neq \varnothing$ é compacto e conexo, $e$ $(g \circ f)^{-1}(K)=f^{-1}\left(g^{-1}(K)\right)$ é compacto, temos que:

$$
\operatorname{deg}_{K}(g \circ f)=\operatorname{deg}_{K}(g) \cdot \operatorname{deg}_{g^{-1}(K)}(f) .
$$

Demonstração. Com efeito, sejam $\theta_{K} \in \mathrm{H}_{n}(M, M \backslash K), \theta_{g^{-1}(K)} \in \mathrm{H}_{n}\left(M^{\prime}, M^{\prime} \backslash g^{-1}(K)\right)$ e $\theta_{(g \circ f)^{-1}(K)} \in \mathrm{H}_{n}\left(M^{\prime \prime}, M^{\prime \prime} \backslash(g \circ f)^{-1}(K)\right)$ as classes fundamentais em $K \subseteq M, g^{-1}(K) \subseteq M^{\prime}$ e $(g \circ f)^{-1}(K) \subseteq M^{\prime \prime}$, respectivamente. Por definição, sabemos que $\operatorname{deg}_{K}(g \circ f)$ é o número inteiro tal que $(g \circ f)_{*}\left(\theta_{(g \circ f)^{-1}(K)}\right)=\operatorname{deg}_{K}(g \circ f) \cdot \theta_{K}$, onde $(g \circ f)_{*}: \mathrm{H}_{n}\left(M^{\prime \prime}, M^{\prime \prime} \backslash\right.$ $\left.(g \circ f)^{-1}(K)\right) \longrightarrow \mathrm{H}_{n}(M, M \backslash K)$ é o homomorfismo induzido por $g \circ f$. Entretanto, sendo $f_{*}: \mathrm{H}_{n}\left(M^{\prime \prime}, M^{\prime \prime} \backslash f^{-1}\left(g^{-1}(K)\right)\right)=\mathrm{H}_{n}\left(M^{\prime \prime}, M^{\prime \prime} \backslash(g \circ f)^{-1}(K)\right) \longrightarrow \mathrm{H}_{n}\left(M^{\prime}, M^{\prime} \backslash\right.$ $\left.g^{-1}(K)\right)$ e $g_{*}: \mathrm{H}_{n}\left(M^{\prime}, M^{\prime} \backslash g^{-1}(K)\right) \longrightarrow \mathrm{H}_{n}(M, M \backslash K)$ os homomorfismos induzidos por $f$ e $g$, respectivamente, sabemos também que $(g \circ f)_{*}=g_{*} \circ f_{*}$. Logo, como $\operatorname{deg}_{K}(g), \operatorname{deg}_{g^{-1}(K)}(f) \in \mathbb{Z}$ são tais que $f_{*}\left(\theta_{(g \circ f)^{-1}(K)}\right)=\operatorname{deg}_{g^{-1}(K)}(f) \cdot \theta_{g^{-1}(K)}$ e $g_{*}\left(\theta_{g^{-1}(K)}\right)=$ $\operatorname{deg}_{K}(g) \cdot \theta_{K}$, segue que:

$$
\begin{aligned}
\operatorname{deg}_{K}(g \circ f) \cdot \theta_{K} & =(g \circ f)_{*}\left(\theta_{(g \circ f)^{-1}(K)}\right)=g_{*}\left(f_{*}\left(\theta_{(g \circ f)^{-1}(K)}\right)\right) \\
& =g_{*}\left(\operatorname{deg}_{g^{-1}(K)}(f) \cdot \theta_{g^{-1}(K)}\right)=\operatorname{deg}_{g^{-1}(K)}(f) \cdot g_{*}\left(\theta_{g^{-1}(K)}\right) \\
& =\operatorname{deg}_{g^{-1}(K)}(f) \cdot \operatorname{deg}_{K}(g) \cdot \theta_{K},
\end{aligned}
$$

e, portanto, $\operatorname{deg}_{K}(g \circ f)=\operatorname{deg}_{K}(g) \cdot \operatorname{deg}_{g^{-1}(K)}(f)$, conforme queríamos mostrar. 
Corolário 1.3.1. Sejam Me N variedades topológicas orientáveis de dimensãon (sem fronteira), e considere um homeomorfismo $h: M \longrightarrow N$. Dado um subconjunto compacto, conexo e nãovazio $K \subseteq N$, temos que $\operatorname{deg}_{K} h= \pm 1$.

DemonstraçÃo. De fato, sendo $h^{-1}: N \longrightarrow M$ o homeomorfismo inverso de $h$, sabemos que a composição $h \circ h^{-1}: N \longrightarrow N$ é a identidade Id $: N \longrightarrow N$. Logo, dado um subconjunto compacto, conexo e não-vazio $K \subseteq N$, segue (da proposição anterior e do exemplo 1.3.6) que $\operatorname{deg}_{K} h \cdot \operatorname{deg}_{h^{-1}(K)}\left(h^{-1}\right)=\operatorname{deg}_{K}(\mathrm{Id})=1$. Assim, como $\operatorname{deg}_{K} h \in \mathbb{Z}$ e $\operatorname{deg}_{h^{-1}(K)}\left(h^{-1}\right) \in \mathbb{Z}$, podemos concluir que $\operatorname{deg}_{K} h=\operatorname{deg}_{h^{-1}(K)}\left(h^{-1}\right)=1$ ou $\operatorname{deg}_{K} h=\operatorname{deg}_{h^{-1}(K)}\left(h^{-1}\right)=-1$, isto é, $\operatorname{deg}_{K} h= \pm 1$.

Proposição 1.3.4 . Sejam $M$ e $N$ variedades topológicas orientáveis de dimensão $n$ (sem fronteira), e considere uma aplicação contínua própria $f: M \longrightarrow N$. Se $N$ é conexa, então o número inteiro $\operatorname{deg}_{K} f$ é independente do subconjunto compacto e conexo $K \subseteq N, K \neq \varnothing$ (em outras palavras, para quaisquer conjuntos compactos, conexos e não-vazios $K_{1}, K_{2} \subseteq N$, temos $\left.\operatorname{deg}_{K_{1}} f=\operatorname{deg}_{K_{2}} f\right)$.

DemonstraçÃo. Com efeito, dados $K_{1}, K_{2} \subseteq N$, compactos, conexos e não-vazios, existe $K \subseteq N$, compacto e conexo, tal que $K_{1}, K_{2} \subseteq K$ : basta tomar $K=K_{1} \cup K_{2} \cup \alpha(I)$, onde $\alpha: I \longrightarrow N$ é um caminho ligando um ponto $x_{1} \in K_{1}$ a um outro ponto $x_{2} \in K_{2}$. Considere então o seguinte diagrama comutativo:

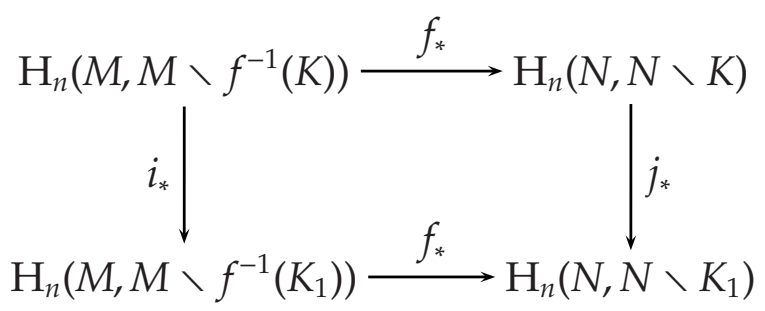

(onde $i_{*}$ e $j_{*}$ são os homomorfismos induzidos pelas respectivas inclusões). Denotando por $\theta_{K} \in \mathrm{H}_{n}(N, N \backslash K), \theta_{f^{-1}(K)} \in \mathrm{H}_{n}\left(M, M \backslash f^{-1}(K)\right), \theta_{K_{1}} \in \mathrm{H}_{n}\left(N, N \backslash K_{1}\right)$ e $\theta_{f^{-1}\left(K_{1}\right)} \in$ $\mathrm{H}_{n}\left(M, M \backslash f^{-1}\left(K_{1}\right)\right)$ as classes fundamentais, temos que $\left(j_{*} \circ f_{*}\right)\left(\theta_{f^{-1}(K)}\right)=\left(f_{*} \circ i_{*}\right)\left(\theta_{f^{-1}(K)}\right)$. Assim, como:

$$
\left(j_{*} \circ f_{*}\right)\left(\theta_{f^{-1}(K)}\right)=j_{*}\left(\operatorname{deg}_{K} f \cdot \theta_{K}\right)=\operatorname{deg}_{K} f \cdot j_{*}\left(\theta_{K}\right)=\operatorname{deg}_{K} f \cdot \theta_{K_{1}}
$$

$\mathrm{e}$

$$
\left(f_{*} \circ i_{*}\right)\left(\theta_{f^{-1}(K)}\right)=f_{*}\left(\theta_{f^{-1}\left(K_{1}\right)}\right)=\operatorname{deg}_{K_{1}} f \cdot \theta_{K_{1}},
$$

segue que $\operatorname{deg}_{K} f=\operatorname{deg}_{K_{1}} f$. De maneira análoga, pode-se verificar também que $\operatorname{deg}_{K} f=\operatorname{deg}_{K_{2}} f$, d'onde concluímos que $\operatorname{deg}_{K_{1}} f=\operatorname{deg}_{K_{2}} f$, conforme queríamos provar. 
Definição 1.3.5 . Sejam $M$ e $N$ variedades topológicas orientáveis (sem fronteira), de mesma dimensão, e suponha $N$ conexa. Dado um homeomorfismo $h: M \longrightarrow N$, sabemos que $\operatorname{deg}_{K} h=$ \pm 1 , qualquer que seja o subconjunto compacto e conexo $K \subseteq N$, com $K \neq \varnothing$ (corolário 1.3.1). Logo, pela proposição anterior, segue que, ou $\operatorname{deg}_{K} h=1$, ou $\operatorname{deg}_{K} h=-1$, para todo subconjunto compacto, conexo e não-vazio $K \subseteq N$; de acordo com estes dois casos, dizemos (respectivamente) que h preserva ou inverte orientação.

Proposição 1.3.5 (Propriedade aditiva do grau local). Sejam $f: M \longrightarrow N$ e $K \subseteq N$ conforme a definição 1.3.4, e suponha que $M$ é uma união finita de conjuntos abertos $M_{\lambda}$, $\lambda \in\{1,2, \ldots, r\}$, tais que os conjuntos $L_{\lambda}=f^{-1}(K) \cap M_{\lambda}$ são dois a dois disjuntos. Se, para cada $\lambda \in\{1,2, \ldots, r\}$, denotarmos por $f_{\lambda}$ a restrição de $f$ a $M_{\lambda} \subseteq M$, temos que:

$$
\operatorname{deg}_{K} f=\sum_{\lambda=1}^{r} \operatorname{deg}_{K} f_{\lambda}
$$

(note que cada $L_{\lambda}$ é compacto, pois $f^{-1}(K)$ é a união disjunta destes conjuntos).

DemonstraçÃo. Dado $p \in f^{-1}(K)$, considere o diagrama

$$
\bigoplus_{\lambda=1}^{r} \mathrm{H}_{n}\left(M_{\lambda}, M_{\lambda} \backslash L_{\lambda}\right) \stackrel{\left\{j_{*}^{\lambda}\right\}}{\longrightarrow} \mathrm{H}_{n}\left(M, M \backslash f^{-1}(K)\right) \stackrel{\left(j_{p}\right)_{*}}{\longrightarrow} \mathrm{H}_{n}(M, M \backslash\{p\})
$$

onde $j_{*}^{\lambda}: \mathrm{H}_{n}\left(M_{\lambda}, M_{\lambda} \backslash L_{\lambda}\right) \longrightarrow \mathrm{H}_{n}\left(M, M \backslash f^{-1}(K)\right)$ e $\left(j_{p}\right)_{*}: \mathrm{H}_{n}\left(M, M \backslash f^{-1}(K)\right) \longrightarrow$ $\mathrm{H}_{n}(M, M \backslash\{p\})$ são os homomorfismos induzidos pelas respectivas inclusões. Temos então que a composição destes homomorfismos leva todas as componentes da classe fundamental $\left\{\theta_{L_{\lambda}}\right\}$ no elemento nulo de $\mathrm{H}_{n}(M, M \backslash\{p\})$, com exceção da componente $\theta_{L_{\lambda}}$ para a qual tem-se $p \in L_{\lambda}$, que é levada na classe fundamental $\theta_{p} \in \mathrm{H}_{n}(M, M \backslash\{p\})$. Daí, segue que $\left(j_{p}\right)_{*}\left[\left\{j_{*}^{\lambda}\right\}\left(\left\{\theta_{L_{\lambda}}\right\}\right)\right]=\theta_{p}$ para todo $p \in f^{-1}(K)$, e, portanto, $\left\{j_{*}^{\lambda}\right\}\left(\left\{\theta_{L_{\lambda}}\right\}\right)=\theta_{f^{-1}(K)}$, uma vez que a classe fundamental $\theta_{f^{-1}(K)}$ é o único elemento de $\mathrm{H}_{n}\left(M, M \backslash f^{-1}(K)\right)$ levado por $\left(j_{p}\right)_{*}$ em $\theta_{p}$. Por outro lado, sendo $f_{*}: \mathrm{H}_{n}\left(M, M \backslash f^{-1}(K)\right) \longrightarrow \mathrm{H}_{n}(N, N \backslash K)$ e $\left(f_{\lambda}\right)_{*}: \mathrm{H}_{n}\left(M_{\lambda}, M_{\lambda} \backslash L_{\lambda}\right) \longrightarrow \mathrm{H}_{n}(N, N \backslash K)$ os homomorfismos induzidos por $f$ e $f_{\lambda}$, respectivamente, sabemos que o diagrama abaixo é comutativo, para todo $\lambda \in$ $\{1,2, \ldots, r\}$ :

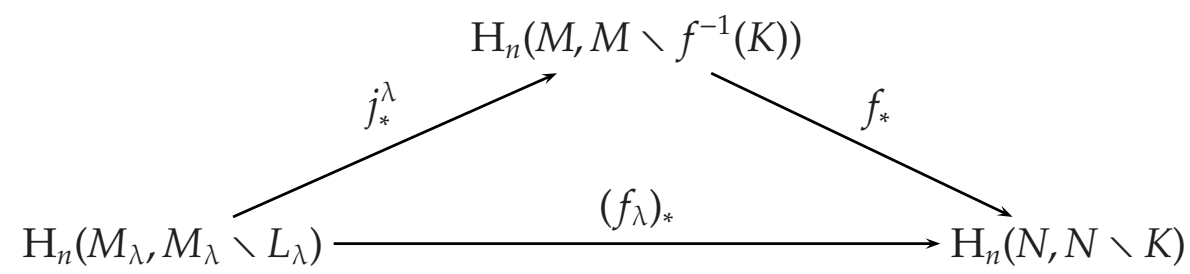


Assim, temos que $f_{*} \circ\left(j_{*}^{\lambda}\right)=\left(f_{\lambda}\right)_{*}$ para cada $\lambda \in\{1,2, \ldots, r\}$, ou seja, $f_{*} \circ\left\{j_{*}^{\lambda}\right\}=\left\{\left(f_{\lambda}\right)_{*}\right\}$. Deste modo, obtemos que:

$$
\begin{aligned}
\left(\operatorname{deg}_{K} f\right) \cdot \theta_{K} & =f_{*}\left(\theta_{f^{-1}(K)}\right)=f_{*}\left(\left\{j_{*}^{\lambda}\right\}\left(\left\{\theta_{L_{\lambda}}\right\}\right)\right)=\left\{\left(f_{\lambda}\right)_{*}\right\}\left(\left\{\theta_{L_{\lambda}}\right\}\right) \\
& =\sum_{\lambda=1}^{r}\left(f_{\lambda}\right)_{*}\left(\theta_{L_{\lambda}}\right)=\left(\sum_{\lambda=1}^{r} \operatorname{deg}_{K} f_{\lambda}\right) \cdot \theta_{K},
\end{aligned}
$$

e, portanto,

$$
\operatorname{deg}_{K} f=\sum_{\lambda=1}^{r} \operatorname{deg}_{K} f_{\lambda},
$$

como queríamos demonstrar.

Corolário 1.3.2 . Seja M uma variedade topológica conexa de dimensão $n$, orientável e sem fronteira. Se $p: M^{\prime} \longrightarrow M$ é um recobrimento que possui um número finito $k>0$ de folhas, então, dado $c \in M$ arbitrário, temos que $\operatorname{deg}_{c}(p)= \pm k$ (note que, pelo exemplo 1.3.2, $p: M^{\prime} \longrightarrow$ Mé uma aplicação própria, e, portanto, o conjunto $p^{-1}(c)$ é compacto).

DemonstraçÃo. De fato, dado $c \in M$, seja $U \subseteq M$ uma vizinhança aberta contrátil (e, portanto, orientável) de $c$, que constitui uma vizinhança elementar em relação ao recobrimento $p: M^{\prime} \longrightarrow M$. Temos então que $p^{-1}(U)$ é a união de uma família $\left\{U_{1}^{\prime}, U_{2}^{\prime}, \ldots, U_{k}^{\prime}\right\}$ de abertos em $M^{\prime}$, dois a dois disjuntos, cada qual é levado homeomorficamente por $p$ sobre $U$ (e, conseqüentemente, cada $U_{i}^{\prime}, i \in\{1,2, \ldots k\}$, é contrátil). Seja, pois, $p^{-1}(c)=\left\{x_{1}, x_{2}, \ldots, x_{k}\right\}, \operatorname{com} x_{i} \in U_{i}^{\prime}$ para cada $i \in\{1,2, \ldots, k\}$, e denote por

$$
U^{\prime}=\bigcup_{i=1}^{k} U_{i}^{\prime} .
$$

Como as restrições $p_{i}=\left.p\right|_{U_{i}^{\prime}}: U_{i}^{\prime} \longrightarrow U$ são homeomorfismos sobre $U$, segue que os homomorfismos induzidos $\left(p_{i}\right)_{*}: \mathrm{H}_{n}\left(U_{i}^{\prime}, U_{i}^{\prime} \backslash\left\{x_{i}\right\}\right) \longrightarrow \mathrm{H}_{n}(U, U \backslash\{c\})$ são isomorfismos; além disso, como $\overline{M^{\prime} \backslash U^{\prime}}=M^{\prime} \backslash U^{\prime} \subseteq \operatorname{Int}\left(M^{\prime} \backslash p^{-1}(c)\right)=M^{\prime} \backslash p^{-1}(c)$ e $\overline{M \backslash U}=M \backslash U \subseteq$ $\operatorname{Int}(M \backslash\{c\})=M \backslash\{c\}$, temos (do teorema da excisão em homologia singular) que os homomorfismos $j_{*}: \mathrm{H}_{n}(U, U \backslash\{c\}) \longrightarrow \mathrm{H}_{n}(M, M \backslash\{c\})$ e $j_{*}^{\prime}: \mathrm{H}_{n}\left(U^{\prime}, U^{\prime} \backslash p^{-1}(c)\right) \longrightarrow$ $\mathrm{H}_{n}\left(M^{\prime}, M^{\prime} \backslash p^{-1}(c)\right)$, induzidos pelas respectivas inclusões, constituem isomorfismos. Assim, denotando por $p_{*}: \mathrm{H}_{n}\left(M^{\prime}, M^{\prime} \backslash p^{-1}(c)\right) \longrightarrow \mathrm{H}_{n}(M, M \backslash\{c\})$ e $\left(\left.p\right|_{U^{\prime}}\right)_{*}: \mathrm{H}_{n}\left(U^{\prime}, U^{\prime} \backslash\right.$ $\left.p^{-1}(c)\right) \longrightarrow \mathrm{H}_{n}(U, U \backslash\{c\})$ os homomorfismos induzidos por $p: M^{\prime} \longrightarrow M$ e $\left.p\right|_{U^{\prime}}: U^{\prime} \longrightarrow$ $U$, respectivamente, obtemos o seguinte diagrama comutativo: 


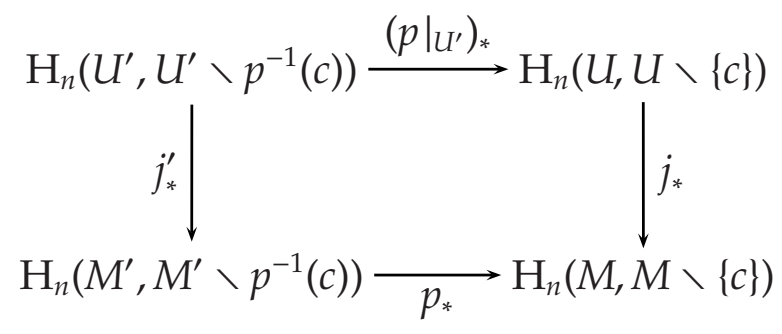

Agora, observe que, como $\left\{x_{i}\right\}=U_{i}^{\prime} \cap p^{-1}(c)$ para cada $i \in\{1,2, \ldots, k\}$, a proposição 1.3.5 nos garante que:

$$
\operatorname{deg}_{c}\left(\left.p\right|_{U^{\prime}}\right)=\sum_{i=1}^{k} \operatorname{deg}_{c}\left(p_{i}\right)
$$

Logo, sendo $\theta_{c}^{M} \in \mathrm{H}_{n}(M, M \backslash\{c\}), \theta_{c}^{U} \in \mathrm{H}_{n}(U, U \backslash\{c\}), \theta_{p^{-1}(c)}^{U^{\prime}} \in \mathrm{H}_{n}\left(U^{\prime}, U^{\prime} \backslash p^{-1}(c)\right) \mathrm{e}$ $\theta_{p^{-1}(c)}^{M^{\prime}} \in \mathrm{H}_{n}\left(M^{\prime}, M^{\prime} \backslash p^{-1}(c)\right)$ as classes fundamentais, e lembrando que $j_{*}^{\prime}\left(\theta_{p^{-1}(c)}^{U^{\prime}}\right)=\theta_{p^{-1}(c)}^{M^{\prime}}$ e $j_{*}\left(\theta_{c}^{U}\right)=\theta_{c}^{M}$ (vide lema 1.3.1), segue (da comutatividade do diagrama acima) que:

$$
\begin{aligned}
p_{*}\left(j_{*}^{\prime}\left(\theta_{p^{-1}(c)}^{U^{\prime}}\right)\right) & =j_{*}\left[\left(\left.p\right|_{U^{\prime}}\right)_{*}\left(\theta_{p^{-1}(c)}^{U^{\prime}}\right)\right]=j_{*}\left[\operatorname{deg}_{c}\left(\left.p\right|_{U^{\prime}}\right) \cdot \theta_{c}^{U}\right]=\operatorname{deg}_{c}\left(\left.p\right|_{U^{\prime}}\right) \cdot j_{*}\left(\theta_{c}^{U}\right) \\
& =\operatorname{deg}_{c}\left(\left.p\right|_{U^{\prime}}\right) \cdot \theta_{c}^{M}=\left[\sum_{i=1}^{k} \operatorname{deg}_{c}\left(p_{i}\right)\right] \cdot \theta_{c}^{M},
\end{aligned}
$$

isto é, $\operatorname{deg}_{c}(p)=\sum_{i=1}^{k} \operatorname{deg}_{c}\left(p_{i}\right)$ (uma vez que $\left.p_{*}\left(j_{*}^{\prime}\left(\theta_{p^{-1}(c)}^{U^{\prime}}\right)\right)=p_{*}\left(\theta_{p^{-1}(c)}^{M^{\prime}}\right)=\operatorname{deg}_{c}(p) \cdot \theta_{c}^{M}\right)$. Isto posto, vamos mostrar então que $\operatorname{deg}_{c}\left(p_{i_{1}}\right)=\operatorname{deg}_{c}\left(p_{i_{2}}\right)$ para quaisquer $i_{1}, i_{2} \in\{1,2, \ldots, k\}$.

Com efeito, dados $i_{1}, i_{2} \in\{1,2, \ldots, k\}$ arbitrários, considere as inclusões $j_{i_{1}}^{\prime}:\left(U_{i_{1}}^{\prime}, U_{i_{1}}^{\prime} \backslash\right.$ $\left.\left\{x_{i_{1}}\right\}\right) \longrightarrow\left(U^{\prime}, U^{\prime} \backslash p^{-1}(c)\right)$ e $j_{i_{2}}^{\prime}:\left(U_{i_{2}}^{\prime}, U_{i_{2}}^{\prime} \backslash\left\{x_{i_{2}}\right\}\right) \longrightarrow\left(U^{\prime}, U^{\prime} \backslash p^{-1}(c)\right)$; como $\overline{U^{\prime} \backslash U_{i_{1}}^{\prime}}=$ $U^{\prime} \backslash U_{i_{1}}^{\prime} \subseteq \operatorname{Int}\left(U^{\prime} \backslash p^{-1}(c)\right)=U^{\prime} \backslash p^{-1}(c)$ e $\overline{U^{\prime} \backslash U_{i_{2}}^{\prime}}=U^{\prime} \backslash U_{i_{2}}^{\prime} \subseteq \operatorname{Int}\left(U^{\prime} \backslash p^{-1}(c)\right)=U^{\prime} \backslash$ $p^{-1}(c)$ (onde os fechos são relativos a $U^{\prime}$ ), segue, do teorema da excisão em homologia singular, que os homomorfismos $\left(j_{i_{1}}^{\prime}\right)_{*}: \mathrm{H}_{n}\left(U_{i_{1}}^{\prime}, U_{i_{1}}^{\prime} \backslash\left\{x_{i_{1}}\right\}\right) \longrightarrow \mathrm{H}_{n}\left(U^{\prime}, U^{\prime} \backslash p^{-1}(c)\right) \mathrm{e}$ $\left(j_{i_{2}}^{\prime}\right)_{*}: \mathrm{H}_{n}\left(U_{i_{2}}^{\prime}, U_{i_{2}}^{\prime} \backslash\left\{x_{i_{2}}\right\}\right) \rightarrow \mathrm{H}_{n}\left(U^{\prime}, U^{\prime} \backslash p^{-1}(c)\right)$, induzidos respectivamente por $j_{i_{1}}^{\prime}$ e $j_{i_{2}}^{\prime}$, constituem isomorfismos, que comutam o seguinte diagrama:

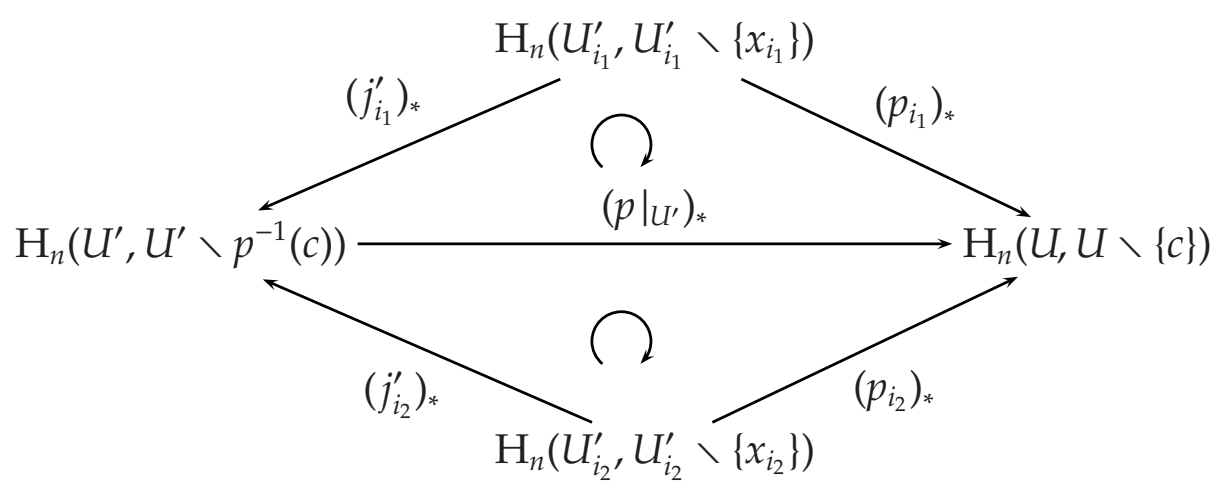


Por outro lado, sendo $\theta_{i_{1}}^{\prime} \in \mathrm{H}_{n}\left(U_{i_{1}}^{\prime}, U_{i_{1}}^{\prime} \backslash\left\{x_{i_{1}}\right\}\right)$ e $\theta_{i_{2}}^{\prime} \in \mathrm{H}_{n}\left(U_{i_{2}}^{\prime}, U_{i_{2}}^{\prime} \backslash\left\{x_{i_{2}}\right\}\right)$ as classes fundamentais, temos (do lema 1.3.1) que $\left(j_{i_{1}}^{\prime}\right)_{*}\left(\theta_{i_{1}}^{\prime}\right)=\left(j_{i_{2}}^{\prime}\right)_{*}\left(\theta_{i_{2}}^{\prime}\right)=\theta_{p^{-1}(c)}^{U^{\prime}}$ (onde $\theta_{p^{-1}(c)}^{U^{\prime}} \in$ $\mathrm{H}_{n}\left(U^{\prime}, U^{\prime} \backslash p^{-1}(c)\right)$ é a classe fundamental). Portanto, da comutatividade do diagrama acima, segue que:

$$
\begin{aligned}
\operatorname{deg}_{c}\left(p_{i_{1}}\right) \cdot \theta_{c}^{U} & =\left(p_{i_{1}}\right)_{*}\left(\theta_{i_{1}}^{\prime}\right)=\left[\left(\left.p\right|_{U^{\prime}}\right)_{*} \circ\left(j_{i_{1}}^{\prime}\right)_{*}\right]\left(\theta_{i_{1}}^{\prime}\right) \\
& =\left[\left(p_{i_{2}}\right)_{*} \circ\left(j_{i_{2}}^{\prime}\right)_{*}^{-1} \circ\left(j_{i_{1}}^{\prime}\right)_{*}\right]\left(\theta_{i_{1}}^{\prime}\right)=\left[\left(p_{i_{2}}\right)_{*} \circ\left(j_{i_{2}}^{\prime}\right)_{*}^{-1}\right]\left(\theta_{p^{-1}(c)}^{U^{\prime}}\right) \\
& =\left(p_{i_{2}}\right)_{*}\left(\theta_{i_{2}}^{\prime}\right)=\operatorname{deg}_{c}\left(p_{i_{2}}\right) \cdot \theta_{c}^{U},
\end{aligned}
$$

ou seja, $\operatorname{deg}_{c}\left(p_{i_{1}}\right)=\operatorname{deg}_{c}\left(p_{i_{2}}\right)$, conforme queríamos mostrar (note que a classe fundamental $\theta_{c}^{U}$ é um gerador de $\left.\mathrm{H}_{n}(U, U \backslash\{c\})\right)$.

Finalmente, lembrando que as restrições $p_{i}=\left.p\right|_{U_{i}^{\prime}}: U_{i}^{\prime} \longrightarrow U$ são homeomorfismos (onde $i \in\{1,2, \ldots, k\}$ ), segue, do corolário 1.3.1, que $\operatorname{deg}_{c}\left(p_{i}\right)= \pm 1$ para cada $i \in\{1,2, \ldots, k\}$. Ora, se $\operatorname{deg}_{c}\left(p_{1}\right)=\operatorname{deg}_{c}\left(p_{2}\right)=\ldots=\operatorname{deg}_{c}\left(p_{k}\right)$ e $\operatorname{deg}_{c}\left(p_{i}\right)= \pm 1$, qualquer que seja $i \in\{1,2, \ldots, k\}$, temos então que:

$$
\sum_{i=1}^{k} \operatorname{deg}_{c}\left(p_{i}\right)= \pm k
$$

o que nos leva a concluir que $\operatorname{deg}_{c}(p)= \pm k$.

Agora, retornando à teoria de raízes, considere uma aplicação contínua própria $f:(M, \partial M) \longrightarrow(N, \partial N)$ entre variedades topológicas conexas de dimensão $n$ (não necessariamente orientáveis), e, dado um ponto $c \in \operatorname{Int} N$, seja $V \subseteq \operatorname{Int} N$ uma vizinhança aberta contrátil de $c$ em $N$ (lembre-se que toda variedade topológica é localmente simplesmente conexa); como $f$ preserva fronteira, temos que $f^{-1}(V) \subseteq \operatorname{Int} M$. Seja, pois, $R$ uma classe de raízes de $f$ em $c$, e $U \subseteq f^{-1}(V)$ um aberto em $M$ tal que $U \cap f^{-1}(c)=R$ (cuja existência é garantida pela proposição 1.3.1). Temos então que $U$ é uma variedade topológica de dimensão $n$, sem fronteira, embora possivelmente não-conexa.

Assim sendo, vamos supor primeiramente que $U$ seja uma variedade orientável (em particular, se $M$ for orientável, consideraremos a orientação de $U$ como sendo a restrição da orientação de $M$ ). Note que, como a vizinhança aberta $V$ é contrátil, segue que $V$ também é uma variedade orientável (de dimensão $n$ e sem fronteira), cuja orientação adotaremos como sendo a restrição da orientação de $N$, caso $N$ seja orientável; deste modo, temos que $\left.f\right|_{U}: U \longrightarrow V$ é uma aplicação contínua entre variedades topológicas orientáveis de dimensão $n$ e sem fronteira. Isto posto, como $\{c\} \subseteq V$ é compacto e conexo, e $f^{-1}(c)$ é um subconjunto compacto de $U$ (pois $f$ é própria), segue que o grau local de $\left.\mathbf{f}\right|_{U}$ em c, denotado por $\operatorname{deg}_{c}\left(\left.f\right|_{U}\right)$, está bem definido, e constitui o número inteiro 
tal que $\left(\left.f\right|_{U}\right)_{*}\left(\theta_{f^{-1}(c)}\right)=\operatorname{deg}_{c}\left(\left.f\right|_{U}\right) \cdot \theta_{c}$, onde $\left(\left.f\right|_{U}\right)_{*}: \mathrm{H}_{n}\left(U, U \backslash f^{-1}(c)\right) \longrightarrow \mathrm{H}_{n}(V, V \backslash\{c\})$ é o homomorfismo induzido por $\left.f\right|_{u}\left(\right.$ e $\theta_{f^{-1}(c)}$ e $\theta_{c}$ são as classes fundamentais em $f^{-1}(c)$ e $\{c\}$, respectivamente). Sem muitas dificuldades, podemos ver que, se $U_{0} \subseteq U$ é um aberto contendo $R$, orientado pela restrição da orientação de $U$, temos $\operatorname{deg}_{c}\left(\left.f\right|_{U_{0}}\right)=$ $\operatorname{deg}_{c}\left(\left.f\right|_{U}\right)$; conseqüentemente, se $U_{1}$ e $U_{2}$ são subconjuntos abertos de $f^{-1}(V)$, ambos contendo $R$ e orientados de maneira que suas orientações coincidam na intersecção $U_{1} \cap U_{2}$, então $\operatorname{deg}_{c}\left(\left.f\right|_{U_{1}}\right)=\operatorname{deg}_{c}\left(\left.f\right|_{U_{2}}\right)$. Além disso, se $V_{0} \subseteq V$ é outra vizinhança aberta contrátil de $c \operatorname{em} N$, com $U \subseteq f^{-1}\left(V_{0}\right)$, segue que $\operatorname{deg}_{c}\left(\left.f\right|_{U}\right)$ possui o mesmo valor, independentemente de considerarmos $\left.f\right|_{u}$ como aplicação em $V$ ou $V_{0}$. Da propriedade aditiva do grau local (proposição 1.3.5), temos ainda que:

$$
\sum\left\{\operatorname{deg}_{c}\left(\left.f\right|_{U}\right): R \text { é classe de raízes de } f \text { em } c\right\}=\operatorname{deg}_{c}\left(f \mid \cup U_{R}\right),
$$

onde, para cada classe de raízes $R$ de $f$ em $c, U_{R} \subseteq f^{-1}(V)$ é um aberto tal que $U_{R} \cap f^{-1}(c)=$ $R$.

Observe então que, devido às condições impostas na definição 1.3.4, tivemos de supor que o aberto $U \subseteq f^{-1}(V)$, contendo a classe de raízes $R$, fosse orientável para podermos definir $\operatorname{deg}_{c}\left(\left.f\right|_{U}\right)$. Agora, vamos admitir que $U$ não seja necessariamente orientável; deste modo, pode não existir qualquer aberto $U \subseteq f^{-1}(V)$, contendo $R$, que seja orientável (como é o caso, por exemplo, se $M$ for uma variedade topológica fechada, não-orientável, e $f$ for a aplicação constante $f: M \longrightarrow\{c\} \subseteq \operatorname{Int} N)$. Entretanto, mostraremos a seguir que, se $f:(M, \partial M) \longrightarrow(N, \partial N)$ for uma aplicação orientável, então o aberto $U$ também é orientável, e vamos descrever um procedimento que será utilizado para orientar $U$ quando $f$ for uma aplicação orientável.

Definição 1.3.6 . Considere $f:(M, \partial M) \longrightarrow(N, \partial N)$ uma aplicação contínua, própria e orientável, entre variedades topológicas conexas de dimensão $n$, e sejam $V$ uma vizinhança aberta contrátil de um ponto $c \in$ Int $N$ em $N$, e $U \subseteq f^{-1}(V)$ um aberto contendo uma classe de raízes $R$ de $f$ em $c$, com $U \cap f^{-1}(c)=R$. Primeiramente, convém mostrarmos que $U$ é uma variedade orientável: de fato, todo laço em $U$ é levado por $f$ num laço do conjunto contrátil $V$, e, portanto, todo laço em U preserva orientação (uma vez que os laços em $V$ são contráteis, e fé orientável); logo, pela proposição 1.1.3, temos que cada componente conexa de U é orientável, o que implica que U é orientável.

Isto posto, a fim de orientar $U$, vamos utilizar o seguinte procedimento, o qual denominaremos processo de orientação: se M for uma variedade orientável, consideramos a orientação de $U$ como sendo a restrição da orientação de $M$. Caso contrário, tomamos $x_{R} \in R \subseteq U$ arbitrário, contido numa componente conexa (aberta) $U_{x_{R}}$ de $U$, e escolhemos uma orientação $s\left(x_{R}\right) \in \tilde{U}_{x_{R}}$ em $x_{R}$ (onde $\tilde{U}_{x_{R}}$ é o fibrado orientado de $U_{x_{R}}$ ). Pela definição de classe de raízes, sabemos que, dado outro ponto $x \in R$, existe um caminho $\omega: I \longrightarrow M, \operatorname{com} \omega(0)=x_{R}$ e $\omega(1)=x$, 
tal que $f \circ \omega: I \longrightarrow N$ é um laço contrátil com base no ponto $c$; além disso, sendo $\left(j_{x_{R}}\right)_{*}$ : $\mathrm{H}_{n}\left(U_{x_{R}}, U_{x_{R}} \backslash\left\{x_{R}\right\}\right) \longrightarrow \mathrm{H}_{n}\left(\operatorname{Int} M\right.$, Int $\left.M \backslash\left\{x_{R}\right\}\right)$ o isomorfismo induzido pela inclusão, temos que s $\left(x_{R}\right)$ determina uma única orientação local de Int $M$ em $x_{R}$, a saber, $s_{M}\left(x_{R}\right)=\left(j_{x_{R}}\right)_{*}\left(s\left(x_{R}\right)\right)$. Assim, orientamos $U$ em $x$ estendendo a orientação local de $x_{\mathbf{R}}$ em Int $\mathbf{M}$ ao longo de $\omega$, e depois restringimos a orientação local obtida, para uma orientação em $U$ : denotando por $\tilde{p}_{M}: \overparen{\operatorname{Int} M} \longrightarrow$ Int $M$ a projeção orientada de Int $M$ (a qual constitui um recobrimento de duas folhas, pois Int $M$ é não-orientável), consideramos a orientação $s_{M}(x)$ de Int $M$ em $x$ como sendo $s_{M}(x)=\tilde{\omega}(1)$, onde $\tilde{\omega}: I \longrightarrow \overparen{\operatorname{Int} M}$ é o único levantamento de $\omega$ (relativamente ao recobrimento $\left.\tilde{p}_{M}\right)$ tal que $\tilde{\omega}(0)=s\left(x_{R}\right) \in \overparen{\operatorname{Int} M}$; então, considerando o isomorfismo $\left(j_{x}\right)_{*}: \mathrm{H}_{n}\left(U_{x}, U_{x} \backslash\{x\}\right) \longrightarrow$ $\mathrm{H}_{n}($ Int $M$, Int $M \backslash\{x\})$, induzido pela inclusão da componente conexa $U_{x} \subseteq U$, que contém $x$, em Int $M$, definimos a orientação de $U$ em $x$ por $s(x)=\left(j_{x}\right)_{*}^{-1}\left(s_{M}(x)\right) \in \mathrm{H}_{n}\left(U_{x}, U_{x} \backslash\{x\}\right)$. Note que esta orientação $s(x)$ é independente da escolha do caminho $\omega: I \longrightarrow M$ : com efeito, dado um outro caminho $\lambda: I \longrightarrow M$, satisfazendo $\lambda(0)=x_{R}$ e $\lambda(1)=x$, tal que $f \circ \lambda: I \longrightarrow$ Né um laço contrátil com base no ponto $c$, e sendo $\omega^{-1} \vee \lambda: I \longrightarrow$ Mo laço constituído da justaposição de $\omega^{-1}$ e $\lambda$, temos que $f \circ\left(\omega^{-1} \vee \lambda\right): I \longrightarrow N$ é um laço contrátil, pois $f \circ\left(\omega^{-1} \vee \lambda\right)=\left(f \circ \omega^{-1}\right) \vee(f \circ \lambda)$; $\operatorname{logo}, f \circ\left(\omega^{-1} \vee \lambda\right)$ é um laço que preserva orientação, o que significa que $\omega^{-1} \vee \lambda$ também o é (uma vez que a aplicação $f$ é orientável). Portanto, temos que $s_{M}(x)=\tilde{\omega}(1)=\tilde{\omega}^{-1}(0)=\tilde{\lambda}(1)$, d'onde concluímos que a orientação s $(x)$ não depende do caminho $\omega: I \longrightarrow M$ que escolhemos acima.

Deste modo, a partir da orientação em $x_{R}$, podemos orientar cada ponto $x \in R$. Agora, considere uma componente conexa $U_{0}$ de $U$ tal que $U_{0} \cap R \neq \varnothing$, e seja $x \in U_{0} \cap R$. A fim de orientarmos os pontos de $U_{0}$ que não pertencem a $R$, procedemos da seguinte maneira: dado $y \in U_{0} \backslash R$, tomamos um caminho $\alpha: I \longrightarrow U_{0}$ tal que $\alpha(0)=x$ e $\alpha(1)=y$ (o qual existe, pois $U_{0}$ é uma variedade conexa, e, portanto, conexa por caminhos), e orientamos $U$ em $x$ de acordo com o procedimento descrito acima; então, sendo $\tilde{p}_{0}: \tilde{C}_{0} \longrightarrow U_{0}$ o recobrimento de $U_{0}$ obtido de uma componente conexa $\tilde{C}_{0}$ do fibrado orientado $\tilde{U}_{0}$, contendo a orientação $s(x)$ de $U$ em $x$, definimos a orientação de $U_{0}$ em y como sendo $s(y)=\tilde{\alpha}(1)$, onde $\tilde{\alpha}: I \longrightarrow \tilde{C}_{0}$ é o unico levantamento de $\alpha$ (relativamente a $\tilde{p}_{0}$ ) tal que $\tilde{\alpha}(0)=s(x) \in \tilde{C}_{0}$ (em outras palavras, orientamos $U_{0}$ em y estendendo a orientação de $x$ ao longo de $\alpha$, conforme ilustrado na figura 1.3 a seguir). Para verificar que s(y) não depende do caminho $\alpha$ escolhido, basta observar que, se $\beta: I \longrightarrow U_{0}$ é outro caminho satisfazendo $\beta(0)=x$ e $\beta(1)=y$, então $\alpha^{-1} \vee \beta: I \longrightarrow U_{0}$ é um laço que preserva orientação (uma vez que $U_{0}$ é orientável), isto é, $s(y)=\tilde{\alpha}(1)=\tilde{\alpha}^{-1}(0)=\tilde{\beta}(1)$.

Conseqüentemente, temos que s(y) também não depende do ponto $x \in U_{0} \cap R$ que tomamos inicialmente: de fato, dado outro ponto $x^{\prime} \in U_{0} \cap R$, e sendo $\gamma, \delta: I \longrightarrow U_{0}$ caminhos tais que $\gamma(0)=x, \gamma(1)=\delta(0)=x^{\prime}$ e $\delta(1)=y$, segue que o caminho $\beta=\gamma \vee \delta: I \longrightarrow U_{0}$ satisfaz $\beta(0)=x$ e $\beta(1)=y$, e, portanto, $s(y)=\tilde{\alpha}(1)=\tilde{\beta}(1)=\tilde{\delta}(1)$. Assim, a orientação de $x_{R}$ também nos permite orientar os pontos de cada componente conexa de U que intercepta $R$. Logo, orientando as demais componentes de $U$ (que não interceptam $R$ ) arbitrariamente, tornamos $U$ 
uma variedade orientada.

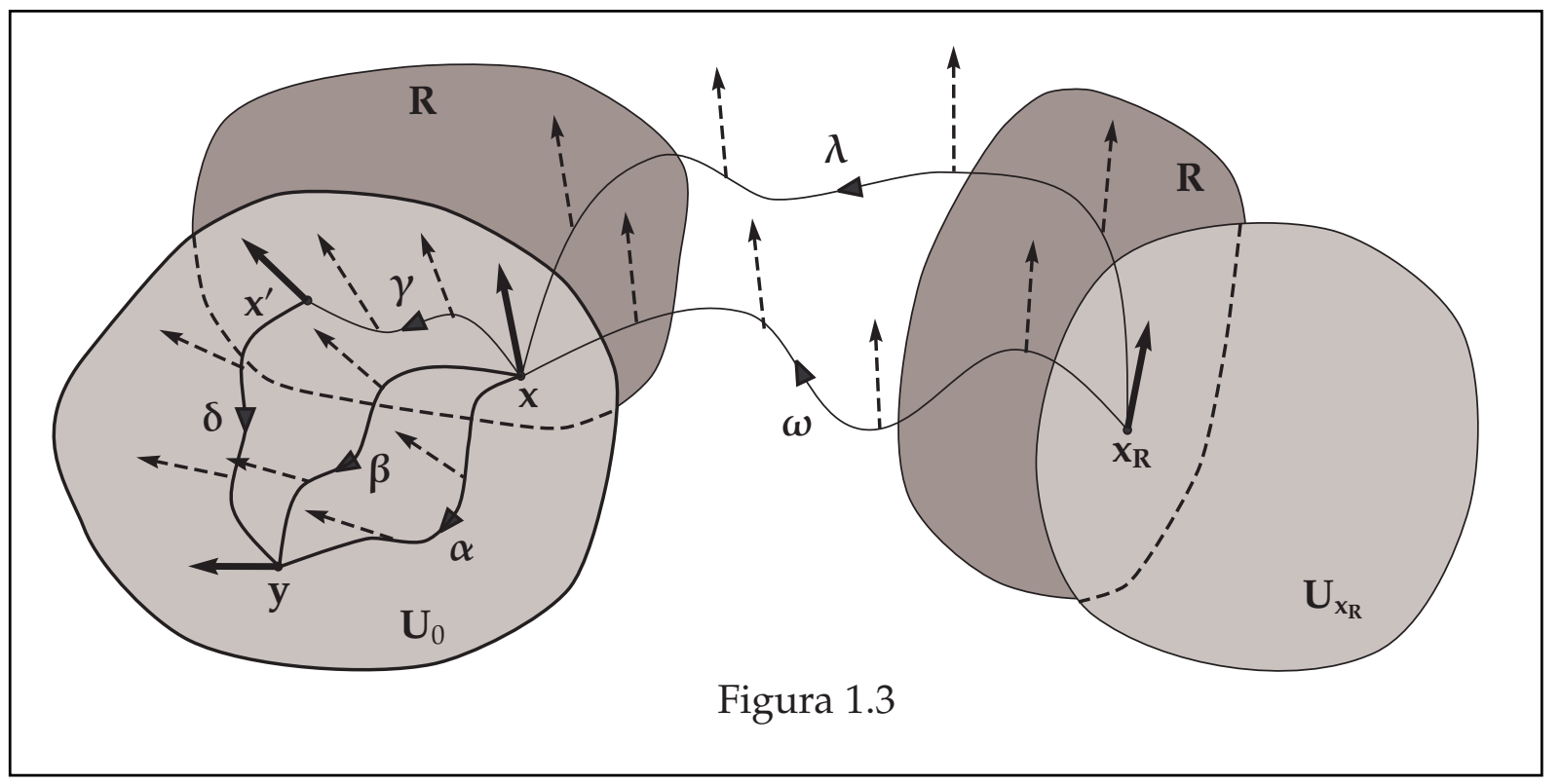

Agora, observe que, embora não haja um critério na escolha das orientações de $U$ e $V$ (a menos, é claro, que $M$ e $N$ sejam variedades orientadas), o grau local $\operatorname{deg}_{c}\left(\left.f\right|_{U}\right)$ possui o mesmo valor absoluto, independentemente das orientações de $U$ e $V$. De fato, utilizando as mesmas notações acima, fixemos uma orientação $s_{U}: U \longrightarrow \tilde{U}$ em $U$, e consideremos a classe fundamental $\theta_{f^{-1}(c)}$ em $f^{-1}(c) \subseteq U$; então, adotando uma orientação $s_{V}: V \longrightarrow \tilde{V}$ em $V$, e sendo $\theta_{c}$ a classe fundamental em $\{c\}$, segue que $\operatorname{deg}_{c}\left(\left.f\right|_{U}\right) \in \mathbb{Z}$ é tal que $\left(\left.f\right|_{U}\right)_{*}\left(\theta_{f^{-1}(c)}\right)=\operatorname{deg}_{c}\left(\left.f\right|_{U}\right) \cdot \theta_{c}$, onde $\left(\left.f\right|_{U}\right)_{*}: \mathrm{H}_{n}\left(U, U \backslash f^{-1}(c)\right) \longrightarrow$ $\mathrm{H}_{n}(V, V \backslash\{c\})$ é o homomorfismo induzido por $\left.f\right|_{U}: U \longrightarrow V$. Entretanto, considerando a orientação oposta $-s_{V}: V \longrightarrow \tilde{V}$ em $V$, obtemos outra classe fundamental $\theta_{c}^{\prime}$ em $\{c\}$, satisfazendo $\theta_{c}^{\prime}=-1 \cdot \theta_{c}$ (vide definição 1.3.3), a qual, por sua vez, determina um grau local $\operatorname{deg}_{c}^{\prime}\left(\left.f\right|_{U}\right) \in \mathbb{Z}$ tal que $\left(\left.f\right|_{U}\right)_{*}\left(\theta_{f^{-1}(c)}\right)=\operatorname{deg}_{c}^{\prime}\left(\left.f\right|_{U}\right) \cdot \theta_{c}^{\prime}$. Ora, se $\left(\left.f\right|_{U}\right)_{*}\left(\theta_{f^{-1}(c)}\right)=$ $\operatorname{deg}_{c}\left(\left.f\right|_{u}\right) \cdot \theta_{c}=\operatorname{deg}_{c}^{\prime}\left(\left.f\right|_{U}\right) \cdot \theta_{c}^{\prime}$ e $\theta_{c}^{\prime}=-1 \cdot \theta_{c}$, concluímos que $\operatorname{deg}_{c}^{\prime}\left(\left.f\right|_{U}\right)=-\operatorname{deg}_{c}\left(\left.f\right|_{u}\right)$. Por outro lado, suponhamos agora que a orientação $s_{V}: V \longrightarrow \tilde{V}$ é fixa; então, se $f:(M, \partial M) \longrightarrow(N, \partial N)$ é uma aplicação orientável, e se tomarmos a orientação oposta de $U$ em $x_{R} \in R$, o processo de orientação descrito acima altera a orientação de cada componente conexa de $U$ que intercepta $R$. Logo, o grau local, em $c \in \operatorname{Int} N$, da restrição de $f$ a cada uma destas componentes, também muda de sinal. Ora, como a classe de raízes $R$ é compacta, segue que o número de componentes de $U$, que interceptam $R$, é finito; além disso, se $U_{0}$ é uma componente conexa de $U$ que não intercepta $R$, segue que $U_{0} \cap f^{-1}(c)=\varnothing$ (pois $U_{0} \cap f^{-1}(c) \subseteq U \cap f^{-1}(c)=R$ ), isto é, $\left(\left.f\right|_{U_{0}}\right)^{-1}(c)=\varnothing$, o que implica que $\operatorname{deg}_{c}\left(\left.f\right|_{U_{0}}\right)=0$. Portanto, pela propriedade aditiva do grau local aplicada 
à restrição $\left.f\right|_{U}: U \longrightarrow V$, podemos ver que a mudança de orientação de $U$ também inverte o sinal de $\operatorname{deg}_{c}\left(\left.f\right|_{u}\right)$. Conseqüentemente, a menos de sinal, o grau $\operatorname{local} \operatorname{deg}_{c}\left(\left.f\right|_{u}\right)$ é independente das orientações de $U$ e $V$, conforme queríamos mostrar.

Finalmente, antes de definirmos o conceito de multiplicidade de uma classe de raízes, convém observarmos também que, se $f$ não for uma aplicação orientável, o aberto $U$ ainda pode ser orientável (é o que ocorre, por exemplo, se a classe de raízes $R$ é finita e $U$ é tomado como sendo uma união de vizinhanças euclidianas). Entretanto, a não-orientabilidade de $f$ implica que a orientação de $U$, obtida pelo processo de orientação, dependerá não somente da orientação de $U$ em $x_{R}$ (como era o caso, se $f$ fosse orientável), mas também da escolha dos caminhos entre $x_{R}$ e os demais pontos de $R$; isto significa que o grau local $\operatorname{deg}_{c}\left(\left.f\right|_{u}\right)$ pode não estar bem definido, pois uma dada orientação de $U$ em $x_{R}$ pode definir duas possíveis orientações na componente conexa de $U$ que contém $x_{R}$, sendo que cada uma delas determinará um valor diferente para o grau local nesta componente. Assim, para evitar esta inconveniência, consideramos que, se $f$ não é orientável, o grau $\operatorname{local} \operatorname{deg}_{c}\left(\left.f\right|_{U}\right)$ deve ser calculado utilizando-se coeficientes em $\mathbb{Z}_{2}$, pois, deste modo, o aberto $U$ torna-se unicamente orientado.

Tendo em vista as observações acima, podemos então introduzir a seguinte

Definição 1.3.7 . Seja $f:(M, \partial M) \longrightarrow(N, \partial N)$ uma aplicação própria entre variedades topológicas conexas, de dimensão $n$, e sejam $c \in \operatorname{Int} N$ e $R$ uma classe de raízes de $f$ em $c$. Dada uma vizinhança aberta contrátil $V \subseteq \operatorname{Int} N$ de c em $N$, e sendo $U \subseteq f^{-1}(V)$ um aberto tal que $U \cap f^{-1}(c)=R$, definimos a multiplicidade de $R$, denotada por $|m(R)|$, como sendo

$$
|m(R)|=\left|\operatorname{deg}_{c}\left(\left.f\right|_{U}\right)\right|,
$$

onde:

- se $f$ é uma aplicação orientável (isto é, dos tipos I ou II), $\operatorname{deg}_{c}\left(\left.f\right|_{u}\right)$ é o grau local, com coeficientes em $\mathbb{Z}$, e U é orientado de acordo com o processo de orientação;

- se fé uma aplicação não-orientável (isto é, do tipo III), $\operatorname{deg}_{c}(f \mid u)$ éo grau local com coeficientes em $\mathbb{Z}_{2}$.

Exemplo 1.3.8 . Seja $f:(M, \partial M) \longrightarrow(N, \partial N)$ uma aplicação própria entre variedades topológicas conexas (de dimensão $n$ ), e sejam $c \in \operatorname{Int} N$ e $V \subseteq N$ uma vizinhança aberta contrátil de $c$ em $N$. Supondo $f^{-1}(c)$ finito e não-vazio, considere uma classe de raízes $R=\left\{x_{1}, x_{2}, \ldots, x_{k}\right\}$ de $f$ em $c$, e, para cada $i \in\{1,2, \ldots, k\}$, denote por $U_{i}$ uma vizinhança aberta de $x_{i}$ em $f^{-1}(V)$, tal que $U_{i} \cap f^{-1}(c)=\left\{x_{i}\right\}$. Orientando o aberto $U=\bigcup_{i=1}^{k} U_{i} \subseteq f^{-1}(V)$ pelo processo de orientação, segue (da propriedade aditiva do 
grau local, aplicada a $\left.f\right|_{U}: U \longrightarrow V$ ), que:

$$
\operatorname{deg}_{c}\left(\left.f\right|_{U}\right)=\sum_{i=1}^{k} \operatorname{deg}_{c}\left(\left.f\right|_{U_{i}}\right) \text {. }
$$

Assim, se $f$ for uma aplicação orientável, concluímos que:

$$
|m(R)|=\left|\sum_{i=1}^{k} \operatorname{deg}_{c}\left(\left.f\right|_{U_{i}}\right)\right| .
$$

Note que, caso $f$ seja não-orientável, a expressão acima ainda contínua válida: basta tomarmos coeficientes em $\mathbb{Z}_{2}$ nos cálculos dos graus locais.

\section{4 (O) Graur Cohomológico}

O objetivo desta seção é definir o grau cohomológico para aplicações próprias $f:(M, \partial M) \longrightarrow(N, \partial N)$, e mostrar sua relação com o conceito de grau local introduzido na seção anterior. Primeiramente, no entanto, convém apresentarmos uma revisão da cohomologia de Čech com suporte compacto para variedades com fronteira, sendo que, para tanto, utilizaremos alguns resultados sobre limites diretos (o apêndice A contém um breve resumo sobre este assunto).

Definição 1.4.1 . Seja $M$ uma variedade topológica de dimensão n e $A \subseteq M$ um subconjunto localmente compacto (por exemplo, aberto ou fechado). Denotando por $\Lambda$ a família de todos os subconjuntos compactos $K \subseteq M$, tais que $A \subseteq M \backslash K$, temos que $\Lambda$ constitui um conjunto dirigido pela relação de inclusão direta, dada por $K_{1} \leqslant K_{2} \Longleftrightarrow K_{1} \subseteq K_{2}$, onde $K_{1}, K_{2} \in \Lambda$ (vide exemplo A.1). Isto posto, dados $K_{1}, K_{2} \in \Lambda$, com $K_{1} \leqslant K_{2}$, seja $i_{K_{1}, K_{2}}^{*}: \mathrm{H}^{k}(M, M \backslash$ $\left.K_{1}\right) \longrightarrow \mathrm{H}^{k}\left(M, M \backslash K_{2}\right)$ o homomorfismo em cohomologia singular induzido pela inclusão $i:\left(M, M \backslash K_{2}\right) \longrightarrow\left(M, M \backslash K_{1}\right)$. Como $i_{K_{1}, K_{3}}^{*}=i_{K_{2}, K_{3}}^{*} \circ i_{K_{1}, K_{2}}^{*}$ para quaisquer $K_{1}, K_{2}, K_{3} \in \Lambda$, com $K_{1} \leqslant K_{2} \leqslant K_{3}$, segue então que $\left\{\mathrm{H}^{k}(M, M \backslash K), i_{K_{1}, K_{2}}^{*}\right\}_{K_{1}, K_{2} \in \Lambda}$ é um sistema direto de grupos abelianos e homomorfismos (note que $i_{K_{1}, K_{1}}^{*}$ é o homomorfismo identidade, para todo $K_{1} \in \Lambda$ ). Assim, ao grupo abeliano $\underset{\lim }{\longrightarrow}\left\{\mathrm{H}^{k}(M, M \backslash K): K \in \Lambda\right\}$, denominamos $\boldsymbol{k}$-ésimo grupo de cohomologia de Čech com suporte compacto (e coeficientes em $\mathbb{Z}$ ) do par $(M, A)$, e o denotamos por $\check{\mathrm{H}}_{c}^{k}(M, A)$. Como usualmente, se $A=\varnothing$, escrevemos $\check{\mathrm{H}}_{c}^{k}(M, \varnothing)=\check{\mathrm{H}}_{c}^{k}(M)$ (neste caso, $\Lambda$ é constituído de todos os subconjuntos compactos de $M$ ).

Proposição 1.4.1. Sendo $M$ uma variedade topológica de dimensão n, com fronteira $\partial M$, então existe um isomorfismo $\check{\varphi}: \check{\mathrm{H}}_{c}^{k}(M, \partial M) \longrightarrow \check{\mathrm{H}}_{c}^{k}(\operatorname{Int} M)$. 
DemonstraçÃo. Com efeito, denotemos por $\Lambda$ a família de todos os subconjuntos compactos $K \subseteq M$, tais que $\partial M \subseteq M \backslash K$, dirigida pela relação de inclusão direta. Como todo subconjunto compacto $L \subseteq M \backslash \partial M=\operatorname{Int} M$ constitui um subconjunto compacto de $M$, tal que $\partial M \subseteq M \backslash L$, e como $K \subseteq M \backslash \partial M=\operatorname{Int} M$ para todo compacto $K \in \Lambda$, segue que $\Lambda$ coincide com a família dos subconjuntos compactos de Int $M$ (também dirigida pela relação de inclusão direta). Isto posto, da$\operatorname{dos} K_{1}, K_{2} \in \Lambda$, com $K_{1} \leqslant K_{2}$, sejam $i_{K_{1}, K_{2}}^{*}: \mathrm{H}^{k}\left(M, M \backslash K_{1}\right) \rightarrow \mathrm{H}^{k}\left(M, M \backslash K_{2}\right)$ e $j_{K_{1}, K_{2}}^{*}: \mathrm{H}^{k}\left(\operatorname{Int} M, \operatorname{Int} M \backslash K_{1}\right) \longrightarrow \mathrm{H}^{k}\left(\operatorname{Int} M, \operatorname{Int} M \backslash K_{2}\right)$ os homomorfismos em cohomologia singular induzidos pelas inclusões $i_{K_{1}, K_{2}}:\left(M, M \backslash K_{2}\right) \longrightarrow\left(M, M \backslash K_{1}\right)$ e $j_{K_{1}, K_{2}}$ : (Int $\left.M, \operatorname{Int} M \backslash K_{2}\right) \longrightarrow\left(\operatorname{Int} M\right.$, Int $\left.M \backslash K_{1}\right)$; por definição, temos então que $\check{\mathrm{H}}_{c}^{k}(M, \partial M)$ e $\widetilde{\mathrm{H}}_{c}^{k}(\operatorname{Int} M)$ são os limites diretos dos sistemas $\mathcal{S}(M, \partial M)=\left\{\mathrm{H}^{k}(M, M \backslash K), i_{K_{1}, K_{2}}^{*}\right\}_{K_{1}, K_{2} \in \Lambda}$ e $\mathcal{S}(\operatorname{Int} M)=\left\{\mathrm{H}^{k}(\operatorname{Int} M, \operatorname{Int} M \backslash K), j_{K_{1}, K_{2}}^{*}\right\}_{K_{1}, K_{2} \in \Lambda}$, respectivamente. Considere, pois, a aplicação identidade $\operatorname{Id}_{\Lambda}: \Lambda \longrightarrow \Lambda$ (dada por $\operatorname{Id}_{\Lambda}(K)=K$ para todo $K \in \Lambda$ ), e, para cada $K \in \Lambda$, seja $i_{K}^{*}: H^{k}(M, M \backslash K) \longrightarrow \mathrm{H}^{k}(\operatorname{Int} M$, Int $M \backslash K)$ o homomorfismo em cohomologia singular induzido pela inclusão $i_{K}:(\operatorname{Int} M$, Int $M \backslash K) \longrightarrow(M, M \backslash K)$. Dados $K_{1}, K_{2} \in \Lambda$, com $K_{1} \leqslant K_{2}$, sabemos que o diagrama abaixo é comutativo:

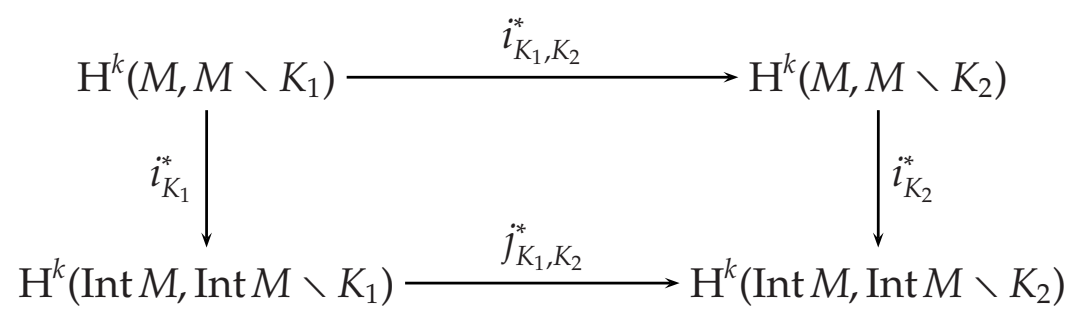

Daí, segue que $\Phi=\left\{\operatorname{Id}_{\Lambda}, i_{K}^{*}\right\}_{K \in \Lambda}$ é uma aplicação entre os sistemas diretos $\mathcal{S}(M, \partial M)$ e $\mathcal{S}(\operatorname{Int} M)$, a qual induz um homomorfismo $\check{\varphi}=\stackrel{\Phi}{\rightarrow}: \check{\mathrm{H}}_{c}^{k}(M, \partial M) \longrightarrow \check{\mathrm{H}}_{c}^{k}(\operatorname{Int} M)$. Agora, observe que $\partial M=\overline{\partial M} \subseteq M \backslash K=\operatorname{Int}(M \backslash K)$ para todo $K \in \Lambda$; pela excisão em cohomologia singular (vide [Vi], teorema 3.12, página 81), isto significa que $i_{K}^{*}$ : $\mathrm{H}^{k}(M, M \backslash K) \longrightarrow \mathrm{H}^{k}(\operatorname{Int} M$, Int $M \backslash K)$ é um isomorfismo, qualquer que seja $K \in \Lambda$. Logo, pela proposição A.3, podemos concluir que $\check{\varphi}$ é um isomorfismo entre os grupos $\check{\mathrm{H}}_{c}^{k}(M, \partial M)$ e $\tilde{\mathrm{H}}_{c}^{k}(\operatorname{Int} M)$.

Proposição 1.4.2. Se Mé uma variedade topológica conexa de dimensão n, então $\check{\mathrm{H}}_{c}^{n}(M, \partial M) \simeq$ $\mathbb{Z}$ se $M$ for orientável, ou $\check{\mathrm{H}}_{c}^{n}(M, \partial M) \simeq \mathbb{Z}_{2}$ caso contrário.

DemonstraÇÃo. Como $\check{\mathrm{H}}_{c}^{n}(M, \partial M) \simeq \check{\mathrm{H}}_{c}^{n}(\operatorname{Int} M)$, podemos supor, sem perda de generalidade, que $\partial M=\varnothing$. Seja, pois, $\Lambda_{0}$ a família de todos os subconjuntos compactos e conexos $K \subseteq M$; no exemplo A.3, vimos que $\Lambda_{0}$ é um subconjunto cofinal da família $\Lambda$ de todos os subconjuntos compactos de $M$, ambas dirigidas pela relação de inclusão 
direta. Portanto:

$$
\check{\mathrm{H}}_{c}^{n}(M)=\underset{\lim }{\longrightarrow}\left\{\mathrm{H}^{n}(M, M \backslash K): K \in \Lambda\right\} \simeq \underset{\lim }{\longrightarrow}\left\{\mathrm{H}^{n}(M, M \backslash K): K \in \Lambda_{0}\right\}
$$

(vide item (iii) da proposição A.2). Entretanto, do teorema dos coeficientes universais para cohomologia singular, temos que:

$$
\mathrm{H}^{n}(M, M \backslash K ; \mathbb{Z}) \simeq \operatorname{Hom}\left(\mathrm{H}_{n}(M, M \backslash K), \mathbb{Z}\right) \oplus \operatorname{Ext}\left(\mathrm{H}_{n-1}(M, M \backslash K), \mathbb{Z}\right)
$$

para todo $K \in \Lambda_{0}$ (vide [Vi], teorema 3.14, página 84); por outro lado, sabemos também que:

$$
\operatorname{Hom}\left(\mathrm{H}_{n}(M, M \backslash K), \mathbb{Z}\right) \simeq \begin{cases}\mathbb{Z} & \text { se } M \text { é orientável } \\ \{0\} & \text { se } M \text { é não-orientável }\end{cases}
$$

e

$$
\operatorname{Ext}\left(\mathrm{H}_{n-1}(M, M \backslash K), \mathbb{Z}\right) \simeq \begin{cases}\{0\} & \text { se } M \text { é orientável } \\ \mathbb{Z}_{2} & \text { se } M \text { é não-orientável }\end{cases}
$$

(vide [D], corolários 3.4 e 3.5, páginas 260 e 261). Logo, para todo $K \in \Lambda_{0}$, segue que:

$$
\mathrm{H}^{n}(M, M \backslash K ; \mathbb{Z}) \simeq\left\{\begin{array}{ll}
\mathbb{Z} & \text { se } M \text { é orientável } \\
\mathbb{Z}_{2} & \text { se } M \text { é não-orientável }
\end{array} .\right.
$$

Isto posto, consideremos os sistemas diretos $\left\{G_{K}, f_{K_{1}, K_{2}}\right\}_{K_{1}, K_{2} \in \Lambda_{0}}$ e $\left\{\mathrm{H}_{K}, g_{K_{1}, K_{2}}\right\}_{K_{1}, K_{2} \in \Lambda_{0}}$, onde $G_{K}=\mathbb{Z}$ e $H_{K}=\mathbb{Z}_{2}$ para cada $K \in \Lambda_{0}$, e $f_{K_{1}, K_{2}}: G_{K_{1}} \longrightarrow G_{K_{2}}$ e $g_{K_{1}, K_{2}}: H_{K_{1}} \longrightarrow \mathrm{H}_{K_{2}}$ são os homomorfismos identidade, quaisquer que sejam $K_{1}, K_{2} \in \Lambda_{0}$ tais que $K_{1} \leqslant K_{2}$; sem muitas dificuldades, podemos ver que $\underset{\lim }{\longrightarrow}\left\{G_{K}: K \in \Lambda_{0}\right\} \simeq \mathbb{Z}$ e $\underset{\lim _{\longrightarrow}}{\longrightarrow}\left\{\mathrm{H}_{K}: K \in \Lambda_{0}\right\} \simeq \mathbb{Z}_{2}$. Assim, supondo $M$ orientável, temos que $H^{n}(M, M \backslash K ; \mathbb{Z}) \simeq G_{K}$ para todo $K \in \Lambda_{0}$, e, conseqüentemente (da proposição A.3), segue que:

$$
\check{\mathrm{H}}_{c}^{n}(M) \simeq \underset{\lim }{\longrightarrow}\left\{\mathrm{H}^{n}(M, M \backslash K ; \mathbb{Z}): K \in \Lambda_{0}\right\} \simeq \underline{\lim }_{\longrightarrow}\left\{G_{K}: K \in \Lambda_{0}\right\} \simeq \mathbb{Z} .
$$

Por outro lado, se $M$ é não-orientável, temos que $\mathrm{H}^{n}(M, M \backslash K ; \mathbb{Z}) \simeq \mathrm{H}_{K}$ para todo $K \in \Lambda_{0}$, o que implica que:

$$
\check{\mathrm{H}}_{c}^{n}(M) \simeq \underset{\lim }{\longrightarrow}\left\{\mathrm{H}^{n}(M, M \backslash K ; \mathbb{Z}): K \in \Lambda_{0}\right\} \simeq \underset{\lim }{\longrightarrow}\left\{\mathrm{H}_{K}: K \in \Lambda_{0}\right\} \simeq \mathbb{Z}_{2} .
$$

Deste modo, concluímos que:

$$
\check{\mathrm{H}}_{c}^{n}(M, \partial M) \simeq \check{\mathrm{H}}_{c}^{n}(\operatorname{Int} M) \simeq\left\{\begin{array}{ll}
\mathbb{Z} & \text { se } \operatorname{Int} M \text { é orientável } \\
\mathbb{Z}_{2} & \text { se } \operatorname{Int} M \text { é não-orientável }
\end{array},\right.
$$

como queríamos demonstrar. 
Definição 1.4.2 . Sejam $M e N$ variedades topológicas de dimensão $n, e$, dados subconjuntos localmente compactos $A \subseteq M e B \subseteq N$, considere uma aplicação contínua própria $f:(M, A) \longrightarrow$ $(N, A)$. Sejam ainda $\Lambda$ a família dos conjuntos compactos $K \subseteq M$ tais que $A \subseteq M \backslash K$, e $\Omega$ a família dos conjuntos compactos $L \subseteq N$ tais que $B \subseteq N \backslash L$, ambas dirigidas pela relação de inclusão direta. Note então que $f^{-1}(L) \in \Lambda$ para todo $L \in \Omega$ : com efeito, se $L \in \Omega$ (isto é, se $L \subseteq N$ é compacto, com $B \subseteq N \backslash L)$, segue que $f^{-1}(L)$ é um subconjunto compacto de $M$ (pois $f$ é própria), tal que $A \subseteq f^{-1}(B) \subseteq f^{-1}(N \backslash L)=M \backslash f^{-1}(L)$. Isto posto, seja $\phi: \Omega \longrightarrow \Lambda$ a função dada por $\phi(L)=f^{-1}(L)$ para cada $L \in \Omega$. Evidentemente, temos que $\phi$ preserva ordem, pois, dados $L_{1}, L_{2} \in \Omega$ tais que $L_{1} \leqslant L_{2}$, segue que $L_{1} \subseteq L_{2}$, o que implica que $f^{-1}\left(L_{1}\right) \subseteq f^{-1}\left(L_{2}\right)$, ou seja, $\phi\left(L_{1}\right) \leqslant \phi\left(L_{2}\right)$. Agora, dado $L \in \Omega$, considere o homomorfismo $f_{L}^{*}: H^{k}(N, N \backslash L) \longrightarrow H^{k}\left(M, M \backslash f^{-1}(L)\right)$ induzido em cohomologia singular pela restrição de f a $M \backslash f^{-1}(L)$. Então, dados $L_{1}, L_{2} \in \Omega$, com $L_{1} \leqslant L_{2}$, e sendo $j_{L_{1}, L_{2}}^{*}: \mathrm{H}^{k}\left(N, N \backslash L_{1}\right) \longrightarrow$ $\mathrm{H}^{k}\left(N, N \backslash L_{2}\right)$ e $i_{f^{-1}\left(L_{1}\right), f^{-1}\left(L_{2}\right)}^{*}: \mathrm{H}^{k}\left(M, M \backslash f^{-1}\left(L_{1}\right)\right) \longrightarrow \mathrm{H}^{k}\left(M, M \backslash f^{-1}\left(L_{2}\right)\right)$ os homomorfismos induzidos pelas inclusões $N \backslash L_{2} \subseteq N \backslash L_{1}$ e $M \backslash f^{-1}\left(L_{2}\right) \subseteq M \backslash f^{-1}\left(L_{1}\right)$, temos o seguinte diagrama comutativo:

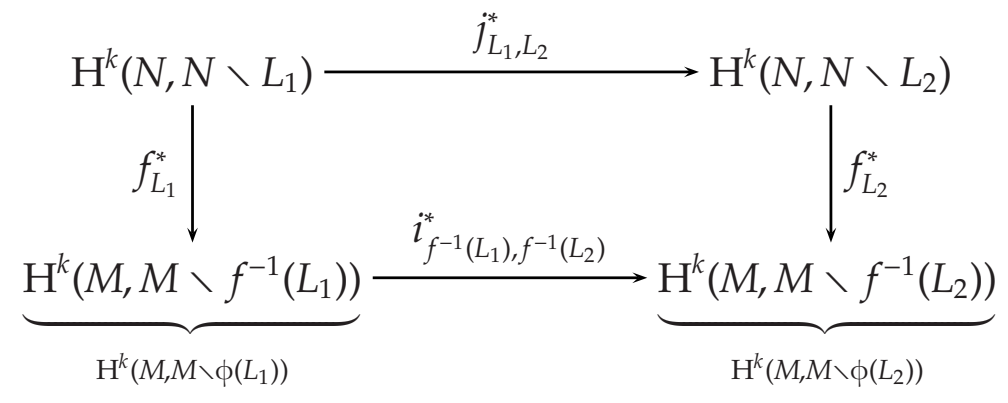

Portanto, $\Phi=\left\{\phi, f_{L}^{*}\right\}_{L \in \Omega}$ é uma aplicação entre os sistemas diretos $\mathcal{S}(N)=\left\{\mathrm{H}^{k}(N, N \backslash\right.$ $\left.L), j_{L_{1}, L_{2}}^{*}\right\}_{L_{1}, L_{2} \in \Omega}$ e $\mathcal{S}(M)=\left\{\mathrm{H}^{k}(M, M \backslash K), i_{K_{1}, K_{2}}^{*}\right\}_{K_{1}, K_{2} \in \Lambda}$. Deste modo, definimos o homomorfismo induzido (em cohomologia de Čech com suporte compacto), pela função $f$, como sendo o limite direto

$$
\check{f_{k}^{*}}=\stackrel{\Phi}{\rightarrow}: \check{\mathrm{H}}_{c}^{k}(N, B) \longrightarrow \check{\mathrm{H}}_{c}^{k}(M, A) .
$$

Note que, dados $L \in \Omega$ e $\mu_{L} \in \mathrm{H}^{k}(N, N \backslash L)$, o homomorfismo $\check{f}_{k}^{*}$ leva $\llbracket \mu_{L} \rrbracket \in \check{\mathrm{H}}_{c}^{k}(N, B)$ na classe $\llbracket f_{L}^{*}\left(\mu_{L}\right) \rrbracket \in \check{\mathrm{H}}_{c}^{k}(M, A)$.

Proposição 1.4.3 . Sejam $M, M^{\prime}$ e $M^{\prime \prime}$ variedades topológicas de dimensão $n$, e considere subconjuntos localmente compactos $A \subseteq M, A^{\prime} \subseteq M^{\prime}$ e $A^{\prime \prime} \subseteq M^{\prime \prime}$. Se $f:(M, A) \longrightarrow\left(M^{\prime}, A^{\prime}\right)$ e $g:\left(M^{\prime}, A^{\prime}\right) \longrightarrow\left(M^{\prime \prime}, A^{\prime \prime}\right)$ são aplicações contínuas próprias, então (lembrando que a composta de duas aplicações próprias também é própria) temos que:

$$
(\widetilde{g \circ f})_{k}^{*}=\check{f}_{k}^{*} \circ \check{g}_{k}^{*} .
$$


DemonstraçÃo. De fato, sejam $\Lambda$ a família dos conjuntos compactos $K \subseteq M$ tais que $A \subseteq M \backslash K, \Lambda^{\prime}$ a família dos compactos $K^{\prime} \subseteq M^{\prime}$ tais que $A^{\prime} \subseteq M^{\prime} \backslash K^{\prime}$, e $\Lambda^{\prime \prime}$ a família dos compactos $K^{\prime \prime} \subseteq M^{\prime \prime}$ tais que $A^{\prime \prime} \subseteq M^{\prime \prime} \backslash K^{\prime \prime}$, todas dirigidas pela relação de inclusão direta. Conforme observamos na definição 1.4.2, temos que $f^{-1}\left(K^{\prime}\right) \in \Lambda$ e $g^{-1}\left(K^{\prime \prime}\right) \in \Lambda^{\prime}$ para quaisquer $K^{\prime} \in \Lambda^{\prime}$ e $K^{\prime \prime} \in \Lambda^{\prime \prime}$; sejam, pois, $\phi: \Lambda^{\prime} \longrightarrow \Lambda$ e $\phi^{\prime}: \Lambda^{\prime \prime} \longrightarrow \Lambda^{\prime}$ as aplicações dadas por $\phi\left(K^{\prime}\right)=f^{-1}\left(K^{\prime}\right)$ e $\phi^{\prime}\left(K^{\prime \prime}\right)=g^{-1}\left(K^{\prime \prime}\right)$, e, para cada par $K^{\prime} \in \Lambda^{\prime}$ e $K^{\prime \prime} \in \Lambda^{\prime \prime}$, sejam $f_{K^{\prime}}^{*}: H^{k}\left(M^{\prime}, M^{\prime} \backslash K^{\prime}\right) \longrightarrow \mathrm{H}^{k}\left(M, M \backslash f^{-1}\left(K^{\prime}\right)\right)$ e $g_{K^{\prime \prime}}^{*}: \mathrm{H}^{k}\left(M^{\prime \prime}, M^{\prime \prime} \backslash K^{\prime \prime}\right) \longrightarrow \mathrm{H}^{k}\left(M^{\prime}, M^{\prime} \backslash\right.$ $\left.g^{-1}\left(K^{\prime \prime}\right)\right)$ os homomorfismos em cohomologia singular induzidos pelas restrições $f_{K^{\prime}}$ : $\left(M, M \backslash f^{-1}\left(K^{\prime}\right)\right) \longrightarrow\left(M^{\prime}, M^{\prime} \backslash K^{\prime}\right)$ e $g_{K^{\prime \prime}}:\left(M^{\prime}, M^{\prime} \backslash g^{-1}\left(K^{\prime \prime}\right)\right) \longrightarrow\left(M^{\prime \prime}, M^{\prime \prime} \backslash K^{\prime \prime}\right)$, de $f$ e $g$, respectivamente. Por definiç̧ão, temos então que $f_{k}^{*}(\llbracket x \rrbracket)=\llbracket f_{K^{\prime}}^{*}(x) \rrbracket$ e $\dot{g}_{k}^{*}(\llbracket y \rrbracket)=\llbracket g_{K^{\prime \prime}}^{*}(y) \rrbracket$, para quaisquer $x \in \mathrm{H}^{k}\left(M^{\prime}, M^{\prime} \backslash K^{\prime}\right)$ e $y \in \mathrm{H}^{k}\left(M^{\prime \prime}, M^{\prime \prime} \backslash K^{\prime \prime}\right)$, com $K^{\prime} \in \Lambda^{\prime}$ e $K^{\prime \prime} \in \Lambda^{\prime \prime}$. Por outro lado, como $(g \circ f)^{-1}\left(K^{\prime \prime}\right) \in \Lambda$ para todo $K^{\prime \prime} \in \Lambda^{\prime \prime}$, segue que a aplicação $\psi: \Lambda^{\prime \prime} \longrightarrow \Lambda$, dada por $\psi\left(K^{\prime \prime}\right)=(g \circ f)^{-1}\left(K^{\prime \prime}\right)$, está bem definida; assim, para cada $K^{\prime \prime} \in \Lambda^{\prime \prime}$, seja $(g \circ f)_{K^{\prime \prime}}^{*}: \mathrm{H}^{k}\left(M^{\prime \prime}, M^{\prime \prime} \backslash K^{\prime \prime}\right) \longrightarrow \mathrm{H}^{k}\left(M, M \backslash(g \circ f)^{-1}\left(K^{\prime \prime}\right)\right)$ o homomorfismo induzido pela restrição $(g \circ f)_{K^{\prime \prime}}:\left(M, M \backslash(g \circ f)^{-1}\left(K^{\prime \prime}\right)\right) \longrightarrow\left(M^{\prime \prime}, M^{\prime \prime} \backslash K^{\prime \prime}\right)$, de $g \circ f$ a $M \backslash(g \circ f)^{-1}\left(K^{\prime \prime}\right)$. Dado $y \in \mathrm{H}^{k}\left(M^{\prime \prime}, M^{\prime \prime} \backslash K^{\prime \prime}\right)$, com $K^{\prime \prime} \in \Lambda^{\prime \prime}$, sabemos que:

$$
(\overline{g \circ f})_{k}^{*}(\llbracket y \rrbracket)=\llbracket(g \circ f)_{K^{\prime}}^{*}(y) \rrbracket .
$$

Agora, observe que, para todo $K^{\prime \prime} \in \Lambda^{\prime \prime}$, temos $(g \circ f)_{K^{\prime \prime}}^{*}=f_{g^{-1}\left(K^{\prime \prime}\right)}^{*} \circ g_{K^{\prime \prime}}^{*}$, conforme ilustra o diagrama abaixo:

$$
\mathrm{H}^{k}\left(M^{\prime \prime}, M^{\prime \prime} \backslash K^{\prime \prime}\right) \stackrel{g_{K^{\prime \prime}}^{*}}{\longrightarrow} \mathrm{H}^{k}\left(M^{\prime}, M^{\prime} \backslash g^{-1}\left(K^{\prime \prime}\right)\right) \stackrel{f_{g^{-1}\left(K^{\prime \prime}\right)}}{\longrightarrow} \underbrace{\mathrm{H}^{k}\left(M, M \backslash f^{-1}\left(g^{-1}\left(K^{\prime \prime}\right)\right)\right)}_{\mathrm{H}^{k}\left(M, M \backslash(g \circ f)^{-1}\left(K^{\prime \prime}\right)\right)}
$$

Portanto, para todo $y \in \mathrm{H}^{k}\left(M^{\prime \prime}, M^{\prime \prime} \backslash K^{\prime \prime}\right)$, com $K^{\prime \prime} \in \Lambda^{\prime \prime}$, temos que:

$$
\begin{aligned}
(\widetilde{g \circ f})_{k}^{*}(\llbracket y \rrbracket) & =\llbracket(g \circ f)_{K^{\prime}}^{*}(y) \rrbracket=\llbracket\left(f_{g^{-1}\left(K^{\prime \prime}\right)}^{*} \circ g_{K^{\prime \prime}}^{*}\right)(y) \rrbracket=\llbracket f_{g^{-1}\left(K^{\prime \prime}\right)}^{*}\left(g_{K^{\prime \prime}}^{*}(y)\right) \rrbracket \\
& =\tilde{f}_{k}^{*}\left(\llbracket g_{K^{\prime \prime}}^{*}(y) \rrbracket\right)=\tilde{f}_{k}^{*}\left(\check{g}_{k}^{*}(\llbracket y \rrbracket)\right)=\left(\tilde{f}_{k}^{*} \circ \check{g}_{k}^{*}\right)(\llbracket y \rrbracket),
\end{aligned}
$$

d'onde concluímos que $(\widetilde{g \circ f})_{k}^{*}=\check{f_{k}^{*}} \circ \check{g}_{k^{\prime}}^{*}$ como queríamos provar.

Proposição 1.4.4. Sejam Me N variedades topológicas de dimensão n, e considere subconjuntos localmente compactos $A \subseteq M e B \subseteq M$. Dadas aplicações contínuas próprias $f, g:(M, A) \longrightarrow$ $(N, B)$, suponha que $h:(M \times I, A \times I) \longrightarrow(N, B)$ seja uma homotopia própria entre $f$ e $g$ (isto é, h é uma aplicação contínua própria tal que $h(x, 0)=f(x)$ e $h(x, 1)=g(x)$, para todo $x \in M)$. Neste caso, sendo $\check{f}_{k}^{*}, \check{g}_{k}^{*}: \check{\mathrm{H}}_{c}^{k}(N, B) \longrightarrow \check{\mathrm{H}}_{c}^{k}(M, A)$ os homomorfismos induzidos por $f$ e g, respectivamente, temos que $\check{f}_{k}^{*}=\check{g}_{k}^{*}$ (em outras palavras, os homomorfismos induzidos em cohomologia de Čech com suporte compacto são invariantes por homotopias próprias). 
DemonstraÇÃo. Sejam $u, v:(M, A) \longrightarrow(M \times I, A \times I)$ as aplicações definidas por $u(x)=(x, 0)$ e $v(x)=(x, 1)$, para cada $x \in M$; como todo subconjunto compacto de $M \times I$ é da forma $K \times J$, onde $K \subseteq M$ é compacto e $J \subseteq I$ é fechado, e como

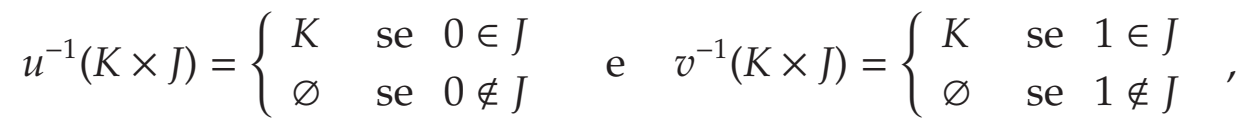

segue que $u$ e $v$ são aplicações próprias. Observe então que $(h \circ u)(x)=h(u(x))=$ $h(x, 0)=f(x)$ e $(h \circ v)(x)=h(v(x))=h(x, 1)=g(x)$, para todo $x \in M$, isto é, $h \circ u=f$ e $h \circ v=g$. Agora, como $h:(M \times I, A \times I) \longrightarrow(N, B)$ é própria, segue que o homomorfismo $\check{h}_{k}^{*}: \check{\mathrm{H}}_{c}^{k}(N, B) \longrightarrow \check{\mathrm{H}}_{c}^{k}(M \times I, A \times I)$ está bem definido; logo, da proposição anterior, temos que $\check{f}_{k}^{*}=\check{u}_{k}^{*} \circ \check{h}_{k}^{*}$ e $\check{g}_{k}^{*}=\check{v}_{k}^{*} \circ \check{h}_{k}^{*}$. Deste modo, para provarmos que $\check{f}_{k}^{*}=\check{g}_{k}^{*}$, basta mostrarmos que $\breve{u}_{k}^{*}=\check{v}_{k}^{*}$.

Com efeito, sejam $\Omega$ a família dos subconjuntos compactos $K \times I \subseteq M \times I$ tais que $A \times I \subseteq(M \times I) \backslash(K \times I)$, e $\Lambda$ a família dos compactos $K \subseteq M$ tais que $A \subseteq M \backslash K$, ambas dirigidas pela relação de inclusão direta. Como $u^{-1}(K \times I), v^{-1}(K \times I) \in \Lambda$ para todo $K \times I \in \Omega$ (pois $u$ e $v$ são próprias), segue que as aplicações $\phi, \psi: \Omega \longrightarrow \Lambda$, dadas por $\phi(K \times I)=u^{-1}(K \times I)$ e $\psi(K \times I)=v^{-1}(K \times I)$, estão bem definidas; além disso, como $u^{-1}(K \times I)=K=v^{-1}(K \times I)$, temos que $\phi=\psi$. Isto posto, para cada $K \times I \in \Omega$, sejam $u_{K}^{*}: \mathrm{H}^{k}(M \times I,(M \times I) \backslash(K \times I)) \longrightarrow \mathrm{H}^{k}(M, M \backslash K)=\mathrm{H}^{k}(M, M \backslash \phi(K \times I))$ e $v_{K}^{*}: \mathrm{H}^{k}(M \times I,(M \times I) \backslash(K \times I)) \longrightarrow \mathrm{H}^{k}(M, M \backslash K)=\mathrm{H}^{k}(M, M \backslash \psi(K \times I))$ os homomorfismos em cohomologia singular induzidos pelas restrições $u_{K}:(M, M \backslash K) \longrightarrow(M \times I,(M \times I) \backslash$ $(K \times I))$ e $v_{K}:(M, M \backslash K) \longrightarrow(M \times I,(M \times I) \backslash(K \times I))$, de $u$ e $v$ a $M \backslash K$, respectivamente, e considere a aplicação contínua $h_{K}:(M \times I,(M \backslash K) \times I) \longrightarrow(M \times I,(M \times I) \backslash(K \times I))$ dada por $h_{K}(x, t)=(x, t)$, onde $(x, t) \in M \times I$. Como $h_{K}(x, 0)=(x, 0)=u_{K}(x)$ e $h_{K}(x, 1)=(x, 1)=v_{K}(x)$ para todo $x \in M$, segue que $h_{K}$ é uma homotopia entre $u_{K}$ e $v_{K}$, o que implica que $u_{K}^{*}=v_{K}^{*}$. Portanto, as aplicações $\Phi=\left\{\phi, u_{K}^{*}\right\}_{K \times I \in \Omega}$ e $\Psi=\left\{\psi, v_{K}^{*}\right\}_{K \times I \in \Omega}$ (entre os sistemas diretos associados às famílias $\left\{\mathrm{H}^{k}(M \times I,(M \times I) \backslash(K \times I))\right\}_{K \times I \in \Omega}$ e $\left.\left\{\mathrm{H}^{k}(M, M \backslash K)\right\}_{K \in \Lambda}\right)$ são iguais, e, conseqüentemente, os limites diretos $\check{u}_{k}^{*}=\underset{\Phi}{\rightarrow}$ e $\check{v}_{k}^{*}=\underset{\Psi}{\rightarrow}$ também o são.

Agora, considere uma variedade topológica $M$, de dimensão $n$, conexa, orientável, e sem fronteira; sendo $\Lambda_{0}$ a família de todos os subconjuntos compactos e conexos de $M$, dirigida pela relação de inclusão inversa, sabemos que:

$$
\check{\mathrm{H}}_{c}^{n} \simeq \underset{\lim }{\longrightarrow}\left\{\mathrm{H}^{n}(M, M \backslash K): K \in \Lambda_{0}\right\}
$$

(pois $\Lambda_{0}$ é cofinal em relação à família de todos os subconjuntos compactos de $M$ ). Isto posto, dado $K \in \Lambda_{0}$, seja $\theta_{K} \in \mathrm{H}_{n}(M, M \backslash K)$ a classe fundamental em $K$. Como $M$ é orientável e $K \subseteq M$ é compacto e conexo, temos que $\mathrm{H}_{n}(M, M \backslash K) \simeq \mathbb{Z} \mathrm{eH}_{n-1}(M, M \backslash K) \simeq$ $\{0\}$ (vide [D], corolários 3.4 e 3.5, páginas 260 e 261); daí, segue que $\operatorname{Hom}\left(\mathrm{H}_{n}(M, M \backslash\right.$ 
$K), \mathbb{Z}) \simeq \operatorname{Hom}(\mathbb{Z}, \mathbb{Z}) \simeq \mathbb{Z} \operatorname{e} \operatorname{Ext}\left(\mathrm{H}_{n-1}(M, M \backslash K), \mathbb{Z}\right) \simeq \operatorname{Ext}(\{0\}, \mathbb{Z}) \simeq\{0\}$. Assim, seja $\kappa: \mathrm{H}^{n}(M, M \backslash K) \longrightarrow \operatorname{Hom}\left(\mathrm{H}_{n}(M, M \backslash K), \mathbb{Z}\right)$ a aplicação de Kronecker (vide [Mu], página 276), que, a cada $[\omega] \in \mathrm{H}^{n}(M, M \backslash K)$, associa o homomorfismo $h_{\omega}: \mathrm{H}_{n}(M, M \backslash K) \longrightarrow \mathbb{Z}$ dado por $h_{\omega}([\alpha])=\langle\omega, \alpha\rangle$, onde $[\alpha] \in \mathrm{H}_{n}(M, M \backslash K)$; do teorema dos coeficientes universais (vide [Vi], teorema 3.14, página 84 ), sabemos que existe uma seqüência exata

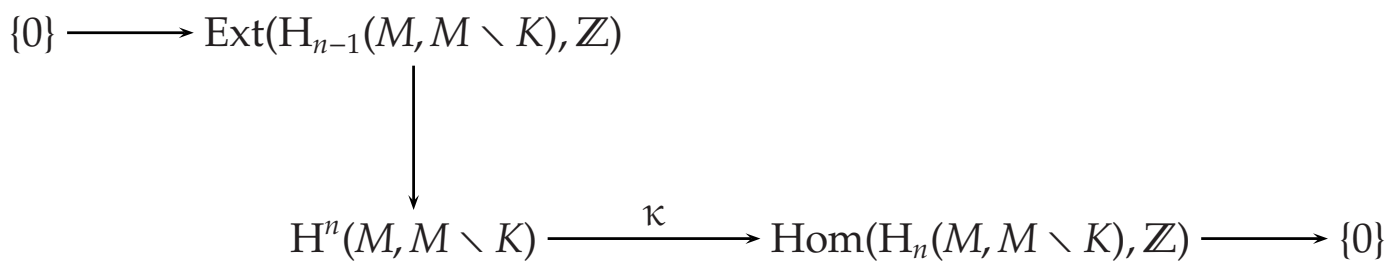

Ora, como $\operatorname{Ext}\left(\mathrm{H}_{n-1}(M, M \backslash K), \mathbb{Z}\right) \simeq\{0\}$, a exatidão desta seqüência nos garante que $\kappa$ é um isomorfismo. Portanto, sendo $\varphi_{K}: \mathrm{H}_{n}(M, M \backslash K) \longrightarrow \mathbb{Z}$ o isomorfismo que leva o gerador $\theta_{K} \in \mathrm{H}_{n}(M, M \backslash K) \simeq \mathbb{Z}$ em $1 \in \mathbb{Z}$, segue que existe uma única classe $\mu_{K} \in \mathrm{H}^{n}(M, M \backslash K)$ tal que $\kappa\left(\mu_{K}\right)=\varphi_{K}$. Observe que, como $\varphi_{K}$ é um gerador de $\operatorname{Hom}\left(\mathrm{H}_{n}(M, M \backslash K), \mathbb{Z}\right) \simeq \mathbb{Z}$ e $\kappa$ é um isomorfismo, temos que $\mu_{K}$ é um dos dois geradores de $\mathrm{H}^{n}(M, M \backslash K) \simeq \mathbb{Z}$.

Note então que $\mathrm{H}^{n}(M, M \backslash K)$ e $\mathrm{H}_{n}(M, M \backslash K)$ são isomorfos (uma vez que $\mathrm{H}^{n}(M, M \backslash$ $K) \simeq \mathbb{Z}$ e $\left.\mathrm{H}_{n}(M, M \backslash K) \simeq \mathbb{Z}\right)$; seja, pois, $\psi_{K}: \mathrm{H}^{n}(M, M \backslash K) \longrightarrow \mathrm{H}_{n}(M, M \backslash K)$ o isomorfismo que leva o gerador $\mu_{K} \in \mathrm{H}^{n}(M, M \backslash K)$ no gerador $\theta_{K} \in \mathrm{H}_{n}(M, M \backslash K)$. Como $\varphi_{K}: \mathrm{H}_{n}(M, M \backslash K) \longrightarrow \mathbb{Z}$ é o isomorfismo tal que $\varphi_{K}\left(\theta_{K}\right)=1$, segue que a aplicação $\phi_{K}: \mathrm{H}^{n}(M, M \backslash K) \longrightarrow \mathbb{Z}$, dada por $\phi_{K}=\varphi_{K} \circ \psi_{K}$, é o isomorfismo que leva $\mu_{K}$ em 1, conforme ilustra o diagrama abaixo:

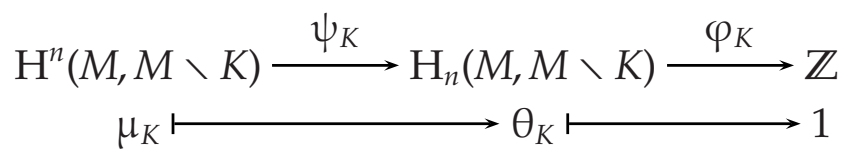

Assim, passando ao limite direto, obtemos um isomorfismo

$$
\stackrel{\Phi}{\rightarrow} \underset{\lim }{\longrightarrow}\left\{\mathrm{H}^{n}(M, M \backslash K): K \in \Lambda_{0}\right\} \longrightarrow \mathbb{Z}
$$

que, para qualquer que seja $K \in \Lambda_{0}$, leva a classe $\llbracket \mu_{K} \rrbracket \in \underset{\lim }{\longrightarrow}\left\{\mathrm{H}^{n}(M, M \backslash K): K \in \Lambda_{0}\right\}$ em $\phi_{K}\left(\mu_{K}\right)=1 \in \mathbb{Z}$; ora, se cada $\llbracket \mu_{K} \rrbracket, K \in \Lambda_{0}$, é levado no gerador $1 \in \mathbb{Z}$, e $\Phi$ é um isomorfismo, temos que $\llbracket \mu_{K} \rrbracket$ é um gerador de $\underset{\longrightarrow}{\lim }\left\{\mathrm{H}^{n}(M, M \backslash K): K \in \Lambda_{0}\right\}$ (qualquer que seja $\left.K \in \Lambda_{0}\right)$, o qual, por sua vez, corresponde a um gerador $\llbracket M \rrbracket \in \check{\mathrm{H}}_{c}^{n}(M)$ pelo isomorfismo $\check{\mathrm{H}}_{c}^{n}(M) \simeq \underset{\lim }{\longrightarrow}\left\{\mathrm{H}^{n}(M, M \backslash K): K \in \Lambda_{0}\right\}$ (note que, pela proposição 1.4.2, temos $\left.\check{\mathrm{H}}_{c}^{n}(M) \simeq \mathbb{Z}\right)$. Deste modo, podemos introduzir a seguinte 
Definição 1.4.3 . O gerador $\llbracket M \rrbracket \in \check{\mathrm{H}}_{c}^{n}(M) \simeq \mathbb{Z}$ obtido acima é denominado classe fundamental (em cohomologia de Čech com suporte compacto) de $M$.

Finalmente, com base nos resultados apresentados, podemos definir o conceito de grau cohomológico.

Definição 1.4.4 . Sejam $M$ e $N$ variedades topológicas conexas de dimensão n, e considere os isomorfismos

$$
\check{\varphi}_{M}: \check{\mathrm{H}}_{c}^{n}(M, \partial M) \longrightarrow \check{\mathrm{H}}_{c}^{n}(\operatorname{Int} M) \text { e } \check{\varphi}_{N}: \check{\mathrm{H}}_{c}^{n}(N, \partial N) \longrightarrow \check{\mathrm{H}}_{c}^{n}(\operatorname{Int} N),
$$

dados pela proposição 1.4.1. Supondo $M$ e $N$ orientáveis, segue que existe uma classe $\llbracket M \rrbracket \in$ $\check{\mathrm{H}}_{c}^{n}(M, \partial M)$, correspondente à classe fundamental $\llbracket \operatorname{Int} M \rrbracket \in \widetilde{\mathrm{H}}_{c}^{n}(\operatorname{Int} M)$ pelo isomorfismo $\check{\varphi}_{M}$, bem como existe uma classe $\llbracket N \rrbracket \in \check{\mathrm{H}}_{c}^{n}(N, \partial N)$, correspondente à classe fundamental $\llbracket \operatorname{Int} N \rrbracket \in \check{\mathrm{H}}_{c}^{n}(\operatorname{Int} N)$ pelo isomorfismo $\check{\varphi}_{N}$. Como $\operatorname{Int} M \rrbracket e \llbracket \operatorname{Int} N \rrbracket$ são geradores de $\check{\mathrm{H}}_{c}^{n}(\operatorname{Int} M)$ e $\tilde{\mathrm{H}}_{c}^{n}(\operatorname{Int} N)$, respectivamente, temos que $\llbracket M \rrbracket e \llbracket N \rrbracket$ são geradores dos respectivos grupos $\widetilde{\mathrm{H}}_{c}^{n}(M, \partial M)$ e $\overline{\mathrm{H}}_{c}^{n}(N, \partial N)$. Logo, dada uma aplicação contínua própria $f:(M, \partial M) \longrightarrow$ $(N, \partial N)$, e sendo $\widetilde{f}_{n}^{*}: \check{\mathrm{H}}_{c}^{n}(N, \partial N) \longrightarrow \check{\mathrm{H}}_{c}^{n}(M, \partial M)$ o homomorfismo em cohomologia de Čech com suporte compacto induzido por $f$, segue que existe $k \in \mathbb{Z}$ tal que $\check{f_{n}^{*}}(\llbracket N \rrbracket)=k \cdot \llbracket M \rrbracket$. A este inteiro $k$, denominamos grau cohomológico de $f$, e o denotamos por $\operatorname{deg}(f)$. Observe que, tomando-se coeficientes em $\mathbb{Z}_{2}$, as variedades $M$ e $N$ tornam-se sempre orientáveis; neste caso, procedendo de maneira análoga ao que fizemos acima, obtemos classes $\llbracket M \rrbracket \in \check{\mathrm{H}}_{c}^{n}\left(M, \partial M ; \mathbb{Z}_{2}\right) e \llbracket N \rrbracket \in$ $\check{\mathrm{H}}_{c}^{n}\left(N, \partial N ; \mathbb{Z}_{2}\right)$, que constituem geradores destes grupos, e definimos o grau cohomológico mod 2, denotado por $\operatorname{deg}(f, 2)$, como sendo o número inteiro tal que $\tilde{f}_{n}^{*}(\llbracket N \rrbracket)=\operatorname{deg}(f, 2) \cdot \llbracket M \rrbracket$.

Como conseqüência imediata da proposição 1.4.4, temos a seguinte

Proposição 1.4.5. Sejam $M$ e N variedades topológicas conexas de dimensão n, e considere duas aplicações contínuas próprias $f, g:(M, \partial M) \longrightarrow(N, \partial N)$. Se $h:(M \times I, \partial M \times I) \longrightarrow(N, \partial N)$ é uma homotopia própria entre $f$ e $g$, então $\operatorname{deg}(f)=\operatorname{deg}(g)$ se $M$ e $N$ forem orientáveis, ou $\operatorname{deg}(f, 2)=\operatorname{deg}(g, 2)$ caso contrário (em outras palavras, o grau cohomológico é invariante por homotopias próprias).

Proposição 1.4.6 . Sejam $M, M^{\prime}$ e $M^{\prime \prime}$ variedades topológicas de dimensão $n$, conexas $e$ orientáveis. Se $f:\left(M^{\prime \prime}, \partial M^{\prime \prime}\right) \longrightarrow\left(M^{\prime}, \partial M^{\prime}\right)$ e $g:\left(M^{\prime}, \partial M^{\prime}\right) \longrightarrow(M, \partial M)$ são aplicações contínuas próprias, então $\operatorname{deg}(g \circ f)=\operatorname{deg}(g) \cdot \operatorname{deg}(f)$.

Demonstração. De fato, sejam

$$
\check{\varphi}: \check{\mathrm{H}}_{c}^{n}(M, \partial M) \longrightarrow \check{\mathrm{H}}_{c}^{n}(\operatorname{Int} M),
$$




$$
\begin{gathered}
\check{\varphi}^{\prime}: \check{\mathrm{H}}_{c}^{n}\left(M^{\prime}, \partial M^{\prime}\right) \longrightarrow \check{\mathrm{H}}_{c}^{n}\left(\operatorname{Int} M^{\prime}\right) \mathrm{e} \\
\check{\varphi}^{\prime \prime}: \check{\mathrm{H}}_{c}^{n}\left(M^{\prime \prime}, \operatorname{Int} M^{\prime \prime}\right) \longrightarrow \check{\mathrm{H}}_{c}^{n}\left(\operatorname{Int} M^{\prime \prime}\right)
\end{gathered}
$$

os isomorfismos dados pela proposição 1.4.1, e considere as classes $\llbracket M \rrbracket \in \check{\mathrm{H}}_{c}^{n}(M, \partial M)$, $\llbracket M^{\prime} \rrbracket \in \check{\mathrm{H}}_{c}^{n}\left(M^{\prime}, \partial M^{\prime}\right)$ e $\llbracket M^{\prime \prime} \rrbracket \in \widetilde{\mathrm{H}}_{c}^{n}\left(M^{\prime \prime}, \partial M^{\prime \prime}\right)$ correspondentes às classes fundamentais $\llbracket \operatorname{Int} M \rrbracket \in \check{\mathrm{H}}_{c}^{n}(\operatorname{Int} M), \llbracket \operatorname{Int} M^{\prime} \rrbracket \in \check{\mathrm{H}}_{c}^{n}\left(\operatorname{Int} M^{\prime}\right)$ e $\llbracket \operatorname{Int} M^{\prime \prime} \rrbracket \in \check{\mathrm{H}}_{c}^{n}\left(\operatorname{Int} M^{\prime \prime}\right)$ pelos isomorfismos $\check{\varphi}, \check{\varphi}^{\prime}$ e $\check{\varphi}^{\prime \prime}$, respectivamente. Pela definição 1.4.4, sabemos que os graus cohomológicos $\operatorname{deg}(f), \operatorname{deg}(g)$ e $\operatorname{deg}(g \circ f)$ são os números inteiros tais que:

$$
\check{f_{n}^{*}}\left(\llbracket M^{\prime} \rrbracket\right)=\operatorname{deg}(f) \cdot \llbracket M^{\prime \prime} \rrbracket, \check{g}_{n}^{*}(\llbracket M \rrbracket)=\operatorname{deg}(g) \cdot \llbracket M^{\prime} \rrbracket
$$

e

$$
(\overline{g \circ f})_{n}^{*}(\llbracket M \rrbracket)=\operatorname{deg}(g \circ f) \cdot \llbracket M^{\prime \prime} \rrbracket,
$$

onde $\widetilde{f}_{n}^{*}: \check{\mathrm{H}}_{c}^{n}\left(M^{\prime}, \partial M^{\prime}\right) \longrightarrow \check{\mathrm{H}}_{c}^{n}\left(M^{\prime \prime}, \partial M^{\prime \prime}\right), \check{g}_{n}^{*}: \check{\mathrm{H}}_{c}^{n}(M, \partial M) \longrightarrow \check{\mathrm{H}}_{c}^{n}\left(M^{\prime}, \partial M^{\prime}\right)$ e $(\overline{g \circ f})_{n}^{*}:$ $\check{\mathrm{H}}_{c}^{n}(M, \partial M) \longrightarrow \check{\mathrm{H}}_{c}^{n}\left(M^{\prime \prime}, \partial M^{\prime \prime}\right)$ são os homomorfismos em cohomologia de Čech com suporte compacto induzidos por $f, g$ e $g \circ f$, respectivamente (note que a composta de duas aplicações próprias também é própria). Logo, da proposição 1.4.3, segue que:

$$
\begin{aligned}
\operatorname{deg}(g \circ f) \cdot \llbracket M^{\prime \prime} \rrbracket & =\widetilde{(g \circ f})_{n}^{*}(\llbracket M \rrbracket)=\left(\check{f}_{n}^{*} \circ \check{g}_{n}^{*}\right)(\llbracket M \rrbracket)=\check{f_{n}^{*}}\left(\operatorname{deg}(g) \cdot \llbracket M^{\prime} \rrbracket\right) \\
& =\operatorname{deg}(g) \cdot \widetilde{f_{n}^{*}}\left(\llbracket M^{\prime} \rrbracket\right)=\operatorname{deg}(g) \cdot \operatorname{deg}(f) \cdot \llbracket M^{\prime \prime} \rrbracket .
\end{aligned}
$$

Daí, concluímos que $\operatorname{deg}(g \circ f)=\operatorname{deg}(g) \cdot \operatorname{deg}(f)$, conforme queríamos mostrar.

Agora, vamos mostrar a relação entre o grau cohomológico e o grau local, sendo que, para tanto, necessitamos de alguns resultados preliminares. Seja, pois, $M$ uma variedade topológica de dimensão $n$ (não necessariamente conexa), orientável e sem fronteira; por definição, temos que:

$$
\check{\mathrm{H}}_{c}^{n}(M)=\underline{\lim }\left\{\mathrm{H}^{n}(M, M \backslash K): K \in \Lambda\right\},
$$

onde $\Lambda$ é a família de todos os subconjuntos compactos de $M$, dirigida pela relação de inclusão direta. Então, dado $K \in \Lambda$, e sendo $\theta_{K} \in \mathrm{H}_{n}(M, M \backslash K)$ a classe fundamental em $K$, considere o homomorfismo $D_{K}: \mathrm{H}^{n}(M, M \backslash K) \longrightarrow \mathrm{H}_{0}(M)$, dado por $D_{K}(x)=x \cap \theta_{K}$ para cada $x \in \mathrm{H}^{n}\left(M, M \backslash K\right.$ ) (onde $\cap$ denota o produto cap); dados $K_{1}, K_{2} \in \Lambda$, com $K_{1} \leqslant K_{2}$, e sendo $j_{K_{1}, K_{2}}^{*}: \mathrm{H}^{n}\left(M, M \backslash K_{1}\right) \longrightarrow \mathrm{H}^{n}\left(M, M \backslash K_{2}\right)$ o homomorfismo induzido pela inclusão $M \backslash K_{2} \subseteq M \backslash K_{1}$, segue (da naturalidade do produto cap) que o diagrama abaixo é comutativo: 


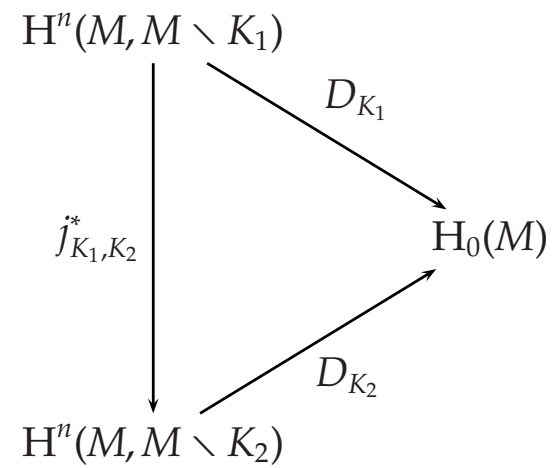

Logo, passando ao limite direto, obtemos um homomorfismo

$$
\check{\mathrm{D}}_{M}: \check{\mathrm{H}}_{c}^{n}(M)=\underline{\lim }\left\{\mathrm{H}^{n}(M, M \backslash K): K \in \Lambda\right\} \longrightarrow \mathrm{H}_{0}(M),
$$

o qual é definido por $\check{\mathrm{D}}_{M}(\llbracket x \rrbracket)=x \cap \theta_{K}$, para cada $x \in \mathrm{H}^{n}(M, M \backslash K)$, com $K \in \Lambda$. A respeito deste homomorfismo, temos então o seguinte teorema (cuja demonstração pode ser encontrada em [Ma2], páginas 360 a 364):

Teorema 1.4.1 (Dualidade de Poincaré). Sendo M uma variedade topológica de dimensão $n$ (não necessariamente conexa), orientável e sem fronteira, a aplicação $\check{\mathrm{D}}_{M}: \breve{\mathrm{H}}_{c}^{n}(M) \longrightarrow \mathrm{H}_{0}(M)$, dada por $\check{\mathrm{D}}_{M}(\llbracket x \rrbracket)=x \cap \theta_{K}$ para cada $x \in \mathrm{H}^{n}(M, M \backslash K)$, onde $K \subseteq M$ é compacto (e $\theta_{K} \in \mathrm{H}_{n}(M, M \backslash K)$ é a classe fundamental em $\left.K\right)$, é um isomorfismo.

Definição 1.4.5 . A aplicação $\breve{\mathrm{D}}_{M}: \breve{\mathrm{H}}_{c}^{n}(M) \longrightarrow \mathrm{H}_{0}(M)$, dada no teorema anterior, é denominada isomorfismo de Poincaré relativo a $M$.

Isto posto, podemos introduzir a seguinte

Definição 1.4.6. Sejam $M e N$ variedades topológicas de dimensão n, orientáveis e sem fronteira, e considere uma aplicação própria $f: M \longrightarrow N$. Denotando por $\check{\mathrm{D}}_{M}: \breve{\mathrm{H}}_{c}^{n}(M) \longrightarrow \mathrm{H}_{0}(M)$ $e \check{\mathrm{D}}_{N}: \breve{\mathrm{H}}_{c}^{n}(N) \longrightarrow \mathrm{H}_{0}(N)$ os isomorfismos de Poincaré relativos a $M$ e $N$, respectivamente, e sendo $\tilde{f}_{n}^{*}: \breve{\mathrm{H}}_{c}^{n}(N) \longrightarrow \breve{\mathrm{H}}_{c}^{n}(M)$ o homomorfismo (em cohomologia de Čech com suporte compacto) induzido por $f$, definimos o homomorfismo transfer em homologia (associado a f) como sendo o homomorfismo $f_{!}: \mathrm{H}_{0}(N) \longrightarrow \mathrm{H}_{0}(M)$ obtido pela seguinte composição:

$$
\mathrm{H}_{0}(N) \stackrel{\check{\mathrm{D}}_{N}^{-1}}{\longrightarrow} \check{\mathrm{H}}_{c}^{n}(N) \stackrel{\check{f}_{n}^{*}}{\longrightarrow} \check{\mathrm{H}}_{c}^{n}(M) \stackrel{\check{\mathrm{D}}_{M}}{\longrightarrow} \mathrm{H}_{0}(M)
$$

(isto é, $f_{!}=\check{\mathrm{D}}_{M} \circ \check{f_{n}^{*}} \circ \check{\mathrm{D}}_{\mathrm{N}}^{-1}$ ). Por outro lado, sendo $f_{*}: \mathrm{H}_{0}(M) \longrightarrow \mathrm{H}_{0}(N)$ o homomorfismo em homologia singular induzido por $f$, denominamos homomorfismo transfer em cohomologia (associado a f) ao homomorfismo $f^{!}: \check{\mathrm{H}}_{c}^{n}(M) \longrightarrow \breve{\mathrm{H}}_{c}^{n}(N)$ dado pela seguinte composição: 


$$
\check{\mathrm{H}}_{c}^{n}(M) \stackrel{\check{\mathrm{D}}_{M}}{\longrightarrow} \mathrm{H}_{0}(M) \stackrel{f_{*}}{\longrightarrow} \mathrm{H}_{0}(N) \stackrel{\check{\mathrm{D}}_{N}^{-1}}{\longrightarrow} \check{\mathrm{H}}_{c}^{n}(N)
$$

(isto é, $f^{!}=\check{\mathrm{D}}_{N}^{-1} \circ f_{*} \circ \check{\mathrm{D}}_{M}$ ).

A proposição abaixo, mostra-nos que o homomorfismo transfer em homologia está diretamente relacionado ao grau local.

Proposição 1.4.7 . Sejam $M$ e $N$ variedades topológicas de dimensão $n$, orientáveis e sem fronteira. Se $f: M \longrightarrow N$ é uma aplicação contínua própria, e $N$ é conexa, segue que o grau local $\operatorname{deg}_{K}(f)$ assume o mesmo valor $r \in \mathbb{Z}$ em todo subconjunto compacto e conexo $K \subseteq N$ (vide proposição 1.3.4); conseqüentemente, a composição

$$
\mathrm{H}_{0}(N) \stackrel{f_{!}}{\longrightarrow} \mathrm{H}_{0}(M) \stackrel{f_{*}}{\longrightarrow} \mathrm{H}_{0}(N)
$$

é tal que $\left(f_{*} \circ f_{!}\right)(x)=r \cdot x$, para cada $x \in \mathrm{H}_{0}(N)$.

DemonstraçÃo. Com efeito, dado $x \in \mathrm{H}_{0}(N)$, sabemos que existe $\llbracket y \rrbracket \in \check{\mathrm{H}}_{c}^{n}(N)$, digamos $y \in \mathrm{H}^{n}(N, N \backslash L)$ com $L \subseteq N$ compacto, satisfazendo $\check{\mathrm{D}}_{N}(\llbracket y \rrbracket)=x=y \cap \theta_{L}$, onde $\check{\mathrm{D}}_{N}: \check{\mathrm{H}}_{c}^{n}(N) \longrightarrow \mathrm{H}_{0}(N)$ é o isomorfismo de Poincaré relativo a $N$, e $\theta_{L} \in \mathrm{H}_{n}(N, N \backslash L)$ é a classe fundamental em $L$; note que, como a família de todos os subconjuntos compactos e conexos de $N$ é cofinal em relação à família dos subconjuntos compactos de $N$, podemos supor $L$ conexo. Por outro lado, sendo $\check{f}_{n}^{*}: \check{\mathrm{H}}_{c}^{n}(N) \longrightarrow \check{\mathrm{H}}_{c}^{n}(M)$ o homomorfismo em cohomologia de Čech com suporte compacto induzido por $f$, temos que $\tilde{f}_{n}^{*}(\llbracket y \rrbracket)=\llbracket f_{L}^{*}(y) \rrbracket$, onde $f_{L}^{*}: \mathrm{H}^{n}(N, N \backslash L) \longrightarrow \mathrm{H}^{n}\left(M, M \backslash f^{-1}(L)\right)$ é o homomorfismo em cohomologia singular induzido pela restrição de $f$ a $\left(M, M \backslash f^{-1}(L)\right)$. Isto posto, sendo $\theta_{f^{-1}(L)} \in \mathrm{H}_{n}\left(M, M \backslash f^{-1}(L)\right)$ a classe fundamental em $f^{-1}(L)$, segue que:

$$
f_{!}(x)=\left(\check{\mathrm{D}}_{M} \circ \check{f}_{n}^{*} \circ \check{\mathrm{D}}_{N}^{-1}\right)(x)=\left(\check{\mathrm{D}}_{M} \circ \check{f}_{n}^{*}\right)(\llbracket y \rrbracket)=\check{\mathrm{D}}_{M}\left(\llbracket f_{L}^{*}(y) \rrbracket\right)=f_{L}^{*}(y) \cap \theta_{f^{-1}(L)}
$$

(onde $\check{\mathrm{D}}_{M}: \check{\mathrm{H}}_{c}^{n}(M) \longrightarrow \mathrm{H}_{0}(M)$ é o isomorfismo de Poincaré relativo a $M$ ). Entretanto, da naturalidade do produto cap (vide [D], página 239), temos que:

$$
f_{*}\left(f_{L}^{*}(y) \cap \theta_{f^{-1}(L)}\right)=y \cap f_{*}\left(\theta_{f^{-1}(L)}\right)
$$

isto é, $\left(f_{*} \circ f_{!}\right)(x)=y \cap f_{*}\left(\theta_{f^{-1}(L)}\right)$; além disso, da definição de grau local, sabemos que $r=\operatorname{deg}_{L}(f)$ é o número inteiro tal que $f_{*}\left(\theta_{f^{-1}(L)}\right)=r \cdot \theta_{L}$. Portanto:

$$
\left(f_{*} \circ f_{!}\right)(x)=y \cap f_{*}\left(\theta_{f^{-1}(L)}\right)=y \cap\left(r \cdot \theta_{L}\right)=r \cdot\left(y \cap \theta_{L}\right)=r \cdot x
$$

Deste modo, concluímos que $\left(f_{*} \circ f_{!}\right)(x)=r \cdot x$ para todo $x \in \mathrm{H}_{0}(N)$, conforme queríamos provar. 
Por outro lado, as próximas duas proposições têm um caráter mais técnico, e serão utilizadas na demonstração do teorema posterior.

Proposição 1.4.8 . Sejam Me $N$ variedades topológicas de dimensão n, conexas e orientáveis, e considere uma aplicação contínua própria $f:(M, \partial M) \longrightarrow(N, \partial N)$. Sendo $W=M \backslash f^{-1}(\partial N)$, temos que $W$ é um subconjunto aberto de Int $M$ tal que, para toda componente conexa $U$ de $W$, a restrição $\left.f\right|_{U}: U \longrightarrow$ Int $N$ é uma aplicação própria. Além disso, denotando por $i^{!}: \overline{\mathrm{H}}_{c}^{n}(U) \longrightarrow \overline{\mathrm{H}}_{c}^{n}(\operatorname{Int} M)$ o homomorfismo transfer em cohomologia associado à inclusão $i: U \longrightarrow$ Int $M$, onde $U$ é uma componente conexa de $W$, temos que $i$ ! é um isomorfismo que leva a classe fundamental $\llbracket U \rrbracket \in \check{\mathrm{H}}_{c}^{n}(U)$ na classe fundamental $\llbracket \operatorname{Int} M \rrbracket \in \check{\mathrm{H}}_{c}^{n}(\operatorname{Int} M)$, e comuta o seguinte diagrama:

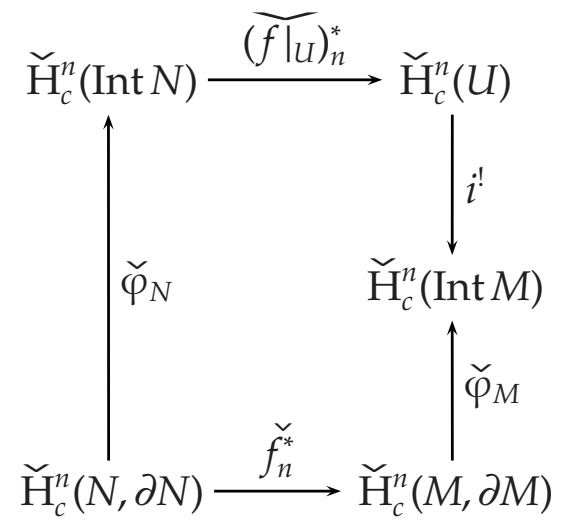

(onde $\check{\varphi}_{M}: \check{\mathrm{H}}_{c}^{n}(M, \partial M) \longrightarrow \check{\mathrm{H}}_{c}^{n}(\operatorname{Int} M)$ e $\widetilde{\varphi_{N}}: \check{\mathrm{H}}_{c}^{n}(N, \partial N) \longrightarrow \check{\mathrm{H}}_{c}^{n}(\operatorname{Int} N)$ são os isomorfismos dados na proposição 1.4.1). Conseqüentemente, sendo $\operatorname{deg}(f) \in \mathbb{Z}$ o grau cohomológico de $f$, temos que:

$$
\widetilde{\left(\left.f\right|_{U}\right)_{n}^{*}}(\llbracket \operatorname{Int} N \rrbracket)=\operatorname{deg}(f) \cdot \llbracket U \rrbracket,
$$

onde $\llbracket \operatorname{Int} N \rrbracket \in \check{\mathrm{H}}_{c}^{n}(\operatorname{Int} N)$ é a classe fundamental em $\operatorname{Int} N$.

DemonstraçÃo. Como $f^{-1}(\partial N)$ é fechado em $M$, e $f$ preserva fronteira, segue que $W=M \backslash f^{-1}(\partial N)$ é de fato um subconjunto aberto de Int $M$. Seja, pois, $U \subseteq W$ uma componente conexa de $W$; vamos mostrar que a restrição $\left.f\right|_{U}: U \longrightarrow \operatorname{Int} N$ é uma aplicação própria.

Primeiramente, verifiquemos que $\left.f\right|_{W}: W \longrightarrow \operatorname{Int} N$ é própria. Com efeito, dado $K \subseteq$ Int $N$ compacto, temos que $f^{-1}(K) \subseteq M$ é compacto (pois $f$ é própria); por outro lado, sabemos que:

$$
\left(\left.f\right|_{W}\right)^{-1}(K)=f^{-1}(K) \cap W=f^{-1}(K) \cap\left(M \backslash f^{-1}(\partial N)\right)=f^{-1}(K) \backslash f^{-1}(\partial N) .
$$

Note então que $f^{-1}(K) \cap f^{-1}(\partial N)=\varnothing$ : de fato, se $x \in f^{-1}(K) \cap f^{-1}(\partial N)$, segue que $f(x) \in f\left(f^{-1}(K)\right)=K$ e $f(x) \in f\left(f^{-1}(\partial N)\right)=\partial N$, ou seja, $f(x) \in K \cap \partial N$, o que é impossível 
(uma vez que $K \subseteq \operatorname{Int} N$ ). Assim, temos que:

$$
\left(\left.f\right|_{W}\right)^{-1}(K)=f^{-1}(K) \backslash f^{-1}(\partial N)=f^{-1}(K)
$$

isto é, $\left(\left.f\right|_{W}\right)^{-1}(K)$ é compacto, o que prova que $\left.f\right|_{W}: W \longrightarrow$ Int $N$ é uma aplicação própria. Conseqüentemente, como $U$ é uma componente conexa de $W$, segue que a restrição $\left.f\right|_{U}: U \longrightarrow$ Int $N$ também é uma aplicação própria (pois, dado um compacto $K \subseteq \operatorname{Int} N$, a imagem inversa $\left(\left.f\right|_{U}\right)^{-1}(K)$ é, ou o conjunto vazio, ou uma união finita de componentes conexas do compacto $\left.\left(\left.f\right|_{W}\right)^{-1}(K) \subseteq W\right)$.

Agora, considere a inclusão $i: U \longrightarrow$ Int $M$; por definição, sabemos que o homomorfismo transfer em cohomologia $i^{!}: \check{\mathrm{H}}_{c}^{n}(U) \longrightarrow \check{\mathrm{H}}_{c}^{n}(\operatorname{Int} M)$ é o homomorfismo obtido pela seguinte composição:

$$
\check{\mathrm{H}}_{c}^{n}(U) \stackrel{\check{\mathrm{D}}_{U}}{\longrightarrow} \mathrm{H}_{0}(U) \stackrel{i_{*}}{\longrightarrow} \mathrm{H}_{0}(\operatorname{Int} M) \stackrel{\check{\mathrm{D}}_{\operatorname{Int} M}^{-1}}{\longrightarrow} \check{\mathrm{H}}_{c}^{n}(\operatorname{Int} M)
$$

(onde $\check{\mathrm{D}}_{U}: \check{\mathrm{H}}_{c}^{n}(U) \longrightarrow \mathrm{H}_{0}(U)$ e $\check{\mathrm{D}}_{\text {Int } M}: \check{\mathrm{H}}_{c}^{n}(\operatorname{Int} M) \longrightarrow \mathrm{H}_{0}(\operatorname{Int} M)$ são os isomorfismos de Poincaré relativos a $U$ e Int $M$, respectivamente). Como $U$ e Int $M$ são variedades conexas, orientáveis e sem fronteira, temos que o homomorfismo em homologia singular $i_{*}: \mathrm{H}_{0}(U) \longrightarrow \mathrm{H}_{0}$ (Int $\left.M\right)$ é o isomorfismo identidade, o que implica que $i^{!}$é um isomorfismo. Vamos mostrar então que $i$ l leva a classe fundamental $\llbracket U \rrbracket \in \widetilde{\mathrm{H}}_{c}^{n}(U)$ na classe fundamental $\llbracket \operatorname{Int} M \rrbracket \in \check{\mathrm{H}}_{c}^{n}(\operatorname{Int} M)$. Com efeito, dado $K \subseteq U$ compacto, seja $x \in \mathrm{H}^{n}(U, U \backslash K)$ tal que $x \in \llbracket U \rrbracket$; temos, pois, que $\check{\mathrm{D}}_{U}(\llbracket U \rrbracket)=x \cap \theta_{K}$, onde $\theta_{K} \in \mathrm{H}_{n}(U, U \backslash K)$ é a classe fundamental em $K$. Assim, seja $y \in \mathrm{H}^{n}(\operatorname{Int} M$, Int $M \backslash K)$ tal que $x=i_{K}^{*}(y)$ e $y \in \llbracket \operatorname{Int} M \rrbracket$, onde $i_{K}^{*}:: \mathrm{H}^{n}(\operatorname{Int} M$, Int $M \backslash K) \longrightarrow \mathrm{H}^{n}(U, U \backslash K)$ é o homomorfismo induzido pela inclusão $i_{K}:(U, U \backslash K) \longrightarrow(\operatorname{Int} M, \operatorname{Int} M \backslash K)$. Sendo $\theta_{K}^{\prime} \in \mathrm{H}_{n}(\operatorname{Int} M$, Int $M \backslash K)$ a classe fundamental em $K$ (relativa a Int $M$ ), sabemos que $\theta_{K}^{\prime}=\left(i_{K}\right)_{*}\left(\theta_{K}\right)$, onde $\left(i_{K}\right)_{*}: \mathrm{H}_{n}(U, U \backslash K) \longrightarrow \mathrm{H}_{n}(\operatorname{Int} M$, Int $M \backslash K)$ é o homomorfismo induzido pela inclusão $i_{K}$. Logo, como $\check{D}_{\operatorname{Int} M}(\llbracket \operatorname{Int} M \rrbracket)=y \cap \theta_{K}^{\prime}$, segue, da naturalidade do produto cap (vide [D], página 239), que:

$$
\begin{aligned}
i_{*}\left(\check{\mathrm{D}}_{U}(\llbracket U \rrbracket)\right) & =i_{*}\left(x \cap \theta_{K}\right)=i_{*}\left(i_{K}^{*}(y) \cap \theta_{K}\right)=y \cap\left(i_{K}\right)_{*}\left(\theta_{K}\right) \\
& =y \cap \theta_{K}^{\prime}=\check{D}_{\operatorname{Int} M}(\llbracket \operatorname{Int} M \rrbracket)
\end{aligned}
$$

d'onde concluímos que $i !(\llbracket U \rrbracket)=\left(\check{\mathrm{D}}_{\text {Int } M}^{-1} \circ i_{*} \circ \check{\mathrm{D}}_{U}\right)(\llbracket U \rrbracket)=\llbracket \operatorname{Int} M \rrbracket$, como queríamos provar.

Isto posto, vamos mostrar que o diagrama abaixo é comutativo (onde $\breve{\varphi}_{M}$ e $\check{\varphi}_{N}$ são os isomorfismos dados na proposição 1.4.1): 


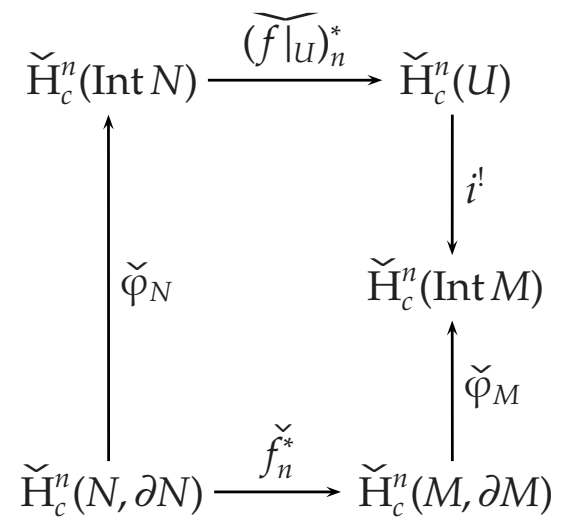

De fato, dado $K \subseteq \operatorname{Int} N$ compacto, sejam $D_{1}: \mathrm{H}^{n}\left(U, U \backslash\left(\left.f\right|_{U}\right)^{-1}(K)\right) \longrightarrow \mathrm{H}_{0}(U)$ e $D_{2}: \mathrm{H}^{n}\left(\operatorname{Int} M, \operatorname{Int} M \backslash f^{-1}(K)\right) \longrightarrow \mathrm{H}_{0}(\operatorname{Int} M)$ os homomorfismos dados por $D_{1}(x)=x \cap \theta_{1}$ e $D_{2}(y)=y \cap \theta_{2}$, para cada $x \in \mathrm{H}^{n}\left(U, U \backslash\left(\left.f\right|_{U}\right)^{-1}(K)\right)$ e cada $y \in \mathrm{H}^{n}(\operatorname{Int} M$, Int $M \backslash$ $\left.f^{-1}(K)\right)$, onde $\theta_{1} \in \mathrm{H}_{n}\left(U, U \backslash\left(\left.f\right|_{U}\right)^{-1}(K)\right)$ e $\theta_{2} \in \mathrm{H}_{n}\left(\operatorname{Int} M\right.$, Int $\left.M \backslash f^{-1}(K)\right)$ são as classes fundamentais em $\left(\left.f\right|_{U}\right)^{-1}(K)$ e $f^{-1}(K)$, respectivamente. Assim, denotando por $i_{*}$ : $\mathrm{H}_{0}(U) \longrightarrow \mathrm{H}_{0}(\operatorname{Int} M)$ o homomorfismo induzido pela inclusão $U \subseteq \operatorname{Int} M$, segue (da naturalidade do produto cap) que o diagrama abaixo é comutativo:

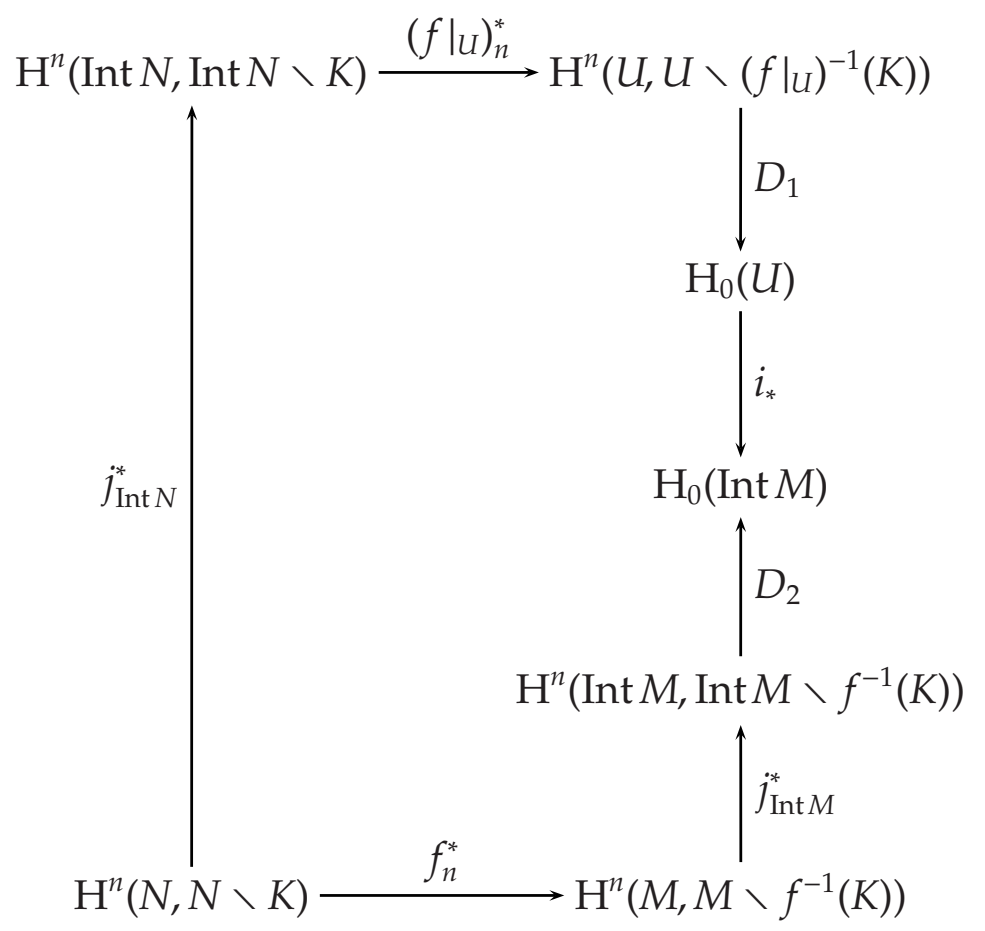

(onde $j_{\operatorname{Int} N}^{*}$ e $j_{\operatorname{Int} M}^{*}$ são os homomorfismos induzidos pelas inclusões $\operatorname{Int} N \subseteq N$ e Int $M \subseteq M$, respectivamente). Logo, passando ao limite direto (vide proposição A.4), 
obtemos a comutatividade do diagrama desejado:

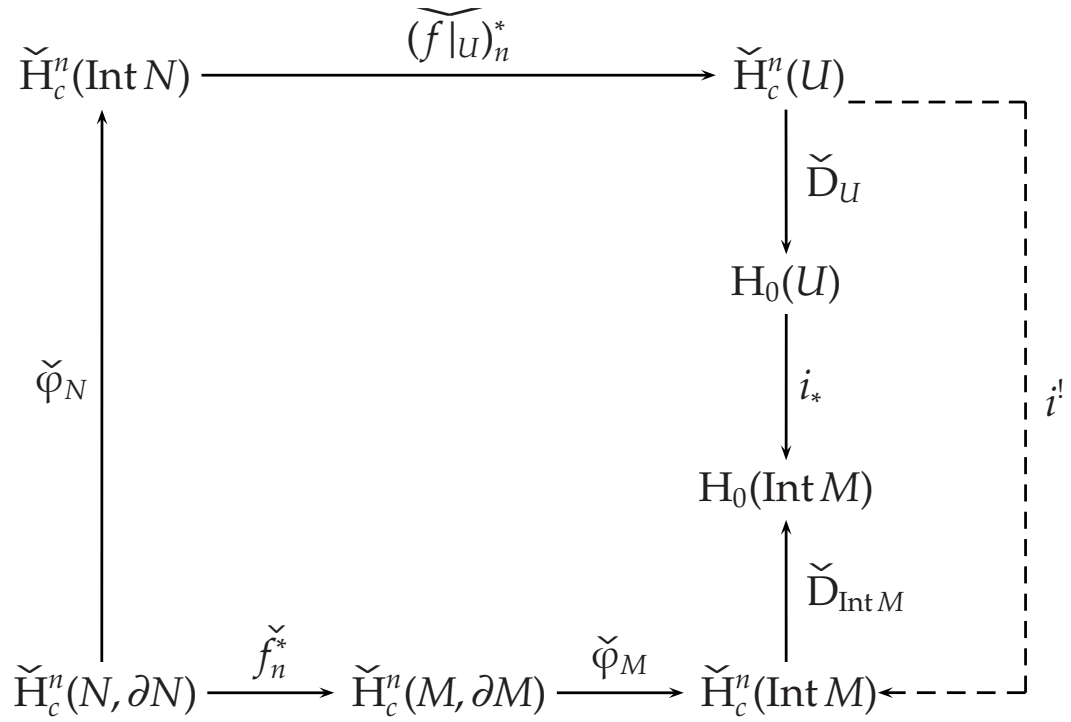

Finalmente, sejam $\llbracket M \rrbracket \in \check{\mathrm{H}}_{c}^{n}(M, \partial M)$ e $\llbracket N \rrbracket \in \check{\mathrm{H}}_{c}^{n}(N, \partial N)$ as classes correspondentes às classes fundamentais $\llbracket \operatorname{Int} M \rrbracket \in \check{\mathrm{H}}_{c}^{n}(\operatorname{Int} M)$ e $\llbracket \operatorname{Int} N \rrbracket \in \check{\mathrm{H}}_{c}^{n}(\operatorname{Int} N)$, pelos isomorfismos $\check{\varphi}_{M}$ e $\check{\varphi}_{N}$, respectivamente; por definição, sabemos que o grau cohomológico de $f$ é o número $\operatorname{deg}(f) \in \mathbb{Z}$ tal que $\tilde{f}_{n}^{*}(\llbracket N \rrbracket)=\operatorname{deg}(f) \cdot \llbracket M \rrbracket$. Portanto, a comutatividde do diagrama acima nos garante que:

$$
\begin{aligned}
\left(i !{ }^{!} \circ\left(\widetilde{\left.f\right|_{U}}\right)_{n}^{*}\right)(\llbracket \operatorname{Int} N \rrbracket) & \left.=\left(i ! \widetilde{\left(\left.f\right|_{U}\right.}\right)_{n}^{*} \circ \check{\varphi}_{N}\right)(\llbracket N \rrbracket)=\left(\check{\varphi}_{M} \circ \check{f}_{n}^{*}\right)(\llbracket N \rrbracket) \\
& =\check{\varphi}_{M}(\operatorname{deg}(f) \cdot \llbracket M \rrbracket)=\operatorname{deg}(f) \cdot \check{\varphi}_{M}(\llbracket M \rrbracket) \\
& =\operatorname{deg}(f) \cdot \llbracket \operatorname{Int} M \rrbracket .
\end{aligned}
$$

Conseqüentemente, como $i^{!}: \check{\mathrm{H}}_{c}^{n}(U) \longrightarrow \check{\mathrm{H}}_{c}^{n}(\operatorname{Int} M)$ é um isomorfismo que leva a classe fundamental $\llbracket U \rrbracket \in \check{\mathrm{H}}_{c}^{n}(U)$ na classe fundamental $\llbracket \operatorname{Int} M \rrbracket \in \check{\mathrm{H}}_{c}^{n}(\operatorname{Int} M)$, podemos concluir que $\left(\widetilde{\left.f\right|_{U}}\right)_{n}^{*}(\llbracket \operatorname{Int} N \rrbracket)=\operatorname{deg}(f) \cdot \llbracket U \rrbracket$.

Proposição 1.4.9 . Sejam $M$ e $N$ variedades topológicas de dimensão $n$, orientáveis e sem fronteira, e considere uma aplicação contínua própria $f: M \longrightarrow N$. Se $U \subseteq M$ é um aberto, tal que a restrição $\left.f\right|_{U}: U \longrightarrow N$ é própria, então, denotando por $i_{*}: \mathrm{H}_{0}(U) \longrightarrow \mathrm{H}_{0}(M)$ o homomorfismo em homologia singular induzido pela inclusão $U \subseteq M$, temos o seguinte diagrama comutativo:

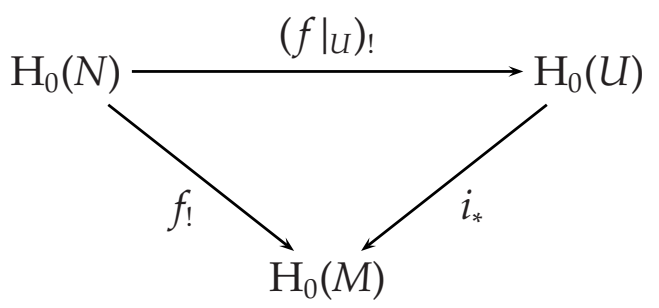


(onde $f_{!} e\left(\left.f\right|_{U}\right)_{!}$são os homomorfismos transfer em homologia, associados a $f$ e $\left.f\right|_{U}$, respectivamente).

DemonstraÇÃo. Com efeito, dado $K \subseteq N$ compacto, considere os homomorfismos $D_{M}: \mathrm{H}^{n}\left(M, M \backslash f^{-1}(K)\right) \longrightarrow \mathrm{H}_{0}(M)$ e $D_{U}: \mathrm{H}^{n}\left(U, U \backslash\left(\left.f\right|_{U}\right)^{-1}(K)\right) \longrightarrow \mathrm{H}_{0}(U)$, dados por $D_{M}(x)=x \cap \theta_{M}$ e $D_{U}(y)=y \cap \theta_{U}$ para cada $x \in \mathrm{H}^{n}\left(M, M \backslash f^{-1}(K)\right)$ e cada $y \in$ $\mathrm{H}^{n}\left(U, U \backslash\left(\left.f\right|_{U}\right)^{-1}(K)\right)$, onde $\theta_{M} \in \mathrm{H}_{n}\left(M, M \backslash f^{-1}(K)\right)$ e $\theta_{U} \in \mathrm{H}_{n}\left(U, U \backslash\left(\left.f\right|_{U}\right)^{-1}(K)\right)$ são as classes fundamentais. Da naturalidade do produto cap, temos então o seguinte diagrama comutativo:

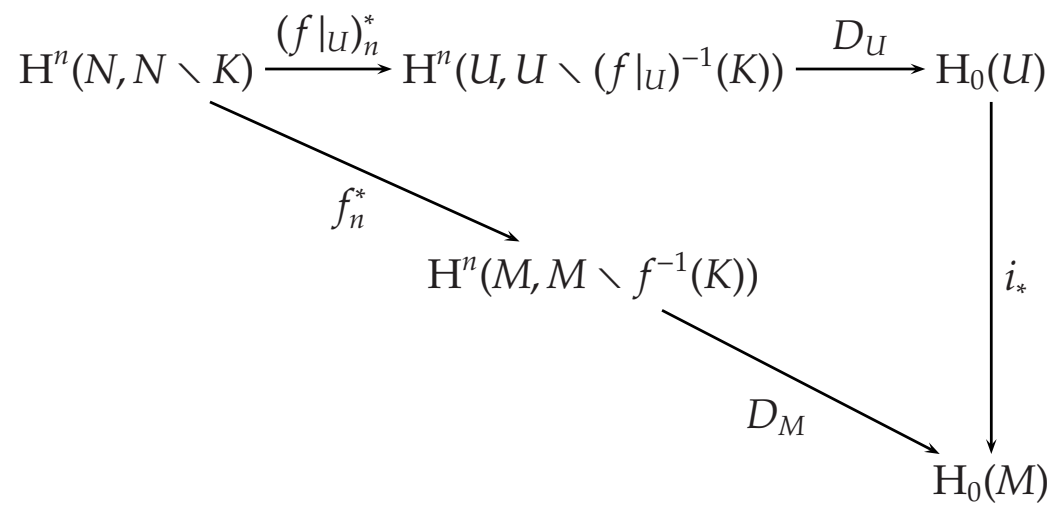

Logo, passando ao limite direto (vide proposição A.4), obtemos o diagrama comutativo abaixo (onde $\check{\mathrm{D}}_{M}, \check{\mathrm{D}}_{N}$ e $\check{\mathrm{D}}_{U}$ são os isomorfismos de Poincaré relativos a $M, N$ e $U$, respectivamente):

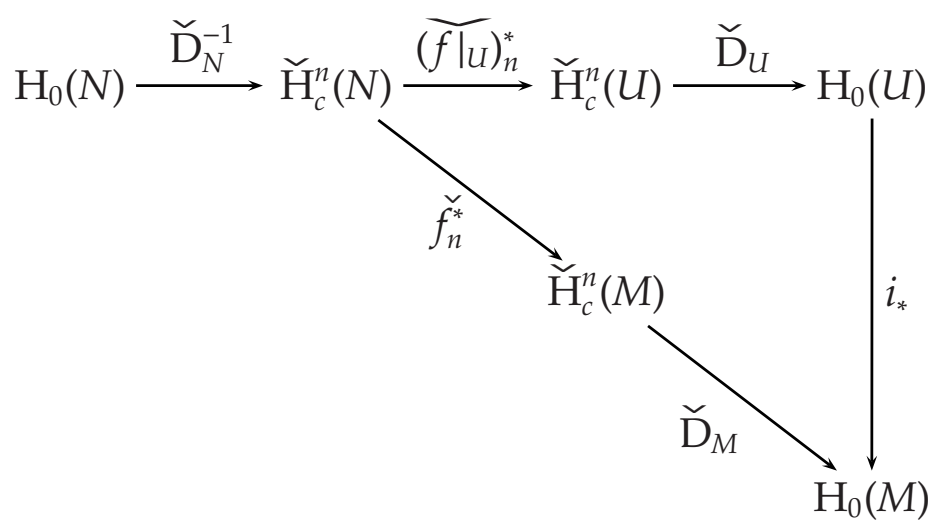

Portanto, como $f_{!}=\check{\mathrm{D}}_{M} \circ \check{f}_{n}^{*} \circ \check{\mathrm{D}}_{N}^{-1}$ e $\left(\left.f\right|_{U}\right)_{!}=\check{\mathrm{D}}_{U} \circ \widetilde{\left(\left.f\right|_{U}\right)_{n}^{*}} \circ \check{\mathrm{D}}_{N}^{-1}$, segue a comutatividade do diagrama desejado.

Finalmente, com base nas proposições anteriores, podemos demonstrar o seguinte

Teorema 1.4.2 . Sejam $M$ e $N$ variedades topológicas de dimensão $n$, conexas e orientáveis, e considere uma aplicação contínua própria $f:(M, \partial M) \longrightarrow(N, \partial N)$. Sendo $W=M \backslash f^{-1}(\partial N)$, 
temos então que $\operatorname{deg}(f)=\operatorname{deg}_{c}\left(\left.f\right|_{W}\right)$, qualquer que sejac $\in \operatorname{Int} N$. Em particular, orientando-se M e $N$ com coeficientes em $\mathbb{Z}_{2}$, temos que $\operatorname{deg}(f, 2)=\operatorname{deg}_{c}\left(\left.f\right|_{W}\right)$ para todo $c \in \operatorname{Int} N$, onde $\operatorname{deg}_{c}\left(\left.f\right|_{W}\right)$ é o grau local (de $\left.f\right|_{W}$ em c) a coeficientes em $\mathbb{Z}_{2}$.

DemonstraçÃo. Com efeito, na proposição 1.4.8, vimos que $W=M \backslash f^{-1}(\partial N)$ é um subconjunto aberto de Int $M$, tal que, para toda componente conexa $U$ de $W$, as restrições $\left.f\right|_{W}: W \longrightarrow \operatorname{Int} N$ e $\left.f\right|_{U}: U \longrightarrow$ Int $N$ são aplicações próprias; além disso, sendo $\llbracket \operatorname{Int} N \rrbracket \in \check{\mathrm{H}}_{c}^{n}(\operatorname{Int} N)$ e $\llbracket U \rrbracket \in \check{\mathrm{H}}_{c}^{n}(U)$ as classes fundamentais em Int $N$ e $U$, respectivamente, temos que:

$$
\widetilde{\left(\left.f\right|_{U}\right)_{n}^{*}}(\llbracket \operatorname{Int} N \rrbracket)=\operatorname{deg}(f) \cdot \llbracket U \rrbracket,
$$

onde deg $(f)$ é o grau cohomológico de $f$. Assim sendo, consideremos as classes [Int $N] \in$ $\mathrm{H}_{0}(\operatorname{Int} N)$ e $[U] \in \mathrm{H}_{0}(U)$, correspondentes às classes fundamentais $\llbracket \operatorname{Int} N \rrbracket$ e $\llbracket U \rrbracket$ pelos isomorfismos de Poincaré $\check{D}_{\text {Int } N}: \check{\mathrm{H}}_{c}^{n}(\operatorname{Int} N) \rightarrow \mathrm{H}_{0}(\operatorname{Int} N)$ e $\check{\mathrm{D}}_{U}: \check{\mathrm{H}}_{c}^{n}(U) \longrightarrow \mathrm{H}_{0}(U)$, respectivamente. Sabemos que o homomorfismo transfer em homologia, associado a $\left.f\right|_{U}: U \longrightarrow \operatorname{Int} N$, é o homomorfismo $\left(\left.f\right|_{U}\right)_{!}: \mathrm{H}_{0}(\operatorname{Int} N) \longrightarrow \mathrm{H}_{0}(U)$ dado pela seguinte composição:

$$
\mathrm{H}_{0}(\operatorname{Int} N) \stackrel{\check{\mathrm{D}}_{\text {Int } N}^{-1}}{\longrightarrow} \check{\mathrm{H}}_{c}^{n}(\operatorname{Int} N) \stackrel{\left(\widetilde{\left.f\right|_{U}}\right)_{n}^{*}}{\longrightarrow} \check{\mathrm{H}}_{c}^{n}(U) \stackrel{\check{\mathrm{D}}_{U}}{\longrightarrow} \mathrm{H}_{0}(U)
$$

Logo, temos que:

$$
\begin{aligned}
\left(\left.f\right|_{U}\right)_{!}([\operatorname{Int} N]) & =\left(\check{\mathrm{D}}_{U} \circ\left(\widetilde{\left.f\right|_{U}}\right)_{n}^{*} \circ \check{\mathrm{D}}_{\operatorname{Int} N}^{-1}\right)([\operatorname{Int} N])=\left(\check{\mathrm{D}}_{U} \circ\left(\widetilde{\left.f\right|_{U}}\right)_{n}^{*}\right)(\llbracket \operatorname{Int} N \rrbracket) \\
& =\check{\mathrm{D}}_{U}(\operatorname{deg}(f) \cdot \llbracket U \rrbracket)=\operatorname{deg}(f) \cdot \check{\mathrm{D}}_{U}(\llbracket U \rrbracket)=\operatorname{deg}(f) \cdot[U] .
\end{aligned}
$$

Por outro lado, como o homomorfismo $\left(\left.f\right|_{U}\right)_{*}: \mathrm{H}_{0}(U) \longrightarrow \mathrm{H}_{0}(\operatorname{Int} N)$, induzido por $\left.f\right|_{U}$, leva a classe $[U] \in \mathrm{H}_{0}(U)$ na classe $[\operatorname{Int} N] \in \mathrm{H}_{0}(\operatorname{Int} N)$, obtemos que:

$$
\left(\left(\left.f\right|_{U}\right)_{*} \circ\left(\left.f\right|_{U}\right)_{!}\right)([\operatorname{Int} N])=\operatorname{deg}(f) \cdot[\operatorname{Int} N] .
$$

Agora, pela proposição 1.4.9, sabemos que o diagrama abaixo é comutativo:

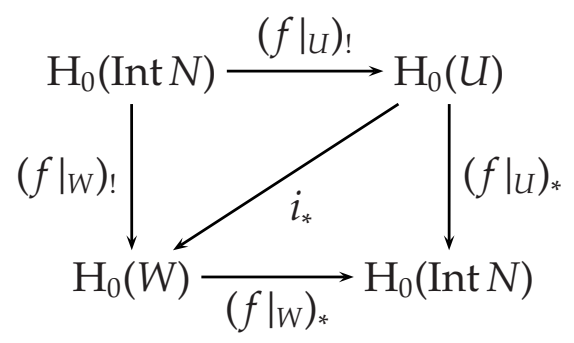

(onde $i_{*}$ : é o homomorfismo em homologia singular induzido pela inclusão $U \subseteq W$ ). Conseqüentemente, temos que:

$$
\left(\left(\left.f\right|_{W}\right)_{*} \circ\left(\left.f\right|_{W}\right)_{!}\right)([\operatorname{Int} N])=\left(\left(\left.f\right|_{U}\right)_{*} \circ\left(\left.f\right|_{U}\right)_{!}\right)([\operatorname{Int} N])=\operatorname{deg}(f) \cdot[\operatorname{Int} N] .
$$


Finalmente, observe então que, da proposição 1.4.7, também temos:

$$
\left(\left(\left.f\right|_{W}\right)_{*} \circ\left(\left.f\right|_{W}\right)_{!}\right)([\operatorname{Int} N])=\operatorname{deg}_{c}\left(\left.f\right|_{W}\right) \cdot[\operatorname{Int} N]
$$

qualquer que seja $c \in \operatorname{Int} N$ (lembre-se que Int $N$ é uma variedade orientável, conexa e sem fronteira). Deste modo, concluímos que $\operatorname{deg}(f)=\operatorname{deg}_{c}\left(\left.f\right|_{W}\right)$ para todo $c \in \operatorname{Int} N$, conforme queríamos provar.

Em particular, tomando-se coeficientes em $\mathbb{Z}_{2}$, as variedades $M$ e $N$ tornam-se unicamente orientadas, e, neste caso, podemos utilizar o mesmo raciocínio da demonstração acima para concluir que $\operatorname{deg}(f, 2)=\operatorname{deg}_{c}\left(\left.f\right|_{W}\right)$, qualquer que seja $c \in \operatorname{Int} N$, onde $\operatorname{deg}_{c}\left(\left.f\right|_{W}\right)$ é o grau local a coeficientes em $\mathbb{Z}_{2}$.

Corolário 1.4.1. Sejam $M$ e $N$ variedades topológicas de dimensão n, conexas e orientáveis. Se $h: M \longrightarrow N$ é um homeomorfismo (e, conseqüentemente, uma aplicação contínua própria que preserva fronteira), então $\operatorname{deg}(h)= \pm 1$.

DemonstraçÃo. Com efeito, sendo $W=M \backslash h^{-1}(\partial N)=M \backslash \partial M=\operatorname{Int} M$, considere a restrição $\left.h\right|_{\operatorname{Int} M}: \operatorname{Int} M \longrightarrow \operatorname{Int} N$; pelo teorema anterior, sabemos que $\operatorname{deg}(h)=$ $\operatorname{deg}_{c}\left(\left.h\right|_{\text {Int } M}\right)$, qualquer que seja $c \in \operatorname{Int} N$. Por outro lado, observando que $\left.h\right|_{\text {Int } M}$ é um homeomorfismo sobre Int $N$, segue (do corolário 1.3.1) que $\operatorname{deg}_{c}\left(\left.h\right|_{\operatorname{Int} M}\right)= \pm 1$ para todo $c \in \operatorname{Int} N$, d'onde concluímos que $\operatorname{deg}(h)= \pm 1$, como queríamos provar.

Corolário 1.4.2 . Sejam $M$ e $N$ variedades topológicas de dimensão $n$, conexas e orientáveis, e considere uma aplicação contínua própria $f:(M, \partial M) \longrightarrow(N, \partial N)$. Dado um ponto $c \in$ Int $N$, seja ainda $V \subseteq$ Int $N$ uma vizinhança aberta contrátil de c em $N$ (lembre-se que toda variedade topológica é localmente simplesmente conexa). Se, para cada classe de raízes $R$ de $f$ em $c$, denotarmos por $U_{R} \subseteq f^{-1}(V)$ um aberto tal que $U_{R} \cap f^{-1}(c)=R$ (cuja existência é garantida pela proposição 1.3.1), então:

$$
\sum\left\{\operatorname{deg}_{c}\left(\left.f\right|_{U_{R}}\right): R \text { é classe de raízes de } f \text { em } c\right\}=\operatorname{deg}(f) \text {. }
$$

DemonstraÇÃo. De fato, considere o aberto

$$
U=\bigcup\left\{U_{R}: R \text { é classe de raízes de } f \operatorname{em} c\right\} \subseteq \operatorname{Int} M ;
$$

pela propriedade aditiva do grau local (proposição 1.3.5), temos então que:

$$
\sum\left\{\operatorname{deg}_{c}\left(\left.f\right|_{U_{R}}\right): R \text { é classe de raízes de } f \operatorname{em} c\right\}=\operatorname{deg}_{c}\left(\left.f\right|_{U}\right) \text {. }
$$

Por outro lado, sendo $W=M \backslash f^{-1}(\partial N)$, sabemos que $W$ é um subconjunto aberto de Int $M$; além disso, como $U_{R} \subseteq W$ para toda classe de raízes $R$ de $f$ em $c$ (pois $U_{R} \subseteq f^{-1}(V)$ 
e $V \subseteq \operatorname{Int} N)$, segue que $U \subseteq W$. Agora, observe que:

$$
\begin{aligned}
f^{-1}(c) & =\bigcup\{R: R \text { é classe de raízes de } f \text { em } c\} \\
& =\bigcup\left\{U_{R} \cap f^{-1}(c): R \text { é classe de raízes de } f \text { em } c\right\} \\
& \subseteq \bigcup\left\{U_{R}: R \text { é classe de raízes de } f \text { em } c\right\}=U,
\end{aligned}
$$

isto é, $W \backslash U \subseteq W \backslash f^{-1}(c)$. Logo, pela excisão em homologia singular, temos que o homomorfismo $i_{*}: \mathrm{H}_{n}\left(U, U \backslash f^{-1}(c)\right)=\mathrm{H}_{n}\left(W \backslash(W \backslash U),\left(W \backslash f^{-1}(c)\right) \backslash(W \backslash U)\right) \longrightarrow$ $\mathrm{H}_{n}\left(W, W \backslash f^{-1}(c)\right)$, induzido pela inclusão $i:\left(U, U \backslash f^{-1}(c)\right) \longrightarrow\left(W, W \backslash f^{-1}(c)\right)$, é um isomorfismo, que leva a classe fundamental $\theta_{U} \in \mathrm{H}_{n}\left(U, U \backslash f^{-1}(c)\right)$ na classe fundamental $\theta_{W} \in \mathrm{H}_{n}\left(W, W \backslash f^{-1}(c)\right)$, e comuta o seguinte diagrama:

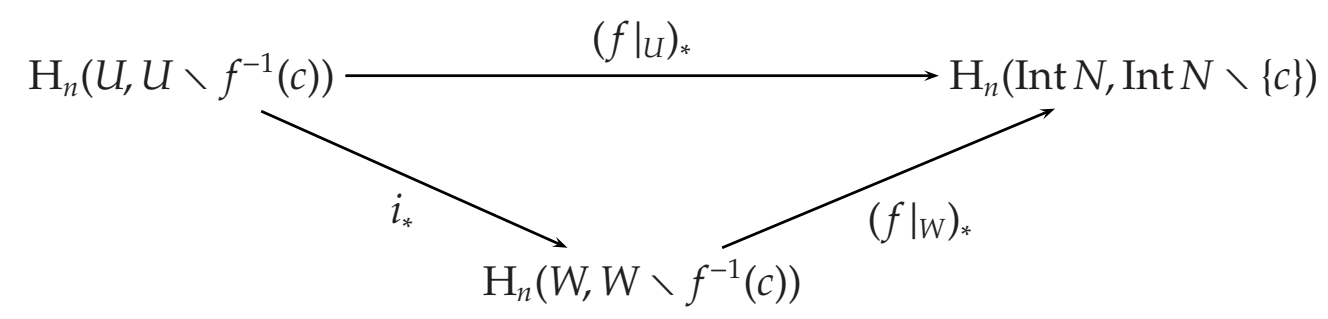

(note que $f(U) \subseteq \operatorname{Int} N$, pois $U \subseteq W=M \backslash f^{-1}(\partial N)$ ). Assim, denotando por $\theta_{c} \in$ $\mathrm{H}_{n}(\operatorname{Int} N$, Int $N \backslash\{c\})$ a classe fundamental em $\{c\}$, temos que:

$$
\left(\left.f\right|_{U}\right)_{*}\left(\theta_{U}\right)=\left(\left(\left.f\right|_{W}\right)_{*} \circ i_{*}\right)\left(\theta_{U}\right)=\left(\left.f\right|_{W}\right)_{*}\left(\theta_{W}\right)=\operatorname{deg}_{c}\left(\left.f\right|_{W}\right) \cdot \theta_{c} .
$$

Ora, como o grau $\operatorname{local}_{\operatorname{deg}_{c}}\left(\left.f\right|_{U}\right)$ é definido como sendo o número inteiro tal que

$$
\left(\left.f\right|_{U}\right)_{*}\left(\theta_{U}\right)=\operatorname{deg}_{c}\left(\left.f\right|_{U}\right) \cdot \theta_{c},
$$

segue então que $\operatorname{deg}_{c}\left(\left.f\right|_{U}\right)=\operatorname{deg}_{c}\left(\left.f\right|_{W}\right)$. Deste modo, utilizando o teorema anterior, podemos concluir que:

$\sum\left\{\operatorname{deg}_{c}\left(\left.f\right|_{U_{R}}\right): R\right.$ é classe de raízes de $\left.f \operatorname{em} c\right\}=\operatorname{deg}_{c}\left(\left.f\right|_{U}\right)=\operatorname{deg}_{c}\left(\left.f\right|_{W}\right)=\operatorname{deg}(f)$, conforme queríamos demonstrar.

No próximo capítulo (seção 2.1), veremos mais alguns resultados que relacionarão o conceito de grau cohomológico à multiplicidade de uma classe de raízes. 


\section{Capiturilo 2}

\section{Teoria de Nielsen de Raízes}

Neste capítulo, dada uma classe de raízes $R$ de uma aplicação própria $f:(M, \partial M) \longrightarrow$ $(N, \partial N)$ em $c \in \operatorname{Int} N$, vamos utilizar a multiplicidade $|m(R)|$ para definir o número de Nielsen de raízes e o número de Nielsen transverso de raízes $(\operatorname{de} f$ em $c$ ). Primeiramente, no entanto, convém estudarmos algumas propriedades de $|m(R)|$.

\subsection{A Propriedadle Fundamental da Teoria de Raízes}

No que faremos a seguir, vamos utilizar o conceito de recobrimento associado a um subgrupo, apresentado na definição 1.2.1.

Proposição 2.1.1 . Sendo $M$ e $N$ variedades topológicas conexas de dimesão n (não necessariamente orientáveis), considere uma aplicação contínua própria $f:(M, \partial M) \longrightarrow(N, \partial N)$. Dado um ponto $c \in \operatorname{Int} N$, seja $\hat{q}: \hat{N} \longrightarrow N$ o recobrimento de $N$ associado ao subgrupo $f_{\#}\left(\pi_{1}\left(M, x_{0}\right)\right) \subseteq \pi_{1}(N, c)$, onde $f_{\#}: \pi_{1}\left(M, x_{0}\right) \longrightarrow \pi_{1}(N, c)$ é o homomorfismo induzido por $f$, e $x_{0} \in M$ é tal que $f\left(x_{0}\right)=c$. Seja ainda $\hat{f}: M \longrightarrow \hat{N}$ o levantamento de $f$ relativamente a $\hat{q}$ (isto é, $f=\hat{q} \circ \hat{f}$ ), que leva o ponto $x_{0} \in M$ na classe de equivalência $\left\langle\varepsilon_{c}\right\rangle$, onde $\varepsilon_{c}: I \longrightarrow N$ éo caminho constante igual a c (cuja existência é garantida pelo lema 1.2.1). Então, dado ĉ $\in \hat{q}^{-1}(c)$, o conjunto $\hat{f}^{-1}(\hat{c})$, ou é vazio ou constitui uma classe de raízes de $f$ em $c$.

Demonstração. Primeiramente, observe que, dados $z_{0}, z_{1} \in M$ e $c_{0}, c_{1} \in N$, tais que $f\left(z_{0}\right)=c_{0}$ e $f\left(z_{1}\right)=c_{1}$, e sendo $\gamma: I \longrightarrow M$ um caminho ligando $z_{0}$ a $z_{1}$, temos que $\delta=f \circ \gamma: I \longrightarrow N$ é um caminho ligando $c_{0}$ a $c_{1}$, e as aplicações $\bar{\gamma}: \pi_{1}\left(M, z_{0}\right) \longrightarrow \pi_{1}\left(N, z_{1}\right)$ e $\bar{\delta}: \pi_{1}\left(N, c_{0}\right) \longrightarrow \pi_{1}\left(N, c_{1}\right)$, definidas por $\bar{\gamma}([\alpha])=\left[\gamma^{-1} \vee \alpha \vee \gamma\right]$ e $\bar{\delta}([\beta])=\left[\delta^{-1} \vee \beta \vee \delta\right]$, para $[\alpha] \in \pi_{1}\left(M, z_{0}\right)$ e $[\beta] \in \pi_{1}\left(N, c_{0}\right)$, são isomorfismos (vide [Li], proposição 4 , página 
34). Além disso, denotando por $f_{\#}^{0}: \pi_{1}\left(M, z_{0}\right) \longrightarrow \pi_{1}\left(N, c_{0}\right)$ e $f_{\#}^{1}: \pi_{1}\left(M, z_{1}\right) \longrightarrow \pi_{1}\left(M, c_{1}\right)$ os homomorfismos induzidos por $f$, segue que:

$$
\begin{aligned}
\left(\bar{\delta} \circ f_{\#}^{0}\right)([\alpha]) & =\bar{\delta}([f \circ \alpha])=\left[\delta^{-1} \vee(f \circ \alpha) \vee \delta\right]=\left[(f \circ \gamma)^{-1} \vee(f \circ \alpha) \vee(f \circ \gamma)\right] \\
& =\left[f \circ\left(\gamma^{-1} \vee \alpha \vee \gamma\right)\right]=f_{\#}^{1}\left(\left[\gamma^{-1} \vee \alpha \vee \gamma\right]\right)=\left(f_{\#}^{1} \circ \bar{\gamma}\right)([\alpha])
\end{aligned}
$$

para todo $[\alpha] \in \pi_{1}\left(M, z_{0}\right)$, ou seja, o diagrama abaixo é comutativo:

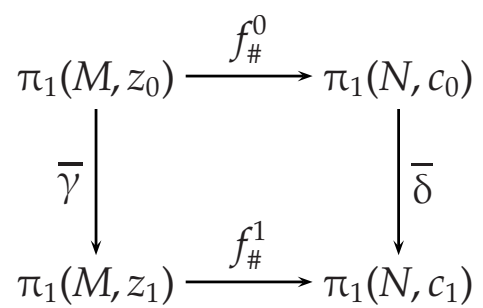

Em particular, se $c_{0}=c_{1}$, isto é, se $f\left(z_{0}\right)=f\left(z_{1}\right)=y \in N$, temos o seguinte diagrama comutativo:

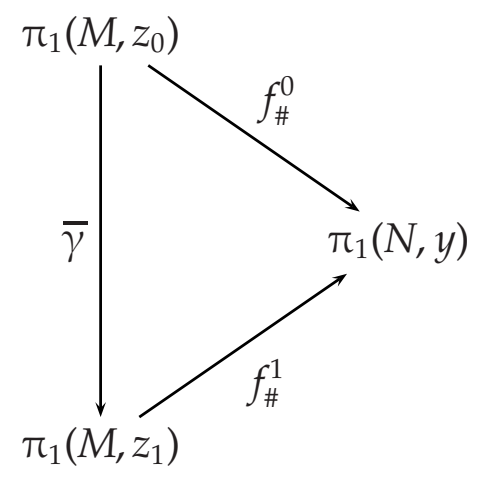

Isto posto, supondo $\hat{f}^{-1}(\hat{c}) \neq \varnothing$, considere $x_{1}, x_{2} \in \hat{f}^{-1}(\hat{c})$, e seja $\omega: I \longrightarrow M$ um caminho tal que $\omega(0)=x_{1}$ e $\omega(1)=x_{2}$; como $(f \circ \omega)(0)=(f \circ \omega)(1)=\hat{q}(\hat{c})=c$ e $(\hat{f} \circ \omega)(0)=(\hat{f} \circ \omega)(1)=\hat{c}$, segue que $f \circ \omega: I \longrightarrow N$ e $\hat{f} \circ \omega: I \longrightarrow \hat{N}$ são laços com base nos pontos $c \in N$ e $\hat{c} \in \hat{N}$, respectivamente. Assim, como $\hat{q}_{\#}(\hat{f} \circ \omega)=[\hat{q} \circ \hat{f} \circ \omega]=[f \circ \omega]$ e $\hat{q}_{\#}\left(\pi_{1}\left(\hat{N},\left\langle\varepsilon_{c}\right\rangle\right)\right)=f_{\#}\left(\pi_{1}\left(M, x_{0}\right)\right)$, temos que $[f \circ \omega] \in f_{\#}\left(\pi_{1}\left(M, x_{0}\right)\right)$, isto é, $[f \circ \omega]=$ $f_{\#}([\lambda])=[f \circ \lambda]$ para algum laço $\lambda: I \longrightarrow M$ com base em $x_{0}$. Por outro lado, dado um caminho $\gamma: I \longrightarrow M$ tal que $\gamma(0)=x_{0}$ e $\gamma(1)=x_{1}$, sabemos (de acordo com o que acabamos de mostrar acima) que a aplicação $\bar{\gamma}: \pi_{1}\left(M, x_{0}\right) \longrightarrow \pi_{1}\left(M, x_{1}\right)$, definida por $\bar{\gamma}([\alpha])=\left[\gamma^{-1} \vee \alpha \vee \gamma\right]$, é um isomorfismo que comuta o seguinte diagrama:

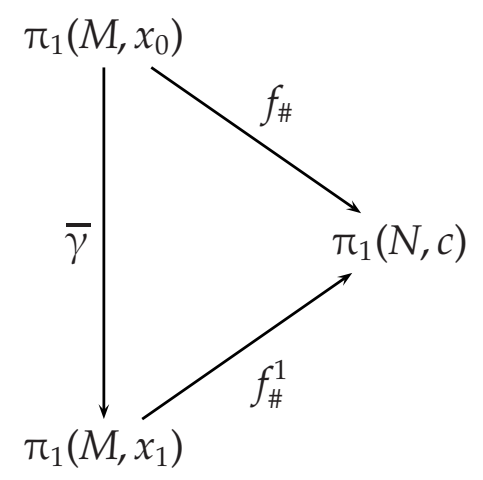


(onde $f_{\#}^{1}: \pi_{1}\left(M, x_{1}\right) \longrightarrow \pi_{1}(N, c)$ é o homomorfismo induzido por $f$ ). Deste modo, temos que:

$$
[f \circ \omega]=[f \circ \lambda]=f_{\#}([\lambda])=f_{\#}^{1}(\bar{\gamma}([\lambda]))=f_{\#}^{1}\left(\left[\gamma^{-1} \vee \lambda \vee \gamma\right]\right)=\left[f \circ\left(\gamma^{-1} \vee \lambda \vee \gamma\right)\right],
$$

isto é, o laço $\mu: I \longrightarrow M$ (com base no ponto $x_{1}$ ) dado por $\mu=\gamma^{-1} \vee \lambda \vee \gamma$ é tal que $[f \circ \omega]=[f \circ \mu]$ (vide figura 2.1 a seguir). Conseqüentemente, o produto $\left[f \circ \mu^{-1}\right] *[f \circ \omega]$ é igual à classe de homotopia do caminho constante $\varepsilon_{c}$, ou seja,

$$
\left[\varepsilon_{c}\right]=\left[f \circ \mu^{-1}\right] *[f \circ \omega]=\left[\left(f \circ \mu^{-1}\right) \vee(f \circ \omega)\right]=\left[f \circ\left(\mu^{-1} \vee \omega\right)\right] .
$$
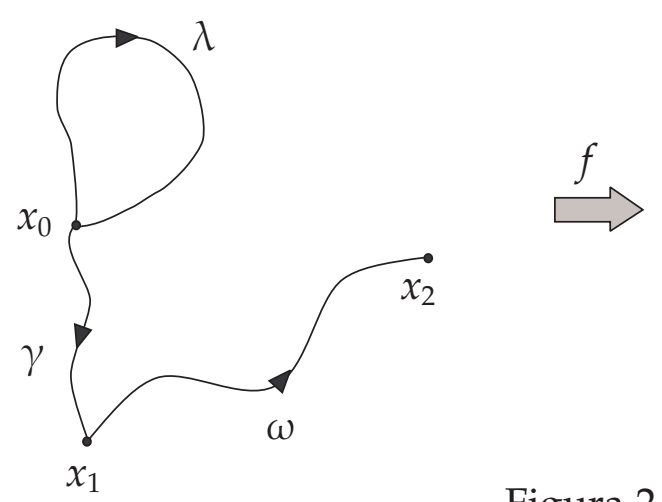

Figura 2.1

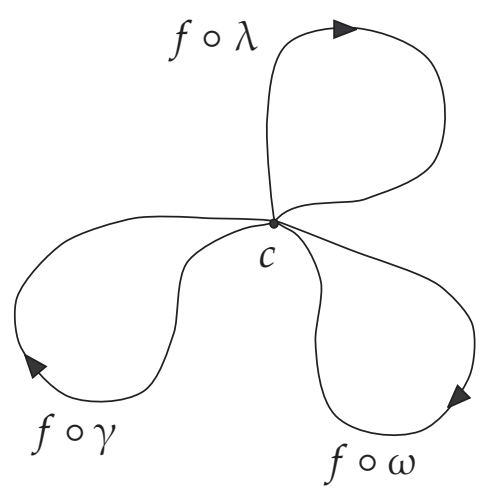

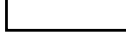

Portanto, $\mu^{-1} \vee \omega: I \longrightarrow M$ é um caminho ligando os pontos $x_{1}$ e $x_{2}$, tal que $f \circ\left(\mu^{-1} \vee \omega\right)$ é um laço contrátil em $c$, o que mostra que quaisquer dois pontos de $\hat{f}^{-1}(\hat{c})$ pertencem à mesma classe de raízes de $f$ em $c$; em outras palavras, existe uma classe de raízes $R$, de $f$ em $c$, tal que $\hat{f}^{-1}(\hat{c}) \subseteq R$.

Agora, dados $x \in \hat{f}^{-1}(\hat{c}) \subseteq R$ e $y \in R$, seja $\omega: I \longrightarrow M$ um caminho ligando $x$ e $y$, tal que $f \circ \omega: I \longrightarrow N$ é um laço contrátil em $c$; temos então que o levantamento $\hat{f} \circ \omega: I \longrightarrow \hat{N}$ de $f \circ \omega$ (relativamente ao recobrimento $\hat{q}$ ) é um laço contrátil em $\hat{c} \in \hat{N}$. Em particular, $\hat{f} \circ \omega$ é um caminho fechado, d'onde segue que $\hat{f}(y)=(\hat{f} \circ \omega)(1)=$ $(\hat{f} \circ \omega)(0)=\hat{f}(x)=\hat{c}$, isto é, $y \in \hat{f}^{-1}(\hat{c})$. Assim, podemos concluir que $\hat{f}^{-1}(\hat{c})=R$, ou seja, $\hat{f}^{-1}(\hat{c})$ é uma classe de raízes de $f$ em $c$, conforme queríamos mostrar.

Corolário 2.1.1 . Nas condições da proposição anterior, dada uma classe de raízes $R$ de $f$ em $c$, existe um elemento $\hat{c} \in \hat{q}^{-1}(c)$ tal que $R=\hat{f}^{-1}(\hat{c})$.

DemonstraÇÃo. De fato, tomando um ponto $x \in R \subseteq f^{-1}(c)$, considere o elemento $\hat{c}=\hat{f}(x) \in \hat{N}$; temos então que $\hat{c} \in \hat{q}^{-1}(c)$, pois $\hat{q}(\hat{c})=\hat{q}(\hat{f}(x))=f(x)=c$. Assim, como 
$x \in \hat{f}^{-1}(\hat{c})$, segue, da proposição anterior, que o conjunto $R^{\prime}=\hat{f}^{-1}(\hat{c})$ é uma classe de raízes de $f$ em $c$. Entretanto, sabemos que quaisquer duas classes de raízes de $f$ em $c$, ou coincidem ou são disjuntas. Logo, como $x \in R \cap R^{\prime}$, concluímos que $R=R^{\prime}=\hat{f}^{-1}(\hat{c})$, conforme queríamos provar.

Assim sendo, utilizando as mesmas notações da proposição 2.1.1, vamos descrever a multiplicidade de $R=\hat{f}^{-1}(\hat{c})$ em função do levantamento $\hat{f}: M \longrightarrow \hat{N}$.

Seja, pois, $V \subseteq$ Int $N$ uma vizinhança aberta contrátil de $c$, que constitui uma vizinhança elementar em relação ao espaço de recobrimento $\hat{N}$, isto é, tal que $\hat{q}^{-1}(V)$ é uma união disjunta de abertos em $\hat{N}$, cada um dos quais se aplica por $\hat{q}$ homeomorficamente sobre $V$. Considere ainda uma classe de raízes $R=\hat{f}^{-1}(\hat{c})$ de $f$ em $c$ (onde $\hat{c} \in \hat{q}^{-1}(c)$ é tal que $\left.\hat{f}^{-1}(\hat{c}) \neq \varnothing\right)$, e seja $\hat{V}$ a componente conexa de $\hat{q}^{-1}(V)$ que contém $\hat{c}$, de modo que a restrição $\left.\hat{q}\right|_{\hat{V}}$ é, portanto, um homeomorfismo sobre $V$. Como $\hat{q}(\partial \hat{N}) \subseteq \partial N$ e $\hat{q}(\operatorname{Int} \hat{N})=\operatorname{Int} N$ (uma vez que $\hat{q}$ é um homeomorfismo local), e $f(\partial M) \subseteq \partial N$, temos que $\hat{f}$ preserva fronteira, pois:

$$
\hat{f}(\partial M) \subseteq \hat{q}^{-1}((\hat{q} \circ \hat{f})(\partial M)) \subseteq \hat{q}^{-1}(f(\partial M)) \subseteq \hat{q}^{-1}(\partial N)=\partial \hat{N} .
$$

Logo, como $\hat{V}$ é um subconjunto aberto de $\operatorname{Int} \hat{N}$ (uma vez que $\hat{V} \subseteq \hat{q}^{-1}(V) \subseteq \operatorname{Int} \hat{N}$ ), segue que $\hat{f}^{-1}(\hat{V})$ é um subconjunto aberto de Int $M$. Além disso, note que $R=\hat{f}^{-1}(\hat{V}) \cap f^{-1}(c)$ : de fato, se $x \in R=\hat{f}^{-1}(\hat{c})$, temos que $\hat{f}(x)=\hat{c} \in \hat{V}$, e, portanto, $x \in \hat{f}^{-1}(\hat{V})$ e $f(x)=$ $\hat{q}(\hat{f}(x))=\hat{q}(\hat{c})=c$, isto é, $x \in \hat{f}^{-1}(\hat{V}) \cap f^{-1}(c)$; reciprocamente, dado $x \in \hat{f}^{-1}(\hat{V}) \cap f^{-1}(c)$, segue que $f(x)=c=(\hat{q} \circ \hat{f})(x)$ e $\hat{f}(x) \in \hat{V}$, o que, pelo fato de $\hat{f}(x)$ ser o único ponto de $\hat{V}$ tal que $\hat{q}(\hat{f}(x))=c$, implica que $x \in \hat{f}^{-1}(\hat{c})=R$ (lembre-se que $\left.\hat{q}\right|_{\hat{V}}$ é um homeomorfismo sobre $V$ ). Isto posto, seja $U$ um subconjunto aberto de $\hat{f}^{-1}(\hat{V})$, contendo $R$; se $f:(M, \partial M) \longrightarrow(N, \partial N)$ for orientável, podemos orientar $U$ utilizando o processo de orientação dado na definição 1.3.6, e então, escolhendo-se uma orientação para $\hat{V}$ (o que é possível, pois, se $V$ é contrátil, $\hat{V}$ é orientável), o número inteiro $\operatorname{deg}_{\hat{c}}\left(\left.\hat{f}\right|_{U}\right)$ estará bem definido. Se, no entanto, $f$ não for orientável, podemos utilizar coeficientes em $\mathbb{Z}_{2}$ para definir o grau local $\operatorname{deg}_{\hat{c}}\left(\left.\hat{f}\right|_{U}\right)$. Da comutatividade do diagrama abaixo (e da proposição 1.3.3), temos ainda que:

$$
\operatorname{deg}_{c}\left(\left.f\right|_{U}\right)=\operatorname{deg}_{c}\left(\left.\hat{q}\right|_{\hat{V}}\right) \cdot \operatorname{deg}_{\left(\left.\hat{q}\right|_{\hat{V}}\right)^{-1}(c)}\left(\left.\hat{f}\right|_{U}\right)=\operatorname{deg}_{c}\left(\left.\hat{q}\right|_{\hat{V}}\right) \cdot \operatorname{deg}_{\hat{c}}\left(\left.\hat{f}\right|_{U}\right)
$$

(note que $\left(\left.\hat{q}\right|_{\hat{V}}\right)^{-1}(c)=\hat{c}$, pois $\left.\hat{q}\right|_{\hat{V}}$ é um homeomorfismo sobre $V$ ).

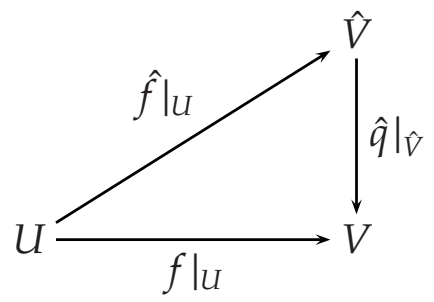


Agora, lembrando que o aberto $V$ é orientável (pois é contrátil), fixemos uma orientação para ele. Se o homeomorfismo $\left.\hat{q}\right|_{\hat{V}}: \hat{V} \longrightarrow V$ preserva orientação, temos que $\operatorname{deg}_{c}\left(\left.\hat{q}\right|_{\hat{V}}\right)=$ 1, e, portanto, $\operatorname{deg}_{c}\left(\left.f\right|_{U}\right)=\operatorname{deg}_{\hat{c}}\left(\left.\hat{f}\right|_{U}\right)$; por outro lado, se $\left.\hat{q}\right|_{\hat{V}}$ inverte orientação, segue que $\operatorname{deg}_{c}\left(\left.\hat{q}\right|_{\hat{V}}\right)=-1$, o que implica que $\operatorname{deg}_{c}\left(\left.f\right|_{U}\right)=-\operatorname{deg}_{\hat{c}}\left(\left.\hat{f}\right|_{U}\right)$. Deste modo, tendo em vista que $|m(R)|=\left|\operatorname{deg}_{c}\left(\left.f\right|_{U}\right)\right|$, obtemos a seguinte descrição alternativa para a multiplicidade de uma classe de raízes:

Teorema 2.1.1. Sendo $M$ e $N$ variedades topológicas conexas de mesma dimensão, considere uma aplicação contínua própria $f:(M, \partial M) \longrightarrow(N, \partial N)$, e, dado $c \in$ Int $N$, seja $R$ uma classe de raízes de $f$ em $c$. Denotando por $\hat{q}: \hat{N} \longrightarrow N$ o recobrimento de $N$ associado ao subgrupo $f_{\#}\left(\pi_{1}\left(M, x_{0}\right)\right) \subseteq \pi_{1}(N, c)$, onde $x_{0} \in f^{-1}(c)$, seja $\hat{f}: M \longrightarrow \hat{N}$ o levantamento de $f$ relativo a $\hat{q}$, que leva o ponto $x_{0} \in M$ na classe de equivalência do caminho constante igual a c (vide lema 1.4.1), e considere um elemento $\hat{c} \in \hat{q}^{-1}(c)$ tal que $R=\hat{f}^{-1}(\hat{c})$. Sendo $V \subseteq \operatorname{Int} N$ uma vizinhança aberta contrátil de $c$, que constitui uma vizinhança elementar em relação ao espaço de recobrimento $\hat{N}$, considere ainda a componente conexa $\hat{V}$ de $\hat{q}^{-1}(V)$ contendo $\hat{c}$, e seja $U$ um subconjunto aberto de $\hat{f}^{-1}(\hat{V})$, contendo R. Então:

$$
|m(R)|=\left|\operatorname{deg}_{\hat{c}}\left(\left.\hat{f}\right|_{U}\right)\right|
$$

onde:

- se f é orientável, $\operatorname{deg}_{\hat{c}}\left(\left.\hat{f}\right|_{u}\right)$ é o grau local com coeficientes em $\mathbb{Z}$, e U é orientado de acordo com o processo de orientação;

- se fé não-orientável, $\operatorname{deg}_{\hat{c}}\left(\left.\hat{f}\right|_{u}\right)$ é o grau local com coeficientes em $\mathbb{Z}_{2}$.

Finalmente, vamos demonstrar o seguinte resultado fundamental:

Teorema 2.1.2. Sejam Me N variedades topológicas conexas de mesma dimensão n, e considere uma aplicação contínua própria $f:(M, \partial M) \longrightarrow(N, \partial N)$. Se $R_{1}$ e $R_{2}$ são classes de raízes de $f$ num ponto $c \in \operatorname{Int} N$, então $\left|m\left(R_{1}\right)\right|=\left|m\left(R_{2}\right)\right|$.

DemonstraçÃo. Com efeito, denotando por $\hat{q}: \hat{N} \longrightarrow N$ o recobrimento de $N$ associado ao subgrupo $f_{\#}\left(\pi_{1}\left(M, x_{0}\right)\right) \subseteq \pi_{1}(N, c)$, onde $x_{0} \in f^{-1}(c)$, e sendo $\hat{f}: M \longrightarrow \hat{N}$ o levantamento de $f$ relativo a $\hat{q}$, que leva o ponto $x_{0} \in M$ na classe de equivalência do caminho constante igual a $c$, considere $\hat{c}_{1}, \hat{c}_{2} \in \hat{q}^{-1}(c) \subseteq \hat{N}$ tais que $R_{1}=\hat{f}^{-1}\left(\hat{c}_{1}\right)$ e $R_{2}=\hat{f}^{-1}\left(\hat{c}_{2}\right)$. Suponha ainda que $M$ e $N$, e conseqüentemente $\hat{N}$, sejam orientáveis (lembre-se que a proposição 1.1.1 nos garante a orientabilidade de $\hat{N}$, uma vez que $N$ é orientável e $\hat{q}: \hat{N} \longrightarrow N$ é um homeomorfismo local). Como $\hat{N}$ é conexo, temos (pela proposição 1.3.4) que $\operatorname{deg}_{\hat{c}_{1}}(\hat{f})=\operatorname{deg}_{\hat{c}_{2}}(\hat{f})$. Considere então uma vizinhança aberta contrátil $V \subseteq \operatorname{Int} N$ de $c$, que constitui uma vizinhança elementar em relação 
ao espaço de recobrimento $\hat{N}$, de modo que as restrições $\left.\hat{q}\right|_{\hat{V}_{1}}$ e $\left.\hat{q}\right|_{\hat{V}_{2}}$ são, portanto, homeomorfismos sobre $V$, onde $\hat{V}_{1}$ e $\hat{V}_{2}$ são as componentes de $\hat{q}^{-1}(V)$ contendo $\hat{c}_{1}$ e $\hat{c}_{2}$, respectivamente. Sendo $U_{1} \subseteq M$ o aberto dado por $U_{1}=\hat{f}^{-1}\left(\hat{V}_{1}\right)$, orientado pela restrição da orientação de $M$, temos que $R_{1} \subseteq U_{1}$, pois $R_{1}=\hat{f}^{-1}\left(\hat{c}_{1}\right)$ e $\hat{c}_{1} \in \hat{V}_{1} ;$ logo, como $\overline{M \backslash U_{1}} \subseteq M \backslash U_{1} \subseteq M \backslash R_{1}=\operatorname{Int}\left(M \backslash R_{1}\right)$, segue (do teorema da excisão em homologia singular) que o homomorfismo $\left(i_{1}\right)_{*}: \mathrm{H}_{n}\left(U_{1}, U_{1} \backslash R_{1}\right) \rightarrow \mathrm{H}_{n}\left(M, M \backslash R_{1}\right)$, induzido pela inclusão $i_{1}:\left(U_{1}, U_{1} \backslash R_{1}\right)=\left(M \backslash\left(M \backslash U_{1}\right),\left(M \backslash R_{1}\right) \backslash\left(M \backslash U_{1}\right)\right) \longrightarrow\left(M, M \backslash R_{1}\right)$, constitui um isomorfismo. Além disso, denotando por $\theta_{U_{1}} \in \mathrm{H}_{n}\left(U_{1}, U_{1} \backslash R_{1}\right)$ e $\theta_{M} \in \mathrm{H}_{n}\left(M, M \backslash R_{1}\right)$ as classes fundamentais, temos (do lema 1.3.1) que $\left(i_{1}\right)_{*}\left(\theta_{U_{1}}\right)=\theta_{M}$.

Agora, lembrando que $R_{1}=\hat{f}^{-1}\left(\hat{c}_{1}\right) \subseteq U_{1}$, note que o seguinte diagrama é comutativo:

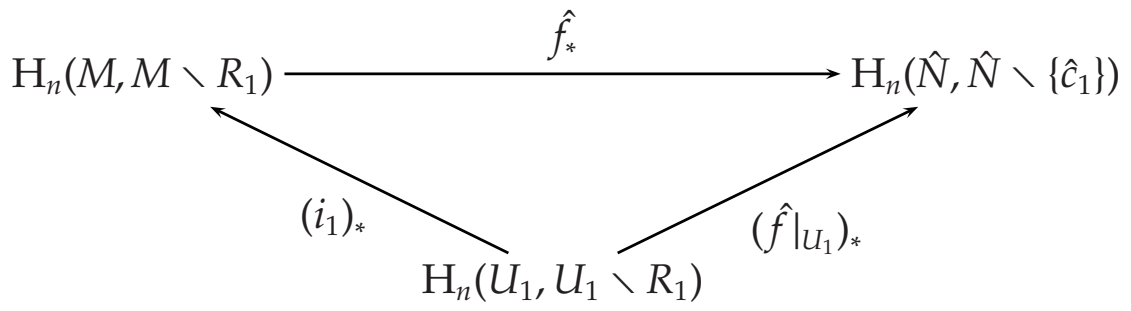

Como $\hat{f}_{*}\left(\theta_{M}\right)=\operatorname{deg}_{\hat{c}_{1}}(\hat{f}) \cdot \theta_{\hat{c}_{1}}$ e $\left(\left.\hat{f}\right|_{U_{1}}\right)_{*}\left(\theta_{U_{1}}\right)=\operatorname{deg}_{\hat{c}_{1}}\left(\left.\hat{f}\right|_{U_{1}}\right) \cdot \theta_{\hat{c}_{1}}$, onde $\theta_{\hat{c}_{1}} \in \mathrm{H}_{n}\left(\hat{N}, \hat{N} \backslash\left\{\hat{c}_{1}\right\}\right)$ é a classe fundamental, segue então que:

$$
\operatorname{deg}_{\hat{c}_{1}}\left(\left.\hat{f}\right|_{U_{1}}\right) \cdot \theta_{\hat{c}_{1}}=\left(\left.\hat{f}\right|_{U_{1}}\right)_{*}\left(\theta_{U_{1}}\right)=\hat{f}_{*}\left(\left(i_{1}\right)_{*}\left(\theta_{U_{1}}\right)\right)=\hat{f}_{*}\left(\theta_{M}\right)=\operatorname{deg}_{\hat{c}_{1}}(\hat{f}) \cdot \theta_{\hat{c}_{1}},
$$

isto é, $\operatorname{deg}_{\hat{c}_{1}}\left(\left.\hat{f}\right|_{U_{1}}\right)=\operatorname{deg}_{\hat{c}_{1}}(\hat{f})$. De maneira análoga, podemos mostar também que o aberto $U_{2}=\hat{f}^{-1}\left(\hat{V}_{2}\right) \subseteq M$, orientado pela restrição da orientação de $M$, satisfaz $\operatorname{deg}_{\hat{c}_{2}}\left(\left.\hat{f}\right|_{U_{2}}\right)=\operatorname{deg}_{\hat{c}_{2}}(\hat{f})$, o que, pelo fato de termos $\operatorname{deg}_{\hat{c}_{1}}(\hat{f})=\operatorname{deg}_{\hat{c}_{2}}(\hat{f}), \operatorname{implica}_{\hat{c}}$ que $\operatorname{deg}_{\hat{c}_{1}}\left(\left.\hat{f}\right|_{U_{1}}\right)=\operatorname{deg}_{\hat{c}_{2}}\left(\left.\hat{f}\right|_{U_{2}}\right)$. Isto posto, considere os seguintes diagramas comutativos:
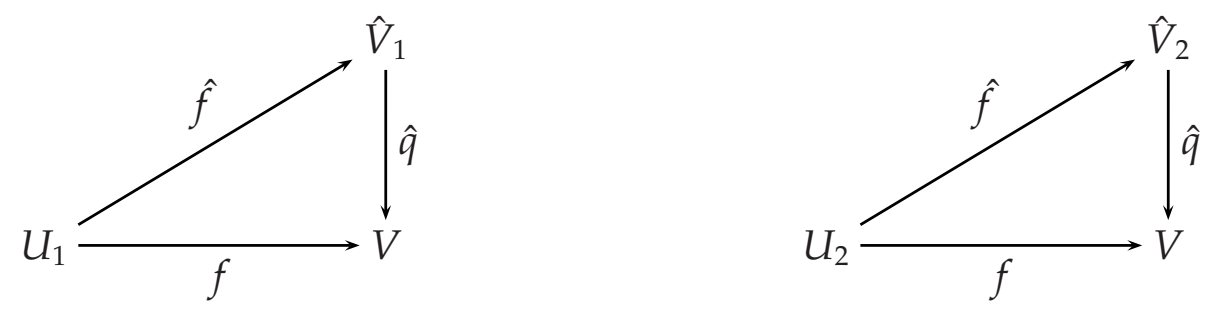

Como $\left(\left.\hat{q}\right|_{\hat{V}_{1}}\right)^{-1}(c)=\hat{c}_{1}$ e $\left(\left.\hat{q}\right|_{\hat{V}_{2}}\right)^{-1}(c)=\hat{c}_{2}$ (pois $\left.\hat{q}\right|_{\hat{V}_{1}}$ e $\left.\hat{q}\right|_{\hat{V}_{2}}$ são homeomorfismos sobre $V$ ), segue, da proposição 1.3.3 e do corolário 1.3.1, que:

$$
\left|\operatorname{deg}_{c}\left(\left.f\right|_{U_{1}}\right)\right|=\left|\operatorname{deg}_{\hat{c}_{1}}\left(\left.\hat{f}\right|_{U_{1}}\right) \cdot \operatorname{deg}_{c}\left(\left.\hat{q}\right|_{\hat{V}_{1}}\right)\right|=\left|\operatorname{deg}_{\hat{c}_{1}}\left(\left.\hat{f}\right|_{U_{1}}\right) \cdot( \pm 1)\right|=\left|\operatorname{deg}_{\hat{c}_{1}}\left(\left.\hat{f}\right|_{U_{1}}\right)\right|
$$

e

$$
\left|\operatorname{deg}_{c}\left(\left.f\right|_{U_{2}}\right)\right|=\left|\operatorname{deg}_{\hat{c}_{2}}\left(\left.\hat{f}\right|_{U_{2}}\right) \cdot \operatorname{deg}_{c}\left(\left.\hat{q}\right|_{\hat{V}_{2}}\right)\right|=\left|\operatorname{deg}_{\hat{c}_{2}}\left(\left.\hat{f}\right|_{U_{2}}\right) \cdot( \pm 1)\right|=\left|\operatorname{deg}_{\hat{c}_{2}}\left(\left.\hat{f}\right|_{U_{2}}\right)\right| .
$$


Logo, como $\operatorname{deg}_{\hat{c}_{1}}\left(\left.\hat{f}\right|_{U_{1}}\right)=\operatorname{deg}_{\hat{c}_{2}}\left(\left.\hat{f}\right|_{U_{2}}\right)$, temos que $\left|\operatorname{deg}_{c}\left(\left.f\right|_{U_{1}}\right)\right|=\left|\operatorname{deg}_{c}\left(\left.f\right|_{U_{2}}\right)\right| ;$ conseqüentemente, observando que $U_{1} \cap f^{-1}(c)=R_{1}$ e $U_{2} \cap f^{-1}(c)=R_{2}$, concluímos que:

$$
\left|m\left(R_{1}\right)\right|=\left|\operatorname{deg}_{c}\left(\left.f\right|_{U_{1}}\right)\right|=\left|\operatorname{deg}_{c}\left(\left.f\right|_{U_{2}}\right)\right|=\left|m\left(R_{2}\right)\right|
$$

(vide definição 1.3.7). Em particular, como toda variedade topológica é orientável quando consideramos coeficientes em $\mathbb{Z}_{2}$, segue que, se $f:(M, \partial M) \longrightarrow(N, \partial N)$ é uma aplicação não-orientável, temos $\left|m\left(R_{1}\right)\right|=\left|m\left(R_{2}\right)\right|$.

Suponhamos agora que pelo menos uma das variedades $M$ ou $N$ é não-orientável, e $f$ é uma aplicação orientável. Consideremos novamente $\hat{c}_{1}, \hat{c}_{2} \in \hat{q}^{-1}(c) \subseteq \hat{N}$ tais que $R_{1}=\hat{f}^{-1}\left(\hat{c}_{1}\right)$ e $R_{2}=\hat{f}^{-1}\left(\hat{c}_{2}\right)$, e, dado um subconjunto euclidiano $E$ em $\operatorname{Int} \hat{N}$ (isto é, um subconjunto $E \subseteq \operatorname{Int} \hat{N}$ homeomorfo ao espaço euclidiano $\mathbb{R}^{n}$ ), tomemos $\hat{b}_{1}, \hat{b}_{2} \in E$ arbitrários. Como $\hat{c}_{1}, \hat{c}_{2} \in \hat{q}^{-1}(c)$ e $c \in \operatorname{Int} N$, temos que $\hat{c}_{1}, \hat{c}_{2} \in \operatorname{Int} \hat{N}$ (lembre-se que $\hat{q}(\partial \hat{N})=\partial N$, pois $\hat{q}$ é um homeomorfismo local); logo, existe um homeomorfismo $h: \hat{N} \longrightarrow \hat{N}$ tal que $h\left(\hat{c}_{1}\right)=\hat{b}_{1}$ e $h\left(\hat{c}_{2}\right)=\hat{b}_{2}$ (vide teorema B.1, do apêndice B). Assim, $S=h(E)$ é um subconjunto aberto de Int $\hat{N}$, homeomorfo ao espaço euclidiano $\mathbb{R}^{n}$, que contém os pontos $\hat{c}_{1}$ e $\hat{c}_{2}$; além disso, como $E$ é homeomorfo a $\mathbb{R}^{n}$, temos que $S=h(E)$ é conexo e contrátil. Isto posto, observe que, como $f$ é uma aplicação orientável, o levantamento $\hat{f}:(M, \partial M) \longrightarrow(\hat{N}, \partial \hat{N})$ também o é (note que $\hat{f}$ preserva fronteira, pois $f(\partial M) \subseteq \partial N$ e $\hat{q}(\partial \hat{N})=\partial N)$ : de fato, sendo $\omega: I \longrightarrow M$ um laço que inverte orientação relativamente ao recobrimento orientado de $M$, e supondo que a imagem $\hat{\omega}=\hat{f} \circ \omega: I \longrightarrow \hat{N}$ seja um laço contrátil (isto é, supondo que $\hat{f}$ seja uma aplicação nãoorientável), temos que $\hat{q} \circ \hat{\omega}=\hat{q} \circ(\hat{f} \circ \omega)=f \circ \omega$ é um laço contrátil em $N$, o que contradiz o fato de $f$ ser uma aplicação orientável. Em particular, isto implica que $\hat{f}^{-1}(S) \subseteq \operatorname{Int} M$ é orientável, pois, como $S$ é contrátil, não existem laços $\omega: I \longrightarrow \hat{f}^{-1}(S)$ que invertem orientação (do contrário $\hat{f}$ não seria orientável, uma vez que a imagem $\hat{f} \circ \omega$ é um laço contrátil em $S$ ). Portanto, $\hat{f}^{-1}(S)$ é uma subvariedade topológica orientável de $\operatorname{Int} M$.

Assim sendo, vamos estender o processo de orientação (dado na definição 1.3.6) para orientar $\hat{f}^{-1}(S)$. Primeiramente, suponhamos que não exista componente conexa de $\hat{f}^{-1}(S)$ que intercepte $R_{1}$ e $R_{2}$ simultaneamente; neste caso, tomando-se dois pontos quaisquer $x_{1} \in R_{1}$ e $x_{2} \in R_{2}$, escolhemos orientações em $x_{1}$ e $x_{2}$, orientamos as componentes de $\hat{f}^{-1}(S)$ que interceptam $R_{1}$ ou $R_{2}$ de acordo com o processo de orientação, e depois orientamos as demais componentes de $\hat{f}^{-1}(S)$ arbitrariamente. Por outro lado, suponhamos agora que $C_{0}$ seja uma componente conexa de $\hat{f}^{-1}(S)$ que intercepta $R_{1}$ e $R_{2}$ simultaneamente; neste caso, escolhemos uma orientação para $C_{0}$, e tomamos dois pontos $x_{1} \in R_{1}$ e $x_{2} \in R_{2}$ tais que $x_{1}, x_{2} \in C_{0}$. Então, orientamos as componentes de $\hat{f}^{-1}(S)$ que interceptam pelo menos uma das classes de raízes $R_{1}$ ou $R_{2}$, estendendo as orientações de $x_{1}$ e $x_{2}$ (obtidas da orientação de $C_{0}$ ) de acordo com o processo de orientação, e depois orientamos as outras componentes de $\hat{f}^{-1}(S)$ arbitrariamente. 
Assim, precisamos mostrar apenas que este procedimento de orientação está bem definido, isto é, precisamos mostrar que, se $C$ é uma componente conexa de $\hat{f}^{-1}(S)$ que intercepta $R_{1}$ e $R_{2}$ simultaneamente, então a sua orientação (obtida pelo procedimento acima) é independente da escolha dos pontos $x_{1}$ ou $x_{2}$.

De fato, dados $x_{1}^{\prime} \in R_{1} \cap C$ e $x_{2}^{\prime} \in R_{2} \cap C$, considere dois caminhos $\alpha_{1}, \alpha_{2}: I \longrightarrow \operatorname{Int} M$, com $\alpha_{1}(0)=x_{1}, \alpha_{1}(1)=x_{1}^{\prime}, \alpha_{2}(0)=x_{2}$ e $\alpha_{2}(1)=x_{2}^{\prime}$, tais que $f \circ \alpha_{1}$ e $f \circ \alpha_{2}$ são laços contráteis no ponto $c$. Sejam ainda $\beta: I \longrightarrow C_{0}$ e $\gamma: I \longrightarrow C$ caminhos tais que $\beta(0)=x_{1}$, $\beta(1)=x_{2}, \gamma(0)=x_{1}^{\prime}$ e $\gamma(1)=x_{2}^{\prime}$; como as orientações em $x_{1}$ e $x_{2}$ são determinadas pela orientação de $C_{0}$, ao estendermos a orientação de $x_{1}$ a $x_{2}$, ao longo de $\beta$, obtemos a mesma orientação em $x_{2}$ (lembre-se que, por ser orientável, todo laço em $C_{0}$ preserva orientação). Por outro lado, utilizando o processo de orientação, as orientações de $x_{1}^{\prime}$ e $x_{2}^{\prime}$ são obtidas estendendo-se as orientações de $x_{1}$ e $x_{2}$ ao longo de $\alpha_{1}$ e $\alpha_{2}$, respectivamente. Isto posto, suponhamos que a orientação de $C$, obtida pelo processo de orientação a partir da orientação de $x_{1}^{\prime}$, seja diferente da orientação de $C$ obtida por este mesmo processo a partir da orientação de $x_{2}^{\prime}$. Neste caso, estendendo-se a orientação de $x_{1}^{\prime}$ ao longo de $\omega$ (de acordo com o que é feito no processo de orientação), a orientação de $x_{2}^{\prime}$ obtida será diferente daquela que obtivemos pelo processo de orientação. Logo, o laço $\lambda: I \longrightarrow$ Int $M$, com base em $x_{2}^{\prime}$, definido por $\lambda=\alpha_{2}^{-1} \vee \beta^{-1} \vee \alpha_{1} \vee \gamma$, será um laço que inverte orientação. Entretanto, como $f \circ \alpha_{1}$ e $f \circ \alpha_{2}$ são laços contráteis em $N$, ambos com base no ponto $c$, segue que $\hat{f} \circ \alpha_{1}$ e $\hat{f} \circ \alpha_{2}$ (e, conseqüentemente, $\hat{f} \circ \alpha_{2}^{-1}$ ) são laços contráteis com base em $\hat{c}_{1}$ e $\hat{c}_{2}$, respectivamente (pois $\hat{f} \circ \alpha_{1}$ é um levantamento de $f \circ \alpha_{1}$, enquanto que $\hat{f} \circ \alpha_{2}$ é um levantamento de $\left.f \circ \alpha_{2}\right)$; portanto, temos que:

$$
[\hat{f} \circ \lambda]=\left[\left(\hat{f} \circ \alpha_{2}^{-1}\right) \vee\left(\hat{f} \circ \beta^{-1}\right) \vee\left(\hat{f} \circ \alpha_{1}\right) \vee(\hat{f} \circ \gamma)\right]=\left[\left(\hat{f} \circ \beta^{-1}\right) \vee(\hat{f} \circ \gamma)\right],
$$

ou seja, o laço $\hat{f} \circ \lambda$ é homotópico a $\left(\hat{f} \circ \beta^{-1}\right) \vee(\hat{f} \circ \gamma)$. Assim, como $\left(\hat{f} \circ \beta^{-1}\right) \vee(\hat{f} \circ \gamma)$ é um laço em $S$ (pois $\left(\hat{f} \circ \beta^{-1}\right) \vee(\hat{f} \circ \gamma)=\hat{f} \circ\left(\beta^{-1} \vee \gamma\right)$, e $\beta^{-1} \vee \gamma$ é um laço em $\hat{f}^{-1}(S)$ ), e como $S$ é contrátil, temos que $\hat{f} \circ \lambda$ é um laço contrátil em Int $\hat{N}$. Resumindo, temos então que $\hat{f}$ leva o laço $\lambda: I \longrightarrow$ Int $M$, que inverte orientação, no laço contrátil $\hat{f} \circ \lambda$, o que evidentemente contradiz o fato de $\hat{f}$ ser uma aplicação orientável. Deste modo, concluímos que a orientação de $C$, obtida pelo processo de orientação a partir da orientação de $x_{1}^{\prime}$, deve ser igual àquela obtida a partir da orientação de $x_{2}^{\prime}$, como queríamos mostrar.

Agora, escolhendo-se uma orientação para $S$, temos a aplicação própria $\hat{f}: \hat{f}^{-1}(S) \longrightarrow$ $S$ entre variedades orientadas; logo, como $S$ é conexa, segue que:

$$
\operatorname{deg}_{\hat{c}_{1}}(\hat{f})=\operatorname{deg}_{\hat{c}_{2}}(\hat{f})
$$

(vide proposição 1.3.4). Sejam, pois, $S_{1}$ e $S_{2}$ vizinhanças abertas de $\hat{c}_{1}$ e $\hat{c}_{2}$ em $S$, respectivamente, tais que $\left.\hat{q}\right|_{S_{1}}$ e $\left.\hat{q}\right|_{S_{2}}$ são homeomorfismos sobre suas respectivas imagens 
(note que tais $S_{1}$ e $S_{2}$ de fato existem, pois $\hat{q}: \hat{N} \longrightarrow N$ é um homeomorfismo local). Considere ainda uma vizinhança euclidiana elementar $V \operatorname{de} c$ em $\hat{q}\left(S_{1}\right) \cap \hat{q}\left(S_{2}\right)$, e sejam $\hat{V}_{1}$ e $\hat{V}_{2}$ as componentes conexas de $\hat{q}^{-1}(V)$ contendo $\hat{c}_{1}$ e $\hat{c}_{2}$, respectivamente, de modo que as restrições $\left.\hat{q}\right|_{\hat{V}_{1}}$ e $\left.\hat{q}\right|_{\hat{V}_{2}}$ são, portanto, homeomorfismos sobre $V$. Sendo $U_{1}=\hat{f}^{-1}\left(\hat{V}_{1}\right)$ e $U_{2}=\hat{f}^{-1}\left(\hat{V}_{2}\right)$, temos então que $\hat{V}_{1} \cup \hat{V}_{2} \subseteq S$, pois:

$$
\hat{V}_{1} \cup \hat{V}_{2} \subseteq \hat{q}^{-1}(V) \subseteq \hat{q}^{-1}\left(\hat{q}\left(S_{1}\right) \cap \hat{q}\left(S_{2}\right)\right)=\hat{q}^{-1}\left(\hat{q}\left(S_{1}\right)\right) \cap \hat{q}^{-1}\left(\hat{q}\left(S_{2}\right)\right)=S_{1} \cap S_{2} \subseteq S .
$$

Logo, $U_{1} \cup U_{2} \subseteq \hat{f}^{-1}\left(\hat{V}_{1}\right) \cup \hat{f}^{-1}\left(\hat{V}_{2}\right)=\hat{f}^{-1}\left(\hat{V}_{1} \cup \hat{V}_{2}\right) \subseteq \hat{f}^{-1}(S)$. Isto posto, consideremos $U_{1}$ e $U_{2}$ orientados pela restrição da orientação de $\hat{f}^{-1}(S)$ (a qual, conforme vimos acima, é obtida pelo processo de orientação), e orientemos $\hat{V}_{1}$ e $\hat{V}_{2}$ pela restrição da orientação de $S$. Então, considerando as aplicações $\left.\hat{f}\right|_{U_{1}}: U_{1} \longrightarrow \hat{V}_{1}$ e $\left.\hat{f}\right|_{U_{2}}: U_{2} \longrightarrow \hat{V}_{2}$, temos (conforme demonstramos anteriormente, para o caso em que $M$ e $N$ eram orientáveis) que $\operatorname{deg}_{\hat{c}_{1}}\left(\left.\hat{f}\right|_{u_{1}}\right)=\operatorname{deg}_{\hat{c}_{1}}(\hat{f})$ e $\operatorname{deg}_{\hat{c}_{2}}\left(\left.\hat{f}\right|_{U_{2}}\right)=\operatorname{deg}_{\hat{c}_{2}}(\hat{f})$. Assim, como $\operatorname{deg}_{\hat{c}_{1}}(\hat{f})=\operatorname{deg}_{\hat{c}_{2}}(\hat{f})$, segue que $\operatorname{deg}_{\hat{c}_{1}}\left(\left.\hat{f}\right|_{U_{1}}\right)=\operatorname{deg}_{\hat{c}_{2}}\left(\left.\hat{f}\right|_{U_{2}}\right)$, o que, pelo teorema 2.1.1, significa que:

$$
\left|m\left(R_{1}\right)\right|=\left|\operatorname{deg}_{\hat{c}_{1}}\left(\left.\hat{f}\right|_{U_{1}}\right)\right|=\left|\operatorname{deg}_{\hat{c}_{2}}\left(\hat{f} \mid u_{2}\right)\right|=\left|m\left(R_{2}\right)\right| .
$$

Deste modo, concluímos que, se pelo menos uma das variedades $M$ ou $N$ é nãoorientável, e $f:(M, \partial M) \longrightarrow(N, \partial N)$ é uma aplicação orientável, também temos $\left|m\left(R_{1}\right)\right|=\left|m\left(R_{2}\right)\right|$, o que encerra a demonstração.

Na seção 2.3, veremos que os números de Nielsen de raízes, de uma aplicação contínua própria $f:(M, \partial M) \longrightarrow(N, \partial N)$ num ponto $c \in \operatorname{Int} N$, são definidos a partir das multiplicidades das classes de raízes de $f$ em $c$; conseqüentemente, uma vez conhecida a multiplicidade de uma classe de raízes $R$ de $f$ em $c$, o teorema acima nos permite calcular estes números diretamente de suas definições, uma vez que toda classe de raízes de $f$ em $c$ possui a mesma multiplicidade. Assim, tendo como objetivo o cálculo dos números de Nielsen, veremos agora como determinar $|m(R)|$.

\section{2 (1) Cålcullo da Munltiplicidladle dle unma Classe de Rai̊zes}

No que segue, dada uma aplicação contínua própria $f:(M, \partial M) \longrightarrow(N, \partial N)$ (onde $M$ e $N$ serão consideradas variedades topológicas conexas de dimensão $n$ ), continuaremos a denotar por $\hat{q}: \hat{N} \longrightarrow N$ o recobrimento de $N$ associado ao subgrupo $f_{\#}\left(\pi_{1}\left(M, x_{0}\right)\right) \subseteq$ $\pi_{1}(N, c)$, onde $c \in \operatorname{Int} N$ e $x_{0} \in f^{-1}(c)$, sendo que $\hat{f}: M \longrightarrow \hat{N}$ denotará o levantamento de $f$, relativamente a $\hat{q}$, que leva o ponto $x_{0} \in M$ na classe de equivalência do caminho 
constante igual a $c$ (cuja existência é garantida pelo lema 1.2.1). Lembramos ainda (conforme já mencionado anteriormente) que $\hat{q}(\partial \hat{N})=\partial N$ e $\hat{f}(\partial M) \subseteq \partial \hat{N}$; além disso, note que $\hat{f}$ é uma aplicação própria, pois, dado $K \subseteq \hat{N}$ compacto, temos que:

$$
K \subseteq \hat{q}^{-1}(\hat{q}(K)) \Longrightarrow \hat{f}^{-1}(K) \subseteq \hat{f}^{-1}\left(\hat{q}^{-1}(\hat{q}(K))\right)=\left((\hat{q} \circ \hat{f})^{-1} \circ \hat{q}\right)(K)=\left(f^{-1} \circ \hat{q}\right)(K),
$$

isto é, $\hat{f}^{-1}(K)$ é um subconjunto fechado de $M$, contido no compacto $\left(f^{-1} \circ \hat{q}\right)(K)$.

Proposição 2.2.1 . Se $f:(M, \partial M) \longrightarrow(N, \partial N)$ é uma aplicação contínua própria do tipo III, então, para toda classe de raízes $R$ de $f$ num ponto $c \in \operatorname{Int} N$, temos que:

$$
|m(R)|=\operatorname{deg}(\hat{f}, 2) \in \mathbb{Z}_{2},
$$

onde $\operatorname{deg}(\hat{f}, 2)$ é o grau cohomológico mod 2 da aplicação própria $\hat{f}:(M, \partial M) \longrightarrow(\hat{N}, \partial \hat{N})$.

DemonstraçÃo. De fato, seja $R$ uma classe de raízes de $f$ num ponto $c \in \operatorname{Int} N$, e considere $\hat{c} \in \hat{q}^{-1}(c) \subseteq \operatorname{Int} \hat{N}$ tal que $R=\hat{f}^{-1}(\hat{c})$. Utilizando coeficientes em $\mathbb{Z}_{2}$ para orientar $M$ e $\hat{N}$, temos (pelo teorema 1.4.2) que $\operatorname{deg}(\hat{f}, 2)=\operatorname{deg}_{\hat{c}}\left(\left.\hat{f}\right|_{W}\right)$, onde $W=$ $M \backslash f^{-1}(\partial \hat{N})$ e $\operatorname{deg}_{\hat{c}}\left(\left.\hat{f}\right|_{W}\right)$ é o grau local de $\left.\hat{f}\right|_{W}: W \longrightarrow \operatorname{Int} \hat{N}$ em $\hat{c}$ a coeficientes em $\mathbb{Z}_{2}$. Isto posto, sendo $V \subseteq \operatorname{Int} N$ uma vizinhança aberta contrátil de $c$, que constitui uma vizinhança elementar em relação ao espaço de recobrimento $\hat{N}$, considere a componente conexa $\hat{V}$ de $\hat{q}^{-1}(V)$ contendo $\hat{c}$, e seja $U$ um subconjunto aberto de $\hat{f}^{-1}(\hat{V})$, contendo R. Pelo teorema 2.1.1, temos então que $|m(R)|=\left|\operatorname{deg}_{\hat{c}}\left(\left.\hat{f}\right|_{U}\right)\right|=\operatorname{deg}_{\hat{c}}\left(\left.\hat{f}\right|_{U}\right) \in \mathbb{Z}_{2}$, onde $\operatorname{deg}_{\hat{c}}\left(\left.\hat{f}\right|_{U}\right)$ é o grau local com coeficientes em $\mathbb{Z}_{2}$. Agora, note que $\hat{V} \subseteq \operatorname{Int} \hat{N}$, pois $V \subseteq \operatorname{Int} N$ e $\hat{V} \subseteq \hat{q}^{-1}(V)$ (lembre-se que $\left.\hat{q}(\partial \hat{N})=\partial N\right)$; conseqüentemente, temos que:

$$
\hat{f}^{-1}(\hat{V}) \subseteq \hat{f}^{-1}(\operatorname{Int} \hat{N})=\hat{f}^{-1}(\hat{N} \backslash \partial \hat{N})=\hat{f}^{-1}(\hat{N}) \backslash \hat{f}^{-1}(\partial \hat{N})=M \backslash \hat{f}^{-1}(\partial \hat{N})=W,
$$

e, portanto, $R \subseteq U \subseteq \hat{f}^{-1}(\hat{V}) \subseteq W$. Assim, como $\overline{W \backslash U}=W \backslash U \subseteq W \backslash R=\operatorname{Int}(W \backslash$ $R$ ), segue (do teorema da excisão em homologia singular) que o homomorfismo $i_{*}$ : $\mathrm{H}_{n}(U, U \backslash R) \longrightarrow \mathrm{H}_{n}(W, W \backslash R)$, induzido pela inclusão $i:(U, U \backslash R)=(W \backslash(W \backslash$ $U),(W \backslash R) \backslash(W \backslash U)) \longrightarrow(W, W \backslash R)$, constitui um isomorfismo. Além disso, denotando por $\theta_{U} \in \mathrm{H}_{n}(U, U \backslash R)$ e $\theta_{W} \in \mathrm{H}_{n}(W, W \backslash R)$ as classes fundamentais, temos (do lema 1.3.1) que $i_{*}\left(\theta_{U}\right)=\theta_{W}$. Agora, lembrando que $R=\hat{f}^{-1}(\hat{c}) \subseteq U$, observe que o seguinte diagrama é comutativo:

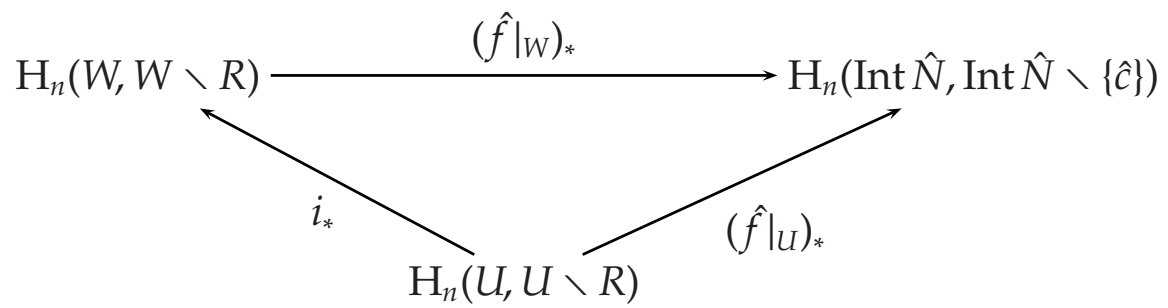

Então, como $\left(\left.\hat{f}\right|_{W}\right)_{*}\left(\theta_{W}\right)=\operatorname{deg}_{\hat{c}}\left(\left.\hat{f}\right|_{W}\right) \cdot \theta_{\hat{c}}$ e $\left(\left.\hat{f}\right|_{U}\right)_{*}\left(\theta_{U}\right)=\operatorname{deg}_{\hat{c}}\left(\left.\hat{f}\right|_{U}\right) \cdot \theta_{\hat{c}}$, onde $\theta_{\hat{c}} \in$ 
$\mathrm{H}_{n}(\operatorname{Int} \hat{N}, \operatorname{Int} \hat{N} \backslash\{\hat{c}\})$ é a classe fundamental, obtemos que:

$$
\operatorname{deg}_{\hat{c}}\left(\left.\hat{f}\right|_{U}\right) \cdot \theta_{\hat{c}}=\left(\left.\hat{f}\right|_{U}\right)_{*}\left(\theta_{U}\right)=\left(\left.\hat{f}\right|_{W}\right)_{*}\left(i_{*}\left(\theta_{U}\right)\right)=\left(\left.\hat{f}\right|_{W}\right)_{*}\left(\theta_{W}\right)=\operatorname{deg}_{\hat{c}}\left(\left.\hat{f}\right|_{W}\right) \cdot \theta_{\hat{c}},
$$

isto é, $\operatorname{deg}_{\hat{c}}\left(\left.\hat{f}\right|_{U}\right)=\operatorname{deg}_{\hat{c}}\left(\left.\hat{f}\right|_{W}\right)$. Portanto, $\operatorname{comodeg}(\hat{f}, 2)=\operatorname{deg}_{\hat{c}}\left(\left.\hat{f}\right|_{W}\right) \mathrm{e}|m(R)|=\operatorname{deg}_{\hat{c}}\left(\left.\hat{f}\right|_{U}\right)$, segue que $|m(R)|=\operatorname{deg}(\hat{f}, 2)$, conforme queríamos provar.

Proposição 2.2.2 . Seja $f:(M, \partial M) \longrightarrow(N, \partial N)$ uma aplicação contínua própria, e, dado $c \in \operatorname{Int} N$, denote por $\mathrm{j}$ a cardinalidade de $\hat{q}^{-1}(c)$ [isto é, $\mathrm{j}$ é a cardinalidade do grupo quociente $\left.\pi_{1}(N, c) / f_{\#}\left(\pi_{1}\left(M, x_{0}\right)\right)\right]$. Então, se jé infinito, temos que $|m(R)|=0$ para toda classe de raízes $R$ de $f$ em $c$.

DemonstraÇÃo. Com efeito, seja $R_{1}$ uma classe de raízes de $f$ em $c$, $\operatorname{com} R_{1}=\hat{f}^{-1}\left(\hat{c}_{1}\right)$ para algum $\hat{c}_{1} \in \hat{q}^{-1}(c)$, e, dada uma vizinhança aberta contrátil $V \subseteq \operatorname{Int} N$ de $c$, que constitui uma vizinhança elementar em relação ao espaço de recobrimento $\hat{N}$, seja $\hat{V}_{1}$ a componente conexa de $\hat{q}^{-1}(V)$ que contém $\hat{c}_{1}$. Isto posto, supondo $\left|m\left(R_{1}\right)\right| \neq 0$, o teorema 2.1.1 nos garante que $\operatorname{deg}_{\hat{c}_{1}}\left(\left.\hat{f}\right|_{U_{1}}\right) \neq 0$, onde $U_{1}=\hat{f}^{-1}\left(\hat{V}_{1}\right) \supseteq R_{1}$. Conseqüentemente, dado outro ponto $\hat{c}_{2} \in \hat{q}^{-1}(c)$, e sendo $\hat{V}_{2}$ a componente conexa de $\hat{q}^{-1}(V)$ contendo $\hat{c}_{2}$, a demonstração do teorema 2.1.2 nos mostra que $\operatorname{deg}_{\hat{c}_{2}}\left(\left.\hat{f}\right|_{U_{2}}\right) \neq 0$, onde $U_{2}=\hat{f}^{-1}\left(\hat{V}_{2}\right)$ (pois $\operatorname{deg}_{\hat{c}_{1}}\left(\left.\hat{f}\right|_{U_{1}}\right)=\operatorname{deg}_{\hat{c}_{2}}\left(\left.\hat{f}\right|_{U_{2}}\right)$ ). Ora, se $\operatorname{deg}_{\hat{c}_{2}}\left(\left.\hat{f}\right|_{U_{2}}\right) \neq 0$, temos que $\hat{f}^{-1}\left(\hat{c}_{2}\right) \neq \varnothing$ (uma vez que $\hat{f}^{-1}\left(\hat{c}_{2}\right)=\varnothing$ implica $\operatorname{deg}_{\hat{c}_{2}}\left(\left.\hat{f}\right|_{U_{2}}\right)=0$ ); logo, pela proposição 2.1.2, o conjunto $R_{2}=\hat{f}^{-1}\left(\hat{c}_{2}\right)$ é uma classe de raízes de $f$ em $c$. Portanto, se existe uma classe de raízes de $f$ em $c$, cuja multiplicidade é não-nula, então, para todo $\hat{c} \in \hat{q}^{-1}(c)$, existe uma classe de raízes $R$ de $f$ em $c$ tal que $R=\hat{f}^{-1}(\hat{c})$ (note que esta conclusão é a recíproca do corolário 2.1.1). Neste caso, temos que o número de classes de raízes de $f$ em $c$ é igual à cardinalidade de $\hat{q}^{-1}(c)$, isto é, j. Entretanto, sabemos que $f^{-1}(c)$ é a união disjunta das classes de raízes de $f$ em $c$, as quais, por serem subconjuntos abertos de $f^{-1}(c)$ (proposição 1.3.1), constituem uma cobertura aberta de $f^{-1}(c)$. Assim, como $f^{-1}(c)$ é compacto (pois $f$ é própria), segue que a quantidade de classes de raízes de $f$ em $c$ é finita, o que significa que j é finito. Deste modo, podemos concluir que, se j é infinito, então $|m(R)|=0$ para toda classe de raízes $R$ de $f$ em $c$.

Como conseqüência das proposições 2.2.1 e 2.2.2, podemos agora nos restringir ao cálculo de $|m(R)|$ nos casos em que $f:(M, \partial M) \longrightarrow(N, \partial N)$ é orientável e a cardinalidade j de $\hat{q}^{-1}(c)$ é finita.

Doravante, dada uma aplicação contínua própria $f:(M, \partial M) \longrightarrow(N, \partial N)$ e um ponto $c \in \operatorname{Int} N$, vamos utilizar (no restante desta seção e ao longo da próxima) os espaços de recobrimento representados na figura 2.2 abaixo: 


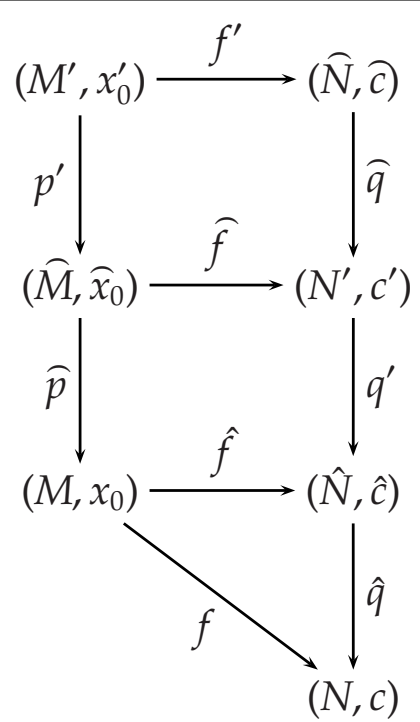

Figura 2.2

Os espaços e aplicações ilustrados acima são obtidos da seguinte maneira:

- $\widehat{p}: \widehat{M} \longrightarrow M$ é o recobrimento orientado de $M$;

- $q^{\prime}: N^{\prime} \longrightarrow \hat{N}$ é o recobrimento de $\hat{N}$ associado ao subgrupo $(\hat{f} \circ \hat{p})_{\#}\left(\pi_{1}\left(\widehat{M}, \widehat{x}_{0}\right)\right) \subseteq$ $\pi_{1}(\hat{N}, \hat{c})$, e $\hat{f}: \widehat{M} \longrightarrow N^{\prime}$ é um levantamento de $\hat{f} \circ \hat{p}$ relativamente a $q^{\prime}$ (vide lema 1.2.1);

- $\widehat{q}: \widehat{N} \longrightarrow N^{\prime}$ é o recobrimento orientado de $N^{\prime}$;

- $p^{\prime}: M^{\prime} \longrightarrow \widehat{M}$ é o pullback de $\widehat{q}: \widehat{N} \longrightarrow N^{\prime}$ via $\widehat{f}$ (isto é, $M^{\prime}=\{(\widehat{x}, \widehat{y}) \in \widehat{M} \times \widehat{N}$ : $\widehat{q}(\widehat{y})=\widehat{f}(\widehat{x})\}$ e $p^{\prime}: M^{\prime} \longrightarrow \widehat{M}$ é tal que $p^{\prime}(\widehat{x}, \widehat{y})=\widehat{x}$ para cada $\left.(\widehat{x}, \widehat{y}) \in M^{\prime}\right)$, enquanto que $f^{\prime}: M^{\prime} \longrightarrow \widehat{N}$ é a aplicação dada por $f^{\prime}(\widehat{x}, \widehat{y})=\widehat{y}$ para cada $(\widehat{x}, \widehat{y}) \in M^{\prime}$; note que $p^{\prime}$ é um recobrimento de $\widehat{M}$, pois, tomando-se $\widehat{x} \in \widehat{M}$ arbitrário, e sendo $V^{\prime} \subseteq N^{\prime}$ uma vizinhança elementar de $\widehat{f}(\widehat{x})$ relativamente ao recobrimento $\widehat{q}: \widehat{N} \longrightarrow N^{\prime}$, temos que $\widehat{U}=\widehat{f}^{-1}(\widehat{V}) \subseteq \widehat{M}$ é uma vizinhança elementar de $\widehat{x}$ relativamente a $p^{\prime}$; por outro lado, como $\left(\hat{q} \circ f^{\prime}\right)(\widehat{x}, \widehat{y})=\widehat{q}(\widehat{y})=\widehat{f}(\widehat{x})=\left(\widehat{f} \circ p^{\prime}\right)(\widehat{x}, \widehat{y})$ para todo $(\widehat{x}, \widehat{y}) \in M^{\prime}$, segue que $f^{\prime}: M^{\prime} \longrightarrow \widehat{N}$ é um levantamento de $\widehat{f} \circ p^{\prime}: M^{\prime} \longrightarrow N^{\prime}$ relativamente ao recobrimento $\widehat{q}: \widehat{N} \longrightarrow N^{\prime}$.

Observe ainda que:

(i) o fato de $M, N$ e $\hat{N}$ serem variedades topológicas conexas de dimensão $n$, conexas e com fronteira, nos garante que os demais espaços $\widehat{M}, \widehat{N}, M^{\prime}$ e $N^{\prime}$ também o são, uma vez que $\hat{p}: \widehat{M} \longrightarrow M, q^{\prime}: N^{\prime} \longrightarrow \hat{N}, p^{\prime}: M^{\prime} \longrightarrow \widehat{M}$ e $\widehat{q}: \widehat{N} \longrightarrow N^{\prime}$ são homeomorfismos locais; 
(ii) como $\hat{p}, \widehat{q}, p^{\prime}$ e $q^{\prime}$ são homeomorfismos locais, segue (do teorema da invariância do domínio) que $\widehat{p}(\partial \widehat{M})=\partial M, q^{\prime}\left(\partial N^{\prime}\right)=\partial \hat{N}, p^{\prime}\left(\partial M^{\prime}\right)=\partial \widehat{M}$ e $\widehat{q}(\partial \widehat{N})=\partial N^{\prime}$, e, conseqüentemente, como $\hat{f}: M \longrightarrow \hat{N}$ preserva fronteira, temos $\widehat{f}(\partial \widehat{M}) \subseteq \partial N^{\prime}$ e $f^{\prime}\left(\partial M^{\prime}\right) \subseteq \partial \widehat{N}$ (isto é, $\widehat{f}$ e $f^{\prime}$ também preservam fronteira);

(iii) as aplicações $\widehat{f}:(\widehat{M}, \partial \widehat{M}) \longrightarrow\left(N^{\prime}, \partial N^{\prime}\right)$ e $f^{\prime}:\left(M^{\prime}, \partial M^{\prime}\right) \longrightarrow(\widehat{N}, \partial \widehat{N})$ são próprias (assim como $f$ e $\hat{f}$ ): de fato, dados $K^{\prime} \subseteq N^{\prime}$ e $\widehat{K} \subseteq \widehat{N}$ compactos, temos que:

$$
\begin{array}{r}
K^{\prime} \subseteq q^{-1}\left(q^{\prime}\left(K^{\prime}\right)\right) \Longrightarrow \widehat{f}^{-1}\left(K^{\prime}\right) \subseteq \widehat{f}^{-1}\left(q^{-1}\left(q^{\prime}\left(K^{\prime}\right)\right)\right)=\left(q^{\prime} \circ \widehat{f}\right)^{-1}\left(q^{\prime}\left(K^{\prime}\right)\right) \\
=(\hat{f} \circ \hat{p})^{-1}\left(q^{\prime}\left(K^{\prime}\right)\right)=\hat{p}^{-1}\left(\hat{f}^{-1}\left(q^{\prime}\left(K^{\prime}\right)\right)\right)
\end{array}
$$

$\mathrm{e}$

$$
\begin{aligned}
\widehat{K} \subseteq \widehat{q}^{-1}(\widehat{q}(\widehat{K})) \Longrightarrow f^{\prime-1}(\widehat{K}) \subseteq & f^{\prime-1}\left(\widehat{q}^{-1}(\widehat{q}(\widehat{K}))\right)=\left(\widehat{q} \circ f^{\prime}\right)^{-1}(\widehat{q}(\widehat{K})) \\
& =\left(\widehat{f} \circ p^{\prime}\right)^{-1}(\widehat{q}(\widehat{K}))=p^{\prime-1}\left(\hat{f}^{-1}(\widehat{q}(\widehat{K}))\right),
\end{aligned}
$$

o que implica que $\widehat{f}^{-1}\left(K^{\prime}\right)$ é compacto (pois é um subconjunto fechado do compacto $\left.\widehat{p}^{-1}\left(\hat{f}^{-1}\left(q^{\prime}\left(K^{\prime}\right)\right)\right) \subseteq \widehat{M}\right)$, ou seja, $\widehat{f}$ é própria; daí, segue que $p^{-1}\left(\widehat{f}^{-1}(\widehat{q}(\widehat{K}))\right)$ é compacto, e, portanto, $f^{\prime-1}\left(K^{\prime}\right)$ também o é (pois constitui um subconjunto fechado $\left.\operatorname{de} p^{\prime-1}\left(\widehat{f}^{-1}(\widehat{q}(\widehat{K}))\right) \subseteq M^{\prime}\right) ;$

(iv) as variedades $\widehat{M}, \widehat{N}$ e $M^{\prime}$ são sempre orientáveis (independentemente da orientabilidade de $M$ e $N)$, de maneira que o grau cohomológico $\operatorname{deg}\left(f^{\prime}\right) \in \mathbb{Z}$ da aplicação própria $f^{\prime}:\left(M^{\prime}, \partial M^{\prime}\right) \longrightarrow(\widehat{N}, \partial \widehat{N})$ encontra-se bem definido (vide definição 1.4.4).

Proposição 2.2.3 . Seja $f:(M, \partial M) \longrightarrow(N, \partial N)$ uma aplicação contínua própria e orientável, $e$, dado $c \in \operatorname{Int} N$, suponha que a cardinalidade $\mathrm{j}$ de $\hat{q}^{-1}(c)$ é finita. Então, para toda classe de raízes $R$ de $f$ em $c$, temos:

$$
|m(R)|=\left|\operatorname{deg}\left(f^{\prime}\right)\right|,
$$

onde $\operatorname{deg}\left(f^{\prime}\right)$ é o grau cohomológico de $f^{\prime}:\left(M^{\prime}, \partial M^{\prime}\right) \longrightarrow(\widehat{N}, \partial \widehat{N})$.

DemonstraçÃo. Dada uma classe de raízes $R$ de $f$ em $c \in \operatorname{Int} N$, seja $V \subseteq \operatorname{Int} N$ uma vizinhança aberta contrátil de $c$, que constitui uma vizinhança elementar em relação ao espaço de recobrimento $\hat{N}$, e considere a componente conexa $\hat{V}$ de $\hat{q}^{-1}(V)$ que contém $\hat{c}$, de modo que a restrição $\left.\hat{q}\right|_{\hat{V}}: \hat{V} \longrightarrow V$ é, portanto, um homeomorfismo sobre $V$ (onde $\hat{c} \in \hat{q}^{-1}(c)$ é tal que $\left.R=\hat{f}^{-1}(\hat{c})\right)$. Seja ainda $U \subseteq \hat{f}^{-1}(\hat{V})$ um aberto tal que $U \cap f^{-1}(c)=R$, e considere a orientação $s: U \longrightarrow \widehat{M}$ de $U$, induzida no recobrimento orientado de $M$ pela orientação de $U$, obtida pelo processo de orientação a partir de um ponto $x_{R} \in R \subseteq U$ (vide definição 1.3.6). Definindo $\widehat{U}=s(U), \widehat{R}=s(R)$ e $\widehat{x}_{R}=s\left(x_{R}\right)$, temos então que $c^{\prime}=\widehat{f}\left(\widehat{x}_{R}\right) \in N^{\prime}$ satisfaz $c^{\prime} \in q^{\prime-1}(\hat{c})$, uma vez que:

$$
q^{\prime}\left(c^{\prime}\right)=q^{\prime}\left(\widehat{f}\left(\widehat{x}_{R}\right)\right)=\hat{f}\left(\widehat{p}\left(\widehat{x}_{R}\right)\right)=\hat{f}\left(x_{R}\right)=\hat{c}
$$


(observe que $\hat{f}\left(x_{R}\right)=\hat{c}$ qualquer que seja $x_{R} \in R$, pois $R=\hat{f}^{-1}(\hat{c})$ ). Isto posto, suponhamos que $\hat{V}$ seja uma vizinhança elementar em relação ao espaço de recobrimento $N^{\prime}$, e consideremos a componente conexa $V^{\prime}$ de $q^{\prime-1}(\hat{V})$ contendo $c^{\prime}$ (de maneira que a restrição $\left.q^{\prime}\right|_{V^{\prime}}: V^{\prime} \longrightarrow \hat{V}$ é, portanto, um homeomorfismo sobre $\left.\hat{V}\right)$. Note que $\widehat{f}(\widehat{U}) \subseteq V^{\prime}$ : de fato, como $\hat{f}(U) \subseteq \hat{V}$ e $\hat{f}(U)=\left(q^{\prime} \circ \widehat{f} \circ s\right)(U)=\left(q^{\prime} \circ \widehat{f}\right)(\hat{U})$, temos que $\left(q^{\prime} \circ \widehat{f}\right)(\widehat{U}) \subseteq \hat{V}$, e, conseqüentemente, como $q^{-1}(\hat{V})$ é uma união disjunta de abertos em $N^{\prime}$, cada um dos quais é levado homeomorficamente por $q^{\prime}$ sobre $\hat{V}$, segue que cada componente conexa que compõe $q^{-1}(\hat{V})$ contém uma cópia homeomorfa de $\left(q^{\prime} \circ \widehat{f}\right)(\widehat{U})$, sendo que uma delas coincide com $\widehat{f}(\widehat{U})$; assim, como $c^{\prime} \in \widehat{f}(\widehat{U})$ (pois $c^{\prime}=\widehat{f}\left(\widehat{x}_{R}\right)$ e $\widehat{x}_{R} \in \widehat{U}$ ), podemos concluir que a componente de $q^{-1}(\hat{V})$ que contém $\widehat{f}(\widehat{U})$ é $V^{\prime}$, isto é, $\widehat{f}(\widehat{U}) \subseteq V^{\prime}$. Deste modo, tomando $\widehat{S}=\widehat{f}^{-1}\left(c^{\prime}\right)$, vamos provar que $\widehat{S}=\widehat{R}$.

Primeiramente, a fim de mostrarmos que $\widehat{R} \subseteq \widehat{S}$, observe que $\widehat{x}_{R} \in \widehat{S}$ (uma vez que $\left.\widehat{f}\left(\widehat{x}_{R}\right)=c^{\prime}\right)$; considere, pois, um outro ponto qualquer $\widehat{x} \in \widehat{R}$, e seja $x=\widehat{p}(\widehat{x}) \in M$. Como $\widehat{R}=s(R)$, temos que $x \in \widehat{p}(\widehat{R})=\widehat{p}(s(R))=R$. Além disso, como $s: U \longrightarrow \widehat{M}$ é a induzida em $\widehat{M}$ pela orientação de $U$ obtida pelo processo de orientação, segue que $\widehat{x}=s(x)$ é definido da seguinte forma: dado um caminho $\omega: I \longrightarrow M$ ligando $x_{R}$ a $x$, tal que $f \circ \omega: I \longrightarrow N$ é um laço contrátil com base no ponto $c$, tomamos $\widehat{x}$ como sendo a extensão da orientação $\widehat{x}_{R}$ de $x_{R}$ ao longo de $\omega$, isto é, $\widehat{x}=\widehat{\omega}(1)$, onde $\widehat{\omega}: I \longrightarrow \widehat{M}$ é o único levantamento de $\omega$ (relativamente ao recobrimento orientado $\widehat{p}: \widehat{M} \longrightarrow M$ ) tal que $\widehat{\omega}(0)=\widehat{x}_{R}$ (para mais detalhes, vide definição 1.3.6). Isto posto, como

$$
\left(\hat{q} \circ q^{\prime}\right) \circ(\widehat{f} \circ \widehat{\omega})=\hat{q} \circ\left(q^{\prime} \circ \widehat{f}\right) \circ \widehat{\omega}=\hat{q} \circ(\hat{f} \circ \hat{p}) \circ \widehat{\omega}=(\hat{q} \circ \hat{f}) \circ(\hat{p} \circ \widehat{\omega})=f \circ \omega,
$$

segue que $\hat{f} \circ \widehat{\omega}: I \longrightarrow N^{\prime}$ é um levantamento de $f \circ \omega: I \longrightarrow N$, relativamente ao recobrimento $\hat{q} \circ q^{\prime}: N^{\prime} \longrightarrow N$ (note que $\hat{q} \circ q^{\prime}$ é de fato um recobrimento de $N$, conforme se verifica em [Li], corolário da proposição 12, página 139). Assim, como $f \circ \omega$ é um laço contrátil, temos que $\widehat{f} \circ \widehat{\omega}$ também o é, o que, em particular, implica que:

$$
c^{\prime}=\widehat{f}\left(\widehat{x}_{R}\right)=(\widehat{f} \circ \widehat{\omega})(0)=(\widehat{f} \circ \widehat{\omega})(1)=\widehat{f}(\widehat{x}),
$$

isto é, $\widehat{x} \in \widehat{f}^{-1}\left(c^{\prime}\right)=\widehat{S}$.

Agora, vamos mostrar que $\widehat{S} \subseteq \widehat{R}$. Para tanto, suponhamos (por absurdo) que exista um ponto $\widehat{x} \in \widehat{S}$ tal que $\widehat{x} \notin \widehat{S}$. Como $\widehat{x} \in \widehat{S}=\widehat{f}^{-1}\left(c^{\prime}\right)$ e $c^{\prime} \in q^{-1}(\hat{c})$, segue que $(\hat{f} \circ \hat{p})(\hat{x})=\left(q^{\prime} \circ \widehat{f}\right)(\widehat{x})=q^{\prime}\left(c^{\prime}\right)=\hat{c}$, ou seja, o ponto $x=\widehat{p}(\widehat{x}) \in M$ é tal que $x \in \hat{f}^{-1}(\hat{c})=R$; logo, existe um caminho $\omega: I \longrightarrow M$, ligando $x_{R}$ a $x$, tal que $f \circ \omega: I \longrightarrow N$ é um laço contrátil em $c$. Seja, pois, $\omega^{-1}: I \longrightarrow M$ o caminho inverso de $\omega$ (ligando $x$ a $x_{R}$ ), e considere o levantamento $\widehat{\omega}^{-1}: I \longrightarrow \widehat{M}$ de $\omega^{-1}$ (relativamente ao recobrimento orientado $\hat{p}: \widehat{M} \longrightarrow M$ ), com $\widehat{\omega}^{-1}(0)=\widehat{x}$. Temos então que o ponto $\widehat{x}_{R}^{\prime}=\widehat{\omega}^{-1}(1) \in \widehat{M}$ é tal que $\widehat{x}_{R}^{\prime} \in \widehat{p}^{-1}\left(x_{R}\right)$, pois $\widehat{p}\left(\widehat{x}_{R}^{\prime}\right)=\widehat{p}\left(\widehat{\omega}^{-1}(1)\right)=\omega^{-1}(1)=x_{R}$. Entretanto, note que $\widehat{x}_{R}^{\prime} \neq \widehat{x}_{R}$ : 
com efeito, sendo $\widehat{\omega}=\left(\widehat{\omega}^{-1}\right)^{-1}: I \longrightarrow \widehat{M}$ o inverso do levantamento $\widehat{\omega}^{-1}$ de $\omega^{-1}$, temos que:

$$
(\widehat{p} \circ \widehat{\omega})(t)=\left[\widehat{p} \circ\left(\widehat{\omega}^{-1}\right)^{-1}\right](t)=\left(\widehat{p} \circ \widehat{\omega}^{-1}\right)(1-t)=\omega^{-1}(1-t)=\omega(t)
$$

para todo $t \in I$, ou seja, $\widehat{\omega}$ é um levantamento de $\omega$ (relativamente ao recobrimento orientado $\hat{p})$, que satisfaz $\widehat{\omega}(0)=\left(\widehat{\omega}^{-1}\right)^{-1}(0)=\widehat{\omega}^{-1}(1)=\widehat{x}_{R}^{\prime}$ e $\widehat{\omega}(1)=\left(\widehat{\omega}^{-1}\right)^{-1}(1)=\widehat{\omega}^{-1}(0)=$ $\widehat{x} ; \operatorname{logo}$, se $\widehat{x}_{R}^{\prime}=\widehat{x}_{R}$, teríamos que $\widehat{\omega}(0)=\widehat{x}_{R}$, isto é, $\widehat{x}=\widehat{\omega}(1)$ coincidiria com a orientação $s(x)$ de $x$, obtida pelo processo de orientação a partir de $\widehat{x}_{R}$, o que mostraria que $\widehat{x}=s(x) \in s(R)=\widehat{R}$, contradizendo nossa hipótese de que $\widehat{x} \notin \widehat{R}$. Por outro lado, como $\widehat{f} \circ \widehat{\omega}: I \longrightarrow N^{\prime}$ é um levantamento de $f \circ \omega$ relativamente ao recobrimento $\hat{q} \circ q^{\prime}: N^{\prime} \longrightarrow N$ (onde $\left.\widehat{\omega}=\left(\widehat{\omega}^{-1}\right)^{-1}\right)$, e como $f \circ \omega$ é um laço contrátil em $c$, segue que $\widehat{f} \circ \widehat{\omega}$ é um laço contrátil em $c^{\prime}$, e, conseqüentemente, $\widehat{f}\left(\widehat{x}_{R}^{\prime}\right)=(\widehat{f} \circ \widehat{\omega})(0)=c^{\prime}$, isto é, $\widehat{x}_{R}^{\prime} \in \widehat{f}^{-1}\left(c^{\prime}\right)=\widehat{S}$. Agora, observe que $\widehat{S}=\widehat{f^{-1}}\left(c^{\prime}\right)$ é uma classe de raízes de $\hat{f} \circ \widehat{p}: \widehat{M} \longrightarrow \hat{N}$ em $\hat{c} \in \operatorname{Int} \hat{N}$ (basta aplicar a proposição 2.1.1, substituindo $f$, $\hat{q}$ e $\hat{f}$ por $\hat{f} \circ \hat{p}, q^{\prime}$ e $\hat{f}$, respectivamente); logo, como $\widehat{x}_{R}, \widehat{x}_{R}^{\prime} \in \widehat{S}$, segue que existe um caminho $\widehat{\mu}: I \longrightarrow \widehat{M}$, com $\hat{\mu}(0)=\widehat{x}_{R}$ e $\widehat{\mu}(1)=\widehat{x}_{R}^{\prime}$, tal que $\hat{f} \circ \hat{p} \circ \widehat{\mu}: I \longrightarrow \hat{N}$ é um laço contrátil em $\hat{c}$. No entanto, o laço $\mu=\hat{p} \circ \widehat{\mu}: I \longrightarrow M$, com base em $x_{R}=\hat{p}\left(\widehat{x}_{R}\right)=\hat{p}\left(\widehat{x}_{R}^{\prime}\right)$, não pode ser levantado para um laço em $\widehat{M}$ (relativamente ao recobrimento $\hat{p}: \widehat{M} \longrightarrow M$ ), uma vez que $\widehat{x}_{R} \neq \widehat{x}_{R}^{\prime}$; isto, por sua vez, significa que $\mu: I \longrightarrow M$ é um laço que inverte orientação relativamente ao recobrimento orientado de $M$. Assim, como $\hat{f} \circ \hat{p} \circ \hat{\mu}$ é um laço contrátil em $\hat{c}$, e $\hat{q} \circ \hat{f} \circ \mu=\hat{q} \circ(\hat{f} \circ \hat{p} \circ \hat{\mu})$, segue que $\hat{q} \circ \hat{f} \circ \mu$ é um laço contrátil em $c$, isto é, a aplicação $f=\hat{q} \circ \hat{f}$ leva o laço $\mu: I \longrightarrow M$, que inverte orientação relativamente ao recobrimento orientado de $M$, num laço contrátil em $N$, contradizendo a hipótese de $f$ ser uma aplicação orientável. Deste modo, concluímos que $\widehat{x} \in \widehat{R}$ para todo $\widehat{x} \in \widehat{S}$, ou seja, $\widehat{S} \subseteq \widehat{R}$, e, portanto, $\widehat{S}=\widehat{R}$.

Isto posto, note que $\left.\widehat{p}\right|_{\widehat{U}}: \widehat{U} \longrightarrow U$ é um homeomorfismo, pois é uma aplicação contínua e bijetora, cuja inversa é a aplicação contínua $s: U \longrightarrow \widehat{U}$. Logo, como $\hat{f} \circ \hat{p}=q^{\prime} \circ \widehat{f}$ e $\widehat{f}(\widehat{U}) \subseteq V^{\prime}$, segue que $\left.\widehat{p}\right|_{\widehat{U}}: \widehat{U} \longrightarrow U$ e $\left.q^{\prime}\right|_{V^{\prime}}: V^{\prime} \longrightarrow \hat{V}$ são homeomorfismos tais que $\left(\left.\hat{f}\right|_{U}\right) \circ\left(\left.\hat{p}\right|_{\widehat{U}}\right)=\left(\left.q^{\prime}\right|_{V^{\prime}}\right) \circ\left(\left.\widehat{f}\right|_{\widehat{U}}\right)$; conseqüentemente, da proposição 1.3.3, do corolário 1.3.1 e do teorema 2.1.1, temos que:

$$
\begin{aligned}
|m(R)| & =\left|\operatorname{deg}_{\hat{c}}\left(\left.\hat{f}\right|_{U}\right)\right|=\left|\operatorname{deg}_{\hat{c}}\left(\left.\hat{f}\right|_{U}\right)\right| \cdot 1=\left|\operatorname{deg}_{\hat{c}}\left(\left.\hat{f}\right|_{U}\right)\right| \cdot\left|\operatorname{deg}_{x_{0}}\left(\left.\hat{p}\right|_{\widehat{U}}\right)\right| \\
& =\left|\operatorname{deg}_{\hat{c}}\left(\left.\hat{f}\right|_{U}\right) \cdot \operatorname{deg}_{\left(\left.\hat{f}\right|_{U}\right)^{-1}(\hat{c})}\left(\left.\hat{p}\right|_{\widehat{U}}\right)\right|=\left|\operatorname{deg}_{\hat{c}}\left[\left(\left.\hat{f}\right|_{U}\right) \circ\left(\left.\hat{p}\right|_{\widehat{U}}\right)\right]\right| \\
& =\left|\operatorname{deg}_{\hat{c}}\left[\left(\left.q^{\prime}\right|_{V^{\prime}}\right) \circ\left(\left.\hat{f}\right|_{\widehat{U}}\right)\right]\right|=\left|\operatorname{deg}_{\hat{c}}\left(\left.q^{\prime}\right|_{V^{\prime}}\right) \cdot \operatorname{deg}_{\left(\left.q^{\prime}\right|_{V^{\prime}}\right)^{-1(\hat{c})}}\left(\left.\hat{f}\right|_{\widehat{U}}\right)\right| \\
& =\left|\operatorname{deg}_{\hat{c}}\left(\left.q^{\prime}\right|_{V^{\prime}}\right)\right| \cdot\left|\operatorname{deg}_{c^{\prime}}\left(\left.\widehat{f}\right|_{\widehat{U}}\right)\right|=1 \cdot\left|\operatorname{deg}_{c^{\prime}}\left(\left.\hat{f}\right|_{\widehat{U}}\right)\right| \\
& =\left|\operatorname{deg}_{c^{\prime}}\left(\left.\hat{f}\right|_{\widehat{U}}\right)\right| .
\end{aligned}
$$

Agora, lembremos que o recobrimento $p^{\prime}: M^{\prime} \longrightarrow \widehat{M}$ é o pullback de $\widehat{q}: \widehat{N} \longrightarrow N^{\prime}$ via $\widehat{f}$, isto é, $M^{\prime}=\{(\widehat{x}, \widehat{y}) \in \widehat{M} \times \widehat{N}: \widehat{q}(\widehat{y})=\widehat{f}(\widehat{x})\}$ e $p^{\prime}(\widehat{x}, \widehat{y})=\widehat{x}$ para cada $(\widehat{x}, \widehat{y}) \in M^{\prime}$, 
enquanto que a aplicação $f^{\prime}: M^{\prime} \longrightarrow \widehat{N}$, dada por $f^{\prime}(\widehat{x}, \widehat{y})=\widehat{y}$, é o levantamento de $\widehat{f} \circ p^{\prime}$ relativamente ao recobrimento $\widehat{q}: \widehat{N} \longrightarrow N^{\prime}$. Observando que $V^{\prime}$ é orientável (pois é contrátil), consideremos uma orientação $\widehat{s}: V^{\prime} \longrightarrow \widehat{N}$ de $V^{\prime}$, e definamos $\widehat{V}=\widehat{S}\left(V^{\prime}\right)$. Temos então que $\left.\widehat{q}\right|_{\widehat{V}}: \widehat{V} \longrightarrow V^{\prime}$ é um homeomorfismo, cuja inversa é $\widehat{s}: V^{\prime} \longrightarrow \widehat{V}$. Seja ainda $\widehat{c}=\widehat{s}\left(c^{\prime}\right) \in \widehat{q}^{-1}\left(c^{\prime}\right) \cap \widehat{V}$ (lembre-se que $c^{\prime} \in V^{\prime}$ ), e denotemos por $U^{\prime}$ o subconjunto aberto de $M^{\prime}$ dado por:

$$
U^{\prime}=\left\{(\widehat{x}, \widehat{y}) \in M^{\prime}: \widehat{x} \in \widehat{U} \text { e } \widehat{y} \in \widehat{V}\right\}
$$

Como os pontos $\widehat{x}_{0}=s\left(x_{0}\right) \in \widehat{U}$ e $\widehat{c} \in \widehat{V}$ são tais que $\widehat{f}\left(\widehat{x}_{0}\right)=\widehat{q}(\widehat{c})=c^{\prime}$, segue que $\left(\widehat{x}_{0}, \widehat{c}\right) \in U^{\prime}$, e, portanto, $U^{\prime} \neq \varnothing$. Deste modo, sendo $h: U^{\prime} \longrightarrow \widehat{U}$ a restrição de $p^{\prime}$ a $U^{\prime}, \mathrm{e}$ denotando por $h^{-1}: \widehat{U} \longrightarrow U^{\prime}$ a aplicação dada por $h^{-1}(\widehat{x})=(\widehat{x}, \widehat{s}(\widehat{f}(\hat{x})))$ para cada $\widehat{x} \in \widehat{U}$ (a qual, de fato, está bem definida, uma vez que $\widehat{f}(\widehat{U}) \subseteq V^{\prime}$ e $\left.\widehat{q}(\widehat{s}(\widehat{f}(\widehat{x})))=\widehat{f}(\widehat{x})\right)$, temos que $\left(h \circ h^{-1}\right)(\widehat{x})=h(\widehat{x}, \widehat{s}(\widehat{f}(\widehat{x})))=\widehat{x} \mathrm{e}$

$$
\left(h^{-1} \circ h\right)(\widehat{x}, \widehat{y})=h^{-1}(\widehat{x})=(\widehat{x}, \widehat{s}(\widehat{f}(\widehat{x})))=(\widehat{x}, \widehat{s}(\widehat{q}(\widehat{y})))=(\widehat{x}, \widehat{y}),
$$

para quaisquer $\widehat{x} \in \widehat{U}$ e $(\widehat{x}, \widehat{y}) \in U^{\prime}$ (note que $\widehat{f}(\widehat{x})=\widehat{q}(\widehat{y})$ e $\widehat{s}(\widehat{q}(\widehat{y}))=\widehat{y}$ para todo $\left.(\widehat{x}, \widehat{y}) \in U^{\prime}\right)$. Em outras palavras, $h: U^{\prime} \longrightarrow \widehat{U}$ e $h^{-1}: \widehat{U} \longrightarrow U^{\prime}$ são aplicações contínuas tais que $h \circ h^{-1}: \widehat{U} \longrightarrow \widehat{U}$ e $h^{-1} \circ h: U^{\prime} \longrightarrow U^{\prime}$ são as identidades em $\widehat{U}$ e $U^{\prime}$, respectivamente, o que implica que $h$ é um homeomorfismo, cuja inversa é $h^{-1}$. Isto posto, sendo $R^{\prime}=\left\{(\widehat{x}, \widehat{y}) \in M^{\prime}: \widehat{x} \in \widehat{R}\right.$ e $\left.\widehat{y} \in \widehat{V}\right\}$ e $S^{\prime}=f^{\prime-1}(\widehat{c})$, vamos mostrar que $R^{\prime}=S^{\prime}$.

Com efeito, como $\widehat{R}=\widehat{S}=\widehat{f}^{-1}\left(c^{\prime}\right)$ (conforme vimos há pouco), então, dado $(\widehat{x}, \widehat{y}) \in R^{\prime}$, temos que $\widehat{x} \in \widehat{R}=\widehat{f}^{-1}\left(c^{\prime}\right)$, isto é, $\widehat{f}(\hat{x})=c^{\prime}$. Logo, como $(\widehat{x}, \widehat{y}) \in M^{\prime}$, segue que $\widehat{f}(\widehat{x})=\widehat{q}(\widehat{y})$, e, portanto, $\widehat{q}(\widehat{y})=c^{\prime}$, ou seja, $f^{\prime}(\widehat{x}, \widehat{y})=\widehat{y} \in \widehat{q}^{-1}\left(c^{\prime}\right)$. Entretanto, como $\left.\widehat{q}\right|_{\widehat{V}}: \widehat{V} \longrightarrow V^{\prime}$ é uma bijeção $($ e $\widehat{y} \in \widehat{V})$, devemos ter $\widehat{y}=\widehat{c}$ (uma vez que $\left.\widehat{q}(\widehat{y})=\widehat{q}(\widehat{c})=c^{\prime}\right)$, d'onde concluímos que $f^{\prime}(\widehat{x}, \widehat{y})=\widehat{c}$, isto é, $(\widehat{x}, \widehat{y}) \in f^{\prime-1}(\widehat{c})=S^{\prime}$. Reciprocamente, se $(\widehat{x}, \widehat{y}) \in S^{\prime}=f^{\prime-1}(\hat{c})$, segue que $\widehat{y}=f^{\prime}(\widehat{x}, \widehat{y})=\widehat{c}$; assim, como $\widehat{f}(\widehat{x})=\widehat{q}(\widehat{y})$ (pois $\left.(\widehat{x}, \widehat{y}) \in M^{\prime}\right)$, temos que $\widehat{f}(\widehat{x})=\widehat{q}(\hat{c})=c^{\prime}$, e, portanto, $\widehat{x} \in \widehat{f}^{-1}\left(c^{\prime}\right)=\widehat{S}=\widehat{R}$. Conseqüentemente, como $\widehat{y}=\widehat{c} \in \widehat{V}$, obtemos que $(\widehat{x}, \widehat{y}) \in R^{\prime}$, o que prova que $R^{\prime}=S^{\prime}$.

Finalmente, observe que os homeomorfismos $h=\left.p^{\prime}\right|_{U^{\prime}}: U^{\prime} \longrightarrow \widehat{U}$ e $\left.\widehat{q}\right|_{\widehat{V}}: \widehat{V} \longrightarrow V^{\prime}$ satisfazem $\left(\left.\widehat{f}\right|_{\widehat{U}}\right) \circ\left(\left.p^{\prime}\right|_{U^{\prime}}\right)=\left(\left.\widehat{q}\right|_{\widehat{V}}\right) \circ\left(\left.f^{\prime}\right|_{U^{\prime}}\right)$, pois $\widehat{f} \circ p^{\prime}=\widehat{q} \circ f^{\prime}$ e $f^{\prime}\left(U^{\prime}\right) \subseteq \widehat{V}$. Logo, da proposição 1.3.3 e do corolário 1.3.1, segue que:

$$
\begin{aligned}
\left|\operatorname{deg}_{c^{\prime}}\left(\left.\widehat{f}\right|_{\widehat{U}}\right)\right| & =\left|\operatorname{deg}_{c^{\prime}}\left(\left.\widehat{f}\right|_{\widehat{U}}\right)\right| \cdot 1=\left|\operatorname{deg}_{c^{\prime}}\left(\left.\widehat{f}\right|_{\widehat{U}}\right)\right| \cdot\left|\operatorname{deg}_{\widehat{x} 0}\left(\left.p^{\prime}\right|_{U^{\prime}}\right)\right| \\
& =\left|\operatorname{deg}_{c^{\prime}}\left(\left.\widehat{f}\right|_{\widehat{U}}\right) \cdot \operatorname{deg}_{\left(\left.\widehat{f}\right|_{\widehat{U}}\right)^{-1}\left(c^{\prime}\right)}\left(\left.p^{\prime}\right|_{U^{\prime}}\right)\right|=\left|\operatorname{deg}_{c^{\prime}}\left[\left(\left.\widehat{f}\right|_{\widehat{U}}\right) \circ\left(\left.p^{\prime}\right|_{U^{\prime}}\right)\right]\right| \\
& =\left|\operatorname{deg}_{c^{\prime}}\left[\left(\left.\widehat{q}\right|_{\widehat{V}}\right) \circ\left(\left.f^{\prime}\right|_{U^{\prime}}\right)\right]\right|=\left|\operatorname{deg}_{c^{\prime}}\left(\left.\widehat{q}\right|_{\widehat{V}}\right) \cdot \operatorname{deg}_{\left(\left.\widehat{q}\right|_{\widehat{V}}\right)^{-1}\left(c^{\prime}\right)}\left(\left.f^{\prime}\right|_{U^{\prime}}\right)\right| \\
& =\left|\operatorname{deg}_{c^{\prime}}\left(\left.\widehat{q}\right|_{\widehat{V}}\right)\right| \cdot\left|\operatorname{deg}_{\widehat{c}}\left(\left.f^{\prime}\right|_{U^{\prime}}\right)\right|=1 \cdot\left|\operatorname{deg}_{\widehat{c}}\left(\left.f^{\prime}\right|_{U^{\prime}}\right)\right|=\left|\operatorname{deg}_{\widehat{c}}\left(\left.f^{\prime}\right|_{U^{\prime}}\right)\right| .
\end{aligned}
$$


Por outro lado, sendo $W^{\prime}=M^{\prime} \backslash f^{\prime-1}(\partial \widehat{N}) \subseteq \operatorname{Int} M^{\prime}$, temos que:

$$
W^{\prime}=\left\{(\widehat{x}, \widehat{y}) \in M^{\prime}: f^{\prime}(\widehat{x}, \widehat{y}) \notin \partial \widehat{N}\right\}=\left\{(\widehat{x}, \widehat{y}) \in M^{\prime}: \widehat{y} \notin \partial \widehat{N}\right\} ;
$$

conseqüentemente, como $U^{\prime}=\left\{(\widehat{x}, \widehat{y}) \in M^{\prime}: \widehat{x} \in \widehat{U}\right.$ e $\left.\widehat{y} \in \widehat{V}\right\}$ e $\widehat{V} \subseteq \operatorname{Int} \widehat{N}$ (pois $\widehat{V}=\widehat{S}\left(V^{\prime}\right)$ e $\left.V^{\prime} \subseteq \operatorname{Int} N^{\prime}\right)$, segue que $U^{\prime} \subseteq W^{\prime}$. Além disso, como $\widehat{R} \subseteq \widehat{U}$, temos que $R^{\prime}=\left\{(\widehat{x}, \widehat{y}) \in M^{\prime}: \widehat{x} \in \widehat{R}\right.$ e $\left.\widehat{y} \in \widehat{V}\right\} \subseteq U^{\prime}$, ou seja, $f^{\prime-1}(\mathcal{c}) \subseteq U^{\prime}$ (uma vez que $\left.S^{\prime}=f^{\prime-1}(\mathcal{c})=R^{\prime}\right)$, e, portanto, $W^{\prime} \backslash U^{\prime} \subseteq W^{\prime} \backslash f^{\prime-1}(\mathcal{c})$. Assim, como $\overline{W^{\prime} \backslash U^{\prime}}=W^{\prime} \backslash U^{\prime} \subseteq$ $W^{\prime} \backslash f^{\prime-1}(\mathcal{c})=\operatorname{Int}\left(W^{\prime} \backslash f^{\prime-1}(\mathcal{C})\right.$ ) (onde o fecho é relativo a $W^{\prime}$ ), segue, do teorema da excisão em homologia singular, que o homomorfismo $i_{*}^{\prime}: H_{n}\left(U^{\prime}, U^{\prime} \backslash f^{-1}(\mathcal{c})\right) \longrightarrow$ $\mathrm{H}_{n}\left(W^{\prime}, W^{\prime} \backslash f^{\prime-1}(\mathcal{c})\right)$, induzido pela inclusão $i^{\prime}:\left(U^{\prime}, U^{\prime} \backslash f^{\prime-1}(\mathcal{c})\right) \longrightarrow\left(W^{\prime}, W^{\prime} \backslash f^{\prime-1}(\bar{c})\right)$, é um isomorfismo, que leva a classe fundamental $\theta_{U^{\prime}} \in \mathrm{H}_{n}\left(U^{\prime}, U^{\prime} \backslash f^{\prime-1}(\mathcal{c})\right)$ na classe fundamental $\theta_{W^{\prime}} \in \mathrm{H}_{n}\left(W^{\prime}, W^{\prime} \backslash f^{\prime-1}(\mathcal{c})\right)$, e comuta o seguinte diagrama:

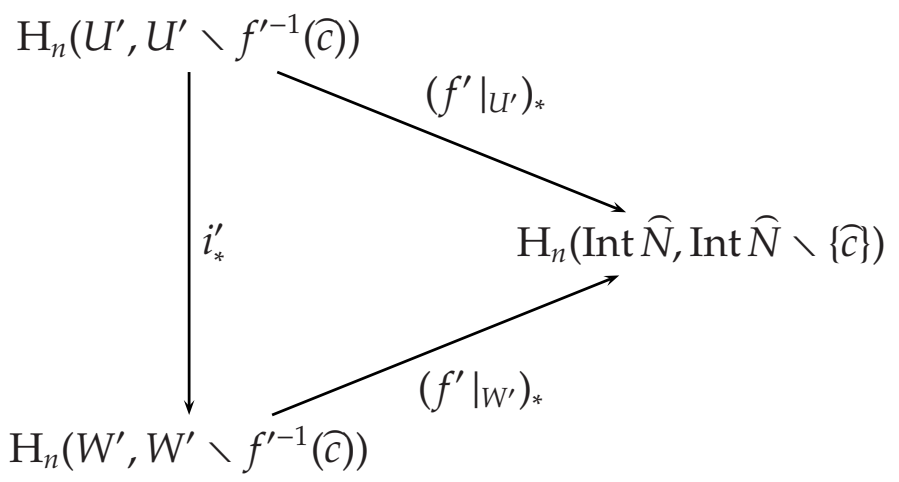

(note que $f^{\prime}\left(U^{\prime}\right) \subseteq \operatorname{Int} \widehat{N}$, pois $U^{\prime} \subseteq W^{\prime} \subseteq f^{\prime-1}(\partial \widehat{N})$ ). Logo, denotando por $\theta_{\widehat{c}} \in$ $\mathrm{H}_{n}(\operatorname{Int} \widehat{N}$, Int $\widehat{N} \backslash\{\hat{c}\})$ a classe fundamental em $\{\hat{c}\}$, temos que:

$$
\left(\left.f^{\prime}\right|_{U^{\prime}}\right)_{*}\left(\theta_{U^{\prime}}\right)=\left(\left.f^{\prime}\right|_{W^{\prime}}\right)_{*}\left(i_{*}^{\prime}\left(\theta_{U^{\prime}}\right)\right)=\left(\left.f^{\prime}\right|_{W^{\prime}}\right)_{*}\left(\theta_{W^{\prime}}\right)=\operatorname{deg}_{\widehat{c}}\left(\left.f^{\prime}\right|_{W^{\prime}}\right) \cdot \theta_{\widehat{c}} .
$$

Ora, como o grau local deg ${ }_{\widehat{c}}\left(\left.f^{\prime}\right|_{u^{\prime}}\right)$ é definido como sendo o número inteiro tal que

$$
\left(\left.f^{\prime}\right|_{U^{\prime}}\right)_{*}\left(\theta_{U^{\prime}}\right)=\operatorname{deg}_{\widehat{c}}\left(\left.f^{\prime}\right|_{U^{\prime}}\right) \cdot \theta_{\widehat{c}},
$$

obtemos então que $\operatorname{deg}_{\widehat{c}}\left(\left.f^{\prime}\right|_{U^{\prime}}\right)=\operatorname{deg}_{\widehat{c}}\left(\left.f^{\prime}\right|_{W^{\prime}}\right)$. Deste modo, como

$$
|m(R)|=\left|\operatorname{deg}_{c^{\prime}}\left(\left.\widehat{f}\right|_{\widehat{u}}\right)\right|,\left|\operatorname{deg}_{c^{\prime}}\left(\widehat{f}_{\widehat{U}}\right)\right|=\left|\operatorname{deg}_{\widehat{c}}\left(\left.f^{\prime}\right|_{U^{\prime}}\right)\right| \mathrm{e} \operatorname{deg}_{\widehat{c}}\left(\left.f^{\prime}\right|_{U^{\prime}}\right)=\operatorname{deg}_{\widehat{c}}\left(\left.f^{\prime}\right|_{W^{\prime}}\right),
$$

segue que $|m(R)|=\left|\operatorname{deg}_{\widehat{c}}\left(\left.f^{\prime}\right|_{W^{\prime}}\right)\right|$. Entretanto, como $M^{\prime}$ e $\widehat{N}$ são variedades topológicas conexas e orientáveis, e $f^{\prime}:\left(M^{\prime}, \partial M^{\prime}\right) \longrightarrow(\widehat{N}, \partial \widehat{N})$ é uma aplicação contínua própria, o teorema 1.4.2 nos garante que $\operatorname{deg}_{\widehat{c}}\left(\left.f^{\prime}\right|_{W^{\prime}}\right)=\operatorname{deg}\left(f^{\prime}\right)$, d'onde concluímos que $|m(R)|=$ $\left|\operatorname{deg}\left(f^{\prime}\right)\right|$, conforme queríamos demonstrar. 
No que segue, a fim de não sobrecarregarmos o texto, vamos omitir o termo "relativamente ao(s) recobrimento(s) orientado(s) de ..." das definições 1.2 .3 e 1.2.4, deixando subentendido que estamos nos referindo apenas a levantamentos relativos aos recobrimentos orientados (deste modo, quando dissermos, por exemplo, que um laço $\omega: I \longrightarrow \hat{N}$ preserva orientação, deve-se entender que $\omega$ preserva orientação relativamente ao recobrimento orientado de $\hat{N}$ ).

Corolário 2.2.1. Seja $f:(M, \partial M) \longrightarrow(N, \partial N)$ uma aplicação contínua própria do tipo I (vide definição 1.2.5), e suponha que a cardinalidade j de $\hat{q}^{-1}(c)$ é finita. Então, a variedade topológica $N^{\prime}$ é orientável (e, portanto, o grau cohomológico $\operatorname{deg}(\widehat{f})$ de $\widehat{f}:(\widehat{M}, \partial \widehat{M}) \longrightarrow\left(N^{\prime}, \partial N^{\prime}\right)$ está bem definido), e, para toda classe de raízes $R$ de f em c, temos $|m(R)|=|\operatorname{deg}(\widehat{f})|$.

DemonstraÇÃo. De fato, consideremos novamente o diagrama comutativo abaixo:

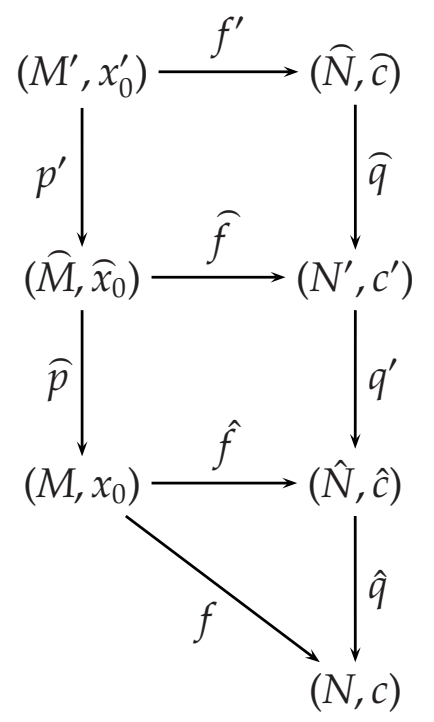

Como $\hat{q}: \hat{N} \longrightarrow N, q^{\prime}: N^{\prime} \longrightarrow \hat{N}$ e $\hat{p}: \widehat{M} \longrightarrow M$ são aplicações de recobrimento, segue (da proposição 1.2.7) que $\hat{q}, q^{\prime}$ e $\hat{p}$ são fiéis à orientação. Isto posto, seja $\alpha: I \longrightarrow M$ um laço com base em $x_{0}$, e consideremos o laço $\hat{\beta}: I \longrightarrow \hat{N}$ (com base em $\hat{c}$ ) dado por $\hat{\beta}=\hat{f} \circ \alpha$. Temos então que $\alpha$ preserva orientação se, e somente se, $\hat{\beta}$ também o faz: com efeito, sabemos que $\hat{\beta}$ preserva orientação se, e somente se, o laço $\gamma=\hat{q} \circ \hat{\beta}: I \longrightarrow N$ preserva orientação (pois $\hat{q}$ é fiel à orientação); logo, como $\hat{q} \circ \hat{\beta}=\hat{q} \circ(\hat{f} \circ \alpha)=f \circ \alpha$, temos que $\hat{\beta}$ preserva orientação se, e só se, $f \circ \alpha$ preserva orientação, o que, por sua vez, ocorre se, e só se, $\alpha$ preserva orientação (uma vez que $f$ é do tipo I, isto é, fiel à orientação). Daí, concluímos que a aplicação $\hat{f}: M \longrightarrow \hat{N}$ também é fiel à orientação. Por outro lado, seja $\widehat{\alpha}: I \longrightarrow \widehat{M}$ um laço com base em $\widehat{x}_{0}$, e considere o laço $\beta^{\prime}: I \longrightarrow N^{\prime}$ (com base em $c^{\prime}$ ) dado por $\beta^{\prime}=\widehat{f} \circ \widehat{\alpha}$. Temos, pois, que $\widehat{\alpha}$ preserva orientação se, e somente se, $\beta^{\prime}$ também o faz: de fato, como $q^{\prime}: N^{\prime} \longrightarrow \hat{N}$ é fiel à orientação, segue 
que $\beta^{\prime}$ preserva orientação se, e só se, o laço $q^{\prime} \circ \beta^{\prime}=q^{\prime} \circ(\hat{f} \circ \widehat{\alpha})=\hat{f} \circ \hat{p} \circ \widehat{\alpha}$ preserva orientação, o que, por sua vez, ocorre se, e somente se, $\widehat{\alpha}$ preserva orientação (uma vez que $\hat{f}: M \longrightarrow \hat{N}$ e $\hat{p}: \widehat{M} \longrightarrow M$ são fiéis à orientação). Logo, temos que $\widehat{f}: \widehat{M} \longrightarrow N^{\prime}$ também é uma aplicação fiel à orientação.

Agora, note que o homomorfismo $\widehat{f_{\#}}: \pi_{1}\left(\widehat{M}, \widehat{x}_{0}\right) \longrightarrow \pi_{1}\left(N^{\prime}, c^{\prime}\right)$, induzido por $\widehat{f}$, é sobrejetivo: com efeito, dado um laço $\omega^{\prime}: I \longrightarrow N^{\prime}$ com base em $c^{\prime}$, temos que o laço $\hat{\omega}=q^{\prime} \circ \omega^{\prime}$ é tal que $[\hat{\omega}] \in\left(\hat{f} \circ \widehat{p}_{\#}\left(\pi_{1}\left(\widehat{M}, \widehat{x}_{0}\right)\right)\right.$ (uma vez que $q^{\prime}: N^{\prime} \longrightarrow \hat{N}$ é o recobrimento de $\hat{N}$ associado ao subgrupo $\left(\hat{f} \circ \widehat{p}_{\#}\left(\pi_{1}\left(\hat{M}, \widehat{x}_{0}\right)\right) \subseteq \pi_{1}(\hat{N}, \hat{c})\right)$, o que implica que existe um laço $\widehat{\omega}: I \longrightarrow \widehat{M}$, com base em $\widehat{x}_{0}$, tal que $[\hat{\omega}]=(\hat{f} \circ \widehat{p})_{\#}([\hat{\omega}])$; logo, como $\left(\hat{f} \circ \widehat{p}_{\#}([\widehat{\omega}])=\right.$ $\left(q^{\prime} \circ \widehat{f}\right)_{\#}([\widehat{\omega}])=q_{\#}^{\prime}\left(\widehat{f_{\#}}([\widehat{\omega}])\right)$, segue que $q_{\#}^{\prime}\left(\widehat{f_{\#}}([\widehat{\omega}])\right)=[\hat{\omega}]=q_{\#}^{\prime}\left(\left[\omega^{\prime}\right]\right)$, ou seja, $\widehat{f}_{\#}([\widehat{\omega}])=$ $\left[\omega^{\prime}\right]$ (pois, como $q^{\prime}: N^{\prime} \longrightarrow \hat{N}$ é um recobrimento, o homomorfismo induzido $q_{\#}^{\prime}:$ $\pi_{1}\left(N^{\prime}, c^{\prime}\right) \longrightarrow \pi_{1}(\hat{N}, \hat{c})$ é injetivo), demonstrando que $\widehat{f}_{\#}$ é um homomorfismo sobrejetivo. Conseqüentemente, dado um laço qualquer $\gamma^{\prime}: I \longrightarrow N^{\prime}$, com base em $c^{\prime}$, segue que existe um laço $\widehat{\gamma}: I \longrightarrow \widehat{M}$, com base em $\widehat{x}_{0}$, tal que $\left[\gamma^{\prime}\right]=[\widehat{f} \circ \widehat{\gamma}]=\widehat{f}_{\#}([\widehat{\gamma}])$. Entretanto, como $\widehat{M}$ é uma variedade orientável, temos que todo laço em $\widehat{M}$ preserva orientação; em particular, segue então que $\widehat{\gamma}$ preserva orientação, o que (pelo fato de $\widehat{f}: \widehat{M} \longrightarrow N^{\prime}$ ser fiel à orientação) implica que o laço $\hat{f} \circ \widehat{\gamma}: I \longrightarrow N^{\prime}$ (com base em $c^{\prime}$ ) preserva orientação. Assim, como $\left[\gamma^{\prime}\right]=[\widehat{f} \circ \widehat{\gamma}]$ e $\widehat{f} \circ \widehat{\gamma}$ preserva orientação, temos que $\gamma^{\prime}$ também preserva orientação, uma vez que $\widehat{f} \circ \widehat{\gamma}$ pode ser levantado para um laço $\widehat{\delta}: I \longrightarrow \widehat{N}$, relativamente ao recobrimento orientado $\widehat{q}: \widehat{N} \longrightarrow N^{\prime}$, e toda homotopia entre $\gamma^{\prime}$ e $\widehat{f} \circ \widehat{\gamma}$ pode ser levantada para uma homotopia entre $\widehat{\delta}$ e um levantamento de $\gamma^{\prime}$ (relativamente a q), o qual, por conseguinte, será um laço em $\widehat{N}$. Deste modo, podemos concluir que todo laço $\gamma^{\prime}: I \longrightarrow N^{\prime}$, com base em $c^{\prime}$, preserva orientação, o que (pela proposição 1.2.5) significa que $N^{\prime}$ é uma variedade orientável, e, portanto, o grau cohomológico $\operatorname{deg}(\widehat{f})$ da aplicação própria $\widehat{f}:(\widehat{M}, \partial \widehat{M}) \longrightarrow\left(N^{\prime}, \partial N^{\prime}\right)$ encontra-se bem definido (vide definição 1.4.4). Além disso, pela definição 1.4.2, a orientabilidade de $N^{\prime}$ nos garante que o recobrimento orientado $\widehat{q}: \widehat{N} \longrightarrow N^{\prime}$ é a identidade, isto é, $\widehat{N}=N^{\prime}$. Logo, como $p^{\prime}: M^{\prime} \longrightarrow \widehat{M}$ é o pullback de $\widehat{q}$ via $\widehat{f}$, segue que:

$$
M^{\prime}=\{(\widehat{x}, \widehat{y}) \in \widehat{M} \times \widehat{N}: \widehat{f}(\widehat{x})=\widehat{q}(\widehat{y})=\widehat{y}\}=\{(\widehat{x}, \widehat{f}(\widehat{x})): \widehat{x} \in \widehat{M}\},
$$

e, conseqüentemente, as aplicações $p^{\prime}: M^{\prime} \longrightarrow \widehat{M}$ e $f^{\prime}: M^{\prime} \longrightarrow \widehat{N}$ são dadas por $p^{\prime}((\widehat{x}, \widehat{f}(\widehat{x})))=\widehat{x}$ e $f^{\prime}((\widehat{x}, \widehat{f}(\widehat{x})))=\widehat{f}(\widehat{x})$ para cada $\widehat{x} \in \widehat{M}$. Note então que, deste modo, o recobrimento $p^{\prime}: M^{\prime} \longrightarrow \widehat{M}$ é um homeomorfismo, cuja inversa é a aplicação contínua $p^{\prime-1}: \widehat{M} \longrightarrow M^{\prime}$ definida por $p^{\prime-1}(\widehat{x})=(\widehat{x}, \widehat{f}(\widehat{x}))$, para cada $\widehat{x} \in \widehat{M}$. Portanto, como $f^{\prime}=\widehat{q} \circ f^{\prime}=\widehat{f} \circ p^{\prime}$, segue (da proposição 1.4.6 e do corolário 1.4.1) que:

$$
\begin{aligned}
\left|\operatorname{deg}\left(f^{\prime}\right)\right| & =\left|\operatorname{deg}\left(\widehat{f} \circ p^{\prime}\right)\right|=\left|\operatorname{deg}(\widehat{f}) \cdot \operatorname{deg}\left(p^{\prime}\right)\right| \\
& =|\operatorname{deg}(\widehat{f})| \cdot\left|\operatorname{deg}\left(p^{\prime}\right)\right|=|\operatorname{deg}(\widehat{f})| \cdot 1=|\operatorname{deg}(\widehat{f})|,
\end{aligned}
$$


d'onde concluímos (utilizando a proposição anterior) que $|m(R)|=|\operatorname{deg}(\widehat{f})|$, para toda classe de raízes $R$ de $f$ em $c$.

Agora, antes de demonstrarmos o próximo resultado, convém observarmos alguns fatos sobre laços que preservam ou invertem orientação.

Observação 2.2.1. A fim de apresentarmos uma descrição alternativa para o fibrado orientado, considere uma variedade topológica conexa $X$, de dimensão $n$ e sem fronteira, e seja $x_{0} \in X$; da teoria de recobrimentos, sabemos que existem uma variedade topológica simplesmente conexa $X^{U}$, bem como uma aplicação de recobrimento $p^{U}: X^{U} \longrightarrow X$, dita universal, tais que, para todo recobrimento $\bar{p}: \bar{X} \longrightarrow X$, existe um outro recobrimento $\bar{p}^{U}: X^{U} \longrightarrow \bar{X}$ satisfazendo $\bar{p} \circ \bar{p}^{U}=p^{U}$ (isto é, $X^{U}$ recobre qualquer outro recobrimento $\bar{X}$ de $X)$. Por outro lado, se $\bar{p}: \bar{X} \longrightarrow X$ é um recobrimento qualquer de $X$, dizemos que um homeomorfismo $\bar{D}: \bar{X} \longrightarrow \bar{X}$ é uma transformação de deck, relativamente a $\bar{p}$, se $\bar{p} \circ \bar{D}=\bar{p}$. Além disso, sabemos também que, dado um laço $\omega: I \longrightarrow X$, com base em $x_{0}$, existe uma única transformação de deck $\bar{D}_{\omega}: \bar{X} \longrightarrow \bar{X}$ relativamente ao recobrimento $\bar{p}: \bar{X} \longrightarrow X$, denominada transformação de deck induzida por $\omega$ em $\bar{X}$, definida da seguinte maneira: para cada $\bar{x} \in \bar{X}$, consideramos $\bar{D}_{\omega}(\bar{x})=\bar{\omega}(1)$, onde $\bar{\omega}: I \longrightarrow \bar{X}$ é o único levantamento de $\omega$, relativamente ao recobrimento $\bar{p}$, tal que $\bar{\omega}(0)=\bar{x}$ (para maiores detalhes sobre estes assuntos, vide [Bd], páginas 147 a 150). Isto posto, seja $D_{\omega}^{U}: X^{U} \longrightarrow X^{U}$ a transformação de deck induzida por $\omega$ no espaço de recobrimento universal $X^{U}$ de $X$; seguindo o raciocínio dado na observação 5.9 de [Bk3] (página 406), pode-se verificar que o laço $\omega: I \longrightarrow X$ preserva ou inverte orientação conforme o grau local $\operatorname{deg}_{y}\left(D_{\omega}^{U}\right)$ é respectivamente igual a 1 ou -1 , onde $y \in X^{U}$ é tal que $D_{\omega}^{U}(x)=y$ para algum $x \in\left(p^{U}\right)^{-1}\left(x_{0}\right)$ (note que, como $D_{\omega}^{U}: X^{U} \longrightarrow X^{U}$ é um homeomorfismo, temos, do corolário 1.3.1, que $\operatorname{deg}_{y}\left(D_{\omega}^{U}\right)= \pm 1$ ). Deste modo, segue que o fibrado orientado de $X$ é o espaço de recobrimento de $X$ associado ao subgrupo $G$ de $\pi_{1}\left(X, x_{0}\right)$, constituído das classes de homotopia dos laços $\omega: I \longrightarrow X$, para os quais a transformação de deck induzida por $\omega$, no espaço de recobrimento universal de $X$, é um homeomorfismo que preserva orientação.

Observação 2.2.2 . Tendo em vista os conceitos apresentados na observação acima, e adotando as mesmas notações lá utilizadas, suponhamos agora que $\bar{p}: \bar{X} \longrightarrow X$ seja um recobrimento de $X$, com $\bar{X}$ orientável, e considere a transformação de deck $\bar{D}_{\omega}: \bar{X} \longrightarrow X$ induzida por $\omega$ em $\bar{X}$; pela definição de recobrimento universal, sabemos que existe um recobrimento $\bar{p}^{U}: X^{U} \longrightarrow \bar{X}$ tal que $p^{U}=\bar{p} \circ \bar{p}^{U}$. Logo, como $\bar{p} \circ \bar{D}_{\omega}=\bar{p}$ e $p^{U} \circ D_{\omega}^{U}=p^{U}$, obtemos que $\bar{p} \circ\left(\bar{D}_{\omega} \circ \bar{p}^{U}\right)=\bar{p} \circ \bar{p}^{U}=p^{U}$ e $\bar{p} \circ\left(\bar{p}^{U} \circ D_{\omega}^{U}\right)=p^{U} \circ D_{\omega}^{U}=p^{U}$, isto é, 
$\bar{D}_{\omega} \circ \bar{p}^{U}: X^{U} \longrightarrow \bar{X}$ e $\bar{p}^{U} \circ D_{\omega}^{U}: X^{U} \longrightarrow \bar{X}$ constituem levantamentos de $p^{U}$ relativamente ao recobrimento $\bar{p}: \bar{X} \longrightarrow X$, conforme ilustra o diagrama abaixo:

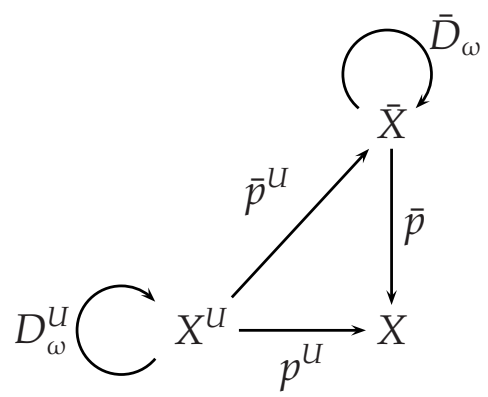

Em contrapartida, sendo $\bar{x}, \bar{y} \in \bar{X}$ tais que $\bar{p}^{U}(x)=\bar{x}$ e $\bar{D}_{\omega}(\bar{x})=\bar{y}$ (onde $x, y \in X^{U}$ são tais que $p^{U}(x)=x_{0}$ e $\left.D_{\omega}^{U}(x)=y\right)$, sabemos que $\bar{D}_{\omega}(\bar{x})=\bar{\omega}(1)=\bar{y}$ e $D_{\omega}^{U}(x)=\omega^{U}(1)=y$, onde $\bar{\omega}: I \longrightarrow \bar{X}$ e $\omega^{U}: I \longrightarrow X^{U}$ são, respectivamente, os únicos levantamentos de $\omega$, relativamente aos recobrimentos $\bar{p}$ e $p^{U}$, tais que $\bar{\omega}(0)=\bar{x}$ e $\omega^{U}(0)=x$; conseqüentemente, denotando por $\bar{\omega}^{U}: I \longrightarrow X^{U}$ o único levantamento de $\bar{\omega}$ relativamente a $\bar{p}^{U}$ (isto é, $\left.\bar{\omega}=\bar{p}^{U} \circ \bar{\omega}^{U}\right)$, com $\bar{\omega}^{U}(0)=x$, temos que $p^{U} \circ \bar{\omega}^{U}=\left(\bar{p} \circ \bar{p}^{U}\right) \circ \bar{\omega}^{U}=\bar{p} \circ \bar{\omega}=\omega \mathrm{e}$ $\bar{\omega}^{U}(0)=x=\omega^{U}(0)$. Portanto, da unicidade do levantamento $\omega^{U}: I \longrightarrow X^{U}$, segue que $\bar{\omega}^{U}=\omega^{U}$, o que implica que:

$$
\bar{p}^{U}(y)=\bar{p}^{U}\left(\omega^{U}(1)\right)=\left(\bar{p}^{U} \circ \bar{\omega}^{U}\right)(1)=\bar{\omega}(1)=\bar{y} .
$$

Assim, temos que

$$
\left(\bar{D}_{\omega} \circ \bar{p}^{U}\right)(x)=\bar{D}_{\omega}(\bar{x})=\bar{y} \text { e }\left(\bar{p}^{U} \circ D_{\omega}^{U}\right)(x)=\bar{p}^{U}(y)=\bar{y},
$$

ou seja, $\bar{D}_{\omega} \circ \bar{p}^{U}: X^{U} \longrightarrow \bar{X}$ e $\bar{p}^{U} \circ D_{\omega}^{U}: X^{U} \longrightarrow \bar{X}$ são levantamentos de $p^{U}$ (relativamente a $\bar{p}$ ) que coincidem no ponto $x \in X^{U}$. Daí, segue (da unicidade destes levantamentos) que $\bar{D}_{\omega} \circ \bar{p}^{U}=\bar{p}^{U} \circ D_{\omega}^{U}$, o que (pela proposição 1.3.3) significa que:

$$
\operatorname{deg}_{\bar{y}}\left(\bar{D}_{\omega}\right) \cdot \operatorname{deg}_{\left(\bar{D}_{\omega}\right)^{-1}(\bar{y})}\left(\bar{p}^{U}\right)=\operatorname{deg}_{\bar{y}}\left(\bar{p}^{U} \circ D_{\omega}^{U}\right)
$$

(lembre-se que as variedades $X^{U}$ e $\bar{X}$ são orientáveis). Logo, como $\left(\bar{D}_{\omega}\right)^{-1}(\bar{y})=\{\bar{x}\}$ (pois $\bar{D}_{\omega}: \bar{X} \longrightarrow \bar{X}$ é um homeomorfismo), obtemos que:

$$
\text { (I) } \operatorname{deg}_{\bar{y}}\left(\bar{D}_{\omega}\right) \cdot \operatorname{deg}_{\bar{x}}\left(\bar{p}^{U}\right)=\operatorname{deg}_{\bar{y}}\left(\bar{p}^{U} \circ D_{\omega}^{U}\right) .
$$

Agora, observe que, como $\left(\bar{p}^{U}\right)^{-1}(\bar{y})$ não é necessariamente conexo, não podemos utilizar diretamente a proposição 1.3.3 para concluir que

$$
\operatorname{deg}_{\bar{y}}\left(\bar{p}^{U} \circ D_{\omega}^{U}\right)=\operatorname{deg}_{\bar{y}}\left(\bar{p}^{U}\right) \cdot \operatorname{deg}_{(\bar{p} U)^{-1}(\bar{y})}\left(D_{\omega}^{U}\right),
$$

uma vez que o grau local $\operatorname{deg}_{K}\left(D_{\omega}^{U}\right)$ encontra-se bem definido somente se, além de compacto, $K$ também é conexo; entretanto, denotando por $K=\left(\bar{p}^{U}\right)^{-1}(\bar{y})$, segue, da 
proposição 4.5 de [D] (página 268), que a igualdade $\left(D_{\omega}^{U}\right)_{*}\left(\theta_{\left(D_{\omega}^{U}\right)^{-1}(K)}\right)=\operatorname{deg}_{y}\left(D_{\omega}^{U}\right) \cdot \theta_{K}$ é válida ainda que $K$ não seja conexo (onde, como usualmente, $\theta_{\left(D_{\omega}^{U}\right)^{-1}(K)} \in \mathrm{H}_{n}\left(X^{U}\right.$, $X^{U} \backslash$ $\left.\left(D_{\omega}^{U}\right)^{-1}(K)\right)$ e $\theta_{K} \in \mathrm{H}_{n}\left(X^{U}, X^{U} \backslash K\right)$ são as classes fundamentais em $\left(D_{\omega}^{U}\right)^{-1}(K)$ e $K$, respectivamente, e $\left(D_{\omega}^{U}\right)_{*}: \mathrm{H}_{n}\left(X^{U}, X^{U} \backslash\left(D_{\omega}^{U}\right)^{-1}(K)\right) \longrightarrow \mathrm{H}_{n}\left(X^{U}, X^{U} \backslash K\right)$ é o isomorfismo induzido pelo homeomorfimo $\left.D_{\omega}^{U}: X^{U} \longrightarrow X^{U}\right)$. Deste modo, não é difícil mostrar que

$$
\operatorname{deg}_{\bar{y}}\left(\bar{p}^{U} \circ D_{\omega}^{U}\right)=\operatorname{deg}_{\bar{y}}\left(\bar{p}^{U}\right) \cdot \operatorname{deg}_{y}\left(D_{\omega}^{U}\right)
$$

(basta construir um diagrama com os homomorfismos em homologia induzidos por $\bar{p}^{U}, D_{\omega}^{U}$ e $\bar{p}^{U} \circ D_{\omega}^{U}$, e verificar a relação entre as classes fundamentais). Assim, utilizando a proposição 1.3.4, temos que $\operatorname{deg}_{\bar{y}}\left(\bar{p}^{U}\right)=\operatorname{deg}_{\bar{x}}\left(\bar{p}^{U}\right)$, e, conseqüentemente (de (I), (II) e do fato de que $\operatorname{deg}_{\bar{x}}\left(\bar{p}^{U}\right)>0$ ), obtemos que $\operatorname{deg}_{\bar{y}}\left(\bar{D}_{\omega}\right)=\operatorname{deg}_{y}\left(D_{\omega}^{U}\right)$. Finalmente, note então que, se o laço $\omega: I \longrightarrow X$ preserva ou inverte orientação, temos respectivamente que $\operatorname{deg}_{\bar{y}}\left(\bar{D}_{\omega}\right)=\operatorname{deg}_{y}\left(D_{\omega}^{U}\right)=1$ ou $\operatorname{deg}_{\bar{y}}\left(\bar{D}_{\omega}\right)=\operatorname{deg}_{y}\left(D_{\omega}^{U}\right)=-1$. Em resumo, dado qualquer recobrimento $\bar{p}: \bar{X} \longrightarrow X$, com $\bar{X}$ orientável, a transformação de deck $\bar{D}_{\omega}: \bar{X} \longrightarrow \bar{X}$, induzida por um laço $\omega: I \longrightarrow X$ em $\bar{X}$, é um homeomorfismo que preserva ou inverte orientação conforme $\omega$ respectivamente preserva ou inverte orientação.

Corolário 2.2.2 . Se $f:(M, \partial M) \longrightarrow(N, \partial N)$ é uma aplicação contínua própria do tipo II (vide definição 1.4.5), tal que a cardinalidade $\mathrm{j} d e \hat{q}^{-1}(c)$ é finita, então $\operatorname{deg}\left(f^{\prime}\right)=0$, e, conseqüentemente, $|m(R)|=0$ para toda classe de raízes $R$ de $f$ em $c \in \operatorname{Int} N$.

DemonstraçÃo. Como $f$ não é fiel à orientação, segue que existem laços $\omega: I \longrightarrow M$ e $\mu=f \circ \omega: I \longrightarrow N$, com base nos pontos $x_{0} \in \operatorname{Int} M$ e $c \in \operatorname{Int} N$, respectivamente, tal que um deles preserva orientação e o outro inverte orientação. Sem perda de generalidade, podemos supor ainda que $\omega(I) \subseteq \operatorname{Int} M$ e $\mu(I) \subseteq$ Int $N$, pois, caso contrário, basta deformarmos $\omega$ e $\mu$ em vizinhanças contráteis de cada ponto de $\omega(I) \cap \partial M$ e $\mu(I) \cap \partial N$, de modo a obter laços $\omega^{\prime}: I \longrightarrow \operatorname{Int} M$ e $\mu^{\prime}: I \longrightarrow \operatorname{Int} N$, homotópicos a $\omega$ e $\mu$, respectivamente, tais que $\mu^{\prime}=f \circ \omega^{\prime}$, e que preservam ou invertem orientação conforme $\omega$ e $\mu$ também o fazem. Isto posto, sendo $p: \operatorname{Int} M^{\prime} \longrightarrow \operatorname{Int} M$ e $q: \operatorname{Int} \widehat{N} \longrightarrow$ Int $N$ os recobrimentos dados por $p=\left.\left(\hat{p} \circ p^{\prime}\right)\right|_{\operatorname{Int} M^{\prime}}$ e $q=\left.\left(\hat{q} \circ q^{\prime} \circ \hat{q}\right)\right|_{\text {Int } \hat{N}}$, sejam $D_{\omega}^{\prime}: \operatorname{Int} M^{\prime} \longrightarrow \operatorname{Int} M^{\prime}$ e $\widehat{D}_{\mu}:$ Int $\widehat{N} \longrightarrow$ Int $\widehat{N}$ as transformações de deck induzidas por $\omega$ e $\mu=f \circ \omega$ em Int $M^{\prime}$ e Int $\widehat{N}$, respectivamente (vide observação 2.2.1), e considere as aplicações $g_{1}=f^{\prime} \circ D_{\omega}^{\prime}:$ Int $M^{\prime} \longrightarrow \widehat{N}$ e $g_{2}=\widehat{D}_{\mu} \circ f^{\prime}: M^{\prime} \longrightarrow \widehat{N}$. Denotando por $W^{\prime}=M^{\prime} \backslash\left(f^{\prime}\right)^{-1}(\partial \widehat{N})=\operatorname{Int} M^{\prime} \backslash\left(f^{\prime}\right)^{-1}(\partial \widehat{N})$, temos então que:

$$
\operatorname{deg}_{\widehat{c}}\left(\left.g_{1}\right|_{W^{\prime}}\right)=\operatorname{deg}_{\widehat{c}}\left[\left.\left(f^{\prime} \circ D_{\omega}^{\prime}\right)\right|_{W^{\prime}}\right]=\operatorname{deg}_{\widehat{c}}\left[\left(\left.f^{\prime}\right|_{W^{\prime}}\right) \circ D_{\omega}^{\prime}\right] .
$$

Contudo, apesar de $\left(\left.f^{\prime}\right|_{W^{\prime}}\right)^{-1}(\mathcal{C})=\left(f^{\prime}\right)^{-1}(\mathcal{C})$ não ser necessariamente conexo (de maneira que o grau local $\operatorname{deg}_{\left(f^{\prime} \mid W^{\prime}\right)^{-1}(\widehat{c})}\left(D_{\omega}^{\prime}\right)$ pode não estar bem definido), podemos utilizar o 
mesmo raciocínio dado na observação 2.2.2 para mostrar que $\operatorname{deg}_{\widehat{c}}\left[\left(\left.f^{\prime}\right|_{W^{\prime}}\right) \circ D_{\omega}^{\prime}\right]=$ $\operatorname{deg}_{\widehat{c}}\left(\left.f^{\prime}\right|_{W^{\prime}}\right) \cdot \operatorname{deg}_{x_{0}^{\prime}}\left(D_{\omega}^{\prime}\right)$, d'onde obtemos que:

$$
\operatorname{deg}_{\widehat{c}}\left(\left.g_{1}\right|_{W^{\prime}}\right)=\operatorname{deg}_{\widehat{c}}\left(\left.f^{\prime}\right|_{W^{\prime}}\right) \cdot \operatorname{deg}_{x_{0}^{\prime}}\left(D_{\omega}^{\prime}\right) .
$$

Por outro lado, também temos que:

$$
\begin{aligned}
\operatorname{deg}_{\widehat{c}}\left(\left.g_{2}\right|_{W^{\prime}}\right) & =\operatorname{deg}_{\widehat{c}}\left[\left.\left(\widehat{D}_{\mu} \circ f^{\prime}\right)\right|_{W^{\prime}}\right] \\
& =\operatorname{deg}_{\widehat{c}}\left(\widehat{D}_{\mu}\right) \cdot \operatorname{deg}_{\left(\widehat{D}_{\mu}\right)^{-1}(\widehat{c})}\left(\left.f^{\prime}\right|_{W^{\prime}}\right) \\
& =\operatorname{deg}_{\widehat{c}}\left(\widehat{D}_{\mu}\right) \cdot \operatorname{deg}_{\widehat{c}}\left(\left.f^{\prime}\right|_{W^{\prime}}\right) .
\end{aligned}
$$

Além disso, da comutatividade do diagrama ilustrado na figura 2.2 (e do fato de que as transformações de deck $D_{\omega}^{\prime}$ e $\widehat{D}_{\mu}$ satisfazem $p \circ D_{\omega}^{\prime}=p$ e $\left.q \circ \widehat{D}_{\mu}=q\right)$, segue que:

$$
\begin{aligned}
q \circ\left(\left.g_{1}\right|_{W^{\prime}}\right) & =q \circ\left[\left.\left(f^{\prime} \circ D_{\omega}^{\prime}\right)\right|_{W^{\prime}}\right]=q \circ\left(\left.f^{\prime}\right|_{W^{\prime}}\right) \circ D_{\omega}^{\prime} \\
& =\hat{q} \circ q^{\prime} \circ \hat{q} \circ\left(\left.f^{\prime}\right|_{W^{\prime}}\right) \circ D_{\omega}^{\prime}=\hat{q} \circ q^{\prime} \circ\left(\left.\hat{f}\right|_{\widehat{W}}\right) \circ p^{\prime} \circ D_{\omega}^{\prime} \\
& =\hat{q} \circ\left(\left.\hat{f}\right|_{\hat{W}}\right) \circ \hat{p} \circ p^{\prime} \circ D_{\omega}^{\prime}=\left(\left.f\right|_{W}\right) \circ \hat{p} \circ p^{\prime} \circ D_{\omega}^{\prime} \\
& =\left(\left.f\right|_{W}\right) \circ p \circ D_{\omega}^{\prime}=\left(\left.f\right|_{W}\right) \circ p,
\end{aligned}
$$

e:

$$
\begin{aligned}
q \circ\left(\left.g_{2}\right|_{W^{\prime}}\right) & =q \circ\left[\left.\left(\widehat{D}_{\mu} \circ f^{\prime}\right)\right|_{W^{\prime}}\right]=\left(q \circ \widehat{D}_{\mu}\right) \circ\left(\left.f^{\prime}\right|_{W^{\prime}}\right) \\
& =q \circ\left(\left.f^{\prime}\right|_{W^{\prime}}\right)=\left(\left.f\right|_{W}\right) \circ p,
\end{aligned}
$$

onde $W=M \backslash f^{-1}(\partial N), \hat{W}=M \backslash \hat{f}^{-1}(\partial \hat{N})$ e $\widehat{W}=\widehat{M} \backslash \widehat{f}-1\left(\partial N^{\prime}\right)$. Logo, $\left.g_{1}\right|_{W^{\prime}}$ e $\left.g_{2}\right|_{W^{\prime}}$ são levantamentos de $\left(\left.f\right|_{W}\right) \circ p$ relativamente ao recobrimento $q: \operatorname{Int} \widehat{N} \longrightarrow \operatorname{Int} N$. Ademais, sendo $\omega^{\prime}: I \longrightarrow$ Int $M$ o único levantamento de $\omega$, relativamnte a $p$, tal que $\omega^{\prime}(0)=x_{0}^{\prime}$, e considerando $\widehat{\mu}=f^{\prime} \circ \omega^{\prime}: I \longrightarrow \operatorname{Int} \widehat{N}$, temos que:

$$
q \circ \widehat{\mu}=q \circ f^{\prime} \circ \omega^{\prime}=f \circ p \circ \omega^{\prime}=f \circ \omega=\mu,
$$

isto é, $\widehat{\mu}$ é o único levantamento de $\mu$ relativamente ao recobrimento $q$, que começa em $\widehat{\mu}(0)=\left(f^{\prime} \circ \omega^{\prime}\right)(0)=f^{\prime}\left(x_{0}^{\prime}\right)=\widehat{c}$. Assim, como (por definição) $D_{\omega}^{\prime}\left(x_{0}^{\prime}\right)=\omega^{\prime}(1)$ e $\widehat{D}_{\mu}(\mathcal{c})=\widehat{\mu}(1)$, obtemos que:

$$
\begin{aligned}
\left(\left.g_{1}\right|_{W^{\prime}}\right)\left(x_{0}^{\prime}\right) & =g_{1}\left(x_{0}^{\prime}\right)=\left(f^{\prime} \circ D_{\omega}^{\prime}\right)\left(x_{0}^{\prime}\right)=\left(f^{\prime} \circ \omega^{\prime}\right)(1)=\widehat{\mu}(1) \\
& =\widehat{D}_{\mu}(\mathcal{C})=\left(\widehat{D}_{\mu} \circ f^{\prime}\right)\left(x_{0}^{\prime}\right)=g_{2}\left(x_{0}^{\prime}\right)=\left(\left.g_{2}\right|_{W^{\prime}}\right)\left(x_{0}^{\prime}\right) .
\end{aligned}
$$

Portanto, $\left.g_{1}\right|_{W^{\prime}}$ e $\left.g_{2}\right|_{W^{\prime}}$ são levantamentos de $\left(\left.f\right|_{W}\right) \circ p$ (relativamente a q) que coincidem no ponto $x_{0}^{\prime} \in \operatorname{Int} M^{\prime}$, o que, da unicidade destes levantamentos, implica que $\left.g_{1}\right|_{W^{\prime}}=$ $\left.g_{2}\right|_{W^{\prime}}$, e, conseqüentemente, $\operatorname{deg}_{\widehat{c}}\left(\left.g_{1}\right|_{W^{\prime}}\right)=\operatorname{deg}_{\widehat{c}}\left(\left.g_{2}\right|_{W^{\prime}}\right)$, ou seja,

$$
\operatorname{deg}_{\widehat{c}}\left(\left.f^{\prime}\right|_{W^{\prime}}\right) \cdot \operatorname{deg}_{x_{0}^{\prime}}\left(D_{\omega}^{\prime}\right)=\operatorname{deg}_{\widehat{c}}\left(\widehat{D}_{\mu}\right) \cdot \operatorname{deg}_{\widehat{c}}\left(\left.f^{\prime}\right|_{W^{\prime}}\right) \text {. }
$$


Enfim, como apenas um dos laços $\omega$ ou $\mu=f \circ \omega$ preserva orientação, segue, da observação 2.2.2, que $\operatorname{deg}_{x_{0}^{\prime}}\left(D_{\omega}^{\prime}\right) \cdot \operatorname{deg}_{\widehat{c}}\left(\widehat{D}_{\omega}\right)=-1$, o que, pela igualdade acima, significa que $\operatorname{deg}_{\widehat{c}}\left(\left.f^{\prime}\right|_{W^{\prime}}\right)=-\operatorname{deg}_{\widehat{c}}\left(\left.f^{\prime}\right|_{W^{\prime}}\right)$, isto é, $\operatorname{deg}_{\widehat{c}}\left(\left.f^{\prime}\right|_{W^{\prime}}\right)=0$. Deste modo, utilizando o teorema 1.4.2, temos que $\operatorname{deg}\left(f^{\prime}\right)=\operatorname{deg}_{\widehat{c}}\left(\left.f^{\prime}\right|_{W^{\prime}}\right)=0$; por conseguinte, como a cardinalidade j de $\hat{q}^{-1}(c)$ é finita (por hipótese), podemos concluir, pela proposição 2.2.3, que $|m(R)|=0$ para toda classe de raízes $R$ de $f$ em $c$, conforme queríamos demonstrar.

Finalmente, com os resultados obtidos acima, podemos agora calcular $|m(R)|$ para qualquer aplicação própria $f:(M, \partial M) \longrightarrow(N, \partial N)$, num ponto $c \in \operatorname{Int} N$ arbitrário; em particular, isto nos permitirá calcular os números de Nielsen de raízes de $f$ em $c$, pois, conforme veremos na próxima seção, estes números são definidos a partir das multiplicidades $|m(R)|$, as quais, pelo que vimos no teorema 2.1.2, dependem apenas da aplicação $f$ e do ponto $c$.

\subsection{Os Nưmeros de Nielsem de Raízes}

Definição 2.3.1. Dada uma aplicação contínua própria $f:(M, \partial M) \longrightarrow(N, \partial N)$, dizemos que uma classe de raízes $R$ de $f$ em $c \in \operatorname{Int} N$ é essencial se sua multiplicidade $|m(R)|$ é não-nula, e definimos o número de Nielsen de raízes de $f$ em $c$, denotado por $\mathrm{N}(f, c)$, como sendo o número de classes de raízes essenciais de $f$ em c. Por outro lado, se $|m(R)|=0$, dizemos que Ré uma classe de raízes inessencial.

Observe que, como o número de classes de raízes essenciais de $f:(M, \partial M) \longrightarrow$ $(N, \partial N)$ em $c \in \operatorname{Int} N$ é menor ou igual ao número de classes de raízes de $f$ em $c$, e como este, por sua vez, é menor ou igual ao número de raízes de $f$ em $c$ (pois cada classe de raízes de $f$ em $c$ contém pelo menos uma raiz de $f$ em $c$ ) segue que $\mathrm{N}(f, c)$ é um limitante inferior para o número de raízes de $f$ em $c$. Além disso, se $f^{-1}(c)=\varnothing$, segue que não existem classes de raízes de $f$ em $c$ (o que, em particular, implica que $f$ não possui classes de raízes essenciais em $c$ ), e, conseqüentemente, $\mathrm{N}(f, c)=0$.

Definição 2.3.2. Dada uma aplicação contínua própria $f:(M, \partial M) \longrightarrow(N, \partial N)$, e um ponto $c \in \operatorname{Int} N$, dizemos que fé transversa a c se existe uma vizinhança euclidiana $V$ de c em Int $N$, tal que $f^{-1}(V)$ é uma união finita e disjunta de vizinhanças euclidianas em Int $M$, cada uma das quais é levada homeomorficamente por $f$ sobre $V$.

A respeito da definição acima, note que, se $f^{-1}(c)=\varnothing$, segue (do fato de $N$ ser uma variedade topológica e $c \in \operatorname{Int} N$ ) que $c$ admite uma vizinhança euclidiana $V$ em Int $N$ 
tal que $f^{-1}(V)=\varnothing ; \operatorname{logo}, f^{-1}(V)$ é a união de uma família vazia de subconjuntos $\operatorname{de}$ Int $M$ (isto é, uma família sem qualquer conjunto). Ora, como os membros de uma família vazia de conjuntos possuem qualquer propriedade que desejemos (simplesmente por não existirem elementos na família, para os quais a propriedade em questão não se verifica), inclusive àquela que afirma que seus membros são vizinhanças euclidianas levadas homeomorficamente por $f$ sobre $V$, podemos adotar (por "vacuidade") que, se $f^{-1}(c)=\varnothing$, então $f$ é transversa a $c$.

Exemplo 2.3.1. Se $M$ é uma variedade topológica conexa, sem fronteira, e $\bar{p}: \bar{M} \longrightarrow$ Mé um recobrimento que possui um número finito de folhas, então, dado $c \in M$ arbitrário, temos que $\bar{p}$ é uma aplicação transversa a $c$ : com efeito, do exemplo 1.2.3, sabemos que $\bar{p}: \bar{M} \longrightarrow M$ é uma aplicação própria; além disso, como $\bar{p}$ é um recobrimento de, digamos, $k$ folhas, segue que o ponto $c \in M$ admite uma vizinhança euclidiana $V \subseteq M$, tal que $\bar{p}^{-1}(V)$ é a união de uma coleção finita $\left\{U_{1}, U_{2}, \ldots, U_{k}\right\} \subseteq \bar{M}$, de abertos dois a dois disjuntos, onde, para cada $i \in\{1, \ldots, k\}$, a restrição de $\bar{p}$ a $U_{i}$ é um homeomorfismo sobre $V$ (e, portanto, $U_{1}, U_{2}, \ldots, U_{k}$ são vizinhanças euclidianas em $\bar{M}$ ).

Definição 2.3.3 . Dada uma aplicação contínua própria $f:(M, \partial M) \longrightarrow(N, \partial N)$, e um ponto $c \in \operatorname{Int} N$, definimos o número de Nielsen transverso de raízes (de $f$ em $c$ ), denotado por $\mathrm{N}_{\phi}(f, c)$, como sendo:

- $\mathrm{N}_{\phi}(f, c)=0$ se $f^{-1}(c)=\varnothing$;

- $\mathrm{N}_{\phi}(f, c)=\sum\{|m(R)|$ : Ré classe de raízes de $f$ em $c\}$ se $f^{-1}(c) \neq \varnothing$ (isto é, caso $f^{-1}(c) \neq$ $\varnothing, \mathrm{N}_{\pitchfork}(f, c)$ é a soma das multiplicidades de todas as classes de raízes de $f$ em $\left.c\right)$.

Note que, conforme sugere o nome, o número de Nielsen transverso de raízes está diretamente relacionado com o conceito de transversalidade introduzido na definição 2.3.2: de fato, se uma aplicação própria $f:(M, \partial M) \longrightarrow(N, \partial N)$ é transversa a um ponto $c \in \operatorname{Int} N$ (com $f^{-1}(c) \neq \varnothing$ ), segue de imediato que $f^{-1}(c)$ é um conjunto finito, pois, sendo $V \subseteq$ Int $N$ uma vizinhança euclidiana de $c$ tal que $V=\bigcup U_{1} \cup U_{2} \ldots \cup U_{k}$, onde $U_{1}, U_{2}, \ldots, U_{k}$ são vizinhanças euclidianas disjuntas em Int $M$, cada qual levada homeomorficamente por $f$ sobre $V$, temos que cada ponto de $f^{-1}(c)$ está contido num único $U_{i}, i \in\{1,2, \ldots, k\}$; por outro lado, como as restrições $\left.f\right|_{U_{i}}: U_{i} \longrightarrow V$, com $i \in$ $\{1,2, \ldots, k\}$, são homeomorfismos sobre $V$, segue (do corolário 1.3.1) que $\operatorname{deg}_{c}\left(\left.f\right|_{U_{i}}\right)= \pm 1$, e, portanto, pelo que vimos no exemplo 1.3.7, obtemos que:

$$
|m(R)|=\left|\sum_{j=1}^{l} \operatorname{deg}_{c}\left(\left.f\right|_{U_{i}}\right)\right| \leqslant \sum_{j=1}^{l}\left|\operatorname{deg}_{c}\left(\left.f\right|_{U_{i j}}\right)\right|=l,
$$


onde $R=\left\{x_{i_{1}}, x_{i_{2}}, \ldots, x_{i_{l}}\right\} \subseteq f^{-1}(c)$ é uma classe de raízes de $f$ em $c$, e $U_{i_{1}}, U_{i_{2}}, \ldots, U_{i_{l}} \in$ $\left\{U_{1}, \ldots, U_{k}\right\}$. Logo, o número $l$ de raízes na classe $R$ é, no mínimo, igual à multiplicidade de $R$, ou seja, cada classe de raízes $R$ de $f$ em $c$ contém pelo menos $|m(R)|$ raízes de $f$ em $c$. Conseqüentemente, como $\mathrm{N}_{\pitchfork}(f, c)$ é a soma das multiplicidades de todas as classes de raízes de $f$ em $c$, segue que, se $f$ é transversa a $c$, então $\mathrm{N}_{\pitchfork}(f, c)$ é um limitante inferior para o número de classes de raízes de $f$ em $c$.

Isto posto, tendo em vista as interpretações para $\mathrm{N}(f, c)$ e $\mathrm{N}_{\pitchfork}(f, c)$ dadas acima, é natural surgir a seguinte pergunta: se uma aplicação própria $f:(M, \partial M) \longrightarrow(N, \partial N)$ é transversa a um ponto $c \in \operatorname{Int} N$, qual dos dois números $\mathrm{N}(f, c)$ ou $\mathrm{N}_{巾}(f, c)$, é o limitante inferior mais preciso para o número de raízes de $f$ em $c$ ? Sem muitas dificuldades, podemos verificar que a resposta é $\mathrm{N}_{\phi}(f, c)$, pois $\mathrm{N}(f, c) \leqslant \mathrm{N}_{\phi}(f, c)$ : com efeito, tanto $\mathrm{N}(f, c)$, quanto $\mathrm{N}_{\phi}(f, c)$, são calculados utilizando somente as classes de raízes essenciais de $f$ em $c$; entretanto, se $R$ é uma classe de raízes essencial de $f$ em $c$, temos que a "contribuição" de $R$ no cálculo de $\mathrm{N}(f, c)$ é +1 , enquanto que, no cálculo de $\mathrm{N}_{\uparrow}(f, c)$, a "contribuição" de $R$ é $|m(R)| \geqslant 1$ (observe que, como $|m(R)| \in \mathbb{Z}_{+}$é não-nulo, então $|m(R)| \geqslant 1)$, d'onde concluímos que $\mathrm{N}(f, c) \leqslant \mathrm{N}_{\phi}(f, c)$. Nos exemplos apresentados mais adiante, veremos que, em geral, a igualdade $\mathrm{N}(f, c)=\mathrm{N}_{\pitchfork}(f, c)$ não é válida, uma vez que ela ocorre somente quando as classes de raízes de $f$ em $c$ têm multiplicidade igual a 1 (ou quando $f^{-1}(c)=\varnothing$ ).

Agora, sabendo que as classes de raízes de uma aplicação própria $f:(M, \partial M) \longrightarrow$ $(N, \partial N)$, num ponto $c \in \operatorname{Int} N$, têm todas a mesma multiplicidade (conforme vimos no teorema 2.1.2), podemos calcular os números $\mathrm{N}(f, c)$ e $\mathrm{N}_{巾}(f, c)$ diretamente de suas definições, utilizando para tanto os resultados obtidos na seção anterior (bem como o diagrama ilustrado na figura 2.2). No que segue, vamos supor obviamente que $f^{-1}(c) \neq \varnothing$ (pois, caso contrário, sabemos que $\mathrm{N}(f, c)=\mathrm{N}_{巾}(f, c)=0$ ).

Teorema 2.3.1. Seja $f:(M, \partial M) \longrightarrow(N, \partial N)$ uma aplicação contínua própria (entre variedades topológicas conexas de mesma dimensão $n), e$, dado $c \in \operatorname{Int} N$, denote por $\mathrm{j}$ a cardinalidade de $\hat{q}^{-1}(c)$. Se $f$ é uma aplicação do tipo I (isto é, fiel à orientação), com j finito e $\operatorname{deg}(\widehat{f}) \neq 0$, então $\mathrm{N}(f, c)=\mathrm{j}$; por outro lado, se $f$ é do tipo III, com j finito e $\operatorname{deg}(\hat{f}, 2)=1$, também temos $\mathrm{N}(f, c)=\mathrm{j}$. Em todos os demais casos, vale que $\mathrm{N}(f, c)=0$.

DemonstraçÃo. Com efeito, se $f$ é do tipo I e j é finito, temos (do teorema 2.1.2 e do corolário 2.2.1) que $|m(R)|=|\operatorname{deg}(\widehat{f})|$ para toda classe de raízes $R$ de $f$ em $c ; \operatorname{logo}$, supondo que $\operatorname{deg}(\widehat{f}) \neq 0$, segue que toda classe de raízes de $f$ em $c$ é essencial, e, portanto, o número de Nielsen $\mathrm{N}(f, c)$ coincide com o número de classes de raízes de $f$ em $c$. No entanto, do corolário 2.1.1, sabemos que, dada uma classe de raízes $R$ de 
$f$ em $c$, existe um elemento $\hat{c} \in \hat{q}^{-1}(c)$ tal que $R=\hat{f}^{-1}(\hat{c})$; assim, como quaisquer duas classes de raízes de $f$ em $c$ são disjuntas, segue que o número de classes de raízes de $f$ em $c$ é igual à cardinalidade $\mathrm{j}$ de $\hat{q}^{-1}(c)$, d'onde concluímos que $\mathrm{N}(f, c)=\mathrm{j}$.

Analogamente, se $f$ é do tipo III e j é finito, a proposição 2.2.1 (juntamente com o teorema 2.1.2) nos garante que $|m(R)|=\operatorname{deg}(\hat{f}, 2) \in \mathbb{Z}_{2}$, para toda classe de raízes $R$ de $f$ em $c$; deste modo, $\operatorname{se} \operatorname{deg}(\hat{f}, 2) \neq 0 \in \mathbb{Z}_{2}$ (isto é, $\operatorname{se} \operatorname{deg}(\hat{f}, 2)=1$ ), temos que toda classe de raízes de $f$ em $c$ é essencial, e, conseqüentemente, $\mathrm{N}(f, c)$ coincide com o número de classes de raízes de $f$ em $c$, que, conforme vimos acima, é igual à cardinalidade $\mathrm{j}$ de $\hat{q}^{-1}(c)$.

Finalmente, observe que, se $f$ for do tipo I e j finito, mas $\operatorname{deg}(\widehat{f})=0$, então $|m(R)|=$ $|\operatorname{deg}(\widehat{f})|=0$ para toda classe de raízes $R$ de $f$ em $c$, o que implica que $\mathrm{N}(f, c)=0$, sendo que o mesmo ocorre se $f$ for do tipo III, com j finito e $\operatorname{deg}(\hat{f}, 2)=0 \in \mathbb{Z}_{2}$, pois, neste caso, também teremos $|m(R)|=\operatorname{deg}(\hat{f}, 2)=0$ para toda classe de raízes $R$ de $f$ em $c$. Já nos casos em que $f$ é do tipo II ou j é infinito, segue de imediato (da proposição 2.2.2 e do corolário 2.2.2) que $|m(R)|=0$ para toda classe de raízes $R$ de $f$ em $c$, e, portanto, $\mathrm{N}(f, c)=0$.

Teorema 2.3.2. Seja $f:(M, \partial M) \longrightarrow(N, \partial N)$ uma aplicação contínua própria (entre variedades topológicas conexas de mesma dimensão $n), e$, dado $c \in \operatorname{Int} N$, denote por $\mathrm{j}$ a cardinalidade de $\hat{q}^{-1}(c)$. Se $f$ é uma aplicação do tipo I e jé finito, temos que $\mathrm{N}_{\phi}(f, c)=\mathrm{j} \cdot|\operatorname{deg}(\widehat{f})|$, enquanto que, se $f$ é do tipo III e jé finito, temos $\mathrm{N}_{\phi}(f, c)=\mathrm{j} \cdot \operatorname{deg}(\hat{f}, 2)$; nos demais casos (isto é, se $f$ for do tipo II ou j for infinito), tem-se que $\mathrm{N}_{\pitchfork}(f, c)=0$.

DEMOnstraÇÃo. De fato, conforme vimos na demonstração do teorema anterior, se $f$ é do tipo I e j é finito, temos $|m(R)|=|\operatorname{deg}(\widehat{f})|$ para toda classe de raízes $R$ de $f$ em $c$, sendo que j coincide com o número de classes de raízes de $f$ em $c$; logo, por definição, temos que:

$$
\mathrm{N}_{\phi}(f, c)=\sum\{|m(R)|: R \text { é classe de raízes de } f \text { em } c\}=\mathrm{j} \cdot|\operatorname{deg}(\widehat{f})| .
$$

Por outro lado, se $f$ é do tipo III e j é finito, segue que $|m(R)|=\operatorname{deg}(\hat{f}, 2) \in \mathbb{Z}_{2}$ para toda classe de raízes $R$ de $f$ em $c$, e, portanto (como j é igual ao número de classes de raízes de $f$ em $c), \mathrm{N}_{\phi}(f, c)=\mathrm{j} \cdot \operatorname{deg}(\hat{f}, 2)$. Enfim, se $f$ for do tipo II ou $\mathrm{j}$ for infinito, teremos que $|m(R)|=0$ para toda classe de raízes $R$ de $f$ em $c$ (conforme nos garante a proposição 2.2.2 e o corolário 2.2.2), de modo que $\mathrm{N}_{\phi}(f, c)=0$.

Assim sendo, os teoremas 2.3.1 e 2.3.2 nos permitem calcular os números de Nielsen $\mathrm{N}(f, c)$ e $\mathrm{N}_{\phi}(f, c)$ para todo tipo de aplicação própria $f:(M, \partial M) \longrightarrow(N, \partial N)$, em qualquer ponto $c \in \operatorname{Int} N$. Contudo, se, em particular, $M$ e $N$ forem variedades orientáveis 
(conexas e de mesma dimensão $n$ ), este teoremas podem ser simplificados utilizando-se o grau cohomológico $\operatorname{deg}(f)$ nos cálculos de $\mathrm{N}(f, c)$ e $\mathrm{N}_{\phi}(f, c)$. Mais especificamente, temos o seguinte

Corolário 2.3.1. Seja $f:(M, \partial M) \longrightarrow(N, \partial N)$ uma aplicação contínua própria, entre variedades topológicas conexas, orientáveis e de mesma dimensão $n$. Dado $c \in \operatorname{Int} N$, e denotando por $\mathrm{j}$ a cardinalidade de $\hat{q}^{-1}(c)$, temos que:

$$
\mathrm{N}(f, c)=\left\{\begin{array}{lll}
\mathrm{j} & \text { se } & \operatorname{deg}(f) \neq 0 \\
0 & \text { se } & \operatorname{deg}(f)=0
\end{array},\right.
$$

enquanto que $\mathrm{N}_{\phi}(f, c)=|\operatorname{deg}(f)|$.

DemonstraçÃo. Primeiramente, suponhamos que a cardinalidade $\mathrm{j}$ de $\hat{q}^{-1}(c)$ seja finita; neste caso, como $\hat{q}: \hat{N} \longrightarrow N$ é uma aplicação de recobrimento e $N$ é uma variedade topológica conexa (e, portanto, todas as fibras $\hat{q}^{-1}(y), y \in N$, têm a mesma cardinalidade), segue que $\hat{q}$ possui um número finito de folhas, o que, pelo exemplo 1.3.3, significa que $\hat{q}: \hat{N} \longrightarrow N$ é uma aplicação própria. Agora, lembrando que $\hat{q}(\partial \hat{N})=$ $\partial N$ (pois $\hat{q}$ é um homeomorfismo local), temos que a restrição $\left.\hat{q}\right|_{\operatorname{Int} \hat{N}}: \operatorname{Int} \hat{N} \longrightarrow \operatorname{Int} N$ é um recobrimento de $\operatorname{Int} N$ que possui o mesmo número de folhas de $\hat{q}$ (a saber, j); assim, como Int $N$, e, conseqüentemente, Int $\hat{N}$, são orientáveis, segue (do teorema 1.4.2) que $\operatorname{deg}(\hat{q})=\operatorname{deg}_{c}\left(\left.\hat{q}\right|_{\operatorname{Int} \hat{N}}\right)$, isto é, $\operatorname{deg}(\hat{q})= \pm \mathrm{j}($ vide corolário 1.3.2). Por outro lado, como $M$ é uma variedade topológica orientável, temos que o recobrimento orientado $\widehat{p}: \widehat{M} \longrightarrow M$ é a identidade (ou seja, $\widehat{M}=M$ ); logo, como $q^{\prime}: N^{\prime} \longrightarrow \hat{N}$ é o recobrimento de $\hat{N}$ associado ao subgrupo $(\hat{f} \circ \hat{p})_{\#}\left(\pi_{1}\left(\widehat{M}, \widehat{x}_{0}\right)\right)=\hat{f}_{\#}\left(\pi_{1}\left(M, x_{0}\right)\right) \subseteq \pi_{1}(\hat{N}, \hat{c})$, segue que $\hat{f_{\#}}\left(\pi_{1}\left(M, x_{0}\right)\right)=q_{\#}^{\prime}\left(\pi_{1}\left(N^{\prime}, c^{\prime}\right)\right)$, enquanto que $\hat{f}: \widehat{M}=M \longrightarrow N^{\prime}$ é tal que $\hat{f}=q^{\prime} \circ \hat{f}$ (uma vez que $\hat{f}$ é o levantamento de $\hat{f} \circ \hat{p}=\hat{f}$ relativamente ao recobrimento $\left.q^{\prime}: N^{\prime} \longrightarrow \hat{N}\right)$. Observe então que o homomorfismo $\hat{f_{\#}}: \pi_{1}\left(M, x_{0}\right) \longrightarrow \pi_{1}(\hat{N}, \hat{c})$ é sobrejetivo: com efeito, dada uma classe $[\hat{\alpha}] \in \pi_{1}(\hat{N}, \hat{c})$, considere $[\beta]=\hat{q}_{\#}([\hat{\alpha}]) \in \hat{q}_{\#}\left(\pi_{1}(\hat{N}, \hat{c})\right)$; como $\hat{q}_{\#}\left(\pi_{1}(\hat{N}, \hat{c})\right)=f_{\#}\left(\pi_{1}\left(M, x_{0}\right)\right.$ ) (pois $\hat{q}: \hat{N} \longrightarrow N$ é o recobrimento de $N$ associado ao subgrupo $\left.f_{\#}\left(\pi_{1}\left(M, x_{0}\right)\right) \subseteq \pi_{1}(N, c)\right)$, segue que existe $[\alpha] \in \pi_{1}\left(M, x_{0}\right)$ tal que $f_{\#}([\alpha])=$ $[\beta]=\hat{q}_{\#}([\hat{\alpha}])$, e, portanto, $\hat{q}_{\#}\left(\hat{f}_{\#}([\alpha])\right)=f_{\#}([\alpha])=[\beta]=\hat{q}_{\#}([\hat{\alpha}])$, o que (pelo fato do homomorfismo $\hat{q}_{\#}: \pi_{1}(\hat{N}, \hat{c}) \longrightarrow \pi_{1}(N, c)$, induzido pelo recobrimento $\hat{q}: \hat{N} \longrightarrow N$, ser injetivo), implica que $\hat{f_{\#}}([\alpha])=[\hat{\alpha}]$, mostrando que $\hat{f_{\#}}: \pi_{1}\left(M, x_{0}\right) \longrightarrow \pi_{1}(\hat{N}, \hat{c})$ é sobrejetivo. Deste modo, obtemos que:

$$
q_{\#}^{\prime}\left(\pi_{1}\left(N^{\prime}, c^{\prime}\right)\right)=\hat{f_{\#}}\left(\pi_{1}\left(M, x_{0}\right)\right)=\pi_{1}(\hat{N}, \hat{c})
$$

isto é, o homomorfismo $q_{\#}^{\prime}: \pi_{1}\left(N^{\prime}, c^{\prime}\right) \longrightarrow \pi_{1}(\hat{N}, \hat{c})$ também é sobrejetivo. Isto, por sua vez, significa que o índice do subgrupo $q_{\#}^{\prime}\left(\pi_{1}\left(N^{\prime}, c^{\prime}\right)\right) \subseteq \pi_{1}(\hat{N}, \hat{c})$, em $\pi_{1}(\hat{N}, \hat{c})$, é 
igual a 1, e, conseqüentemente, o número de folhas do recobrimento $q^{\prime}: N^{\prime} \longrightarrow \hat{N}$ é igual a 1 (pois, conforme se verifica em [Li], corolário 1, página 152, o número de folhas de $q^{\prime}$ é igual ao índice do subgrupo $q_{\#}^{\prime}\left(\pi_{1}\left(N^{\prime}, c^{\prime}\right)\right)$ em $\left.\pi_{1}(\hat{N}, \hat{c})\right)$. Portanto,a restrição $\left.q^{\prime}\right|_{\text {Int } N^{\prime}}: \operatorname{Int} N^{\prime} \longrightarrow \operatorname{Int} \hat{N}$ é um recobrimento de uma única folha, e (pelo exemplo 1.3.3, corolário 1.3.2 e teorema 1.4.2) $\operatorname{deg}\left(q^{\prime}\right)=\operatorname{deg}_{c^{\prime}}\left(\left.q^{\prime}\right|_{\operatorname{lnt} N^{\prime}}\right)= \pm 1$ (note que $N$, e conseqüentemente $\hat{N}$ e $N^{\prime}$, são variedades orientáveis, conforme nos garante a proposição 1.1.1). Logo, sendo $\operatorname{deg}(\hat{q})= \pm \mathrm{j}$ e $\operatorname{deg}\left(q^{\prime}\right)= \pm 1$, concluímos (utilizando a proposição 1.4.6) que:

$$
\begin{aligned}
\operatorname{deg}(f) & =\operatorname{deg}(\hat{q} \circ \hat{f})=\operatorname{deg}(\hat{q}) \cdot \operatorname{deg}(\hat{f})=\operatorname{deg}(\hat{q}) \cdot \operatorname{deg}\left(q^{\prime} \circ \hat{f}\right) \\
& =\operatorname{deg}(\hat{q}) \cdot \operatorname{deg}\left(q^{\prime}\right) \cdot \operatorname{deg}(\hat{f})= \pm \mathbf{j} \cdot \operatorname{deg}(\widehat{f}) .
\end{aligned}
$$

Assim, como $\mathrm{j}>0$, temos que $|\operatorname{deg}(f)|=\mathrm{j} \cdot|\operatorname{deg}(\widehat{f})|$, sendo que $\operatorname{deg}(f) \neq 0$ se, e somente se, $\operatorname{deg}(\widehat{f}) \neq 0$. Por conseguinte, dos teoremas 2.3 .1 e 2.3.2, obtemos que $\mathrm{N}(f, c)=\mathrm{j}$ se $\operatorname{deg}(f) \neq 0, \mathrm{~N}(f, c)=0$ se $\operatorname{deg}(f)=0$, e $\mathrm{N}_{\phi}(f, c)=|\operatorname{deg}(f)|$.

Finalmente, suponhamos agora que a cardinalidade j de $\hat{q}^{-1}(c)$ é infinita. Neste caso, pela proposição 2.2.2, temos que $|m(R)|=0$ para toda classe de raízes $R$ de $f$ em $c$; conseqüentemente, dada uma classe de raízes $R$ de $f$ em $c$, e sendo $V \subseteq \operatorname{Int} N$ uma vizinhança aberta contrátil de $c$, temos (pela definição 1.3.7) que $\operatorname{deg}_{c}\left(\left.f\right|_{U_{R}}\right)=0$, onde $U_{R} \subseteq f^{-1}(V)$ é um aberto tal que $U_{R} \cap f^{-1}(c)=R$. Logo, do corolário 1.4.2, obtemos que:

$$
\operatorname{deg}(f)=\sum\left\{\operatorname{deg}_{c}\left(\left.f\right|_{u_{R}}\right): R \text { é classe de raízes de } f \text { em } c\right\}=0 .
$$

Portanto, como $f:(M, \partial M) \longrightarrow(N, \partial N)$ é uma aplicação própria do tipo I (pois $M$ e $N$ são orientáveis), concluímos (a partir dos teoremas 2.3.1 e 2.3.2) que, se jé infinito, então $\mathrm{N}(f, c)=0$ e $\mathrm{N}_{\pitchfork}(f, c)=0=|\operatorname{deg}(f)|$. Resumindo, temos, pois, que $\mathrm{N}_{\phi}(f, c)=|\operatorname{deg}(f)|$, $\mathrm{N}(f, c)=\mathrm{j}$ se $\operatorname{deg}(f) \neq 0$, e $\mathrm{N}(f, c)=0$ se $\operatorname{deg}(f)=0$, como queríamos demonstrar.

Da invariância do grau cohomológico por homotopias próprias (proposição 1.4.5), obtemos ainda o seguinte

Corolário 2.3.2 . Seja $f:(M, \partial M) \longrightarrow(N, \partial N)$ uma aplicação contínua própria (entre variedades topológicas conexas de mesma dimensão $n)$. Dado $c \in \operatorname{Int} N$, temos que os números de Nielsen $\mathrm{N}(f, c)$ e $\mathrm{N}_{\phi}(f, c)$ são invariantes por homotopias próprias, isto é, se $H:(M \times I, \partial M \times I) \longrightarrow(N, \partial N)$ é uma homotopia própria entre $f$ e outra aplicação contínua própria $g:(M, \partial M) \longrightarrow(N, \partial N)$, então $\mathrm{N}(f, c)=\mathrm{N}(g ; c)$ e $\mathrm{N}_{\phi}(f, c)=\mathrm{N}_{巾}(g ; c)$. Além disso, tanto $\mathrm{N}(f, c)$, quanto $\mathrm{N}_{\phi}(f, c)$, são independentes da escolha do ponto $c \in \operatorname{Int} N$.

DemonstraÇÃo. Com efeito, dado $c \in \operatorname{Int} N$, sejam $x_{0}, x_{1} \in \operatorname{Int} M$ tais que $f\left(x_{0}\right)=c$ e $g\left(x_{1}\right)=c$, onde $g:(M, \partial M) \longrightarrow(N, \partial N)$ é uma aplicação contínua própria, homotópica 
a $f$ por uma homotopia própria $H:(M \times I, \partial M \times I) \longrightarrow(N, \partial N)$. Como $M$ é uma variedade conexa (e, portanto, conexa por caminhos), sabemos que existe um isomorfismo $\gamma: \pi_{1}\left(M, x_{0}\right) \longrightarrow \pi_{1}\left(M, x_{1}\right)$ tal que $f_{\#}=g_{\#} \circ \gamma$, onde $f_{\#}: \pi_{1}\left(M, x_{0}\right) \longrightarrow \pi_{1}(N, c)$ e $g_{\#}: \pi_{1}\left(M, x_{1}\right) \longrightarrow \pi_{1}(N, c)$ são os homomorfismos induzidos por $f$ e $g$, respectivamente; logo, como $\gamma\left(\pi_{1}\left(M, x_{0}\right)\right)=\pi_{1}\left(M, x_{1}\right)$, segue que $f_{\#}\left(\pi_{1}\left(M, x_{0}\right)\right)=\left(g_{\#} \circ \gamma\right)\left(\pi_{1}\left(M, x_{0}\right)\right)=$ $g_{\#}\left(\pi_{1}\left(M, x_{1}\right)\right)$, e, conseqüentemente, o recobrimento $\hat{q}: \hat{N} \longrightarrow N$ associado ao subgrupo $f_{\#}\left(\pi_{1}\left(M, x_{0}\right)\right)$ de $\pi_{1}(N, c)$ coincide com o recobrimento associado ao subgrupo $g_{\#}\left(\pi_{1}\left(M, x_{1}\right)\right)$. Além disso, sem muitas dificuldades, pode-se verificar que os levantamentos $\hat{f}: M \longrightarrow \hat{N}$ e $\hat{g}: M \longrightarrow \hat{N}$ (de $f$ e $g$, respectivamente), pelo recobrimento $\hat{q}: \hat{N} \longrightarrow N$, são propriamente homotópicos, isto é, existe uma homotopia própria $\hat{H}:(M \times I, \partial M \times I) \longrightarrow(\hat{N}, \partial \hat{N})$ entre $\hat{f}$ e $\hat{g}$ (basta tomar $\hat{H}$ como sendo o levantamento de $H$ relativamente a $\hat{q}$, observando que o fato de $H$ ser própria nos garante que $\hat{H}$ também o é). Deste modo, segue que as aplicações $\hat{f} \circ \hat{p}: \widehat{M} \longrightarrow \hat{N}$ e $\hat{q} \circ \hat{p}: \widehat{M} \longrightarrow \hat{N}$ são propriamente homotópicas, e, portanto (por um raciocínio análogo ao que utilizamos acima), o recobrimento $q^{\prime}: N^{\prime} \longrightarrow \hat{N}$ associado ao subgrupo $(\hat{f} \circ \hat{p})_{\#}\left(\pi_{1}\left(\widehat{M}, \widehat{x}_{0}\right)\right) \subseteq \pi_{1}(\hat{N}, \hat{c})$ coincide com o recobrimento de $\hat{N}$ associado ao subgrupo $(\hat{q} \circ \hat{p}) \#\left(\pi_{1}\left(\widehat{M}, \widehat{x}_{1}\right)\right) \subseteq \pi_{1}(\hat{N}, \hat{c})$, onde $\widehat{x}_{1} \in \widehat{p}^{-1}\left(x_{1}\right)$. Daí, segue que os levantamentos $\widehat{f}: \widehat{M} \longrightarrow N^{\prime}$ e $\widehat{q}: \widehat{M} \longrightarrow N^{\prime}$, de $\hat{f} \circ \hat{p}$ e $\hat{q} \circ \hat{p}$, respectivamente, relativos a $q^{\prime}: N^{\prime} \longrightarrow \hat{N}$, também são propriamente homotópicos, o que (pela proposição 1.4.5) nos permite concluir que $\operatorname{deg}(\widehat{f})=\operatorname{deg}(\widehat{q})$ e $\operatorname{deg}(\hat{f}, 2)=\operatorname{deg}(\hat{g}, 2)$, desde que estes números estejam bem definidos. Portanto, dos teoremas 2.3.1 e 2.3.2, obtemos que $\mathrm{N}(f, c)=\mathrm{N}(g ; c)$ e $\mathrm{N}_{巾}(f, c)=\mathrm{N}_{\pitchfork}(g ; c)$, ou seja, o números de Nielsen $\mathrm{N}(f, c)$ e $\mathrm{N}_{\phi}(f, c)$ são invariantes por homotopias próprias.

Finalmente, para mostrarmos que $\mathrm{N}(f, c)$ e $\mathrm{N}_{\phi}(f, c)$ não dependem da escolha do ponto $c \in \operatorname{Int} N$, basta observarmos que, para quaisquer $c_{1}, c_{2} \in \operatorname{Int} N$, a cardinalidade dos conjuntos $\hat{q}^{-1}\left(c_{1}\right)$ e $\hat{q}^{-1}\left(c_{2}\right)$ é a mesma (uma vez que $N$ é uma variedade topológica conexa), além do que, os graus cohomológicos $\operatorname{deg}(\widehat{f})$ e $\operatorname{deg}(\hat{f}, 2)$ são independentes de $c \in \operatorname{Int} N$; logo, pelos teoremas 2.3.1 e 2.3.2, segue que os números de Nielsen $\mathrm{N}(f, c)$ e $\mathrm{N}_{\phi}(f, c)$ são, de fato, independentes de $c \in \operatorname{Int} N$.

Definição 2.3.4 . Dadas aplicações contínuas próprias $f, g:(M, \partial M) \longrightarrow(N, \partial N)$, entre variedades topológicas conexas de mesma dimensão $n$, escreveremos $f \tilde{p} g$ para indicar que $f$ e g são propriamente homotópicas. Além disso, dado $c \in \operatorname{Int} N$, denotaremos por $\operatorname{MR}[f ; c] o$ número mínimo de raízes na classe de homotopia própria de $f$, ou seja:

$$
\operatorname{MR}[f ; c]=\min \{\text { número de raízes de } g \text { em } c: g \widetilde{p} f\} \text {. }
$$

Definimos ainda o número mínimo transverso de raízes, denotado por $\operatorname{MR}_{\pitchfork}[f ; c]$, como sendo o menor número de raízes na classe de homotopia própria de $f$, com a restrição de que as 
aplicações $g:(M, \partial M) \longrightarrow(N, \partial N)$, tais que $g_{\tilde{p}} f$, sejam transversas a $c$, isto é:

$$
\operatorname{MR}_{\phi}[f ; c]=\min \{\text { número de raízes de g em } c: g \tilde{p} f \text { e gétransversa a } c\} \text {. }
$$

Como conseqüência imediata do corolário 2.3.2, segue que, se $f:(M, \partial M) \longrightarrow(N, \partial N)$ é uma aplicação contínua própria e $c \in \operatorname{Int} N$, então $\mathrm{N}(f, c) \leqslant \operatorname{MR}[f ; c]$ e $\mathrm{N}_{\pitchfork}(f, c) \leqslant$ $\operatorname{MR}_{\phi}[f ; c]$. Na próxima seção, veremos que, se a dimensão das variedades $M$ e $N$ for diferente de 2, os números de Nielsen $\mathrm{N}(f, c)$ e $\mathrm{N}_{\phi}(f, c)$ são realizáveis, ou seja, existem aplicações contínuas próprias $g, g^{\prime}:(M, \partial M) \longrightarrow(N, \partial N)$, com $g \widetilde{p} f, g^{\prime} \tilde{p} f$ e $g^{\prime}$ transversa a $c$, tais que as cardinalidades de $g^{-1}(c)$ e $g^{\prime-1}(c)$ são respectivamente iguais a $\mathrm{N}(f, c)$ e $\mathrm{N}_{\phi}(f, c)$, o que mostrará que $\mathrm{N}(f, c)=\operatorname{MR}[f ; c]$ e $\mathrm{N}_{巾}(f, c)=\operatorname{MR}_{\phi}[f ; c]$.

Entretanto, antes de encerrarmos esta seção, convém apresentarmos alguns exemplos que ilustrem os cálculos dos números de Nielsen; em particular, os três primeiros exemplos a seguir mostram, inclusive, que os números de Nielsen são realizáveis para alguns tipos específicos de aplicações entre variedades de dimensão 1.

Exemplo 2.3.2 . Dada uma aplicação contínua própria $f: \mathbb{R} \rightarrow \mathbb{R}$, seja $c \in \mathbb{R}$ tal que $f^{-1}(c) \neq \varnothing$; temos então que $f^{-1}(c)$ é um subconjunto compacto de $\mathbb{R}$, o que implica que existe um intervalo aberto $] a, b\left[\subseteq \mathbb{R}\right.$ (com $a<b$, obviamente), tal que $\left.f^{-1}(c) \subseteq\right] a, b$. Isto posto, considere a aplicação $g: \mathbb{R} \longrightarrow \mathbb{R}$ dada por:

$$
g(x)=\left\{\begin{array}{lll}
f(x) & \text { se } & x \in \mathbb{R} \backslash] a, b[ \\
{\left[\frac{f(a)-f(b)}{a-b}\right] \cdot x+\left[\frac{a \cdot f(b)-b \cdot f(a)}{a-b}\right]} & \text { se } & x \in] a, b[
\end{array} .\right.
$$

Sem muitas dificuldades, pode-se ver que $g$ está bem definida e é contínua em $\mathbb{R}$, sendo que o gráfico da restrição $\left.g\right|_{[a, b]}$ é o segmento de reta que liga os pontos $(a, f(a))$ e $(b, f(b))$, conforme ilustra a figura 2.3 (note que $a, b \notin f^{-1}(c)$, pois $f^{-1}(c)$ é um subconjunto fechado de $\mathbb{R}$, contido no aberto $] a, b[$ ). Além disso, temos que $g$ também é uma aplicação própria, uma vez que $f$ é própria e $g$ coincide com $f$ exceto no conjunto compacto $[a, b] \subseteq \mathbb{R}$.

Agora, denotando por $\varphi:[a, b] \longrightarrow[f(a), f(b)]$ o homeomorfismo dado por

$$
\varphi(x)=\left[\frac{f(a)-f(b)}{a-b}\right] \cdot x+\left[\frac{a \cdot f(b)-b \cdot f(a)}{a-b}\right],
$$

observe que a aplicação $h:[a, b] \times I \longrightarrow \mathbb{R}$, definida por

$$
h(x, t)=t \cdot \varphi(x)+(1-t) \cdot f(x)
$$

para cada $(x, t) \in[a, b] \times I$, constitui uma homotopia entre a restrição $\left.f\right|_{[a, b]}$ e o segmento de reta dado por $\varphi:[a, b] \longrightarrow \mathbb{R}$; como $[a, b] \times I$ é compacto, temos ainda que $h$ é uma 
homotopia própria. Considere, pois, a aplicação $H: \mathbb{R} \times I \longrightarrow \mathbb{R}$, definida por:

$$
H(x, t)=\left\{\begin{array}{lll}
h(x, t) & \text { se } & x \in[a, b] \\
g(x)=f(x) & \text { se } & x \in \mathbb{R} \backslash] a, b[
\end{array} .\right.
$$

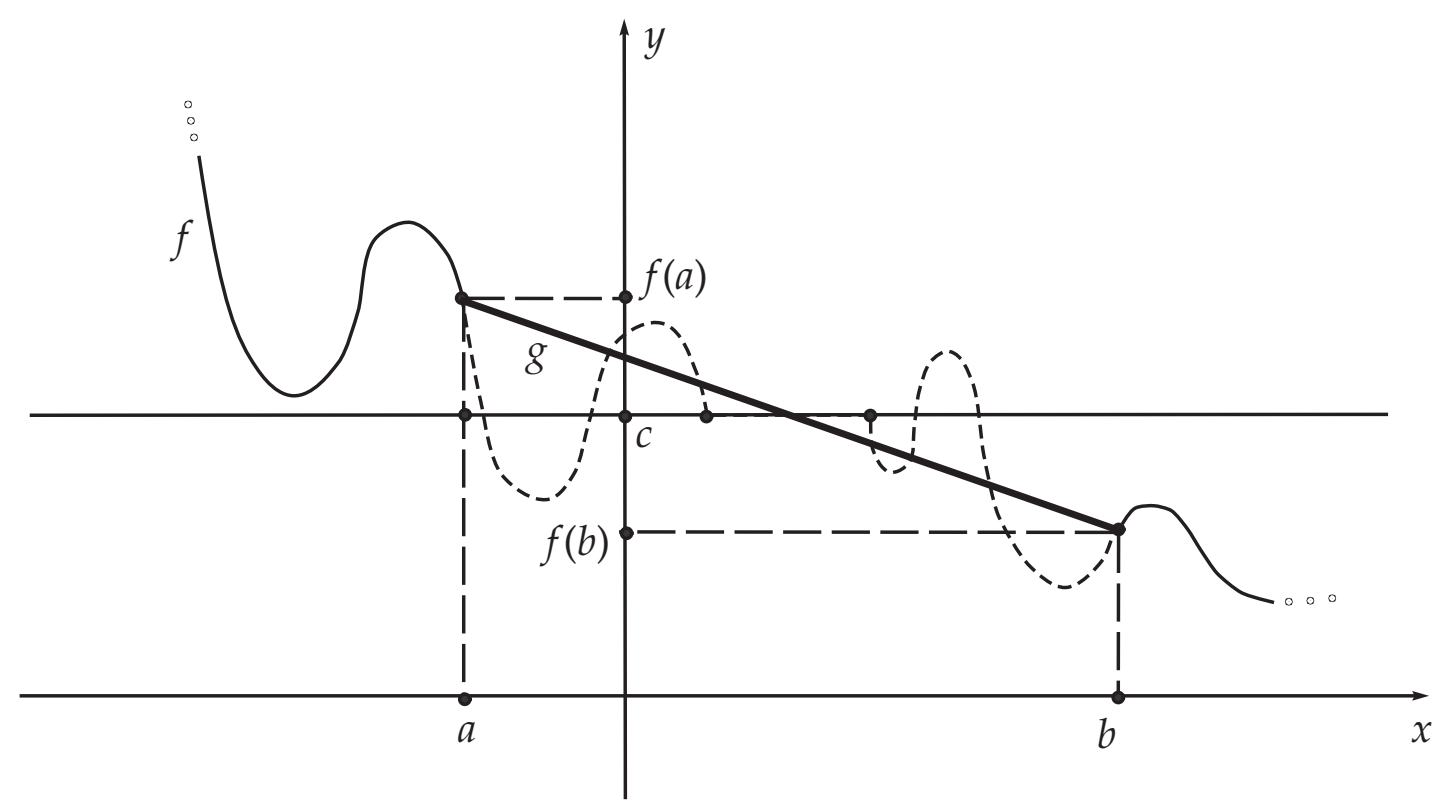

Figura 2.3

Como $H(a, t)=h(a, t)=f(a)=g(a)$ e $H(b, t)=h(b, t)=f(b)=g(b)$ para todo $t \in I$, segue que $H$ encontra-se bem definida e é contínua em $\mathbb{R} \times I$; ademais, como

$$
H(x, 0)=\left\{\begin{array}{lll}
h(x, 0)=f(x) & \text { se } & x \in[a, b] \\
g(x)=f(x) & \text { se } & x \in \mathbb{R} \backslash] a, b[
\end{array}=f(x)\right.
$$

$\mathrm{e}$

$$
H(x, 1)=\left\{\begin{array}{lll}
h(x, 1)=\varphi(x) & \text { se } & x \in[a, b] \\
g(x)=f(x) & \text { se } & x \in \mathbb{R} \backslash] a, b[
\end{array}=g(x),\right.
$$

temos que $H$ contitui uma homotopia entre $f$ e $g$, a qual também é própria, uma vez que $f, g$ e $h$ são aplicações próprias.

Finalmente, do corolário 2.3.2, obtemos então que $\mathrm{N}(f, c)=\mathrm{N}(g, c)$ e $\mathrm{N}_{巾}(f, c)=$ $\mathrm{N}_{\phi}(g, c)$. No entanto, note que $\mathrm{N}(g, c)=\mathrm{N}_{\phi}(g, c)=0$ ou $\mathrm{N}(g, c)=\mathrm{N}_{\phi}(g, c)=1$ : com efeito, se os pontos $(a, f(a))$ e $(b, f(b))$ estão localizados no mesmo semi-plano de $\mathbb{R}^{2}$ determinado pela reta $y=c$, segue que o segmento de reta dado por $\varphi:[a, b] \longrightarrow \mathbb{R}$ não intercepta a reta $y=c$, o que implica que $g$ não possuirá raízes em $c \in \mathbb{R}$ (pois, 
para $x \in \mathbb{R} \backslash] a, b\left[\right.$, temos $g(x)=f(x)$, e, portanto, $g(x) \neq c$, uma vez que $\left.f^{-1}(c) \subseteq\right] a, b[$ ), e, conseqüentemente, teremos $\mathrm{N}(g, c)=\mathrm{N}_{\uparrow}(g, c)=0$. Por outro lado, se os pontos $(a, f(a))$ e $(b, f(b))$ estão localizados em lados opostos do plano $\mathbb{R}^{2}$, relativamente à reta dada por $y=c$, segue que o segmento de reta dado por $\varphi:[a, b] \longrightarrow \mathbb{R}$ intercepta a reta $y=c$ num único ponto, o que, por sua vez, significa que $g$ terá exatamente uma raiz em $c$, a saber, o ponto $z \in[a, b]$ tal que $\varphi(z)=c$. Neste caso, $R=\{z\}$ será a única classe de raízes de $g$ em $c$, e, denotando por $V \subseteq \mathbb{R}$ um intervalo aberto tal que $c \in V \subseteq[f(a), f(b)]$, teremos que $U=\varphi^{-1}(V) \subseteq[a, b]$ é um intervalo aberto de $\mathbb{R}$, contendo $z=\varphi^{-1}(c)$, que satisfaz $U \cap g^{-1}(c)=R$; logo, como $\varphi:[a, b] \longrightarrow \mathbb{R}$ é um homeomorfismo sobre $[f(a), f(b)]$, e $\left.g\right|_{U}=\left.\varphi\right|_{U}$, segue (da definição 1.3 .7 e do corolário 1.3.1) que:

$$
|m(R)|=\left|\operatorname{deg}_{c}\left(\left.g\right|_{U}\right)\right|=\left|\operatorname{deg}_{c}\left(\left.\varphi\right|_{U}\right)\right|=| \pm 1|=1,
$$

o que (pelas definições 2.3.1 e 2.3.3) implica que $\mathrm{N}(g, c)=\mathrm{N}_{\phi}(g, c)=1$. Deste modo, se $\mathrm{N}(f, c)=\mathrm{N}(g, c)$ e $\mathrm{N}_{\phi}(f, c)=\mathrm{N}_{\phi}(g, c), \operatorname{com} \mathrm{N}(g, c)=\mathrm{N}_{\phi}(g, c)=0$ ou $\mathrm{N}(g, c)=\mathrm{N}_{\phi}(g, c)=$ 1 , concluímos que $\mathrm{N}(f, c)=\mathrm{N}_{\phi}(f, c)=0$ ou $\mathrm{N}(f, c)=\mathrm{N}_{\phi}(f, c)=1$.

Observe ainda que a aplicação $g: \mathbb{R} \longrightarrow \mathbb{R}$, utilizada nos cálculos dos números de Nielsen $\mathrm{N}(f, c)$ e $\mathrm{N}_{\phi}(f, c)$, é transversa ao ponto $c$ (por vacuidade, caso $g$ não tenha raízes em $c$, ou pelo fato de $\varphi=\left.g\right|_{[a, b]}$ ser um homeomorfismo sobre $[f(a), f(b)]$, se $g$ possuir uma única raiz $z \in[a, b]$ em $c$ ); assim, para toda aplicação contínua própria $f: \mathbb{R} \longrightarrow \mathbb{R}$ e todo ponto $c \in \mathbb{R}$, os números de Nielsen $\mathrm{N}(f, c)$ e $\mathrm{N}_{\uparrow}(f, c)$, ambos iguais a zero ou ambos iguais a 1 , são realizados por uma aplicação contínua própria $g: \mathbb{R} \longrightarrow \mathbb{R}$, que é transversa a $c$ e propriamente homotópica à $f$.

Exemplo 2.3.3. Seja $f: \mathbb{S}^{1} \longrightarrow \mathbb{R}$ uma aplicação contínua qualquer, e consideremos um ponto $c \in \mathbb{R}$ tal que $f^{-1}(c) \neq \varnothing$; como $\mathbb{S}^{1}$ é compacto e $\mathbb{R}$ é um espaço de Hausdorff, segue (do exemplo 1.3.2) que $f$ é uma aplicação própria. Além disso, como $\mathbb{R}$ é contrátil, temos que $f$ é homotópica a qualquer outra aplicação $g: \mathbb{S}^{1} \longrightarrow \mathbb{R}$, sendo que toda homotopia $H: \mathbb{S}^{1} \times I \longrightarrow \mathbb{R}$ entre $f$ e $g$ também será própria. Isto posto, dado $c^{\prime} \in \mathbb{R}$ tal que $c^{\prime} \neq c$, considere a aplicação (contínua e própria) $g: \mathbb{S}^{1} \longrightarrow \mathbb{R}$ dada por $g(z)=c^{\prime}$ para todo $z \in \mathbb{S}^{1}$; como $g$ não possui raízes em $c$, temos de imediato que $g$ é transversa a $c$, e $\mathrm{N}(g, c)=\mathrm{N}_{\pitchfork}(g, c)=0$. Logo, como $g$ é propriamente homotópica à $f$, concluímos (do corolário 2.3.2) que $\mathrm{N}(f, c)=\mathrm{N}_{\uparrow}(f, c)=0$.

Exemplo 2.3.4 . Sendo $\mathbb{S}^{1}$ a circunferência unitária no plano complexo, considere uma aplicação contínua $f: \mathbb{S}^{1} \longrightarrow \mathbb{S}^{1}$; como $\mathbb{S}^{1}$ é uma variedade topológica compacta, segue (do exemplo 1.3.2) que $f$ é uma aplicação própria. Além disso, sabemos também que, qualquer que seja $f: \mathbb{S}^{1} \longrightarrow \mathbb{S}^{1}$, existe um inteiro $r \geqslant 0$ tal que $f$ é homotópica à aplicação 
$g: \mathbb{S}^{1} \longrightarrow \mathbb{S}^{1}$, dada por $g(z)=z^{r}$ para cada $z \in \mathbb{S}^{1}$ (vide [Sp], lema 13, página 55), sendo que $r \neq 0$ se, e somente se, $f^{-1}(z) \neq \varnothing$ para todo $z \in \mathbb{S}^{1}$. Agora, utilizando o conceito de grau introduzido no exemplo 1.3.7, segue que $\mathrm{d}(g)=\mathrm{d}(f)=r$ (conforme nos garante o corolário 4.7, página 65, de [D]), e, conseqüentemente, (do teorema 1.4.2, da proposição 1.3.4 e do exemplo 1.3.7), temos que o grau cohomológico de $g$ é:

$$
\operatorname{deg}(g)=\operatorname{deg}_{c}(g)=\operatorname{deg}_{\mathbb{S}^{1}}(g)=\mathrm{d}(g)=r,
$$

para qualquer que seja $c \in \mathbb{S}^{1}$. Logo, como toda homotopia $H: \mathbb{S}^{1} \times I \longrightarrow \mathbb{S}^{1}$, entre $f$ e $g$, é uma aplicação própria (pois $\mathbb{S}^{1} \times I$ é compacto), segue, do corolário 1.4 .1 e da proposição 1.4.5, que:

$$
\mathrm{N}_{\phi}(f, c)=|\operatorname{deg}(f)|=|\operatorname{deg}(g)|=r,
$$

onde $c \in \mathbb{S}^{1}$ é arbitrário. Por outro lado, suponhamos que $r \neq 0$ (pois, caso contrário, temos $\operatorname{deg}(f)=\operatorname{deg}(g)=r=0$, o que, pelo corolário 2.3.1, implica de imediato que $\mathrm{N}(f, c)=0)$; do exemplo 1.3.5, segue então que o conjunto das raízes de $g$ num ponto $c \in \mathbb{S}^{1}$ é:

$$
g^{-1}(c)=\left\{\exp \left(\frac{2 \pi i k}{r}\right): k=1,2, \ldots, r\right\},
$$

sendo que cada $z \in g^{-1}(c)$ constitui, por si só, uma classe de raízes de $g$ em $c$. Isto posto, observe que $g$ é transversa a $c$, pois constitui um recobrimento de $r$ folhas (vide [Li], exemplo 6, página 122); assim, considere uma vizinhança euclidiana $V \subseteq \mathbb{S}^{1}$ de $c$, tal que $g^{-1}(V)$ é a união disjunta de vizinhanças euclidianas $U_{1}, U_{2}, \ldots, U_{r}$, cada uma das quais contém um único ponto de $g^{-1}(c)$, e é levada homeomorficamente por $g$ sobre $V$. Do corolário 1.3 .1 e da definição 1.3.7, temos que a multiplicidade de uma classe de raízes $R=\{z\}$, com $z \in U_{i} \cap g^{-1}(c)$ para algum $i \in\{1,2, \ldots, r\}$, é:

$$
|m(R)|=\left|\operatorname{deg}_{c}\left(\left.g\right|_{U_{i}}\right)\right|=| \pm 1|=1 .
$$

Portanto, do teorema 2.1.2, segue que todas as $r$ classes de raízes de $g$ em $c$ têm multiplicidade igual a 1 (ou seja, são todas classes de raízes essenciais), o que, pelo corolário 2.3.2, implica que $\mathrm{N}(f, c)=\mathrm{N}(g, c)=r$. Deste modo, concluímos que, para toda aplicação contínua (própria) $f: \mathbb{S}^{1} \longrightarrow \mathbb{S}^{1}$ e todo ponto $c \in \mathbb{S}^{1}$, tem-se $\mathrm{N}(f, c)=$ $\mathrm{N}_{\phi}(f, c)=r$, onde $r=\mathrm{d}(f)$.

Exemplo 2.3.5. Dado $n \geqslant 2$, seja $f: \mathbb{S}^{n} \longrightarrow \mathbb{S}^{n}$ uma aplicação contínua de grau $\mathrm{d}(f) \neq 0$. Pelo que vimos no exemplo 1.3.7, o grau de $f$ coincide com o grau local de $f$ em $\mathbb{S}^{n}$; por outro lado, como $\mathbb{S}^{n}$ é uma variedade topológica compacta, conexa, orientável e sem fronteira, temos que $f$ é uma aplicação própria, $\operatorname{edeg}_{\mathbb{S}^{n}}(f)=\operatorname{deg}_{c}(f)$, qualquer que 
seja $c \in \mathbb{S}^{n}$ (vide exemplo 1.3.2 e proposição 1.3.4). Assim, como $W=\mathbb{S}^{n} \backslash f^{-1}\left(\partial \mathbb{S}^{n}\right)=$ $\mathbb{S}^{n} \backslash f^{-1}(\varnothing)=\mathbb{S}^{n}$, segue (do teorema 1.4.2) que o grau cohomológico deg $(f)$ é tal que $\operatorname{deg}(f)=\mathrm{d}(f) \neq 0$. Agora, observe que, como $\mathbb{S}^{n}$ é simplesmente conexa para $n \geqslant 2$, temos que $\pi_{1}\left(\mathbb{S}^{n}, x_{0}\right) \simeq\{0\}$ para qualquer $x_{0} \in f^{-1}(c) ; \log , f_{\#}\left(\pi_{1}\left(\mathbb{S}^{n}, x_{0}\right)\right) \simeq\{0\}$, o que, pelo fato de $\hat{q}: \hat{N} \longrightarrow \mathbb{S}^{n}$ ser o recobrimento de $\mathbb{S}^{n}$ associado ao subgrupo $f_{\#}\left(\pi_{1}\left(\mathbb{S}^{n}, x_{0}\right)\right) \subseteq$ $\pi_{1}\left(\mathbb{S}^{n}, c\right)$, implica que $\hat{q}_{\#}\left(\pi_{1}(\hat{N}, \hat{c})\right)=f_{\#}\left(\pi_{1}\left(\mathbb{S}^{n}, x_{0}\right)\right) \simeq\{0\}$. Conseqüentemente, como o homomorfismo $\hat{q}_{\#}: \pi_{1}(\hat{N}, \hat{c}) \longrightarrow \pi_{1}\left(\mathbb{S}^{n}, c\right)$ é injetivo, segue que $\pi_{1}(\hat{N}, \hat{c}) \simeq\{0\}$, ou seja, $\hat{N}$ é simplesmente conexo. Deste modo, concluímos que $\hat{N}$ é o espaço de recobrimento universal de $\mathbb{S}^{n}$, que, a menos de homeomorfismos, coincide com $\mathbb{S}^{n}$ (vide [Bd], corolário 4.6, página 145). Portanto, $\hat{q}: \hat{N}=\mathbb{S}^{n} \longrightarrow \mathbb{S}^{n}$ é um recobrimento de uma única folha (isto é, um homeomorfismo), o que significa que a cardinalidade j de $\hat{q}^{-1}(c)$ é igual a 1. Logo, do corolário 2.3.1, obtemos que $\mathrm{N}(f, c)=1$ e $\mathrm{N}_{\pitchfork}(f, c)=|\mathrm{d}(f)|$; em particular, se $|\mathrm{d}(f)|>1$, temos $\mathrm{N}_{巾}(f, c) \supsetneqq \mathrm{N}(f, c)$.

Exemplo 2.3.6 . Generalizando o exemplo anterior, segue (do corolário 2.3.1) que $\mathrm{N}_{\phi}(f, c)=|\operatorname{deg}(f)|$ é estritamente maior que $\mathrm{N}(f, c)=1$, para toda aplicação contínua própria $f:(M, \partial M) \longrightarrow(N, \partial N)$, entre variedades topológicas orientáveis de mesma dimensão, desde que o homomorfismo induzido $f_{\#}: \pi_{1}\left(M, x_{0}\right) \longrightarrow \pi_{1}(N, c)$ seja sobrejetivo (onde $c \in \operatorname{Int} N$ e $x_{0} \in f^{-1}(c)$ ) e $|\operatorname{deg}(f)|>1$ (note que, se $f_{\#}$ é sobrejetivo, temos que $\hat{q}_{\#}\left(\pi_{1}(\hat{N}, \hat{c})\right)=f_{\#}\left(\pi_{1}\left(M, x_{0}\right)\right)=\pi_{1}(N, c)$, ou seja, o homomorfismo $\hat{q}_{\#}: \pi_{1}(\hat{N}, \hat{c}) \longrightarrow \pi_{1}(N, c)$, além de injetivo, também é sobrejetivo, o que mostra que, neste caso, o recobrimento $\hat{q}: \hat{N} \longrightarrow N$ é um homeomorfismo, e, portanto, a cardinalidade $\mathrm{j}=\mathrm{N}(f, c)$ de $\hat{q}^{-1}(c)$ é igual a 1).

Exemplo 2.3.7. Sendo $g:(M, \partial M) \longrightarrow(N, \partial N)$ uma aplicação contínua própria, entre variedades topológicas conexas de mesma dimensão $n$, considere uma outra variedade topológica conexa $X$, também de dimensão $n$ (com ou sem fronteira), e denote por $f: M \times X \longrightarrow N \times X$ a aplicação contínua dada por $f=g \times I d$, onde Id $: X \longrightarrow X$ é a identidade (isto é, $f\left(x_{0}, x\right)=\left(g\left(x_{0}\right), x\right)$ para cada $\left.\left(x_{0}, x\right) \in M \times X\right)$. Utilizando diretamente as definições dos números de Nielsen de raízes, pode-se demonstrar que $\mathrm{N}(f, c)=\mathrm{N}\left(g, c^{\prime}\right)$ e $\mathrm{N}_{\phi}(f, c)=\mathrm{N}_{\phi}\left(g, c^{\prime}\right)$, onde $c=\left(c^{\prime}, c^{\prime \prime}\right) \in \operatorname{Int} N \times \operatorname{Int} X$ (vide [BBS], teorema 2.1, páginas 9 e 10). Em particular, se $f: \mathbb{S}^{2} \times \mathbb{R} P^{2} \longrightarrow \mathbb{S}^{2} \times \mathbb{R} P^{2}$ é a aplicação dada por $f=g \times$ Id, onde Id $: \mathbb{R} P^{2} \longrightarrow \mathbb{R P}^{2}$ é a identidade e $g: \mathbb{S}^{2} \longrightarrow \mathbb{S}^{2}$ é uma aplicação contínua de grau $\mathrm{d}(g)=d$, com $|d|>1$, temos que $\mathrm{N}(f, c)=\mathrm{N}\left(g, c^{\prime}\right)=1$ e $\mathrm{N}_{\phi}(f, c)=\mathrm{N}_{\phi}\left(g, c^{\prime}\right)=|d|>1$ para qualquer que seja $c=\left(c^{\prime}, c^{\prime \prime}\right) \in \mathbb{S}^{2} \times \mathbb{R} P^{2}$ (note que $g$ é uma aplicação própria tal que $\mathrm{N}\left(g, c^{\prime}\right)=1$ e $\mathrm{N}_{\phi}\left(g, c^{\prime}\right)=|d|$, conforme vimos no exemplo 2.3.5). 
Exemplo 2.3.8 . Dada uma variedade topológica conexa $N$, sem fronteira e não-orientável, seja $f: M \longrightarrow N$ o recobrimento orientado de $N$ (de modo que $M$ constitui uma variedade topológica conexa, orientável e sem fronteira), e considere o recobrimento $\hat{q}: \hat{N} \longrightarrow N$ associado ao subgrupo $f_{\#}\left(\pi_{1}\left(M, x_{0}\right)\right) \subseteq \pi_{1}(N, c)$, onde $c \in N$ e $x_{0} \in f^{-1}(c)$. Denote ainda por $\hat{f}: M \longrightarrow \hat{N}$ o levantamento de $f$, relativamente ao recobrimento $\hat{q}$, tal que $\hat{f}\left(x_{0}\right)=\hat{c}$, onde $\hat{c} \in \hat{q}^{-1}(c)$. Como $f=\hat{q} \circ \hat{f}$ e $\hat{q}_{\#}\left(\pi_{1}(\hat{N}, \hat{c})\right)=f_{\#}\left(\pi_{1}\left(M, x_{0}\right)\right)$, temos que:

$$
\left(\hat{q}_{\#} \circ \hat{f_{\#}}\right)\left(\pi_{1}\left(M, x_{0}\right)\right)=(\hat{q} \circ \hat{f})_{\#}\left(\pi_{1}\left(M, x_{0}\right)\right)=f_{\#}\left(\pi_{1}\left(M, x_{0}\right)\right)=\hat{q}_{\#}\left(\pi_{1}(\hat{N}, \hat{c})\right) .
$$

Logo, como o homomorfismo $\hat{q}_{\#}: \pi_{1}(\hat{N}, \hat{c}) \longrightarrow \pi_{1}(N, c)$, induzido pelo recobrimento $\hat{q}: \hat{N} \longrightarrow N$, é injetivo, obtemos que $\hat{f}_{\#}\left(\pi_{1}\left(M, x_{0}\right)\right)=\pi_{1}(\hat{N}, \hat{c})$, isto é, o homomorfismo $\hat{f_{\#}}: \pi_{1}\left(M, x_{0}\right) \longrightarrow \pi_{1}(\hat{N}, \hat{c})$ é sobrejetivo. Conseqüentemente, temos que $\hat{f}: M \longrightarrow \hat{N}$ é um homeomorfismo (vide corolário da página 162 de [Li]), e, portanto, $|\operatorname{deg}(\hat{f})|=1$ (note que, pelo fato de $\hat{f}$ ser um homeomorfismo e $M$ ser orientável, temos que $\hat{N}$ também é orientável, o que nos garante que o grau cohomológico $\operatorname{deg}(\hat{f})$ encontra-se bem definido). Agora, da orientabilidade de $M$, segue que o recobrimento orientado de $M, \widehat{p}: \widehat{M} \longrightarrow M$, é a identidade (isto é, $\widehat{M}=M$ ); por conseguinte, dado $c^{\prime} \in\left(q^{\prime}\right)^{-1}\left(c^{\prime}\right)$, onde $q^{\prime}: N^{\prime} \longrightarrow \hat{N}$ é o recobrimento de $\hat{N}$ associado ao subgrupo $(\hat{p} \circ \hat{f})_{\#}\left(\pi_{1}\left(\hat{M}, x_{0}\right)\right)=$ $\hat{f}_{\#}\left(\pi_{1}\left(M, x_{0}\right)\right)=\pi_{1}(\hat{N}, \hat{c})$, temos que:

$$
q_{\#}^{\prime}\left(\pi_{1}\left(N^{\prime}, c^{\prime}\right)\right)=\hat{f_{\#}}\left(\pi_{1}\left(M, x_{0}\right)\right)=\pi_{1}(\hat{N}, \hat{c})
$$

ou seja, $q_{\#}^{\prime}: \pi_{1}\left(N^{\prime}, c^{\prime}\right) \longrightarrow \pi_{1}(\hat{N}, \hat{c})$ é um homomorfismo sobrejetivo, o que, conforme vimos na demonstração do corolário 2.3.1, implica que $q^{\prime}$ é um recobrimento de uma única folha. Assim, temos que $q^{\prime}: N^{\prime} \longrightarrow \hat{N}$ também é um homeomorfismo, d'onde segue que $N^{\prime}$ é orientável e $\left|\operatorname{deg}\left(q^{\prime}\right)\right|=1$. Deste modo, denotando por $\widehat{f}: \widehat{M}=M \longrightarrow N^{\prime}$ o levantamento de $\hat{f} \circ \hat{p}=\hat{f}$, relativamente ao recobrimento $q^{\prime}$, tal que $\hat{f}\left(x_{0}\right)=c^{\prime}$, obtemos (da proposição 1.4.6) que:

$$
1=|\operatorname{deg}(\hat{f})|=\left|\operatorname{deg}\left(q^{\prime} \circ \widehat{f}\right)\right|=\left|\operatorname{deg}\left(q^{\prime}\right)\right| \cdot|\operatorname{deg}(\widehat{f})|=1 \cdot|\operatorname{deg}(\widehat{f})|
$$

isto é, $|\operatorname{deg}(\hat{f})|=1$. Finalmente, como $f: M \longrightarrow N$ é uma aplicação de recobrimento, temos (da proposição 1.1.5) que $f$ é do tipo I; além disso, como $f$ é um recobrimento de duas folhas e $\hat{f}$ é um homeomorfismo, segue que $\hat{q}$ também constitui um recobrimento de duas folhas, o que significa que a cardinalidade de $\hat{q}^{-1}(c)$ é $\mathrm{j}=2$. Logo, utilizando os teoremas 2.3.1 e 2.3.2, concluímos que $\mathrm{N}(f, c)=\mathrm{j}=2$ e $\mathrm{N}_{\phi}(f, c)=\mathrm{j} \cdot|\operatorname{deg}(\widehat{f})|=2$.

Exemplo 2.3.9. Denotando por $\mathbb{K}$ a garrafa de Klein, considere uma aplicação contínua $f: \mathbb{K} \longrightarrow \mathbb{K}$, e sejam $c \in \mathbb{K}$ e $x_{0} \in f^{-1}(c)$; como $\mathbb{K}$ é uma variedade topológica compacta não-orientável, segue que $f$ é uma aplicação própria, e (pelo exemplo 19, página 179, 
de [Li]) existem geradores $\alpha, \beta \in \pi_{1}\left(\mathbb{K}, x_{0}\right)$ que satisfazem a relação $\alpha * \beta=\beta^{-1} * \alpha$. Além disso, da proposição 6.4 de [BO] (páginas 416 e 417), sabemos que existem $b, d, e \in \mathbb{Z}$ tais que $f_{\#}(\alpha)=\alpha^{b} * \beta^{d}, f_{\#}(\beta)=\beta^{e}$ e $\mathrm{N}(f, c)=|b \cdot e|$, com $e=0$ se, e somente se, $|b|$ for um número par. Assim, supondo $e \neq 0$, temos que $|b|$ é ímpar, e, portanto, $\mathrm{N}(f, c)=|b \cdot e| \neq 0$; coseqüentemente, utilizando o diagrama ilustrado na figura $2.2(\operatorname{com} M=N=\mathbb{K}$ ), e denotando por $\mathrm{j}$ a cardinalidade de $\hat{q}^{-1}(c)$, o teorema 2.3.1 nos garante que $f$ não é do tipo II, e $\mathrm{j}=\mathrm{N}(f, c)=|b \cdot e|$. Por outro lado, se $e \neq 0$, a demonstração da proposição 6.4 de $[\mathrm{BO}]$ nos mostra que $f_{\#}: \pi_{1}\left(\mathbb{K}, x_{0}\right) \longrightarrow \pi_{1}(\mathbb{K}, c)$ é um homomorfismo injetivo, ou seja, $\operatorname{Ker}\left(f_{\#}\right) \simeq \mathbb{Z}$; daí, segue que $f$ não pode ser do tipo III, pois, neste caso, existiria um laço que inverte orientação (e, portanto, não-contrátil) que é levado por $f$ num laço contrátil em $c$ (lembre-se que $[\omega] \in \operatorname{Ker}\left(f_{\#}\right)$ se, e só se, $f \circ \omega$ é um laço contrátil em $c$ ). Deste modo, se $e \neq 0$, segue que $f: \mathbb{K} \longrightarrow \mathbb{K}$ é uma aplicação do tipo I. Isto posto, vamos mostrar que $|\operatorname{deg}(\widehat{f})|=1$ (vide diagrama da figura 2.2).

Primeiramente, note que, como $f: M=\mathbb{K} \longrightarrow N=\mathbb{K}$ é fiel à orientação e $\mathbb{K}$ é não-orientável, então $\hat{N}$ também é não-orientável: com efeito, sendo $\omega: I \longrightarrow M=\mathbb{K}$ um laço que inverte orientação, temos que $f \circ \omega=\hat{q} \circ \hat{f} \circ \omega: I \longrightarrow N=\mathbb{K}$ é um laço que inverte orientação; conseqüentemente, como $\hat{q}: \hat{N} \longrightarrow N$ é do tipo I (pois constitui uma aplicação de recobrimento), segue que $\hat{f} \circ \omega: I \longrightarrow \hat{N}$ é um laço que inverte orientação, o que, pela proposição 1.1.3, implica que $\hat{N}$ é não-orientável. Agora, observe que $q^{\prime}: N^{\prime} \longrightarrow \hat{N}$ é um recobrimento de duas folhas: de fato, sendo $\hat{\omega}: I \longrightarrow \hat{N}$ um laço (com base em $\hat{c}$ ) tal que $[\hat{\omega}] \in q_{\#}^{\prime}\left(\pi_{1}\left(N^{\prime}, c^{\prime}\right)\right)$, temos que $\hat{\omega}$ preserva orientação, pois, pelo corolário 2.3.1, sabemos que $N^{\prime}$ é orientável (e, portanto, todo laço em $N^{\prime}$ preserva orientação), enquanto que, por ser uma aplicação de recobrimento, $q^{\prime}: N^{\prime} \longrightarrow \hat{N}$ é fiel à orientação; logo, denotando por $\bar{q}: \bar{N} \longrightarrow \hat{N}$ o recobrimento orientado de $\hat{N}$ (o qual possui duas folhas, uma vez que $\hat{N}$ é não-orientável), segue que $\hat{\omega}$ pode ser levantado para um laço $\bar{\omega}: I \longrightarrow \bar{N}$ relativamente a $q^{\prime}$, o que implica que $[\hat{\omega}] \in \bar{q}_{\#}\left(\pi_{1}(\bar{N}, \bar{c})\right)$, onde $\bar{c}=\bar{\omega}(0) \in \bar{q}^{-1}(\hat{c})$. Deste modo, temos que $q_{\#}^{\prime}\left(\pi_{1}\left(N^{\prime}, c^{\prime}\right)\right) \subseteq \bar{q}_{\#}\left(\pi_{1}(\bar{N}, \bar{c})\right)$, e, por conseguinte (da proposição 8 , página 162, de [Li]), existe uma aplicação contínua $\bar{q}^{\prime}: N^{\prime} \longrightarrow \bar{N}$, com $\bar{q}^{\prime}\left(c^{\prime}\right)=\bar{c}$, que torna comutativo o seguinte diagrama:

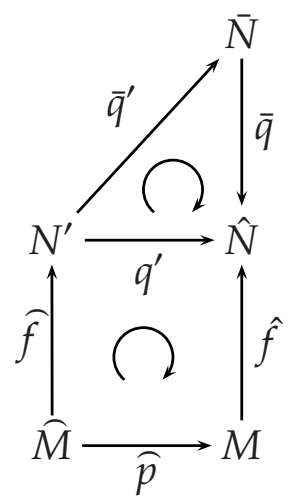


Em contrapartida, sendo $\hat{\gamma}: I \longrightarrow \hat{N}$ um laço tal que $[\hat{\gamma}] \in \bar{q}_{\#}\left(\pi_{1}(\bar{N}, \bar{c})\right) \subseteq \pi_{1}(\hat{N}, \hat{c})$, temos que $\hat{\gamma}$ preserva orientação (uma vez que $\bar{q}$ é fiel à orientação e $\bar{N}$ é orientável). No entanto, como $\hat{q}_{\#}\left(\pi_{1}(\hat{N}, \hat{c})\right)=f_{\#}\left(\pi_{1}\left(M, x_{0}\right)\right)$ e $f=\hat{q} \circ \hat{f}$, segue que $\hat{q}_{\#}\left(\hat{f}_{\#}\left(\pi_{1}\left(M, x_{0}\right)\right)\right)=$ $f_{\#}\left(\pi_{1}\left(M, x_{0}\right)\right)=\hat{q}_{\#}\left(\pi_{1}(\hat{N}, \hat{c})\right)$, ou seja, $\hat{f}_{\#}: \pi_{1}\left(M, x_{0}\right) \longrightarrow \pi_{1}(\hat{N}, \hat{c})$ é um homomorfismo sobrejetivo (pois $\hat{q}_{\#}$ é injetivo); logo, como $\hat{f}: M \longrightarrow \hat{N}$ é fiel à orientação (conforme vimos na demonstração do corolário 2.2.1), segue que $[\hat{\gamma}]=\hat{f}_{\#}([\gamma])$, onde $\gamma: I \longrightarrow M$ é um laço com base em $x_{0}$ que preserva orientação. Conseqüentemente, $\gamma$ pode ser levantado para um laço $\hat{\gamma}: I \longrightarrow \widehat{M}$ relativamente ao recobrimento orientado $\widehat{p}: \widehat{M} \longrightarrow$ $M$; assim, considerando o laço $\gamma^{\prime}: I \longrightarrow N^{\prime}$ dado por $\gamma^{\prime}=\widehat{f} \circ \widehat{\gamma}$, temos que:

$$
q_{\#}^{\prime}\left(\left[\gamma^{\prime}\right]\right)=\left[q^{\prime} \circ \gamma^{\prime}\right]=\left[q^{\prime} \circ(\widehat{f} \circ \widehat{\gamma})\right]=[\hat{f} \circ(\widehat{p} \circ \widehat{\gamma})]=[\hat{f} \circ \gamma]=\hat{f}_{\#}([\gamma])=[\hat{\gamma}],
$$

isto é, $[\hat{\gamma}] \in q_{\#}^{\prime}\left(\pi_{1}\left(N^{\prime}, c^{\prime}\right)\right)$. Daí, concluímos que $\bar{q}_{\#}\left(\pi_{1}(\bar{N}, \bar{c})\right) \subseteq q_{\#}^{\prime}\left(\pi_{1}\left(N^{\prime}, c^{\prime}\right)\right)$, e, portanto, $\bar{q}_{\#}\left(\pi_{1}(\bar{N}, \bar{c})\right)=q_{\#}^{\prime}\left(\pi_{1}\left(N^{\prime}, c^{\prime}\right)\right)$, o que significa que a aplicação $\bar{q}^{\prime}: N^{\prime} \longrightarrow \bar{N}$ é um homeomorfismo, provando que $q^{\prime}=\bar{q} \circ \bar{q}^{\prime}$ é um recobrimento de duas folhas.

Agora, denotando por $q: N^{\prime} \longrightarrow N$ o recobrimento dado por $q=\hat{q} \circ q^{\prime}$, note que a cardinalidade de $q^{-1}(c)$ é igual a $2 \cdot \mathrm{j}=2 \cdot|b \cdot e|$, pois $q^{-1}(c)=\left(q^{\prime}\right)^{-1}\left(\hat{q}_{-1}(c)\right)$, sendo que $\hat{q}_{-1}(c)$ possui cardinalidade $\mathrm{j}=|b \cdot e|$ e $q^{\prime}: N^{\prime} \longrightarrow \hat{N}$ é um recobrimento duplo de $\hat{N}$. Isto posto, seja $q^{0}: N^{0} \longrightarrow N=\mathbb{K}$ o recobrimento orientado de $\mathbb{K}$ (isto é, $q^{0}=\hat{p}$ ); se $\omega: I \longrightarrow N$ é uma laço tal que $[\omega] \in q_{\#}\left(\pi_{1}\left(N^{\prime}, c^{\prime}\right)\right.$ ), segue que $\omega$ preserva orientação (pois $N^{\prime}$ é orientável e o recobrimento $q=\hat{q} \circ q^{\prime}: N^{\prime} \longrightarrow N$ é uma aplicação fiel à orientação), e, portanto, $\omega$ pode ser levantado para um laço $\omega^{0}: I \longrightarrow N^{0}$ relativamente a $q^{0}$. Assim, temos que $[\omega] \in q_{\#}^{0}\left(\pi_{1}\left(N^{0}, c^{0}\right)\right)$, onde $c^{0} \in\left(q^{0}\right)^{-1}(c)$, isto é, $q_{\#}\left(\pi_{1}\left(N^{\prime}, c^{\prime}\right)\right) \subseteq q_{\#}^{0}\left(\pi_{1}\left(N^{0}, c^{0}\right)\right)$, o que (pela proposição 8 , página 162 , de [Li]) significa que existe um recobrimento $\check{q}: N^{\prime} \longrightarrow N^{0}, \operatorname{com} \check{q}\left(c^{\prime}\right)=c^{0}$, que comuta o seguinte diagrama:

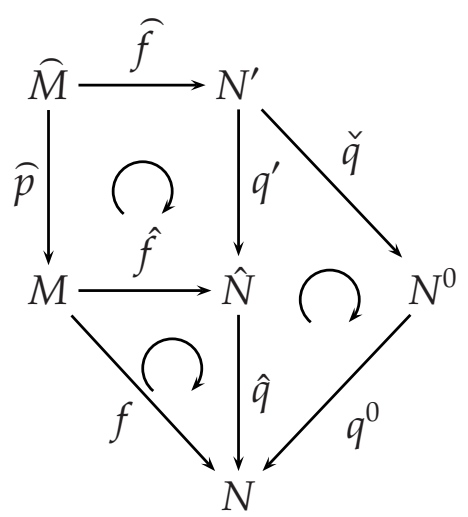

Como $q^{-1}(c)$ possui cardinalidade $2 \cdot|b \cdot e|$, e $q^{0}: N^{0} \longrightarrow N$ é um recobrimento de duas folhas, $\operatorname{com} q=q^{0} \circ \check{q}$, segue que $\check{q}: N^{\prime} \longrightarrow N^{0}$ é um recobrimento de $|b \cdot e|$ folhas; logo, lembrando que $N^{\prime}$ e $N^{0}$ são variedades topológicas conexas, orientáveis e sem fronteira (de modo, que o grau cohomológico $\operatorname{deg}(\breve{q})$ encontra-se bem definido), obtemos, do 
teorema 1.4.2 e do corolário 1.3.2, que:

$$
|\operatorname{deg}(\check{q})|=\left|\operatorname{deg}_{c^{0}}(\check{q})\right|=|b \cdot e| .
$$

Finalmente, observe então que a comutatividade do diagrama acima nos garante que a aplicação $f^{0}: \widehat{M} \longrightarrow N^{0}$, dada por $f^{0}=\check{q} \circ \widehat{f}$, satisfaz $f \circ \widehat{p}=q^{0} \circ f^{0}$; conseqüentemente da demonstração da proposição 6.4 de [BO], temos que $\operatorname{deg}\left(f^{0}\right)=b \cdot e$ (note que $\widehat{M}$ e $N^{0}$ são orientáveis), e, portanto (utilizando a proposição 1.4.6), concluímos que:

$$
|b \cdot e|=\left|\operatorname{deg}\left(f^{0}\right)\right|=|\operatorname{deg}(\check{q})| \cdot|\operatorname{deg}(\widehat{f})|=|b \cdot e| \cdot|\operatorname{deg}(\widehat{f})|,
$$

ou seja, $|\operatorname{deg}(\widehat{f})|=1$, conforme queríamos provar.

Deste modo, como $f: M=\mathbb{K} \longrightarrow N=\mathbb{K}$ é uma aplicação contínua própria do tipo $\mathrm{I}$, com $\mathrm{j}=|b \cdot e|$ e $|\operatorname{deg}(\widehat{f})|=1$, segue (do teorema 2.3.2) que:

$$
\mathrm{N}_{\pitchfork}(f, c)=\mathrm{j} \cdot|\operatorname{deg}(\hat{f})|=|b \cdot e|=\mathrm{N}(f, c) .
$$

Exemplo 2.3.10 . Denotando por $\mathbb{T}^{2}$ o toro, considere uma aplicação contínua $f$ : $\mathbb{T}^{2} \longrightarrow \mathbb{T}^{2}$, e sejam $c \in \mathbb{T}^{2}$ e $x_{0} \in f^{-1}(c)$; como $\mathbb{T}^{2}$ é uma variedade topológica compacta e orientável, temos que $f$ é uma aplicação própria do tipo I. Lembrando que $\mathrm{H}_{1}\left(\mathbb{T}^{2}\right) \simeq$ $\mathbb{Z} \oplus \mathbb{Z}$, seja ainda $A=\left\{a_{i j}\right\}$ a matriz quadrada de ordem 2 do homomorfismo induzido $\left(f_{*}\right)_{1}: \mathrm{H}_{1}\left(\mathbb{T}^{2}\right) \longrightarrow \mathrm{H}_{1}\left(\mathbb{T}^{2}\right)$, relativamente a uma base $\mathcal{B}=\left\{b_{1}, b_{2}\right\}$ de $\mathrm{H}_{1}\left(\mathbb{T}^{2}\right)$; do exemplo 4.26 de [Bk3] (páginas 402 e 403), temos que $\operatorname{deg}(f)=\operatorname{det} A$ e $\mathrm{N}(f, c)=|\operatorname{det} A| . \operatorname{Logo}$, do corolário 2.3.1, obtemos que $\mathrm{N}_{\pitchfork}(f, c)=\mathrm{N}(f, c)=|\operatorname{det} A|$.

Exemplo 2.3.11 . Seja $f: \mathbb{T}^{2} \# \mathbb{R} P^{2} \longrightarrow \mathbb{T}^{2}$ a aplicação contínua (própria) dada no item (b) do exemplo 1.2.3, e considere um ponto $c \in \mathbb{T}^{2} \backslash D_{1}$. Como $D_{1}$ é contrátil, segue que todo laço $\omega: I \longrightarrow \mathbb{T}^{2}$, com base em $c$, é homotópico a um laço $\omega^{\prime}: I \longrightarrow \mathbb{T}^{2} \backslash \operatorname{Int} D_{1}$; logo, como $f$ coincide com a aplicação identidade Id : $\mathbb{T}^{2} \backslash \operatorname{Int} D_{1} \longrightarrow \mathbb{T}^{2} \backslash \operatorname{Int} D_{1}$ em $\mathbb{T}^{2} \backslash$ Int $D_{1}$, segue que $f(c)=c$ e

$$
f_{\#}\left(\left[\omega^{\prime}\right]\right)=\left[f \circ \omega^{\prime}\right]=\left[\omega^{\prime}\right]=[\omega],
$$

onde $f_{\#}: \pi_{1}\left(\mathbb{T}^{2} \# \mathbb{R} P^{2}, c\right) \longrightarrow \pi_{1}\left(\mathbb{T}^{2}, c\right)$ é o homomorfismo induzido por $f$ (note que $\left[\omega^{\prime}\right] \in \pi_{1}\left(\mathbb{T}^{2} \# \mathbb{R} P^{2}, c\right)$, pois $\left.\mathbb{T}^{2} \backslash \operatorname{Int} D_{1} \subseteq \mathbb{T}^{2} \# \mathbb{R} P^{2}\right)$. Daí, obtemos que $f_{\#}\left(\pi_{1}\left(\mathbb{T}^{2} \# \mathbb{R} P^{2}, c\right)\right)=$ $\pi_{1}\left(\mathbb{T}^{2}, c\right)$ (isto é, $f_{\#}$ é um homomorfismo sobrejetivo), o que implica que o recobrimento $\hat{q}: \hat{N} \longrightarrow \mathbb{T}^{2}$, associado ao subgrupo $f_{\#}\left(\pi_{1}\left(\mathbb{T}^{2} \# \mathbb{R} P^{2}, c\right)\right)=\pi_{1}\left(\mathbb{T}^{2}, c\right)$, é um homeomorfismo: com efeito, dado $\hat{c} \in \hat{N}$ tal que $\hat{q}(\hat{c})=c$, temos que o homomorfismo induzido $\hat{q}_{\#}: \pi_{1}\left(\mathbb{T}^{2} \# \mathbb{R} P^{2}, c\right) \longrightarrow \pi_{1}\left(\mathbb{T}^{2}, c\right)$, além de injetivo, também é sobrejetivo (pois $\left.\hat{q}_{\#}\left(\pi_{1}\left(\mathbb{T}^{2} \# \mathbb{R} P^{2}, c\right)\right)=f_{\#}\left(\pi_{1}\left(\mathbb{T}^{2} \# \mathbb{R} P^{2}, c\right)\right)=\pi_{1}\left(\mathbb{T}^{2}, c\right)\right)$, e, portanto (do corolário 3 , páginas 
152 e 153, de [Li]), o recobrimento $\hat{q}: \hat{N} \longrightarrow \mathbb{T}^{2}$ constitui um homeomorfismo. Assim, temos que a cardinalidade j de $\hat{q}^{-1}(c)$ é igual a 1 . Agora, utilizando coeficientes em $\mathbb{Z}_{2}$ a fim de orientar $\mathbb{T}^{2} \# \mathbb{R} P^{2}$ e $\mathbb{T}^{2}$, observe que $\operatorname{deg}(f, 2)=1$ : de fato, pelo teorema 1.4.2, temos que $\operatorname{deg}(f, 2)=\operatorname{deg}_{c}(f)$, onde $\operatorname{deg}_{c}(f)$ é o grau local de $f$ em $c$ a coeficientes em $\mathbb{Z}_{2}$; logo, como $\left.f\right|_{\mathbb{T}^{2} \backslash \text { Int } D_{1}}$ é a identidade e $c=f(c) \in \mathbb{T}^{2} \backslash D_{1} \subseteq \mathbb{T}^{2} \backslash \operatorname{Int} D_{1}$, segue que $\operatorname{deg}_{c}(f)=1$, e, portanto, $\operatorname{deg}(f, 2)=1$. Ora, se $\operatorname{deg}(f, 2)=1$ e o recobrimento $\hat{q}: \hat{N} \longrightarrow \mathbb{T}^{2}$ é um homeomorfismo, segue que o grau cohomológico a coeficientes em $\mathbb{Z}_{2}$, do levantamento $\hat{f}: \mathbb{T}^{2} \# \mathbb{R} P^{2} \longrightarrow \hat{N}$ de $f$, relativamente a $\hat{q}$, é $\operatorname{deg}(\hat{f}, 2)=1$, uma vez que $\operatorname{deg}(f, 2)=\operatorname{deg}(\hat{g}, 2) \cdot \operatorname{deg}(\hat{f}, 2)$ e $\operatorname{deg}(\hat{g}, 2)=1$ (vide corolário 1.4.1 e proposição 1.4.6). Deste modo, como $f: \mathbb{T}^{2} \# \mathbb{R} P^{2} \longrightarrow \mathbb{T}^{2}$ é uma aplicação contínua própria do tipo III (vide item (b) do exemplo 1.2.3), com $\mathrm{j}=1 \mathrm{e} \operatorname{deg}(\hat{f}, 2)=1$, temos, dos teoremas 2.3.1 e 2.3.2, que $\mathrm{N}(f, c)=\mathrm{j}=1$ e $\mathrm{N}_{\phi}(f, c)=\mathrm{j} \cdot \operatorname{deg}(\hat{f}, 2)=1$.

\subsection{A Realização dos $\mathbb{N}$ ưmeros de $\mathbb{N}$ ielsen de $\mathbb{R}$ aiizes}

Conforme mencionamos na seção anterior, vamos mostrar agora que, dada uma aplicação contínua própria $f:(M, \partial M) \longrightarrow(N, \partial N)$ e um ponto $c \in \operatorname{Int} N$, os números de Nielsen $\mathrm{N}(f, c)$ e $\mathrm{N}_{\phi}(f, c)$ são realizáveis, isto é, $\mathrm{N}(f, c)=\operatorname{MR}[f ; c]$ e $\mathrm{N}_{\phi}(f, c)=$ $\operatorname{MR}_{\phi}[f ; c]$. Para tanto, contudo, precisaremos introduzir o conceito de transversalidade de microfibrados.

Definição 2.4.1 . Um microfibrado $\xi=\xi^{n}=\left(E(\xi), X, r_{\xi}\right)$, sobre um espaço topológico $X$ (denominado base), consiste de um espaço total $E(\xi) \supseteq X$, juntamente com uma retração $r_{\xi}: E(\xi) \longrightarrow X$ (ou seja, uma aplicação contínua $r_{\xi}: E(\xi) \longrightarrow X$ satisfazendo $r_{\xi}(x)=x$ para todo $x \in X \subseteq E(\xi))$, tais que:

(i) para cada $x \in X$, o conjunto $\xi_{x}=r_{\xi}^{-1}(x)$, denominado fibra de $\xi$ sobre $x$, é uma variedade topológica de dimensão $n$, sem fronteira;

(ii) para cada $x \in X$, existem uma vizinhança aberta $U_{x} \subseteq X$ de $x$, e um homeomorfismo $\varphi_{x}: U_{x} \times \xi_{x} \longrightarrow r_{\xi}^{-1}\left(U_{x}\right)$, tais que a aplicação $r_{\xi} \circ \varphi_{x}: U_{x} \times \xi_{x} \longrightarrow U_{x} \subseteq X$ é a projeção de $U_{x} \times \xi_{x}$ sobre $U_{x}$, isto é, $\left(r_{\xi} \circ \varphi_{x}\right)(u, y)=u$ para todo $(u, y) \in U_{x} \times \xi_{x}$.

Exemplo 2.4.1 . Remetendo à Teoria dos Espaços Fibrados, lembremos que uma aplicação contínua $\pi: E \longrightarrow B$ (entre espaços topológicos) é um fibrado trivial, com espaço total $E$ e base $B$, se existem um espaço topológico $F$, denominado fibra, e um homeomorfismo $\gamma: B \times F \longrightarrow E$, tais que a aplicação $\pi \circ \gamma: B \times F \longrightarrow B$ é a projeção de 
$B \times F$ sobre $B$. Deste modo, se $\xi=\xi^{n}=\left(E(\xi), X, r_{\xi}\right)$ é um microfibrado sobre $X$, então $r_{\xi}: E(\xi) \longrightarrow X$ é localmente um fibrado trivial, pois, para cada $x \in E(\xi)$, existe uma vizinhança $V_{x} \subseteq E(\xi)$ de $x$ tal que a restrição $r_{x}=\left.r_{\xi}\right|_{V_{x}}: V_{x} \longrightarrow r_{\xi}\left(V_{x}\right)$ é um fibrado trivial. Em particular, note que todo fibrado trivial $\pi: E \longrightarrow B$, cujas fibras $\pi^{-1}(x), x \in B$, são variedades topológicas de dimensão $n$ sem fronteira, constitui um microfibrado sobre $B$.

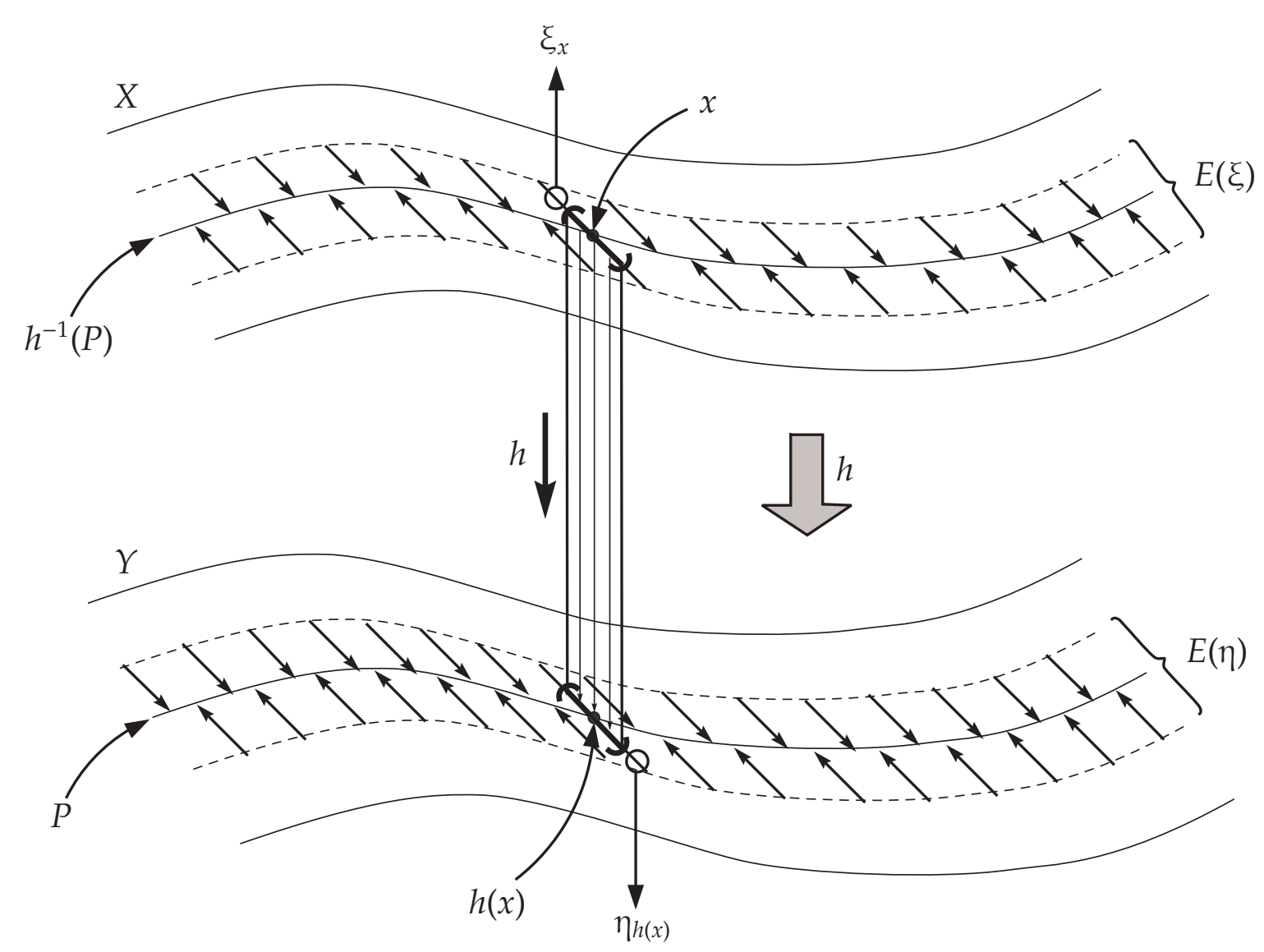

Figura 2.4

Definição 2.4.2. Seja $(Y, P)$ um par de espaços topológicos, onde Pé um subconjunto fechado de $Y$. Dizemos que um microfibrado $\eta=\left(E(\eta), P, r_{\eta}\right)$, sobre $P$, é normal se o espaço total $E(\eta) \supseteq P$ é um subconjunto aberto de $Y$. Além disso, se X é uma variedade topológica de dimensão $m$, sem fronteira, e $\eta=\eta^{n}=\left(E(\eta), P, r_{\eta}\right)$ é um microfibrado normal sobre $P \subseteq E(\eta) \subseteq Y$, dizemos que uma aplicação contínua $h: X \longrightarrow Y$ é topologicamente transversa a $\eta \operatorname{se}^{-1}(P) \subseteq X$ é uma subvariedade topológica (de dimensão $\ell \leqslant m$ ), que admite um microfibrado normal $\xi=\xi^{n}=\left(X, h^{-1}(P), r_{\xi}\right)$ satisfazendo a seguinte propriedade: para cada $x \in h^{-1}(P)$, existe 
uma vizinhança aberta $U_{x}$ de $x$ na fibra $\xi_{x}$, que é levada homeomorficamente por h sobre uma vizinhança aberta $U_{h(x)}$ de $h(x)$ na fibra $\eta_{h(x)}$ (vide figura 2.4). Em particular, se $h^{-1}(P)=\varnothing$, consideraremos, por vacuidade, que $h: X \longrightarrow$ Yé topologicamente transversa a $\eta$.

Na definição acima, convém observarmos que as dimensões dos microfibrados $\eta$ e $\xi$ têm de ser necessariamente iguais, pois, como $U_{x} \subseteq \xi_{x}$ é uma variedade topológica de dimensão igual à da fibra $\xi_{x}$, levada homeomorficamente na variedade $U_{h(x)} \subseteq \eta_{h(x)}$ (que, por sua vez, possui dimensão igual à da fibra $\left.\eta_{h(x)}\right)$, segue, do teorema da invariância da dimensão (vide [Bd], corolário 19.10, página 235) que $U_{x}$ e $U_{h(x)}$ têm a mesma dimensão, e, conseqüentemente, as dimensões de $\xi_{x}$ e $\eta_{h(x)}$ também são iguais.

A fim de evitarmos uma digressão excessivamente longa, que nos desviaria do objetivo principal desta seção, não vamos nos deter num estudo mais aprofundado destes conceitos. Para nossos propósitos, convém enunciarmos apenas o seguinte teorema, cuja demonstração, por exigir uma gama muito extensa de resultados preliminares, será omitida (para mais detalhes, vide [KS], teorema 1.1, páginas 85 a 90, juntamente com [Sch], teorema 5.3, página 10 , e [F], teorema 17, página 371 ).

Teorema 2.4.1. Sejam $X, Y$ e $P \subseteq Y$ variedades topológicas conexas e sem fronteira, cujas dimensões $m$, n e $p$, respectivamente, satisfazem $n+p=m, n \geqslant 2$ e $2 \leqslant p \leqslant m$, e considere um microfibrado normal (sobre $P$ ) $\eta=\eta^{n}=\left(Y, P, r_{\eta}\right)$, de dimensão igual à de X. Sejam ainda $C, D \subseteq X$ subconjuntos fechados, e $V_{C}, V_{D} \subseteq X$ vizinhanças abertas de $C$ e $D$, respectivamente, e suponha que $h: X \longrightarrow Y$ seja uma aplicação contínua, tal que a restrição $\left.h\right|_{V_{C}}: V_{C} \longrightarrow Y$ é topologicamente transversa à $\eta$. Então, lembrando que toda variedade topológica é metrizável, e denotando por $d_{Y}: Y \longrightarrow \mathbb{R}_{+}$uma métrica em $Y$, segue que, dada uma aplicação contínua arbitrária $\epsilon: X \longrightarrow \mathbb{R}_{+}$, existem uma vizinhança aberta $V_{\epsilon} \subseteq X$ de $C \cup D$, bem como uma homotopia $H_{\epsilon}: X \times I \longrightarrow Y$, entre he outra aplicação contínua $h^{\prime}: X \longrightarrow Y\left(\operatorname{com} V_{\epsilon}\right.$ e $H_{\epsilon}$ dependendo de $\epsilon)$, tais que:

(i) $H_{\epsilon}(x, t)=h(x)$ para todo $x \in C \cup\left(X \backslash V_{D}\right)$ e todo $t \in I$;

(ii) a restrição $h_{\epsilon}^{\prime}=\left.h^{\prime}\right|_{V_{\epsilon}}: V_{\epsilon} \longrightarrow Y$ é topologicamente transversa à $\eta$;

(iii) $d_{Y}\left(H_{\epsilon}(x, t), h(x)\right) \leqslant \epsilon(x)$ para todo $(x, t) \in X \times I$.

Em outras palavras, existe uma aplicação contínua $h^{\prime}: X \longrightarrow Y$, homotópica à h e topologicamente transversa à $\eta$ numa vizinhança de $C \cup D$, tal que $h$ ' encontra-se "tão próxima" de $h$ quanto se queira.

Agora, sejam $M$ e $N$ variedades topológicas conexas, de dimensão $n$ e com fronteira (eventualmente vazias); ao empregarmos o conceito de transversalidade de microfibrados para uma aplicação contínua própria $\mathcal{F}:(M, \partial M) \longrightarrow(N, \partial N)$, teremos de assumir 
que $\mathcal{F}$ também satisfaz $\mathcal{F}(\operatorname{Int} M) \subseteq \operatorname{Int} N$, sendo que, neste caso, denotaremos por $\mathcal{F}^{0}: \operatorname{Int} M \longrightarrow \operatorname{Int} N$ a restrição de $\mathcal{F}$ à $\operatorname{Int} M$. Além disso, dado $c \in \operatorname{Int} N$, consideraremos (no decorrer desta seção) $X=\operatorname{Int} M, Y=\operatorname{Int} M \times \operatorname{Int} N$ e $P=\operatorname{Int} M \times\{c\}$. Isto posto, sendo $r: Y \longrightarrow P$ a retração dada por $r(x, y)=(x, c)$ para cada $(x, y) \in Y=\operatorname{Int} M \times \operatorname{Int} N$, temos que $r$ constitui um fibrado trivial, com espaço total $Y$, base $P$ e fibra $F=\operatorname{Int} N$ (vide exemplo 2.4.1): com efeito, sendo $\gamma: P \times F \longrightarrow Y$ o homeomorfismo definido por $\gamma(x, c, y)=(x, y)$ para cada $(x, c, y) \in P \times F=\operatorname{Int} M \times\{c\} \times \operatorname{Int} N$, temos que a composição $r \circ \gamma: P \times F \longrightarrow P$ é a projeção de $P \times F$ sobre $P$, pois $(r \circ \gamma)(x, c, y)=r(x, y)=(x, c)$ para quaisquer $(x, c) \in P=\operatorname{Int} M \times\{c\}$ e $y \in F=\operatorname{Int} N$. Assim, como $P=\operatorname{Int} M \times\{c\}$ é um subconjunto fechado de $Y=\operatorname{Int} M \times \operatorname{Int} N$, e, para cada $\left(x_{0}, c\right) \in P$, a imagem inversa $r^{-1}\left(x_{0}, c\right)=\left\{x_{0}\right\} \times$ Int $N$ é uma variedade topológica de dimensão $n$ sem fronteira (na topologia induzida pela topologia produto de $Y=\operatorname{Int} M \times \operatorname{Int} N$ ), segue (pelo que vimos no exemplo 2.4.1) que $r: Y \longrightarrow P$ define um microfibrado normal sobre $P$, o qual denotaremos por $\eta=\eta^{n}=(Y, P, r)$.

Observe então que, se $h: X=\operatorname{Int} M \longrightarrow Y=\operatorname{Int} M \times \operatorname{Int} N$ é a aplicação definida por $h=\left(e, \mathcal{F}^{0}\right)$, onde $e: \operatorname{Int} M \longrightarrow \operatorname{Int} M$ é uma aplicação qualquer, segue que $h^{-1}(P)=$ $\mathcal{F}^{-1}(c)$, pois:

$$
\begin{aligned}
h^{-1}(P) & =\{x \in \operatorname{Int} M: h(x)=(e(x), \mathcal{F}(x)) \in P=\operatorname{Int} M \times\{c\}\} \\
& =\left\{x \in \operatorname{Int} M: \mathcal{F}^{0}(x)=\mathcal{F}(x)=c\right\}=\mathcal{F}^{-1}(c)
\end{aligned}
$$

(note que $\mathcal{F}^{-1}(c) \subseteq \operatorname{Int} M$, uma vez que $\mathcal{F}(\partial M) \subseteq \partial N$ ). Por outro lado, sabemos (por definição) que $\mathcal{F}$ é transversa a $c \in \operatorname{Int} N$ se existe uma vizinhança euclidiana $V$ de $c$ em Int $N$, tal que $\mathcal{F}^{-1}(V)$ é uma união finita e disjunta de vizinhanças euclidianas em Int $M$, cada uma das quais é levada homeomorficamente por $\mathcal{F}$ sobre $V$. Assim, temos que a transversalidade topológica de $h$ ao microfibrado normal $\eta=\eta^{n}$ está diretamente relacionada à transversalidade $\operatorname{de} \mathcal{F}$ em $c$, conforme nos mostra a seguinte

Proposição 2.4.1 . Uma aplicação contínua própria $\mathcal{F}:(M, \partial M, \operatorname{Int} M) \longrightarrow(N, \partial N, \operatorname{Int} N)$ é transversa a um ponto $c \in \operatorname{Int} N$ se, e somente se, existe uma aplicação contínua e $:$ Int $M \longrightarrow$ Int $M$ tal que a aplicação $h:$ Int $M \longrightarrow \operatorname{Int} M \times \operatorname{Int} N$, dada por $h=\left(e, \mathcal{F}^{0}\right)$, é topologicamente transversa ao microfibrado normal $\eta=\eta^{n}$.

DemonstraÇão. Com efeito, suponhamos que exista $e:$ Int $M \longrightarrow$ Int $M$ contínua tal que a aplicação $h=\left(e, \mathcal{F}^{0}\right): \operatorname{Int} M \longrightarrow \operatorname{Int} M \times \operatorname{Int} N$ é topologicamente transversa ao microfibrado normal $\eta=\eta^{n}=(Y, P, r)$; neste caso, temos que $h^{-1}(P)=\mathcal{F}^{-1}(c) \subseteq$ Int $M$ é uma subvariedade topológica de dimensão $\ell \leqslant n$, que admite um microfibrado normal $\xi=\xi^{n}=\left(\operatorname{Int} M, h^{-1}(P), r_{\xi}\right)$ satisfazendo a seguinte propriedade: dado $x_{0} \in h^{-1}(P)=$ $\mathcal{F}^{-1}(c)$ arbitrário, existe uma vizinhança aberta $U_{x_{0}}$ de $x_{0}$ na fibra $\xi_{x_{0}}$, que é levada 
homeomorficamente por $h$ sobre uma vizinhança aberta $U_{h\left(x_{0}\right)}$ de $h\left(x_{0}\right)$ na fibra $\eta_{h\left(x_{0}\right)}$. Ora, se $\xi=\xi^{n}$ é um microfibrado normal sobre $h^{-1}(P)$, segue, em particular, que tanto a fibra $\xi_{x_{0}}$, quanto a vizinhança aberta $U_{x_{0}} \subseteq \xi_{x_{0}}$, são variedades topológicas de dimensão $n$ (sem fronteira); $\operatorname{logo}$, como Int $M \supseteq \xi_{x_{0}}$ é uma variedade de dimensão $n$, temos que $U_{x_{0}}$ é uma vizinhança aberta de $x_{0}$ em Int $M$. Agora, observe que, como a aplicação $r: Y=\operatorname{Int} M \times \operatorname{Int} N \longrightarrow P=\operatorname{Int} M \times\{c\}$, dada por $r(x, y)=(x, c)$ para cada $(x, y) \in Y=\operatorname{Int} M \times \operatorname{Int} N$, é a retração com a qual se encontra munido o microfibrado normal $\eta=\eta^{n}$, temos que:

$$
\begin{aligned}
\eta_{h\left(x_{0}\right)} & =r^{-1}\left(h\left(x_{0}\right)\right)=r^{-1}\left(e\left(x_{0}\right), \mathcal{F}\left(x_{0}\right)\right)=r^{-1}\left(e\left(x_{0}\right), c\right) \\
& =\left\{(x, y) \in \operatorname{Int} M \times \operatorname{Int} N: r(x, y)=\left(e\left(x_{0}\right), c\right)\right\} \\
& =\left\{(x, y) \in \operatorname{Int} M \times \operatorname{Int} N:(x, c)=\left(e\left(x_{0}\right), c\right)\right\} \\
& =\left\{e\left(x_{0}\right)\right\} \times \operatorname{Int} N,
\end{aligned}
$$

qualquer que seja $x_{0} \in h^{-1}(P)=\mathcal{F}^{-1}(c)$. Portanto, se $U_{h\left(x_{0}\right)}$ é uma vizinhança aberta de $h\left(x_{0}\right)$ na fibra $\eta_{h\left(x_{0}\right)}$, segue que $U_{h\left(x_{0}\right)}$ é da forma $\left\{e\left(x_{0}\right)\right\} \times V_{c}$, onde $V_{c}$ é uma vizinhança aberta de $\mathcal{F}\left(x_{0}\right)=\mathcal{F}^{0}\left(x_{0}\right)=c$ em Int $N$. Deste modo, como $U_{x_{0}}$ é aplicado homeomorficamente por $h$ sobre $U_{h\left(x_{0}\right)}=\left\{e\left(x_{0}\right)\right\} \times V_{c}$, segue que a aplicação $e: \operatorname{Int} M \longrightarrow \operatorname{Int} M$ é constante (e igual a $e\left(x_{0}\right) \in \operatorname{Int} M$ ) na vizinhança $U_{x_{0}} \subseteq \operatorname{Int} M$ de $x_{0}$, e, conseqüentemente, $\mathcal{F}^{0}:$ Int $M \longrightarrow$ Int $N$ leva $U_{x_{0}}$ homeomorficamente sobre $V_{c}$. Daí, concluímos que, para cada $x_{0} \in h^{-1}(P)=\mathcal{F}^{-1}(c)$, existe uma vizinhança aberta $U_{x_{0}} \subseteq$ Int $M$ de $x_{0}$ que é levada homeomorficamente por $\mathcal{F}$ numa vizinhança aberta $V_{c} \subseteq \operatorname{Int} N$ de $\mathcal{F}\left(x_{0}\right)=c$; em particular, isto significa que $h^{-1}(P)=\mathcal{F}^{-1}(c)$ é um conjunto discreto, e, portanto, finito (uma vez que $\mathcal{F}^{-1}(c)$ é compacto). Isto posto, considere $h^{-1}(P)=\mathcal{F}^{-1}(c)=\left\{x_{1}, x_{2}, \ldots, x_{r}\right\}$, e, para cada $i \in\{1,2, \ldots, r\}$, sejam $U_{i} \subseteq \operatorname{Int} M$ e $V_{c}^{i} \subseteq \operatorname{Int} N$ vizinhanças abertas de $x_{i}$ e $\mathcal{F}\left(x_{i}\right)=c$, respectivamente, tais que $U_{i}$ é levada homeomorficamente por $\mathcal{F}$ sobre $V_{c}^{i}$; considere ainda $W_{1}, W_{2}, \ldots, W_{r} \subseteq$ Int $M$ vizinhanças euclidianas tais que, para cada $i \in\{1,2, \ldots, r\}, x_{i} \in W_{i} \subseteq U_{i}$ e $W_{i} \cap W_{j}=\varnothing$ se $j \neq i$. Sendo $V \subseteq \operatorname{Int} N$ uma vizinhança euclidiana de $c$, tal que

$$
V \subseteq \bigcap_{i=1}^{r} \mathcal{F}\left(W_{i}\right) \subseteq \bigcap_{i=1}^{r} V_{c}^{i}
$$

segue que:

$$
\mathcal{F}^{-1}(V)=\bigsqcup_{i=1}^{r} V_{i},
$$

onde, para cada $i \in\{1,2, \ldots, r\}, V_{i} \subseteq \operatorname{Int} M$ é a vizinhança euclidiana de $x_{i}$, contida em $W_{i}$, que é levada homeomorficamente por $\mathcal{F}$ sobre $V$ (observe que estamos utilizando o símbolo $\sqcup$ para denotar união disjunta). Em resumo, temos então que existe uma 
vizinhança euclidiana $V$ de $c$ em $\operatorname{Int} N$, tal que $\mathcal{F}^{-1}(V)$ é uma união finita e disjunta de vizinhanças euclidianas em Int $M$, cada uma das quais é aplicada homeomorficamente por $\mathcal{F}$ sobre $V$, o que mostra que a aplicação $\mathcal{F}:(M, \partial M, \operatorname{Int} M) \longrightarrow(N, \partial N, \operatorname{Int} N)$ é transversa à $c$.

Reciprocamente, suponhamos agora que $\mathcal{F}$ seja transversa à $c \in \operatorname{Int} N$, e seja $V \subseteq$ Int $N$ uma vizinhança euclidiana de $c$ tal que

$$
\mathcal{F}^{-1}(V)=\bigsqcup_{i=1}^{r} V_{i},
$$

onde, para cada $i \in\{1,2, \ldots, r\}, V_{i}$ é uma vizinhança euclidiana em $\operatorname{Int} M$, aplicada homeomorficamente por $\mathcal{F}$ sobre $V$. Neste caso, temos que cada ponto de $\mathcal{F}^{-1}(c)$ está contido num único $V_{i}$, onde $i \in\{1,2, \ldots, r\}$, o que implica que $\mathcal{F}^{-1}(c)$ é um conjunto discreto e finito, contendo exatamente $r$ pontos, digamos $\mathcal{F}^{-1}(c)=\left\{x_{1}, x_{2}, \ldots, x_{r}\right\}$, com $x_{i} \in V_{i}$ para cada $i \in\{1,2, \ldots, r\}$. Isto posto, considere a aplicação

$$
r_{\xi}: \mathcal{F}^{-1}(V)=\bigsqcup_{i=1}^{r} V_{i} \longrightarrow \mathcal{F}^{-1}(c),
$$

dada por $r_{\xi}(x)=x_{i}$ para cada $x \in V_{i}$, onde $i \in\{1,2, \ldots, r\}$ (note que todo ponto $x \in$ $\mathcal{F}^{-1}(V)$ está contido num único $\left.V_{i}\right)$; como $r_{\xi}\left(x_{i}\right)=x_{i}$ para todo $x_{i} \in \mathcal{F}^{-1}(c)$, temos que $r_{\xi}$ é uma retração de $\mathcal{F}^{-1}(V)$ sobre $\mathcal{F}^{-1}(c)$. Além disso, dado $x \in \mathcal{F}^{-1}(V)$, digamos $x \in V_{i}$, com $i \in\{1,2, \ldots, r\}$, temos que a aplicação $\varphi_{i}:\left\{x_{i}\right\} \times V_{i} \longrightarrow V_{i}=r_{\xi}^{-1}\left(x_{i}\right)$, dada por $\varphi_{i}\left(x_{i}, y\right)=y$ para cada $\left(x_{i}, y\right) \in\left\{x_{i}\right\} \times V_{i}$, constitui um homeomorfismo tal que a composição $r_{\xi} \circ \varphi_{i}:\left\{x_{i}\right\} \times V_{i} \longrightarrow\left\{x_{i}\right\}$ é a projeção de $\left\{x_{i}\right\} \times V_{i}$ sobre $\left\{x_{i}\right\}$ (evidentemente, estamos supondo $\mathcal{F}^{-1}(c)$ munido da topologia discreta, de modo que $\left\{x_{i}\right\} \subseteq \mathcal{F}^{-1}(c)$ é, portanto, uma vizinhança aberta de $x_{i}=r_{\xi}(x)$ em $\left.\mathcal{F}^{-1}(c)\right)$. Assim, como $r_{\xi}^{-1}(x)=V_{i} \subseteq$ Int $M$ é uma variedade topológica de dimensão $n$ (sem fronteira) para todo $x \in V_{i}$, e como $\mathcal{F}^{-1}(V)$ é um subconjunto aberto de $\operatorname{Int} M$ contendo $\mathcal{F}^{-1}(c)$, segue que $r_{\xi}$ : $\mathcal{F}^{-1}(V) \longrightarrow \mathcal{F}^{-1}(c)$ define um microfibrado normal sobre $\mathcal{F}^{-1}(c)$, o qual denotaremos por $\xi=\xi^{n}=\left(\mathcal{F}^{-1}(V), \mathcal{F}^{-1}(c), r_{\xi}\right)$. Isto posto, considere então uma aplicação contínua $e:$ Int $M \longrightarrow$ Int $M$ tal que $e\left(V_{i}\right)=\left\{x_{i}\right\}$ para cada $i \in\{1,2, \ldots, r\}$ (tome, por exemplo, a aplicação identidade $\operatorname{Id}_{M}: \operatorname{Int} M \longrightarrow \operatorname{Int} M$, e deforme $\operatorname{Id}_{M}$ continuamente numa vizinhança compacta $C$ de $\mathcal{F}^{-1}(V)$ em Int $M$ a fim de obter a aplicação $e: \operatorname{Int} M \longrightarrow \operatorname{Int} M$ requerida); sendo $h: \operatorname{Int} M \longrightarrow \operatorname{Int} M \times \operatorname{Int} N$ a aplicação dada por $h=\left(e, \mathcal{F}^{0}\right)$, sabemos que $h^{-1}(P)=\mathcal{F}^{-1}(c)$, onde $P=\operatorname{Int} M \times\{c\}$. Logo, como $\mathcal{F}^{-1}(c)$ é discreto e finito, temos que $h^{-1}(P) \subseteq$ Int $M$ é uma subvariedade topológica de dimensão zero. Por outro lado, lembrando que $\eta=\eta^{n}=(Y, P, r)$ é o microfibrado normal sobre $P$, com espaço total $Y=\operatorname{Int} M \times \operatorname{Int} N$ e retração $r: Y \longrightarrow P$, dada por $r(x, y)=(x, c)$ para cada $(x, y) \in Y$, 
segue que:

$$
\eta_{h\left(x_{i}\right)}=r^{-1}\left(h\left(x_{i}\right)\right)=r^{-1}\left(e\left(x_{i}\right), \mathcal{F}^{0}\left(x_{i}\right)\right)=r^{-1}\left(x_{i}, c\right)=\left\{x_{i}\right\} \times \operatorname{Int} N
$$

para todo $x_{i} \in h^{-1}(P)=\mathcal{F}^{-1}(c)$. Deste modo, como $V \subseteq \operatorname{Int} N$ é uma vizinhança euclidiana de $c$, temos que o conjunto $V_{c}^{i}=\left\{x_{i}\right\} \times V$ é uma vizinhança aberta de $h\left(x_{i}\right)=\left(x_{i}, c\right)$ na fibra $\eta_{h\left(x_{i}\right)}$; ademais, como $\xi_{x_{i}}=r_{\xi}^{-1}\left(x_{i}\right)=V_{i}$, segue que $V_{i}$ é uma vizinhança aberta de $x_{i}$ na fibra $\xi_{x_{i}}$. Conseqüentemente, como $\mathcal{F}$ leva $V_{i} \subseteq \operatorname{Int} M$ homeomorficamente sobre $V \subseteq \operatorname{Int} N$, e a aplicação $e: \operatorname{Int} M \longrightarrow \operatorname{Int} M$ é constante e igual a $x_{i}$ em $V_{i}$, temos que $V_{i}$ é aplicado homeomorficamente por $h=\left(e, \mathcal{F}^{0}\right)$ sobre $V_{c}^{i}=\left\{x_{i}\right\} \times V$. Portanto, para todo $x_{i} \in h^{-1}(P)=\mathcal{F}^{-1}(c)=\left\{x_{1}, x_{2}, \ldots, x_{r}\right\}$, existe uma vizinhança aberta $V_{x_{i}}$ de $x_{i}$ na fibra $\xi_{x_{i}}$ que é levada homeomorficamente por $h=\left(e, \mathcal{F}^{0}\right)$ numa vizinhança aberta $V_{c}^{i}$ de $h\left(x_{i}\right)=\left(x_{i}, c\right)$ na fibra $\eta_{h\left(x_{i}\right)}$, d'onde concluímos que a aplicação $h=\left(e, \mathcal{F}^{0}\right): \operatorname{Int} M \longrightarrow \operatorname{Int} M \times \operatorname{Int} N$ é topologicamente tranversa à $c$, conforme queríamos provar.

Isto posto, seja $f:(M, \partial M) \longrightarrow(N, \partial N)$ uma aplicação contínua própria (entre variedades topológicas conexas de dimensão $n$ ), e considere um ponto $c \in \operatorname{Int} N$. Mais adiante, veremos que a etapa mais importante na demonstração de que os números de Nielsen $\mathrm{N}(f, c)$ e $\mathrm{N}_{\uparrow}(f, c)$ são realizáveis, consiste de "criar" e "remover" pontos de $f^{-1}(c)$, por meio de homotopias próprias, a fim de encontrarmos aplicações contínuas $g, g^{\prime}:(M, \partial M) \longrightarrow(N, \partial N)$, propriamente homotópicas à $f$, que possuem respectivamente $\mathrm{N}(f, c)$ e $\mathrm{N}_{\phi}(f, c)$ raízes em $c$. Para descrevermos as condições em que tais procedimentos são possíveis, precisaremos introduzir o conceito de Nielsen-relação.

Definição 2.4.3. Sejam $X, Y e P \subseteq Y$ variedades topológicas conexas, e considere uma aplicação contínua $h: X \longrightarrow Y$. Dados dois pontos $x_{1}, x_{2} \in h^{-1}(P)$, dizemos que $x_{1}$ e $x_{2}$ estão Nielsenrelacionados, e escrevemos $x_{1} \stackrel{N}{\sim} x_{2}$, se existe um caminho $\omega: I \longrightarrow X, \operatorname{com} \omega(0)=x_{1} e$ $\omega(1)=x_{2}$, tal que $h \circ \omega: I \longrightarrow Y$ é homotópico a um caminho em P; neste caso, dizemos ainda que $\omega$ Nielsen-relaciona os pontos $x_{1}$ e $x_{2}$.

O próximo lema nos mostra que, para aplicações da forma $h=\left(e, \mathcal{F}^{0}\right): \operatorname{Int} M \longrightarrow$ $\operatorname{Int} M \times \operatorname{Int} N$, onde $e: \operatorname{Int} M \longrightarrow \operatorname{Int} M$ e $\mathcal{F}:(M, \partial M, \operatorname{Int} M) \longrightarrow(N, \partial N, \operatorname{Int} N)$ são aplicações contínuas quaisquer, o conceito de Nielsen-relação, para pontos na imagem inversa $h^{-1}(\operatorname{Int} M \times\{c\}), \operatorname{com} c \in \operatorname{Int} N$, equivale à relação de pertencer à mesma classe de raízes de $\mathcal{F}$ em $c$.

Lema 2.4.1 . Seja $\mathcal{F}:(M, \partial M, \operatorname{Int} M) \longrightarrow(N, \partial N, \operatorname{Int} N)$ uma aplicação contínua (entre variedades topológicas conexas de mesma dimensão $n$ ), e considere um ponto $c \in \operatorname{Int} N$. Sendo 
$h: X=\operatorname{Int} M \longrightarrow Y=\operatorname{Int} M \times \operatorname{Int} N$ a aplicação dada por $h=\left(e, \mathcal{F}^{0}\right)$, onde e $: \operatorname{Int} M \longrightarrow \operatorname{Int} M$ é uma aplicação contínua qualquer, e denotando por $P=\operatorname{Int} M \times\{c\} \subseteq Y$, temos que dois pontos $x_{1}, x_{2} \in h^{-1}(P)$ estão Nielsen-relacionados se, e somente se, eles pertencem à mesma classe de raízes de $\mathcal{F}$ em $c$.

DemonstraÇÃo. Com efeito, se $x_{1}, x_{2} \in R$, onde $R$ é uma classe de raízes de $\mathcal{F}$ em $c$, segue (por definição) que existe um caminho $\omega: I \longrightarrow \operatorname{Int} M, \operatorname{com} \omega(0)=x_{1}$ e $\omega(1)=x_{2}$, tal que $\mathcal{F} \circ \omega: I \longrightarrow$ Int $N$ é um laço contrátil em $c$. Seja, pois, $H: I \times I \longrightarrow \operatorname{Int} N$ uma homotopia entre $\mathcal{F} \circ \omega$ e o caminho constante $\epsilon_{c}: I \longrightarrow\{c\} \subseteq \operatorname{Int} N$ (isto é, $H: I \times I \longrightarrow$ Int $N$ é uma aplicação contínua tal que $H(s, 0)=(\mathcal{F} \circ \omega)(0), H(s, 1)=\epsilon_{c}(s)=c$, $H(0, t)=(\mathcal{F} \circ \omega)(0)=\epsilon_{c}(0)=c$ e $H(1, t)=(\mathcal{F} \circ \omega)(1)=\epsilon_{c}(1)=c$, para quaisquer $(s, t) \in I \times I)$, e considere o caminho $\lambda: I \longrightarrow P=\operatorname{Int} M \times\{c\}$ dado por $\lambda(t)=((e \circ \omega)(t), c)$ para cada $t \in I$. Sendo $K: I \times I \longrightarrow Y=\operatorname{Int} M \times \operatorname{Int} N$ a aplicação contínua definida por $K(s, t)=((e \circ \omega)(s), H(s, t))$ para cada $(s, t) \in I \times I$, temos então que $K$ é uma homotopia entre os caminhos $h \circ \omega: I \longrightarrow Y$ e $\lambda: I \longrightarrow P$, uma vez que:

$$
\begin{gathered}
K(s, 0)=((e \circ \omega)(s), H(s, 0))=((e \circ \omega)(s),(\mathcal{F} \circ \omega)(s))=(h \circ \omega)(s), \\
K(s, 1)=((e \circ \omega)(s), H(s, 1))=((e \circ \omega)(s), c)=\lambda(s), \\
K(0, t)=((e \circ \omega)(0), H(0, t))=((e \circ \omega)(0),(\mathcal{F} \circ \omega)(0))=(h \circ \omega)(0)=\lambda(0) \text { e } \\
K(1, t)=((e \circ \omega)(1), H(1, t))=((e \circ \omega)(1),(\mathcal{F} \circ \omega)(1))=(h \circ \omega)(1)=\lambda(1),
\end{gathered}
$$

quaisquer que sejam $s, t \in I$. Logo, temos que o caminho $h \circ \omega: I \longrightarrow Y$ é homotópico a um caminho em $P$, o que implica que $\omega$ Nielsen-relaciona os pontos $x_{1}$ e $x_{2}$, isto é, $x_{1} \stackrel{N}{\sim} x_{2}$.

Reciprocamente, suponhamos que $x_{1}, x_{2} \in h^{-1}(P)$ sejam tais que $x_{1} \stackrel{N}{\sim} x_{2}$; neste caso, segue que existe um caminho $\omega: I \longrightarrow \operatorname{Int} M, \operatorname{com} \omega(0)=x_{1}$ e $\omega(1)=x_{2}$, tal que $h \circ \omega: I \longrightarrow Y=\operatorname{Int} M \times \operatorname{Int} N$ é homotópico a um caminho em $P=\operatorname{Int} M \times\{c\}$, digamos $\lambda=\left(\lambda_{1}, c\right): I \longrightarrow \operatorname{Int} M \times\{c\}$, com $\lambda_{1}: I \longrightarrow$ Int $M$. Por outro lado, se $x_{1}, x_{2} \in h^{-1}(P) \subseteq$ Int $M$, temos que $h\left(x_{1}\right)=\left(e\left(x_{1}\right), \mathcal{F}\left(x_{1}\right)\right)$ e $h\left(x_{2}\right)=\left(e\left(x_{2}\right), \mathcal{F}\left(x_{2}\right)\right)$ pertencem a $P=\operatorname{Int} M \times\{c\}$, isto é, $\mathcal{F}\left(x_{1}\right)=\mathcal{F}\left(x_{2}\right)=c$; logo, $\mathcal{F} \circ \omega: I \longrightarrow$ Int $M$ é um laço com base em $c=(\mathcal{F} \circ \omega)(0)=(\mathcal{F} \circ \omega)(1)$. Agora, considere uma homotopia $H: I \times I \longrightarrow$ $Y=\operatorname{Int} M \times \operatorname{Int} N$ entre $h \circ \omega=(e \circ \omega, \mathcal{F} \circ \omega)$ e $\lambda=\left(\lambda_{1}, c\right)$; temos então que $H$ é uma aplicação contínua tal que $H(s, 0)=(h \circ \omega)(s)=((e \circ \omega)(s),(\mathcal{F} \circ \omega)(s)), H(s, 1)=$ $\lambda(s)=\left(\lambda_{1}(s), c\right), H(0, t)=(h \circ \omega)(0)=\lambda(0)$ e $H(1, t)=(h \circ \omega)(1)=\lambda(1)$, para quaisquer $(s, t) \in I \times I$. Conseqüentemente, denotando por $\pi_{N}: \operatorname{Int} M \times \operatorname{Int} N \longrightarrow \operatorname{Int} N$ a projeção de Int $M \times \operatorname{Int} N$ sobre $\operatorname{Int} N\left(\right.$ dada por $\pi_{N}(x, y)=y$ para cada $\left.(x, y) \in \operatorname{Int} M \times \operatorname{Int} N\right)$, segue que a aplicação $K=\pi_{N} \circ H: I \times I \longrightarrow$ Int $N$ é contínua e satisfaz:

$$
K(s, 0)=\left(\pi_{N} \circ H\right)(s, 0)=\pi_{N}((e \circ \omega)(s),(\mathcal{F} \circ \omega)(s))=(\mathcal{F} \circ \omega)(s),
$$




$$
\begin{gathered}
K(s, 1)=\left(\pi_{N} \circ H\right)(s, 1)=\pi_{N}\left(\lambda_{1}(s), c\right)=c \\
K(0, t)=\left(\pi_{N} \circ H\right)(0, t)=\pi_{N}(\lambda(0))=\pi_{N}\left(\lambda_{1}(0), c\right)=c=(\mathcal{F} \circ \omega)(0) \mathrm{e} \\
K(1, t)=\left(\pi_{N} \circ H\right)(1, t)=\pi_{N}(\lambda(1))=\pi_{N}\left(\lambda_{1}(1), c\right)=c=(\mathcal{F} \circ \omega)(1)
\end{gathered}
$$

para todo $(s, t) \in I \times I$. Deste modo, concluímos que $K$ é uma homotopia entre o laço $\mathcal{F} \circ \omega: I \longrightarrow$ Int $N$ e o caminho constante $\epsilon_{c}: I \longrightarrow\{c\} \subseteq \operatorname{Int} N$, ou seja, $\mathcal{F} \circ \omega$ é um laço contrátil em $c$, o que prova que $x_{1}$ e $x_{2}$ pertencem a uma mesma classe de raízes de $\mathcal{F}$ em $c$.

Isto posto, lembremos então que, dada uma variedade topológica $X$, de dimensão $n$, definimos uma orientação local de $X$ num ponto $x \in \operatorname{Int} X$ como sendo um gerador $\mathcal{O}(x)$ de $\mathrm{H}_{n}(X, X \backslash\{x\}) \simeq \mathbb{Z}$. Considere, pois, $h: X \longrightarrow Y$ uma aplicação contínua entre variedades topológicas conexas e sem fronteira, e seja $P$ uma subvariedade topológica conexa de $Y$. Suponha ainda que as dimensões $n, m, p \geqslant 0$ de $X, Y$ e $P \subseteq Y$, respectivamente, satisfaçam $n+p=m$ e $p<m$ (de modo que $P$ é, portanto, um subconjunto fechado de $Y$ ), e considere um microfibrado normal $\delta=\delta^{n}=\left(Y, P, r_{\delta}\right)$, de dimensão igual à de $X$, tal que $h: X \longrightarrow Y$ é topologicamente transversa à $\delta$. Isto significa que $h^{-1}(P)$ é uma subvariedade topológica de $X$, de dimensão $\ell \leqslant n$, que admite um microfibrado normal $\xi=\xi^{n}=\left(X, h^{-1}(P), r_{\xi}\right)$ satisfazendo a seguinte propriedade: para cada $x \in h^{-1}(P)$, existe uma vizinhança aberta de $x$ na fibra $\xi_{x}$, que é levada homeomorficamente por $h$ numa vizinhança aberta de $h(x)$ na fibra $\delta_{h(x)}$. Assim, dado um ponto $x \in h^{-1}(P)$, sejam $U_{x} \subseteq \xi_{x}$ e $U_{h(x)} \subseteq \delta_{h(x)}$ vizinhanças abertas de $x$ e $h(x)$, respectivamente, tais que a restrição $\left.h\right|_{U_{x}}: U_{x} \longrightarrow U_{h(x)}$ é um homeomorfismo sobre $U_{h(x)}$. Como $\xi_{x}$ e $\delta_{h(x)}$ são variedades topológicas de dimensão $n$ (sem fronteira), segue que os abertos $U_{x} \subseteq \xi_{x}$ e $U_{h(x)} \subseteq \delta_{h(x)}$ também o são; logo, sendo $\left(j_{x}\right)_{*}: \mathrm{H}_{n}\left(\xi_{x}, \xi_{x} \backslash\{x\}\right) \longrightarrow \mathrm{H}_{n}(X, X \backslash\{x\}),\left(j_{\xi_{x}}\right)_{*}: \mathrm{H}_{n}\left(U_{x}, U_{x} \backslash\{x\}\right) \longrightarrow \mathrm{H}_{n}\left(\xi_{x}, \xi_{x} \backslash\{x\}\right)$ e $\left(j_{\delta_{h(x)}}\right)_{*}: \mathrm{H}_{n}\left(U_{h(x)}, U_{h(x)} \backslash\{h(x)\}\right) \longrightarrow \mathrm{H}_{n}\left(\delta_{h(x)}, \delta_{h(x)} \backslash\{h(x)\}\right)$ os isomorfismos induzidos pelas respectivas inclusões, obtemos a seguinte cadeia de isomorfismos:

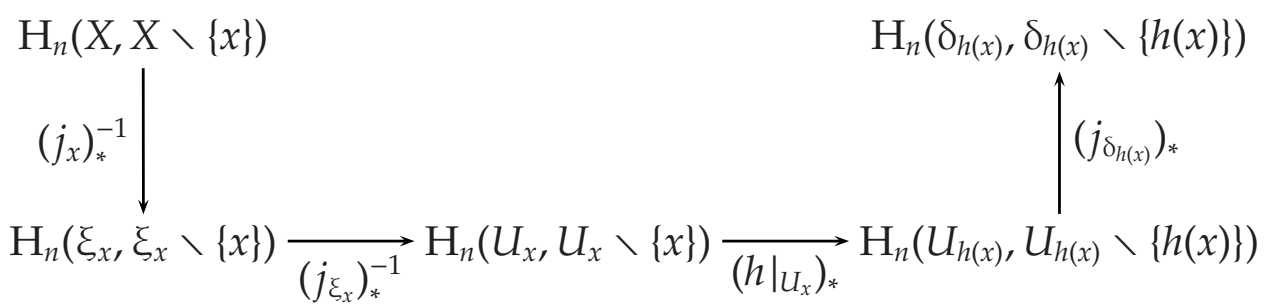

Dada uma orientação local $\mathcal{O}(x) \in \mathrm{H}_{n}(X, X \backslash\{x\})$ de $X$ em $x$, temos então que os isomorfismos acima determinam uma orientação local da fibra $\delta_{h(x)}$ em $h(x) \in P$, a 
qual (utilizando um abuso de notação) denotaremos por $h_{*}(\mathcal{O}(x))$, isto é,

$$
h_{*}(\mathcal{O}(x))=\left[\left(j_{\delta_{h(x)}}\right)_{*} \circ\left(\left.h\right|_{U_{x}}\right)_{*} \circ\left(j_{\xi_{x}}\right)_{*}^{-1} \circ\left(j_{x}\right)_{*}^{-1}\right](\mathcal{O}(x)) .
$$
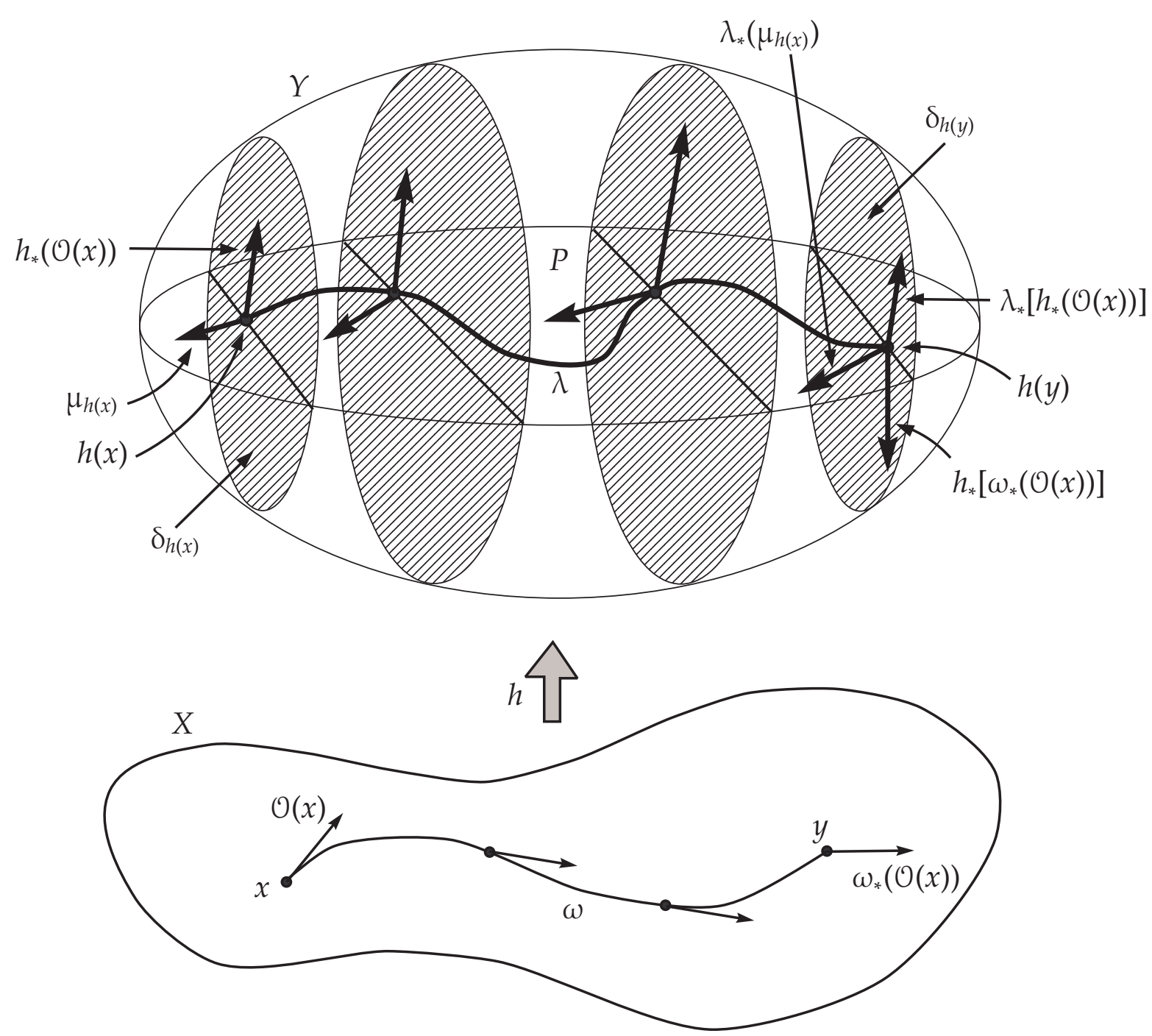

Figura 2.5

Por outro lado, dado $y \in h^{-1}(P)$, e supondo que $x$ e $y$ estão Nielsen-relacionados, sabemos que existe um caminho $\omega: I \longrightarrow X$, com $\omega(0)=x$ e $\omega(1)=y$, tal que $h \circ \omega: I \longrightarrow Y$ é homotópico a um caminho $\lambda: I \longrightarrow P, \operatorname{com} \lambda(0)=h(x) \in P$ e $\lambda(1)=h(y) \in P$. Entretanto, como $X$ é uma variedade topológica conexa e sem fronteira, segue que o fibrado orientado $\tilde{X}$ de $X$ encontra-se bem definido (sendo a variedade topológica constituída das orientações locais de todos os pontos de $X$ ), de maneira que, se $\tilde{p}_{X}: \tilde{C}_{X} \longrightarrow X$ é o recobrimento obtido da componente conexa $\tilde{C}_{X}$ de $\tilde{X}$ que contém 
$\mathcal{O}(x)$, podemos levantar o caminho $\omega: I \longrightarrow X$, relativamente a $\tilde{p}_{X}$, para um único caminho $\tilde{\omega}: I \longrightarrow \tilde{C}_{X}$ com início em $\tilde{\omega}(0)=\mathcal{O}(x)$. Logo, denotando por $\omega_{*}(\mathcal{O}(x))$ o ponto final do levantamento $\tilde{\omega}$ (isto é, $\omega_{*}(\mathcal{O}(x))=\tilde{\omega}(1) \in \mathrm{H}_{n}(X, X \backslash\{y\})$ ), temos que $\omega_{*}(\mathcal{O}(x))$ é a orientação local de $X$ no ponto $y$, obtida estendendo-se a orientação local $\mathcal{O}(x)$ ao longo do caminho $\omega$. Agora, vamos descrever um procedimento para transladar a orientação $h_{*}(\mathcal{O}(x))$ de $\delta_{h(x)}$ em $h(x)$, ao longo do caminho $\lambda$, a fim de obtermos uma orientação $\lambda_{*}\left[h_{*}(\mathcal{O}(x))\right]$, da fibra $\delta_{h(x)}$ no ponto $h(y)$ (vide figura 2.5).

Primeiramente, observe que, como $h(x) \in P$ e $\delta=\delta^{n}=\left(Y, P, r_{\delta}\right)$ é um microfibrado normal sobre $P$, segue que existem uma vizinhança aberta $V_{h(x)} \subseteq P$ de $h(x)$, e um homeomorfismo $\varphi_{h(x)}: V_{h(x)} \times \delta_{h(x)} \longrightarrow r_{\delta}^{-1}\left(V_{h(x)}\right)$, tais que a aplicação $r_{\delta} \circ \varphi_{h(x)}: V_{h(x)} \times$ $\delta_{h(x)} \longrightarrow V_{h(x)}$ é a projeção de $V_{h(x)} \times \delta_{h(x)}$ sobre $V_{h(x)}$. Como $r_{\delta}: Y \longrightarrow P$ é contínua e $V_{h(x)}$ é aberto em $P$, temos que $W_{h(x)}=r_{\delta}^{-1}\left(V_{h(x)}\right)$ é um subconjunto aberto de $Y$, e, portanto, constitui uma variedade topológica de dimensão $m=p+n$, da mesma forma que o produto $V_{h(x)} \times \delta_{h(x)}$ (note que, por ser aberto em $P, V_{h(x)}$ possui dimensão $p$, enquanto que a fibra $\delta_{h(x)}$ é de dimensão $\left.n\right)$. Isto posto, seja $w_{h(x)} \in W_{h(x)}$ tal que $\varphi_{h(x)}(h(x), h(x))=w_{h(x)}$; como $\overline{Y \backslash W_{h(x)}}=Y \backslash W_{h(x)} \subseteq Y \backslash\left\{w_{h(x)}\right\}=\operatorname{Int}(Y \backslash\{h(x)\})$, segue (do teorema da excisão em homologia singular) que a inclusão $i_{h(x)}:\left(W_{h(x)}, W_{h(x)} \backslash\left\{w_{h(x)}\right\}\right) \longrightarrow\left(Y, Y \backslash\left\{w_{h(x)}\right\}\right)$ induz um isomorfismo $\left(i_{h(x)}\right)_{*}: \mathrm{H}_{p+n}\left(W_{h(x)}, W_{h(x)} \backslash\left\{w_{h(x)}\right\}\right) \longrightarrow \mathrm{H}_{p+n}\left(Y, Y \backslash\left\{w_{h(x)}\right\}\right)$, uma vez que $\left(W_{h(x)}, W_{h(x)} \backslash\left\{w_{h(x)}\right\}\right)=\left(Y \backslash\left(Y \backslash W_{h(x)}\right),\left(Y \backslash\left\{w_{h(x)}\right\}\right) \backslash\left(Y \backslash W_{h(x)}\right)\right.$. Por outro lado, como $Y$ é uma variedade topológica conexa e sem fronteira, segue que existe um homeomorfismo $\rho_{h(x)}: Y \longrightarrow Y$, homotópico à identidade, tal que $\rho_{h(x)}\left(w_{h(x)}\right)=$ $h(x)$ (vide [Vi], lema 6.4, página 150); logo, sendo $\left(\varphi_{h(x)}\right)_{*}: \mathrm{H}_{p+n}\left(V_{h(x)} \times \delta_{h(x)},\left(V_{h(x)} \times\right.\right.$ $\left.\left.\delta_{h(x)}\right) \backslash\{(h(x), h(x))\}\right) \longrightarrow \mathrm{H}_{p+n}\left(W_{h(x)}, W_{h(x)} \backslash\left\{w_{h(x)}\right\}\right)$ e $\left(\rho_{h(x)}\right)_{*}: \mathrm{H}_{p+n}\left(Y, Y \backslash\left\{w_{h(x)}\right\}\right) \longrightarrow$ $\mathrm{H}_{p+n}(Y, Y \backslash\{h(x)\})$ os isomorfismos induzidos por $\varphi_{h(x)}$ e $\rho_{h(x)}$, temos a seguinte cadeia de isomorfismos:

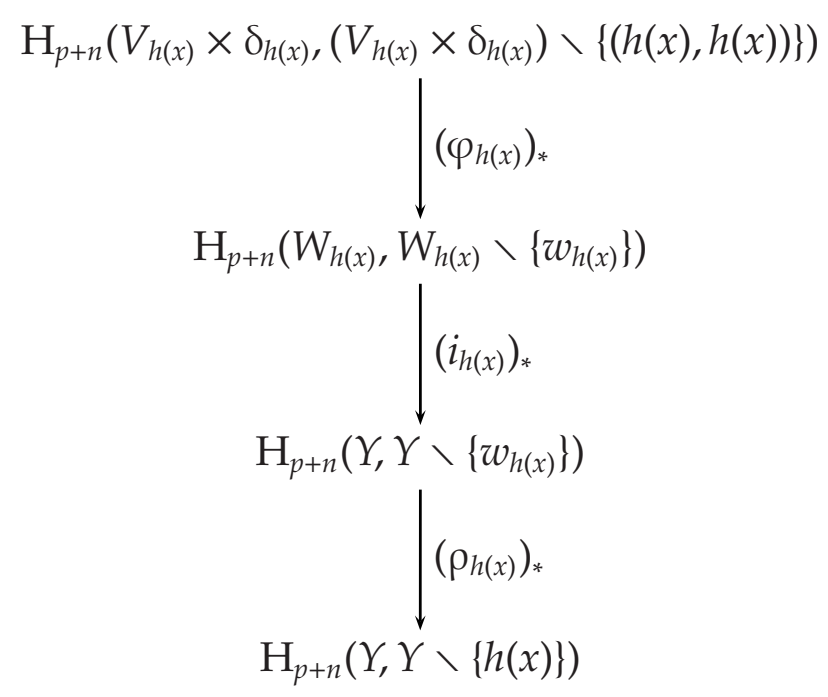


Agora, sendo $A=V_{h(x)}, A_{0}=V_{h(x)} \backslash\{h(x)\} \subseteq A, B=\delta_{h(x)}$ e $B_{0}=\delta_{h(x)} \backslash\{h(x)\} \subseteq B$, temos que:

$$
\begin{aligned}
\left(A_{0} \times B\right) \cup\left(A \times B_{0}\right)= & {\left[\left(V_{h(x)} \backslash\{h(x)\}\right) \times \delta_{h(x)}\right] \cup\left[V_{h(x)} \times\left(\delta_{h(x)} \backslash\{h(x)\}\right)\right] } \\
= & \left\{(v, d) \in V_{h(x)} \times \delta_{h(x)}: v \neq h(x)\right\} \bigcup \\
& \left\{(v, d) \in V_{h(x)} \times \delta_{h(x)}: d \neq h(x)\right\} \\
= & \left\{(v, d) \in V_{h(x)} \times \delta_{h(x)}:(v, d) \neq(h(x), h(x))\right\} \\
= & \left(V_{h(x)} \times \delta_{h(x)}\right) \backslash\{(h(x), h(x))\}
\end{aligned}
$$

o que, em particular, implica que $A_{0} \times B=\left(V_{h(x)} \backslash\{h(x)\}\right) \times \delta_{h(x)}$ e $A \times B_{0}=V_{h(x)} \times\left(\delta_{h(x)} \backslash\{h(x)\}\right)$ são abertos em $\left(A_{0} \times B\right) \cup\left(A \times B_{0}\right)$; portanto, do teorema de Künneth em homologia singular (vide [Ma1], página 302, teorema 6.2), obtemos que:

$$
\begin{aligned}
\mathrm{H}_{p+n}\left(A \times B,\left(A_{0} \times B\right) \times\left(A \times B_{0}\right)\right) \simeq & \left(\bigoplus_{i+j=p+n}\left[\mathrm{H}_{i}\left(A, A_{0}\right) \otimes \mathrm{H}_{j}\left(B, B_{0}\right)\right]\right) \bigoplus \\
& \left(\bigoplus_{i+j=p+n}\left[\operatorname{Tor}\left(\mathrm{H}_{i}\left(A, A_{0}\right), \mathrm{H}_{j}\left(B, B_{0}\right)\right)\right]\right) .
\end{aligned}
$$

No entanto, como $A=V_{h(x)}$ e $B=\delta_{h(x)}$ são variedades topológicas de dimensões respectivamente iguais a $p$ e $n$, temos que $\mathrm{H}_{i}\left(A, A_{0}\right) \simeq\{0\}$ e $\mathrm{H}_{j}\left(B, B_{0}\right) \simeq\{0\}$ para $i \neq p$ e $j \neq n$, sendo que $\mathrm{H}_{p}\left(A, A_{0}\right) \simeq \mathbb{Z}$ e $\mathrm{H}_{n}\left(B, B_{0}\right) \simeq \mathbb{Z}$; assim, a expressão acima se reduz a:

$$
\begin{aligned}
\mathrm{H}_{p+n}\left(A \times B,\left(A_{0} \times B\right) \times\left(A \times B_{0}\right)\right) \simeq \quad & {\left[\mathrm{H}_{p}\left(A, A_{0}\right) \otimes \mathrm{H}_{n}\left(B, B_{0}\right)\right] \oplus } \\
& \operatorname{Tor}\left(\mathrm{H}_{p}\left(A, A_{0}\right), \mathrm{H}_{n-1}\left(B, B_{0}\right)\right) \oplus \\
& \operatorname{Tor}\left(\mathrm{H}_{p-1}\left(A, A_{0}\right), \mathrm{H}_{n}\left(B, B_{0}\right)\right) \\
\simeq \quad & {\left[\mathrm{H}_{p}\left(A, A_{0}\right) \otimes \mathrm{H}_{n}\left(B, B_{0}\right)\right] \oplus } \\
& \operatorname{Tor}(\mathbb{Z},\{0\}) \oplus \operatorname{Tor}(\{0\}, \mathbb{Z}) \\
\simeq & \mathrm{H}_{p}\left(A, A_{0}\right) \otimes \mathrm{H}_{n}\left(B, B_{0}\right),
\end{aligned}
$$

isto é, existe um isomorfismo

$$
\begin{gathered}
\mathrm{H}_{p+n}\left(V_{h(x)} \times \delta_{h(x),}\left(V_{h(x)} \times \delta_{h(x)}\right) \backslash\{h(x), h(x)\}\right) \\
\left(\psi_{h(x)}\right)_{*} \\
\mathrm{H}_{p}\left(V_{h(x)}, V_{h(x)} \backslash\{h(x)\}\right) \otimes \mathrm{H}_{n}\left(\delta_{h(x)}, \delta_{h(x)} \backslash\{h(x)\}\right)
\end{gathered}
$$

Deste modo, escolhida uma orientação local $\mu_{h(x)} \in \mathrm{H}_{p}\left(V_{h(x)}, V_{h(x)} \backslash\{h(x)\}\right)$, de $V_{h(x)}$ em $h(x)$, seja $v_{h(x)} \in \mathrm{H}_{p+n}(Y, Y \backslash\{h(x)\})$ a orientação local de $Y$ em $h(x)$ correspondente a $\mu_{h(x)} \otimes h_{*}(\mathcal{O}(x)) \in \mathrm{H}_{p}\left(V_{h(x)}, V_{h(x)} \backslash\{h(x)\}\right) \otimes \mathrm{H}_{n}\left(\delta_{h(x)}, \delta_{h(x)} \backslash\{h(x)\}\right)$ pela seguinte cadeia de isomorfismos: 


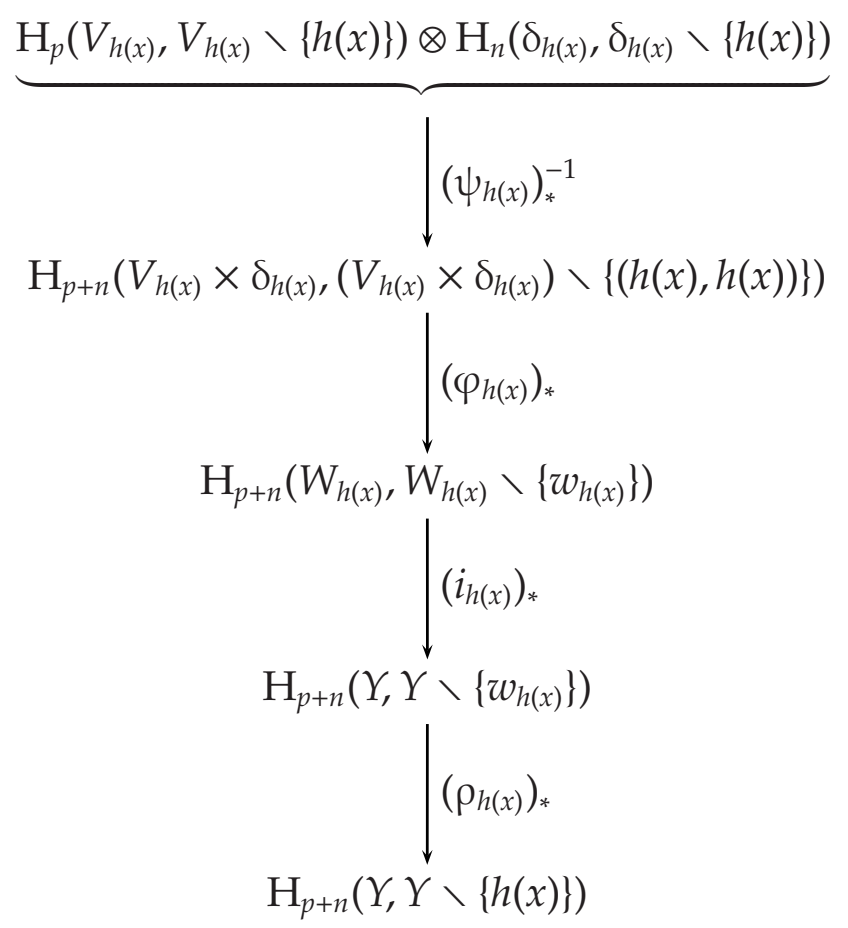

(note que, pelas propriedades do produto tensorial, o elemento $\mu_{h(x)} \otimes h_{*}(\mathcal{O}(x))$ é um gerador de $\left.\mathrm{H}_{p}\left(V_{h(x)}, V_{h(x)} \backslash\{h(x)\}\right) \otimes \mathrm{H}_{n}\left(\delta_{h(x)}, \delta_{h(x)} \backslash\{h(x)\}\right)\right)$. Então, lembrando que $Y$ é uma variedade topológica conexa e sem fronteira (de modo que o fibrado orientado $\tilde{Y}$ de $Y$ encontra-se bem definido), podemos estender a orientação $v_{h(x)}$ ao longo do caminho $h \circ \omega: I \longrightarrow Y$ : sendo $\tilde{C}_{Y}$ a componente conexa do fibrado orientado $\tilde{Y}$, que contém $v_{h(x)} \in \mathrm{H}_{p+n}(Y, Y \backslash\{h(x)\})$, e denotando por $h \tilde{o} \omega: I \longrightarrow \tilde{C}_{Y}$ o único levantamento de $h \circ \omega$, relativamente ao recobrimento $\tilde{p}_{Y}: \tilde{C}_{Y} \longrightarrow Y$ obtido da projeção orientada de $Y$, com $(h \tilde{o} \omega)(0)=v_{h(x)}$, tomamos $(h \circ \omega)_{*}\left(v_{h(x)}\right) \in \mathrm{H}_{p+n}(Y, Y \backslash\{h(x)\})$ como sendo a orientação local de $Y$ em $h(y)=(h \circ \omega)(1)$, dada por $(h \circ \omega)_{*}\left(v_{h(x)}\right)=(h \tilde{\circ} \omega)(1)$. Por outro lado, considere uma vizinhança aberta $V_{h(y)} \subseteq P$ de $h(y) \in P$ tal que existe um homeomorfismo $\varphi_{h(y)}: V_{h(y)} \times \delta_{h(y)} \longrightarrow W_{h(y)}=r_{\delta}^{-1}\left(V_{h(y)}\right)$, cuja composição $r_{\delta} \circ \varphi_{h(y)}$ : $V_{h(y)} \times \delta_{h(y)} \longrightarrow V_{h(y)}$ é a projeção de $V_{h(y)} \times \delta_{h(y)}$ sobre $V_{h(y)}$ (lembre-se que $\delta=\delta^{n}=\left(Y, P, r_{\delta}\right)$ é um microfibrado sobre $P)$, e sejam $\left(j_{V_{h(x)}}\right)_{*}: \mathrm{H}_{p}\left(V_{h(x)}, V_{h(x)} \backslash\{h(x)\}\right) \longrightarrow \mathrm{H}_{p}(P, P \backslash\{h(x)\})$

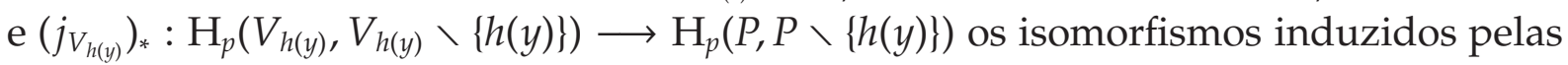
respectivas inclusões; denotando por $\mu_{h(x)}^{P} \in \mathrm{H}_{p}(P, P \backslash\{h(x)\})$ a orientação local de $P \mathrm{em}$

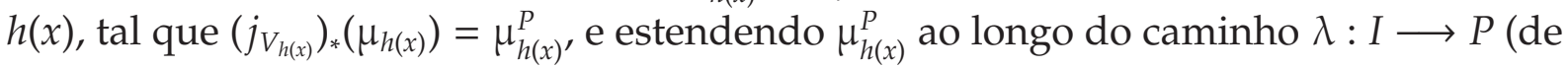
maneira análoga ao que fizemos para o caminho $h \circ \omega$ acima), obtemos uma orientação local $\lambda_{*}\left(\mu_{h(x)}^{P}\right) \in \mathrm{H}_{p}(P, P \backslash\{h(y)\})$ de $P$ em $h(y)=\lambda(1)$, a qual determina uma única orientação local $\mu_{h(y)} \in \mathrm{H}_{p}\left(V_{h(y)}, V_{h(y)} \backslash\{h(y)\}\right)$ pelo isomorfismo $\left(j_{V_{h(y)}}\right)_{*}$. Assim sendo, consideremos então a cadeia de isomorfismos representada a seguir: 


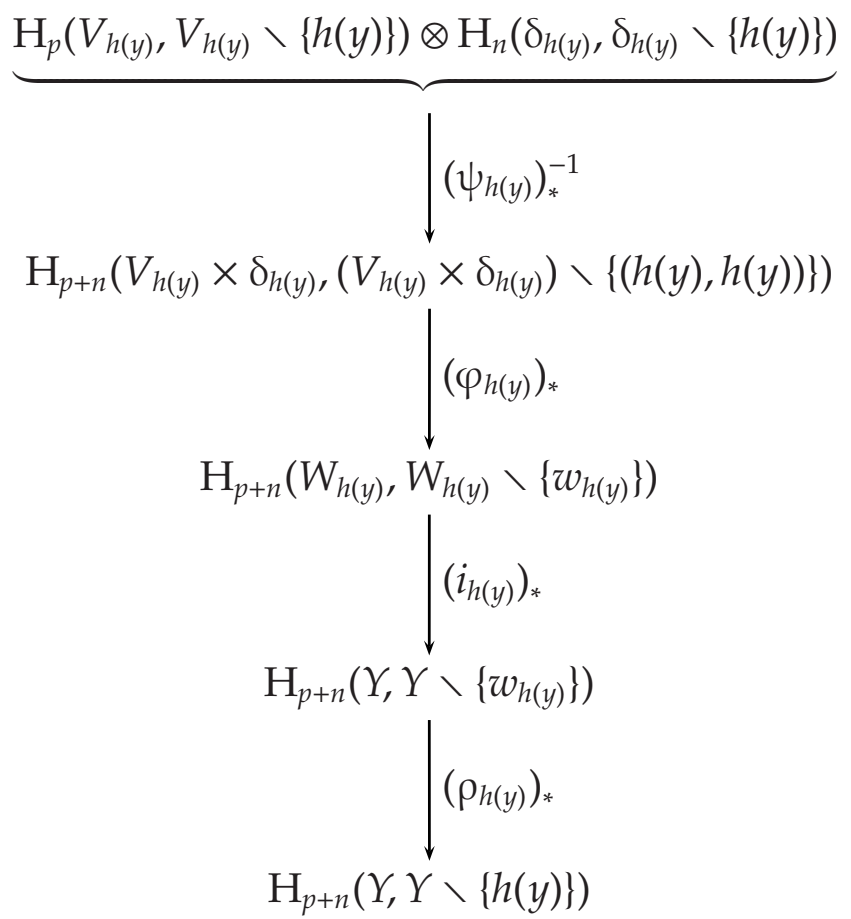

onde:

- $\left(\psi_{h(y)}\right)_{*}: \mathrm{H}_{p+n}\left(V_{h(y)} \times \delta_{h(y)},\left(V_{h(y)} \times \delta_{h(y)}\right) \backslash\{(h(y), h(y))\}\right) \longrightarrow \mathrm{H}_{p}\left(V_{h(y)}, V_{h(y)} \backslash\{h(y)\}\right) \otimes$ $\mathrm{H}_{n}\left(\delta_{h(y)}, \delta_{h(y)} \backslash\{h(y)\}\right)$ é o isomorfismo obtido pelo teorema de Künneth aplicado ao produto $V_{h(y)} \times \delta_{h(y)}$;

- $\left(\varphi_{h(y)}\right)_{*}: \mathrm{H}_{p+n}\left(V_{h(y)} \times \delta_{h(y)},\left(V_{h(y)} \times \delta_{h(y)}\right) \backslash\{(h(y), h(y))\}\right) \longrightarrow \mathrm{H}_{p+n}\left(W_{h(y)}, W_{h(y)} \backslash\left\{w_{h(y)}\right\}\right)$ éo isomorfismo induzido pelo homeomorfismo $\varphi_{h(y)}: V_{h(y)} \times \delta_{h(y)} \longrightarrow W_{h(y)}=r_{\delta}^{-1}\left(V_{h(y)}\right)$, $\operatorname{com} w_{h(y)} \in W_{h(y)}$ tal que $\varphi_{h(y)}(h(y), h(y))=w_{h(y)} ;$

- $\left(i_{h(y)}\right)_{*}: \mathrm{H}_{p+n}\left(W_{h(y)}, W_{h(y)} \backslash\left\{w_{h(y)}\right\}\right) \longrightarrow \mathrm{H}_{p+n}\left(Y, Y \backslash\left\{w_{h(y)}\right\}\right)$ é o isomorfismo induzido pela inclusão de $W_{h(y)}$ em $Y$;

- $\left(\rho_{h(y)}\right)_{*}: \mathrm{H}_{p+n}\left(Y, Y \backslash\left\{w_{h(y)}\right\}\right) \longrightarrow \mathrm{H}_{p+n}(Y, Y \backslash\{h(y)\})$ é o isomorfismo induzido por um homeomorfismo $\rho_{h(y)}: Y \longrightarrow Y$, homotópico à identidade, que leva $w_{h(y)}$ em $h(y)$ (cuja existência é garantida pelo lema 6.4, página 150, de [Vi]).

Deste modo, definimos a translação da orientação local $h_{*}(\mathcal{O}(x)) \in \mathrm{H}_{n}\left(\delta_{h(x)}, \delta_{h(x)} \backslash\{h(x)\}\right)$, de $h(x)$ a $h(y)$ ao longo do caminho $\lambda: I \longrightarrow P$, como a orientação local $\lambda_{*}\left[h_{*}(\mathcal{O}(x))\right] \in$ $\mathrm{H}_{n}\left(\delta_{h(y)}, \delta_{h(y)} \backslash\{h(y)\}\right)$, da fibra $\delta_{h(y)}$ no ponto $h(y)=\lambda(1)$, tal que o gerador $\mu_{h(y)} \otimes$ $\lambda_{*}\left[h_{*}(\mathcal{O}(x))\right] \in \mathrm{H}_{p}\left(V_{h(y)}, V_{h(y)} \backslash\{h(y)\}\right) \otimes \mathrm{H}_{n}\left(\delta_{h(y)}, \delta_{h(y)} \backslash\{h(y)\}\right)$ corresponde a $(h \circ \omega)_{*}\left(v_{h(x)}\right) \in$ $\mathrm{H}_{p+n}(Y, Y \backslash\{h(y)\})$ pelos isomorfismos mencionados acima.

Observe que $\lambda_{*}\left[h_{*}(\mathcal{O}(x))\right]$ não depende da orientação local $\mu_{h(x)} \in \mathrm{H}_{p}\left(V_{h(x)}, V_{h(x)} \backslash\right.$ $\{h(x)\})$ escolhida inicialmente: de fato, repetindo o mesmo procedimento com o outro 
gerador $-\mu_{h(x)} \in \mathrm{H}_{p}\left(V_{h(x)}, V_{h(x)} \backslash\{h(x)\}\right) \simeq \mathbb{Z}$, obteríamos a orientação local $-v_{h(x)} \in$ $\mathrm{H}_{p+n}(Y, Y \backslash\{h(x)\})$, oposta à $v_{h(x)}$, pois (pelas propriedades do produto tensorial) $\mathrm{o}$ gerador $\left(-\mu_{h(x)}\right) \otimes h_{*}(\mathcal{O}(x)) \in \mathrm{H}_{p}\left(V_{h(x)}, V_{h(x)} \backslash\{h(x)\}\right) \otimes \mathrm{H}_{n}\left(\delta_{h(x)}, \delta_{h(x)} \backslash\{h(x)\}\right) \simeq \mathbb{Z} \otimes \mathbb{Z}$ coincide com o gerador $-\left(\mu_{h(x)} \otimes h_{*}(\mathcal{O}(x))\right)$, oposto à $\mu_{h(x)} \otimes h_{*}(\mathcal{O}(x))$, e, conseqüentemente, ao aplicarmos o isomorfismo $\left(\rho_{h(x)}\right)_{*} \circ\left(i_{h(x)}\right)_{*} \circ\left(\varphi_{h(x)}\right)_{*} \circ\left(\psi_{h(x)}\right)_{*}^{-1}$ sobre $\left(-\mu_{h(x)}\right) \otimes h_{*}(O(x))$, obtemos a orientação local $-v_{h(x)} \in \mathrm{H}_{p+n}(Y, Y \backslash\{h(x)\})$, uma vez que:

$$
\left[\left(\rho_{h(x)}\right)_{*} \circ\left(i_{h(x)}\right)_{*} \circ\left(\varphi_{h(x)}\right)_{*} \circ\left(\psi_{h(x)}\right)_{*}^{-1}\right]\left(\mu_{h(x)} \otimes h_{*}(\mathcal{O}(x))\right)=v_{h(x)}
$$

Daí, estendendo a orientação $-v_{h(x)} \in \mathrm{H}_{p+n}(Y, Y \backslash\{h(x)\})$ ao longo do caminho $h \circ \omega: I \longrightarrow$ $Y$, chegaríamos à orientação local $-(h \circ \omega)_{*}\left(v_{h(x)}\right) \in \mathrm{H}_{p+n}(Y, Y \backslash\{h(y)\})$, oposta à orientação $(h \circ \omega)_{*}\left(v_{h(x)}\right)$ que obtivemos ao estender $v_{h(x)}$; note que, ao estender $-v_{h(x)}$ ao longo de $h \circ \omega$, não podemos chegar à mesma orientação $(h \circ \omega)_{*}\left(v_{h(x)}\right)$ estendida de $v_{h(x)}$, pois, neste caso, existiriam dois levantamentos distintos de $(h \circ \omega)^{-1}$, relativamente ao recobrimento $\tilde{p}_{Y}: \tilde{C}_{Y} \longrightarrow Y$, com início no mesmo ponto $(h \circ \omega)_{*}\left(v_{h(x)}\right)$ (um deles terminando em $v_{h(x)}$, e o outro em $\left.-v_{h(x)}\right)$. Analogamente, sendo $-\mu_{h(x)}^{P} \in \mathrm{H}_{n}(P, P \backslash\{h(x)\})$ a orientação local de $P$ em $h(x)$, tal que $\left(j_{V_{h(x)}}\right)_{*}\left(-\mu_{h(x)}\right)=-\mu_{h(x)}^{P}$ (onde $\left(j_{V_{h(x)}}\right)_{*}: \mathrm{H}_{p}\left(V_{h(x)}, V_{h(x)} \backslash\{h(x)\}\right) \longrightarrow \mathrm{H}_{p}(P, P \backslash$ $\{h(x)\})$ é o isomorfismo induzido pela inclusão), segue que, ao estendermos $-\mu_{h(x)}^{P}$ ao longo do caminho $\lambda: I \longrightarrow P$, obteremos a orientação local $-\lambda_{*}\left(\mu_{h(x)}^{P}\right) \in \mathrm{H}_{p}(P, P \backslash\{h(y)\})$, oposta àquela a que chegamos ao estender $\mu_{h(x)}^{P}$, e esta, por sua vez, determinará a orientação local $-\mu_{h(y)} \in \mathrm{H}_{p}\left(V_{h(y)}, V_{h(y)} \backslash\{h(y)\}\right)$, oposta à $\mu_{h(y)}$, pelo isomorfismo $\left(j_{V_{h(y)}}\right)_{*}: \mathrm{H}_{p}\left(V_{h(y)}, V_{h(y)} \backslash\{h(y)\}\right) \longrightarrow \mathrm{H}_{p}(P, P \backslash\{h(y)\})$ induzido pela inclusão. Logo, a orientação local $\zeta_{h(y)} \in \mathrm{H}_{n}\left(\delta_{h(y)}, \delta_{h(y)} \backslash\{h(y)\}\right)$, tal que $\left(-\mu_{h(y)}\right) \otimes \zeta_{h(y)} \in \mathrm{H}_{p}\left(V_{h(y)}, V_{h(y)} \backslash\right.$ $\{h(y)\}) \otimes \mathrm{H}_{n}\left(\delta_{h(y)}, \delta_{h(y)} \backslash\{h(y)\}\right)$, correspondente a $-(h \circ \omega)_{*}\left(v_{h(x)}\right) \in \mathrm{H}_{p+n}(Y, Y \backslash\{h(y)\})$ pelos isomorfismos ilustrados no último diagrama acima, deve coincidir com $\lambda_{*}\left[h_{*}(\mathcal{O}(x))\right]$, uma vez que estes isomorfismos levam $\left(-\mu_{h(y)}\right) \otimes \lambda_{*}\left[h_{*}(\mathcal{O}(x))\right]=-\left(\mu_{h(y)} \otimes \lambda_{*}\left[h_{*}(\mathcal{O}(x))\right]\right)$ em $-(h \circ \omega)_{*}\left(v_{h(x)}\right)$. Portanto, $\lambda_{*}\left[h_{*}(\mathcal{O}(x))\right]$ é, de fato, independente da escolha da orientação $\mu_{h(x)} \in \mathrm{H}_{p}\left(V_{h(x)}, V_{h(x)} \backslash\{h(x)\}\right)$, conforme queríamos provar.

Resumindo, temos então que, adotada uma orientação local $\mathcal{O}(x) \in \mathrm{H}_{n}(X, X \backslash\{x\})$, obtemos orientações locais $\omega_{*}(\mathcal{O}(x)) \in \mathrm{H}_{n}(X, X \backslash\{y\}), h_{*}(\mathcal{O}(x)) \in \mathrm{H}_{n}\left(\delta_{h(x)}, \delta_{h(x)} \backslash\{h(x)\}\right)$ e $\lambda_{*}\left[h_{*}(\mathcal{O}(x))\right] \in \mathrm{H}_{n}\left(\delta_{h(y)}, \delta_{h(y)} \backslash\{h(y)\}\right)$, a primeira estendendo-se $\mathcal{O}(x)$ ao longo do caminho $\omega: I \longrightarrow X$, a segunda pela transversalidade topológica da aplicação $h: X \longrightarrow Y$ relativamente ao microfibrado $\delta=\delta^{n}=\left(Y, P, r_{\delta}\right)$, e a terceira pela translação de $h_{*}(\mathcal{O}(x))$ ao longo do caminho $\lambda: I \longrightarrow P$. Entretanto, utilizando novamente a transversalidade topológica de $h: X \longrightarrow Y$, sabemos que existem vizinhanças abertas $U_{y} \subseteq \xi_{y}$ e $U_{h(y)} \subseteq \delta_{h(y)}$, de $y$ e $h(y)$, respectivamente, tais que a restrição $\left.h\right|_{U_{y}}: U_{y} \longrightarrow U_{h(y)}$ é um homeomorfismo sobre $U_{h(y)}$; assim, denotando por $\left(j_{y}\right)_{*}: \mathrm{H}_{n}\left(\xi_{y}, \xi_{y} \backslash\{y\}\right) \longrightarrow \mathrm{H}_{n}(X, X \backslash\{y\})$, $\left(j_{\xi_{y}}\right)_{*}: \mathrm{H}_{n}\left(U_{y}, U_{y} \backslash\{y\}\right) \longrightarrow \mathrm{H}_{n}\left(\xi_{y}, \xi_{y} \backslash\{y\}\right)$ e $\left(j_{\delta_{h(y)}}\right)_{*}: \mathrm{H}_{n}\left(U_{h(y)}, U_{h(y)} \backslash\{h(y)\}\right) \longrightarrow$ $\mathrm{H}_{n}\left(\delta_{h(y)}, \delta_{h(y)} \backslash\{h(y)\}\right)$ os isomorfismos induzidos pelas respectivas inclusões, segue 
que a orientação local $\omega_{*}(\mathcal{O}(x)) \in \mathrm{H}_{n}(X, X \backslash\{y\})$, obtida estendendo-se $\mathcal{O}(x)$ ao longo de $\omega$, corresponde a uma orientação local $h_{*}\left[\omega_{*}(\mathcal{O}(x))\right] \in \mathrm{H}_{n}\left(\delta_{h(y)}, \delta_{h(y)} \backslash\{h(y)\}\right)$, da fibra $\delta_{h(y)}$ no ponto $h(y) \in P$, pela seguinte cadeia de isomorfismos:

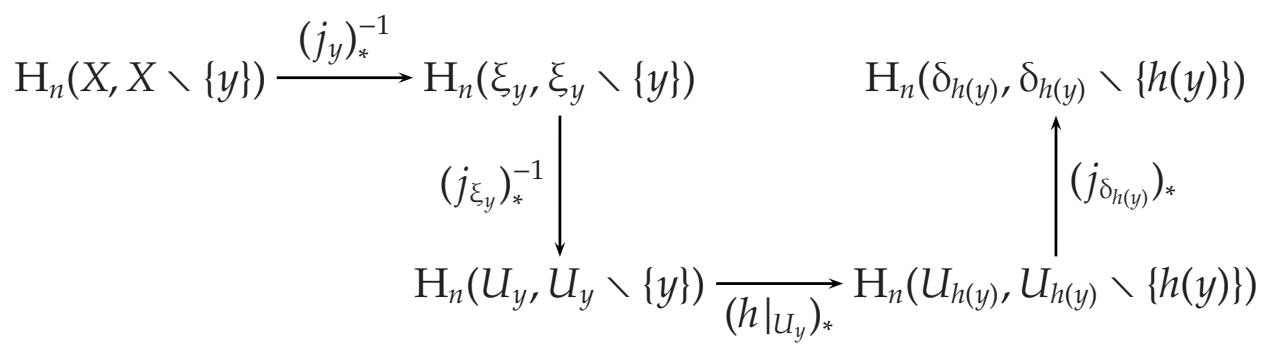

(note que, por serem respectivamente abertos em $\xi_{y}$ e $\delta_{h(y)}, U_{y}$ e $U_{h(y)}$ constituem variedades topológicas de dimensão $n)$. Deste modo, como tanto $\lambda_{*}\left[h_{*}(\mathcal{O}(x))\right]$, quanto $h_{*}\left[\omega_{*}(\mathcal{O}(x))\right]$ são geradores de $\mathrm{H}_{n}\left(\delta_{h(y)}, \delta_{h(y)} \backslash\{h(y)\}\right) \simeq \mathbb{Z}$, temos duas possibilidades: $\lambda_{*}\left[h_{*}(\mathcal{O}(x))\right]=h_{*}\left[\omega_{*}(\mathcal{O}(x))\right]$ ou $\lambda_{*}\left[h_{*}(\mathcal{O}(x))\right]=-h_{*}\left[\omega_{*}(\mathcal{O}(x))\right]$, onde, evidentemente, estamos denotando por $-h_{*}\left[\omega_{*}(\mathcal{O}(x))\right]$ o gerador de $\mathrm{H}_{n}\left(\delta_{h(y)}, \delta_{h(y)} \backslash\{h(y)\}\right) \simeq \mathbb{Z}$ que é distinto de $h_{*}\left[\omega_{*}(\mathcal{O}(x))\right]$. Por conseguinte, isto nos leva à seguinte

Definição 2.4.4 . Sejam $X, Y$ e $P \subseteq Y$ variedades topológicas conexas, sem fronteira, de dimensões respectivamente iguais a $n, m$ e $p, \operatorname{com} n+p=m$ e $p<m$. Considere ainda um microfibrado normal $\delta=\delta^{n}=\left(Y, P, r_{\delta}\right)$, de dimensão igual à de $X$, e suponha que $h: X \longrightarrow Y$ seja uma aplicação contínua topologicamente transversa a $\delta$. Utilizando as mesmas notações adotadas acima, dizemos que dois pontos $x, y \in h^{-1}(P) \subseteq X$ estão $\mathcal{R}$-relacionados com respeito à $h$, e escrevemos $x \stackrel{h}{\sim} y$, se existe um caminho $\omega: I \longrightarrow X$, Nielsen-relacionando $x$ e $y$, tal que, para qualquer que seja a orientação local $\mathcal{O}(x) \in \mathrm{H}_{n}(X, X \backslash\{x\})$ escolhida, temos

$$
\lambda_{*}\left[h_{*}(\mathcal{O}(x))\right]=-h_{*}\left[\omega_{*}(\mathcal{O}(x))\right],
$$

onde $\lambda: I \longrightarrow P$ é um caminho homotópico a $h \circ \omega: I \longrightarrow Y, \lambda_{*}\left[h_{*}(\mathcal{O}(x))\right]$ é a translação da orientação local $h_{*}(\mathcal{O}(x)) \in \mathrm{H}_{n}\left(\delta_{h(x)}, \delta_{h(x)} \backslash\{h(x)\}\right)$ ao longo de $\lambda$, e $\omega_{*}(\mathcal{O}(x))$ é a orientação local de X em y obtida estendendo-se $\mathcal{O}(x)$ ao longo de $\omega$.

A definição de $\mathcal{R}$-relação nos permite então caracterizar os pontos de $h^{-1}(P)$ que podem ser "removidos" por uma homotopia; mais especificamente, temos o seguinte

Lema 2.4.2. Sejam $X, Y$ e $P \subseteq Y$ variedades topológicas conexas e sem fronteira, cujas dimensões $n$, m e $p$, respectivamente, satisfazem $n+p=m, n \geqslant 2$ e $2 \leqslant p<m$, e considere um microfibrado normal $\delta=\delta^{n}=\left(Y, P, r_{\delta}\right)$, de dimensão igual à de $X$. Suponha ainda que $B \subseteq X$ seja uma n-bola própria em $X$, e que $h: \bar{B} \longrightarrow Y$ é uma aplicação contínua topologicamente transversa a $\delta$ (onde o fecho de $B$ é relativo à variedade $X$ ). Se $h^{-1}(P)=\left\{x_{0}, x_{1}\right\} \subseteq B$ e $x_{0} \stackrel{h}{\sim} x_{1}$, 
segue que hé homotópica a uma aplicação contínua $g: \bar{B} \longrightarrow Y \backslash P \subseteq Y$, relativamente a $\partial B=\bar{B} \backslash B$ (isto é, existe uma aplicação contínua $g: \bar{B} \longrightarrow Y \backslash P$, homotópica à $h$ por uma homotopia $H: \bar{B} \times I \longrightarrow Y$ tal que $H(x, t)=h(x)=g(x)$, para todo $(x, t) \in \partial B \times I)$.

Por razões históricas, resultados como o do lema acima (que fornecem condições sob as quais podemos "remover" pontos da imagem de uma aplicação contínua, por meio de homotopias) são conhecidos como do tipo Whitney; sua demonstração pode ser encontrada em [Je] (lema 3.1, páginas 175 e 176), e será omitida aqui devido à tecnicidade envolvida.

A seguir, veremos então como interpretar o conceito de $\mathcal{R}$-relação para aplicações do tipo $h: \operatorname{Int} M \longrightarrow \operatorname{Int} M \times \operatorname{Int} N$, onde $P=\operatorname{Int} M \times\{c\}$ e $c \in \operatorname{Int} N$. Primeiramente, no entanto, convém adotarmos mais uma notação: dada uma aplicação contínua própria $\mathcal{F}:(M, \partial M, \operatorname{Int} M) \longrightarrow(N, \partial N$, Int $N)$, entre variedades topológicas conexas de dimensão $n$, tal que $\mathcal{F}$ é transversa a um ponto $c \in \operatorname{Int} N$, e sendo $x \in \operatorname{Int} M$ uma raiz de $\mathcal{F}$ em $c$, sabemos que existem vizinhanças abertas $U_{x} \subseteq \operatorname{Int} M$ e $V_{c} \subseteq \operatorname{Int} N$, de $x$ e $c$, respectivamente, tais que a restrição $\left.\mathcal{F}\right|_{U_{x}}: U_{x} \longrightarrow V_{c}$ é um homeomorfismo sobre $V_{c}$; logo, denotando por $\left(j_{U_{x}}\right)_{*}: \mathrm{H}_{n}\left(U_{x}, U_{x} \backslash\{x\}\right) \longrightarrow \mathrm{H}_{n}(\operatorname{Int} M, \operatorname{Int} M \backslash\{x\})$ e $\left(j_{V_{c}}\right)_{*}$ : $\mathrm{H}_{n}\left(V_{c}, V_{c} \backslash\{c\}\right) \longrightarrow \mathrm{H}_{n}(\operatorname{Int} N$, Int $N \backslash\{c\})$ os isomorfismos induzidos pelas respectivas inclusões, temos que, dada uma orientação local $\mathcal{O}(x) \in \mathrm{H}_{n}(\operatorname{Int} M$, Int $M \backslash\{x\})$, existe uma única orientação local de $N$ em $c$, correspondente a $\mathcal{O}(x)$ pela seguinte cadeia de isomorfismos:

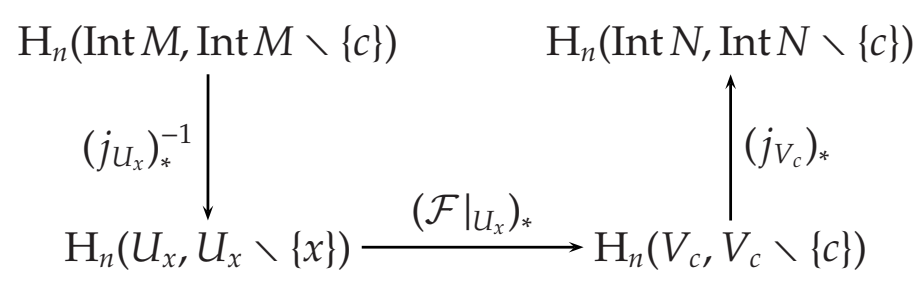

Assim, utilizando um abuso de notação, vamos representar esta orientação local de $N$ em $c$ por $\mathcal{F}_{*}(\mathcal{O}(x))$, isto é,

$$
\mathcal{F}_{*}(\mathcal{O}(x))=\left[\left(j_{V_{c}}\right)_{*} \circ\left(\left.\mathcal{F}\right|_{U_{x}}\right)_{*} \circ\left(j_{U_{x}}\right)_{*}^{-1}\right](\mathcal{O}(x)) .
$$

Lema 2.4.3 . Seja $\mathcal{F}:(M, \partial M, \operatorname{Int} M) \longrightarrow(N, \partial N, \operatorname{Int} N)$ uma aplicação contínua própria (entre variedades topológicas conexas de mesma dimensão $n$ ), e considere novamente $X=\operatorname{Int} M$, $Y=\operatorname{Int} M \times \operatorname{Int} N$ e $P=\operatorname{Int} M \times\{c\}$, onde $c \in \operatorname{Int} N$. Suponha ainda que e $: \operatorname{Int} M \longrightarrow \operatorname{Int} M$ seja uma aplicação contínua, tal que a aplicação $h=\left(e, \mathcal{F}^{0}\right): X=\operatorname{Int} M \longrightarrow Y=\operatorname{Int} M \times \operatorname{Int} N$ é topologicamente transversa ao microfibrado normal $\eta=\eta^{n}=(Y, P, r)$, onde $r: Y \longrightarrow P$ é a retração definida por $r(x, y)=(x, c)$ para cada $(x, y) \in Y=\operatorname{Int} M \times \operatorname{Int} N$. Dados $x_{1}, x_{2} \in h^{-1}(P)$, 
e um caminho $\omega: I \longrightarrow X=\operatorname{Int} M, \operatorname{com} \omega(0)=x_{1}$ e $\omega(1)=x_{2}$, tal que $\mathcal{F} \circ \omega: I \longrightarrow \operatorname{Int} N$ é um laço contrátil em $c$, temos que $x_{1}$ e $x_{2}$ estão $\mathcal{R}$-relacionados com respeito à $h$ se, e somente se, $\mathcal{F}_{*}\left(\mathcal{O}\left(x_{1}\right)\right)=-\mathcal{F}_{*}\left[\omega_{*}\left(\mathcal{O}\left(x_{1}\right)\right)\right]$, onde $\mathcal{O}\left(x_{1}\right) \in \mathrm{H}_{n}\left(X, X \backslash\left\{x_{1}\right\}\right)$ é uma orientação local arbitrária de $X$ em $x_{1}$, e $\omega_{*}\left(\mathcal{O}\left(x_{1}\right)\right) \in \mathrm{H}_{n}\left(X, X \backslash\left\{x_{2}\right\}\right)$ é a orientação local de X em $x_{2}$ obtida estendendo-se $\mathcal{O}\left(x_{1}\right)$ ao longo do caminho $\omega$.

DemonstraçÃo. De fato, sabemos que $h^{-1}(P)=\mathcal{F}^{-1}(c)$; além disso, a existência do caminho $\omega: I \longrightarrow X=\operatorname{Int} M$, ligando $x_{1}$ a $x_{2}$, tal que $\mathcal{F} \circ \omega: I \longrightarrow$ Int $N$ é uma laço contrátil em $c$, significa que $x_{1}$ e $x_{2}$ pertencem à mesma classe de raízes de $\mathcal{F}$ em $c$, o que, pelo lema 2.4.1, implica que $x_{1}$ e $x_{2}$ estão Nielsen-relacionados (condição necessária para que tenhamos $x_{1} \stackrel{h}{\sim} x_{2}$ ). Por outro lado, conforme vimos na demonstração da proposição 2.4.1, a transversalidade topológica de $h=\left(e, \mathcal{F}^{0}\right): X \longrightarrow Y$, em relação ao microfibrado normal $\eta=\eta^{n}=(Y, P, r)$, nos garante que existem vizinhanças abertas $U_{x_{1}}, U_{x_{2}} \subseteq X=\operatorname{Int} M$ e $V_{c} \subseteq \operatorname{Int} N$, de $x_{1}, x_{2}$ e $c$, respectivamente, tais que a aplicação $e: X \longrightarrow X$ satifaz $e\left(z_{1}\right)=e\left(x_{1}\right)$ e $e\left(z_{2}\right)=e\left(x_{2}\right)$ para quaisquer $z_{1} \in U_{x_{1}}$ e $z_{2} \in U_{x_{2}}$, e as restrições $\left.\mathcal{F}\right|_{U_{x_{1}}}: U_{x_{1}} \longrightarrow V_{c}$ e $\left.\mathcal{F}\right|_{U_{x_{2}}}: U_{x_{2}} \longrightarrow V_{c}$ são homeomorfismos sobre $V_{c}$. Assim, como $\eta_{h(x)}=\{e(x)\} \times \operatorname{Int} N$ para todo $x \in h^{-1}(P)$, segue que $W_{x_{1}}=\left\{e\left(x_{1}\right)\right\} \times V_{c} \subseteq \eta_{h\left(x_{1}\right)}$ e $W_{x_{2}}=\left\{e\left(x_{2}\right)\right\} \times V_{c} \subseteq \eta_{h\left(x_{2}\right)}$ são vizinhanças abertas dos pontos $h\left(x_{1}\right)=\left(e\left(x_{1}\right), c\right) \mathrm{e}$ $h\left(x_{2}\right)=\left(e\left(x_{2}\right), c\right)$, respectivamente, tais que as restrições $\left.h\right|_{U_{x_{1}}}: U_{x_{1}} \longrightarrow W_{x_{1}}$ e $\left.h\right|_{U_{x_{2}}}$ : $U_{x_{2}} \longrightarrow W_{x_{2}}$ são homeomorfismos sobre $W_{x_{1}}$ e $W_{x_{2}}$ (note que todos os abertos $U_{x_{1}}$, $U_{x_{2}}, V_{c}, W_{x_{1}}$ e $W_{x_{2}}$ são variedades topológicas de dimensão $\left.n\right)$. Agora, considere as aplicações $\pi_{x_{1}}: \eta_{h\left(x_{1}\right)} \longrightarrow \operatorname{Int} N$ e $\pi_{x_{2}}: \eta_{h\left(x_{2}\right)} \longrightarrow \operatorname{Int} N$, definidas por $\pi_{x_{1}}\left(e\left(x_{1}\right), y_{1}\right)=y_{1}$ e $\pi_{x_{2}}\left(e\left(x_{2}\right), y_{2}\right)=y_{2}$ para cada $\left(e\left(x_{1}\right), y_{1}\right) \in \eta_{h\left(x_{1}\right)}=\left\{e\left(x_{1}\right)\right\} \times \operatorname{Int} N$ e cada $\left(e\left(x_{2}\right), y_{2}\right) \in \eta_{h\left(x_{2}\right)}=$ $\left\{e\left(x_{2}\right)\right\} \times \operatorname{Int} N$ (em outras palavras, $\pi_{x_{1}}$ e $\pi_{x_{2}}$ são as projeções de $\eta_{h\left(x_{1}\right)}=\left\{e\left(x_{1}\right)\right\} \times \operatorname{Int} N$ e $\eta_{h\left(x_{2}\right)}=\left\{e\left(x_{2}\right)\right\} \times \operatorname{Int} N$ sobre Int $\left.N\right)$; denotando por $j_{h\left(x_{1}\right)}: W_{x_{1}} \longrightarrow \eta_{h\left(x_{1}\right)}, j_{h\left(x_{2}\right)}: W_{x_{2}} \longrightarrow$ $\eta_{h\left(x_{2}\right), j_{U_{1}}}: U_{x_{1}} \longrightarrow X, j_{U_{x_{2}}}: U_{x_{2}} \longrightarrow X$ e $j_{V_{c}}: V_{c} \longrightarrow$ Int $N$ as inclusões, obtemos o diagrama comutativo ilustrado na figura 2.6. Logo, sendo $\mathcal{O}\left(x_{1}\right) \in \mathrm{H}_{n}\left(X, X \backslash\left\{x_{1}\right\}\right)$ uma orientação local arbitrária de $X$ em $x_{1}$, e $\omega_{*}\left(\mathcal{O}\left(x_{1}\right)\right) \in \mathrm{H}_{n}\left(X, X \backslash\left\{x_{2}\right\}\right)$ a orientação local obtida estendendo-se $\mathcal{O}\left(x_{1}\right)$ ao longo do caminho $\omega: I \longrightarrow X$, consideremos as orientações locais $h_{*}\left(\mathcal{O}\left(x_{1}\right)\right) \in \mathrm{H}_{n}\left(\eta_{h\left(x_{1}\right)}, \eta_{h\left(x_{1}\right)} \backslash\left\{h\left(x_{1}\right)\right\}\right), h_{*}\left[\omega_{*}\left(\mathcal{O}\left(x_{1}\right)\right)\right] \in \mathrm{H}_{n}\left(\eta_{h\left(x_{2}\right)}, \eta_{h\left(x_{2}\right)} \backslash\right.$ $\left.\left\{h\left(x_{2}\right)\right\}\right), \mathcal{F}_{*}\left(\mathcal{O}\left(x_{1}\right)\right) \in \mathrm{H}_{n}(\operatorname{Int} N, \operatorname{Int} N \backslash\{c\})$ e $\mathcal{F}_{*}\left[\omega_{*}\left(\mathcal{O}\left(x_{1}\right)\right)\right] \in \mathrm{H}_{n}(\operatorname{Int} N, \operatorname{Int} N \backslash\{c\})$, que, de acordo com as notações que estamos adotando, são definidas por:

$$
\begin{aligned}
h_{*}\left(\mathcal{O}\left(x_{1}\right)\right) & =\left[\left(j_{h\left(x_{1}\right)}\right)_{*} \circ\left(\left.h\right|_{U_{x_{1}}}\right)_{*} \circ\left(j{U_{x_{1}}}_{*}\right)_{*}^{-1}\right]\left(\mathcal{O}\left(x_{1}\right)\right), \\
h_{*}\left(\omega_{*}\left(\mathcal{O}\left(x_{1}\right)\right)\right) & =\left[\left(j_{h\left(x_{2}\right)}\right)_{*} \circ\left(\left.h\right|_{U_{x_{2}}}\right)_{*} \circ\left(j_{U_{x_{2}}}\right)_{*}^{-1}\right]\left(\omega_{*}\left(\mathcal{O}\left(x_{1}\right)\right)\right), \\
\mathcal{F}_{*}\left(\mathcal{O}\left(x_{1}\right)\right) & =\left[\left(j_{V_{c}}\right)_{*} \circ\left(\left.\mathcal{F}\right|_{U_{x_{1}}}\right)_{*} \circ\left(j_{U_{x_{1}}}\right)_{*}^{-1}\right]\left(\mathcal{O}\left(x_{1}\right)\right) \mathrm{e} \\
\mathcal{F}_{*}\left(\omega_{*}\left(\mathcal{O}\left(x_{1}\right)\right)\right) & =\left[\left(j_{V_{c}}\right)_{*} \circ\left(\left.\mathcal{F}\right|_{U_{x_{2}}}\right)_{*} \circ\left(j_{U_{x_{2}}}\right)_{*}^{-1}\right]\left(\omega_{*}\left(\mathcal{O}\left(x_{1}\right)\right)\right) .
\end{aligned}
$$




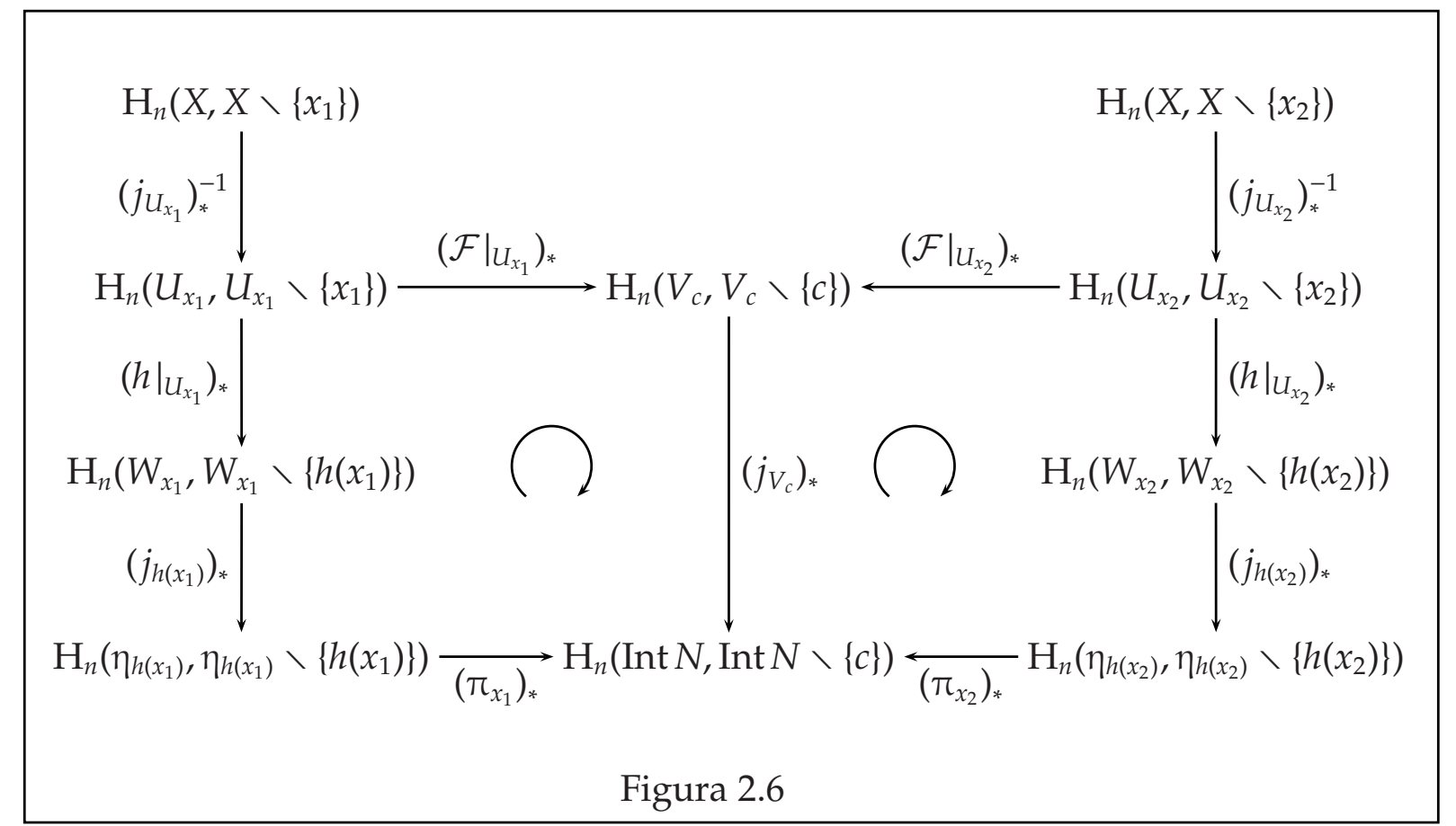

De maneira análoga ao que fizemos na discussão que precedeu a definição 2.4.5, podemos utilizar o teorema de Künneth em homologia singular para concluir que existem isomorfismos

$$
\left(\psi_{h\left(x_{1}\right)}^{Y}\right)_{*}: \mathrm{H}_{2 n}\left(Y, Y \backslash\left\{h\left(x_{1}\right)\right\}\right) \longrightarrow \mathrm{H}_{n}\left(X, X \backslash\left\{e\left(x_{1}\right)\right\}\right) \otimes \mathrm{H}_{n}(\operatorname{Int} N, \operatorname{Int} N \backslash\{c\})
$$

e

$$
\begin{gathered}
\mathrm{H}_{2 n}\left(P \times \eta_{h\left(x_{1}\right)},\left(P \times \eta_{h\left(x_{1}\right)}\right) \backslash\left\{\left(h\left(x_{1}\right), h\left(x_{1}\right)\right)\right\}\right) \\
\left(\psi_{\left.h\left(x_{1}\right)\right)}\right)_{*} \\
\left.\mathrm{H}_{n}\left(P, P \backslash\left\{h\left(x_{1}\right)\right\}\right) \otimes \mathrm{H}_{n}\left(\eta_{h\left(x_{1}\right)}\right), \eta_{h\left(x_{1}\right)} \backslash\left\{h\left(x_{1}\right)\right\}\right)
\end{gathered}
$$

(lembre-se que $h\left(x_{1}\right)=\left(e\left(x_{1}\right), c\right)$ é tal que $h\left(x_{1}\right) \in P=\operatorname{Int} M \times\{c\}$ e $h\left(x_{1}\right) \in \eta_{h\left(x_{1}\right)}=$ $\left.\left\{e\left(x_{1}\right)\right\} \times \operatorname{Int} N\right)$. Por outro lado, note que a aplicação $\varphi_{x_{1}}: P \times \eta_{h\left(x_{1}\right)} \longrightarrow Y$, dada por $\varphi_{x_{1}}\left(x, c, e\left(x_{1}\right), y\right)=(x, y)$ para cada $\left(x, c, e\left(x_{1}\right), y\right) \in P \times \eta_{h\left(x_{1}\right)}=(\operatorname{Int} M \times\{c\}) \times\left(\left\{e\left(x_{1}\right)\right\} \times \operatorname{Int} N\right)$, constitui um homeomorfismo sobre $Y$, que leva o ponto $\left(h\left(x_{1}\right), h\left(x_{1}\right)\right)=\left(e\left(x_{1}\right), c, e\left(x_{1}\right), c\right) \in$ $P \times \eta_{h\left(x_{1}\right)}$ em $h\left(x_{1}\right)=\left(e\left(x_{1}\right), c\right) \in Y=\operatorname{Int} M \times \operatorname{Int} N ;$ assim, denotando por $p_{x_{1}}: P=\operatorname{Int} M \times$ $\{c\} \longrightarrow X=\operatorname{Int} M$ o homeomorfismo dado por $p_{x_{1}}(x, c)=x$ para cada $(x, c) \in P$, segue que a aplicação $p_{x_{1}} \times \pi_{x_{1}}: P \times \eta_{h\left(x_{1}\right)} \longrightarrow Y$, definida por $\left(p_{x_{1}} \times \pi_{x_{1}}\right)\left(x_{P}, x_{\eta}\right)=\left(p_{x_{1}}\left(x_{P}\right), \pi_{x_{1}}\left(x_{\eta}\right)\right)$ para cada $\left(x_{P}, x_{\eta}\right) \in P \times \eta_{h\left(x_{1}\right)}$, coincide com $\varphi_{x_{1}}$, pois, sendo $x_{P}=(x, c) \in P=\operatorname{Int} M \times\{c\}$ 
e $x_{\eta}=\left(e\left(x_{1}\right), y\right) \in \eta_{h\left(x_{1}\right)}=\left\{e\left(x_{1}\right)\right\} \times \operatorname{Int} N$, temos que:

$$
\begin{aligned}
\varphi_{x_{1}}\left(x_{P}, x_{\eta}\right) & =\varphi_{x_{1}}\left(x, c, e\left(x_{1}\right), y\right)=(x, y) \\
& =\left(p_{x_{1}}\left(x_{P}\right), \pi_{x_{1}}\left(x_{\eta}\right)\right) \\
& =\left(p_{x_{1}} \times \pi_{x_{1}}\right)\left(x_{P}, x_{\eta}\right) .
\end{aligned}
$$

Considere então o homomorfismo

$$
\begin{array}{r}
\mathrm{H}_{n}\left(P, P \backslash\left\{x_{1}\right\}\right) \otimes \mathrm{H}_{n}\left(\eta_{h\left(x_{1}\right)}, \eta_{h\left(x_{1}\right)} \backslash\left\{h\left(x_{1}\right)\right\}\right) \\
\left(p_{x_{1}}\right)_{*} \otimes\left(\pi_{x_{1}}\right)_{*} \\
\mathrm{H}_{n}\left(X, X \backslash\left\{e\left(x_{1}\right)\right\}\right) \otimes \mathrm{H}_{n}(\operatorname{Int} N, \operatorname{Int} N \backslash\{c\})
\end{array}
$$

dado por:

$$
\left[\left(p_{x_{1}}\right)_{*} \otimes\left(\pi_{x_{1}}\right)_{*}\right]\left(z_{P} \otimes z_{\eta}\right)=\left[\left(p_{x_{1}}\right)_{*}\left(z_{P}\right)\right] \otimes\left[\left(\pi_{x_{1}}\right)_{*}\left(z_{\eta}\right)\right]
$$

para cada $z_{P} \otimes z_{\eta} \in \mathrm{H}_{n}\left(P, P \backslash\left\{x_{1}\right\}\right) \otimes \mathrm{H}_{n}\left(\eta_{h\left(x_{1}\right)}, \eta_{h\left(x_{1}\right)} \backslash\left\{h\left(x_{1}\right)\right\}\right)$; como os isomorfismos $\left(\psi_{h\left(x_{1}\right)}\right)_{*}$ e $\left(\psi_{h\left(x_{1}\right)}^{Y}\right)_{*}$, dados pelo teorema de Künneth, são definidos pelo teorema de Eilenberg-Zilber (vide [Ma1], páginas 300 a 302), segue, da naturalidade das aplicações de Eilenberg-Zilber (novamente, vide [Ma1], lema 5.6, página 290), que o seguinte diagrama é comutativo:

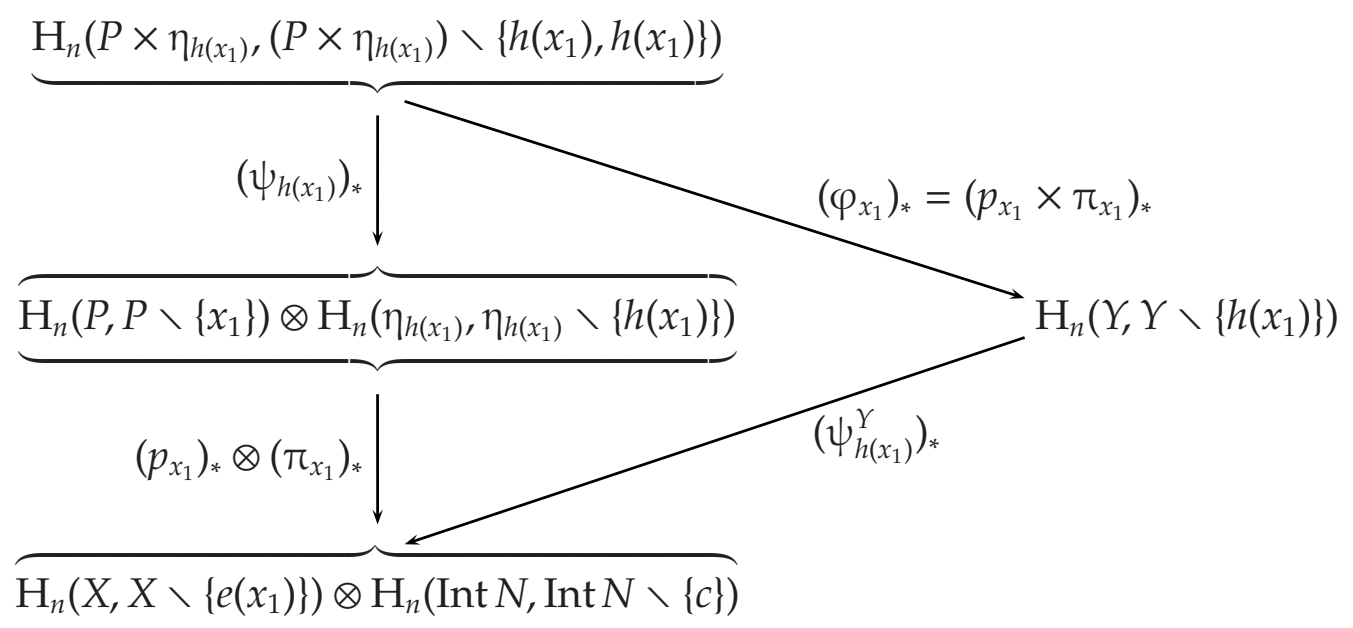

Por conseguinte, temos que $\left(\psi_{h\left(x_{1}\right)}^{Y}\right)_{*} \circ\left(\varphi_{x_{1}}\right)_{*} \circ\left(\psi_{h\left(x_{1}\right)}\right)_{*}=\left(p_{x_{1}}\right)_{*} \otimes\left(\pi_{x_{1}}\right)_{*}$, isto é,

$$
\left[\left(\psi_{h\left(x_{1}\right)}^{Y}\right)_{*} \circ\left(\varphi_{x_{1}}\right)_{*} \circ\left(\psi_{h\left(x_{1}\right)}\right)_{*}\right]\left(z_{P} \otimes z_{\eta}\right)=\left[\left(p_{x_{1}}\right)_{*}\left(z_{P}\right)\right] \otimes\left[\left(\pi_{x_{1}}\right)_{*}\left(z_{\eta}\right)\right]
$$

para cada $x_{P} \in \mathrm{H}_{n}\left(P, P \backslash\left\{h\left(x_{1}\right)\right\}\right)$ e cada $z_{\eta} \in \mathrm{H}_{n}\left(\eta_{h\left(x_{1}\right)}, \eta_{h\left(x_{1}\right)} \backslash\left\{h\left(x_{1}\right)\right\}\right)$, de modo que o diagrama abaixo é comutativo: 


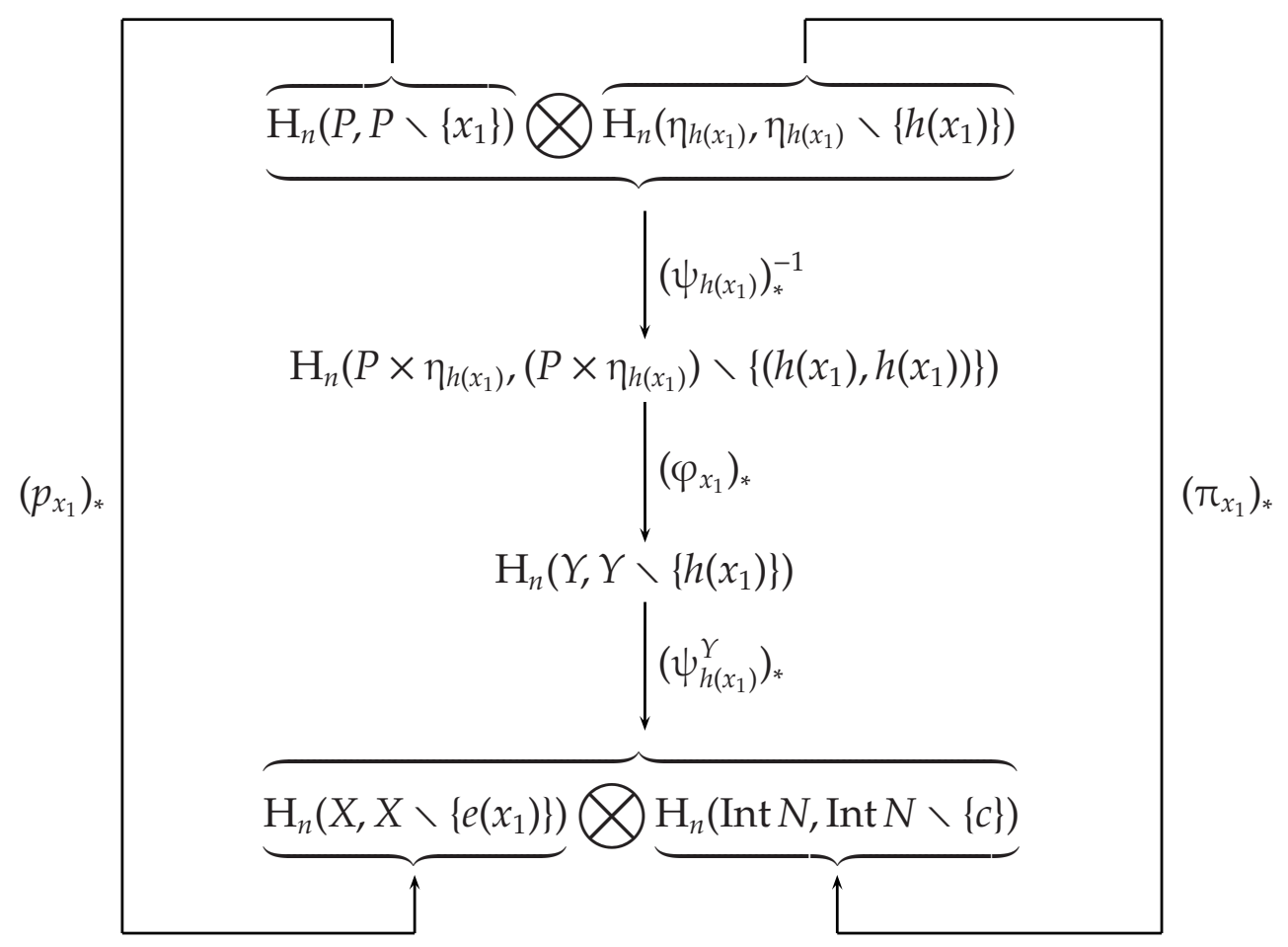

Em contrapartida, fazendo as mesmas considerações para $x_{2}$ no lugar de $x_{1}$, obtemos também o diagrama comutativo a seguir:

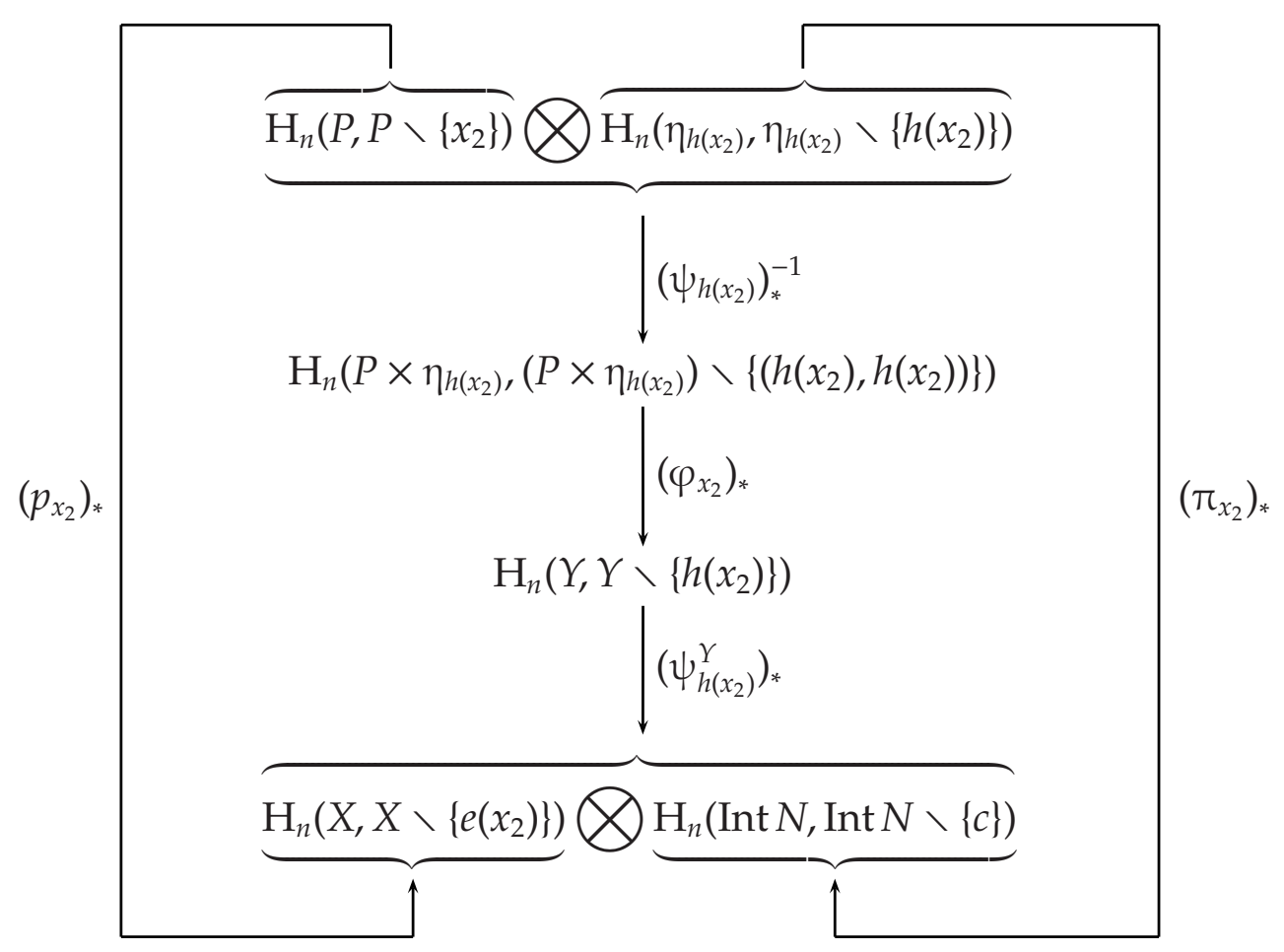


Isto posto, sendo $\lambda: I \longrightarrow P=\operatorname{Int} M \times\{c\}$ o caminho dado por $\lambda=(e \circ \omega, c)$, temos que $\lambda$ é homotópico ao caminho $h \circ \omega=(e \circ \omega, \mathcal{F} \circ \omega): I \longrightarrow Y=\operatorname{Int} M \times \operatorname{Int} N$ (uma vez que $\mathcal{F} \circ \omega: I \longrightarrow$ Int $N$ é um laço contrátil em $c)$. Lembremos então que a translação de $h_{*}\left(\mathcal{O}\left(x_{1}\right)\right) \in \mathrm{H}_{n}\left(\eta_{h\left(x_{1}\right)}, \eta_{h\left(x_{1}\right)} \backslash\left\{h\left(x_{1}\right)\right\}\right)$, de $h\left(x_{1}\right)=\left(e\left(x_{1}\right), c\right)$ a $h\left(x_{2}\right)=\left(e\left(x_{2}\right), c\right)$ ao longo de $\lambda=(e \circ \omega, c)$, é a orientação local $\lambda_{*}\left[h_{*}\left(\mathcal{O}\left(x_{1}\right)\right)\right] \in \mathrm{H}_{n}\left(\eta_{h\left(x_{2}\right)}, \eta_{h\left(x_{2}\right)} \backslash\left\{h\left(x_{2}\right)\right\}\right)$ obtida pelo seguinte procedimento: escolhendo-se uma orientação local $\mu_{h\left(x_{1}\right)} \in \mathrm{H}_{n}\left(P, P \backslash\left\{h\left(x_{1}\right)\right\}\right)$, tomamos a orientação local

$$
\left[\left(\varphi_{x_{1}}\right)_{*} \circ\left(\psi_{h\left(x_{1}\right)}\right)_{*}^{-1}\right]\left(\mu_{h\left(x_{1}\right)} \otimes h_{*}\left(\mathcal{O}\left(x_{1}\right)\right)\right) \in \mathrm{H}_{2 n}\left(Y, Y \backslash\left\{h\left(x_{1}\right)\right\}\right),
$$

estendêmo-la ao longo do caminho $h \circ \omega: I \longrightarrow Y$ para uma orientação

$$
v_{h\left(x_{2}\right)}=(h \circ \omega)_{*}\left(\left[\left(\varphi_{x_{1}}\right)_{*} \circ\left(\psi_{h\left(x_{1}\right)}\right)_{*}^{-1}\right]\left(\mu_{h\left(x_{1}\right)} \otimes h_{*}\left(\mathcal{O}\left(x_{1}\right)\right)\right)\right) \in \mathrm{H}_{2 n}\left(Y, Y \backslash\left\{h\left(x_{2}\right)\right\}\right),
$$

e finalmente tomamos $\lambda_{*}\left[h_{*}\left(\mathcal{O}\left(x_{1}\right)\right)\right] \in \mathrm{H}_{n}\left(\eta_{h\left(x_{2}\right)}, \eta_{h\left(x_{2}\right)} \backslash\left\{h\left(x_{2}\right)\right\}\right)$ tal que

$$
\left[\left(\varphi_{x_{2}}\right)_{*} \circ\left(\psi_{h\left(x_{2}\right)}\right)_{*}^{-1}\right]\left(\lambda_{*}\left(\mu_{h\left(x_{1}\right)}\right) \otimes \lambda_{*}\left[h_{*}\left(\mathcal{O}\left(x_{1}\right)\right)\right]\right)=v_{h\left(x_{2}\right)},
$$

onde $\lambda_{*}\left(\mu_{h\left(x_{1}\right)}\right) \in \mathrm{H}_{n}\left(P, P \backslash\left\{h\left(x_{2}\right)\right\}\right)$ é a orientação local obtida estendendo-se $\mu_{h\left(x_{1}\right)}$ ao longo do caminho $\lambda: I \longrightarrow P$. No entanto, observe que, como $h \circ \omega=(e \circ \omega, \mathcal{F} \circ \omega)$, segue (do penúltimo diagrama acima, e da definição de fibrado orientado para espaços produto, dada em [D], páginas 255 e 256) que o processo de estender a orientação local $\left[\left(\varphi_{x_{1}}\right)_{*} \circ\left(\psi_{h\left(x_{1}\right)}\right)_{*}^{-1}\right]\left(\mu_{h\left(x_{1}\right)} \otimes h_{*}\left(\mathcal{O}\left(x_{1}\right)\right)\right) \in \mathrm{H}_{2 n}\left(Y, Y \backslash\left\{h\left(x_{1}\right)\right\}\right)$ ao longo de $h \circ \omega$, a fim de obter $v_{h\left(x_{2}\right)} \in \mathrm{H}_{2 n}\left(Y, Y \backslash\left\{h\left(x_{2}\right)\right\}\right)$, equivale a estender as orientações locais $\left(p_{x_{1}}\right)_{*}\left(\mu_{h\left(x_{1}\right)}\right) \in$ $\mathrm{H}_{n}\left(X, X \backslash\left\{e\left(x_{1}\right)\right\}\right)$ e $\left(\pi_{x_{1}}\right)_{*}\left(h_{*}\left(\mathcal{O}\left(x_{1}\right)\right)\right) \in \mathrm{H}_{n}(\operatorname{Int} N, \operatorname{Int} N \backslash\{c\})$, ao longo de $e \circ \omega: I \longrightarrow$ $X=\operatorname{Int} M$ e $\mathcal{F} \circ \omega: I \longrightarrow$ Int $N$, respectivamente, determinando assim orientações locais $\tau_{x_{2}}=(e \circ \omega)_{*}\left[\left(p_{x_{1}}\right)_{*}\left(\mu_{h\left(x_{1}\right)}\right)\right] \in \mathrm{H}_{n}\left(X, X \backslash\left\{e\left(x_{2}\right)\right\}\right)$ e $\tau_{c}=(\mathcal{F} \circ \omega)_{*}\left[\left(\pi_{x_{1}}\right)_{*}\left(h_{*}\left(\mathcal{O}\left(x_{1}\right)\right)\right)\right] \in$ $\mathrm{H}_{n}($ Int $N$, Int $N \backslash\{c\})$, e depois tomar $v_{h\left(x_{2}\right)} \in \mathrm{H}_{2 n}\left(Y, Y \backslash\left\{h\left(x_{2}\right)\right\}\right)$ como sendo $v_{h\left(x_{2}\right)}=$ $\left(\psi_{h\left(x_{2}\right)}^{Y}\right)_{*}^{-1}\left(\tau_{x_{2}} \otimes \tau_{c}\right)$; contudo, procedendo desta maneira, teremos (pela comutatividade do último diagrama acima) que $\lambda_{*}\left[h_{*}\left(\mathcal{O}\left(x_{1}\right)\right)\right]=\left(\pi_{x_{2}}\right)_{*}^{-1}\left(\tau_{c}\right)$, pois:

$$
\left[\left(\varphi_{x_{2}}\right)_{*} \circ\left(\psi_{h\left(x_{2}\right)}\right)_{*}^{-1}\right]\left(\left(p_{x_{2}}\right)_{*}^{-1}\left(\tau_{x_{2}}\right) \otimes\left(\pi_{x_{2}}\right)_{*}^{-1}\left(\tau_{c}\right)\right)=\left(\psi_{h\left(x_{2}\right)}^{Y}\right)_{*}^{-1}\left(\tau_{x_{2}} \otimes \tau_{c}\right)=v_{h\left(x_{2}\right)}
$$

(note que $\left.\left(p_{x_{2}}\right)_{*}^{-1}\left(\tau_{x_{2}}\right)=\lambda_{*}\left(\mu_{h\left(x_{1}\right)}\right)\right)$. Deste modo, podemos concluir que o ato de transladar $h_{*}\left(\mathcal{O}\left(x_{1}\right)\right) \in \mathrm{H}_{n}\left(\eta_{h\left(x_{1}\right)}, \eta_{h\left(x_{1}\right)} \backslash\left\{h\left(x_{1}\right)\right\}\right)$ ao longo do caminho $\lambda: I \longrightarrow P$, equivale a estender a orientação local $\left(\pi_{x_{1}}\right)_{*}\left(h_{*}\left(\mathcal{O}\left(x_{1}\right)\right)\right) \in \mathrm{H}_{n}(\operatorname{Int} N, \operatorname{Int} N \backslash\{c\})$ ao longo de $\mathcal{F} \circ \omega: I \longrightarrow \operatorname{Int} N$, obtendo a orientação local $\tau_{c}=(\mathcal{F} \circ \omega)_{*}\left[\left(\pi_{x_{1}}\right)_{*}\left(h_{*}\left(\mathcal{O}\left(x_{1}\right)\right)\right)\right] \in \mathrm{H}_{n}(\operatorname{Int} N$, Int $N \backslash\{c\})$, e depois tomar $\lambda_{*}\left[h_{*}\left(\mathcal{O}\left(x_{1}\right)\right)\right]=\left(\pi_{x_{2}}\right)_{*}^{-1}\left(\tau_{c}\right)$. Ora, como $\mathcal{F} \circ \omega$ é um laço contrátil em $c$ (e, portanto, preserva orientação), temos que $\tau_{c}=\left(\pi_{x_{1}}\right)_{*}\left(h_{*}\left(\mathcal{O}\left(x_{1}\right)\right)\right)$, isto é, ao estendermos a orientação local $\left(\pi_{x_{1}}\right)_{*}\left(h_{*}\left(\mathcal{O}\left(x_{1}\right)\right)\right)$ ao longo de $\mathcal{F} \circ \omega$, "chegaremos" à mesma orientação local. Logo, temos que:

$$
\lambda_{*}\left[h_{*}\left(\mathcal{O}\left(x_{1}\right)\right)\right]=\left[\left(\pi_{x_{2}}\right)_{*}^{-1} \circ\left(\pi_{x_{1}}\right)_{*}\right]\left(h_{*}\left(\mathcal{O}\left(x_{1}\right)\right)\right) ;
$$


conseqüentemente, das definições de $h_{*}\left(\mathcal{O}\left(x_{1}\right)\right), h_{*}\left[\omega_{*}\left(\mathcal{O}\left(x_{1}\right)\right)\right], \mathcal{F}_{*}\left(\mathcal{O}\left(x_{1}\right)\right)$ e $\mathcal{F}_{*}\left[\omega_{*}\left(\mathcal{O}\left(x_{1}\right)\right)\right]$, e da comutatividade do diagrama ilustrado na figura 2.6, segue que:

$$
\begin{aligned}
x_{1} \stackrel{h}{\sim} x_{2} & \Longleftrightarrow \lambda_{*}\left[h_{*}\left(\mathcal{O}\left(x_{1}\right)\right)\right]=-h_{*}\left[\omega_{*}\left(\mathcal{O}\left(x_{1}\right)\right)\right] \\
& \Longleftrightarrow\left[\left(\pi_{x_{2}}\right)_{*}^{-1} \circ\left(\pi_{x_{1}}\right)_{*}\right]\left(h_{*}\left(\mathcal{O}\left(x_{1}\right)\right)\right)=-h_{*}\left[\omega_{*}\left(\mathcal{O}\left(x_{1}\right)\right)\right] \\
& \Longleftrightarrow\left(\pi_{x_{1}}\right)_{*}\left(h_{*}\left(\mathcal{O}\left(x_{1}\right)\right)\right)=\left(\pi_{x_{2}}\right)_{*}\left(-h_{*}\left[\omega_{*}\left(\mathcal{O}\left(x_{1}\right)\right)\right]\right)=-\left(\pi_{x_{2}}\right)_{*}\left(h_{*}\left[\omega_{*}\left(\mathcal{O}\left(x_{1}\right)\right)\right]\right) \\
& \Longleftrightarrow \mathcal{F}_{*}\left(\mathcal{O}\left(x_{1}\right)\right)=-\mathcal{F}_{*}\left[\omega_{*}\left(\mathcal{O}\left(x_{1}\right)\right)\right],
\end{aligned}
$$

ou seja, $x_{1}$ e $x_{2}$ estão $\mathcal{R}$-relacionados com respeito a $h=\left(e, \mathcal{F}^{0}\right)$ se, e somente se, $\mathcal{F}_{*}\left(\mathcal{O}\left(x_{1}\right)\right)=-\mathcal{F}_{*}\left[\omega_{*}\left(\mathcal{O}\left(x_{1}\right)\right)\right]$, conforme queríamos demonstrar.

O próximo lema nos fornece informações a respeito do número de pontos, numa classe de raízes de uma aplicação própria $\mathcal{F}:(M, \partial M, \operatorname{Int} M) \longrightarrow(N, \partial N, \operatorname{Int} N)$, que estão $\mathcal{R}$-relacionados entre si com respeito à uma aplicação $h=\left(e, \mathcal{F}^{0}\right): \operatorname{Int} M \longrightarrow \operatorname{Int} M \times \operatorname{Int} N$. Tais pontos, por sua vez, serão exatamente aqueles que poderemos "remover" por meio de homotopias próprias, a fim de minimizar o conjunto de raízes de $\mathcal{F}$.

Lema 2.4.4. Seja $\mathcal{F}:(M, \partial M, \operatorname{Int} M) \longrightarrow(N, \partial N$, Int $N)$ uma aplicação contínua própria (entre variedades topológicas de dimensão $n)$, e considere novamente $X=\operatorname{Int} M, Y=\operatorname{Int} M \times \operatorname{Int} N$ e $P=\operatorname{Int} M \times\{c\}$, onde $c \in \operatorname{Int} N$. Supondo que $\mathcal{F}$ é transversa a $c$ (de modo que o conjunto $\mathcal{F}^{-1}(c)$ é, portanto, finito), seja ainda $R=\left\{x_{1}, x_{2}, \ldots, x_{k}\right\}$ uma classe de raízes de $\mathcal{F}$ em $c$. Se $k>|m(R)|$, então existem dois pontos em $R$ que estão $\mathcal{R}$-relacionados com respeito a qualquer aplicação contínua $h=\left(e, \mathcal{F}^{0}\right): X \longrightarrow Y$, desde que $h$ seja topologicamente transversa ao microfibrado normal $\eta=\eta^{n}=(Y, P, r)$.

Demonstração. Primeiramente, suponhamos que $\mathcal{F}$ seja uma aplicação orientável, e, dada uma vizinhança aberta contrátil $V \subseteq \operatorname{Int} N$ de $c$, considere, para cada $i \in\{1,2$, $\ldots, k\}$, uma vizinhança aberta $U_{i} \subseteq \operatorname{Int} M$ de $x_{i}$ em $\mathcal{F}^{-1}(V)$, tal que $U_{i} \cap \mathcal{F}^{-1}(V)=\left\{x_{i}\right\}$ (a qual existe, pois $\mathcal{F}^{-1}(c)$ é finito). Como $\mathcal{F}$ é transversa a $c$, podemos supor ainda que $V$ é uma vizinhança euclidiana em Int $N$, e $U_{1}, U_{2}, \ldots, U_{k}$ são vizinhanças euclidianas duas a duas disjuntas, tais que $\left.\mathcal{F}\right|_{U_{i}}: U_{i} \longrightarrow V$ é um homeomorfismo sobre $V$ para cada $i \in\{1,2, \ldots, k\}$. Assim, orientando o aberto $U=\bigcup_{i=1}^{k} U_{i}=\mathcal{F}^{-1}(V)$ pelo processo de orientação dado na definição 1.3.6, segue (do exemplo 1.3.8) que:

$$
|m(R)|=\left|\sum_{i=1}^{k} \operatorname{deg}_{c}\left(\left.\mathcal{F}\right|_{U_{i}}\right)\right|,
$$

onde, para cada $i \in\{1,2, \ldots, k\}, \operatorname{deg}_{c}\left(\left.\mathcal{F}\right|_{U_{i}}\right)$ denota o grau local a coeficientes em $\mathbb{Z}$. Ora, como cada uma das restrições $\left.\mathcal{F}\right|_{U_{i}}$ é um homeomorfismo sobre $V$, e os 
abertos $U_{1}, U_{2}, \ldots, U_{k}, V$ são variedades topológicas orientáveis (pois constituem vizinhanças euclidianas), temos, do corolário 1.3.1, que $\operatorname{deg}_{c}\left(\left.\mathcal{F}\right|_{U_{i}}\right)= \pm 1$, qualquer que seja $i \in\{1,2, \ldots, k\}$; logo, como $k>|m(R)|$, segue que nem todos os graus locais $\operatorname{deg}_{c}\left(\left.\mathcal{F}\right|_{U_{i}}\right)$ são iguais. Deste modo, sem perda de generalidade, podemos supor que $\operatorname{deg}_{c}\left(\left.\mathcal{F}\right|_{U_{1}}\right)=-\operatorname{deg}_{c}\left(\left.\mathcal{F}\right|_{U_{2}}\right)$. Entretanto, sendo $\left(j_{U_{1}}\right)_{*}: \mathrm{H}_{n}\left(U_{1}, U_{1} \backslash\left\{x_{1}\right\}\right) \longrightarrow \mathrm{H}_{n}\left(X, X \backslash\left\{x_{1}\right\}\right)$ $\mathrm{e}\left(j_{U_{2}}\right)_{*}: \mathrm{H}_{n}\left(U_{2}, U_{2} \backslash\left\{x_{2}\right\}\right) \longrightarrow \mathrm{H}_{n}\left(X, X \backslash\left\{x_{2}\right\}\right)$ os isomorfismos induzidos pelas respectivas inclusões, temos que as orientações $s_{U_{1}}\left(x_{1}\right) \in \mathrm{H}_{n}\left(U_{1}, U_{1} \backslash\left\{x_{1}\right\}\right)$ e $s_{U_{2}}\left(x_{2}\right) \in \mathrm{H}_{n}\left(U_{2}, U_{2} \backslash\left\{x_{2}\right\}\right)$, de $U_{1}$ e $U_{2}$ em $x_{1}$ e $x_{2}$, respectivamente, definem orientações locais $\mathcal{O}\left(x_{1}\right) \in \mathrm{H}_{n}\left(X, X \backslash\left\{x_{1}\right\}\right)$ e $\mathcal{O}\left(x_{2}\right) \in \mathrm{H}_{n}\left(X, X \backslash\left\{x_{2}\right\}\right)$, a saber, $\mathcal{O}\left(x_{1}\right)=\left(j_{U_{1}}\right)_{*}\left(s_{U_{1}}\left(x_{1}\right)\right)$ e $\mathcal{O}\left(x_{2}\right)=\left(j_{U_{2}}\right)_{*}\left(s_{U_{2}}\left(x_{2}\right)\right)$; além disso, como $U=\bigcup_{i=1}^{k} U_{i}$ é orientado pelo processo de orientação, temos que a orientação local $\mathcal{O}\left(x_{2}\right)$ é obtida estendendo-se $\mathcal{O}\left(x_{1}\right)$ ao longo de um caminho $\omega: I \longrightarrow X=\operatorname{Int} M$, ligando $x_{1} \in U_{1}$ a $x_{2} \in U_{2}$, tal que $\mathcal{F} \circ \omega: I \longrightarrow$ Int $N$ é um laço contrátil em $c$ (vide definição 1.3.6). Agora, sendo $\left(\left.\mathcal{F}\right|_{U_{1}}\right)_{*}: \mathrm{H}_{n}\left(U_{1}, U_{1} \backslash\left\{x_{1}\right\}\right) \longrightarrow \mathrm{H}_{n}(V, V \backslash\{c\}) \mathrm{e}$ $\left(\left.\mathcal{F}\right|_{U_{2}}\right)_{*}: \mathrm{H}_{n}\left(U_{2}, U_{2} \backslash\left\{x_{2}\right\}\right) \longrightarrow \mathrm{H}_{n}(V, V \backslash\{c\})$ os isomorfismos induzidos por $\left.\mathcal{F}\right|_{U_{1}} \mathrm{e}$

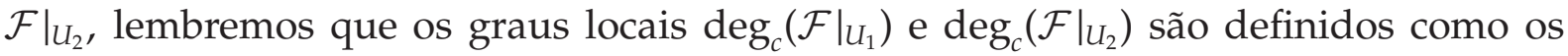
números inteiros tais que $\left.\left.\mathcal{F}\right|_{U_{1}}\right)_{*}\left(\theta_{x_{1}}\right)=\operatorname{deg}_{c}\left(\left.\mathcal{F}\right|_{U_{1}}\right) \cdot \theta_{c}$ e $\left.\left.\mathcal{F}\right|_{U_{2}}\right)_{*}\left(\theta_{x_{2}}\right)=\operatorname{deg}_{c}\left(\left.\mathcal{F}\right|_{U_{2}}\right) \cdot \theta_{c}$, onde $\theta_{x_{1}} \in \mathrm{H}_{n}\left(U_{1}, U_{1} \backslash\left\{x_{1}\right\}\right), \theta_{x_{2}} \in \mathrm{H}_{n}\left(U_{2}, U_{2} \backslash\left\{x_{2}\right\}\right)$ e $\theta_{c} \in \mathrm{H}_{n}(V, V \backslash\{c\})$ são as classes fundamentais. Por outro lado, de acordo com as notações que estamos adotando, temos que $\mathcal{F}_{*}\left(\mathcal{O}\left(x_{1}\right)\right) \in \mathrm{H}_{n}(\operatorname{Int} N$, Int $N \backslash\{c\})$ e $\mathcal{F}_{*}\left(\mathcal{O}\left(x_{2}\right)\right) \in \mathrm{H}_{n}(\operatorname{Int} N$, Int $N \backslash\{c\})$ são as orientações locais obtidas de $\mathcal{O}\left(x_{1}\right) \in \mathrm{H}_{n}\left(X, X \backslash\left\{x_{1}\right\}\right)$ e $\mathcal{O}\left(x_{2}\right) \in \mathrm{H}_{n}\left(X, X \backslash\left\{x_{2}\right\}\right)$, respectivamente, pelas seguintes cadeias de isomorfismos (onde $\left(J_{V}\right)_{*}: \mathrm{H}_{n}(V, V \backslash\{c\}) \longrightarrow \mathrm{H}_{n}(\operatorname{Int} N, \operatorname{Int} N \backslash\{c\}$ ) é o isomorfismo induzido pela inclusão de $V$ em $\operatorname{Int} N)$ :

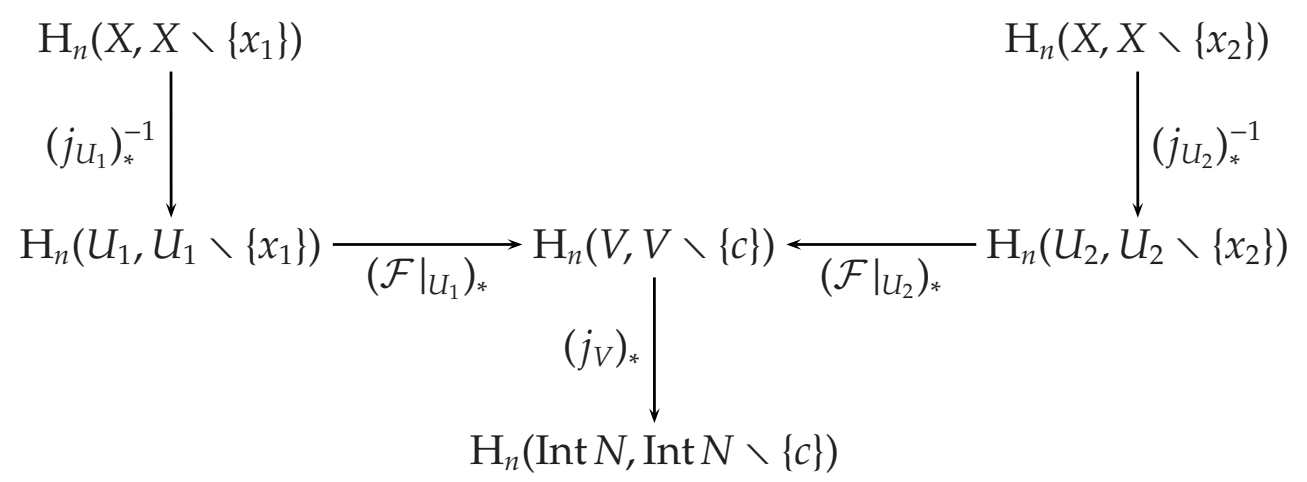

Em outras palavras, temos que:

$$
\mathcal{F}_{*}\left(\mathcal{O}\left(x_{1}\right)\right)=\left[\left(j_{V}\right)_{*} \circ\left(\left.\mathcal{F}\right|_{U_{1}}\right)_{*} \circ\left(j_{U_{1}}\right)_{*}^{-1}\right]\left(\mathcal{O}\left(x_{1}\right)\right)
$$

e

$$
\mathcal{F}_{*}\left(\mathcal{O}\left(x_{2}\right)\right)=\left[\left(j_{V}\right)_{*} \circ\left(\left.\mathcal{F}\right|_{U_{2}}\right)_{*} \circ\left(j_{U_{2}}\right)_{*}^{-1}\right]\left(\mathcal{O}\left(x_{2}\right)\right) .
$$


Portanto, se $\operatorname{deg}_{c}\left(\left.\mathcal{F}\right|_{U_{1}}\right)=-\operatorname{deg}_{c}\left(\left.\mathcal{F}\right|_{U_{2}}\right)$, segue que:

$$
\left(\left.\mathcal{F}\right|_{U_{1}}\right)_{*}\left(\theta_{x_{1}}\right)=\operatorname{deg}_{c}\left(\left.\mathcal{F}\right|_{U_{1}}\right) \cdot \theta_{c}=-\operatorname{deg}_{c}\left(\left.\mathcal{F}\right|_{U_{2}}\right) \cdot \theta_{c}=-\left(\left.\mathcal{F}\right|_{U_{2}}\right)_{*}\left(\theta_{x_{2}}\right)
$$

e, conseqüentemente,

$$
\left[\left(j_{V}\right)_{*} \circ\left(\left.\mathcal{F}\right|_{U_{1}}\right)_{*}\right]\left(\theta_{x_{1}}\right)=-\left[\left(j_{V}\right)_{*} \circ\left(\left.\mathcal{F}\right|_{U_{2}}\right)_{*}\right]\left(\theta_{x_{2}}\right) .
$$

No entanto, pela definição de classe fundamental, temos que $\theta_{x_{1}}$ e $\theta_{x_{2}}$ coincidem com as orientações de $U_{1}$ e $U_{2}$ em $x_{1}$ e $x_{2}$, respectivamente, isto é, $\theta_{x_{1}}=s_{U_{1}}\left(x_{1}\right)$ e $\theta_{x_{2}}=s_{U_{2}}\left(x_{2}\right)$. Logo, como $s_{U_{1}}\left(x_{1}\right)=\left(j_{U_{1}}\right)_{*}^{-1}\left(\mathcal{O}\left(x_{1}\right)\right)$ e $s_{U_{2}}\left(x_{2}\right)=\left(j_{U_{2}}\right)_{*}^{-1}\left(\mathcal{O}\left(x_{2}\right)\right)$, a última igualdade acima equivale a:

$$
\left[\left(j_{V}\right)_{*} \circ\left(\left.\mathcal{F}\right|_{U_{1}}\right)_{*} \circ\left(j_{U_{1}}\right)_{*}^{-1}\right]\left(\mathcal{O}\left(x_{1}\right)\right)=-\left[\left(j_{V}\right)_{*} \circ\left(\left.\mathcal{F}\right|_{U_{2}}\right)_{*} \circ\left(j_{U_{2}}\right)_{*}^{-1}\right]\left(\mathcal{O}\left(x_{2}\right)\right)
$$

ou seja, $\mathcal{F}_{*}\left(\mathcal{O}\left(x_{1}\right)\right)=-\mathcal{F}_{*}\left(\mathcal{O}\left(x_{2}\right)\right)$. Assim, pelo lema 2.4.3, podemos concluir que os pontos $x_{1}$ e $x_{2}$ estão $\mathcal{R}$-relacionados com respeito a qualquer aplicação contínua $h: X \longrightarrow Y$ da forma $h=\left(e, \mathcal{F}^{0}\right)$, desde que $h$ seja topologicamente transversa ao microfibrado normal $\eta=\eta^{n}=(Y, P, r)$.

Isto posto, suponhamos agora que a aplicação $\mathcal{F}$ seja não-orientável (isto é, do tipo III); utilizando as mesmas vizinhanças euclidianas $U_{1}, U_{2}, \ldots, U_{k}$ e $V$, segue (do exemplo 1.3.8 com coeficientes em $\mathbb{Z}_{2}$ ) que:

$$
|m(R)|=\left|\sum_{i=1}^{k} \operatorname{deg}_{c}\left(\left.\mathcal{F}\right|_{U_{i}}\right)\right|=\left\{\begin{array}{lll}
1 & \text { se } & k \text { é ímpar } \\
0 & \text { se } & k \text { é par }
\end{array}\right.
$$

(note que, em $\mathbb{Z}_{2}$, temos $\operatorname{deg}_{c}\left(\left.\mathcal{F}\right|_{U_{i}}\right)=1$ para todo $i \in\{1,2, \ldots, k\}$ ). Suponhamos então que $k>|m(R)|=1$; neste caso, existem pelo menos duas raízes em $R$, digamos $x_{1}$ e $x_{2}$, bem como um caminho $\omega: I \longrightarrow X=\operatorname{Int} M$, ligando $x_{1}$ a $x_{2}$, tal que $\mathcal{F} \circ \omega: I \longrightarrow \operatorname{Int} N$ é um laço contrátil em $c$. Deste modo, orientando-se o aberto $U=\bigcup_{i=1}^{k} U_{i}$ com coeficientes em $\mathbb{Z}_{2}$, e utilizando as notações definidas anteriormente, segue que, se a orientação local $\mathcal{O}\left(x_{2}\right) \in \mathrm{H}_{n}\left(X, X \backslash\left\{x_{2}\right\}\right)$, obtida estendendo-se $\mathcal{O}\left(x_{1}\right) \in \mathrm{H}_{n}\left(X, X \backslash\left\{x_{1}\right\}\right)$ ao longo de $\omega$, for tal que $\mathcal{F}_{*}\left(\mathcal{O}\left(x_{1}\right)\right)=-\mathcal{F}_{*}\left(\mathcal{O}\left(x_{2}\right)\right)$, concluímos de imediato (do lema 2.4.3) que $x_{1}$ e $x_{2}$ estão $\mathcal{R}$-relacionados, qualquer que seja $h=\left(e, \mathcal{F}^{0}\right): X \longrightarrow Y$ topologicamente transversa a $\eta$. Caso contrário (ou seja, se $\mathcal{F}_{*}\left(\mathcal{O}\left(x_{1}\right)\right)=\mathcal{F}_{*}\left(\mathcal{O}\left(x_{2}\right)\right)$ ), tomamos um laço $\lambda: I \longrightarrow \operatorname{Int} M$, que inverte orientação, tal que $\mathcal{F} \circ \lambda: I \longrightarrow$ Int $N$ é um laço contrátil (o qual existe, pois $\mathcal{F}$ é supostamente do tipo III), e consideramos um caminho $\alpha: I \longrightarrow \operatorname{Int} M$, ligando $x_{1}$ a $p=\lambda(0)=\lambda(1)$; assim, denotando por $\omega^{\prime}: I \longrightarrow$ Int $M$ o caminho dado pela justaposição $\omega^{\prime}=\alpha \vee \lambda \vee \alpha^{-1} \vee \omega$, temos que $\omega^{\prime}$ é um caminho ligando $x_{1}$ a $x_{2}$ (uma vez 
que $\omega^{\prime}(0)=\alpha(0)=x_{1}$ e $\left.\omega^{\prime}(1)=\alpha(1)=x_{2}\right)$, cuja imagem $\mathcal{F} \circ \omega^{\prime}: I \longrightarrow \operatorname{Int} N$ é um laço contrátil em $c$, pois:

$$
\begin{aligned}
{\left[\mathcal{F} \circ \omega^{\prime}\right] } & =\left[\mathcal{F} \circ\left(\alpha \vee \lambda \vee \alpha^{-1} \vee \omega\right)\right] \\
& =\left[(\mathcal{F} \circ \alpha) \vee(\mathcal{F} \circ \lambda) \vee\left(\mathcal{F} \circ \alpha^{-1}\right) \vee(\mathcal{F} \circ \omega)\right] \\
& =\left[(\mathcal{F} \circ \alpha) \vee\left(\epsilon_{p}\right) \vee\left(\mathcal{F} \circ \alpha^{-1}\right) \vee\left(\epsilon_{c}\right)\right] \\
& =\left[(\mathcal{F} \circ \alpha) \vee\left(\mathcal{F} \circ \alpha^{-1}\right)\right]=\left[\mathcal{F} \circ\left(\alpha \vee \alpha^{-1}\right)\right]=\left[\epsilon_{c}\right]
\end{aligned}
$$

(observe que estamos denotando por $\epsilon_{p}: I \longrightarrow\{p\} \subseteq \operatorname{Int} M$ e $\epsilon_{c}: I \longrightarrow\{c\} \subseteq \operatorname{Int} N$ os caminhos constantes iguais a $p=\lambda(0)=\lambda(1)$ e $c$, respectivamente). Logo, como $\lambda$ é um laço que inverte orientação, ao estendermos a orientação local $\mathcal{O}\left(x_{1}\right) \in \mathrm{H}_{n}\left(X, X \backslash\left\{x_{1}\right\}\right)$ ao longo de $\omega^{\prime}=\alpha \vee \lambda \vee \alpha^{-1} \vee \omega$, obteremos uma orientação local $\mathcal{O}^{\prime}\left(x_{2}\right) \in \mathrm{H}_{n}\left(X, X \backslash\left\{x_{2}\right\}\right)$ que é oposta àquela $\mathcal{O}\left(x_{2}\right)$ que obtivemos estendendo $\mathcal{O}\left(x_{1}\right)$ ao longo de $\omega$. Daí, como $\mathcal{F}_{*}\left(\mathcal{O}\left(x_{1}\right)\right)=\mathcal{F}_{*}\left(\mathcal{O}\left(x_{2}\right)\right)$ e $\mathcal{O}\left(x_{2}\right)=-\mathcal{O}^{\prime}\left(x_{2}\right)$, segue que $\mathcal{F}_{*}\left(\mathcal{O}\left(x_{1}\right)\right)=-\mathcal{F}_{*}\left(\mathcal{O}^{\prime}\left(x_{2}\right)\right)$, o que novamente implica (pelo lema 2.4.3) que $x_{1}$ e $x_{2}$ estão $\mathcal{R}$-relacionados com respeito a toda aplicação contínua $h=\left(e, \mathcal{F}^{0}\right): X \longrightarrow Y$ topologicamente transversa a $\eta$.

Enfim, se $|m(R)|=0$, temos que $k$ é um número par, e, portanto, se $k>|m(R)|$, segue que $k \geqslant 2 ; \operatorname{logo}$, repetindo o procedimento acima, podemos encontrar duas raízes em $R$ que estão $\mathcal{R}$-relacionadas, encerrando a demonstração.

Lema 2.4.5 . Sendo $\phi:(M, \partial M, \operatorname{Int} M) \longrightarrow(N, \partial N$, Int $N)$ uma aplicação contínua própria (entre variedades topológicas conexas de mesma dimensão $n \geqslant 3$ ), transversa a um ponto $c \in$ Int $N$, considere dois pontos $x_{1}, x_{2} \in \phi^{-1}(c)$, pertencentes à uma mesma classe de raízes $R$ de $\phi$ em $c$, e sejam $U_{1}, U_{2} \subseteq \operatorname{Int} M$ e $V_{c} \subseteq \operatorname{Int} N$ vizinhanças euclidianas de $x_{1}, x_{2}$ e c , respectivamente, tais que as restrições $\left.\phi\right|_{U_{1}}: U_{1} \longrightarrow V_{c}$ e $\left.\phi\right|_{U_{2}}: U_{2} \longrightarrow V_{c}$ são homeomorfismos sobre $V_{c}$ (de modo que $\left.\operatorname{deg}_{c}\left(\left.\phi\right|_{U_{1}}\right)= \pm 1 e \operatorname{deg}_{c}\left(\left.\phi\right|_{U_{2}}\right)= \pm 1\right) . \operatorname{Se} \operatorname{deg}_{c}\left(\left.\phi\right|_{U_{1}}\right)=-\operatorname{deg}_{c}\left(\left.\phi\right|_{U_{2}}\right)$, segue que existe uma aplicação contínua própria $\phi^{\prime}:(M, \partial M, \operatorname{Int} M) \longrightarrow(N, \partial N, \operatorname{Int} N)$, transversa à c e propriamente homotópica à $\phi$, tal que:

(i) $\phi^{\prime}$ coincide com $\phi$ em $M \backslash B$, onde $B \subseteq \operatorname{Int} M$ é uma n-bola própria satisfazendo $\bar{B} \subseteq \operatorname{Int} M$ $e \bar{B} \cap \phi^{-1}(c)=\left\{x_{1}, x_{2}\right\} ;$

(ii) $\left(\phi^{\prime}\right)^{-1}(c)=\phi^{-1}(c) \backslash\left\{x_{1}, x_{2}\right\}$;

(iii) $R^{\prime}=R \backslash\left\{x_{1}, x_{2}\right\}$ constitui uma classe de raízes de $\phi^{\prime}$ em $c$.

DemonstraÇÃo. De fato, como $\phi$ é transversa à $c \in \operatorname{Int} N$, segue que existe uma vizinhança euclidiana $V_{c} \subseteq \operatorname{Int} N$ de $c$, tal que $\phi^{-1}\left(V_{c}\right)$ é uma união finita e disjunta de vizinhanças euclidianas em Int $M$, cada uma das quais é levada homeomorficamente por $\phi$ sobre $V_{c}$. Isto posto, dada uma classe de raízes $R=\left\{x_{1}, x_{2}, \ldots, x_{k}\right\}$ de $\phi$ em $c$, sejam 
$U_{1}, U_{2}, \ldots, U_{k} \subseteq \operatorname{Int} M$ vizinhanças euclidianas contendo $x_{1}, x_{2}, \ldots, x_{k}$, respectivamente, tais que, para cada $i \in\{1,2, \ldots, k\}$, a restrição $\left.\phi\right|_{U_{i}}: U_{i} \longrightarrow V_{c}$ é um homeomorfismo sobre $V_{c}$; temos então que o conjunto $U=\bigcup_{i=1}^{k} U_{i}$ é uma vizinhança aberta de $R$ em Int $M$, tal que $U \cap \phi^{-1}(c)=R$. Assim, orientando $U$ de acordo com o processo de orientação descrito na definição 1.3.6, e considerando as componentes conexas $U_{1}, U_{2} \subseteq$ $U$ orientadas pela restrição da orientação de $U$, temos que a orientação $\mu_{x_{2}} \in \mathrm{H}_{n}\left(U_{2}, U_{2} \backslash\right.$ $\left.\left\{x_{2}\right\}\right)$ de $U_{2}$ em $x_{2}$ é obtida da seguinte maneira: sendo $\mu_{x_{1}} \in \mathrm{H}_{n}\left(U_{1}, U_{1} \backslash\left\{x_{1}\right\}\right)$ a orientação de $U_{1}$ em $x_{1}$, consideramos a orientação local $\mathcal{O}\left(x_{1}\right) \in \mathrm{H}_{n}\left(\operatorname{Int} M\right.$, Int $\left.M \backslash\left\{x_{1}\right\}\right)$ dada por $\mathcal{O}\left(x_{1}\right)=\left(j_{U_{1}}\right)_{*}\left(\mu_{x_{1}}\right)$, onde $\left(j_{U_{1}}\right)_{*}: \mathrm{H}_{n}\left(U_{1}, U_{1} \backslash\left\{x_{1}\right\}\right) \longrightarrow \mathrm{H}_{n}\left(\operatorname{Int} M\right.$, Int $\left.M \backslash\left\{x_{1}\right\}\right)$ é o isomorfismo induzido pela inclusão, estendemos $\mathcal{O}\left(x_{1}\right)$ ao longo de um caminho $\omega: I \longrightarrow$ Int $M$ ligando $x_{1}$ a $x_{2}$, tal que $\phi \circ \omega: I \longrightarrow$ Int $N$ é um laço contrátil em $c$, obtendo uma orientação local $\omega_{*}\left(\mathcal{O}\left(x_{1}\right)\right) \in \mathrm{H}_{n}\left(\operatorname{Int} M\right.$, Int $\left.M \backslash\left\{x_{2}\right\}\right)$, e depois tomamos $\mu_{x_{2}} \in \mathrm{H}_{n}\left(U_{2}, U_{2} \backslash\left\{x_{2}\right\}\right)$ de tal forma que $\omega_{*}\left(\mathcal{O}\left(x_{1}\right)\right)=\left(j_{U_{2}}\right)_{*}\left(\mu_{x_{2}}\right)$, onde $\left(j_{U_{2}}\right)_{*}: \mathrm{H}_{n}\left(U_{2}, U_{2} \backslash\right.$ $\left.\left\{x_{2}\right\}\right) \longrightarrow \mathrm{H}_{n}\left(\right.$ Int $M$, Int $\left.M \backslash\left\{x_{2}\right\}\right)$ é o isomorfismo induzido pela inclusão. Agora, observe que, uma vez adotadas estas orientações em $U_{1}$ e $U_{2}$, temos que $\mu_{x_{1}}$ e $\mu_{x_{2}}$ constituem as classes fundamentais em $x_{1}$ e $x_{2}$, respectivamente; logo, escolhida uma orientação para $V_{c} \subseteq \operatorname{Int} N$, e denotando por $\theta_{c} \in \mathrm{H}_{n}\left(V_{c}, V_{c} \backslash\{c\}\right)$ a classe fundamental associada à esta orientação, segue que $\left(\left.\phi\right|_{U_{1}}\right)_{*}\left(\mu_{x_{1}}\right)=\operatorname{deg}_{c}\left(\left.\phi\right|_{U_{1}}\right) \cdot \theta_{c}$ e $\left(\left.\phi\right|_{U_{2}}\right)_{*}\left(\mu_{x_{2}}\right)=\operatorname{deg}_{c}\left(\left.\phi\right|_{U_{2}}\right) \cdot \theta_{c}$, onde $\left(\left.\phi\right|_{U_{1}}\right)_{*}: \mathrm{H}_{n}\left(U_{1}, U_{1} \backslash\left\{x_{1}\right\}\right) \longrightarrow \mathrm{H}_{n}\left(V_{c}, V_{c} \backslash\{c\}\right)$ e $\left(\left.\phi\right|_{U_{2}}\right)_{*}: \mathrm{H}_{n}\left(U_{2}, U_{2} \backslash\left\{x_{2}\right\}\right) \longrightarrow$ $\mathrm{H}_{n}\left(V_{c}, V_{c} \backslash\{c\}\right)$ são os isomorfismos induzidos pelas restrições $\left.\phi\right|_{U_{1}}: U_{1} \longrightarrow V_{c} \mathrm{e}$ $\left.\phi\right|_{U_{2}}: U_{2} \longrightarrow V_{c}$. Deste modo, sendo $\left(j_{V_{c}}\right)_{*}: \mathrm{H}_{n}\left(V_{c}, V_{c} \backslash\{c\}\right) \longrightarrow \mathrm{H}_{n}(\operatorname{Int} N, \operatorname{Int} N \backslash\{c\}) \mathrm{o}$ isomorfismo induzido pela inclusão, considere os elementos $\phi_{*}\left(\mathcal{O}\left(x_{1}\right)\right), \phi_{*}\left[\omega_{*}\left(\mathcal{O}\left(x_{1}\right)\right)\right] \in$ $\mathrm{H}_{n}(\operatorname{Int} N$, Int $N \backslash\{c\})$ dados por:

$$
\phi_{*}\left(\mathcal{O}\left(x_{1}\right)\right)=\left[\left(j_{V_{c}}\right)_{*} \circ\left(\left.\phi\right|_{U_{1}}\right)_{*} \circ\left(j_{U_{1}}\right)_{*}^{-1}\right]\left(\mathcal{O}\left(x_{1}\right)\right)
$$

e

$$
\phi_{*}\left[\omega_{*}\left(\mathcal{O}\left(x_{1}\right)\right)\right]=\left[\left(j_{V_{c}}\right)_{*} \circ\left(\left.\phi\right|_{U_{2}}\right)_{*} \circ\left(j_{U_{2}}\right)_{*}^{-1}\right]\left(\omega_{*}\left(\mathcal{O}\left(x_{1}\right)\right)\right) ;
$$

se $\operatorname{deg}_{c}\left(\phi \mid U_{1}\right)=-\operatorname{deg}_{c}\left(\phi \mid U_{2}\right)$, segue que:

$$
\begin{aligned}
\phi_{*}\left(\mathcal{O}\left(x_{1}\right)\right) & =\left[\left(j_{V_{c}}\right)_{*} \circ\left(\left.\phi\right|_{U_{1}}\right)_{*} \circ\left(j_{U_{1}}\right)_{*}^{-1}\right]\left(\mathcal{O}\left(x_{1}\right)\right)=\left[\left(j_{V_{c}}\right)_{*} \circ\left(\left.\phi\right|_{U_{1}}\right)_{*}\right]\left(\mu_{x_{1}}\right) \\
& =\left(j_{V_{c}}\right)_{*}\left[\operatorname{deg}_{c}\left(\left.\phi\right|_{U_{1}}\right) \cdot \theta_{c}\right]=\left(j_{V_{c}}\right)_{*}\left[-\operatorname{deg}_{c}\left(\left.\phi\right|_{U_{2}}\right) \cdot \theta_{c}\right] \\
& =-\left(j_{V_{c}}\right)_{*}\left[\operatorname{deg}_{c}\left(\left.\phi\right|_{U_{2}}\right) \cdot \theta_{c}\right]=-\left[\left(j_{V_{c}}\right)_{*} \circ\left(\left.\phi\right|_{U_{2}}\right)_{*}\right]\left(\mu_{x_{2}}\right) \\
& =-\left[\left(j_{V_{c}}\right)_{*} \circ\left(\left.\phi\right|_{U_{2}}\right)_{*} \circ\left(j_{U_{2}}\right)_{*}^{-1}\right]\left(\omega_{*}\left(\mathcal{O}\left(x_{1}\right)\right)\right)=-\phi_{*}\left[\omega_{*}\left(\mathcal{O}\left(x_{1}\right)\right)\right],
\end{aligned}
$$

o que, pelo lema 2.4.3, implica que $x_{1}$ e $x_{2}$ estão $\mathcal{R}$-relacionados com respeito à uma aplicação $h=\left(e, \phi^{0}\right): \operatorname{Int} M \longrightarrow \operatorname{Int} M \times \operatorname{Int} N$, que é topologicamente transversa ao 
microfibrado $\eta=\eta^{n}=(Y, P, r)$. No entanto, do fato de $R$ ser discreto e $X=\operatorname{Int} M$ ser uma variedade topológica conexa, sabemos que existe uma n-bola própria $B \subseteq \operatorname{Int} M$, contendo $x_{1}$ e $x_{2}$, tal que $\bar{B} \subseteq \operatorname{Int} M$ e $\bar{B} \cap \phi^{-1}(c)=\left\{x_{1}, x_{2}\right\}$. Ademais, sem perda de generalidade, podemos supor que existe um caminho $\omega: I \longrightarrow B$, com $\omega(0)=x_{1} \mathrm{e}$ $\omega(1)=x_{2}$, tal que $\phi \circ \omega: I \longrightarrow \operatorname{Int} N$ é um laço contrátil em $c:$ com efeito, como $x_{1}$ e $x_{2}$ pertencem à mesma classe de raízes de $\phi$ em $c$, segue que existe um caminho $\omega^{\prime}: I \longrightarrow \operatorname{Int} M$, ligando $x_{1}$ a $x_{2}$, tal que $\phi \circ \omega^{\prime}: I \longrightarrow \operatorname{Int} N$ é um laço contrátil em $c ;$ logo, se a imagem $\omega^{\prime}(I)$ não estiver contida em $B$ (conforme queremos), pode-se deformar $\omega^{\prime}(I)$ numa vizinhança contrátil de cada ponto $y \in \omega^{\prime}(I) \cap(\operatorname{Int} M \backslash B)$, de maneira a obter um caminho $\omega: I \longrightarrow B$, homotópico à $\omega^{\prime}$, com $\phi \circ \omega$ homotópico à $\phi \circ \omega^{\prime}$. Pelo que vimos na demonstração do lema 2.4.3, temos ainda que tal caminho $\omega: I \longrightarrow B$ $\mathcal{R}$-relaciona os pontos $x_{1}$ e $x_{2}$ com respeito à restrição $\left.h\right|_{\bar{B}}: \bar{B} \longrightarrow Y$. Isto posto, note então que:

$$
\begin{aligned}
\left(\left.h\right|_{\bar{B}}\right)^{-1}(P) & =\bar{B} \cap h^{-1}(P)=\bar{B} \cap\{x \in X: h(x) \in P\} \\
& =\bar{B} \cap\left\{x \in X:\left(e(x), \phi^{0}(x)\right) \in \operatorname{Int} M \times\{c\}\right\} \\
& =\bar{B} \cap\{x \in X: \phi(x)=c\}=\bar{B} \cap \phi^{-1}(c) \\
& =\left\{x_{1}, x_{2}\right\} .
\end{aligned}
$$

Em contrapartida, lembrando que $\phi:(M, \partial M, \operatorname{Int} M) \longrightarrow(N, \partial N, \operatorname{Int} N)$ é transversa à $c$, seja $E_{c} \subseteq \operatorname{Int} N$ uma vizinhança euclidiana de $c$, tal que $\phi^{-1}\left(E_{c}\right)=E_{1} \cup E_{2}$, onde $E_{1}, E_{2} \subseteq B$ são vizinhanças euclidianas disjuntas de $x_{1}$ e $x_{2}$, respectivamente, que são levadas homeomorficamente por $\phi$ sobre $E_{c}$ (de modo que a restrição $\left.\phi\right|_{\bar{B}}: \bar{B} \longrightarrow \operatorname{Int} N$ é, portanto, transversa à c); denotando por $r_{\delta}: E=E_{1} \cup E_{2} \longrightarrow\left(\left.h\right|_{\bar{B}}\right)^{-1}(P)=\left\{x_{1}, x_{2}\right\}$ a retração dada por:

$$
r_{\delta}(x)=\left\{\begin{array}{lll}
x_{1} & \text { se } & x \in E_{1} \\
x_{2} & \text { se } & x \in E_{2}
\end{array},\right.
$$

temos que $\delta=\delta^{n}=\left(E,\left(\left.h\right|_{\bar{B}}\right)^{-1}(P), r_{\delta}\right)$ é um microfibrado normal sobre $\left(\left.h\right|_{\bar{B}}\right)^{-1}(P)=\left\{x_{1}, x_{2}\right\}$, tal que $\left.h\right|_{\bar{B}}=\left(\left.e\right|_{\bar{B}},\left.\phi^{0}\right|_{\bar{B}}\right): \bar{B} \longrightarrow Y$ é topologicamente transversa à $\delta$. Logo, do lema 2.4.2, segue que existe uma aplicação contínua $\hbar: \bar{B} \longrightarrow Y \backslash P=\operatorname{Int} M \times(\operatorname{Int} N \backslash\{c\})$, homotópica à $\left.h\right|_{\bar{B}}: \bar{B} \longrightarrow Y$ por uma homotopia $K: \bar{B} \times I \longrightarrow Y$ tal que $K(x, t)=\hbar(x)=$ $\left(\left.h\right|_{\bar{B}}\right)(x)$ para todo $(x, t) \in \partial B \times I$. Assim, considerando a aplicação $\hbar^{\prime}: X=\operatorname{Int} M \longrightarrow$ $Y=\operatorname{Int} M \times \operatorname{Int} N$ dada por:

$$
\hbar^{\prime}(x)=\left\{\begin{array}{lll}
\hbar(x) & \text { se } & x \in \bar{B} \\
h(x) & \text { se } & x \in X \backslash B
\end{array},\right.
$$

temos que $\hbar^{\prime}$ encontra-se bem definida e é contínua em $X$. Além disso, sendo $\bar{e}: \bar{B} \longrightarrow$ $\operatorname{Int} M, \bar{\phi}: \bar{B} \longrightarrow \operatorname{Int} N \backslash\{c\}, e^{\prime}: \operatorname{Int} M \longrightarrow \operatorname{Int} M$ e $\phi_{0}^{\prime}: \operatorname{Int} M \longrightarrow \operatorname{Int} N$ as aplicações 
contínuas tais que $\hbar=(\bar{e}, \bar{\phi})$ e $\hbar^{\prime}=\left(e^{\prime}, \phi_{0}^{\prime}\right)$, temos que:

$$
\phi_{0}^{\prime}(x)=\left\{\begin{array}{lll}
\bar{\phi}(x) & \text { se } & x \in \bar{B} \\
\phi(x) & \text { se } & x \in X \backslash B
\end{array},\right.
$$

e, conseqüentemente, a aplicação $\phi^{\prime}:(M, \partial M, \operatorname{Int} M) \longrightarrow(N, \partial N, \operatorname{Int} N)$, dada por

$$
\phi^{\prime}(x)=\left\{\begin{array}{lll}
\phi_{0}^{\prime}(x) & \text { se } & x \in X=\operatorname{Int} M \\
\phi(x) & \text { se } & x \in \partial M
\end{array}=\left\{\begin{array}{lll}
\bar{\phi}(x) & \text { se } & x \in \bar{B} \\
\phi(x) & \text { se } & x \in M \backslash \bar{B}
\end{array},\right.\right.
$$

também encontra-se bem definida e é contínua em $M$. Como $\bar{\phi}: \bar{B} \longrightarrow \operatorname{Int} N \times\{c\}$ não possui raízes em $c$, e $\phi$ é transversa à $c\left(\operatorname{com} \phi^{-1}(c) \backslash\left\{x_{1}, x_{2}\right\} \subseteq \operatorname{Int}(X \backslash B)=X \backslash \bar{B}\right)$, temos que $\phi^{\prime}$ é transversa à $c$, e satisfaz $\left(\phi^{\prime}\right)^{-1}(c)=\phi^{-1}(c) \backslash\left\{x_{1}, x_{2}\right\}$; observe ainda que $R^{\prime}=R \backslash\left\{x_{1}, x_{2}\right\}$ constitui uma classe de raízes de $\phi^{\prime}$ em $c$, pois, dados $x^{\prime}, x^{\prime \prime} \in$ $R \backslash\left\{x_{1}, x_{2}\right\} \subseteq \operatorname{Int} M \backslash \bar{B}$, e sendo $\alpha: I \longrightarrow M$ um caminho ligando $x^{\prime}$ a $x^{\prime \prime}$, tal que $\phi \circ \alpha: I \longrightarrow N$ é um laço contrátil em $c$, podemos deformar $\alpha$ numa vizinhança de cada ponto $y=\alpha(t) \in \bar{B}$, de modo a encontrar um caminho $\beta: I \longrightarrow X \backslash \bar{B}$, ligando $x^{\prime}$ a $x^{\prime \prime}$, tal que $\phi \circ \beta=\phi^{\prime} \circ \beta$ é homotópico a $\phi \circ \alpha$. Por outro lado, como $\phi^{\prime}$ difere de $\phi$ apenas no compacto $\bar{B} \subseteq$ Int $M$, temos que $\phi^{\prime}$ é uma aplicação própria, enquanto que a aplicação $K^{\prime}:(M \times I, \partial M \times I) \longrightarrow(N, \partial N)$, definida por:

$$
K^{\prime}(x, t)=\left\{\begin{array}{cll}
\left(\pi_{N} \circ K\right)(x, t) & \text { se } x \in \bar{B}, \forall t \in I \\
\phi(x) & \text { se } x \in M \backslash B, \forall t \in I
\end{array},\right.
$$

constitui uma homotopia própria entre $\phi^{\prime}$ e $\phi$, onde $\pi_{N}: Y \longrightarrow \operatorname{Int} N$ é a projeção de $Y=\operatorname{Int} M \times \operatorname{Int} N$ sobre Int $N$ (lembre-se que $K: \bar{B} \times I \longrightarrow Y$ é uma homotopia entre $\hbar=(\bar{e}, \bar{\phi})=\left(\bar{e},\left.\phi^{\prime}\right|_{\bar{B}}\right)$ e $\left.h\right|_{\bar{B}}=\left(\left.e\right|_{\bar{B}},\left.\phi\right|_{\bar{B}}\right)$, tal que $K(x, t)=\hbar(x)=\left(\left.h\right|_{\bar{B}}\right)(x)$ para todo $(x, t) \in \partial B \times I)$. Deste modo, concluímos que $\phi^{\prime}:(M, \partial M, \operatorname{Int} M) \longrightarrow(N, \partial N, \operatorname{Int} N)$ é a aplicação que procurávamos.

Finalmente, vamos mostrar agora que os números de Nielsen de raízes são realizáveis, com respeito a homotopias próprias, desde que a dimensão $n$ das variedades topológicas envolvidas seja diferente de 2 (conforme veremos na seção 3.2 mais adiante, para o caso $n=2$ este resultado geralmente não é válido).

Teorema 2.4.2. Seja $f:(M, \partial M) \longrightarrow(N, \partial N)$ uma aplicação contínua própria, entre variedades topológicas conexas de mesma dimensão $n$, e considere um ponto $c \in \operatorname{Int} N$. Se $n \neq 2$, então existe uma aplicação contínua própria $g:(M, \partial M) \longrightarrow(N, \partial N)$, propriamente homotópica à $f$, que é transversa a c e possui exatamente $\mathrm{N}_{\pitchfork}(f, c)$ raízes em $c$. Em outras palavras, se $n \neq 2,0$ número de Nielsen transverso de raízes é realizável, ou seja, $\mathrm{N}_{\phi}(f, c)=\operatorname{MR}_{\phi}[f ; c]$. 
Demonstração. Primeiramente, suponhamos que $n=1$; neste caso, pelo teorema de classificação das variedades topológicas de dimensão 1 (vide [Le], teorema 6.1, página 118 ), segue que $M$ e $N$ devem ser homeomorfas a subconjuntos conexos de $\mathbb{S}^{1}$ ou $\mathbb{R}$. Logo, sem muitas dificuldades, pode-se verificar que $f$ deve assumir uma das seguintes formas:

(i) $f: \mathbb{R} \longrightarrow \mathbb{R}$;

(ii) $f: \mathbb{S}^{1} \longrightarrow \mathbb{R}$;

(iii) $f: \mathbb{S}^{1} \longrightarrow \mathbb{S}^{1}$;

(iv) $f:(I, \partial I) \longrightarrow(I, \partial I)$;

(v) $f:\left(\mathbb{R}_{+},\{0\}\right) \longrightarrow\left(\mathbb{R}_{+},\{0\}\right)$, onde $\mathbb{R}_{+}=\{x \in \mathbb{R}: x \geqslant 0\}$.

Note que, como $f$ deve ser (por hipótese) uma aplicação contínua própria que preserva fronteira, os demais casos possíveis (isto é, que não contradizem as hipóteses do problema) podem ser reduzidos a um dos cinco listados acima. Deste modo, observando que os casos (i), (ii), e (iii) já foram discutidos respectivamente nos exemplos 2.3.2, 2.3.3 e 2.3.4, enquanto que, em (iv) e (v), a demonstração segue o mesmo raciocínio do exemplo 2.3.2, concluímos que o teorema é válido em dimensão $n=1$.

Isto posto, suponhamos que $n \geqslant 3$; do teorema $C$. 1 do apêndice $C$, sabemos que existe uma aplicação contínua própria $\mathcal{F}:(M, \partial M, \operatorname{Int} M) \longrightarrow(N, \partial N, \operatorname{Int} N)$, propriamente homotópica à $f$, tal que $\mathcal{F}^{-1}(c)=f^{-1}(c)$. Assim, consideremos novamente $X=\operatorname{Int} M$, $Y=\operatorname{Int} M \times \operatorname{Int} N$ e $P=\operatorname{Int} M \times\{c\}$, e seja $\eta=\eta^{n}=(Y, P, r)$ o microfibrado normal (sobre $P$ ) cuja retração $r: Y \longrightarrow P$ é dada por $r(x, y)=(x, c)$ para cada $(x, y) \in Y$; se $W \subseteq \operatorname{Int} M$ é um aberto tal que $f^{-1}(c) \cap W=\varnothing$, segue que a restrição $\left.\mathcal{F}\right|_{W}: W \longrightarrow \operatorname{Int} N$ satisfaz:

$$
\left(\left.\mathcal{F}\right|_{W}\right)^{-1}(c)=W \cap \mathcal{F}^{-1}(c)=W \cap f^{-1}(c)=\varnothing
$$

Logo, por vacuidade, temos que $\left.\mathcal{F}\right|_{W}$ é transversa à $c \in \operatorname{Int} N$, e, conseqüentemente (pela proposição 2.4.1), existe uma aplicação contínua $e: \operatorname{Int} M \longrightarrow \operatorname{Int} M$ tal que a restrição $\left.h\right|_{W}: W \longrightarrow Y$, da aplicação $h=\left(e, \mathcal{F}^{0}\right): X=\operatorname{Int} M \longrightarrow Y=\operatorname{Int} M \times \operatorname{Int} N$, é topologicamente transversa à $\eta$. Por outro lado, sendo $V \subseteq \operatorname{Int} N$ uma n-bola própria contendo $c$, considere $U=\mathcal{F}^{-1}(V) \subseteq \operatorname{Int} M$; como $\bar{V}$ é compacto (uma vez que $\bar{V}$ é homeomorfo ao disco unitário fechado $\mathbb{D}^{n} \subseteq \mathbb{R}^{n}$ ), temos que $\mathcal{F}^{-1}(\bar{V})$ também o é, o que implica que $\bar{U}$ é compacto, pois $\bar{U}=\overline{\mathcal{F}^{-1}(V)} \subseteq \mathcal{F}^{-1}(\bar{V})$. Portanto, temos que $U=\mathcal{F}^{-1}(V)$ é uma vizinhança aberta de $\mathcal{F}^{-1}(c)=f^{-1}(c)$ em $X=\operatorname{Int} M$, tal que $\bar{U}$ é compacto. Agora, lembrando que toda variedade topológica é metrizável, e denotando por $d_{N}: \operatorname{Int} N \longrightarrow \mathbb{R}_{+}$uma métrica em Int $N$, seja $\epsilon: X \longrightarrow \mathbb{R}_{+}$a aplicação contínua definida por:

$$
\epsilon(x)=\frac{d_{N}(c, \mathcal{F}(x))}{2}
$$


para cada $x \in X=\operatorname{Int} M$, e considere a métrica $d_{Y}: Y=\operatorname{Int} M \times \operatorname{Int} N \longrightarrow \mathbb{R}_{+}$dada por:

$$
d_{Y}\left((x, y),\left(x^{\prime}, y^{\prime}\right)\right)=d_{M}\left(x, x^{\prime}\right)+d_{N}\left(y, y^{\prime}\right)
$$

para cada $(x, y),\left(x^{\prime}, y^{\prime}\right) \in Y$, onde $d_{M}:$ Int $M \longrightarrow \mathbb{R}_{+}$é uma métrica em Int $M$. Utilizando o teorema 2.4.1 (com $C=\varnothing, V_{C}=W, D=f^{-1}(c)=\mathcal{F}^{-1}(c)$ e $\left.V_{D}=U\right)$, segue então que existe uma vizinhança aberta $V_{\epsilon} \subseteq X$ de $f^{-1}(c)=\mathcal{F}^{-1}(c)$, bem como uma homotopia $H_{\epsilon}: X \times I \longrightarrow Y$, entre $h=\left(e, \mathcal{F}^{0}\right)$ e outra aplicação contínua $h^{\prime}: X \longrightarrow Y$, tais que:

(I) $H_{\epsilon}(x, t)=h(x)$ para todo $x \in X \backslash U$ e todo $t \in I$;

(II) a restrição $\left.h^{\prime}\right|_{V_{\epsilon}}: V_{\epsilon} \longrightarrow Y$ é topologicamente transversa à $\eta$;

(III) $d_{Y}\left(H_{\epsilon}(x, t), h(x)\right) \leqslant \epsilon(x)$ para todo $(x, t) \in X \times I$.

Em particular, sendo $h_{1}^{\prime}: \operatorname{Int} M \longrightarrow \operatorname{Int} M$ e $h_{2}^{\prime}: \operatorname{Int} M \longrightarrow \operatorname{Int} N$ as aplicações contínuas tais que $h^{\prime}=\left(h_{1}^{\prime}, h_{2}^{\prime}\right): X=\operatorname{Int} M \longrightarrow Y=\operatorname{Int} M \times \operatorname{Int} N$, segue (do item (I) dado acima) que $\left(h_{1}^{\prime}(x), h_{2}^{\prime}(x)\right)=h^{\prime}(x)=h(x)=(e(x), \mathcal{F}(x))$ para todo $x \in X \backslash U$, o que significa que $h_{2}^{\prime}(x)=\mathcal{F}(x)$ para cada $x \in X \backslash U$ (isto é, $h_{2}^{\prime}$ coincide com $\mathcal{F}$ em $X \backslash U$ ). Além disso, se $\left.h^{\prime}\right|_{V_{\epsilon}}=\left(\left.h_{1}^{\prime}\right|_{V_{\epsilon}},\left.h_{2}^{\prime}\right|_{V_{\epsilon}}\right): V_{\epsilon} \longrightarrow Y$ é topologicamente transversa à $\eta$, temos (da proposição 2.4.1) que a restrição $\left.h_{2}^{\prime}\right|_{V_{\epsilon}}: V_{\epsilon} \longrightarrow$ Int $N$ é transversa à $c$. Entretanto, do item (III) acima, segue que:

$$
\begin{aligned}
d_{N}\left(h_{2}^{\prime}(x), \mathcal{F}(x)\right) & =d_{M}\left(h_{1}^{\prime}(x), e(x)\right)+d_{N}\left(h_{2}^{\prime}(x), \mathcal{F}(x)\right) \\
& =d_{Y}\left(\left(h_{1}^{\prime}(x), h_{2}^{\prime}(x)\right),(e(x), \mathcal{F}(x))\right) \\
& =d_{Y}\left(h^{\prime}(x), h(x)\right) \leqslant \frac{d_{N}(c, \mathcal{F}(x))}{2}
\end{aligned}
$$

para todo $x \in X$. Logo, se $x \in\left(h_{2}^{\prime}\right)^{-1}(c)$, temos que

$$
d_{N}(c, \mathcal{F}(x)) \leqslant \frac{d_{N}(c, \mathcal{F}(x))}{2}
$$

ou seja, $d_{N}(c, \mathcal{F}(x))=0$, o que implica que $\mathcal{F}(x)=c$; em contrapartida, se $x \in \mathcal{F}^{-1}(c)$, segue que:

$$
d_{N}\left(h_{2}^{\prime}(x), c\right) \leqslant \frac{d_{N}(c, c)}{2}=0
$$

isto é, $d_{N}\left(h_{2}^{\prime}(x), c\right)=0$, o que, por sua vez, significa que $h_{2}^{\prime}(x)=c$. Deste modo, obtemos que $\left(h_{2}^{\prime}\right)^{-1}(c)=\mathcal{F}^{-1}(c)=f^{-1}(c)$, e, conseqüentemente, como $V_{\epsilon} \subseteq X=\operatorname{Int} M$ é uma vizinhança aberta de $f^{-1}(c)=\left(h_{2}^{\prime}\right)^{-1}(c)$, e a restrição $\left.h_{2}^{\prime}\right|_{V_{\epsilon}}: V_{\epsilon} \longrightarrow \operatorname{Int} N$ é transversa à $c$, segue que $h_{2}^{\prime}: \operatorname{Int} M \longrightarrow \operatorname{Int} N$ é transversa a $c$. Assim, da proposição 2.4.1, concluímos que existe uma aplicação contínua $\lambda: \operatorname{Int} M \longrightarrow \operatorname{Int} M$ tal que $\hbar=$ $\left(\lambda, h_{2}^{\prime}\right): X=\operatorname{Int} M \longrightarrow Y=\operatorname{Int} M \times \operatorname{Int} N$ é topologicamente transversa ao microfibrado 
$\eta$. Ademais, como $h_{2}^{\prime}(x)=\mathcal{F}(x)$ para todo $x \in X \backslash U \supseteq \partial M$, temos que a aplicação $\varphi:(M, \partial M, \operatorname{Int} M) \longrightarrow(N, \partial N, \operatorname{Int} N)$, definida por

$$
\varphi(x)=\left\{\begin{array}{lll}
h_{2}^{\prime}(x) & \text { se } & x \in \operatorname{Int} M \\
\mathcal{F}(x) & \text { se } & x \in \partial M
\end{array},\right.
$$

encontra-se bem definida e é contínua em $M$. Note ainda que $\varphi$ é própria e transversa à $c$ (pois $\varphi$ difere de $\mathcal{F}$ apenas no compacto $\bar{U} \subseteq \operatorname{Int} M$, e $h_{2}^{\prime}=\left.\varphi\right|_{\text {Int } M}$ é transversa à $c$ ), além de ser propriamente homotópica à $\mathcal{F}$, pela homotopia $H:(M \times I, \partial M \times I) \longrightarrow(N, \partial N)$ dada por:

$$
H(x, t)=\left\{\begin{array}{cl}
\left(\pi_{N} \circ H_{\epsilon}\right)(x, t) & \text { se } x \in \operatorname{Int} M \\
\mathcal{F}(x) & \text { se } x \in \partial M
\end{array},\right.
$$

onde $\pi_{N}: Y \longrightarrow$ Int $N$ é a projeção de $Y=\operatorname{Int} M \times \operatorname{Int} N$ sobre Int $N$ (lembre-se que $H_{\epsilon}(x, t)=h(x)=(e(x), \mathcal{F}(x))$ para todo $x \in X \backslash U \supseteq \partial M$, de modo que $H$ está bem definida).

Agora, lembremos que o fato de $\varphi$ ser transversa à $c$, significa que existe uma vizinhança euclidiana $E_{c} \subseteq \operatorname{Int} N$ de $c$ tal que $\varphi^{-1}\left(E_{c}\right)$ é uma união finita e disjunta de vizinhanças euclidianas $E_{1}, E_{2}, \ldots, E_{s} \subseteq$ Int $M$, cada uma das quais é levada homeomorficamente por $\varphi$ sobre $E_{c}$; daí, segue que $\varphi^{-1}(c)$ é um subconjunto discreto e finito de Int $M$, digamos $\varphi^{-1}(c)=\left\{x_{1}, x_{2}, \ldots, x_{s}\right\}, \operatorname{com} x_{i} \in E_{i}$ para cada $i \in\{1,2, \ldots, s\}$. No entanto, sendo $R=\left\{x_{i_{1}}, x_{i_{2}}, \ldots, x_{i_{k}}\right\} \subseteq \varphi^{-1}(c)$ uma classe de raízes de $\varphi$ em $c$, sabemos (pelo que vimos no exemplo 1.3.7) que:

$$
|m(R)|=\left|\sum_{j=1}^{k} \operatorname{deg}_{c}\left(\left.\varphi\right|_{E_{i_{j}}}\right)\right| ;
$$

$\operatorname{logo}$, como $\left.\varphi\right|_{E_{i_{j}}}: E_{i_{j}} \longrightarrow E_{c}$ é um homeomorfismo sobre $E_{c}$ para cada $j \in\{1,2, \ldots, k\}$, temos que $\operatorname{deg}_{c}\left(\left.\varphi\right|_{E_{i_{j}}}\right)= \pm 1$, o que implica que $|m(R)| \leqslant k$. Além disso, se $k>|m(R)|$, segue que existem pelo menos dois pontos $x_{j_{1}}, x_{j_{2}} \in R=\left\{x_{i_{1}}, x_{i_{2}}, \ldots, x_{i_{k}}\right\}$ tais que $\operatorname{deg}_{c}\left(\left.\varphi\right|_{E_{j_{1}}}\right)=$ $-\operatorname{deg}_{c}\left(\left.\varphi\right|_{E_{j_{2}}}\right)$, o que mostra que $k$ deve ser da forma $k=|m(R)|+2 \cdot \ell$, onde $\ell$ é um número natural positivo.

Considere então uma classe de raízes $R$ de $\varphi$ em $c$, contendo uma quantidade (finita) $k>|m(R)|$ pontos; como $\hbar=\left(\lambda, h_{2}^{\prime}\right)=\left(\lambda, \varphi^{0}\right)$ é topologicamente transversa à $\eta$, segue (do lema 2.4.4) que existem pelo menos dois pontos $x_{1}, x_{2} \in R$ que estão $\mathcal{R}$-relacionados com respeito à aplicação $\hbar$. Portanto, de maneira análoga ao que fizemos na demonstração do lema 2.4.5, podemos utilizar o lema 2.4.2 para mostrar que existe uma aplicação contínua própria $\varphi^{\prime}:(M, \partial M, \operatorname{Int} M) \longrightarrow(N, \partial N, \operatorname{Int} N)$, transversa à $c$ e propriamente homotópica à $\varphi$, tal que, exceto por $x_{1}$ e $x_{2}, \varphi^{\prime}$ possui as mesmas raízes de $\varphi$ em $c$ (isto é, $\left.\left(\varphi^{\prime}\right)^{-1}(c)=\varphi^{-1}(c) \backslash\left\{x_{1}, x_{2}\right\}\right)$, e $R^{\prime}=R \backslash\left\{x_{1}, x_{2}\right\}$ é uma clase de raízes de $\varphi^{\prime}$ em $c$. Como 
este processo não altera a composição das demais classes de raízes de $\varphi$ em $c$, podemos aplicá-lo a cada uma das classes de raízes de $\varphi$ em $c$, "removendo" pares de pontos por meio de homotopias próprias até que suas cardinalidades sejam iguais a $|m(R)|$, obtendo enfim uma aplicação contínua própria $g:(M, \partial M$, Int $M) \longrightarrow(N, \partial N, \operatorname{Int} N)$, transversa à $c$ e propriamente homotópica a $\varphi$, cujas classes de raízes em $c$ contêm exatamente $|m(R)|$ pontos; observe que, se $|m(R)|=0$ (e, conseqüentemente, $\mathrm{N}_{\phi}(\varphi, c)=0$, uma vez que todas as classes de raízes de $\varphi$ em $c$ têm a mesma multiplicidade), segue que $g$ não possui raízes em $c$.

Finalmente, note então que o número de classes de raízes de $g$ em $c$ é igual ao número $r \geqslant 0$ de classes de raízes essenciais de $\varphi$ em $c$; logo, a quantidade de raízes de $g$ em $c$ é $r \cdot|m(R)|$. Ora, da definição 2.3.3, sabemos que $\mathrm{N}_{\pitchfork}(\varphi, c)=r \cdot|m(R)|$; no entanto, como $\varphi:(M, \partial M, \operatorname{Int} M) \longrightarrow(N, \partial N, \operatorname{Int} N)$ é propriamente homotópica à aplicação $\mathcal{F}:(M, \partial M, \operatorname{Int} M) \longrightarrow(N, \partial N, \operatorname{Int} N)$, e esta, por sua vez, é propriamente homotópica à $f:(M, \partial M) \longrightarrow(N, \partial N)$, temos (do corolário 2.3.2) que $\mathrm{N}_{\phi}(\varphi, c)=\mathrm{N}_{\phi}(\mathcal{F}, c)=\mathrm{N}_{\phi}(f, c)$, d'onde concluímos que $g:(M, \partial M, \operatorname{Int} M) \longrightarrow(N, \partial N$, Int $N)$ é uma aplicação contínua própria, transversa à $c$ e propriamente homotópica à $f$, que possui exatamente $\mathrm{N}_{\pitchfork}(f, c)$ raízes em $c$, provando que o número de Nielsen transverso $\mathrm{N}_{\phi}(f, c)$ é realizável.

Agora, a fim de mostrarmos que o número de Nielsen $\mathrm{N}(f, c)$ também é realizável, precisaremos do seguinte lema:

Lema 2.4.6. Sendo $\phi:(M, \partial M, \operatorname{Int} M) \longrightarrow(N, \partial N$, Int $N)$ uma aplicação contínua própria (entre variedades topológicas conexas de mesma dimensão $n \geqslant 2$ ), transversa a um ponto $c \in$ Int $N$, considere um ponto $x_{0} \in \phi^{-1}(c)$, pertencente a uma classe de raízes $R$ de $\phi$ em $c$, e sejam $B_{0} \subseteq \operatorname{Int} M$ e $C \subseteq$ Int $N$ n-bolas próprias contendo $x_{0}$ e c respectivamente, com $\overline{B_{0}} \subseteq \operatorname{Int} M e \overline{B_{0}} \cap \phi^{-1}(c)=\left\{x_{0}\right\}$, tais que $B_{0}$ é levada homeomorficamente por $\phi$ sobre $C$. Se $\operatorname{deg}_{c}\left(\left.\phi\right|_{B_{0}}\right)=1$ então, dado um inteiro $\ell \geqslant 1$ arbitrário, existem pontos $x_{1}, x_{2}, \ldots, x_{\ell} \in B_{0}$, bem como uma aplicação contínua própria $\phi^{\prime}:(M, \partial M, \operatorname{Int} M) \longrightarrow(N, \partial N, \operatorname{Int} N)$, propriamente homotópica à $\phi$, tais que:

(i) $\phi^{\prime}(x)=\phi(x)$ para todo $x \in M \backslash B_{0}$;

(ii) $\overline{B_{0}} \cap\left(\phi^{\prime}\right)^{-1}(c)=\left\{x_{0}, x_{1}, x_{2}, \ldots, x_{\ell}\right\}$;

(iii) $R^{\prime}=R \cup\left\{x_{1}, x_{2}, \ldots, x_{\ell}\right\}$ é uma classe de raízes de $\phi^{\prime}$ em $c$;

(iv) $x_{1}$ está contido numa n-bola própria $B_{1} \subseteq B_{0}$, tal que $\overline{B_{1}} \subseteq B_{0}, \overline{B_{1}} \cap\left(\phi^{\prime}\right)^{-1}(c)=\left\{x_{1}\right\}$, $\operatorname{deg}_{c}\left(\left.\phi^{\prime}\right|_{B_{1}}\right)=\ell-1$, e a restrição $\left.\phi^{\prime}\right|_{M \backslash B_{1}}: M \backslash B_{1} \longrightarrow N \backslash C$ é transversa à c, e satisfaz $\left(\left.\phi^{\prime}\right|_{M \backslash B_{1}}\right)\left(\operatorname{Int}\left(M \backslash B_{1}\right)\right) \subseteq \operatorname{Int}(N \backslash C) e\left(\left.\phi^{\prime}\right|_{M \backslash B_{1}}\right)\left(\partial M \cup \partial B_{1}\right) \subseteq \partial N \cup \partial C ;$ 
(v) os pontos $x_{2}, x_{3}, \ldots, x_{\ell}$ estão respectivamente contidos em vizinhanças euclidianas $U_{2}, U_{3}$, $\ldots, U_{\ell} \subseteq \operatorname{Int} M$, que são levadas homeomorficamente por $\phi^{\prime}$ sobre uma mesma vizinhança euclidiana de c em Int $N$, tais que $\operatorname{deg}_{c}\left(\left.\phi^{\prime}\right|_{U_{i}}\right)=-1$ para cada $i \in\{2,3, \ldots, \ell\}$.

DemonstraÇÃo. Vide [Bk3], lema 5.35, páginas 425 e 426.

Teorema 2.4.3 . Seja $f:(M, \partial M) \longrightarrow(N, \partial N)$ uma aplicação contínua própria, entre variedades topológicas conexas de mesma dimensão $n$, e considere um ponto $c \in \operatorname{Int} N$. Se $n \neq 2$, então existe uma aplicação contínua própria $g:(M, \partial M) \longrightarrow(N, \partial N)$, propriamente homotópica à $f$, que possui exatamente $\mathrm{N}(f, c)$ raízes em $c$. Em outras palavras, se $n \neq 2$, o número de Nielsen de raízes é realizável, ou seja, $\mathrm{N}(f, c)=\operatorname{MR}[f ; c]$.

DemonstraÇÃo. Conforme observamos na demonstração do teorema 2.4.2, podemos supor que $n \geqslant 3$, uma vez que o caso $n=1$ segue de imediato dos exemplos 2.3.2, 2.3.3 e 2.3.4. Por outro lado, utilizando o teorema 2.4.2, sabemos que existe uma aplicação contínua própria $\phi_{0}:(M, \partial M, \operatorname{Int} M) \longrightarrow(N, \partial N, \operatorname{Int} N)$, transversa à $c \in \operatorname{Int} N$ e propriamente homotópica à $f$, contendo exatamente $r=\mathrm{N}(f, c)$ classes de raízes em $c$, sendo que cada uma delas, por sua vez, contém o mesmo número $k \geqslant 0$ de raízes (mais precisamente, $\phi_{0}$ é a aplicação $g$ mencionada na demonstração do teorema 2.4.2). Isto posto, se $\mathrm{N}(f, c)=0$, segue que $\phi_{0}$ não possui raízes em $c$, d'onde concluímos que $\phi_{0}$ é a aplicação que procuramos. Suponhamos então que $r=\mathrm{N}(f, c) \geqslant 1$, e seja $R_{0}=\left\{x_{1}, x_{2}, \ldots, x_{k}\right\}$ uma classe de raízes de $\phi_{0}$ em $c$; como $\phi_{0}$ é transversa à $c$, segue que existem vizinhanças euclidianas $U_{1}, U_{2}, \ldots, U_{k} \subseteq$ Int $M$ e $V_{c} \subseteq \operatorname{Int} N$, contendo os pontos $x_{1}, x_{2}, \ldots, x_{k}, c$, respectivamente, tais que, para cada $i \in\{1,2, \ldots, k\}$, a restrição $\left.\phi_{0}\right|_{U_{i}}: U_{i} \longrightarrow V_{c}$ é um homeomorfismo sobre $V_{c}$. Logo, orientando o aberto $U=\bigcup_{i=1}^{k} U_{i}$ pelo processo de orientação (dado na definição 1.3.6), temos que:

$$
\left|m\left(R_{0}\right)\right|=\left|\sum_{i=1}^{k} \operatorname{deg}_{c}\left(\phi_{0} \mid u_{i}\right)\right|
$$

(vide exemplo 1.3.8); assim, como $\left.\phi_{0}\right|_{U_{i}}: U_{i} \longrightarrow V_{c}$ é um homeomorfismo para cada $i \in\{1,2, \ldots, k\}$, temos que $\operatorname{deg}_{c}\left(\left.\phi_{0}\right|_{U_{i}}\right)= \pm 1$, o que implica que $\left|m\left(R_{0}\right)\right| \leqslant k$. Observe então que, se $\left|m\left(R_{0}\right)\right|<k$, segue que existem $i_{1}, i_{2} \in\{1,2, \ldots, k\}$ tais que $\operatorname{deg}_{c}\left(\left.\phi_{0}\right|_{U_{i_{1}}}\right)=-\operatorname{deg}_{c}\left(\left.\phi_{0}\right|_{U_{i_{2}}}\right)$; portanto, pelo lema 2.4.5, existe uma aplicação contínua própria $\phi_{0}^{\prime}:(M, \partial M, \operatorname{Int} M) \longrightarrow(N, \partial N, \operatorname{Int} N)$, transversa à c e propriamente homotópica à $\phi_{0}$, tal que toda classe de raízes $R \neq R_{0}$ de $\phi_{0}$ em $c$ constitui uma classe de raízes de $\phi_{0}^{\prime}$ em $c$, e $R_{0}^{\prime}=R_{0} \backslash\left\{x_{i_{1}}, x_{i_{2}}\right\}$ é uma classe de raízes de $\phi_{0}^{\prime}$ em $c$. Deste modo, aplicando repetidamente o lema 2.4.5, a fim de "remover" os pares de pontos $x_{i}, x_{j} \in R_{0}$ 
tais que $\operatorname{deg}_{c}\left(\left.\phi_{0}\right|_{U_{i}}\right)=-\operatorname{deg}_{c}\left(\left.\phi_{0}\right|_{U_{j}}\right)$, obtemos uma aplicação contínua própria $\phi_{1}$ : $(M, \partial M, \operatorname{Int} M) \longrightarrow(N, \partial N, \operatorname{Int} N)$, transversa à $c$ e propriamente homotópica à $\phi_{0}$, tal que $\phi_{1}$ possui as mesmas classes de raízes de $\phi_{0}$ em $c$, com exceção de $R_{0}$, que é "substituída" por uma classe de raízes $R_{1} \subseteq R_{0}$ contendo exatamente $\ell=\left|m\left(R_{0}\right)\right|$ pontos, digamos $x_{\lambda_{1}}$, $x_{\lambda_{2}}, \ldots, x_{\lambda_{\ell}} \in R_{0}, \operatorname{com} \operatorname{deg}_{c}\left(\left.\phi_{0}\right|_{U_{\lambda_{i}}}\right)=\operatorname{deg}_{c}\left(\left.\phi_{0}\right|_{U_{\lambda_{j}}}\right)$ para quaisquer $i, j \in\{1,2, \ldots, \ell\}$. Note que $\ell \geqslant 1$, pois, se $\ell=\left|m\left(R_{0}\right)\right|=0$, teríamos que $\mathrm{N}(f, c)=\mathrm{N}\left(\phi_{0}, c\right)=0$, uma vez que quaisquer duas classes de raízes de $\phi_{0}$ em $c$ possuem a mesma multiplicidade (teorema 2.1.2).

Agora, observe que, sem perda de generalidade, podemos supor $\operatorname{deg}_{c}\left(\left.\phi_{0}\right|_{U_{\lambda_{i}}}\right)=1$ para cada $i \in\{1,2, \ldots, \ell\}$ (pois, caso contrário, basta "invertermos" a orientação do aberto $U$ adotada inicialmente). Por outro lado, da transversalidade de $\phi_{1}$ em relação ao ponto $c$, sabemos que existem n-bolas próprias $B \subseteq \operatorname{Int} M$ e $C \subseteq \operatorname{Int} N$, contendo $x_{\lambda_{1}}$ e $c$, respectivamente, com $\bar{B} \subseteq U_{\lambda_{1}} \subseteq$ Int $M$ e $\bar{B} \cap \phi_{1}^{-1}(c)=\left\{x_{\lambda_{1}}\right\}$, tais que $B$ é levada homeomorficamente por $\phi_{1}$ sobre $C$. Logo, pelo lema 2.4.6, segue que existem pontos $x_{\lambda_{1}}^{\prime}, x_{\lambda_{2}}^{\prime}, \ldots, x_{\lambda_{\ell+1}}^{\prime} \in B$, bem como uma aplicação contínua própria $\phi_{1}^{\prime}:(M, \partial M, \operatorname{Int} M) \longrightarrow$ $(N, \partial N, \operatorname{Int} N)$, propriamente homotópica à $\phi_{1}$, tais que:

(i) $\phi_{1}^{\prime}(x)=\phi_{1}(x)$ para todo $x \in M \backslash B$;

(ii) $\bar{B} \cap\left(\phi_{1}^{\prime}\right)^{-1}(c)=\left\{x_{\lambda_{1}}, x_{\lambda_{1}}^{\prime}, x_{\lambda_{2}}^{\prime}, \ldots, x_{\lambda_{\ell+1}}^{\prime}\right\}$;

(iii) $R_{1}^{\prime}=R_{1} \cup\left\{x_{\lambda_{1}}^{\prime}, x_{\lambda_{2}}^{\prime}, \ldots, x_{\lambda_{\ell+1}}^{\prime}\right\}=\left\{x_{\lambda_{1}}, x_{\lambda_{2}}, \ldots, x_{\lambda_{\ell}}, x_{\lambda_{1}}^{\prime}, x_{\lambda_{2}}^{\prime}, \ldots, x_{\lambda_{\ell+1}}^{\prime}\right\}$ é uma classe de raízes de $\phi_{1}^{\prime}$ em $c$;

(iv) $x_{\lambda_{\ell+1}}^{\prime}$ está contido numa n-bola própria $B_{1} \subseteq B$, tal que $\overline{B_{1}} \subseteq B, \overline{B_{1}} \cap\left(\phi_{1}^{\prime}\right)^{-1}(c)=\left\{x_{\lambda_{\ell+1}}^{\prime}\right\}$, $\operatorname{deg}_{c}\left(\left.\phi_{1}^{\prime}\right|_{B_{1}}\right)=\ell$, e a restrição $\left.\phi_{1}^{\prime}\right|_{M \backslash B_{1}}: M \backslash B_{1} \longrightarrow N \backslash C$ é transversa à $c$ e satisfaz $\left(\left.\phi_{1}^{\prime}\right|_{M \backslash B_{1}}\right)\left(\operatorname{Int}\left(M \backslash B_{1}\right)\right) \subseteq \operatorname{Int}(N \backslash C)$ e $\left(\left.\phi_{1}^{\prime}\right|_{M \backslash B_{1}}\right)\left(\partial M \cup \partial B_{1}\right) \subseteq \partial N \cup \partial C ;$

(v) os pontos $x_{\lambda_{1}}^{\prime}, x_{\lambda_{2}}^{\prime}, \ldots, x_{\lambda_{\ell}}^{\prime}$ estão respectivamente contidos em vizinhanças euclidianas $U_{1}^{\prime}, U_{2}^{\prime}, \ldots, U_{\ell}^{\prime} \subseteq \operatorname{Int} M$, que são levadas homeomorficamente por $\phi_{1}^{\prime}$ sobre uma mesma vizinhança euclidiana de $c$ em $\operatorname{Int} N$, tais que $\operatorname{deg}_{c}\left(\left.\phi_{1}^{\prime}\right|_{U_{i}^{\prime}}\right)=-1$ para cada $i \in\{1,2, \ldots, \ell\}$.

No entanto, de (i) e (v), temos que $\operatorname{deg}_{c}\left(\left.\phi_{1}^{\prime}\right|_{U_{\lambda_{i}}}\right)=-\operatorname{deg}_{c}\left(\left.\phi_{1}^{\prime}\right|_{U_{\lambda_{i}}^{\prime}}\right)$ para todo $i \in\{1,2, \ldots$, $\ell\}$; assim, como a restrição $\left.\phi_{1}^{\prime}\right|_{M \backslash B_{1}}:\left(M \backslash B_{1}, \operatorname{Int}\left(M \backslash B_{1}\right), \partial M \cup \partial B_{1}\right) \longrightarrow(N \backslash C, \operatorname{Int}(N \backslash$ $C), \partial N \cup \partial C$ ) é uma aplicação contínua própria transversa à $c$, com $\overline{B_{1}} \cap\left(\phi_{1}^{\prime}\right)^{-1}(c)=$ $\left\{x_{\lambda_{\ell+1}}^{\prime}\right\}$, podemos aplicar novamente o lema 2.4.5, "removendo" os pares de pontos $x_{\lambda_{i}}$, $x_{\lambda_{i}}^{\prime} \in R_{1}^{\prime}$, onde $i \in\{1,2, \ldots, \ell\}$, de maneira a obter uma aplicação contínua própria $\psi_{0}^{\prime}:\left(M \backslash B_{1}, \operatorname{Int}\left(M \backslash B_{1}\right), \partial M \cup \partial B_{1}\right) \longrightarrow(N \backslash C, \operatorname{Int}(N \backslash C), \partial N \cup \partial C)$, transversa à $c$ e propriamente homotópica à $\left.\phi_{1}^{\prime}\right|_{M \backslash B_{1}}$, que possui as mesmas classes de raízes de $\left.\phi_{1}^{\prime}\right|_{M \backslash B_{1}}$, com exceção de $R_{1}^{\prime} \backslash\left\{x_{\lambda_{\ell+1}}^{\prime}\right\}=\left\{x_{\lambda_{1}}, x_{\lambda_{2}}, \ldots, x_{\lambda_{\ell}}, x_{\lambda_{1}}^{\prime}, x_{\lambda_{2}}^{\prime}, \ldots, x_{\lambda_{\ell}}^{\prime}\right\}$ (a qual "desaparece"), 
e que coincide com $\left.\phi_{1}^{\prime}\right|_{M \backslash B_{1}}$ em $\left(M \backslash B_{1}\right) \backslash W \supseteq \partial B_{1}$, onde $W \subseteq \operatorname{Int}\left(M \backslash B_{1}\right)$ é uma união de n-bolas próprias $B_{1}^{\prime}, B_{2}^{\prime}, \ldots, B_{\ell}^{\prime} \subseteq \operatorname{Int}\left(M \backslash B_{1}\right)$, tais que $\overline{B_{i}^{\prime}} \subseteq \operatorname{Int}\left(M \backslash B_{1}\right)$ e $\overline{B_{i}^{\prime}} \cap\left(\left.\phi_{1}^{\prime}\right|_{M \backslash B_{1}}\right)^{-1}(c)=\left\{x_{\lambda_{i}}, x_{\lambda_{i}}^{\prime}\right\}$ para cada $i \in\{1,2, \ldots, \ell\}$. Deste modo, considerando a aplicação $\psi_{0}:(M, \partial M, \operatorname{Int} M) \longrightarrow(N, \partial N, \operatorname{Int} N)$ dada por:

$$
\psi_{0}(x)=\left\{\begin{array}{ll}
\psi_{0}^{\prime}(x) & \text { se } x \in M \backslash B_{1} \\
\phi_{1}^{\prime}(x) & \text { se } x \in \overline{B_{1}}
\end{array},\right.
$$

podemos ver que $\psi_{0}$ está bem definida e é contínua em $M$ (pois $\phi_{1}^{\prime}$ coincide com $\psi_{0}^{\prime}$ em $\left.\partial B_{1}=\left(M \backslash B_{1}\right) \cap \overline{B_{1}}\right)$, além de ser própria, transversa à $c$ e propriamente homotópica à $\phi_{1}^{\prime}$ (uma vez que $\psi_{0}$ difere de $\phi_{1}^{\prime}$ apenas no compacto $\bar{W} \cup \overline{B_{1}}$, sendo que $\left.\phi_{1}^{\prime}\right|_{B_{1}}$ e $\psi_{0}^{\prime}$ são transversas à $c)$. Note que, de fato, temos $\psi_{0}(\partial M) \subseteq \partial N$ e $\psi_{0}(\operatorname{Int} M) \subseteq \operatorname{Int} N$, pois $\partial M \subseteq\left(M \backslash B_{1}\right) \backslash W$ e $\psi_{0}^{\prime}(\partial M)=\phi_{1}^{\prime}(\partial M) \subseteq \partial N$, enquanto que:

$$
\begin{aligned}
\psi_{0}(\operatorname{Int} M) & =\psi_{0}(\bar{W}) \cup \psi_{0}\left(\overline{B_{1}}\right) \cup \psi_{0}\left(\operatorname{Int} M \backslash\left(B_{1} \cup W\right)\right) \\
& =\psi_{0}^{\prime}(\bar{W}) \cup \phi_{1}^{\prime}\left(\overline{B_{1}}\right) \cup \phi_{1}^{\prime}\left(\operatorname{Int} M \backslash\left(B_{1} \cup W\right)\right) \\
& \subseteq \psi_{0}^{\prime}\left(\operatorname{Int}\left(M \backslash B_{1}\right)\right) \cup \phi_{1}^{\prime}(\operatorname{Int} M) \\
& \subseteq \operatorname{Int}(N \backslash C) \cup \operatorname{Int} N=\operatorname{Int} N .
\end{aligned}
$$

Observe ainda que as classes de raízes de $\phi_{1}{ }^{\prime} \operatorname{em} c$, distintas de $R_{1}^{\prime}=\left\{x_{\lambda_{1}}, x_{\lambda_{2}}, \ldots, x_{\lambda_{\ell}}, x_{\lambda_{1}}^{\prime}\right.$, $\left.x_{\lambda_{2}}^{\prime}, \ldots, x_{\lambda_{\ell+1}}^{\prime}\right\}$ (que também são classes de raízes de $\phi_{1}$ em $c$, e, portanto, de $\phi_{0}$ em $c$ ), constituem classes de raízes de $\psi_{0}$ em $c$; em contrapartida, a classe $R_{1}^{\prime}$ foi "substituída" por $R_{\psi_{0}}=\left\{x_{\lambda_{\ell+1}}^{\prime}\right\} \subseteq B_{1}$, de modo que $\psi_{0}$ possui o mesmo número $r=\mathrm{N}(f, c) \geqslant 1$ de classes de raízes de $\phi_{0}$ em $c$.

Finalmente, repetindo o mesmo procedimento descrito acima para as demais classes de raízes de $\phi_{0}$ em $c$, obtemos uma aplicação contínua própria $g:(M, \partial M, \operatorname{Int} M) \longrightarrow$ $(N, \partial N, \operatorname{Int} N)$, propriamente homotópica à $\phi_{0}$, que contém exatamente $\mathrm{N}(f, c)$ classes de raízes em $c$, cada uma das quais contendo exatamente uma raiz. Logo, como $\phi_{0}$ é propriamente homotópica à $f$, segue que $g$ é a aplicação que procurávamos.

Para encerrarmos esta seção, veremos agora alguns exemplos que ilustram os teoremas 2.4.2 e 2.4.3.

Exemplo 2.4.2. Seja $f: \mathbb{S}^{n} \longrightarrow \mathbb{S}^{n}$ uma aplicação contínua (própria) de grau $\mathrm{d}(f)=d$, com $|d|>1$, e considere um ponto $c \in \mathbb{S}^{n}$. Se $n \geqslant 3$, segue (do exemplo 2.3 .5 e do teorema 2.4.3) que existe uma aplicação contínua (própria) $g: \mathbb{S}^{n} \longrightarrow \mathbb{S}^{n}$, propriamente homotópica à $f$, que possui exatamente $\mathrm{N}(f, c)=1$ raiz em $c$. Entretanto, como $\mathrm{N}_{\phi}(f, c)=|d|>1$ (vide exemplo 2.3.5), segue que esta aplicação $g: \mathbb{S}^{n} \longrightarrow \mathbb{S}^{n}$ não pode ser transversa à $c$, pois qualquer aplicação transversa à $c$ e propriamente homotópica 
à $f$, deve ter no mínimo $\mathrm{N}_{\phi}(f, c)=|d|$ raízes em $c$. Com efeito, o teorema 2.4.2 nos garante que existe uma aplicação contínua $g^{\prime}: \mathbb{S}^{n} \longrightarrow \mathbb{S}^{n}$, transversa à c e propriamente homotópica à $f$, que possui exatamente $|d|$ raízes em $c$.

Exemplo 2.4.3. Seja $f: \mathbb{S}^{2} \times \mathbb{R} P^{2} \longrightarrow \mathbb{S}^{2} \times \mathbb{R}^{2}$ a aplicação contínua dada por $f=f^{\prime} \times \operatorname{Id}$, onde Id $: \mathbb{R} P^{2} \longrightarrow \mathbb{R} P^{2}$ é a identidade e $f^{\prime}: \mathbb{S}^{2} \longrightarrow \mathbb{S}^{2}$ é uma aplicação contínua de grau $\mathrm{d}\left(f^{\prime}\right)=d$, com $|d|>1$. Temos então que $f$ é uma aplicação própria entre variedades topológicas de dimensão 4 , com $\mathrm{N}(f, c)=1$ e $\mathrm{N}_{\phi}(f, c)=|d|>1$, onde $c \in \mathbb{S}^{2} \times \mathbb{R P}^{2}$ (vide exemplo 2.3.7). Assim, do teorema 2.4.3, segue que existe uma aplicação contínua (própria) $g: \mathbb{S}^{2} \times \mathbb{R} P^{2} \longrightarrow \mathbb{S}^{2} \times \mathbb{R P}^{2}$, propriamente homotópica à $f$, que possui apenas uma raiz em $c$. Entretanto, tal aplicação $g$ não pode ser transversa à $c$, uma vez que qualquer aplicação propriamente homotópica à $f$ e transversa à $c$ deve possuir pelo menos $\mathrm{N}_{\pitchfork}(f, c)=|d|>1$ raízes em $c$. De fato, pelo teorema 2.4.2, sabemos que existe uma aplicação contínua própria $g^{\prime}: \mathbb{S}^{2} \times \mathbb{R P}^{2} \longrightarrow \mathbb{S}^{2} \times \mathbb{R} P^{2}$, transversa à c e propriamente homotópica à $f$, com exatamente $|d|$ raízes em $c$.

Exemplo 2.4.4 . Denotando por $\mathbb{T}^{n}$ o $n$-toro (isto é, o produto cartesiano de $n$ cópias de $\left.\mathbb{S}^{1}\right)$, com $n \geqslant 3$, seja $f: \mathbb{T}^{n} \longrightarrow \mathbb{T}^{n}$ uma aplicação contínua, e considere $c \in \mathbb{T}^{n}$ e $x_{0} \in f^{-1}(c)$. Como $\mathbb{T}^{n}$ é uma variedade topológica compacta e orientável, temos que $f$ é uma aplicação contínua própria do tipo I. Isto posto, lembrando que $\mathrm{H}_{1}\left(\mathbb{T}^{n}\right) \simeq \mathbb{Z}^{n}$, seja $A=\left\{a_{i j}\right\}$ a matriz quadrada de ordem $n$ do homomorfismo induzido $\left(f_{*}\right)_{1}: \mathrm{H}_{1}\left(\mathbb{T}^{n}\right) \longrightarrow$ $\mathrm{H}_{1}\left(\mathbb{T}^{n}\right)$, relativamente a uma base $\mathcal{B}=\left\{b_{1}, b_{2}, \ldots, b_{n}\right\}$ de $\mathrm{H}_{1}\left(\mathbb{T}^{n}\right)$; do exemplo 4.26 de [Bk3] (páginas 402 e 403), temos que $\operatorname{deg}(f)=\operatorname{det} A$ e $\mathrm{N}(f, c)=|\operatorname{det} A|$. Portanto, do corolário 2.3.1, segue que $\mathrm{N}(f, c)=\mathrm{N}_{\pitchfork}(f, c)=|\operatorname{det} A|$, o que, pelos teoremas 2.4 .2 e 2.4.3, implica que existe uma aplicação contínua própria $g: \mathbb{T}^{n} \longrightarrow \mathbb{T}^{n}$, transversa à $c$ e propriamente homotópica à $f$, que realiza ambos os números de Nielsen $\mathrm{N}(f, c)$ e $\mathrm{N}_{\uparrow}(f, c)$.

Observação 2.4.1. Seja $f: M \longrightarrow N$ uma aplicação contínua própria entre variedades topológicas de mesma dimensão $n \neq 2$, sem fronteira, e considere um ponto $c \in N$. Em [Bk2], página 282, R. Brooks define uma classe de raízes $R$ de $f$ em $c$ como sendo essencial se $R$ não pode ser "removida" por meio de homotopias a partir de $f$. No entanto, em [BS4] (observação 3.2, página 255), os autores mostram que, se $M$ e $N$ forem variedades topológicas fechadas (isto é, compactas e sem fronteira) e orientáveis, então $R$ é essencial, segundo a concepção de Brooks, se, e somente se, $|m(R)| \neq 0$. Do teorema 2.4.3, temos que esta equivalência também é válida se $f$ for uma aplicação própria que 
preserva fronteira (com $M$ e $N$ não necessariamente compactas ou orientáveis): de fato, pelos lemas 2.2.1 e 2.2.2, e pelos corolários 2.2.1 e 2.2.2, temos que a multiplicidade $|m(R)|$ é invariante por homotopias próprias (pois o conceito de grau cohomológico é invariante por homotopias próprias, conforme vimos na proposição 1.3.5), e, portanto, uma classe de raízes $R$ de $f$ em $c$, tal que $|m(R)| \neq 0$ é "preservada" por homotopias próprias; $\log$ o, se $f$ é própria e preserva fronteira, o teorema 2.4 .3 nos garante que existe uma homotopia própria entre $f$ e outra aplicação $g: M \longrightarrow N$ que não possui classes de raízes (em $c$ ) com multiplicidade nula, o que implica que uma classe de raízes $R$ de $f$ em $c$, tal que $|m(R)|=0$, não é "preservada" por toda homotopia própria. 


\section{Capitirnlo 3}

\section{Aplicações à Teoria do Grau de Hopf}

O objetivo deste último capítulo é interpretar alguns conceitos e resultados da teoria de Nielsen de raízes na linguagem da teoria do grau de Hopf, bem como apresentar algumas propriedades destas teorias para aplicações entre superfícies.

\subsection{Os Graus Absoluto e Geométrico}

Se $f:(M, \partial M) \longrightarrow(N, \partial N)$ é uma aplicação contínua própria entre variedades topológicas de mesma dimensão $n$, sabemos que o grau cohomológico de $f, \operatorname{deg}(f)$, encontra-se bem definido somente se as variedades $M$ e $N$ forem orientáveis (vide definição 1.4.4). Entretanto, se uma delas for não-orientável, só podemos definir o grau cohomológico utilizando coeficientes em $\mathbb{Z}_{2}$, obtendo o denominado grau cohomológico $\bmod 2, \operatorname{deg}(f, 2)$, o qual geralmente fornece poucas informações a respeito das propriedades geométricas da aplicação $f$; em particular, ele não reflete o conceito geométrico intuitivo de grau, a saber, o "número de vezes que a imagem $f(M)$ cobre o contra-domínio N". Deste modo, a fim de encontrar um invariante algébrico que caracterizasse o conceito geométrico de grau independentemente da orientabilidade, Hopf acabou por dar significativas contribuições à teoria de Nielsen de raízes, introduzindo um diferente tipo de grau (denominado por ele de "Absolutgrad" ou "grau absoluto"), que fornece informações a respeito das propriedades geométricas de $f$ mesmo quando uma das variedades $M$ ou $N$ (ou ambas) forem não-orientáveis (por outro lado, caso $M$ e $N$ sejam orientáveis, o grau absoluto coincide com o valor absoluto 
do grau cohomológico de $f$, conforme veremos nos teoremas 3.1.2 e 3.1.3 a seguir).

Definição 3.1.1 . Dada uma aplicação contínua própria $f:(M, \partial M) \longrightarrow(N, \partial N)$, entre variedades topológicas conexas de mesma dimensão $n$, e considerando-se um ponto $c \in \operatorname{Int} N$, definimos o grau absoluto de $f$, denotado por $\mathcal{A}(f)$, como sendo a soma das multiplicidades das classes de raízes de $f$ em $c$, isto é, $\mathcal{A}(f)=\sum\{|m(R)|:$ Ré classe de raízes de $f$ em $c\}$.

O motivo que levou Hopf a definir o grau absoluto $\mathcal{A}(f)$ da maneira acima é que, deste modo, $\mathcal{A}(f)$ encontra-se diretamente relacionado ao conceito de grau geométrico (conforme veremos no teorema 3.2.1), o qual é dado precisamente pela seguinte

Definição 3.1.2 . Dada uma aplicação contínua própria $f:(M, \partial M) \longrightarrow(N, \partial N)$, entre variedades topológicas conexas de mesma dimensão $n$, definimos o grau geométrico de $f$, denotado por $\mathcal{G}(f)$, como sendo o menor inteiro positivo para o qual existem uma $n$-bola fechada $B^{n} \subseteq$ Int $N$ e uma aplicação própria $g:(M, \partial M) \longrightarrow(N, \partial N)$, propriamente homotópica à $f$, tais que $g^{-1}\left(B^{n}\right)$ contém exatamente $\mathcal{G}(f)$ componentes conexas, cada uma delas levada homeomorficamente por $g$ sobre $B^{n}$.

Note que, como $f:(M, \partial M) \longrightarrow(N, \partial N)$ é própria, o grau geométrico de $f$ de fato existe, pois $\mathcal{G}(f)$ é limitado superiormente pelo número de raízes num ponto $c \in \operatorname{Int} N$ de uma aplicação contínua $g:(M, \partial M) \longrightarrow(N, \partial N)$, transversa à $c$ e propriamente homotópica à $f$.

Pelas definições acima, podemos ver que os graus absoluto e geométrico são conceitos que, em essência, pertencem à teoria de Nielsen de raízes. Com efeito, das definições 2.3 .3 e 2.3.4, temos de imediato o seguinte resultado:

Teorema 3.1.1. Sejam $M$ e $N$ variedades topológicas conexas de mesma dimensão n, e considere uma aplicação contínua própria $f:(M, \partial M) \longrightarrow(N, \partial N)$. Dado $c \in \operatorname{Int} N$, temos então que $\mathcal{A}(f)=\mathrm{N}_{\phi}(f, c) e \mathcal{G}(f)=\operatorname{MR}_{\phi}[f ; c]$.

Como podemos verificar na introdução de seu artigo [H2], Hopf percebeu que o problema de determinar o grau geométrico é equivalente ao de encontrar o número mínimo de pontos fixos na classe de homotopia de uma aplicação, e ele explica também que sua definição de grau absoluto utiliza uma extensão de certos conceitos para teoria de raízes que, à época, haviam sido recentemente pesquisados por Jakob Nielsen (em [N1] e [N2]) no estudo dos conjuntos de pontos fixos de uma aplicação. No entanto, a motivação de Hopf para a introdução do grau absoluto $A(f)$ não é mencionada em artigos que posteriormente trataram deste assunto. Conforme vimos na introdução 
deste trabalho, Epstein (em [E]) interpretou os cálculos realizados por Olum em [O] em termos dos graus de levantamentos de $f$ relativamente a certas aplicações de recobrimento (mais especificamente, os recobrimentos que ilustramos no diagrama da figura 2.2); em [O], Olum obteve os possíveis valores para o número de Nielsen transverso de raízes $\mathrm{N}_{\pitchfork}(f, c)$, calculados aqui no teorema 2.3.2, de uma maneira bastante diferente (utilizando o que ele chamou de "grau do anel de grupo"). Epstein, por sua vez, classificou as aplicações entre variedades topológicas em três tipos distintos, de modo equivalente ao que fizemos na definição 1.2.5, e então definiu separadamente o "grau absoluto" $\mathcal{A}(f)$ para cada tipo de aplicação $f$ (vide [E], página 371); ele observou, contudo, que, apesar de "complicada", sua definição para $\mathcal{A}(f)$ é justificada pelo significado geométrico proveniente da igualdade entre os graus geométrico e absoluto, demonstrada por Hopf (vide [E], página 371), não fazendo qualquer menção sobre a relação entre a teoria de Nielsen de raízes e o grau absoluto, conforme Hopf idealizou inicialmente. Assim, pelo fato de [E] ser a referência mais citada atualmente quando se trata do grau absoluto (pois contém demonstrações mais modernas para alguns dos importantes teoremas estabelecidos por Hopf), a conexão com a teoria de Nielsen de raízes, que possibilitou a unificação do grau absoluto de Hopf com o geométrico, acabou por não ser preservada na teoria do grau de Hopf.

A equivalência entre os graus absoluto e geométrico para aplicações contínuas entre variedades topológicas de mesma dimensão $n$, $\operatorname{com} n \neq 2$, foi demonstrado primeiramente em [H2], página 607 (uma nova demonstração desta igualdade foi o que motivou o artigo de Epstein, apresentado em [E], teorema 4.1, página 376); tendo em vista as identificações dadas no teorema 3.1.1, segue então que o teorema 2.4.2 nos fornece este mesmo resultado:

Teorema 3.1.2 . Dada uma aplicação contínua própria $f:(M, \partial M) \longrightarrow(N, \partial N)$, entre variedades topológicas conexas de mesma dimensão $n$, com $n \neq 2$, temos que o grau absoluto $\mathcal{A}(f)$ é igual ao grau geométrico $\mathcal{G}(f)$.

Por outro lado, se ambas as variedades $M$ e $N$ forem orientáveis, a teoria do grau de Hopf pode ser relacionada com o grau cohomológico a coeficientes inteiros, o qual encontra-se bem definido neste caso; mais especificamente, do corolário 2.3.1 e dos teoremas 3.1.1 e 3.1.2, obtemos o seguinte

Teorema 3.1.3. Seja $f:(M, \partial M) \longrightarrow(N, \partial N)$ uma aplicação contínua própria, entre variedades topológicas conexas de mesma dimensão $n$. Se $M$ e $N$ forem ambas orientáveis, temos que $\mathcal{A}(f)=|\operatorname{deg}(f)| ;$ além disso, se $n \neq 2$, também temos $\mathcal{G}(f)=|\operatorname{deg}(f)|$. 


\subsection{Os $\mathbb{N}$ ưmeros de $\mathbb{N}$ ielsem de $\mathbb{R}$ ailzes para Aplicações emtre Superficieies}

Nesta seção, vamos analisar alguns resultados sobre a realização dos números de Nielsen de raízes para aplicações entre superfícies, uma vez que os teoremas 2.4.2, 2.4.3, 3.1.2 e 3.1.3 excluíram o caso $n=2$.

Embora desconhecido à época da publicação do trabalho de Hopf, sabemos atualmente que, dada uma aplicação contínua $f: M \longrightarrow M$, onde $M$ é uma variedade topológica de dimensão $n$, o número de Nielsen $\mathrm{N}(f)$, para pontos fixos de $f$, pode ser realizado como o menor número de pontos fixos na classe de homotopia de $f$, desde que tenhamos $n \neq 2$, sendo que esta propriedade, contudo, não é válida (neste nível de generalidade) no caso de $M$ ser uma superfície. Deste modo, se $f: M \longrightarrow N$ é uma aplicação contínua entre superfícies, não é de se surpreender que os números de Nielsen de raízes $\mathrm{N}(f, c)$ e $\mathrm{N}_{\pitchfork}(f, c)$ também não possam ser realizados sem alguma hipótese adicional acerca de $f$ (ou das superfícies $M$ e $N$ ); o que é surpreendente, no entanto, é que $\mathrm{N}_{\pitchfork}(f, c)$ pode ser realizável ainda que $\mathrm{N}(f, c)$ não o seja, conforme veremos a seguir.

Para aplicações contínuas $f: M \longrightarrow N$ entre superfícies, vários autores determinaram condições que tornam válida a igualdade $\mathcal{A}(f)=\mathcal{G}(f)$, a qual, pelo que vimos no teorema 3.1.1, é equivalente à afirmação de que o número de Nielsen transverso $\mathrm{N}_{\uparrow}(f, c)$ é realizável. Um resultado desta natureza é dado pelo teorema abaixo, demonstrado pela primeira vez por H. Kneser em [Kn1] e [Kn2] (e que constitui um corolário do teorema 2.1, página 419, de [Sk]):

Teorema 3.2.1. Se $f: M \longrightarrow N$ é uma aplicação contínua entre superfícies fechadas (isto é, compactas e sem fronteira), então, para todo $c \in N$, o número de Nielsen transverso $\mathrm{N}_{\phi}(f, c)$ é realizável, e, conseqüentemente, $\mathcal{A}(f)=\mathcal{G}(f)$.

Exemplo 3.2.1 . Sendo $f: \mathbb{K} \longrightarrow \mathbb{K}$ a aplicação contínua dada no exemplo 2.3.9, vimos que $\mathrm{N}(f, c)=\mathrm{N}_{\pitchfork}(f, c)=|b \cdot e|$, onde $c \in \mathbb{K}$ e $b, e \in \mathbb{Z}$ são tais que $e \neq 0$ e $|b|$ é um número ímpar. Logo, do teorema acima, segue que $\mathcal{A}(f)=\mathcal{G}(f)=\mathrm{N}_{\pitchfork}(f, c)=|b \cdot e|$, e existe uma aplicação contínua $g: \mathbb{K} \longrightarrow \mathbb{K}$, transversa à $c$ e propriamente homotópica à $f$, que contém exatamente $|b \cdot e|$ raízes em $c$.

Por outro lado, em [Sk], Skora mostrou que, sob certas condições, o teorema 3.2.1 pode ser generalizado para aplicações contínuas que preservam fronteira, entre superfícies não necessariamente compactas; mais especificamente, do teorema 2.5 de [Sk], temos o seguinte 
Teorema 3.2.2 . Seja $f:(M, \partial M) \longrightarrow(N, \partial N)$ uma aplicação contínua própria entre superfícies, e considere um ponto $c \in N$. Se $f$ é fiel à orientação (isto é, do tipo I), então o número de Nielsen transverso $\mathrm{N}_{\phi}(f, c)$ é realizável, e, portanto, $\mathcal{A}(f)=\mathcal{G}(f)$.

Além disso, na seção 3 (páginas 421 a 423) de [Sk], Skora construiu uma aplicação contínua $f: M \longrightarrow N$, entre superfícies sem fronteira, tal que $f$ é própria, mas não fiel à orientação, e $\mathcal{G}(f)>\mathcal{A}(f)$, demonstrando que a hipótese de ser fiel à orientação é necessária no teorema 3.2.2.

Agora, quanto à realização do número de Nielsen de raízes $\mathrm{N}(f, c)$, a questão é mais delicada, pois as conclusões dos teoremas 3.2.1 e 3.2.2 não são válidas se substituirmos $\mathrm{N}_{巾}(f, c)$ por $\mathrm{N}(f, c)$. De fato, utlizando alguns resultados obtidos por Hopf, Lin encontrou um exemplo de aplicação contínua $f: \mathbb{T}^{2} \# \mathbb{T}^{2} \longrightarrow \mathbb{T}^{2}$ tal que $\mathrm{N}(f, c)=3$ e $\operatorname{MR}[f ; c]=4$, o que significa que, neste caso, $\mathrm{N}(f, c)$ não é realizável (vide [L], seção 4, páginas 205 e 206); note, contudo, que, pelo teorema 3.2.2, $\mathrm{N}_{\phi}(f, c)$ é realizável, uma vez que $\mathbb{T}^{2} \# \mathbb{T}^{2}$ e $\mathbb{T}^{2}$ são superfícies compactas e orientáveis, e, conseqüentemente, $f: \mathbb{T}^{2} \# \mathbb{T}^{2} \longrightarrow \mathbb{T}^{2}$ é própria e fiel à orientação. Para aplicações contínuas entre superfícies fechadas orientáveis, D. L. Gonçalves e H. Zieschang provaram o seguinte resultado (vide [GZ1] e [GZ2], teoremas 3.6 e 2.1, respectivamente):

Teorema 3.2.3. Sejam Me N superfícies fechadas orientáveis, de gêneros he k, respectivamente, e considere uma aplicação contínua $f: M \longrightarrow N$. Se a cardinalidade j do grupo quociente $\pi_{1}(N) / f_{\#}\left(\pi_{1}(M)\right)$ for finita (onde $f_{\#}: \pi_{1}(M) \longrightarrow \pi_{1}(N)$ é o homomorfismo induzido por $f$ ), então o número de Nielsen $\mathrm{N}(f, c)$ é realizável (com $c \in N)$ se, e somente se,

$$
|\operatorname{deg}(f)| \leqslant \frac{2 \cdot h-2+\mathrm{j}}{2 \cdot k-1} .
$$

Assim, dos cálculos dos números de Nielsen de raízes, apresentados na seção 2.3, podemos obter novos resultados a respeito da realização de $\mathrm{N}(f, c)$ para aplicações entre superfícies. Por exemplo, se $\mathrm{N}_{\phi}(f, c)$ for realizável e $\mathrm{N}(f, c)=\mathrm{N}_{\phi}(f, c)$, teremos que $\mathrm{N}(f, c)$ também é realizável; logo, analisando os teoremas 2.3.1, 2.3.2 e 3.2.1, temos de imediato o seguinte

Teorema 3.2.4. Se $f: M \longrightarrow N$ é uma aplicação contínua entre superfícies fechadas, tal que $f$ não é fiel à orientação, então, para todo $c \in N$, o número de Nielsen de raízes $\mathrm{N}(f, c)$ é realizável.

Exemplo 3.2.2 . Sendo $f: \mathbb{T}^{2} \# \mathbb{R} P^{2} \longrightarrow \mathbb{T}^{2}$ a aplicação contínua dada no item $(b)$ do exemplo 1.2.3, vimos (no exemplo 2.3.11) que $\mathrm{N}(f, c)=\mathrm{N}_{\phi}(f, c)=1$ para qualquer que 
seja $c \in \mathbb{T}^{2} \backslash D_{1}$. Portanto, como $f$ é uma aplicação do tipo III, o teorema acima nos garante que existe uma aplicação contínua $g: \mathbb{T}^{2} \# \mathbb{R} P^{2} \longrightarrow \mathbb{T}^{2}$, homotópica à $f$, que possui exatamente uma raiz em $c$.

Em contrapartida, dos teoremas 2.3.1, 2.3.2 e 3.2.1, também é imediato o seguinte

Teorema 3.2.5. Seja $f: M \longrightarrow N$ uma aplicação contínua, fiel à orientação, entre superfícies fechadas não-orientáveis, e considere um ponto $c \in N$. Utilizando as notações adotadas na seção 2.3 , temos que, se j for finito, ou $|\operatorname{deg}(\widehat{f})| \leqslant 1$, então $\mathrm{N}(f, c)$ é realizável.

Finalmente, observe que os teoremas 2.3.1, 2.3.2 e 3.2.2 nos permitem obter alguns resultados sobre a realização de $\mathrm{N}(f, c)$ quando as fronteiras das superfícies são eventualmente não-vazias; de fato, por um raciocínio análogo ao utilizado quando enunciamos os teoremas 3.2.4 e 3.2.5, temos o teorema dado abaixo:

Teorema 3.2.6 . Seja $f:(M, \partial M) \longrightarrow(N, \partial N)$ uma aplicação contínua própria entre superfícies, e considere um ponto $c \in \operatorname{Int} N$. Utilizando as mesmas notações adotadas na seção 2.3, temos que:

(i) se $f$ é fiel à orientação, com $\mathrm{j}$ infinito ou $|\operatorname{deg}(\widehat{f})| \leqslant 1$, então $\mathrm{N}(f, c)$ é realizável;

(ii) se $f$ não é fiel à orientação, então (independentemente de j e de $\left.\operatorname{deg}(\hat{f}, 2) \in \mathbb{Z}_{2}\right) \mathrm{N}(f, c)$ também é realizável. 


\section{Apêndice $\mathbb{A}$}

\section{Limites Diretos}

O objetivo deste apêndice é apresentar uma breve introdução à teoria de limites diretos (de grupos abelianos e homomorfismos), cujos resultados utilizamos na seção 1.3 .

Definição A.1 . Dizemos que um conjunto $\Lambda$ é dirigido se seus elementos estão munidos de uma relação $\leqslant$, satisfazendo as seguintes propriedades:

(i) $\alpha \leqslant \alpha$ para todo $\alpha \in \Lambda$;

(ii) se $\alpha, \beta, \gamma \in \Lambda$ são tais que $\alpha \leqslant \beta e \beta \leqslant \gamma$, então $\alpha \leqslant \gamma$;

(iii) dados $\alpha, \beta \in \Lambda$, existe $\delta \in \Lambda$ tal que $\alpha \leqslant \delta e \beta \leqslant \delta$.

O elemento $\delta$ mencionado acima é denominado limitante superior para $\alpha$ e $\beta$.

Exemplo A.1 . Seja $A$ um subconjunto de uma variedade topológica $M$, e considere a família $\Lambda$ de todos os subconjuntos compactos $K \subseteq M$ tais que $A \subseteq M \backslash K$. Suponha ainda que os elementos de $\Lambda$ estão munidos da relação $\leqslant$ dada por

$$
K_{1} \leqslant K_{2} \Longleftrightarrow K_{1} \subseteq K_{2}\left(K_{1}, K_{2} \in \Lambda\right),
$$

denominada inclusão direta. Temos então que $\Lambda$ é um conjunto dirigido: com efeito, as propriedades (i) e (ii) da definição A.1 são imediatamente satisfeitas; para verificar (iii), basta observar que, dados $K_{1}, K_{2} \in \Lambda$, o conjunto $K_{3}=K_{1} \cup K_{2}$ é compacto e satisfaz $A \subseteq M \backslash K_{3}$ (pois $K_{1}$ e $K_{2}$ são compactos tais que $A \subseteq M \backslash K_{1}$ e $A \subseteq M \backslash K_{2}$, e, portanto, $\left.A \subseteq\left(M \backslash K_{1}\right) \cap\left(M \backslash K_{2}\right)=M \backslash\left(K_{1} \cup K_{2}\right)=M \backslash K_{3}\right)$, isto é, $K_{3}=K_{1} \cup K_{2}$ é um elemento de $\Lambda$ tal que $K_{1} \leqslant K_{3}$ e $K_{2} \leqslant K_{3}$. 
Definição A.2 . Se $\Lambda$ é um conjunto dirigido, dizemos que um subconjunto $\Lambda_{0} \subseteq \Lambda$ é cofinal em $\Lambda$ se, para cada $\alpha \in \Lambda$, existe $\delta \in \Lambda_{0}$ tal que $\alpha \leqslant \delta$; note que $\Lambda_{0}$ também é um conjunto dirigido com respeito à relação de ordem parcial induzida de $\Lambda$.

Exemplo A.2 . Considerando o conjunto dos números naturais $\mathbb{N}$, munido da relação de ordem usual $\leqslant$, temos que todo subconjunto infinito de $\mathbb{N}$ é cofinal.

Exemplo A.3 . Sendo $M$ uma variedade topológica conexa, considere a família $\Lambda_{0}$ constituída de todos os subconjuntos compactos e conexos $K \subseteq M$. Supondo os elementos de $\Lambda_{0}$ munidos da relação de inclusão direta, temos que $\Lambda_{0}$ é um conjunto dirigido: de fato, as propriedades (i) e (ii) da definição A.1 são imediatas; para verificar (iii), basta observar que, dados $K_{1}, K_{2} \in \Lambda_{0}$, podemos ligar $K_{1}$ e $K_{2}$ por um arco em $M$ (uma vez que $M$ é conexa), obtendo um conjunto compacto e conexo $K_{3} \subseteq M$ tal que $K_{1} \leqslant K_{3}$ e $K_{2} \leqslant K_{3}$. Observe então que todo subconjunto compacto de $M$ está contido num $K \in \Lambda_{0}$ (pois todo compacto em $M$ possui um número finito de componentes conexas, que podem ser ligadas por arcos em $M$ ); logo, temos que $\Lambda_{0}$ é um subconjunto cofinal da família $\Lambda$ de todos os subconjuntos compactos de $M$ (dirigida pela relação de inclusão direta).

Definição A.3 . Um sistema direto de grupos abelianos e homomorfismos, correspondente a um conjunto dirigido $\Lambda$, é uma família $\left\{G_{\alpha}\right\}_{\alpha \in \Lambda}$ de grupos abelianos, indexados por $\Lambda$, para os quais existe uma família de homomorfismos $\left\{f_{\alpha \beta}: G_{\alpha} \longrightarrow G_{\beta}\right\}_{\alpha, \beta \in \Lambda}$, definidos para $\alpha \leqslant \beta$, tais que:

(i) $f_{\alpha \alpha}: G_{\alpha} \longrightarrow G_{\alpha}$ é a identidade, para todo $\alpha \in \Lambda$;

(ii) se $\alpha, \beta, \gamma \in \Lambda$ são tais que $\alpha \leqslant \beta \leqslant \gamma$, então $f_{\alpha \gamma}=f_{\beta \gamma} \circ f_{\alpha \beta}$; em outras palavras, o diagrama abaixo é comutativo:

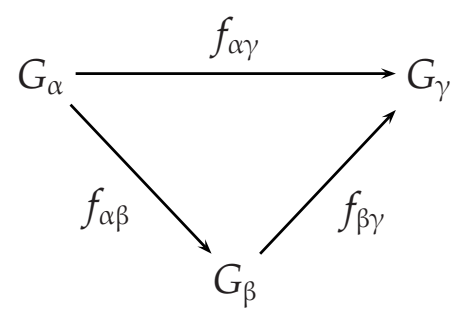

Exemplo A.4 . Sendo $\mathbb{N}$ o conjunto dos números naturais, dirigido pela relação de ordem usual $\leqslant$, considere a família $\left\{G_{n}\right\}_{n \in \mathbb{N}}$ de grupos abelianos, onde $G_{n}=\mathbb{Z}$ para todo $n \in \mathbb{N}$, e, para cada par $m, n \in \mathbb{N}$, com $m \leqslant n$, seja $f_{m n}: \mathbb{Z} \longrightarrow \mathbb{Z}$ o homomorfismo dado por $f_{m n}(k)=2^{n-m} \cdot k$. Temos então que $\left\{G_{n}, f_{m n}\right\}_{m, n \in \mathbb{N}}$ é um sistema direto, pois: 
(i) para todo $n \in \mathbb{N}, f_{n n}: G_{n}=\mathbb{Z} \longrightarrow \mathbb{Z}$ é o homomorfismo identidade, uma vez que $f_{n n}(k)=2^{n-n} \cdot k=k$, para cada $k \in \mathbb{Z}$;

(ii) dados $m, n, p \in \mathbb{N}$, com $m \leqslant n \leqslant p$, segue que:

$$
\begin{aligned}
\left(f_{m n} \circ f_{n p}\right)(k) & =f_{m n}\left(f_{n p}(k)\right)=f_{m n}\left(2^{p-n} \cdot k\right) \\
& =2^{n-m} \cdot\left(2^{p-n} \cdot k\right)=2^{p-m} \cdot k \\
& =f_{m p}(k)
\end{aligned}
$$

qualquer que seja $k \in \mathbb{Z}$ (isto é, $f_{m n} \circ f_{n p}=f_{m p}$ ).

Proposição A.1. Seja $\left\{G_{\alpha}, f_{\alpha \beta}\right\}_{\alpha, \beta \in \Lambda}$ um sistema direto de grupos abelianos e homomorfismos (onde $\Lambda$ é um conjunto dirigido). Sem perda de generalidade podemos supor que os grupos $G_{\alpha}$ cosntituem conjuntos dois a dois disjuntos (pois, caso contrário, podemos substituir qualquer um deles por um outro grupo abeliano isomorfo a ele). Assim, considerando a união disjunta dos grupos $G_{\alpha}$ (como conjuntos), vamos definir a seguinte relação de equivalência: dados $g_{\alpha} \in G_{\alpha}$ e $g_{\beta} \in G_{\beta}$, com $\alpha, \beta \in \Lambda$, diremos que $g_{\alpha} \sim g_{\beta}$ se, e somente se, $f_{\alpha \delta}\left(g_{\alpha}\right)=f_{\beta \delta}\left(g_{\beta}\right)$ para algum limitante superior $\delta \in \Lambda$ de $\alpha$ e $\beta$. Deste modo, sendo $G_{\infty}$ o conjunto das classes de equivalência segundo esta relação, temos que $G_{\infty}$ constitui um grupo abeliano quando munido da operação binária dada por:

$$
\llbracket g_{\alpha} \rrbracket+\llbracket g_{\beta} \rrbracket=\llbracket f_{\alpha \delta}\left(g_{\alpha}\right)+f_{\beta \delta}\left(g_{\beta}\right) \rrbracket,
$$

onde 【 』denota a classe de equivalência segundo a relação $\sim, e \delta \in \Lambda$ é um limitante superior de $\alpha$ e $\beta$. Neste caso, denotando por $0_{\alpha}$ o elemento identidade do grupo $G_{\alpha}$, temos que o elemento identidade do grupo $G_{\infty}$ é a classe $\llbracket 0_{\alpha} \rrbracket$, onde $\alpha \in \Lambda$ pode ser tomado arbitrariamente.

Demonstração. Primeiramente, mostremos que $\sim$ é de fato uma relação de equivalência. Com efeito, dados $g_{\alpha} \in G_{\alpha}, g_{\beta} \in G_{\beta}$ e $g_{\gamma} \in G_{\gamma}$ (onde $\alpha, \beta, \gamma \in \Lambda$ ), podemos ver facilmente que $g_{\alpha} \sim g_{\alpha}$, e que, se $g_{\alpha} \sim g_{\beta}$, então $g_{\beta} \sim g_{\alpha}$. Suponhamos, pois, que $g_{\alpha} \sim g_{\beta}$ e $g_{\beta} \sim g_{\gamma}$; isto significa que existem $\delta, \omega \in \Lambda, \operatorname{com} \alpha, \beta \leqslant \delta$ e $\beta, \gamma \leqslant \omega$, tais que $f_{\alpha \delta}\left(g_{\alpha}\right)=f_{\beta \delta}\left(g_{\beta}\right)$ e $f_{\beta \omega}\left(g_{\beta}\right)=f_{\gamma \omega}\left(g_{\gamma}\right)$. Logo, se $\delta \leqslant \omega$, segue que $\omega$ é um limitante superior de $\alpha$ e $\gamma$, tal que:

$$
f_{\alpha \omega}\left(g_{\alpha}\right)=f_{\delta \omega}\left(f_{\alpha \delta}\left(g_{\alpha}\right)\right)=f_{\delta \omega}\left(f_{\beta \delta}\left(g_{\beta}\right)\right)=f_{\beta \omega}\left(g_{\beta}\right)=f_{\gamma \omega}\left(g_{\gamma}\right)
$$

Portanto, existe um limitante superior $\lambda \in \Lambda$ de $\alpha$ e $\gamma$ satisfazendo $f_{\alpha \lambda}\left(g_{\alpha}\right)=f_{\gamma \lambda}\left(g_{\gamma}\right)$, o que, por definição, implica que $g_{\alpha} \sim g_{\gamma}$.

Agora, observe que a operação binária dada acima está bem definida: de fato, se $g_{\alpha} \in G_{\alpha}, g_{\beta} \in G_{\beta}, g_{\gamma} \in G_{\gamma}$ e $g_{\epsilon} \in G_{\epsilon}$ são tais que $\llbracket g_{\alpha} \rrbracket=\llbracket g_{\gamma} \rrbracket$ e $\llbracket g_{\beta} \rrbracket=\llbracket g_{\epsilon} \rrbracket$, segue que $g_{\alpha} \sim g_{\gamma}$ e $g_{\beta} \sim g_{\epsilon}$, isto é, existem $\delta, \omega \in \Lambda$, $\operatorname{com} \alpha, \gamma \leqslant \delta$ e $\beta, \epsilon \leqslant \omega$, tais que 
$f_{\alpha \delta}\left(g_{\alpha}\right)=f_{\gamma \delta}\left(g_{\gamma}\right)$ e $f_{\beta \omega}\left(g_{\beta}\right)=f_{\epsilon \omega}\left(g_{\epsilon}\right)$. Logo, se $\delta \leqslant \omega$, segue que $\omega$ é um limitante superior de $\alpha, \beta, \gamma$ e $\epsilon$, tal que:

$$
\begin{aligned}
\llbracket g_{\alpha} \rrbracket+\llbracket g_{\beta} \rrbracket & =\llbracket f_{\alpha \omega}\left(g_{\alpha}\right)+f_{\beta \omega}\left(g_{\beta}\right) \rrbracket=\llbracket f_{\alpha \omega}\left(g_{\alpha}\right)+f_{\epsilon \omega}\left(g_{\epsilon}\right) \rrbracket \\
& =\llbracket f_{\delta \omega}\left(f_{\alpha \delta}\left(g_{\alpha}\right)\right)+f_{\epsilon \omega}\left(g_{\epsilon}\right) \rrbracket=\llbracket f_{\delta \omega}\left(f_{\gamma \delta}\left(g_{\gamma}\right)\right)+f_{\epsilon \omega}\left(g_{\epsilon}\right) \rrbracket \\
& =\llbracket f_{\gamma \omega}\left(g_{\gamma}\right)+f_{\epsilon \omega}\left(g_{\epsilon}\right) \rrbracket=\llbracket g_{\gamma} \rrbracket+\llbracket g_{\epsilon} \rrbracket ;
\end{aligned}
$$

por outro lado, se $\omega \leqslant \delta$, temos que $\delta$ é um limitante superior de $\alpha, \beta, \gamma$ e $\epsilon$, tal que:

$$
\begin{aligned}
\llbracket g_{\alpha} \rrbracket+\llbracket g_{\beta} \rrbracket & =\llbracket f_{\alpha \delta}\left(g_{\alpha}\right)+f_{\beta \delta}\left(g_{\beta}\right) \rrbracket=\llbracket f_{\gamma \delta}\left(g_{\gamma}\right)+f_{\beta \delta}\left(g_{\beta}\right) \rrbracket \\
& =\llbracket f_{\gamma \delta}\left(g_{\gamma}\right)+f_{\omega \delta}\left(f_{\beta \omega}\left(g_{\beta}\right)\right) \rrbracket=\llbracket f_{\gamma \delta}\left(g_{\gamma}\right)+f_{\omega \delta}\left(f_{\epsilon \omega}\left(g_{\epsilon}\right)\right) \rrbracket \\
& =\llbracket f_{\gamma \delta}\left(g_{\gamma}\right)+f_{\epsilon \delta}\left(g_{\epsilon}\right) \rrbracket=\llbracket g_{\gamma} \rrbracket+\llbracket g_{\epsilon} \rrbracket .
\end{aligned}
$$

Resumindo, se $\llbracket g_{\alpha} \rrbracket=\llbracket g_{\gamma} \rrbracket$ e $\llbracket g_{\beta} \rrbracket=\llbracket g_{\epsilon} \rrbracket$, segue que $\llbracket g_{\alpha} \rrbracket+\llbracket g_{\beta} \rrbracket=\llbracket g_{\gamma} \rrbracket+\llbracket g_{\epsilon} \rrbracket$, o que prova que a operação binária + está de fato bem definida. Note ainda que esta operação é comutativa, pois, dados $g_{\alpha} \in G_{\alpha}$ e $g_{\beta} \in G_{\beta}$, segue que:

$$
\llbracket g_{\alpha} \rrbracket+\llbracket g_{\beta} \rrbracket=\llbracket f_{\alpha \delta}\left(g_{\alpha}\right)+f_{\beta \delta}\left(g_{\beta}\right) \rrbracket=\llbracket f_{\beta \delta}\left(g_{\beta}\right)+f_{\alpha \delta}\left(g_{\alpha}\right) \rrbracket=\llbracket g_{\beta} \rrbracket+\llbracket g_{\alpha} \rrbracket,
$$

para todo limitante superior $\delta \in \Lambda$ de $\alpha$ e $\beta$ (lembre-se que $G_{\delta}$ é um grupo abeliano, qualquer que seja $\delta \in \Lambda$ ).

Finalmente, vamos mostrar então que o conjunto $G_{\infty}$ (contendo as classes de equivalência dos elementos dos grupos $G_{\alpha}$ ), munido da operação binária +, constitui um grupo (abeliano, devido à comutatividade desta operação). Com efeito, dados $g_{\alpha} \in G_{\alpha}$, $g_{\beta} \in G_{\beta}$ e $g_{\gamma} \in G_{\gamma}$ (onde $\alpha, \beta, \gamma \in \Lambda$ ), sejam $\delta, \epsilon, \omega \in \Lambda$ tais que $\alpha, \beta \leqslant \delta, \beta, \gamma \leqslant \epsilon$ e $\delta, \epsilon \leqslant \omega$ (o que implica que $\delta, \gamma \leqslant \omega$ ). Temos, pois, que:

$$
\begin{aligned}
\left(\llbracket g_{\alpha} \rrbracket+\llbracket g_{\beta} \rrbracket\right)+\llbracket g_{\gamma} \rrbracket & =\llbracket f_{\alpha \delta}\left(g_{\alpha}\right)+f_{\beta \delta}\left(g_{\beta}\right) \rrbracket+\llbracket g_{\gamma} \rrbracket \\
& =\llbracket f_{\delta \omega}\left(f_{\alpha \delta}\left(g_{\alpha}\right)+f_{\beta \delta}\left(g_{\beta}\right)\right)+f_{\gamma \omega}\left(g_{\gamma}\right) \rrbracket \\
& =\llbracket f_{\delta \omega}\left(f_{\alpha \delta}\left(g_{\alpha}\right)\right)+f_{\delta \omega}\left(f_{\beta \delta}\left(g_{\beta}\right)\right)+f_{\gamma \omega}\left(g_{\gamma}\right) \rrbracket \\
& =\llbracket f_{\alpha \omega}\left(g_{\alpha}\right)+f_{\beta \omega}\left(g_{\beta}\right)+f_{\gamma \omega}\left(g_{\gamma}\right) \rrbracket \\
& =\llbracket f_{\alpha \omega}\left(g_{\alpha}\right)+f_{\epsilon \omega}\left(f_{\beta \epsilon}\left(g_{\beta}\right)\right)+f_{\epsilon \omega}\left(f_{\gamma \epsilon}\left(g_{\gamma}\right)\right) \rrbracket \\
& =\llbracket f_{\alpha \omega}\left(g_{\alpha}\right)+f_{\epsilon \omega}\left(f_{\beta \epsilon}\left(g_{\beta}\right)\right)+f_{\gamma \epsilon}\left(g_{\gamma}\right) \rrbracket \\
& =\llbracket g_{\alpha} \rrbracket+\llbracket f_{\beta \epsilon}\left(g_{\beta}\right)+f_{\gamma \epsilon}\left(g_{\gamma}\right) \rrbracket \\
& =\llbracket g_{\alpha} \rrbracket+\left(\llbracket g_{\beta} \rrbracket+\llbracket g_{\gamma} \rrbracket\right),
\end{aligned}
$$

o que prova a associatividade da operação + . Agora, denotando por $0_{\alpha}$ o elemento identidade do grupo $G_{\alpha}$, para cada $\alpha \in \Lambda$, observe que $0_{\alpha} \sim 0_{\beta}$ quaisquer que sejam $\alpha, \beta \in \Lambda$ : de fato, para todo limitante superior $\delta \in \Lambda$ de $\alpha$ e $\beta$, temos que $f_{\alpha \delta}\left(0_{\alpha}\right)=0_{\delta}=$ 
$f_{\beta \delta}\left(0_{\beta}\right)$. Logo, $\llbracket 0_{\alpha} \rrbracket=\llbracket 0_{\beta} \rrbracket$ para quaisquer $\alpha, \beta \in \Lambda$. Por outro lado, dado $\alpha \in \Lambda$, temos que:

$$
\llbracket 0_{\alpha} \rrbracket+\llbracket g_{\beta} \rrbracket=\llbracket g_{\beta} \rrbracket+\llbracket 0_{\alpha} \rrbracket=\llbracket g_{\beta} \rrbracket
$$

para todo $g_{\beta} \in G_{\beta}$ (onde $\beta \in \Lambda$ ), pois, se $\delta \in \Lambda$ é um limitante superior de $\alpha$ e $\beta$, segue que

$$
\llbracket 0_{\alpha} \rrbracket+\llbracket g_{\beta} \rrbracket=\llbracket g_{\beta} \rrbracket+\llbracket 0_{\alpha} \rrbracket=\llbracket f_{\beta \delta}\left(g_{\beta}\right)+f_{\alpha \delta}\left(0_{\alpha}\right) \rrbracket=\llbracket f_{\beta \delta}\left(g_{\beta}\right)+0_{\delta} \rrbracket=\llbracket f_{\beta \delta}\left(g_{\beta}\right) \rrbracket=\llbracket g_{\beta} \rrbracket,
$$

onde a última igualdade provém do fato de que $f_{\beta \delta}\left(g_{\beta}\right) \sim g_{\beta}$ (uma vez que, para todo $\xi \in \Lambda$ tal que $\delta \leqslant \xi$, tem-se $\left.f_{\delta \xi}\left(f_{\beta \delta}\left(g_{\beta}\right)\right)=f_{\beta \xi}\left(g_{\beta}\right)\right)$. Portanto, o conjunto $G_{\infty}$ possui um elemento identidade $\llbracket 0 \rrbracket$ (relativamente à operação binária + ), definido por $\llbracket 0 \rrbracket=\llbracket 0_{\alpha} \rrbracket$, onde $\alpha \in \Lambda$ pode ser tomado arbitrariamente.

Isto posto, para finalizar a demonstração, considere um elemento $g_{\alpha} \in G_{\alpha}$ (onde $\alpha \in \Lambda$ ), e denote por $-g_{\alpha}$ o inverso de $g_{\alpha}$ em $G_{\alpha}$. Temos então que a classe $-\llbracket g_{\alpha} \rrbracket=\llbracket-g_{\alpha} \rrbracket$ é tal que:

$$
\llbracket g_{\alpha} \rrbracket+\left(-\llbracket g_{\alpha} \rrbracket\right)=\left(-\llbracket g_{\alpha} \rrbracket\right)+\llbracket g_{\alpha} \rrbracket=\llbracket 0 \rrbracket,
$$

pois, dado $\delta \in \Lambda$ tal que $\alpha \leqslant \delta$, segue que:

$$
\begin{aligned}
\llbracket g_{\alpha} \rrbracket+\left(-\llbracket g_{\alpha} \rrbracket\right) & =\left(-\llbracket g_{\alpha} \rrbracket\right)+\llbracket g_{\alpha} \rrbracket=\llbracket-g_{\alpha} \rrbracket+\llbracket g_{\alpha} \rrbracket=\llbracket f_{\alpha \delta}\left(-g_{\alpha}\right)+f_{\alpha \delta}\left(g_{\alpha}\right) \rrbracket \\
& =\llbracket f_{\alpha \delta}\left(-g_{\alpha}+g_{\alpha}\right) \rrbracket=\llbracket f_{\alpha \delta}\left(0_{\alpha}\right) \rrbracket=\llbracket 0_{\delta} \rrbracket=\llbracket 0 \rrbracket .
\end{aligned}
$$

Em outras palavras, todo elemento em $G_{\infty}$ possui um inverso (em relação à operação binária + ), d'onde concluímos que $G_{\infty}$ constitui um grupo.

Definição A.4 . Nas condições da proposição anterior, o grupo abeliano $G_{\infty}$ é denominado limite direto do sistema $\left\{G_{\alpha}, f_{\alpha \beta}\right\}_{\alpha, \beta \in \Lambda}$, é denotado por $\lim _{\longrightarrow}\left\{G_{\alpha}: \alpha \in \Lambda\right\}$ (ou, abreviadamente, $\underset{\lim }{\longrightarrow} G_{\alpha}$ ).

Exemplo A.5 . Seja $\left\{G_{n}, f_{m n}\right\}_{m, n \in \mathbb{N}}$ o sistema direto dado no exemplo A.4. Então, para quaisquer $r \in G_{n}=\mathbb{Z}$ e $s \in G_{m}=\mathbb{Z}$, temos que $r \sim s$ se, e somente se, existe $p \in \mathbb{N}$ tal que $n, m \leqslant p$ e

$$
f_{n p}(r)=2^{p-n} \cdot r=f_{m p}(s)=2^{p-m} \cdot s .
$$

Logo, $r \sim s$ se, e somente se, $r / s$ é da forma $2^{k}$, para algum $k \in \mathbb{Z}$. Portanto, a classe de equivalência de um elemento $r \in \mathbb{Z}$ é constituída dos números inteiros da forma $r / 2^{k}$, onde $k \in \mathbb{Z}$. Assim, o limite direto $\underline{\lim } G_{n}$ é isomorfo ao grupo $H$ dos números racionais diádicos (que é o grupo aditivo de todos os números racionais da forma $r / 2^{k}$, onde $r, k \in \mathbb{Z}$ ). 
Proposição A.2 . Sendo $\left\{G_{\alpha}, f_{\alpha \beta}\right\}_{\alpha, \beta \in \Lambda}$ um sistema direto de grupos abelianos e homomorfismos (onde $\Lambda$ é um conjunto dirigido), temos que:

(i) se todos os homomorfismos $f_{\alpha \beta}, \alpha \leqslant \beta$, são isomorfismos, então $\lim _{\longrightarrow} G_{\alpha}$ é isomorfo a cada um dos grupos $G_{\alpha}$;

(ii) se, para cada $\alpha \in \Lambda$, existir um $\beta \in \Lambda$ tal que $\alpha \leqslant \beta$ e $f_{\alpha \beta}: G_{\alpha} \longrightarrow G_{\beta}$ é o homomorfismo identicamente nulo, então $\lim _{\longrightarrow} G_{\alpha}$ é o grupo trivial;

(iii) se $\Lambda_{0} \subseteq \Lambda$ é cofinal em $\Lambda$, então $\underset{\lim }{\longrightarrow}\left\{G_{\alpha}: \alpha \in \Lambda\right\} \simeq \underset{\lim }{\longrightarrow}\left\{G_{\alpha}: \alpha \in \Lambda_{0}\right\}$.

DemonstraçÃo. (i) Da proposição A.1, sabemos que o elemento identidade de $\lim _{\longrightarrow} G_{\alpha}$ é a classe $\llbracket 0_{\alpha} \rrbracket$, onde $\alpha \in \Lambda$ pode ser tomado arbitrariamente. Observe então que $\llbracket 0_{\alpha} \rrbracket$ contém apenas os elementos identidade $0_{\beta} \in G_{\beta}$, onde $\beta \in \Lambda$ : com efeito, dado $g_{\beta} \in G_{\beta}$ tal que $g_{\beta} \in \llbracket 0_{\alpha} \rrbracket$, segue que $g_{\beta} \sim 0_{\alpha}$, isto é, existe um limitante superior $\delta \in \Lambda$ de $\alpha$ e $\beta$, tal que $f_{\alpha \delta}\left(0_{\alpha}\right)=f_{\beta \delta}\left(g_{\beta}\right)$; logo, como $f_{\alpha \delta}\left(0_{\alpha}\right)=0_{\delta}$, temos que $f_{\beta \delta}\left(g_{\beta}\right)=0_{\delta}$, ou seja, $g_{\beta}=0_{\beta}$ (pois $f_{\beta \delta}$ é, por hipótese, um isomorfismo). Isto posto, para cada $\beta \in \Lambda$, consideremos o homomorfismo $\lambda_{\beta}: G_{\beta} \longrightarrow \underset{\lim }{\longrightarrow} G_{\alpha}$ dado por:

$$
\lambda_{\beta}\left(g_{\beta}\right)=\llbracket g_{\beta} \rrbracket, g_{\beta} \in G_{\beta} .
$$

Como

$$
\operatorname{Ker}\left(\lambda_{\beta}\right)=\left\{g_{\beta} \in G_{\beta}: \lambda_{\beta}\left(g_{\beta}\right)=\llbracket g_{\beta} \rrbracket=\llbracket 0_{\alpha} \rrbracket\right\}=\left\{0_{\beta}\right\},
$$

segue que $\lambda_{\beta}$ é um homomorfismo injetor. Vamos mostrar então que $\lambda_{\beta}$ é um homomorfismo sobrejetor; para tanto, dados $\xi \in \Lambda$ e $g_{\xi} \in G_{\xi}$, devemos verificar se existe um elemento $g_{\beta} \in G_{\beta}$ tal que $\lambda_{\beta}\left(g_{\beta}\right)=\llbracket g_{\xi} \rrbracket$. Com efeito, se $\beta \leqslant \xi$, basta tomarmos $g_{\beta}=f_{\beta \xi}^{-1}\left(g_{\xi}\right)$, pois, neste caso, existe $\delta \in \Lambda$ tal que $\beta \leqslant \xi \leqslant \delta$ e $f_{\beta \delta}\left(g_{\beta}\right)=f_{\xi \delta}\left(g_{\xi}\right)$, a saber, $\delta=\xi$ (uma vez que $f_{\beta \xi}\left(g_{\beta}\right)=g_{\xi}=f_{\xi \xi}\left(g_{\xi}\right)$ ); por outro lado, se $\xi \leqslant \beta$, basta tomarmos $g_{\beta}=f_{\xi \beta}\left(g_{\xi}\right)$, pois, neste caso, também existe $\delta \in \Lambda$ tal que $\xi \leqslant \beta \leqslant \delta$ e $f_{\xi \delta}\left(g_{\xi}\right)=f_{\beta \delta}\left(g_{\beta}\right)$, a saber, $\delta=\beta$ (uma vez que $f_{\xi \beta}\left(g_{\xi}\right)=g_{\beta}=f_{\beta \beta}\left(g_{\beta}\right)$ ). Deste modo, concluímos que, para cada $\beta \in \Lambda$, o homomorfimso $\lambda_{\beta}: G_{\beta} \longrightarrow \underset{\lim }{\longrightarrow} G_{\alpha}$ é um isomorfismo, isto é, $\lim _{\longrightarrow} G_{\alpha}$ é isomorfo a cada um dos grupos abelianos $G_{\alpha}$.

(ii) Sendo $\llbracket 0_{\alpha} \rrbracket$ o elemento identidade de $\underline{\lim } G_{\alpha}$ (onde $\alpha \in \Lambda$ é arbitrário), considere um elemento $g_{\beta} \in G_{\beta}, \beta \in \Lambda$; vamos mostrar que $g_{\beta} \in \llbracket 0_{\alpha} \rrbracket$. De fato, supondo $\alpha \leqslant \beta$, segue (por hipótese) que existe $\delta \in \Lambda$ tal que $\alpha \leqslant \beta \leqslant \delta$ e $f_{\beta \delta}: G_{\beta} \longrightarrow G_{\delta}$ é identicamente nulo (isto é, $\operatorname{Im}\left(f_{\beta \delta}\right)=\left\{0_{\delta}\right\}$ ); assim, temos que $f_{\alpha \delta}\left(0_{\alpha}\right)=0_{\delta}$ e $f_{\beta \delta}\left(g_{\beta}\right)=0_{\delta}$, ou seja, $f_{\alpha \delta}\left(0_{\alpha}\right)=f_{\beta \delta}\left(g_{\beta}\right)$, o que significa que $g_{\beta} \sim 0_{\alpha}$, e, portanto, $g_{\beta} \in \llbracket 0_{\alpha} \rrbracket$. De maneira análoga, pode-se verificar que, no caso $\beta \leqslant \alpha$, também tem-se $g_{\beta} \in \llbracket 0_{\alpha} \rrbracket$. Logo, para quaisquer $\beta \in \Lambda$ e $g_{\beta} \in G_{\beta}$, temos que $\llbracket g_{\beta} \rrbracket=\llbracket 0_{\alpha} \rrbracket$, d'onde concluímos que o grupo $\underset{\lim }{\longrightarrow} G_{\alpha}$ é constituído unicamente da classe $\llbracket 0_{\alpha} \rrbracket$, isto é, $\lim _{\longrightarrow} G_{\alpha}$ é o grupo trivial. 
(iii) Denotemos por $\llbracket \rrbracket_{0}$ e $\llbracket \rrbracket$ as classe de equivalência em $\underset{\lim }{\longrightarrow}\left\{G_{\alpha}: \alpha \in \Lambda_{0}\right\}$ e $\underset{\lim }{\longrightarrow}\left\{G_{\alpha}\right.$ : $\alpha \in \Lambda$, respectivamente, e consideremos a função

$$
\varphi: \lim _{\longrightarrow}\left\{G_{\alpha}: \alpha \in \Lambda_{0}\right\} \longrightarrow \lim _{\longrightarrow}\left\{G_{\alpha}: \alpha \in \Lambda\right\}
$$

dada por $\varphi\left(\llbracket g_{\beta} \rrbracket_{0}\right)=\llbracket g_{\beta} \rrbracket$, para cada $g_{\beta} \in G_{\beta}$, com $\beta \in \Lambda_{0}$. Observe que $\varphi$ está bem definida, pois, se $\llbracket g_{\beta} \rrbracket_{0}=\llbracket g_{\gamma} \rrbracket_{0}$ (onde $g_{\beta} \in G_{\beta}$ e $g_{\gamma} \in G_{\gamma}, \beta, \gamma \in \Lambda_{0}$ ), segue que $g_{\beta} \sim g_{\gamma}$ em $\Lambda_{0}$, isto é, existe um limitante superior $\delta \in \Lambda_{0}$ de $\beta$ e $\gamma$ tal que $f_{\beta \delta}\left(g_{\beta}\right)=f_{\gamma \delta}\left(g_{\gamma}\right)$; logo, temos que $g_{\beta} \sim g_{\gamma}$ em $\Lambda$, ou seja, $\varphi\left(\llbracket g_{\beta} \rrbracket_{0}\right)=\llbracket g_{\beta} \rrbracket=\llbracket g_{\gamma} \rrbracket=\varphi\left(\llbracket g_{\gamma} \rrbracket_{0}\right)$. Além disso, temos também que $\varphi$ é um homomorfismo, pois, dados $g_{\beta} \in G_{\beta}$ e $g_{\gamma} \in G_{\gamma}$, $\operatorname{com} \beta, \gamma \in \Lambda_{0}$, e sendo $\delta \in \Lambda_{0}$ um limitante superior de $\beta$ e $\gamma$, segue que:

$$
\begin{aligned}
\varphi\left(\llbracket g_{\beta} \rrbracket_{0}+\llbracket g_{\gamma} \rrbracket_{0}\right) & =\varphi\left(\llbracket f_{\beta \delta}\left(g_{\beta}\right)+f_{\gamma \delta}\left(g_{\gamma}\right) \rrbracket_{0}\right)=\llbracket f_{\beta \delta}\left(g_{\beta}\right)+f_{\gamma \delta}\left(g_{\gamma}\right) \rrbracket \\
& =\llbracket g_{\beta} \rrbracket+\llbracket g_{\gamma} \rrbracket=\varphi\left(\llbracket g_{\beta} \rrbracket_{0}\right)+\varphi\left(\llbracket g_{\gamma} \rrbracket_{0}\right) .
\end{aligned}
$$

Agora, sendo $\llbracket 0_{\alpha} \rrbracket$ o elemento identidade do grupo $\lim _{\longrightarrow}\left\{G_{\alpha}: \alpha \in \Lambda\right\}$ (onde $\alpha \in \Lambda$ pode ser tomado arbitrariamente), temos que:

$$
\operatorname{Ker}(\varphi)=\left\{\llbracket g_{\beta} \rrbracket_{0} \in \lim _{\longrightarrow}\left\{G_{\alpha}: \alpha \in \Lambda_{0}\right\} \quad: \quad \varphi\left(\llbracket g_{\beta} \rrbracket_{0}\right)=\llbracket g_{\beta} \rrbracket=\llbracket 0_{\alpha} \rrbracket\right\} .
$$

Logo, dado $\llbracket g_{\beta} \rrbracket_{0} \in \operatorname{Ker}(\varphi)$, temos que $\llbracket g_{\beta} \rrbracket=\llbracket 0_{\alpha} \rrbracket$, ou seja, $g_{\beta} \sim 0_{\alpha}$ em $\Lambda$; isto, por sua vez, significa que existe um limitante superior $\gamma \in \Lambda$ de $\alpha$ e $\beta$, tal que $f_{\alpha \gamma}\left(0_{\alpha}\right)=f_{\beta \gamma}\left(g_{\beta}\right)$. Entretanto, como $\Lambda_{0}$ é cofinal em $\Lambda$, segue que existe $\delta \in \Lambda_{0}$ tal que $\gamma \leqslant \delta$; assim, como $f_{\beta \delta}=f_{\gamma \delta} \circ f_{\beta \gamma}$, temos que:

$$
f_{\beta \delta}\left(g_{\beta}\right)=f_{\gamma \delta}\left(f_{\beta \gamma}\left(g_{\beta}\right)\right)=f_{\gamma \delta}\left(f_{\alpha \gamma}\left(0_{\alpha}\right)\right)=f_{\gamma \delta}\left(0_{\gamma}\right)=0_{\delta}=f_{\delta \delta}\left(0_{\delta}\right) \text {. }
$$

Em outras palavras, $\delta \in \Lambda_{0}$ é tal que $\beta \leqslant \delta$ e $f_{\beta \delta}\left(g_{\beta}\right)=f_{\delta \delta}\left(0_{\delta}\right)$, o que, por definição, implica que $g_{\beta} \sim 0_{\delta}$ em $\Lambda_{0}$, isto é, $\llbracket g_{\beta} \rrbracket_{0}=\llbracket 0_{\delta} \rrbracket_{0}$. Deste modo, concluímos que o único elemento $\llbracket g_{\beta} \rrbracket_{0} \in \underset{\lim }{\longrightarrow}\left\{G_{\alpha}: \alpha \in \Lambda_{0}\right\}$ tal que $\llbracket g_{\beta} \rrbracket_{0} \in \operatorname{Ker}(\varphi)$ é $\llbracket g_{\beta} \rrbracket_{0}=\llbracket 0_{\delta} \rrbracket_{0}$, que é o elemento identidade de $\underset{\longrightarrow}{\lim }\left\{G_{\alpha}: \alpha \in \Lambda_{0}\right\}$. Portanto, $\varphi$ é um homomorfismo injetor. Finalmente, observe que $\varphi$ também é um homomorfismo sobrejetor: com efeito, dado $\llbracket g_{\beta} \rrbracket \in \lim _{\longrightarrow}\left\{G_{\alpha}: \alpha \in \Lambda\right\}$ (onde $\beta \in \Lambda$ e $g_{\beta} \in G_{\beta}$ ), seja $\delta \in \Lambda_{0}$ tal que $\beta \leqslant \delta$ e considere o elemento $\llbracket f_{\beta \delta}\left(g_{\beta}\right) \rrbracket_{0} \in \underset{\lim }{\longrightarrow}\left\{G_{\alpha}: \alpha \in \Lambda_{0}\right\} ;$ como $f_{\beta \delta}\left(g_{\beta}\right) \sim g_{\beta}$ em $\Lambda$ (pois $\left.f_{\beta \delta}\left(g_{\beta}\right)=f_{\delta \delta}\left(f_{\beta \delta}\left(g_{\beta}\right)\right)\right)$, segue que $\llbracket \overrightarrow{f \beta \delta}_{\beta}\left(g_{\beta}\right) \rrbracket=\llbracket g_{\beta} \rrbracket$, isto é, $\varphi\left(\llbracket f_{\beta \delta}\left(g_{\beta}\right) \rrbracket_{0}\right)=\llbracket g_{\beta} \rrbracket$. Logo, todo elemento de $\underset{\lim }{\longrightarrow}\left\{G_{\alpha}: \alpha \in \Lambda_{0}\right\}$ é a imagem de um elemento de $\underset{\longrightarrow}{\longrightarrow}\left\{G_{\alpha}:\right.$ $\alpha \in \Lambda$ \} pelo homomorfismo $\varphi$, o que implica que $\varphi$ é sobrejetor, e, portanto, é um isomorfismo. 
Definição A.5 . Dados dois conjuntos dirigidos $\Lambda$ e $\Omega$, sejam $\mathcal{S}_{\Lambda}=\left\{G_{\alpha}, f_{\alpha \beta}\right\}_{\alpha, \beta \in \Lambda}$ e $\mathcal{S}_{\Omega}=$ $\left\{H_{\gamma}, g_{\gamma \delta}\right\}_{\gamma, \delta \in \Omega}$ sistemas diretos de grupos abelianos e homomorfismos. Definimos, pois, uma aplicação $\Phi$ entre $\mathcal{S}_{\Lambda}$ e $\mathcal{S}_{\Omega}$ como sendo uma família $\Phi=\left\{\phi, \phi_{\alpha}\right\}_{\alpha \in \Lambda}$, constituída de uma função $\phi: \Lambda \longrightarrow \Omega$ que preserva ordem (isto é, se $\alpha, \beta \in \Lambda$ são tais que $\alpha \leqslant \beta$, então $\phi(\alpha) \leqslant \phi(\beta)$ em $\Omega)$, juntamente com homomorfismos $\phi_{\alpha}: G_{\alpha} \longrightarrow H_{\phi(\alpha)}$ tais que, para cada par $\alpha, \beta \in \Lambda$ com $\alpha \leqslant \beta$, o diagrama a seguir é comutativo:

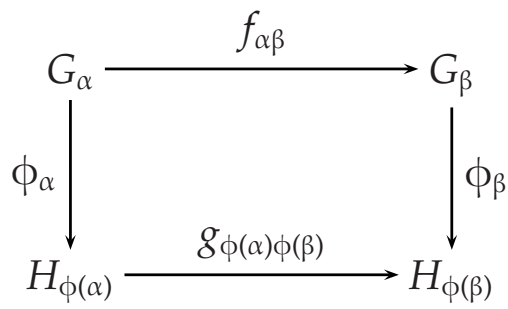

Esta aplicação $\Phi$ induz um homomorfismo

$$
\stackrel{\Phi}{\rightarrow}: \lim _{\longrightarrow}\left\{G_{\alpha}: \alpha \in \Lambda\right\} \longrightarrow \underset{\lim }{\longrightarrow}\left\{H_{\gamma}: \gamma \in \Omega\right\}
$$

denominado limite direto de $\Phi$, que leva a classe de equivalência de um elemento $g_{\alpha} \in G_{\alpha}$ na classe de equivalência de $\phi_{\alpha}\left(g_{\alpha}\right) \in H_{\phi(\alpha)}$, para cada $\alpha \in \Lambda$.

Exemplo A.6 . Dados um sistema direto $\left\{G_{\alpha}, f_{\alpha \beta}\right\}_{\alpha, \beta \in \Lambda}$, de grupos abelianos e homomorfismos, e um conjunto cofinal $\Lambda_{0} \subseteq \Lambda$, seja $\phi: \Lambda_{0} \longrightarrow \Lambda$ a inclusão, e, para cada $\alpha \in \Lambda_{0}$, denote por $\phi_{\alpha}: G_{\alpha} \longrightarrow G_{\phi(\alpha)}$ o homomorfismo identidade em $G_{\alpha}$. Temos então que $\phi$ preserve ordem e $\phi_{\alpha} \circ f_{\alpha \beta}=f_{\phi(\alpha) \phi(\beta)} \circ \phi_{\alpha}$, para quaisquer $\alpha, \beta \in \Lambda_{0}$. Logo, $\Phi=\left\{\phi, \phi_{\alpha}\right\}_{\alpha \in \Lambda_{0}}$ é uma aplicação entre os sistemas diretos $\mathcal{S}_{0}=\left\{G_{\alpha}, f_{\alpha \beta}\right\}_{\alpha, \beta \in \Lambda_{0}}$ e $\mathcal{S}=\left\{G_{\alpha}, f_{\alpha \beta}\right\}_{\alpha, \beta \in \Lambda}$, e o limite direto de $\Phi$ é o homomorfismo

$$
\stackrel{\Phi}{\rightarrow}: \lim _{\longrightarrow}\left\{G_{\alpha}: \alpha \in \Lambda_{0}\right\} \longrightarrow \lim _{\longrightarrow}\left\{G_{\alpha}: \alpha \in \Lambda\right\}
$$

dado por $\underset{\Phi}{\rightarrow}\left(\llbracket g_{\alpha} \rrbracket_{0}\right)=\llbracket g_{\alpha} \rrbracket$ para cada $g_{\alpha} \in G_{\alpha}, \alpha \in \Lambda_{0}$, onde $\llbracket \rrbracket_{0}$ e $\llbracket \rrbracket$ denotam as classes de equivalência em $\underset{\lim }{\longrightarrow}\left\{G_{\alpha}: \alpha \in \Lambda_{0}\right\}$ e $\underset{\lim }{\longrightarrow}\left\{G_{\alpha}: \alpha \in \Lambda\right\}$, respectivamente. Note que $\stackrel{\Phi}{\rightarrow}$ coincide com o homomorfismo $\varphi$, utilizado na demonstração do item (iii) da proposição A.2.

Proposição A.3 . Seja $\Phi=\left\{\phi, \phi_{\alpha}\right\}_{\alpha \in \Lambda}$ uma aplicação entre dois sistemas diretos $\left\{G_{\alpha}, f_{\alpha \beta}\right\}_{\alpha, \beta \in \Lambda}$ e $\left\{H_{\gamma}, k_{\gamma \delta}\right\}_{\gamma, \delta \in \Omega}$. Se $\operatorname{Im}(\phi)=\phi[\Lambda]$ é um subconjunto cofinal em $\Omega$, e, para cada $\alpha \in \Lambda, o$ homomorfismo $\phi_{\alpha}: G_{\alpha} \longrightarrow H_{\phi(\alpha)}$ é um isomorfismo, então os grupos $\lim _{\longrightarrow}\left\{G_{\alpha}: \alpha \in \Lambda\right\}$ e $\underset{\lim }{\longrightarrow}\left\{H_{\gamma}: \gamma \in \Omega\right\}$ são isomorfos; em particular, se $\phi: \Lambda \longrightarrow \Omega$ é sobrejetora, o limite direto $\overleftrightarrow{\Phi}: \underset{\lim }{\longrightarrow}\left\{G_{\alpha}: \alpha \in \Lambda\right\} \longrightarrow \lim _{\longrightarrow}\left\{H_{\gamma}: \gamma \in \Omega\right\}$ é um isomorfismo. 
DemonstraÇÃo. Sendo $\Omega_{0}=\operatorname{Im}(\phi) \subseteq \Omega$, sabemos que o limite direto de $\Phi$ é o homomorfismo

$$
\stackrel{\Phi}{\rightarrow}: \lim _{\longrightarrow}\left\{G_{\alpha}: \alpha \in \Lambda\right\} \longrightarrow \lim _{\longrightarrow}\left\{H_{\gamma}: \gamma \in \Omega_{0}\right\},
$$

dado por $\underset{\Phi}{\rightarrow}\left(\llbracket g_{\alpha} \rrbracket\right)=\llbracket \phi_{\alpha}\left(g_{\alpha}\right) \rrbracket$ para cada $g_{\alpha} \in G_{\alpha}$, onde $\alpha \in \Lambda$. Por outro lado, denotando por $0_{\phi(\alpha)}$ o elemento identidade do grupo $H_{\phi(\alpha)}$, sabemos também que a classe $\llbracket 0_{\phi(\alpha)} \rrbracket$ é o elemento identidade de $\underset{\lim }{\longrightarrow}\left\{H_{\gamma}: \gamma \in \Omega_{0}\right\}$, qualquer que seja $\alpha \in \Lambda$. Assim, temos que:

$$
\operatorname{Ker}(\stackrel{\Phi}{\rightarrow})=\left\{\llbracket g_{\beta} \rrbracket \in \underset{\lim }{\longrightarrow}\left\{G_{\alpha}: \alpha \in \Lambda\right\}: \underset{\Phi}{\rightarrow}\left(\llbracket g_{\beta} \rrbracket\right)=\llbracket \phi_{\beta}\left(g_{\beta}\right) \rrbracket=\llbracket 0_{\phi(\alpha)} \rrbracket\right\} .
$$

Logo, dado $\llbracket g_{\beta} \rrbracket \in \operatorname{Ker}(\underset{\Phi}{\rightarrow})$, segue que $\llbracket \phi_{\beta}\left(g_{\beta}\right) \rrbracket=\llbracket 0_{\phi(\alpha)} \rrbracket$, isto é, $\phi_{\beta}\left(g_{\beta}\right) \sim 0_{\phi(\alpha)}$ em $\Omega_{0}$; isto, por sua vez, significa que existe um limitante superior $\delta \in \Omega_{0}$ de $\phi(\alpha)$ e $\phi(\beta)$, tal que $k_{\phi(\beta) \delta}\left(\phi_{\beta}\left(g_{\beta}\right)\right)=k_{\phi(\alpha) \delta}\left(0_{\phi(\alpha)}\right)=0_{\delta}$. Suponhamos então que $\delta=\phi(\gamma)$, onde $\gamma \in \Lambda$ (lembre-se que $\left.\Omega_{0}=\operatorname{Im}(\phi)\right)$; temos, pois, que:

$$
k_{\phi(\beta) \phi(\gamma)}\left(\phi_{\beta}\left(g_{\beta}\right)\right)=0_{\phi(\gamma)} .
$$

Portanto, como $k_{\phi(\beta) \phi(\gamma)} \circ \phi_{\beta}=\phi_{\gamma} \circ f_{\beta \gamma}$, segue que:

$$
\phi_{\gamma}\left(f_{\beta \gamma}\left(g_{\beta}\right)\right)=0_{\phi(\gamma)}
$$

(note que $\beta \leqslant \gamma$ em $\Lambda$, pois $\phi(\beta) \leqslant \phi(\gamma)$ em $\Omega_{0}$ ). Conseqüentemente, como $\phi_{\gamma}$ é um isomorfismo (por hipótese), temos que $f_{\beta \gamma}\left(g_{\beta}\right)=0_{\gamma}$, o que implica que $g_{\beta} \sim 0_{\gamma}$ em $\Lambda$ (pois $\gamma$ é um limitante superior de $\beta$ tal que $f_{\beta \gamma}\left(g_{\beta}\right)=0_{\gamma}=f_{\gamma \gamma}\left(0_{\gamma}\right)$ ). Deste modo, concluímos que $\llbracket g_{\beta} \rrbracket=\llbracket 0_{\gamma} \rrbracket$ para todo $\llbracket g_{\beta} \rrbracket \in \operatorname{Ker}(\stackrel{\Phi}{\rightarrow})$, ou seja, $\operatorname{Ker}(\stackrel{\Phi}{\rightarrow})$ é o subgrupo trivial de $\underset{\lim }{\longrightarrow}\left\{G_{\alpha}: \alpha \in \Lambda\right\}$ (uma vez que $\llbracket 0_{\gamma} \rrbracket$ é o elemento identidade deste grupo, qualquer que seja $\gamma \in \Lambda$ ), provando, assim, que $\Phi$ é um homomorfismo injetor.

Agora, dado $\llbracket h_{\gamma} \rrbracket \in \underset{\longrightarrow}{\lim }\left\{H_{\gamma}: \gamma \in \Omega_{0}\right\}$ arbitrário, seja $\alpha \in \Lambda$ tal que $\phi(\alpha)=\gamma$, e considere o elemento $g_{\alpha} \in \phi_{\alpha}^{-1}\left(h_{\gamma}\right) \in G_{\alpha}$. Temos então que:

$$
\underset{\Phi}{\rightarrow}\left(\llbracket g_{\alpha} \rrbracket\right)=\llbracket \phi_{\alpha}\left(g_{\alpha}\right) \rrbracket=\llbracket \phi_{\alpha}\left(\phi_{\alpha}^{-1}\left(h_{\gamma}\right)\right) \rrbracket=\llbracket h_{\gamma} \rrbracket,
$$

ou seja, todo elemento de $\lim _{\longrightarrow}\left\{H_{\gamma}: \gamma \in \Omega_{0}\right\}$ é a imagem de um elemento de $\underline{\lim }_{\longrightarrow}\left\{G_{\alpha}:\right.$

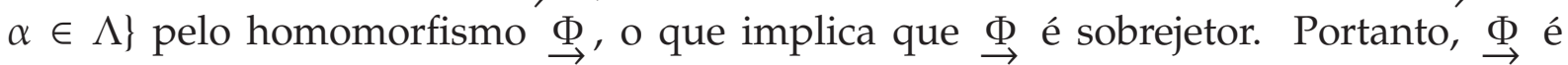
um isomorfismo entre $\underset{\lim }{\longrightarrow}\left\{G_{\alpha}: \alpha \in \Lambda\right\}$ e $\underset{\lim }{\longrightarrow}\left\{H_{\gamma}: \gamma \in \Omega_{0}\right\}$. Finalmente, como $\Omega_{0}$ é cofinal em $\Omega$ (por hipótese), segue, do item (iii) da proposição A.2, que o grupo $\underset{\lim }{\longrightarrow}\left\{H_{\gamma}: \gamma \in \Omega_{0}\right\}$ é isomorfo a $\underset{\lim }{\longrightarrow}\left\{H_{\gamma}: \gamma \in \Omega\right\}$, d'onde concluímos que

$$
\underset{\lim _{\alpha}}{\longrightarrow}\left\{G_{\alpha}: \alpha \in \Lambda\right\} \simeq \lim _{\longrightarrow}\left\{H_{\gamma}: \gamma \in \Omega\right\} \text {. }
$$

Em particular, note que, se $\phi: \Lambda \longrightarrow \Omega$ é sobrejetora, temos $\Omega_{0}=\phi[\Lambda]=\Omega$, e, portanto, o limite direto $\stackrel{\Phi}{\rightarrow}$ é um isomorfismo entre $\lim _{\longrightarrow}\left\{G_{\alpha}: \alpha \in \Lambda\right\}$ e $\underset{\lim }{\longrightarrow}\left\{H_{\gamma}: \gamma \in\right.$ $\left.\Omega_{0}\right\}=\underset{\lim }{\longrightarrow}\left\{H_{\gamma}: \gamma \in \Omega\right\}$. 
Proposição A.4 . Sejam $\left\{K_{\alpha}, f_{\alpha \beta}\right\}_{\alpha, \beta \in \Lambda},\left\{L_{\alpha}, g_{\alpha \beta}\right\}_{\alpha, \beta \in \Lambda},\left\{M_{\gamma}, h_{\gamma \delta}\right\}_{\gamma, \delta \in \Omega}$ e $\left\{N_{\gamma}, i_{\gamma \delta}\right\}_{\gamma, \delta \in \Omega}$ sistemas diretos de grupos abelianos e homomorfismos, correspondentes a conjuntos dirigidos $\Lambda e \Omega, e$ considere aplicações $\mu: \Lambda \longrightarrow \Lambda, \phi, \psi: \Lambda \longrightarrow \Omega e \xi: \Omega \longrightarrow \Omega$ que preservam ordem $e$ comutam no seguinte diagrama:

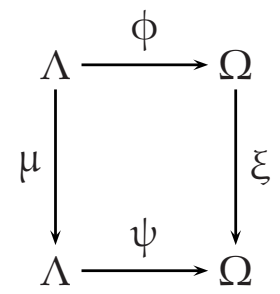

Suponha ainda que $\left\{\phi_{\alpha}: K_{\alpha} \longrightarrow M_{\phi(\alpha)}\right\}_{\alpha \in \Lambda},\left\{\psi_{\alpha}: L_{\alpha} \longrightarrow N_{\psi(\alpha)}\right\}_{\alpha \in \Lambda},\left\{\mu_{\alpha}: K_{\alpha} \longrightarrow L_{\mu(\alpha)}\right\}_{\alpha \in \Lambda} e$ $\left\{\xi_{\gamma}: M_{\gamma} \longrightarrow N_{\xi(\gamma)}\right\}_{\gamma \in \Omega}$ são famílias de homomorfismos, tais que, para cada $\alpha \in \Lambda$, o diagrama abaixo é comutativo:

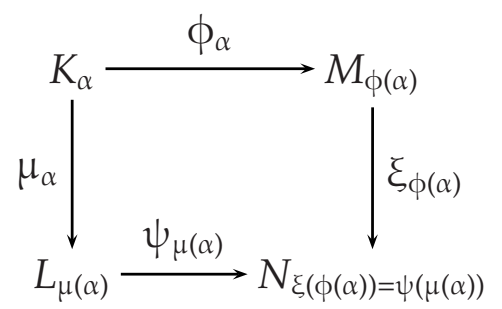

Se $\Phi=\left\{\phi, \phi_{\alpha}\right\}_{\alpha \in \Lambda}, \Psi=\left\{\psi, \psi_{\alpha}\right\}_{\alpha \in \Lambda}, \Gamma=\left\{\mu, \mu_{\alpha}\right\}_{\alpha \in \Lambda}$ e $\Sigma=\left\{\xi, \xi_{\gamma}\right\}_{\gamma \in \Omega}$ são aplicações entre

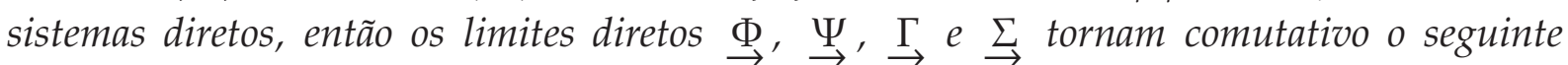
diagrama:

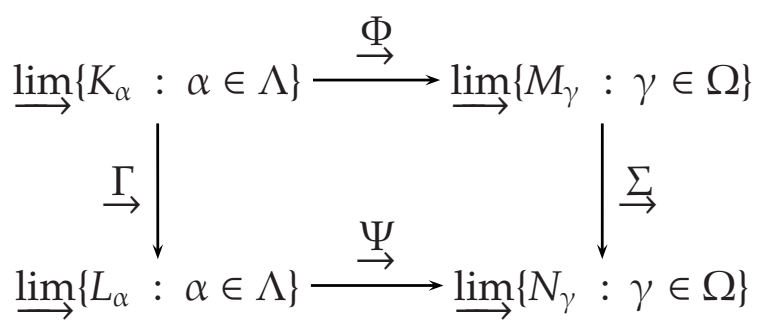

DemonstraçÃo. Com efeito, dados $\alpha \in \Lambda$ e $k \in K_{\alpha}$, temos que:

$$
\begin{aligned}
& \left(\stackrel{\sum}{\rightarrow} \circ \underline{\Phi}\right)(\llbracket k \rrbracket)=\stackrel{\sum}{\rightarrow}(\stackrel{\Phi}{\rightarrow}(\llbracket k \rrbracket))=\stackrel{\sum}{\rightarrow}\left(\llbracket \phi_{\alpha}(k) \rrbracket\right) \\
& =\llbracket \xi_{\phi(\alpha)}\left(\phi_{\alpha}(k)\right) \rrbracket=\llbracket \psi_{\mu(\alpha)}\left(\mu_{\alpha}(k)\right) \rrbracket \\
& =\underset{\Psi}{\rightarrow}\left(\llbracket \mu_{\alpha}(k) \rrbracket\right)=\underline{\Psi}(\underline{\Gamma}(\llbracket k \rrbracket)) \\
& =(\stackrel{\Psi}{\rightarrow} \circ \stackrel{\Gamma}{\rightarrow})(\llbracket k \rrbracket) \text {, }
\end{aligned}
$$

ou seja, $\stackrel{\sum}{\rightarrow} \stackrel{\Phi}{\rightarrow}=\stackrel{\Psi}{\rightarrow} \circ \stackrel{\Gamma}{\rightarrow}$, conforme queríamos provar. 
Proposição A.5 . Sejam

$$
\mathcal{S}_{1}=\left\{K_{\alpha}, f_{\alpha \beta}\right\}_{\alpha, \beta \in \Lambda}, \quad \mathcal{S}_{2}=\left\{L_{\alpha}, g_{\alpha, \beta}\right\}_{\alpha, \beta \in \Lambda} \text { e } \mathcal{S}_{3}=\left\{M_{\alpha}, h_{\alpha \beta}\right\}_{\alpha, \beta \in \Lambda}
$$

sistemas diretos de grupos abelianos e homomorfismos, correspondentes a um mesmo conjunto dirigido $\Lambda$, e, para cada $\alpha \in \Lambda$, sejam $\phi_{\alpha}: L_{\alpha} \longrightarrow K_{\alpha}$ e $\varphi_{\alpha}: K_{\alpha} \longrightarrow M_{\alpha}$ homomorfismos tais que a seguinte seqüência é exata:

$$
L_{\alpha} \stackrel{\phi_{\alpha}}{\longrightarrow} K_{\alpha} \stackrel{\varphi_{\alpha}}{\longrightarrow} M_{\alpha}
$$

Suponha ainda que, para cada par $\alpha, \beta \in \Lambda, \operatorname{com} \alpha \leqslant \beta$, o diagrama abaixo é comutativo:

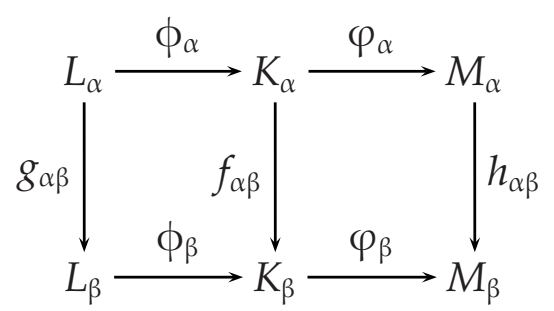

Sendo $\phi: \Lambda \longrightarrow \Lambda$ a função identidade, temos então que $\Phi=\left\{\phi, \phi_{\alpha}\right\}_{\alpha \in \Lambda}$ e $\Psi=\left\{\phi, \varphi_{\alpha}\right\}_{\alpha \in \Lambda}$ são aplicações (de $S_{2}$ em $S_{1}$, e de $S_{1}$ em $\mathcal{S}_{3}$, respectivamente), tais que a seqüência a seguir também é exata:

$$
\stackrel{\lim }{\longrightarrow}\left\{L_{\alpha}: \alpha \in \Lambda\right\} \stackrel{\Phi}{\longrightarrow} \lim _{\longrightarrow}\left\{K_{\alpha}: \alpha \in \Lambda\right\} \stackrel{\Psi}{\longrightarrow} \lim _{\longrightarrow}\left\{M_{\alpha}: \alpha \in \Lambda\right\}
$$

DemonstraçÃo. O fato de $\Phi=\left\{\phi, \phi_{\alpha}\right\}_{\alpha \in \Lambda}$ e $\Psi=\left\{\phi, \varphi_{\alpha}\right\}_{\alpha \in \Lambda}$ serem aplicações entre sistemas diretos segue de imediato da comutatividade do diagrama acima. Assim sendo, vamos mostrar que a seqüência

$$
\stackrel{\lim }{\longrightarrow}\left\{L_{\alpha}: \alpha \in \Lambda\right\} \stackrel{\Phi}{\rightarrow} \lim _{\longrightarrow}\left\{K_{\alpha}: \alpha \in \Lambda\right\} \stackrel{\Psi}{\rightarrow} \underset{\longrightarrow}{\lim }\left\{M_{\alpha}: \alpha \in \Lambda\right\}
$$

é exata, isto é, $\operatorname{Im}(\stackrel{\Phi}{\rightarrow})=\operatorname{Ker}(\underset{\Psi}{\rightarrow})$. Com efeito, dado $\llbracket k_{\alpha} \rrbracket \in \operatorname{Im}(\stackrel{\Phi}{\rightarrow}) \subseteq \underset{\lim }{\rightarrow}\left\{K_{\alpha}: \alpha \in \Lambda\right\}$ (onde $k_{\alpha} \in K_{\alpha}$ ), segue que existe $\llbracket l_{\beta} \rrbracket \in \underset{\lim }{\longrightarrow}\left\{L_{\alpha}: \alpha \in \Lambda\right\}$ (com $l_{\beta} \in L_{\beta}$ ) tal que $\underset{\rightarrow}{\rightarrow}\left(\llbracket l_{\beta} \rrbracket\right)=$ $\llbracket k_{\alpha} \rrbracket$. Como $\underset{\Phi}{\rightarrow}\left(\llbracket l_{\beta} \rrbracket\right)=\llbracket \phi_{\beta}\left(l_{\beta}\right) \rrbracket$, temos então que $\llbracket \phi_{\beta}\left(l_{\beta}\right) \rrbracket=\llbracket k_{\alpha} \rrbracket$, ou seja, $\phi_{\beta}\left(l_{\beta}\right) \sim k_{\alpha}$. Isto, por sua vez, significa que existe um limitante superior $\gamma \in \Lambda$ de $\alpha$ e $\beta$, tal que $f_{\beta \gamma}\left(\phi_{\beta}\left(l_{\beta}\right)\right)=f_{\alpha \gamma}\left(k_{\alpha}\right)$, e, portanto, $f_{\alpha \gamma}\left(k_{\alpha}\right) \in \operatorname{Im}\left(\phi_{\gamma}\right) \subseteq K_{\gamma} . \operatorname{Logo}, \operatorname{como} \operatorname{Im}\left(\phi_{\gamma}\right)=\operatorname{Ker}\left(\varphi_{\gamma}\right)$, temos que $\varphi_{\gamma}\left(f_{\alpha \gamma}\left(k_{\alpha}\right)\right)=0_{M_{\gamma}}$, onde $0_{M_{\gamma}}$ é o elemento identidade do grupo $M_{\gamma}$. Agora, como $\varphi_{\gamma} \circ f_{\alpha \gamma}=h_{\alpha \gamma} \circ \varphi_{\alpha}$, obtemos que $h_{\alpha \gamma}\left(\varphi_{\alpha}\left(k_{\alpha}\right)\right)=0_{M_{\gamma}}=h_{\beta \gamma}\left(0_{M_{\beta}}\right)$. Resumindo, temos que $\gamma$ é um limitante superior de $\alpha$ e $\beta$, tal que $h_{\alpha \gamma}\left(\varphi_{\alpha}\left(k_{\alpha}\right)\right)=h_{\beta \gamma}\left(0_{M_{\beta}}\right)$, o que implica que $\varphi_{\alpha}\left(k_{\alpha}\right) \sim 0_{M_{\beta}}$, isto é, $\llbracket \varphi_{\alpha}\left(k_{\alpha}\right) \rrbracket=\llbracket 0_{M_{\beta}} \rrbracket$. Assim, como $\underset{\Psi}{\Psi}\left(\llbracket k_{\alpha} \rrbracket\right)=\llbracket \varphi_{\alpha}\left(k_{\alpha}\right) \rrbracket$, concluímos que $\underset{\Psi}{\rightarrow}\left(\llbracket k_{\alpha} \rrbracket\right)=\llbracket 0_{M_{\beta}} \rrbracket$, ou seja, $\llbracket k_{\alpha} \rrbracket \in \operatorname{Ker}(\underset{\Psi}{\rightarrow})$ (uma vez que $0_{M_{\beta}}$ é o elemento identidade de $\underset{\lim }{\longrightarrow}\left\{M_{\alpha}: \alpha \Lambda\right\}$, qualquer que seja $\beta \in \Lambda$ ). Deste modo, provamos que $\operatorname{Im}(\underline{\Phi}) \subseteq \operatorname{Ker}(\underset{\Psi}{\rightarrow})$. 
Por outro lado, consideremos um elemento $\llbracket k_{\alpha} \rrbracket \in \operatorname{Ker}\left(\stackrel{\Psi}{\rightarrow} \subseteq \varliminf_{\longrightarrow}\left\{K_{\alpha}: \alpha \in \Lambda\right\}\right.$; temos, pois, que $\underset{\sim}{\Psi}\left(\llbracket k_{\alpha} \rrbracket\right)=\llbracket \varphi_{\alpha}\left(k_{\alpha}\right) \rrbracket=\llbracket 0_{M_{\beta}} \rrbracket$, onde $0_{M_{\beta}}$ é o elemento identidade do grupo $M_{\beta}$ (e $\beta \in \Lambda$ é arbitrário). Logo, segue que $\varphi_{\alpha}\left(k_{\alpha}\right) \sim 0_{M_{\beta}}$, ou seja, existe um limitante superior $\gamma \in \Lambda$ de $\alpha$ e $\beta$, tal que $h_{\alpha \gamma}\left(\varphi_{\alpha}\left(k_{\alpha}\right)\right)=h_{\beta \gamma}\left(0_{M_{\beta}}\right)=0_{M_{\gamma}}$. Como $h_{\alpha \gamma} \circ \varphi_{\alpha}=\varphi_{\gamma} \circ f_{\alpha \gamma}$, temos então que $\varphi_{\gamma}\left(f_{\alpha \gamma}\left(k_{\alpha}\right)\right)=0_{M_{\gamma}}$, isto é, $f_{\alpha \gamma}\left(k_{\alpha}\right) \in \operatorname{Ker}\left(\varphi_{\gamma}\right) \subseteq K_{\gamma}$. Portanto, como $\operatorname{Ker}\left(\varphi_{\gamma}\right)=\operatorname{Im}\left(\phi_{\gamma}\right)$, segue que $f_{\alpha \gamma}\left(k_{\alpha}\right) \in \operatorname{Im}\left(\phi_{\gamma}\right)$, o que significa que existe $l_{\gamma} \in L_{\gamma}$ tal que $\phi_{\gamma}\left(l_{\gamma}\right)=f_{\alpha \gamma}\left(k_{\alpha}\right)$. Agora, sendo $\delta \in \Lambda$ um limitante superior de $\alpha$ e $\gamma$, observe que:

$$
f_{\gamma \delta}\left(\phi_{\gamma}\left(l_{\gamma}\right)\right)=f_{\gamma \delta}\left(f_{\alpha \gamma}\left(k_{\alpha}\right)\right)=f_{\alpha \delta}\left(k_{\alpha}\right) .
$$

Logo, temos que $\phi_{\gamma}\left(l_{\gamma}\right) \sim k_{\alpha}$, ou seja, $\underset{\rightarrow}{\rightarrow}\left(\llbracket l_{\gamma} \rrbracket\right)=\llbracket \phi_{\gamma}\left(l_{\gamma}\right) \rrbracket=\llbracket k_{\alpha} \rrbracket$, d'onde concluímos que $\llbracket k_{\alpha} \rrbracket \in \operatorname{Im}(\underset{\Phi}{\rightarrow})$. Assim, a inclusão $\operatorname{Ker}(\underset{\Psi}{\rightarrow}) \subseteq \operatorname{Im}(\stackrel{\Phi}{\rightarrow})$ também se verifica, de modo que $\operatorname{Im}(\underset{\Phi}{\rightarrow})=\operatorname{Ker}(\stackrel{\Psi}{\rightarrow})$, conforme queríamos demonstrar. 


\section{Apêndlice $\mathbb{B}$}

\section{Um Homeomorfismo para Variedades Topológicas}

Neste apêndice, vamos provar o seguinte resultado (utilizado na demonstração do teorema 2.1.2):

Teorema B.1. Seja M uma variedade topológica conexa de dimensão $n \geqslant 1$. Dados quatro pontos arbitrários $x_{1}, y_{1}, x_{2}, y_{2} \in$ Int $M$, existe um homeomorfismo $h: M \longrightarrow M$ tal que $h\left(x_{1}\right)=y_{1}$ e $h\left(x_{2}\right)=y_{2}$.

Com efeito, utilizando a demonstração do lema 6.4 de [Vi] (páginas 150 a 152), obtemos o seguinte:

Lema B.1 . Seja M uma variedade topológica conexa de dimensão $n$, e, dados $x, y \in \operatorname{Int} M$, considere um caminho $\omega: I \longrightarrow$ Int $M$ tal que $\omega(0)=x$ e $\omega(1)=y$ (o qual existe, pois $M$ é conexa por caminhos). Seja ainda $\left\{U_{1}, U_{2}, \ldots, U_{k}\right\}$ uma cobertura aberta finita do subconjunto compacto $\omega(I) \subseteq$ Int $M$, onde, para cada $i \in\{1,2, \ldots, k\}, U_{i}$ é homeomorfo a uma bola aberta em $\mathbb{R}^{n}$. Então, denotando por $U$ a união dos abertos $U_{i}$, segue que existe um homeomorfismo $\varphi: M \longrightarrow M$ tal que a restrição $\left.\varphi\right|_{M \backslash U}: M \backslash U \longrightarrow M \backslash U$ é a identidade, e $\varphi(x)=y$.

Assim, sob as hipóteses do teorema B.1, considere dois caminhos $\omega_{1}, \omega_{2}: I \longrightarrow \operatorname{Int} M$, $\operatorname{com} \omega_{1}(0)=x_{1}, \omega_{1}(1)=y_{1}, \omega_{2}(0)=x_{2}$ e $\omega_{2}(1)=y_{2}$, e sejam $\mathcal{C}_{1}=\left\{U_{1}, U_{2}, \ldots, U_{k_{1}}\right\}$ e $\mathrm{C}_{2}=\left\{V_{1}, V_{2}, \ldots, V_{k_{2}}\right\}$ coberturas abertas de $\omega_{1}(I)$ e $\omega_{2}(I)$, respectivamente, tais que, para cada $i \in\left\{1,2, \ldots, k_{1}\right\}$ e cada $j \in\left\{1,2, \ldots, k_{2}\right\}$, os conjuntos $U_{i}$ e $V_{j}$ são homeomorfos a 
bolas abertas em $\mathbb{R}^{n}$. Considere ainda

$$
U=\bigcup_{i=1}^{k_{1}} U_{i} \quad \text { e } \quad V=\bigcup_{j=1}^{k_{2}} V_{j},
$$

e sejam $\varphi_{1}: M \longrightarrow M$ e $\varphi_{2}: M \longrightarrow M$ os homeomorfismos que satisfazem $\varphi_{1}\left(x_{1}\right)=y_{1}$ e $\varphi_{2}\left(x_{2}\right)=y_{2}$, tais que as restrições $\left.\varphi_{1}\right|_{M \backslash U}$ e $\left.\varphi_{2}\right|_{M \backslash V}$ coincidem com as aplicações identidade em $M \backslash U$ e $M \backslash V$, respectivamente. Isto posto, a fim de construirmos o homomorfismo $h: M \longrightarrow M$ mencionado no teorema B.1, é natural tentarmos definir

$$
\text { (*) } \quad h(z)=\left\{\begin{array}{lll}
\varphi_{1}(z) & \text { se } & z \in U \\
\varphi_{2}(z) & \text { se } & z \in V
\end{array}\right. \text {. }
$$

Entretanto, para que esta aplicação esteja bem definida, precisamos verificar que as coberturas $\mathcal{C}_{1}$ e $\mathcal{C}_{2}$ podem ser tomadas de tal forma que $U \cap V=\varnothing$ (pois, caso contrário, não há como garantir que $\varphi_{1}(z)=\varphi_{2}(z)$ para $\left.z \in U \cap V\right)$, sendo que isto, por sua vez, depende dos caminhos $\omega_{1}$ e $\omega_{2}$ não se interceptarem (ou seja, $\omega_{1}(I) \cap \omega_{2}(I)=\varnothing$ ); a figura B.1 ilustra uma situação na qual o homomorfismo $h$, dado por $(\star)$, está bem definido.

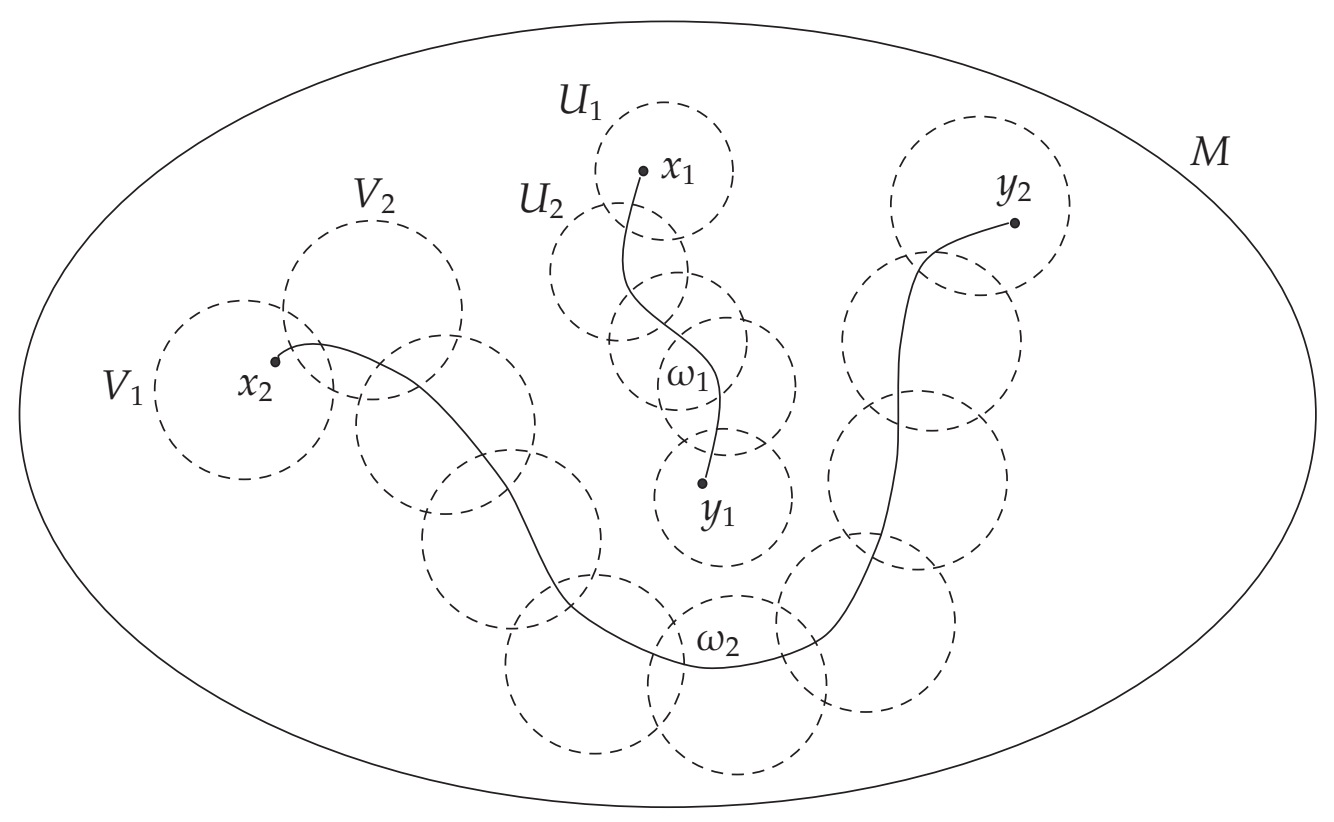

Figura B.1

Resumindo, para concluirmos a demonstração do teorema B.1, basta mostrarmos que, dados quatro pontos $x_{1}, x_{2}, y_{1}$ e $y_{2}$ no interior de uma variedade topológica conexa 
$M$ (de dimensão $n$ ), existem caminhos $\omega_{1}, \omega_{2}: I \longrightarrow \operatorname{Int} M, \operatorname{com} \omega_{1}(0)=x_{1}, \omega_{1}(1)=y_{1}$, $\omega_{2}(0)=x_{2}$ e $\omega_{2}(1)=y_{2}$, tais que $\omega_{1}(I) \cap \omega_{2}(I)=\varnothing$. Para tanto, é suficiente mostrar que existe uma n-célula $W \subseteq \operatorname{Int} M$ (isto é, um subconjunto $W \subseteq \operatorname{Int} M$, homeomorfo a uma bola aberta em $\mathbb{R}^{n}$ ), que contém os pontos $x_{1}, x_{2}, y_{1}$ e $y_{2}$, pois, neste caso, tomando um caminho qualquer $\omega_{1}: I \longrightarrow W$, ligando $x_{1}$ a $y_{1}$, sabemos que existe um caminho $\omega_{2}: I \longrightarrow W$, ligando $x_{2}$ a $y_{2}$, que não intercepta $\omega_{1}$ (uma vez que $W \backslash \omega_{1}(I)$ é conexo por caminhos).

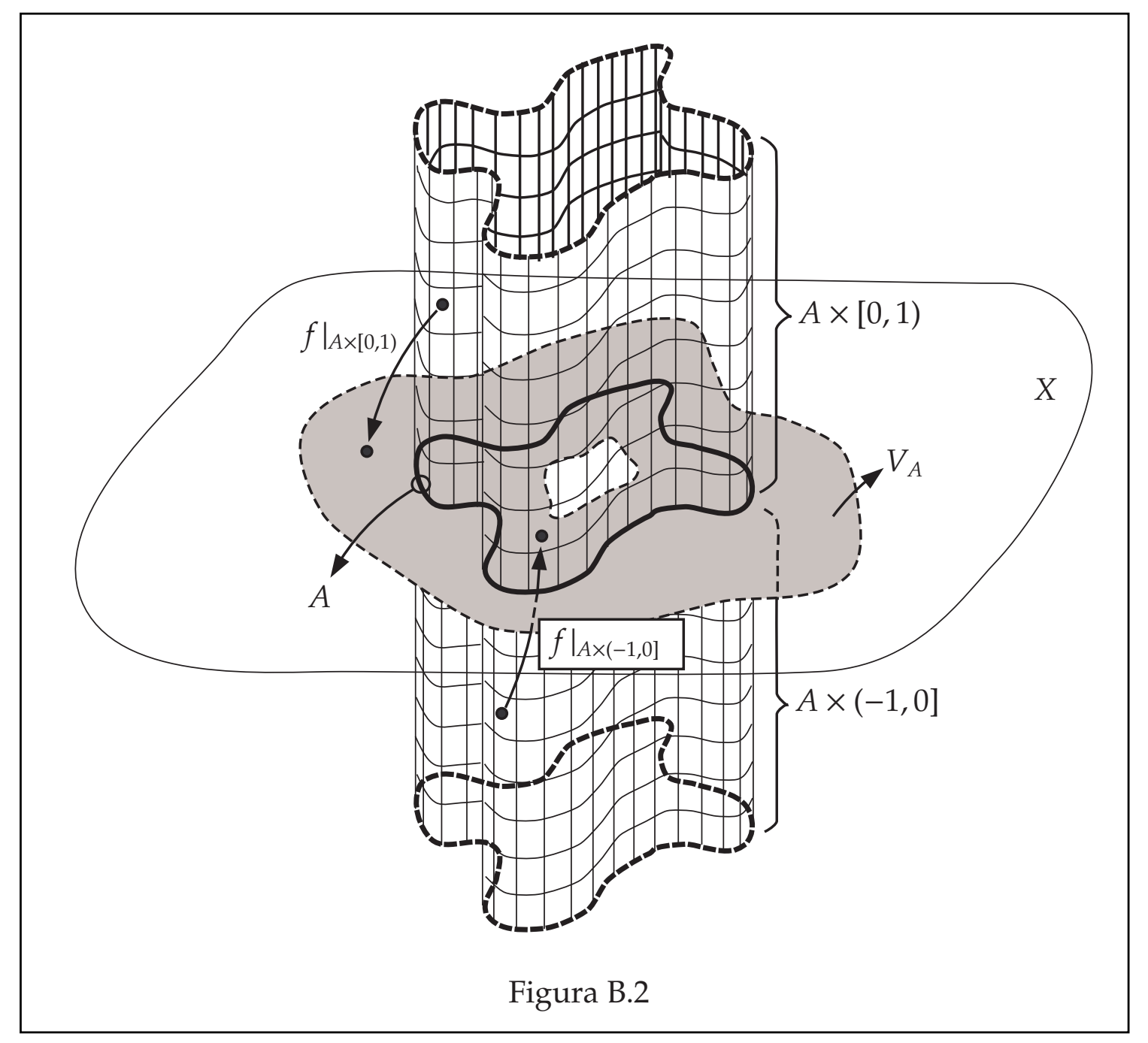

Com este propósito, vamos introduzir então a seguinte

Definição B.1. Seja X um espaço topológico. Dizemos que um subconjunto $A \subseteq X$, possui colarinho duplo em $X$ se existe um homeomorfismo $f$ de $A \times(-1,1)$ sobre uma vizinhança aberta $V_{A} \subseteq X$ de $A$, tal que $f(a, 0)=$ a para todo $a \in A$ (vide figura B.2). 
O lema a seguir constitui um caso particular do teorema 3 de [Bwn1], e nos fornece um exemplo de subconjunto com colarinho duplo (a demonstração será omitida, pois requer uma investigação mais detalhada das propriedades métricas de uma variedade topológica).

Lema B.2 . Seja M uma variedade topológica conexa de dimensão $n$. Se $D \subseteq$ Int $M$ é uma $n$-célula fechada (isto é, um subconjunto de Int $M$, homeomorfo a uma bola fechada em $\mathbb{R}^{n}$ ), então a fronteira $\partial D$ é uma subvariedade topológica de $M$, com dimensão $n-1$, que possui colarinho duplo em $M$.

Por outro lado, o próximo lema (cuja demonstração pode ser encontrada em [DH], páginas 469-470) constitui a peça principal na demonstração do teorema B.1.

Lema B.3 . Sejam M uma variedade topológica conexa de dimensão n (com ou sem fronteira), e $D \subseteq M$ uma n-célula fechada cuja fronteira $\partial D$ possui colarinho duplo em $M$ (por exemplo, uma n-célula fechada em Int $M$ ). Então, dado $p \in M$ arbitrário, existe uma n-célula fechada $C \subseteq M$, tal que $\partial C$ possui colarinho duplo em $M$ e $D \cup\{p\} \subseteq \operatorname{Int} C$.

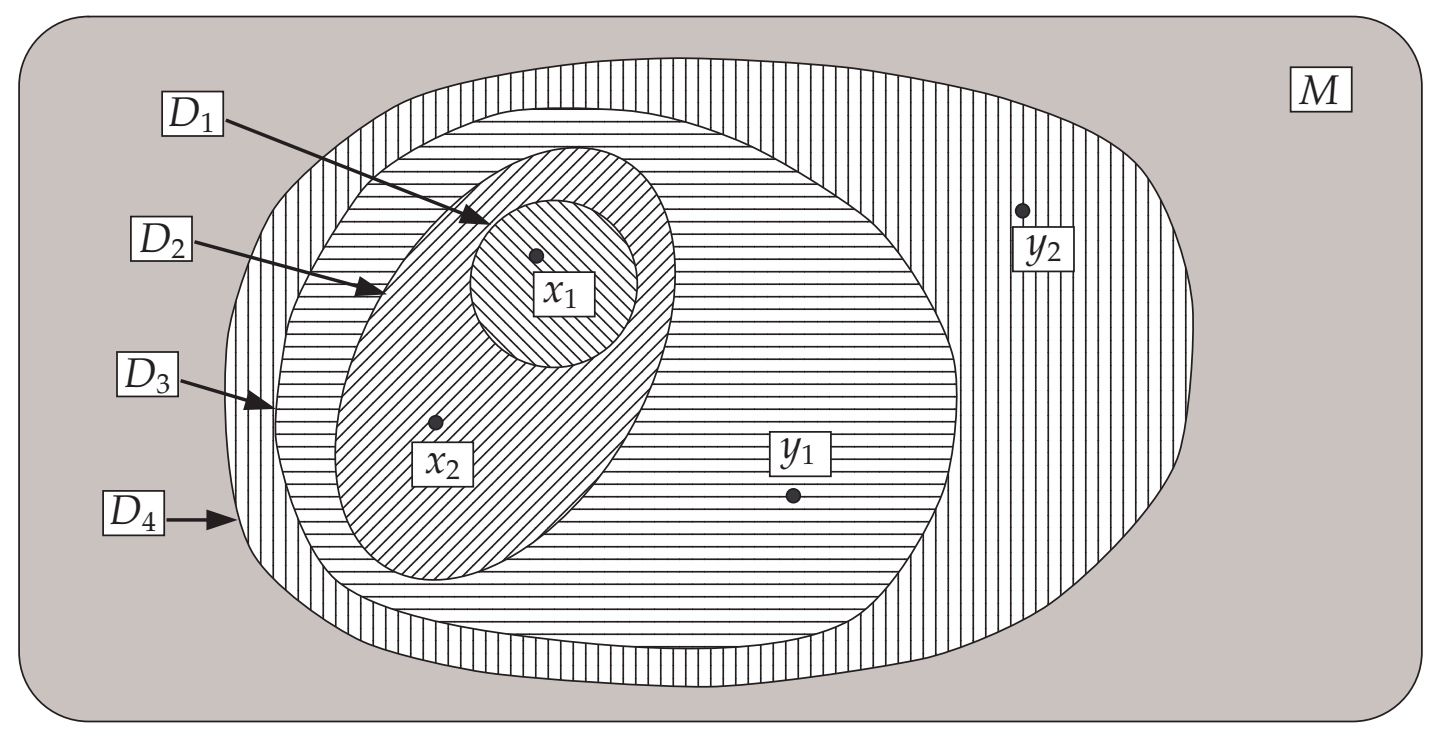

Figura B.3

Finalmente, podemos agora concluir a demonstração do teorema B.1. Considere, 
pois, $x_{1}, x_{2}, y_{1}, y_{2} \in \operatorname{Int} M$ (onde $M$ é uma variedade topológica conexa de dimensão $n$ ), e seja $D_{1} \subseteq$ Int $M$ uma n-célula fechada contendo $x_{1}$. Pelo teorema B.2, sabemos que $\partial D_{1}$ possui colarinho duplo em $M$; logo, do lema B.3, segue que existe uma n-célula fechada $D_{2} \subseteq$ Int $M$, tal que $\partial D_{2}$ possui colarinho duplo em $M$, e $D_{1} \cup\left\{x_{2}\right\} \subseteq \operatorname{Int} D_{2}$. Assim, aplicando mais duas vezes o lema B.3, segue que existem n-células fechadas $D_{3}, D_{4} \subseteq \operatorname{Int} M$, tais que $\partial D_{3}$ e $\partial D_{4}$ possuem colarinho duplo em $M, D_{2} \cup\left\{y_{1}\right\} \subseteq \operatorname{Int} D_{3}$ e $D_{3} \cup\left\{y_{2}\right\} \subseteq$ Int $D_{4}$ (vide figura B.3).

Deste modo, concluímos que existe uma n-célula $W \subseteq \operatorname{Int} M$ (a saber, $W=\operatorname{Int} D_{4}$ ) contendo todos os quatro pontos $x_{1}, x_{2}, y_{1}$ e $y_{2}$, o que, conforme mencionamos anteriormente, é suficiente para garantir que o homeomorfismo $h: M \longrightarrow M$ dado em $(\star)$ está de fato bem definido. 


\section{Deformações Propriamente Homotópicas que Preservam Raízes}

Neste apêndice, nosso objetivo é demonstrar que, dada uma aplicação contínua própria $f:(M, \partial M) \longrightarrow(N, \partial N)$, entre variedades topológicas conexas de mesma dimensão, e dado um ponto $c \in \operatorname{Int} N$ arbitrário, podemos deformar $f$ por meio de uma homotopia própria, a fim de obter uma aplicação contínua própria $\mathcal{F}:(M, \partial M, \operatorname{Int} M) \longrightarrow$ $(N, \partial N, \operatorname{Int} N)$, tal que $\mathcal{F}^{-1}(c)=f^{-1}(c)$.

Com este intuito, vamos introduzir então a seguinte

Definição C.1 . Dado um espaço topológico $X$, dizemos que um subconjunto $A \subseteq X$ possui colarinho em $X$ se existe um homeomorfismo $h$ de $A \times[0,1)$, sobre uma vizinhança aberta $V_{A} \subseteq X$ de $A$, tal que $h(x, 0)=x$ para todo $x \in A$. Por vacuidade, consideraremos ainda que $o$ conjunto vazio sempre possui colarinho, independentemente do espaço topológico $X$.

O próximo lema (cuja demonstração segue de imediato do teorema 1 e do lema 5 de [Bwn1]) constitui o exemplo clássico de subconjunto com colarinho.

Lema C.1 . A fronteira $\partial M$, de uma variedade topológica $M$, possui colarinho em $M$ (vide figura C.1).

Mais especificamente, o lema a seguir nos mostra que a fronteira de uma variedade topológica $M$ possui "colarinho fechado" em $M$. 
Lema C.2 . Dada uma variedade topológica $M$, existem uma vizinhança aberta $W \subseteq M$ da fronteira $\partial M$, bem como um homeomorfismo $h_{M}: \partial M \times I \longrightarrow \bar{W}$, tais que $h_{M}(\partial M \times\{1\})=$ $\bar{W} \backslash W=\partial W \backslash \partial M e h_{M}(x, 0)=x$ para todo $x \in \partial M$.

DemonstraçÃo. De fato, se $\partial M=\varnothing$, o resultado é imediato (por vacuidade). Caso contrário (isto é, se $\partial M \neq \varnothing)$, basta tomarmos $W=h\left(\partial M \times\left[0, \frac{1}{2}\right)\right)$ e $h_{M}(x, t)=h\left(x, \frac{t}{2}\right)$ para todo $(x, t) \in \partial M \times I$, onde $h$ é um homeomorfismo de $\partial M \times[0,1)$ sobre uma vizinhança aberta $V \subseteq M$ de $\partial M$ (o qual existe, pois, pelo lema C.1, sabemos que $\partial M$ possui colarinho em $M$ ).

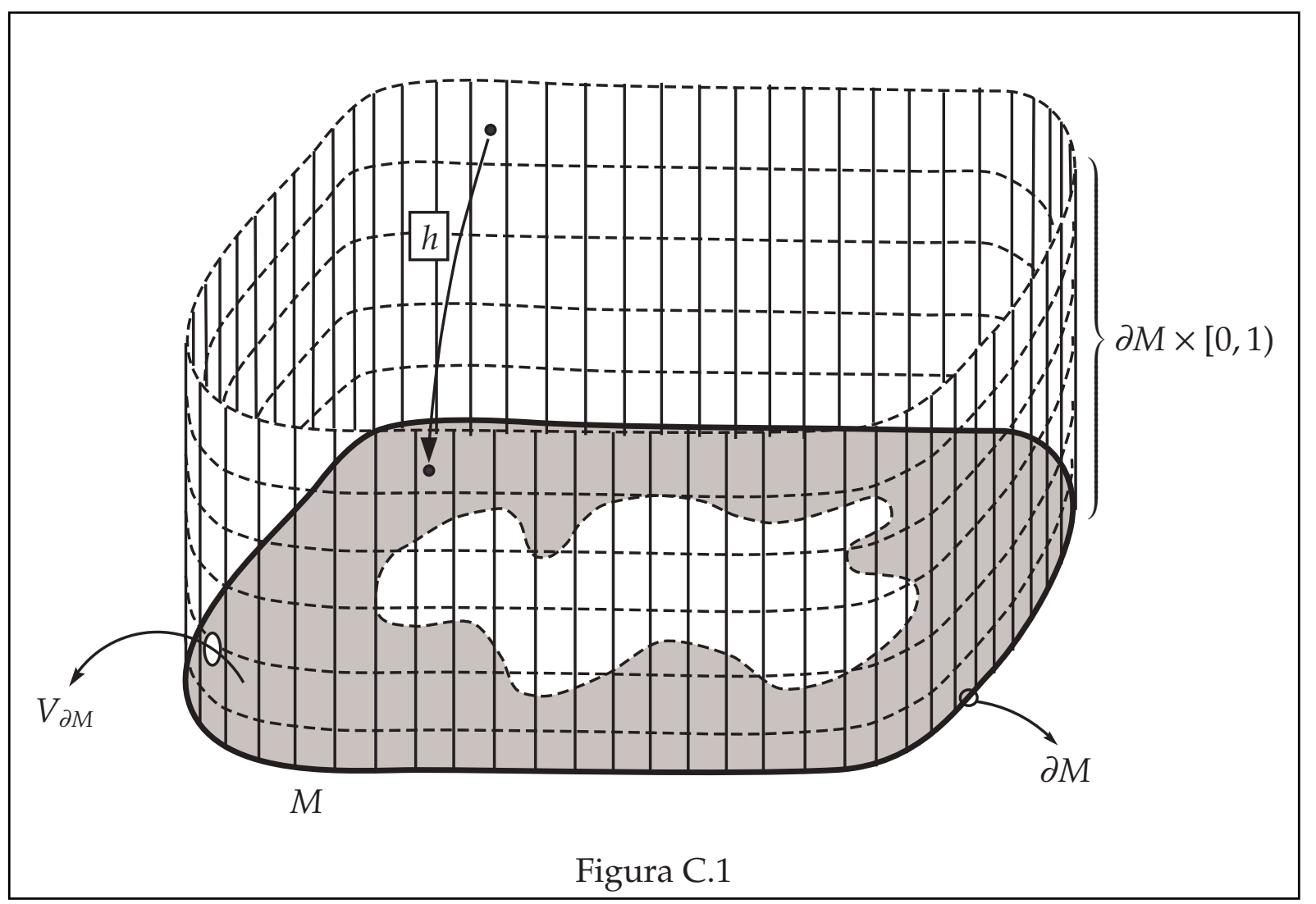

Agora, utilizando o lema acima, podemos demonstrar o principal resultado deste apêndice:

Teorema C.1. Seja $f:(M, \partial M) \longrightarrow(N, \partial N)$ uma aplicação contínua própria, entre variedades topológicas conexas de mesma dimensão $n$. Dado um ponto $c \in \operatorname{Int} N$, existe uma aplicação contínua própria $\mathcal{F}:(M, \partial M, \operatorname{Int} M) \longrightarrow(N, \partial N, \operatorname{Int} N)$, homotópica à $f$ por uma homotopia própria $H:(M \times I, \partial M \times I) \longrightarrow(N, \partial N)$, tal que $\mathcal{F}^{-1}(c)=f^{-1}(c)$. 
DemonstraÇÃo. De fato, como $c \in \operatorname{Int} N$ e $f$ preserva fronteira, temos que $f^{-1}(c) \subseteq$ Int $M$; logo, como $f^{-1}(c)$ é um subconjunto fechado de $M$, segue que $M \backslash f^{-1}(c)$ é aberto em $M$, e, conseqüentemente, constitui uma variedade topológica de dimensão $n$, cuja fronteira coincide com a fronteira de $M$. Analogamente, temos também que $N \backslash\{c\}$ é uma variedade topológica de dimensão $n$, tal que $\partial(N \backslash\{c\})=\partial N$. Isto posto, utilizando o lema C.2, considere vizinhanças abertas $W_{M} \subseteq M \backslash f^{-1}(c)$ e $W_{N} \subseteq N \backslash\{c\}$ de $\partial M$ e $\partial N$, respectivamente, e sejam $h_{M}: \partial M \times I \longrightarrow \overline{W_{M}}$ e $h_{N}: \partial N \times I \longrightarrow \overline{W_{N}}$ homeomorfismos tais que $h_{M}(\partial M \times\{1\})=\overline{W_{M}} \backslash W_{M}=\partial W_{M} \backslash \partial M, h_{N}(\partial N \times\{1\})=\overline{W_{N}} \backslash W_{N}=\partial W_{N} \backslash \partial N$, $h_{M}(x, 0)=x$ e $h_{N}(y, 0)=y$ para quaisquer $x \in \partial M$ e $y \in \partial N$. Temos, pois, que, se $x \in \overline{W_{M}}$ e $f(x) \in \overline{W_{N}}$, existem dois únicos pares $(z, s) \in \partial M \times I$ e $(y, t) \in \partial N \times I$ tais que $x=h_{M}(z, s)$ e $f(x)=h_{N}(y, t)$. Deste modo, vamos definir $\mathcal{F}: M \longrightarrow N$ da seguinte maneira: se $x \in M \backslash W_{M}$, consideraremos

$$
\mathcal{F}(x)=\left\{\begin{array}{cll}
f(x) & \text { se } & f(x) \in N \backslash W_{N} \\
h_{N}\left(y, \frac{t+1}{2}\right) & \text { se } & f(x)=h_{N}(y, t) \in \overline{W_{N}}
\end{array},\right.
$$

enquanto que, se $x=h_{M}(z, s) \in \overline{W_{M}}$, tomamos

$$
\mathcal{F}(x)=\left\{\begin{array}{cl}
f(x) & \text { se } f(x) \in N \backslash W_{N} \\
f(x) & \text { se } f(x)=h_{N}(y, t) \in \overline{W_{N}}, \operatorname{com} t \geqslant s \\
h_{N}\left(y, \frac{s+t}{2}\right) & \text { se } f(x)=h_{N}(y, t) \in \overline{W_{N}}, \operatorname{com} t \leqslant s
\end{array} .\right.
$$

Observe então que, se $x=h_{M}(z, s) \in \overline{W_{M}}$ e $f(x)=h_{N}(y, t) \in \overline{W_{N}}$, com $s=t$, temos $f(x)=h_{N}(y, t)=h_{N}\left(y, \frac{s+t}{2}\right)$. Por outro lado, sabemos que:

$$
\overline{W_{M}} \backslash W_{M}=\partial W_{M} \backslash \partial M=\left(M \backslash W_{M}\right) \cap \overline{W_{M}}
$$

e

$$
\overline{W_{N}} \backslash W_{N}=\partial W_{N} \backslash \partial N=\left(N \backslash W_{N}\right) \cap \overline{W_{N}} ;
$$

assim, como $h_{M}(z, s) \in \overline{W_{M}} \backslash W_{M}$ e $h_{N}(y, t) \in \overline{W_{N}} \backslash W_{N}$ se, e somente se, $s=t=1$, segue que:

(i) se $x \in M \backslash W_{M}$ e $f(x)=h_{N}(y, t) \in \overline{W_{N}} \backslash W_{N}$, temos $t=1$, e, portanto,

$$
\mathcal{F}(x)=f(x)=h_{N}(y, t)=h_{N}\left(y, \frac{t+1}{2}\right) ;
$$

(ii) se $x=h_{M}(z, s) \in \overline{W_{M}} \backslash W_{M}$ e $f(x)=h_{N}(y, t)=\overline{W_{N}} \backslash W_{N}$, temos $s=t=1$, e, portanto,

$$
\mathcal{F}(x)=f(x)=h_{N}(y, t)=h_{N}\left(y, \frac{t+1}{2}\right)=h_{N}\left(y, \frac{s+t}{2}\right) .
$$


Daí, concluímos que as restrições $\left.\mathcal{F}\right|_{M \backslash W_{M}}$ e $\left.\mathcal{F}\right|_{\overline{W_{M}}}$ estão ambas bem definidas e são contínuas, e $\mathcal{F}$ encontra-se bem definida na intersecção $\left(M \backslash W_{M}\right) \cap \overline{W_{M}}=\overline{W_{M}} \backslash W_{M}$, o que mostra que $\mathcal{F}$ está bem definida e é contínua em $M$.

Note ainda que $\mathcal{F}(\partial M) \subseteq \partial N$, pois, se $x \in \partial M$, temos que $f(x) \in \partial N$ (lembre-se que a aplicação $f$ preserva fronteira), isto é, $x=h_{M}(x, 0) \in \overline{W_{M}}$ e $f(x)=h_{N}(f(x), 0) \in \overline{W_{N}}$, o que implica que

$$
\mathcal{F}(x)=h_{N}\left(f(x), \frac{0+0}{2}\right)=h_{N}(f(x), 0)=f(x),
$$

e, conseqüentemente, $\mathcal{F}(x) \in \partial N$. Em contrapartida, também temos que $\mathcal{F}(\operatorname{Int} M) \subseteq$ Int $N$ : com efeito, se $x \in M \backslash W_{M} \subseteq \operatorname{Int} M$, temos que $\mathcal{F}(x)=f(x)$ se $f(x) \in N \backslash W_{N} \subseteq \operatorname{Int} N$ (e, portanto, $\mathcal{F}(x) \in \operatorname{Int} N$ ), ou $\mathcal{F}(x)=h_{N}\left(y, \frac{t+1}{2}\right)$ se $f(x)=h_{N}(y, t) \in \overline{W_{N}}$ (o que também significa que $\mathcal{F}(x) \in \operatorname{Int} N$, pois $h_{N}\left(y, \frac{t+1}{2}\right) \in \partial N$ se, e somente se, $\left.\frac{t+1}{2}=0\right)$. Por outro lado, se $x \in \operatorname{Int} M$ é tal que $x=h_{M}(z, s) \in \overline{W_{M}}$, segue que $s>0$; daí, obtemos que:

(I) $\mathcal{F}(x)=f(x)$ se $f(x) \in N \backslash W_{N} \subseteq \operatorname{Int} N$ (e, portanto, $\left.\mathcal{F}(x) \in \operatorname{Int} N\right)$, ou

(II) $\mathcal{F}(x)=f(x)$ se $f(x)=h_{N}(y, t) \in \overline{W_{N}}$, com $t \geqslant s>0$ (o que implica que $h_{N}(y, t)=$ $f(x)=\mathcal{F}(x) \in \operatorname{Int} N$, posto que $h_{N}(y, t) \in \partial N$ se, e só se, $\left.t=0\right)$, ou

(III) $\mathcal{F}(x)=h_{N}\left(y, \frac{s+t}{2}\right)$ se $f(x)=h_{N}(y, t) \in \overline{W_{N}}, \operatorname{com} t \leqslant s(\mathrm{e}$, por conseguinte, $\mathcal{F}(x) \in \operatorname{Int} N$, uma vez que $h_{N}\left(y, \frac{s+t}{2}\right) \in \operatorname{Int} N$, pois $\left.s>0\right)$.

Assim, temos que $\mathcal{F}(\partial M) \subseteq \partial N$ e $\mathcal{F}(\operatorname{Int} M) \subseteq \operatorname{Int} N$, de modo que podemos denotar $\mathcal{F}: M \longrightarrow N$ por $\mathcal{F}:(M, \partial M, \operatorname{Int} M) \longrightarrow(N, \partial N, \operatorname{Int} N)$. Quanto às raízes de $\mathcal{F}$ em $c \in \operatorname{Int} N$, temos de imediato que $\mathcal{F}^{-1}(c)=f^{-1}(c)$, pois, como $f^{-1}(c) \subseteq M \backslash W_{M}$ e $c \in N \backslash W_{N}$, segue que $\mathcal{F}(x)=f(x)=c$ para todo $x \in M$ tal que $f(x)=c$.

Isto posto, vamos mostrar agora que $\mathcal{F}$ é uma aplicação própria. Considere então um subconjunto compacto $K \subseteq N$; como $N=\left(N \backslash W_{N}\right) \cup \overline{W_{N}}$, segue que $K=K^{\prime} \cup K^{\prime \prime}$, onde $K^{\prime}=\left(N \backslash W_{N}\right) \cap K$ e $K^{\prime \prime}=\overline{W_{N}} \cap K$. Ora, como $N \backslash W_{N}$ é fechado em $N$, temos que $K^{\prime} \subseteq N$ é compacto, pois constitui um subconjunto fechado de $N$, contido no compacto $K$. Entretanto, note que, para todo $x \in M$, temos $\mathcal{F}(x) \in K^{\prime} \subseteq N \backslash W_{N}$ se, e somente se, $f(x) \in N \backslash W_{N}$; logo, como $\mathcal{F}(x)=f(x)$ para todo $x \in M$ tal que $f(x) \in N \backslash W_{N}$, segue que:

$$
\mathcal{F}^{-1}\left(K^{\prime}\right)=\left\{x \in M: \mathcal{F}(x) \in K^{\prime}\right\}=\left\{x \in M: f(x) \in K^{\prime}\right\}=f^{-1}\left(K^{\prime}\right),
$$

o que implica que $\mathcal{F}^{-1}\left(K^{\prime}\right)$ é compacto (uma vez que $f$ é uma aplicação própria). Por outro lado, temos que $K^{\prime \prime}=\overline{W_{N}} \cap K$ também é compacto, pois é um subconjunto fechado de $K$; além disso, denotando por

$$
L_{1}=\left\{x \in M \backslash W_{M}: \mathcal{F}(x) \in K^{\prime \prime} \subseteq \overline{W_{N}}\right\}
$$


e

$$
L_{2}=\left\{x \in \overline{W_{M}}: \mathcal{F}(x) \in K^{\prime \prime} \subseteq \overline{W_{N}}\right\},
$$

segue que:

$$
\mathcal{F}^{-1}\left(K^{\prime \prime}\right)=\left\{x \in M: \mathcal{F}(x) \in K^{\prime \prime}\right\}=L_{1} \cup L_{2} \text {. }
$$

Observe então que, para todo $x \in M \backslash W_{M}$, temos $\mathcal{F}(x) \in K^{\prime \prime} \subseteq \overline{W_{N}}$ se, e somente se, $f(x) \in \overline{W_{N}}$, sendo que, neste caso, $\mathcal{F}(x)=h_{N}\left(y, \frac{t+1}{2}\right)$, onde $y \in \partial N$ e $t \in I$ são tais que $f(x)=h_{N}(y, t) \in \overline{W_{N}}$. Logo, denotando por $\phi: \partial N \times I \longrightarrow \partial N \times I$ o homeomorfismo dado por $\phi(y, t)=\left(y, \frac{t+1}{2}\right)$ para cada $(y, t) \in \partial N \times I$, segue que $\mathcal{F}(x)=\left(h_{N} \circ \phi \circ h_{N}^{-1} \circ f\right)(x)$ para todo $x \in M \backslash W_{M}$ tal que $f(x)=h_{N}(y, t) \in \overline{W_{N}}$; conseqüentemente, temos que:

$$
\begin{aligned}
L_{1} & =\left\{x \in M \backslash W_{M}: \mathcal{F}(x) \in K^{\prime \prime} \subseteq \overline{W_{N}}\right\} \\
& =\left\{x \in M \backslash W_{M}:\left(h_{N} \circ \phi \circ h_{N}^{-1} \circ f\right)(x) \in K^{\prime \prime}\right\} \\
& =\left(M \backslash W_{M}\right) \cap\left[\left(f^{-1} \circ h_{N} \circ \phi^{-1} \circ h_{N}^{-1}\right)\left(K^{\prime \prime}\right)\right] .
\end{aligned}
$$

No entanto, como $K^{\prime \prime} \subseteq \overline{W_{N}}$ é compacto, e as aplicações $h_{N}: \partial N \times I \longrightarrow \overline{W_{N}}$ e $\phi:$ $\partial \mathrm{N} \times I \longrightarrow \partial \mathrm{N} \times I$ são homeomorfismos, segue (juntamente com o fato de $f$ ser própria) que o conjunto $\left(f^{-1} \circ h_{N} \circ \phi^{-1} \circ h_{N}^{-1}\right)\left(K^{\prime \prime}\right)$ é compacto; portanto, como $L_{1}$ é um subconjunto fechado de $\left(f^{-1} \circ h_{N} \circ \phi^{-1} \circ h_{N}^{-1}\right)\left(K^{\prime \prime}\right)$, concluímos que $L_{1}$ é compacto. Finalmente, note que, para todo $x \in \overline{W_{M}}$, também temos $\mathcal{F}(x) \in K^{\prime \prime} \subseteq \overline{W_{N}}$ se, e somente se, $f(x) \in \overline{W_{N}}$, sendo que, neste caso,

$$
\mathcal{F}(x)=\left\{\begin{array}{cl}
f(x) & \text { se } f(x)=h_{N}(y, t) \in \overline{W_{N}}, \operatorname{com} t \geqslant s \\
h_{N}\left(y, \frac{s+t}{2}\right) & \text { se } f(x)=h_{N}(y, t) \in \overline{W_{N}}, \operatorname{com} t \leqslant s
\end{array},\right.
$$

onde $s \in I$ é tal que $x=h_{M}(z, s) \in \overline{W_{M}}$, para algum $z \in \partial M$. Logo, temos que:

$$
L_{2}=\left\{x \in \overline{W_{M}}: \mathcal{F}(x) \in K^{\prime \prime} \subseteq \overline{W_{N}}\right\}=L_{2}^{\prime} \cup L_{2}^{\prime \prime},
$$

onde

$$
L_{2}^{\prime}=\left\{x=h_{M}(z, s) \in \overline{W_{M}}: f(x)=h_{N}(y, t) \in K^{\prime \prime} \text { e } t \geqslant s\right\}
$$

e

$$
L_{2}^{\prime \prime}=\left\{x=h_{M}(z, s) \in \overline{W_{M}}: f(x)=h_{N}(y, t) \in \overline{W_{N}}, h_{n}\left(y, \frac{s+t}{2}\right) \in K^{\prime \prime} \text { e } t \leqslant s\right\} .
$$

Entretanto, denotando por $\pi_{M}: \partial M \times I \longrightarrow I$ e $\pi_{N}: \partial N \times I \longrightarrow I$ as projeções dadas por $\pi_{M}(z, s)=s$ e $\pi_{N}(y, t)=t$ para cada $(z, s) \in \partial M \times I$ e cada $(y, t) \in \partial N \times I$, temos que:

$$
L_{2}^{\prime}=f^{-1}\left(K^{\prime \prime}\right) \cap\left\{x \in \overline{W_{M}}:\left(\pi_{N} \circ h_{N}^{-1}\right)(f(x)) \geqslant\left(\pi_{M} \circ h_{M}^{-1}\right)(x)\right\} .
$$

Assim, como $f^{-1}\left(K^{\prime \prime}\right)$ é compacto (pois $K^{\prime \prime}$ é compacto e $f$ é uma aplicação própria), e como o conjunto

$$
\left\{x \in \overline{W_{M}}:\left(\pi_{N} \circ h_{N}^{-1}\right)(f(x)) \geqslant\left(\pi_{M} \circ h_{M}^{-1}\right)(x)\right\}
$$


é fechado, segue que $L_{2}^{\prime}$ é um subconjunto compacto de $\overline{W_{M}}$. Por outro lado, sendo $\psi_{1}: \partial M \times I \longrightarrow \partial N$ e $\psi_{2}: \partial M \times I \longrightarrow I$ as aplicações contínuas dadas por

$$
\psi_{1}(z, s)=\left(p_{N} \circ h_{N}^{-1} \circ f \circ h_{M}\right)(z, s)
$$

e

$$
\psi_{2}(z, s)=\frac{s+\left(\pi_{N} \circ h_{N}^{-1} \circ f \circ h_{M}\right)(z, s)}{2}
$$

para cada $(z, s) \in \partial M \times I$, onde $p_{N}: \partial N \times I \longrightarrow \partial N$ é a projeção de $\partial N \times I$ sobre $\partial N$ (definida por $p_{N}(y, t)=y$ para cada $\left.(y, t) \in \partial N \times I\right)$, considere a aplicação contínua $\psi=\left(\psi_{1}, \psi_{2}\right): \partial M \times I \longrightarrow \partial N \times I$; se $x \in \overline{W_{M}}$ é tal que $x=h_{M}(z, s)$ e $f(x)=h_{N}(y, t) \in \overline{W_{N}}$, temos que:

$$
\begin{aligned}
\left(h_{N} \circ \psi \circ h_{M}^{-1}\right)(x) & =\left(h_{N} \circ \psi\right)(z, s) \\
& =h_{N}\left(\psi_{1}(z, s), \psi_{2}(z, s)\right) \\
& =h_{n}\left(\left(p_{N} \circ h_{N}^{-1} \circ f \circ h_{M}\right)(z, s), \frac{s+\left(\pi_{N} \circ h_{N}^{-1} \circ f \circ h_{M}\right)(z, s)}{2}\right) \\
& =h_{n}\left(\left(p_{N} \circ h_{N}^{-1} \circ f\right)(x), \frac{s+\left(\pi_{N} \circ h_{N}^{-1} \circ f\right)(x)}{2}\right) \\
& =h_{n}\left(\left(p_{N} \circ h_{N}^{-1}\right)\left(h_{N}(y, t)\right), \frac{s+\left(\pi_{N} \circ h_{N}^{-1}\right)\left(h_{N}(y, t)\right)}{2}\right) \\
& =h_{n}\left(p_{N}(y, t), \frac{s+\pi_{N}(y, t)}{2}\right) \\
& =h_{n}\left(y, \frac{s+t}{2}\right) .
\end{aligned}
$$

Além disso, note que $\psi: \partial M \times I \longrightarrow \partial N \times I$ é uma aplicação própria: com efeito, se $C \subseteq \partial N \times I$ é compacto, temos que $C=C_{1} \times C_{2}$, onde $C_{1} \subseteq \partial N$ e $C_{2} \subseteq I$ são compactos, e, portanto,

$$
\begin{aligned}
\psi^{-1}(C) & =\{(z, s) \in \partial M \times I: \psi(z, s) \in C\} \\
& =\left\{(z, s) \in \partial M \times I:\left(\psi_{1}(z, s), \psi_{2}(z, s)\right) \in C_{1} \times C_{2}\right\} \\
& =\left\{(z, s) \in \partial M \times I: \psi_{1}(z, s) \in C_{1} \text { e } \psi_{2}(z, s) \in C_{2}\right\} \\
& =\psi_{1}^{-1}\left(C_{1}\right) \cap \psi_{2}^{-1}\left(C_{2}\right)
\end{aligned}
$$

$\operatorname{logo}$, como $\psi_{1}: \partial M \times I \longrightarrow \partial N$ é uma aplicação própria (pois $p_{N}, h_{N}^{-1}, f$ e $h_{M}$ são próprias, e $\psi_{1}=p_{N} \circ h_{N}^{-1} \circ f \circ h_{M}$ ), segue que $\psi_{1}^{-1}\left(C_{1}\right)$ é compacto, o que (pelo fato de $\psi_{2}^{-1}\left(C_{2}\right)$ ser um subconjunto fechado de $\partial M \times I)$ implica que $\psi^{-1}(C)=\psi_{1}^{-1}\left(C_{1}\right) \cap \psi_{2}^{-1}\left(C_{2}\right)$ é compacto. 
Deste modo, como:

$$
\begin{aligned}
L_{2}^{\prime \prime}= & \left\{x=h_{M}(z, s) \in \overline{W_{M}}: f(x)=h_{N}(y, t) \in \overline{W_{N}}, h_{N}\left(y, \frac{s+t}{2}\right) \in K^{\prime \prime} \mathrm{e} t \leqslant s\right\} \\
= & \left\{x \in \overline{W_{M}}: f(x) \in \overline{W_{N}},\left(h_{N} \circ \psi \circ h_{M}^{-1}\right)(x) \in K^{\prime \prime} \mathrm{e}\left(\pi_{N} \circ h_{N}^{-1}\right)(f(x)) \leqslant\right. \\
& \left.\left(\pi_{M} \circ h_{M}^{-1}\right)(x)\right\} \\
= & f^{-1}\left(\overline{W_{N}}\right) \cap\left(h_{N} \circ \psi \circ h_{M}^{-1}\right)^{-1}\left(K^{\prime \prime}\right) \cap \\
& \left\{x \in \overline{W_{M}}:\left(\pi_{N} \circ h_{N}^{-1}\right)(f(x)) \leqslant\left(\pi_{M} \circ h_{M}^{-1}\right)(x)\right\},
\end{aligned}
$$

segue que $L_{2}^{\prime \prime}$ é compacto, uma vez que $\left(h_{N} \circ \psi \circ h_{M}^{-1}\right)^{-1}\left(K^{\prime \prime}\right)$ é compacto (pois $h_{N} \circ \psi \circ h_{M}^{-1}$ é própria e $K^{\prime \prime}$ é compacto), e $f^{-1}\left(\overline{W_{N}}\right)$ e $\left\{x \in \overline{W_{M}}:\left(\pi_{N} \circ h_{N}^{-1}\right)(f(x)) \leqslant\left(\pi_{M} \circ h_{M}^{-1}\right)(x)\right\}$ são fechados. Resumindo, temos então que os conjuntos $\mathcal{F}^{-1}\left(K^{\prime}\right), L_{1}, L_{2}$ e $L_{2}^{\prime \prime}$ são compactos, e, conseqüentemente, $\mathcal{F}^{-1}(K)$ também o é, pois:

$$
\begin{aligned}
\mathcal{F}^{-1}(K) & =\mathcal{F}^{-1}\left(K^{\prime} \cup K^{\prime \prime}\right)=\mathcal{F}^{-1}\left(K^{\prime}\right) \cup \mathcal{F}^{-1}\left(K^{\prime \prime}\right)=\mathcal{F}^{-1}\left(K^{\prime}\right) \cup\left(L_{1} \cup L_{2}\right) \\
& =\mathcal{F}^{-1}\left(K^{\prime}\right) \cup\left[L_{1} \cup\left(L_{2}^{\prime} \cup L_{2}^{\prime \prime}\right)\right] .
\end{aligned}
$$

Assim, concluímos que $\mathcal{F}^{-1}(K)$ é compacto para todo subconjunto compacto $K \subseteq N$, o que prova que $\mathcal{F}:(M, \partial M, \operatorname{Int} M) \longrightarrow(N, \partial N, \operatorname{Int} N)$ é uma aplicação própria.

Agora, considere a aplicação $H: M \times I \longrightarrow N$, definida do seguinte modo: se $x \in M \backslash W_{M}$, tomamos

$$
H(x, r)=\left\{\begin{array}{cl}
f(x) & \text { se } f(x) \in N \backslash W_{N}(\forall r \in I) \\
h_{N}\left(y,(1-r) t+r\left(\frac{t+1}{2}\right)\right) & \text { se } f(x)=h_{N}(y, t) \in \overline{W_{N}}
\end{array} ;\right.
$$

por outro lado, se $x=h_{M}(z, s) \in \overline{W_{M}}$, consideramos

$$
H(x, r)=\left\{\begin{array}{cl}
f(x) & \text { se } f(x) \in N \backslash W_{N}(\forall r \in I) \\
f(x) & \text { se } f(x)=h_{N}(y, t) \in \overline{W_{N}}, t \geqslant s(\forall r \in I) \\
h_{N}\left(y,(1-r) t+r\left(\frac{s+t}{2}\right)\right) & \text { se } f(x)=h_{N}(y, t) \in \overline{W_{N}}, t \leqslant s
\end{array} .\right.
$$

De maneira análoga ao que fizemos para a aplicação $\mathcal{F}$, pode-se verificar que $H$ está bem definida e é contínua em $M \times I$; ademais, não é difícil ver também que $H(\partial M \times I) \subseteq \partial N$ (uma vez que, para todo $x \in \partial M$, temos $x=h_{M}(x, 0)$ e $f(x)=h_{N}(f(x), 0) \in \partial N$ ). Logo, como $H(x, 0)=f(x)$ e $H(x, 1)=\mathcal{F}(x)$ para todo $x \in M$, segue que $H:(M \times I, \partial M \times$ $I) \longrightarrow(N, \partial N)$ constitui uma homotopia entre $f$ e $\mathcal{F}$. Portanto, a fim de encerrarmos a demonstração do teorema, basta verificarmos que $H$ é uma aplicação própria.

Considere, pois, as aplicações contínuas $\alpha: f^{-1}\left(\overline{W_{N}}\right) \times I \longrightarrow I$ e $\beta:\left[\overline{W_{M}} \cap f^{-1}\left(\overline{W_{N}}\right)\right] \times$ $I \longrightarrow I$, dadas por:

$$
\alpha\left(x_{1}, r\right)=(1-r) \cdot\left(\pi_{N} \circ h_{N}^{-1} \circ f\right)\left(x_{1}\right)+r \cdot \frac{\left(\pi_{N} \circ h_{N}^{-1} \circ f\right)\left(x_{1}\right)+1}{2}
$$


e

$$
\beta\left(x_{2}, r\right)=(1-r) \cdot\left(\pi_{N} \circ h_{N}^{-1} \circ f\right)\left(x_{2}\right)+r \cdot \frac{\left(\pi_{M} \circ h_{M}^{-1}\right)\left(x_{2}\right)+\left(\pi_{N} \circ h_{N}^{-1} \circ f\right)\left(x_{2}\right)}{2}
$$

para cada $\left(x_{1}, r\right) \in f^{-1}\left(\overline{W_{N}}\right) \times I$ e cada $\left(x_{2}, r\right) \in\left[\overline{W_{M}} \cap f^{-1}\left(\overline{W_{N}}\right)\right] \times I$ (onde $\pi_{M}, \pi_{N}$ e $p_{N}$ são as projeções mencionadas anteriormente), e sejam $\gamma: f^{-1}\left(\overline{W_{N}}\right) \times I \longrightarrow \partial N \times I$ e $\delta:\left[\overline{W_{M}} \cap f^{-1}\left(\overline{W_{N}}\right)\right] \times I \longrightarrow \partial N \times I$ as aplicações contínuas definidas por:

$$
\gamma\left(x_{1}, r\right)=\left(\left(p_{N} \circ h_{N}^{-1} \circ f\right)\left(x_{1}\right), \alpha\left(x_{1}, r\right)\right) \quad \text { e } \quad \delta\left(x_{2}, r\right)=\left(\left(p_{N} \circ h_{N}^{-1} \circ f\right)\left(x_{2}\right), \alpha\left(x_{2}, r\right)\right)
$$

para cada $\left(x_{1}, r\right) \in f^{-1}\left(\overline{W_{N}}\right) \times I$ e cada $\left(x_{2}, r\right) \in\left[\overline{W_{M}} \cap f^{-1}\left(\overline{W_{N}}\right)\right] \times I$. Dado $C=C_{1} \times C_{2} \subseteq \partial N \times I$ compacto, com $C_{1} \subseteq \partial N$ e $C_{2} \subseteq I$ compactos, temos que:

$$
\begin{aligned}
\gamma^{-1}(C) & =\left\{\left(x_{1}, r\right) \in f^{-1}\left(\overline{W_{N}}\right) \times I: \gamma\left(x_{1}, r\right) \in C=C_{1} \times C_{2}\right\} \\
& =\left\{\left(x_{1}, r\right) \in f^{-1}\left(\overline{W_{N}}\right) \times I:\left(p_{N} \circ h_{N}^{-1} \circ f\right)\left(x_{1}\right) \in C_{1} \text { e } \alpha\left(x_{1}, r\right) \in C_{2}\right\} \\
& =\left(p_{N} \circ h_{N}^{-1} \circ f\right)^{-1}\left(C_{1}\right) \cap \alpha^{-1}\left(C_{2}\right)
\end{aligned}
$$

e, analogamente, $\delta^{-1}(C)=\left(p_{N} \circ h_{N}^{-1} \circ f\right)^{-1}\left(C_{1}\right) \cap \beta^{-1}\left(C_{2}\right)$; logo, como $\left(p_{N} \circ h_{N}^{-1} \circ f\right)^{-1}\left(C_{1}\right)$ é compacto (uma vez que $p_{N}, h_{N}^{-1}$ e $f$ são aplicações próprias), e $\alpha^{-1}\left(C_{2}\right)$ e $\beta^{-1}\left(C_{2}\right)$ são fechados, segue que $\gamma^{-1}(C)$ e $\delta^{-1}(C)$ são compactos, o que implica que $\gamma$ e $\delta$ são ambas aplicações próprias. Além disso, se $x=h_{M}(z, s) \in \overline{W_{M}}$ é tal que $f(x)=h_{N}(y, t) \in \overline{W_{N}}$, temos que:

$$
\begin{aligned}
\left(h_{N} \circ \gamma\right)(x, r) & =h_{N}\left(\left(p_{N} \circ h_{N}^{-1} \circ f\right)(x), \alpha(x, r)\right) \\
& =h_{N}\left(\left(p_{N} \circ h_{N}^{-1} \circ f\right)(x),(1-r) \cdot\left(\pi_{N} \circ h_{N}^{-1} \circ f\right)(x)+r \frac{\left(\pi_{N} \circ h_{N}^{-1} \circ f\right)(x)+1}{2}\right) \\
& =h_{N}\left(p_{N}(y, t),(1-r) \cdot \pi_{N}(y, t)+r \frac{\pi_{N}(y, t)+1}{2}\right) \\
& =h_{N}\left(y,(1-r) t+r \frac{t+1}{2}\right)
\end{aligned}
$$

e

$$
\begin{aligned}
\left(h_{N} \circ \delta\right)(x, r)= & h_{N}\left(\left(p_{N} \circ h_{N}^{-1} \circ f\right)(x), \beta(x, r)\right) \\
= & h_{N}\left(\left(p_{N} \circ h_{N}^{-1} \circ f\right)(x),(1-r) \cdot\left(\pi_{N} \circ h_{N}^{-1} \circ f\right)(x)+\right. \\
& \left.r \cdot \frac{\left(\pi_{M} \circ h_{M}^{-1}\right)(x)+\left(\pi_{N} \circ h_{N}^{-1} \circ f\right)(x)}{2}\right) \\
= & h_{N}\left(p_{N}(y, t),(1-r) \cdot \pi_{N}(y, t)+r \frac{\pi_{M}(z, s)+\pi_{N}(y, t)}{2}\right) \\
= & h_{N}\left(y,(1-r) t+r \frac{s+t}{2}\right),
\end{aligned}
$$


para todo $r \in I$. Assim, se $x \in M \backslash W_{M}$, segue que:

$$
H(x, r)=\left\{\begin{array}{cl}
f(x) & \text { se } f(x) \in N \backslash W_{N}(\forall r \in I) \\
\left(h_{N} \circ \gamma\right)(x, r) & \text { se } f(x) \in \overline{W_{N}}
\end{array},\right.
$$

enquanto que, se $x=h_{M}(z, s) \in \overline{W_{M}}$, temos:

$$
H(x, r)=\left\{\begin{array}{cl}
f(x) & \text { se } f(x) \in N \backslash W_{N}(\forall r \in I) \\
f(x) & \text { se } \left.f(x)-h_{N}(y, t) \in \overline{W_{N}}, \operatorname{com} t \geqslant s \quad \forall r \in I\right) \quad . \\
\left(h_{N} \circ \delta\right)(x, r) & \text { se } f(x)=h_{N}(y, t) \in \overline{W_{N}}, \operatorname{com} t \leqslant s
\end{array} .\right.
$$

Isto posto, dado um subconjunto compacto $K \subseteq N$ arbitrário, considere novamente $K^{\prime}=\left(N \backslash W_{N}\right) \cap K$ e $K^{\prime \prime}=\overline{W_{N}} \cap K$ (de modo que $\left.K=K^{\prime} \cup K^{\prime \prime}\right)$; temos então que $K^{\prime}$ é um subconjunto compacto de $N \backslash W_{N}$, e, portanto (como $H(x, r)=f(x)$ para todo $x \in M$ tal que $\left.f(x) \in N \backslash W_{N} \supseteq K^{\prime \prime}\right), H^{-1}\left(K^{\prime}\right)$ é compacto. Por outro lado, temos que $K^{\prime \prime} \subseteq \overline{W_{N}}$ também é compacto, e $H^{-1}\left(K^{\prime \prime}\right)=F_{1} \cup F_{2}$, onde

$$
F_{1}=\left\{(x, r) \in\left(M \backslash W_{M}\right) \times I: H(x, r) \in K^{\prime \prime}\right\}
$$

$\mathrm{e}$

$$
F_{2}=\left\{(x, r) \in \overline{W_{M}} \times I: H(x, r) \in K^{\prime \prime}\right\} ;
$$

no entanto, como $H(x, r)=\left(h_{N} \circ \gamma\right)(x, r)$ para todo $r \in I$ e todo $x \in M \backslash W_{M}$ tal que $f(x) \in \overline{W_{N}} \supseteq K^{\prime \prime}$, segue que $F_{1}=\left[\left(M \backslash W_{M}\right) \times I\right] \cap\left(h_{N} \circ \gamma\right)^{-1}\left(K^{\prime \prime}\right)$, o que implica que $F_{1}$ é compacto (pois, como $h_{N}$ e $\gamma$ são aplicações próprias, o conjunto $\left(h_{N} \circ \gamma\right)^{-1}\left(K^{\prime \prime}\right)$ é compacto). Enfim, note que $F_{2}=F_{2}^{\prime} \cup F_{2}^{\prime \prime}$, onde

$$
\begin{aligned}
F_{2}^{\prime} & =\left\{(x, r) \in \overline{W_{M}} \times I: H(x, r) \in K^{\prime \prime} \text { e }\left(\pi_{N} \circ h_{N}^{-1}\right)(f(x)) \geqslant\left(\pi_{M} \circ h_{M}^{-1}\right)(x)\right\} \\
& =\left\{(x, r) \in \overline{W_{M}} \times I: f(x) \in K^{\prime \prime} \text { e }\left(\pi_{N} \circ h_{N}^{-1}\right)(f(x)) \geqslant\left(\pi_{M} \circ h_{M}^{-1}\right)(x)\right\} \\
& =\left[f^{-1}\left(K^{\prime \prime}\right) \times I\right] \cap\left[\left\{x \in \overline{W_{M}}:\left(\pi_{N} \circ h_{N}^{-1}\right)(f(x)) \geqslant\left(\pi_{M} \circ h_{M}^{-1}\right)(x)\right\} \times I\right]
\end{aligned}
$$

e

$$
\begin{aligned}
F_{2}^{\prime \prime} & =\left\{(x, r) \in \overline{W_{M}} \times I: H(x, r) \in K^{\prime \prime} \mathrm{e}\left(\pi_{N} \circ h_{N}^{-1}\right)(f(x)) \leqslant\left(\pi_{M} \circ h_{M}^{-1}\right)(x)\right\} \\
& =\left\{(x, r) \in \overline{W_{M}} \times I:\left(h_{N} \circ \delta\right)(x, r) \in K^{\prime \prime} \mathrm{e}\left(\pi_{N} \circ h_{N}^{-1}\right)(f(x)) \leqslant\left(\pi_{M} \circ h_{M}^{-1}\right)(x)\right\} \\
& =\left(h_{N} \circ \delta\right)^{-1}\left(K^{\prime \prime}\right) \cap\left[\left\{x \in \overline{W_{M}}:\left(\pi_{N} \circ h_{N}^{-1}\right)(f(x)) \leqslant\left(\pi_{M} \circ h_{M}^{-1}\right)(x)\right\} \times I\right] ;
\end{aligned}
$$

logo, como os conjuntos

$$
\left\{x \in \overline{W_{M}}:\left(\pi_{N} \circ h_{N}^{-1}\right)(f(x)) \geqslant\left(\pi_{M} \circ h_{M}^{-1}\right)(x)\right\} \times I
$$

$\mathrm{e}$

$$
\left\{x \in \overline{W_{M}}:\left(\pi_{N} \circ h_{N}^{-1}\right)(f(x)) \leqslant\left(\pi_{M} \circ h_{M}^{-1}\right)(x)\right\} \times I
$$


são fechados, e $f^{-1}\left(K^{\prime \prime}\right) \times I$ e $\left(h_{N} \circ \delta\right)^{-1}\left(K^{\prime \prime}\right)$ são compactos (pois as aplicações $f, h_{N}$ e $\delta$ são próprias), segue que $F_{2}^{\prime}$ e $F_{2}^{\prime \prime}$ são compactos, e, conseqüentemente, $F_{2}$ também o é. Deste modo, temos que o conjunto

$$
H^{-1}(K)=H^{-1}\left(K^{\prime}\right) \cup H^{-1}\left(K^{\prime \prime}\right)=H^{-1}\left(K^{\prime}\right) \cup F_{1} \cup\left(F_{2}^{\prime} \cup F_{2}^{\prime \prime}\right)
$$

é compacto, o que mostra que a homotopia $H:(M \times I, \partial M \times I) \longrightarrow(N, \partial N)$ é própria, concluindo a demonstração. 


\section{Referências Bibliográficas}

[Bd] G. E. Bredon, Topology and Geometry, Springer-Verlag, New York (1993).

[Bk1] R. B. S. Brooks, On the sharpness of $\Delta_{2}$ and $\Delta_{1}$ Nielsen numbers, J. Reine Angew. Math., vol. 259 (1973), 101-108.

[Bk2] R. B. S. Brooks, Roots of Mappings from Manifolds, Fixed Point Theory and Applications, vol. 2004, 273-307.

[Bk3] R. B. S. Brooks, Nielsen Root Theory, in Handbook of Topological Fixed Point Theory (L. Gorniewicz, ed.), Springer-Verlag, New York (2005).

[BB] R. B. S. Brooks and R. F. Brown, A lower bound for the $\Delta$-Nielsen number, Trans. Amer. Math. Soc., vol. 143 (1969), 555-564.

[BBS] R. B. S. Brooks, R. F. Brown and H. Schirmer, The Absolute Degree and the Nielsen Root Number of Compositions and Cartesian Products of Maps, Topology and its Applications, vol. 116 (2001), 5-27.

[BO] R. B. S. Brooks and Charles Odenthal, Nielsen Numbers for Roots of Maps of Aspherical Manifolds, Pacific Journal of Mathematics, vol. 170 (1995), 405-420.

[Bw] L. E. J. Brouwer, Über Abbildung von Mannigfaltigkeiten, Math. Ann., vol. 71 (1911), 97-115.

[Bwn1] M. Brown, Locally flat imbeddings of topological manifolds, Annals of Math., vol. 75 (1962), 331-341.

[Bwn2] M. Brown, A mapping theorem for untriangulated manifolds, em Topology of 3Manifolds, 92-94, M. K. Fort (editor), Prentice-Hall, Englewood Cliffs, New Jersey (1962).

[BS1] R. F. Brown and H. Schirmer, Nielsen root theory and Hopf degree theory, Pacific Journal of Mathematics, vol. 198 (2001), 49-80. 
[BS2] R. F. Brown and H. Schirmer, Nielsen Coincidence Theory and Coincidence-Producing Maps for Manifolds with Boundary, Topology and its Applications, vol. 46 (1992), 65-79.

[BS3] R. F. Brown and H. Schirmer, Correction to "Nielsen Coincidence Theory and Coincidence-Producing Maps for Manifolds with Boundary", Topology and its Applications, vol. 67 (1995), 233-234.

[BS4] R. F. Brown and H. Schirmer, Nielsen Theory of Roots of Maps of Pairs, Topology and its Applications, vol. 92 (1999), 247-274.

[D] A. Dold, Lectures on Algebraic Topology, Springer-Verlag, Berlim (1972).

[DH] P. H. Doyle and J. G. Hocking, A decomposition theorem for n-dimensional manifolds, Proceedings of American Math. Soc., vol. 13 (1962), 469-471.

[DJ] R. Dobrenko and J. Jezierski, The Coincidence Nielsen Number on Non-Orientable Manifolds, Rocky Mountain Journal of Mathematics, vol. 23, number 1 (1993), 67-85.

[E] D. B. A. Epstein, The degree of a map, Proc. London Math. Soc., vol. 16 (1966), 369-383.

[F] M. H. Freedman, The Topology of Four-Dimensional Manifolds, Journal of Differential Geometry, vol. 17 (1982), 357-453.

[GJ] D. L. Gonçalves and J. Jezierski, Lefschetz Coincidence Formula on Non-orientable Manifolds, Fundamenta Mathematicae, vol. 153 (1997), 1-23.

[GZ1] D. L. Gonçalves and H. Zieschang, Equations in free groups and coincidences of mappings on surfaces, Journal Mathematische Zeitschrift, vol. 237-1 (2001), 1-29.

[GZ2] D. L. Gonçalves and H. Zieschang, Equations in free groups and coincidences of mappings on surfaces II, Fakultät für Mathematik der Ruhr-Universität Bochum, preprint 240 (1998).

[H1] H. Hopf, Zur Topologie der Abbildungen von Mannigfaltigkeiten, Erster Teil, Math. Ann., vol. 100 (1928), 579-608.

[H2] H. Hopf, Zur Topologie der Abbildungen von Mannigfaltigkeiten, Zweiter Teil, Math. Ann., vol. 102 (1930), 562-623.

[Je] J. Jezierski, The Nielsen Coincidence Theory on Topological Manifolds, Fundamenta Mathematicae, vol. 143 (1993), 167-178. 
[Ji1] B. Jiang, Lectures on Nielsen Fixed Point Theory, Contemporary Mathematics (AMS), vol. 14 (1983).

[Ji2] B. Jiang, Fixed Point Classes from a Differentiable Viewpoint, Fixed Point Theory (Proceedings, Sherbrook, Quebéc, 1980), Lecture Notes in Mathematics, vol. 886, Springer-Verlag, Berlin, (1981), 163-170.

[Kn1] H. Kneser, Glättung von Flächenabbildungen, Math. Ann., vol. 100 (1928), 609-616.

[Kn2] H. Kneser, Die kleinste Bedeckungszahl innerhalb einer Klasse von Flächenabbildungen, Math. Ann., vol. 103 (1930), 347-358.

[Ki] T. Kiang, The Theory of Fixed Point Classes, Springer-Verlag e Science Press, Berlim e Beijing (1989).

[KS] R. Kirby and L. Siebenmann, Foundational Essays on Topological Manifolds, Annals of Mathematics Studies, Study 88, Princeton University Press (1977).

[Le] J. M. Lee, Introduction to Topological Manifolds, Springer-Verlag, New York (2000).

[Li] E. L. Lima, Grupo Fundamental e Espaços de Recobrimento, Projeto Euclides, IMPA (1998).

[L] X. Lin, On the Root Classes of Mapping, Acta Mathematica Sinica, New Series, vol. 2, $N^{\circ} 1$ (1986), 199-206.

[Ma1] W. S. Massey, Algebraic Topology: An Introduction, Springer-Verlag, New York (1977).

[Ma2] W. S. Massey, A Basic Course in Algebraic Topology, Springer-Verlag, New York (1991).

[Ma3] W. S. Massey, Homology and Cohomology Theory: An Approach Based on AlexanderSpanier Cochains, Marcel Dekker, Inc., New York (1978).

[Mi] J. Milnor, Microbundles, Part I, Topology, vol. 3 (1964), Supplement 1, 53-80.

[Mu] J. R. Munkres, Elements of Algebraic Topology, Perseus Books (1984).

[N1] J. Nielsen, Untersuchungen zur Topologie der geschlossene zweiseitigen Fläche, Acta Mathematica, vol. 50 (1927), 189-358.

[N2] J. Nielsen, Untersuchungen zur Topologie der geschlossene zweiseitigen Fläche, II, Acta Mathematica, vol. 53 (1929), 1-76. 
[O] P. Olum, Mappings of manifolds and the notion of degree, Annals of Math., vol. 58 (1953), 458-480.

[Q] F. Quinn, Ends of Maps III: Dimensions 4 and 5, Journal of Differential Geometry, vol. 17 (1982), 503-521.

[R] J. J. Rotman, An Introduction to Algebraic Topology, Springer-Verlag, New York (1988).

[RW] Y. Rong and S. Wang, The preimages of submanifolds, Mathematical Proceedings of Cambridge Philosophical Society, vol. 112 (1992), 271-279.

[Sch] M. G. Scharlemann, Transversality Theories in Dimension Four, Inventiones Mathematicae, vol. 33 (1976), 1-14.

[Sk] R. Skora, The degree of a map between surfaces, Math. Ann., vol. 276 (1987), 415-423.

[Sp] E. H. Spanier, Algebraic Topology, Springer-Verlag, New York (1966).

[Ve] D. Vendrúsculo, Coincidence Classes in Nonorientable Manifolds, Fixed Point Theory and Applications, vol. 2006 (Special Issue: Nielsen Theory and Related Topics), $1-9$.

[Vi] J. Vick, Homology Theory: An Introduction to Algebraic Topology, Second Edition, Springer-Verlag, New York (1994).

[X] L. Xiaosong, On the Root Classes of Mappings, Acta Math. Sinica, New Series, vol. 2 (1986), 199-206. 